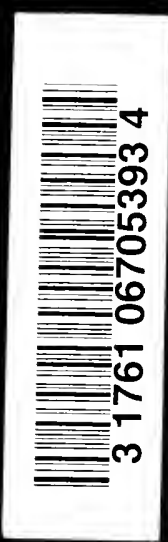




Digitized by the Internet Archive in 2007 with funding from Microsoft Corporation 


\title{
ABDOMINAL SURGERY
}

\section{CLINICAL LECTURES}

FOR STUDENTS AND PHYSICIANS

\author{
BY \\ THORKILD ROVSING \\ PROFESSOR OF CLINICAL SURGERY AT THE UNIVERSITY OF COPENHAGEN
}

EDITED BY

PAUL MONROE PILCHER, A.M., M.D. BROOKLYN, NEW YORK

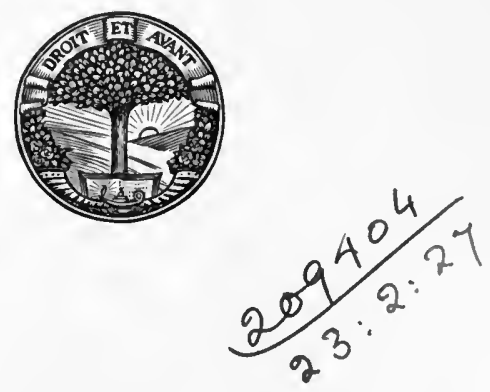

PHILADELPHIA \& LONDON J. B. LIPPINCOTT COMPANY 


\section{Copyright, I9I 4}

BY J. B. Lippincott CoMpany

Electrotyped and Printed by J. B. Lippincott Company The Washington Square Press, Philadelphia, U.S.A. 


\section{AUTHOR'S PREFACE}

Formerly many medical subjects were presented in textbooks in the form of a series of clinical lectures, but this method is less frequently seen to-day for the reason that it is necessary to crowd into compactly written text-books an ever-increasing and overwhelming amount of material which has accumulated. It would, of course, be impossible to present all of the various phases of surgery to the student by means of clinical lectures, as work of this character would prove too voluminous. It seems to me, however, of the greatest importance that the clinical lectures should not entirely disappear from medical text-books.

The clinical lecture unquestionably offers certain advantages and affords a point of view which cannot be found in the systematic compact text-book. It is of advantage to the student because in the clinical lecture we have the personal element of the teacher considering a given case, and the reader lives through and works out further with the author every step in the diagnosis of a specific case and considers with him the therapeutic questions and difficulties.

Particularly when one enters upon debatable ground, especially those conditions which lie on the border line between medicine and surgery, then the study of a living individual patient is far more illuminating and convincing than the dry postulate. For this reason I have presented these questions of diseases of the stomach, which are so much debated by medical men and surgeons, in this book.

The book presents in many respects observations which 
have not yet been brought out in other countries, and also presents methods of examination and treatment which are original and which, perhaps, my foreign colleagues will not accept without question. It is for this reason that $I$ have consented to having this work translated to present to the English-speaking nations. For many of the illustrations I wish to thank the artist, Mogens Gad, and my associate, Dr. Johs Ipsen.

Thorkild Rovsing.

Copenhagen, 1914. 


\section{EDITOR'S PREFACE}

Postgraduate work has been brought to the physician and surgeon in his own office through the published reports of many clinics, especially in this country. It is becoming the custom of some of the great teachers whose elinics are attended by many physicians and surgeons seeking postgraduate work to publish their talks on the studies of individual cases in such a way that they may become available to those less fortunate physicians who cannot attend their clinics. The collected papers of the various clinics and the published elinical lectures of some of our great teachers in this country are a valuable addition to our methods of postgraduate work.

From east to west in this country and in Europe the great question of surgical conditions of the stomach and allied conditions of the esophagus and intestines has occupied the attention of many different schools. There are in England a number of distinet schools ; in Germany others, in many of the other foreign cities still different teachers, all of whom have achieved success in the various surgical diseases of the alimentary tract in their own peculiar way.

We are especially fortunate now in being able to present to the English-speaking public a translation of the works of one of Europe's pioneers in this branch of surgery. His work is unique and individual, his clinical material extensive, and his methods of presenting a subject logical, clear and interesting. He has omitted in his present series of clinical lectures all of the elementary phases of his subject 
and presents in his interesting fashion his own ideas and the results of his work.

In editing this work the original ideas of the author have been preserved sentence for sentence in order that the work may lose none of its individuality.

Paul Monroe Pilcher.

BRookLYN, N. Y., 1914. 


\section{CONTENTS}

LECTURE I

The Basis and Principles of Chinical Subgery ........... 1

LECTURE II

Antiseptic Metiods-Part I: Prominent features in the De-

velopment of the History of Antiseptic Metirods ......... 21

LECTURE III

Antiseptic Methods-Part II: Present Antiseptic Methods

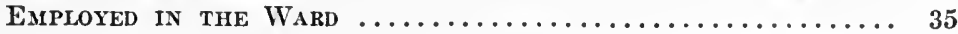

LECTURE IV

Andesthetics: General Axesthesia $\ldots \ldots \ldots \ldots \ldots \ldots \ldots \ldots . \ldots 2$

LECTURE $\mathrm{V}$

Andesthetics (Continued) : Local Anæesthesia ............. 96

LECTURE VI

Foreign Substances Contained in the Esophagus, the Stomach

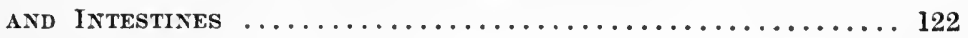

LECTURE VII

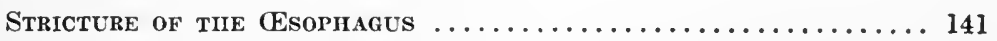

LECTURE VIII

Dilatation of the Esophagus ................... 159

LECTURE IX

The Diagnosis of Diseases of the Stomach ............ 183

LECTURE $\mathrm{X}$

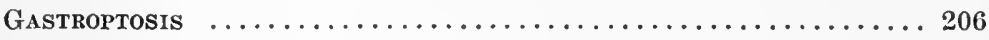

LECTURE XI

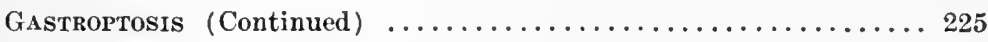

LECTURE XII

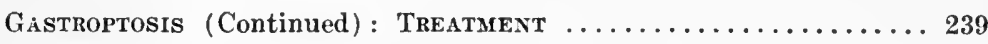

LECTURE XIII

Chronic Ulcer of the Stomach.................... 269

I.ECTURE XIV

Tire Diagnosis of Ulcer of the Stomach............. 287

LECTURE XV

Characteristic Trpes of Ulcer of the Stomach .......... 301

LECTURE XVI

Characteristic Trpes of Ulcer of the Stomach (Continued) ... 311

LECTURE XVII

TUbercular Ulcer of the Stomach $\ldots \ldots \ldots \ldots \ldots \ldots \ldots \ldots \ldots \ldots . \ldots . \ldots . \ldots$ 
LECTURE XVIII

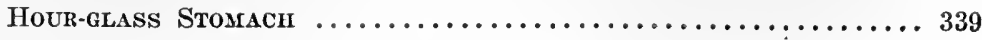

LECTURE XIX

Perilous Ulcer Hemorkhage $\ldots \ldots \ldots \ldots \ldots \ldots \ldots \ldots \ldots \ldots \ldots . \ldots \ldots$

LECTURE XX

Perforating Ulcers of the Stomach $\ldots \ldots \ldots \ldots \ldots \ldots \ldots \ldots \ldots \ldots$

LECTURE XXI

Cancer of the Stomach . . . . . . . . . . . . . . . . . 395

LECTURE XXII

Peptic Uleer of the Jejunuli-Techinic of Gastro-enterostomy.. 423 LECTURE XXIII

ECHINococcus of THE LIVER....................44I

LECTURE XXIV

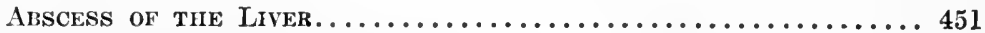

LECTURE XXV

Chronic Jaundice. Xanthoma. Xanthelasma Multiplex..... 464 


\section{ILLUSTRATIONS}

FIG.

1. Water sterilizer with internal spiral PAG

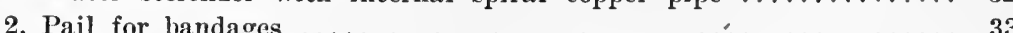

3. Pail for gauze and wadding tampons ................. 33

4. Operation in large operating theatre $\ldots \ldots \ldots \ldots \ldots \ldots \ldots \ldots \ldots$

5. Surgeon with rubber gloves, sterile cap and spectacle mask ...... 41

6. Cingulum dressing after abdominal operation . . . . . . . . . 42

7. Collodion-cotton wool covering being removed . . . . . . . . . 43

8. Aluminum bronze threads seen in wound healed without reaction... 44

9. Line of wound covered with collodion-cotton wool . . . . . . . . 45

10. Sack drainage used in suppurative peritonitis . . . . . . . . . . 47

11. Mikulicz's bag covered with slit mackintosh . . . . . . . . . . 48

12, 13. Colonies of bacteria on gelatine surfaces $\ldots \ldots \ldots \ldots \ldots \ldots \ldots \ldots \ldots$

14. Drainage of empyema by drain conducted through dressing into a

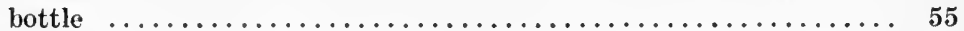

15. Herrfordt's cylinder ......................... 65

16. Component parts of catgut harp $\ldots \ldots \ldots \ldots \ldots \ldots \ldots \ldots$

17. Catgut harp ready for use ....................... 66

18. Author's catgut harp in use ...................... 68

19. Forf's formalin sterilizator for rubber catheters . . . . . . . . .. 69

20. Catheters and bougies in cotton-wool-stopped tubes and transportable

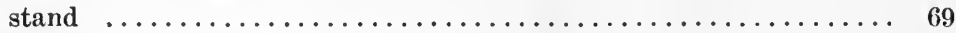

21. Sudeck's ether mask .......................... 82

22. Ether narcosis with Wanscher's apparatus ............... 83

23. Heister's mouth dilator ......................... 86

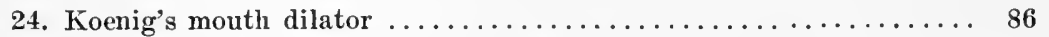

25. Apparatus to draw mucus from throat during narcosis ........ 87

26. Ether bag of Gaudafil ........................ 93

27. Richardson's ether spray for local anæsthesia . . . . . . . . . . 98

28. Tube with ethyl chloride $\ldots \ldots \ldots \ldots \ldots \ldots \ldots \ldots \ldots \ldots \ldots \ldots \ldots \ldots \ldots$

29. Cocaine ansesthesia after Oberst's method ............... 104

30. Formation of bulla by endermatic cocaine injection .......... 106

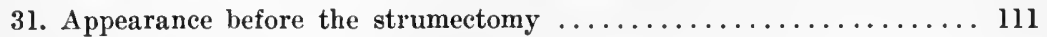

32. Appearance after the strumectomy $\ldots \ldots \ldots \ldots \ldots \ldots \ldots \ldots \ldots \ldots$

33. Braun's syringe for local anmsthesia . . . . . . . . . . . . . . 112

34. Detection of third intervertebral space in line connecting top portions

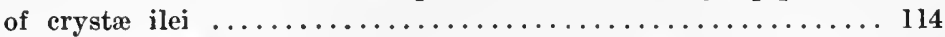

35. Introduction of cannula in direction indicated by dotted line ..... 115

36. Bougies . . . . . . . . . . . . . . . . . . . . . . . . 123

37. Pliable esophagus forceps ....................... 125

38. Gold plate in osophagus removed by œsophagotomy . . . . . . . . . 128

39. An English penny in œsophagus removed by æsophagotomy ........ 129

40. Radiograph of toy bicycle tightly wedged in the esophagus ...... 130 
41. Toy bicycle removed by osophagotomy ................. 131

42. Esophagoscope with Casper's panelectroscope $\ldots \ldots \ldots \ldots \ldots \ldots \ldots 132$

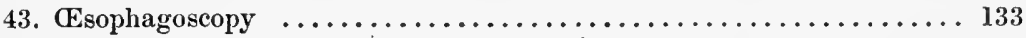

44. Three-inch nail followed day by day by Röntgen illumination ..... 134

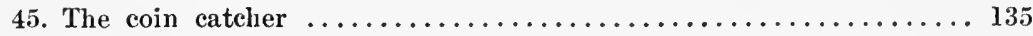

46. Showing healed line of incision after osophagotomy for toy bicycle.. 136

47. Author's gastroscope for introduction of bougie into esophagus.... 151

48. "Idiopathic" dilatation of the cesopliagus ................ 160

49. Röntgen picture of csophagus diverticulum filled with bismuth pulp..161

50. Cancer cardiæ with dilatation of the esophagus $\ldots \ldots \ldots \ldots \ldots \ldots 166$

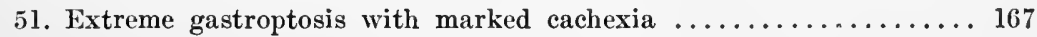

52. Radiograph showing the position of the stomach lying down below

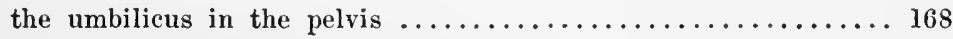

53. Author's apparatus for exhaustion of stagnant contents of the

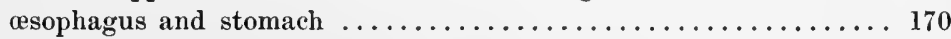

54. Relation of pleura and peritoneum to cardia ............. 171

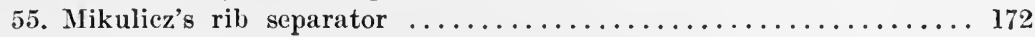

56. Resection of esophagus performed between two clamps ........ 173

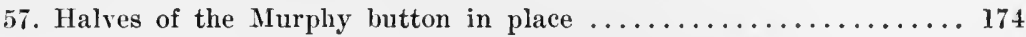

58. Anastomosis formed by joining two portions of the Murphy button. . 175

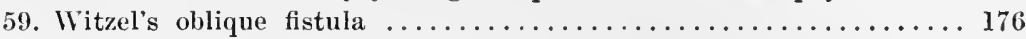

60. Gastrostomy with employment of Pezzer's drain after author's

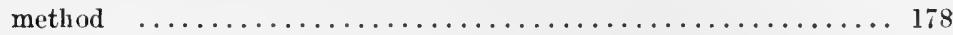

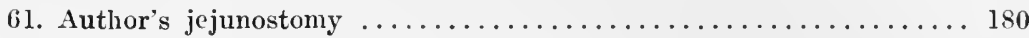

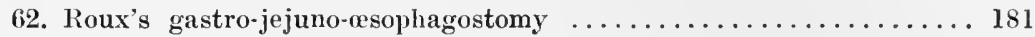

63. Radiograph of stomach filled with lismuth and greatly prolapsed... 191

64. Radiograph from same patient 24 hours later, showing abnormal

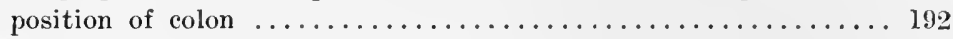

65. Withdrawal of retained meal by aspiration $\ldots \ldots \ldots \ldots \ldots \ldots \ldots$ 195

66. Rinsing out of the stomach $\ldots \ldots \ldots \ldots \ldots \ldots \ldots \ldots \ldots \ldots \ldots \ldots \ldots$

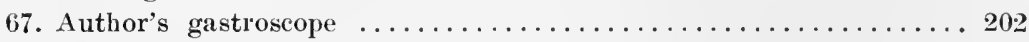

68. Virginal ptosis, tense abdominal wall $\ldots \ldots \ldots \ldots \ldots \ldots \ldots \ldots \ldots 213$

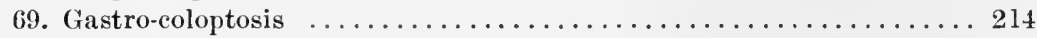

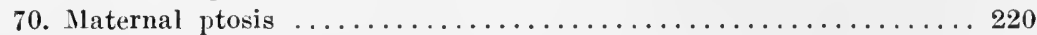

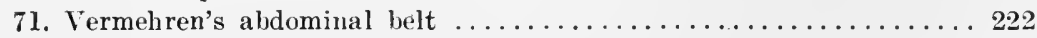

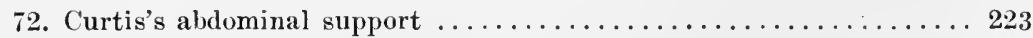

73. Ulcers on a ptosis crease of the mucous membrane of the stomach... 232

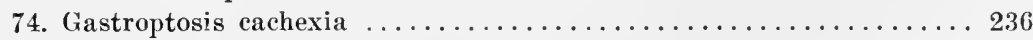

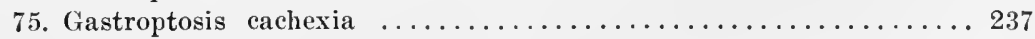

76. Röntgen picture of the bismuth-filled large intestine ........ 242

77. Hepatopexy. The liver fixed to the diaphragm by angular sutures.. 248

78. Hepatopexy by aid of the severed ligamentum teres ......... 248

79. Liver resection with pressure forceps $\ldots \ldots \ldots \ldots \ldots \ldots \ldots \ldots . \ldots \ldots . \ldots \ldots$

80. Case of primary coloptosis with secondary gastroptosis ........ 250

81. Author's method of shortening the gastrocolic ligament ....... 252

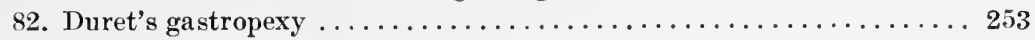

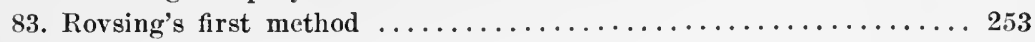

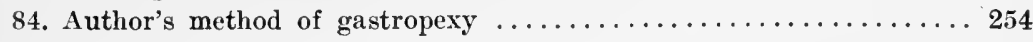




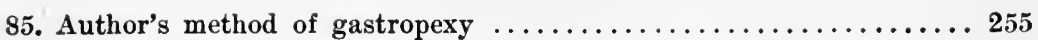

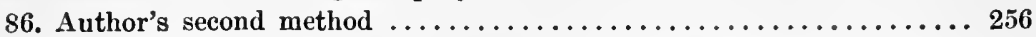

87. Coffey's operation for gastroptosis $\ldots \ldots \ldots \ldots \ldots \ldots \ldots \ldots \ldots . \ldots . \ldots . \ldots 259$

88. Author's method to enlarge the abdominal wall in eases of virginal ptosis ...................................... 266

89. Perforated stomach ulcers removed by excision $\ldots \ldots \ldots \ldots \ldots \ldots 283$

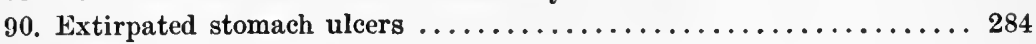

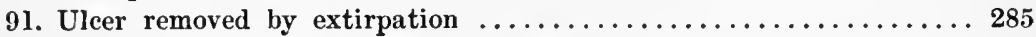

92. Resected pylorus, with adjacent stomach-wall ............... 313

93. Temperature curve after pylorectomy, showing result of silver nitrate

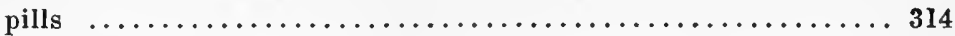

94. Pylorectomy after Billroth's method ................... 318

95. Heinicke-Mikulicz pyloroplasty $\ldots \ldots \ldots \ldots \ldots \ldots \ldots \ldots \ldots \ldots \ldots \ldots \ldots \ldots$

96. Gastro-duodenostomy for stenosis of pylorus $\ldots \ldots \ldots \ldots \ldots \ldots \ldots \ldots$

97. Commeneing hour-glass folding $\ldots \ldots \ldots \ldots \ldots \ldots \ldots \ldots \ldots \ldots \ldots \ldots, \ldots \ldots$

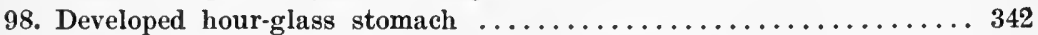

99. Hour-glass stomach, resulting from folds growing together with peritoneal surfaces ................................ 347

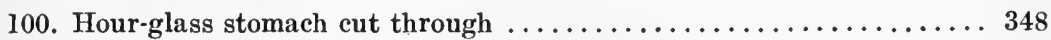

101. Tetanic contraction of normal ptosis stomach ............. 350

102. Radiograph of hour-glass stomach $\ldots \ldots \ldots \ldots \ldots \ldots \ldots \ldots \ldots \ldots \ldots \ldots \ldots \ldots$. 351

103. Radiograph showing result of resection in case of hour-glass stomach 352

104, 105. Showing purpose of gastroplasty in hour-glass stomach $\ldots \ldots 354$

106. Kammerer's operation ............................... 354

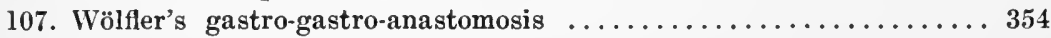

108,109 . Resection of stomach with direct joining of segments ..... 355

110. A simple gastro-anastomosis . ........................ 355

111. Weir's double gastro-enterostomia posterior ................ 355

112. Author's method for gastro-entero-anastomosis $\ldots \ldots \ldots \ldots \ldots \ldots 356$

113. Fowler's bed .................................. 379

114. Subphrenic abscess secondary to perforating ulcer of stomach on

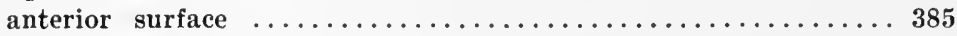

115. Subphrenic abscess secondary to perforating ulcer of stomach on

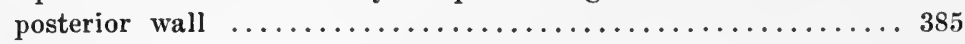

116. Radiograph of a case of extensive cancer of stomach showing irregularity of Röntgen picture $\ldots \ldots \ldots \ldots \ldots \ldots \ldots \ldots \ldots . .396$

117. Adenocarcinoma of anterior wall of stomach removed by resection... 405

118. Sketch of lymphatic glands of stomach $\ldots \ldots \ldots \ldots \ldots \ldots \ldots \ldots, 414$

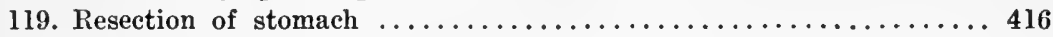

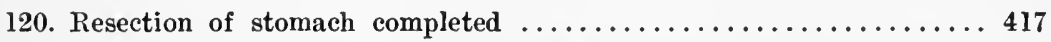

121. Three-quarters of stomach removed together with $5 \mathrm{~cm}$. of duodenum 418

122. Cancer partis cardiacæ ventriculi removed by ring-shaped resection

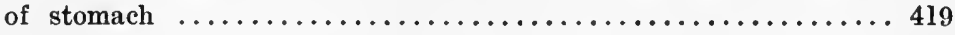

123. Cancer pylori removed by resection ................... 421

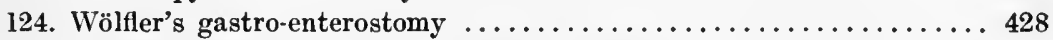

125. Wölfler's gastro-enterostomy, second step, and Braun's entero-

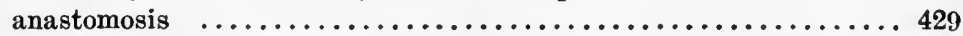

126. Von Hacker's gastro-enterostomia retrocolica ............... 430 
127. Roux's Y-entero-anastomosis. First step ................ 432

128. Roux's Y-shaped entero-anastomosis. Second step $\ldots \ldots \ldots \ldots \ldots 433$

129, 130. The Murphy button and mode of application ........... 439

131. Echinococeus eyst $\ldots \ldots \ldots \ldots \ldots \ldots \ldots \ldots \ldots \ldots \ldots \ldots \ldots \ldots . \ldots \ldots 48$

132. Dilatation of chest cavity by empyema as contrasted with dilatation

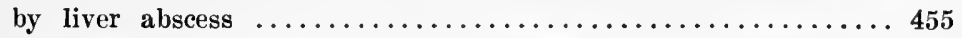

133. Diaphragm raised, and cavity of right lung and pleura compressed by liver abseess and enlarged liver $\ldots \ldots \ldots \ldots \ldots \ldots \ldots \ldots 456$

134. Shows the cicatrix after author's subpleural incision and resection

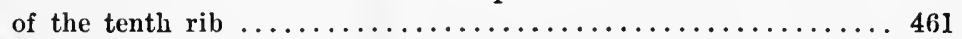

135. Multilocular cystoma in caput pancreatis as a cause of chronic

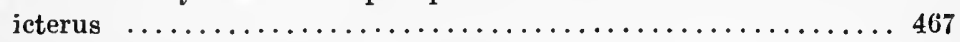

136. Group of xanthoma tumors on patient's elbow ........... 468

\section{PLATES}

I. Non-malignant stricture of thoracic portion of œsophagus with dilatation above caused by the swallowing of lye $\ldots \ldots \ldots \ldots \ldots \ldots \ldots 2$

II. (a) Typical cardio-spasm with the general diffuse dilatation of œsophagus proximal to the constriction ..................... 162

(b) Cardio-spasm of the œesophagus as superimposed upon a carcinoma of the cardiac end of the stomach $\ldots \ldots \ldots \ldots \ldots \ldots \ldots \ldots \ldots \ldots \ldots$

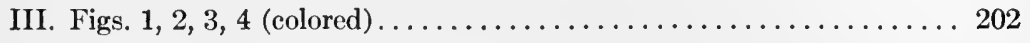

IV. Radiogram of a typical, marked prolapsus of the stomach with extraordinary elongation of the cardiac end of the fundus ....... 208

V. Radiogram of a dilated, markedly atonic and prolapsed stomach . . 218

VI. Radiogram of case with perilous ulcer hemorrhage, showing flattening of duodenum due to adhesions, and stellate filling defect due to

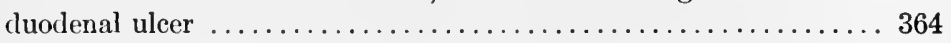

VII. Carcinoma of the stomach showing filling defect to the left of the picture in which there was at operation an extensive carcinoma of

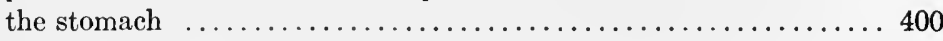

VIII. Radiograph showing filling defects of pyloric antrum and signet ring deformity of duodenum by extension, as shown in dotted outline. 410

IX. (a) Carcinoma of the anterior lesser curvature proven at operation. 414

(b) Most typical picture of anterior pyloric end of stomach carcinoma. 414 


\section{ABDOMINAL SURGERY}

\section{LECTURE I}

\section{THE BASIS AND PRINCIPLES OF CLINICAL SURGERY}

IT seems to me that it will prove more useful and more profitable if, instead of taking up an individual case, I devote this first lecture to certain general observations which may serve as an introduction to our subsequent lessons. First, we will tune up, so to speak, and endeavor to be of one mind as regards the basis and the principles of clinical surgery.

It has often been said, though most often perhaps on festive occasions and when feeling runs high, that the calling and work of the physician are more beautiful than all others. You, of course, must have held this opinion when you chose the medical profession in preference to all others, and I think that this view of, and this enthusiasm for, the medical profession are maintained during the whole course of one's studies. But when you begin the practical work of a physician, then your views experience a change. To many of you the profession appears as beautiful as ever; to some each day brings more joy and happiness by means of it; but to others, many others, on the contrary, it seems a troublesome, prosaic and thankless occupation. And the reason for this is, that the charm of a physician's calling is of a dangerous character. It demands the complete devotion and understanding of its worshipper, and should he fail therein, his calling becomes loathsome to him and he sees only the tiresome and disagreeable side of it. To what, then, is the beauty of this 
work due? It is all due to the power which is a part of this calling. No profession in life, not even that of the statesman or of the judge, has such relative power as that of the physician; for to his care is committed that which mankind holds first and dearest-life and good health. How often are life and death dependent on the timely appearance of the physician upon the scene; while the great charm of this power is that our fellow beings voluntarily submit themselves to it.

The result, however, of this great power inseparably involves tremendous responsibilities, larger and heavier than those incurred in any other profession, because, as surely as we can save human life by instituting the right treatment at the right time, so surely can we forfeit it by faulty treatment.

This power and responsibility of a physician are, of course, more conspicuous in a hospital ; for here all the most serious cases accumulate. And this applies above all to the surgical wards where, in addition to the serious responsibilities associated with operations, one is also answerable, in this modern era of surgery, for conducting the surgical work in such a way that the patient shall not suffer from a lack of proper antiseptic precautions or through faulty operative technic.

The peculiar responsibility of the operator is indeed large and complex, a fact which I shall often reiterate in the future, but the responsibility which I wish especially to mention here is the responsibility of the medical practitioner, which often is lightly esteemed, although it is, in reality, of the utmost importance. You must always remember that the determining decision with regard to diagnosis and treatment, often fundamental as regards the patient's life and well being, always in the first instance lies in the hands of the medical practitioner. No one can expect the physician to be a surgeon of such ability as will enable him to provide his patients with other than elementary surgical assistance, 
but what the patients may with justice expect from the practitioner to whom they intrust themselves in cases of sickness is that he is a sufficiently qualified surgeon to be able to decide when and where surgical assistance is necessary.

Here the practitioner's responsibility is of a two-fold nature. All practitioners properly feel the responsibility of advising patients to undergo operation, and they all feel this because the fears and many scruples of the patient react upon the mind of the practitioner, while on the other hand, he is far less sensible of responsibility if he refrains from advising surgical assistance in cases of certainty or doubt simply because no one, in either case, would saddle him with the responsibility. No one, that is to say, save his own conscience. 'This responsibility is, in consequence, often overlooked and forgotten, more is the pity; because, as a matter of fact, it is a greater and more serious responsibility than that incurred by advising surgical counsel, for in the latter case, if interference be necessary, the fact will be opportunely recognized by the experienced surgeon whose assistance has been sought, and thus no misfortune occurs; while, on the other hand, neglect of a timely call for such surgical help, when needed, often results in loss of life.

From this you will understand that I feel it to be one of my most urgent duties to make you vividly realize this apparently lesser responsibility, so that you will always remember it in connection with your future work.

It should always be every good physician's pride, above all things, to preserve the feeling of this responsibility steadily alight and awake in his conscience, and the more so because, in the world's eye, he can so easily avoid it.

My next task is to teach you how to meet this responsibility, and to put you in the way of using your influence, as a physician, in the right direction.

What concerns you first and foremost is to learn to examine and to diagnose, and afterward to acquire a thor- 
ough acquaintance with the principles of the treatment of wounds, and a complete knowledge of all the surgical methods which every practitioner has at times personally to employ.

To-day I will tell you something about the principal rules for examination and diagnosis. The first lesson which I want to inculcate is : Never delay the thorough examination of a patient to whom you are summoned, and never commence to treat the patient until you have established the diagnosis as completely as possible.

It is, however, by no means easy to formulate in a few words general rules for a thorough surgical examination. The shortest and most famous precept bearing upon this subject is that of the well-known English surgeon, Sir George Humphry, which reads as follows: "Eyes first and much, hands next and little, tongue not at all." Unfortunately, I do not think that this excellently brief direction is so suitable for modern surgery as it was for the surgery of days gone by, when the surgeon's field of activity was verily limited to the exterior of the body and to all external afflictions which could be seen with the eyes and felt with the fingers, while everything internal was a matter for the "physicians" - the interior pathologists. Sir George advised physicians to investigate in silence, because he feared lest the patient's replies to questions should confuse the physician and divert his attention from the essentials, which a precise examination of the patient might reveal to him. Sir George is quite right in saying that a great danger lies in the patients' remarks; that is to say, if they are accepted without sufficient criticism, for the patients' frequent misinterpretations of their own symptoms, and their one-sided emphasis on some particular symptoms,- - generally the unpleasant ones,-and their concealment of others, perhaps more important as regards the diagnosis, frequently mislead the physician. But this rule cannot be accepted in 
these days, since surgery, thanks to antisepsis, has been able to extend its field of usefulness to the remotest organs: to those "internal" diseases which formerly were the undisputed domain of the medical practitioner; it follows naturally that the field of surgical diagnosis has become broader and more complicated, and it must be admitted that in a great number of cases the information obtained from the patient himself is quite indispensable to the diagnosis.

Were I, like Sir George, to endeavor in a few words to establish a principle for clinical investigation, I should say : "First listen and interrogate, then observe, and afterward feel; but, above all, think quickly and logically over what you hear, see, and feel.",

To be able to diagnose correctly, the qualifications of a good detective are indispensable : a lively imagination, but one held in check by a sharp, logical judgment. When I said, first hear and question the patient, I meant that you first allow the patient to talk, and then ask him briefly what is the matter with him; then there generally follows a strong flow of words, including the patient's well-prepared presentment of his illness and many other subjects not connected therewith. Now, while the patient is speaking, you begin to observe him or her, and with a glance you acquire an impression of his or her whole appearance and habitus: small or large, thin or fat, crooked and round shouldered or straight. You next take special notice of the uncovered portion of the patient's body, head, neck, and hands : the color of the skin, the condition of the hair, the eyes, whether they are protuberant, and whether the glance is natural and vigorous; the general expression of the countenance; the condition of the teeth, etc., for by observing the patient in this manner and while he, occupied with his conversation, is quite unaware of the fact that you are already examining him, you obtain valuable data for a diagnosis, and sometimes indeed the diagnosis itself, by comparing your patient's re- 
marks with your own observations. Nothing is so reprehensible, however, as being satisfied with this, no matter how certain one may feel. For it is owing to this premature satisfaction that so many fatal mistakes occur. It may seem superfluous to impress this upon you, but it is unfortunately far otherwise, for again and again the experienced surgeon sees the results of such superficial investigations. In order to make this quite clear to you, and to fix it in your minds, I will illustrate it by detailing the history of two patients who came to me for treatment. During a long period botll these patients were treated for "catarrh of the stomach," a diagnosis so frequently made, as a matter of routine and without further investigation, both by physicians and patients. And herein lies a very dangerous element, for in our "enlightened" times any intelligent person considers that he is quite correct in making a diagnosis of stomach catarrh when he suffers from cardialgia, distaste for food, eructations, and occasional vomiting.

Such patients approach the physician, saying, "I have a stomach catarrh, won't you give me something for it?" and after a few hasty questions, the physician verifies the patient's own diagnosis and proceeds to prescribe diet, powders, etc.; and thus it was with the two cases to which I am referring. The one patient, an elderly man in the sixties, had for a long time suffered from loss of appetite, eructations, flatulency, and distention of the epigastrium. The physician whom he consulted looked at his tongue, slightly pressed his stomach and, remarking that his indisposition was due to too many dinner parties, proceeded to order him a milk diet. But when the attacks grew worse he sent the man to Carlsbad, where the physician at the baths again "verified" the diagnosis as stomach catarrh, and ordered the man to drink plenty of Carlsbad "sprudel;" but, to the patient's astonishment, the symptoms only became worse and the abdominal distention increased. The other patient 
was a young man in the early thirties, who had suffered for a long time from the symptoms mentioned and who also had been treated by his physician for stomach catarrh. In spite of treatment he grew rapidly worse, was unable to retain his food, and became so emaciated and weak that he was unfitted for work.

Both these men eventually consulted me as to whether surgical assistance could benefit their stomach complaint, which both naturally feared was of a serious nature. A systematic examination, however, showed that both these patients were suffering from a maximum distention of the urinary bladder, the cause of their trouble being a retention of the urine. This, in the one case, was due to an hypertrophied prostate, and in the case of the young man was the result of a congenital valvular formation near the orificium internum vesicæ. All of their stomach symptoms, such as loss of appetite, eructations, and vomiting, were due to imperfect elimination of the urine and disappeared as soon as the bladder was drained. You see, therefore, that if the physician had not been satisfied with hearing the patient's own account of his malady, and had he not allowed himself to be influenced by this diagnosis, a serious mistake might have been easily avoided, for by merely questioning them about their other organs he would quickly have ascertained that both passed water more frequently than was normal; while by feeling the abdomen he would quickly have discovered the enormously distended bladder, and a further examination of the urinary organs would have led to a correct diagnosis.

With regard to my first point-listening-the significance of this is, that you are not content with hearing what the patient wishes to tell you but, having satisfied this need of his so far as you think advisable, you cease to listen and begin to interrogate; for by listening to the patient's complaints, while at the same time you keep him in your 
mind's eye and rapidly review in your own thoughts the sufferings which he emphasizes,-your mental faculties are directed toward certain possibilities which may be at the root of the symptoms. First seizing upon that possibility, which for the moment appears to contain most probability, you cross-question the patient quickly and clearly, seeking indications both for and against your theory, and thereby ascertaining the anamnesis and symptoms which the patient has not mentioned, probably because he has attached no importance to them; and if the first thread leads nowhere, try another, etc. To conduct this cross-questioning correctly, in such a manner, so to speak, that it quickly attains its object-the correct diagnosis-is an art which must not be undervalued, for it is here that the skilful physician's ability intrinsically reveals itself. Naturally a thorough knowledge of pathological anatomy is essential, for without this you are not in a position to appreciate the many possibilities which will present themselves. Imagination, presence of mind, criticism, and the gift of logical combination are next called for, though perhaps psychological perceptivity, self-control, and a sense of superiority among human beings are even more important.

You will quickly learn that all patients will not allow themselves to be measured with the same rule. If you wish to learn anything useful from your patients, you must take each in his own particular way. A patient is lively, talkative, imaginative, and speaks fast and loose of all sorts of things at full speed, but seldom remembers to mention that upon which everything depends. Here you must apply the brake as quickly as possible, and ask short, precise questions, while insisting upon short, precise answers; else he breaks away from your examination, and all his remarks become immaterial. Another patient may be reticent and distrustful, so that his evidence has to be hauled forth. But the very greatest drawback with patients is, that so very many 
of them are anxious and nervous, partly from fear of illness, partly from fear of the physician and of what he will do to them. From these patients you will not obtain a sensible word if you meet them with a serious, solemn face, or if you yourself appear nervous and anxious. You must first soothe them with a kindly and cheerful address, and if you are capable of making a little joke or a humorous remark you will often have the good fortune immediately to make them calm and confident.

On the whole, you must remember, when associating with the sick, that these require kindness most of all, and that they first and foremost wish and hope to find a comprehending and sympathetic friend in their physician; and when treated in this manner will, in a few moments, give you their fullest confidence.

As it is with this first principle of hearing and crossexamining, so is it with the second principle of observing, or let us rather say investigating by sight, inasmuch that it is not such a simple affair as it at first appears. I have already stated that, while in the beginning you listen to your patient's explanatory remarks, you should observe him closely, and thus be enabled to proceed rapidly toward a diagnosis, and especially so if you combine that which you see with that which the patient complains of. But you must never allow yourself to be content with this, no matter how busy you may be nor how much you may momentarily imagine that you have arrived at a diagnosis. This statement is best proved by the two cases of urinary retention which I have just mentioned.

You must make it a rule for yourselves always to inspect the patient's bare body, and to search systematically with your eyes for any abnormality. Another rule which I consider of the utmost importance is that you invariably compare the congruous members of the body, because it frequently happens that the unsound portion only betrays its 
abnormality when it is compared with the sound portion; and this is especially so where the extremities are concerned, and where atrophy of the part or difference of posture or increased dimension of the afflicted extremity suggests the diagnosis or shows you how to arrive at it.

This rule applies also in examining the abdomen, the chest, the neck, and the head, and is of great importance. You can learn much from a glance at the different contours of two members. For example, on one side of the body is a tumor, which so far as it is appreciable through the abdominal wall can often tell you by its relative position which organ it springs from and what kind of tumor it is. If you have a homogeneous swelling of the abdomen, the shape will often suggest to you whether it is a free accumulation of fluid or a tumor. Faced with peritonitis, you can often define its limits merely by noticing the movements of the abdominal muscles during respiration, and you can also determine the degree and extent of the meteorism from the design of the intestines, as seen through the abdominal wall.

What a gold mine of information the inspection of the skin may prove to the diagnostician! Now we discover old scars from wounds or fistula which immediately give us a clue to a more complete understanding of the whole subject. Now we find a limited or general design or dilatation of veins on the skin, a certain herald of some more deeply rooted affliction. Then, too, the condition of the skin, if it is slack and thin or stretched or œdematous; if it is pale or red, fresh or sallow, mouldy or hectic.

You see, Sir George had good reason to say, "Use the eyes first and much before you begin to feel,"' because that which one sees with one's eyes is always the most reliable, though it should be added that sight often teaches one how and where to palpate. He would certainly have emphasized this even more strongly could he have foreseen how deeply the eye, later on, would succeed in penetrating the human 
body; because, while in his day one had to content one's self with a contemplation of the outer surface of the body, we, in our day, have learned to peep through the doors and corridors of the human frame. Through the mouth we may examine the larynx by means of a laryngoscope, and with specially constructed tubes look down into the bronchi and œsophagus and stomach; and through the pupil, by the aid of the ophthalmoscope, see what is taking place in the background of the eye, yes, sometimes even read the condition of the brain; and through the anus survey the inside of the rectum, and through the urethra examine the bladder: all with the additional assistance of electric light. But, besides all this, and thanks to Röntgen, we have literally succeeded in observing the human body through and through, and thus detect and diagnose diseases which, in former days, could either not be discovered at all or, if so, only by the more dangerous means of an exploratory operation.

The limits of our diagnostic ability and also of our diagnostic obligations have been enormously extended during the past decade; but these new methods which permit us to explore the interior of our patient possess their seamy side, in so far as they demand an armamentarium and a technic which every medical practitioner cannot possibly acquire.

You will then ask: Has it any special interest for us? Need we study and familiarize ourselves with these new methods? And to this I reply: Naturally, no one can demand or even think that every physician who graduates from our university should be able to master these methods; but what one can expect, or what, more correctly speaking, is an absolute necessity, is that he should know the scope of these methods and the indications for their application, so that, where a diagnosis, which appears obscure to him, may be elucidated by such methods, he sends his patient to be examined by a colleague who has mastered these methods.

In this matter never hesitate, fearing that the recog- 
nition of your own limitations may lower you in your patient's eyes; for sooner or later, as a rule, you will reap the harvest of your patient's gratitude and his recognition of your timely action, always the reward of a good conscience; while, on the other hand, that physician who fails correctly to advise his patient in such cases will some day reap reproach and merited distrust, when the patient, in his need, seeks and finds genuine help elsewhere.

So you must use your eyes and ears before you begin the manual examination, and then you know what you are to feel for, and whether to employ simple palpation with your fingers and hands or to use instruments, probes, or bougies; these in turn will communicate impressions to your tactile nerves.

The time at our disposal does not allow me to enter into the details of the art of feeling, about which so much might be said. These details vary with every single disease and with every single organ, and we will have the opportunity to discuss and practise them at the bedside of the sick and during the lectures on individual cases. Let me lay down only this one fundamental rule: "Feel with a light, soft touch," not only in order to cause the patient as little distress and pain as possible, but because the more lightly and tentatively you use your hands, the more surely and delicately you feel. A hard and coarse hand never arrives at a delicate diagnosis.

And, so little as you may neglect the exterior palpation, so little may you neglect the interior digital exploration, especially as regards the vagina and rectum. Do not allow the consideration of the patient's and your own repulsion toward these examinations to prevent you from always undertaking them when you are in doubt regarding the diagnosis, for in hundreds of cases the simple vaginal or rectal examination immediately gives you the diagnosis and 
fully explains the patient's condition, by disclosing a tumor, exudation, etc.

Finally, I will mention only one golden rule. Never forget, when first examining a patient, to examine the excretions immediately. The urine, in particular, should be examined for albumin, blood, and sugar, if you wish to avoid frequent and serious mistakes; and the excrements should also be made a matter for investigation far more frequently than is usual. It is necessary, however, to admit that very many cases present themselves where even the most painstaking examination fails to lead to a decisive result with regard to diagnosis.

Here we have two modes of procedure toward the acquisition of the diagnosis. In certain cases we can and must endeavor to make it ex juvantibus, which means, to apply that treatment which is suited to the disease which we suppose is presented before us, and this method is especially suitable in the many cases where we are in doubt as to what extent the complaint is of a syphilitic or other nature. Most frequently antisyphilitic treatment causes the tumor, ulceration, or whatever it may be to vanish as dew before the sun if it is syphilis; and here we attain the diagnosis and the cure at one stroke.

The other method is the exploratory incision, a road to diagnosis which this antiseptic era has made too tempting, under the pretext that an exploratory operation, if safeguarded by careful antisepsis, may be regarded as quite harmless. This, however, is a great misapprehension. No operation is quite free from danger, though any operation which is performed at a venture is always more dangerous than one which, goal-assured and accurately planned, is directed against a complaint which has been correctly diagnosed. If you will read through the pages of the yearly records of the wards of different large hospitals, you will be surprised to find a considerable mortality under the head. 
ing of exploratory laparotomy. You will also be surprised by the great variation you will find in the number of operations of exploratory laparotomy performed by the various surgeons; and you can be quite certain that the fewer the operations of exploratory laparotomy so much the cleverer the surgeon, possibly not so much so as operator, but certainly so as diagnostician. Later on we shall often have oceasion to return to this subject, but to-day I will merely impress upon you that it is not only exploratory laparotomy which you must be warned against, as only professional surgeons are tempted to perform it, but also that apparently insignificant and safe operations, if undertaken as an easy short cut to the diagnosis, may involve great unnecessary danger if attempted before all superficial means of ascertaining the diagnosis have been exhausted. Even such a slight operation as the diagnostic puncture of a presumably cystic tumor may prove fatal if the contents of the cyst consist of unexpected and virulent pus, or if, contrary to expectation, the cyst reveals itself as a malignant growth; because the infections matter can then exude through the puncture wound and infect the surrounding tissues, and possibly, if the tumor be intraperitoneal, it may result in fatal peritonitis or in the propagation of cancer. Where an exploratory operation is necessary, all potentialities must be exactly weighed in advance, and the surgeon equipped to meet every eventuality.

Let us take another example. A woman comes to you and presents a somewhat rough, hard tumor in one breast, which you hope is a. non-malignant adenoma, but which you fear is cancer. In the one case an operation is unnecessary, while in the other the whole breast, with the glands, the fat, and the tissues contained in the axilla, must be removed-a mutilating operation and one much feared by the patient. What shall one do? A great number of physicians simply make an incision in such growths, re- 
move a fragment for microscopic investigation, suture the wound, and wait for the verdict of the microscopist-a verdict for which they generally have to wait a week. This so-called "sample" excision, viewed superficially, appears a very adroit, innocuous, and rational encroachment, but nevertheless it is so dangerous that I cannot warn you against it sufficiently; for although, if the growth be nonmalignant, all goes well, yet, if it reveals itself as a malignant tumor, you will have broken its limitations by your incision and have evolved new formations, not only in loco by implantation in the wound, but often in other organs, through the opened blood and lymphatic. tracts, and by your "examination" you will have made a radical recovery of the patient impossible; so, unless you are quite certain about your problem, you should submit the whole growth for microscopic investigation.

From the patient now being brought in, who is anæsthetized and prepared for operation, you will learn that the question of diagnosis, with regard to new formations of malignant tumors, can be even more serious and complicated. He is a married fisherman, who consults us with respect to pains in the right leg. He comes from Bornholm, is thirty-two years old, and tells us that 19 months ago, in Pecember, 1902, he fell on the deck of his boat and struck his right shin-bone against the sharp side of the main hatchway, which struck him in the neighborhood of the tuberosity of the tibia. He does not remember any external wound or swelling, but severe pains at once set in and he was unable to support himself on this leg; but after being confined to his bed for some time, with a treatment of hot bandages and massage, these symptoms subsided, so that he was enabled to get up and attend to his work, though frequently conscious of pain in the damaged limb. Last winter, however, the pain became so unbearable that he was compelled to cease work altogether. 
When you observe the patient, you will notice that he is a strongly-built, well-nourished, and an apparently healthy man. His countenance is weather-beaten and fresh, and, generally speaking, the case presents no other abnormality than the morbid changes in the right lower extremity, caused by the affliction. If you see only this one extremity uncovered, many of you will hardly notice that there is anything abnormal; but follow the rule which I previously impressed upon you as being of such extreme importance, and uncover both extremities and submit them to comparative examination; then even the most incxperienced among you will perceive the difference in the two aspects, for the upper portion of the right leg is distinctly more pronounced than that of the left, and this is due to a swelling, which develops internally and forward, corresponding with the inner side of the tibia; while, at the same time, the right thigh, which in a normal condition is usually rather more pronounced than the left, is here plainly thinner and slacker. You can no longer doubt that it is the right lower extremity which is abnormal and diseased.

On further investigation, we find that the contours of the knee-joint itself are normal, and that there are no symptoms of accumulations of fluid or thickening of the capsule, though immediately beneath the knee and commencing on the tuberositas tibiæ we find a tumefaction, which raises the skin. This, however, is natural growth and can be creased and shifted against its substratum, while tumors appertaining to bones are hard and somewhat uneven to the touch, without softer pliable parts, and do not give a sensation of crackling parchment when pressed.

What have we, then, to consider here? First and foremost, a fracture, for the wound is particularly adapted to cause a direct fracture, which in turn would explain the violent pains and the immediate functional disturbance 
which supervened, and the tumor can then easily be defined as callus. Moreover, the course of the affliction, during its early stage, also points toward the correctness of this theory, for after he had been confined to his bed for some time with a treatment of fomentation and massage, he was relieved of pain and enabled to return to work. But why, a whole year after the accident, has the pain increased so violently, and why is the growth so greatly augmented at this late stage? Had the growth been a simple callus, it would have developed in the first instance and have been greatest immediately after the accident; whereas, at that period, the man himself noticed no growth at all. Yes, all this is inconsistent with a simple fracture, and tells us that there must be something "behind." Now, you all know that a fracture can be "compound," with which word we generally associate that most common complication, a solutio continui, a wound in the skin and flesh corresponding with the fracture; a something we fear, because it can become the avenue for pathogenic microbes, which, if they find their way into the crushed and contused bone tissues, may produce a suppurative osteitis. This, however, does not apply to our present case. First, because the man declares positively that no wound of the skin was caused by the accident, although this declaration is not confirmable, and some slight abrasion of the skin, which he perhaps has overlooked, might have sufficed for the purpose. Second, because any indication of an acute inflammation is entirely missing in the first stage. If we have, indeed, to deal with a case of suppurative osteitis, this must have been caused by a very enfeebled microbe; still, we cannot altogether preclude this possibility, although the whole course of events directs us rather toward the theory of a process of a chronic inflammation or a tumor-like formation. We might imagine that at the time of the accident germs, circulating in the blood, settled in that hæmatoma in the 
crushed bone tissues and stopped the circulation at this spot. But, if so, which germs are we to consider? Experience shows that it is essentially syphilis, tuberculosis, and sarcoma which one sees develop on a traumatic basis of this sort.

Would it not have been simpler and more rational if the patient's physician had made a small incision and chiselled a small hole in the bone, in order to ascertain if it was not a simple abscess, before he sent him to us? It may appear so, but nevertheless it would be quite improper, because, if it should prove to be a malignant tumor, the rational treatment here would be amputatio femoris; and even this would give a chance of a complete recovery only if the tumor were absolutely local. Where you make a sample incision, you open the lymphatic and blood-vessels to the tumor germs, and the wound itself becomes infected. When, a week later, you receive notification from the microscopist, to whom you have sent the removed sample of tissue, that it is indeed cancer, then, generally speaking, all chance of recovery by means of amputation is lost, as a result of the exploratory operation.

If, therefore, you have any suspicion of a tumor, you must immediately do your utmost to arrive at a diagnosis, and preferably should send your patient to a hospital, an infirmary, or some clinical home, and there you should act very much as we are doing in this case.

First of all, you will take a Röntgen picture of the bone, and this will often give you the diagnosis at once. If it is a callus which forms the tumor, you will see the newlymade bone like a sharp shadow on the outside of the otherwise apparently sound bone. If it is an osteitis, with central abscess and hyperostosis, you will see the cavity of the abscess like a sharply-defined light on the thickened bone. If, on the other hand, it is a tumor, you will find destruction of the bone, and therewith the elucidation of the 
thickened portion. Now, the Röntgen picture proves this; for on the swollen part you only see a quite thin normal bone shadow surrounding a well-marked lighter area. Sometimes this picture may be due to tuberculosis or syphilis, and the Röntgen picture leaves us in doubt. This rarely happens, but it must be borne in mind because, in such circumstances, an amputation would prove a fatal mistake.

First you must try an antisyphilitic course of treatment, inunction of mercury and iodide of potassium both being necessary; and if, as in the present case, this treatment is of no avail whatever, only tuberculosis and sarcoma remain to be considered, with an overwhelming probability in favor of the latter.

I have therefore prepared this man for amputatio femoris, though for safety's sake I shall make an exploratory examination and remove a piece of the tumor for immediate microscopic diagnosis after applying an Esmarch bandage to the spot eventually to be amputated and thereby stopping the blood current. You notice that there is no bleeding and that the tumor is fairly soft, resembling fish flesh, so I am convinced that here we have to do with a sarcoma. But as I wish to have absolute proof that there is no tuberculosis, the clinical assistant, Dr. Blad, is now at hand in the laboratory, with a freezing microtome, in order to take a sample of the tumor, and in five to seven minutes we shall know the result.

I now cover the wound with sterile gauze, and await the answer from the laboratory, allowing the Esmarch bandage to remain quietly in position. Were I now to remove it, the blood would immediately commence to flow again, with even increased force, and tumor germs from the wound would become absorbed in the blood, and by this means be conveyed about the body, where, being deposited, they wound produce metastases in other organs. Therefore, 
when one is faced with a sarcoma, it becomes a matter of the greatest importance for the patient that the cessation of the blood current should continue till the amputation is completed.

Now comes the answer from the laboratory-an indubitable sarcoma-so we immediately proceed with an amputation, midway up the thigh and at a respectable distance from the tumor. Had the microscopical examination revealed tuberculosis, a curettement, or at the most a resection, would have been the correct mode of procedure.

This example, then, shows you that in exceptional cases a sample excision may be not only permissible but necessary, in order to provide against fatal error, though you should not resort to such a method until all bloodless methods of arriving at a diagnosis have been exhausted. Should the microscopic verdict read "malignant new formation," you must be thoroughly prepared to undertake a radical operation. 


\section{LECTURE II}

\section{ANTISEPTIC METHODS-PART I}

Prominent Features in the Development of the History of Antiseptic Methods

WE are now approaching the end of the term, and I should like to devote the last two lessons to the supplementing of my introductory lecture. You will remember in that lecture that I impressed upon you that a surgeon's fitness is determined, in the first instance, by two factors: one, the ability and erudition of the surgeon to investigate and diagnose with the utmost care and criticism; the other, the surgeon's complete comprehension and mastery of antiseptic methods. The first comprises, generally speaking, those qualifications which enable us to do the right thing, in the right way, and at the right moment; while it depends upon the second as to whether our clearly indicated action proves of benefit to the patient.

In that first lecture, however, I discussed only the general principles of examination and diagnosis in surgical cases. To-day I wish to speak to you upon the subject of antiseptic treatment. During the past term you have indeed had rich opportunity to observe how antiseptics are utilized in this ward, and how they act. But I have not systematically reviewed with you the fundamental principles of our various modes of procedure, which will certainly not be without significance for you.

My predecessor, Professor Bloch, never mentioned antiseptics in this place without paying his most devout homage to Lister, and in order to impress upon you the enormous debt which surgical science, together with all mankind, owes to this praiseworthy man, he presented us with a fine picture 
of Lister, the one which now hangs on the wall of this amphitheatre.

I cannot do better than emulate Bloch in this practice, but at the same time I wish to impress upon you, and with no less emphasis, the name of another man, who is more apt to be forgotten by you than Lister because he was not a surgeon-the name of Pasteur. Antiseptic methods are the achievement of Pasteur and Lister, so it is incorrect to designate Lister "the father of the antiseptic methods of treatment," since Lister would never have introduced the antiseptic treatment of wounds to the world had not Pasteur's epoch-making discovery of the domain of microbiology fructified his mind. We should always, therefore, associate Pasteur with Lister in our minds when we mention this epoch-making discovery, which, for significance, has no parallel in the war against disease.

Only he who has lived through the times both before and after the introduction of antiseptic methods of treatment is thoroughly able to feel and fully understand the revolution which this indicates. But you also, when as young phrsicians you obtain access to the archives of the large hospitals, will be enabled to acquire a very lively impression of its immediate importance, by turning over the pages of the journal of pre-antiseptic times, when hospital gangrene and pyæmia derastated the surgical wards. It was only a few days ago, in such an old volume, that I paused at a big black cross which stood under the diagnosis " "unguis incarnatus," and found that it referred to a great, strong peasant, twenty-two years of age, who had come to have an ingrown nail removed, and who had died from pyæmia two days after the operation!

This is only one single example among many, and it is only by perusing several such journals, and thereby learning how completely, even during the years immediately following the introduction of Lister's methods, the results of 
wound treatment altered, that you will first rightly understand what a boon this progress was for the surgeons and what a blessing it was for the whole of mankind. One must doubly admire Lister because his method, when produced, was already so accurately thought out and so certain in its effect that it compelled conviction from all who essayed it. And this was of the utmost importance, because you must not imagine that opposition and lack of confidence were wanting in the presence of the clear and simple train of reasoning which formed the foundation of this method; and had not this latter by its so practically striking results immediately gained a foothold it might perhaps never have prevailed.

We have an example of how extraordinarily difficult it may prove for extremely talented men to disabuse their minds of old and deeply-rooted theories and mistaken stand-points in Billroth, who, even in 1880, fourteen years after Lister had announced his method, declared this to be a delusion, maintaining that, though microbes were certainly found in wound inflammations, they were the result of, and not the cause of, the inflammation. Lister may still suffer the affront of hearing a few of his contemporary English colleagues voice these views (Bantock).

Nevertheless, however well thought out Lister's antiseptic method may have been when first produced, perfect it certainly was not. During the forty years which have elapsed since its appearance, it has undergone so many changes and improvements that, when all is said and done, nothing remains of that method in the antiseptic treatment you see to-day practised in this ward, save the principle. But on this principle everything first and foremost depends, and therefore the antiseptic treatment of wounds is and remains Lister's even though the active and valuable labor of numerous other surgeons has transformed and improved it to that high degree of perfection which it 
has now reached, and which enables us successfully to perform operations which could never have succeeded under Lister's first modus operandi.

It would necessitate many lessons to relate to you the complete history of the antiseptic method, its progress and retrogression; all the experiments, investigations, and discussions by means of which the original idea of Lister has developed into its present, though by no means final and completed, state. I believe, however, that I can quite well give you the leading features by merely going through the developments of antiseptic methods here in this ward, as I have followed them during the termination of Saxtorph's and during the whole of Bloch's term of authority, in order finally to sketch for you and to prove to you the motive of that method at which I have temporarily arrived. This ward has ever stood in the foremost ranks with regard to the development of antiseptics, notwithstanding the unfarorable conditions existing in this hundred-andfifty-year-old hospital. It possesses the honor of being the first ward in the world to introduce Lister's antiseptic method, in that Saxtorph, so soon as Lister's announcement appeared, travelled to Edinburgh and made himself familiar with the method, carrying it home with him to his ward, where it was actually adopted in 1867. As already emphasized, the effect on the results of the ward's operations was at once extraordinarily propitious; but soon it was clear that the system was by no means infallible or ideal. In spite of all precautions, serious wound infections now and again appeared, and those demands which we now make upon our antiseptic methods, the latter were far from being able to satisfy. At that time laparotomy was a rare and important event in the ward, and one which was performed under great tension and only by bringing the antiseptic preparations to their finest edge, and yet it was seldom satisfactory; while now, you see us perform hundreds of laparotomies yearly without the after- 
effects leaving anything to be desired, as is the case in other operations, and it is easy enough now to see the errors in Lister's original teachings.

The chief mistake was an exaggerated belief in carbolic acid and its infallible effect as regards impregnated matter, and the lack of appreciation of the necessity and significance of general cleanliness. At that time the antiseptic dressing consisted of eight layers of carbolic gauze, above which was laid a piece of mackintosh, and we as volunteers were entrusted with the making of these bandages. We spread the large pieces of carbolic gauze, prepared by an English chemist and imported from England, over the beds in the various wards, where they often dragged on the dirty floors, while we measured them off and cut them out with uncleansed scissors and hands. We then rolled up the neatly-folded compresses in that piece of mackintosh intended for the outer layer, placed a wrapper of gauze round the parcel, and walked around with our parcels till occasion arose to make use of them. Just as we had absolute faith in the carbolic acid contained in the bandage, so also we regarded our hands as being perfectly antiseptic when we had dipped them in 5 per cent. carbolic solution, without attaching any importance to the mechanical cleansing of the skin. Another mistake was the overestimation of the danger from atmospheric infection, which led to the employment of that, in so many respects, disagreeable, carbolic spray, the employment of which was deemed quite indispensable to every operation, and woe to the unlucky volunteer who had not sufficient spirit in his lamp to enable the spray to exhale its vapor during the operation! We have since learned that the importance of atmospheric infection sinks into insignificance when compared with that of contagious infection.

It appeared, moreover, that the strong 5 per cent. carbolic solution had direct evil effects, in that toxic symptoms sometimes appeared, due to absorption from the wounds, and often caused necrosis of the tissues, which made the 
exposed surfaces an easy prey to infection. This was especially true with regard to the danger from poisoning, in a yet greater degree with the sublimate solutions which were introduced as antiseptics by Robert Koch and on his recommendation most extensively used, especially in Germany, where it superseded carbolic acid. Even in Saxtorph's time such experiences effected a reaction against Lister's antiseptic, a reaction which gave birth to the socalled Aseptic Method, which banished all antiseptics from wound treatment, substituting in their place an extraordinarily thorough cleanliness, by means of which infection was to be excluded from wounds.

The most ardent apostle of the doctrine of the aseptic method was the German surgeon Neuber, of Kiel, who, since 1883, had pursued the most thorough aseptic technic in his private hospital. Even now a visit there will show you the aseptic method in its pure and most perfect form. Soap and clean water-spring water-effect the disinfection, both of the patient and of the surgeon's hands, while the instruments and dressing materials are either boiled or sterilized in gushing steam. During the operation irrigation is employed with quantities of boiled water, and it is part of the system that the patients shall be rigidly separated into three classes-the aseptic, the infected, and the septic-all of whom have their special building and operating accommodations, the pathogenic miçrobes not being allowed to gain access to the aseptic ward. Yes, if only it were so easy to separate the sheep from the goats, the clean from the unclean; then, indeed, it would not be so difficult to uphold asepsis. But it so happens, unfortunately, that at times the thoroughly purged and apparently aseptic skin may conceal pyogenic microbes below the surface, in the hair follicles and gland ducts. We see it often in performing laparotomy, where, after many days of scrupulous preparation, we have worked our way through the aseptically-pre- 
pared skin and through the abdominal wall to the peritoneum, on whose behalf this aseptic work has been performed, we are compelled, just in this spot, to open the way for myriads of pathogenic germs, which are thus let loose upon the clean aseptic wound and are spread about the operating table and the aseptic operating room with pus and rinsing water.

Aseptic treatment, even in this its purest form, is not in reality entirely different from or even opposed to antiseptic treatment in Lister's sense, as is so frequently stated, but is in reality only another form, a form which most certainly does not make use of chemical antiseptics, while it does make use of mechanical and physical means, not only with regard to the removal of bacteria but also their direct annihilation by boiling and sterilizing the instruments and dressing materials. Very few surgeons, however, have refrained, as the consistent Neuber did, from the use of chemical antiseptics in the treatment of wounds. All surgeons now term themselves aseptic, so to speak, though in reality they employ chemical antiseptics on an extended scale. They wash themselves and the patient with ether, alcohol, solutions of sublimate, lysol, carbolic solutions, or any other of the many antiseptics, and do not restrict themselves to these. Even in the first years of asepsis, iodoform smuggled itself in as an auxiliary ; for not only did one sprinkle the line of sutures with it, but it was customary to powder the healthy wound surface with it, in the belief that the iodine would be disintegrated and would have a destructive effect upon the microbes (Binz). The use of this preparation is a fair example of the unscientific way in which medical men sometimes accept theories without proof. The drug was proclaimed by the well-esteemed Viennese surgeon, Mosetig von Moorhoff, as a wonderfully effective antiseptic, with these proud words, "Ubi jodoformium non sepsis," though without any scientific examination what 
ever, and on the basis of only some superficial clinical observations. One thought that because no putrefying odor was developed in wounds treated with iodoform, this could only be explained by the potency of a wonderful antiseptic; and, without opposition or criticism of any kind, this socalled antiseptic made its entry into all hospitals, and superseded the real and good antiseptics. One soon became aware of a great drawback to this remedy-i.e., the danger from frequent fatal poisoning which accompanied its use. This danger was all the more insidious because it did not depend alone on the amount of iodoform used, but far oftener poisoning was due to an incalculable idiosyncrasy in many people in whom doses of even one grain might produce fatal poisoning. This is often demonstrated in a very sinister way, in that the patients suddenly become a prey to psychoses of a maniacal nature; they shout and scream for hours, or, as one knows from a long list of unfortunate cases, they may jump out of bed and fling themselves through the windows. But in addition to this, however, the serious poisonings are characterized by an acute fatty degeneration of the heart and parenchymatous organs. Stubenrauch has also pointed out that the local application of iodoform to the wound surfaces of parenchymatous organs, such as the kidneys or the liver, involves rapid fatty degeneration to a great extent in the adjacent parenchyma. You must, therefore, be carefully warned against applying iodoform to such wounds. It was even more of a shock to discover that its supposed power as a germicide, which was to counterbalance the said drawbacks, did not in reality exist. It was I, as perhaps you know, who in 1886, while a young physician in this hospital, made this sad discovery. I had noticed that the wounds which were systematically sprinkled with iodoform by means of the spray were all more or less suppurating. It then occurred to me to deposit the contents of the iodoform 
spray on gelatin plates, and I found, to my surprise, that numerous colonies of Staphylococcus pyogenes aureus appeared. In other words, this antiseptic, which enjoyed the blind confidence of the whole world, was replete with virulent pus-producing microbes. A series of experimental examinations which I undertook in this hospital, together with Chr. Heyn, a contemporary, proved that in reality this iodoform was quite ineffectual when confronted with the common pus-producing organisms, and in a later essay I showed that iodoform, when used against tubercular bacilli -for which it was generally considered a specific remedywas just as ineffectual. Not only was the announcement of these facts received with mistrust, but our modest suggestion that in the future one ought to disinfect the iodoform before using it was greeted with that wrath which one generally expects will be meted out only to him who attacks some holy faith. However, a long series of investigations soon proved that the facts were as stated, and little by little, though very tardily, the iodoform was expelled from its unmerited position among our true surgical antiseptics.

Many attempts have been made to rehabilitate iodoform. Behring and de Ruyter found that it combined with certain ptomaines of putrefaction, and considered, in consequence, that this might quite outweigh its inefficiency as a microbedestroying agent. Naturally, however, we cannot agree with them in this, because, although this qualification may have a favorable effect locally, and is, perhaps, the reason why the iodoform gauze tampons in the vagina and rectum prevent the odor of putrefaction for a much longer period than ordinary gauze does, yet it has no influence whatever on the virulence of the bacteria or on their further penetration into the body. It is reputed as having good effect upon one single specific microbe-Ducrey's bacillus, the cause of soft chancre. 
The latest attempt to produce the long-missing proof that "iodoform is a true antiseptic" emanates from Heile of Breslau. Heile's ${ }^{1}$ investigations are very interesting, though more destructive for the practical use of iodoform than of anything else, because he shows that iodoform is quite useless as a germicide wherever air is admitted. It is not until the air has been excluded and has blended intimately with certain tissues, such as the liver tissue, that a poison is formed-dijodacetyliden, which is extraordinarily poisonous for the bacteria, but unfortunately, also, for the human organism. Heile himself says that the germicidal effect of iodoform depends upon the production of this substance; but also, unfortunately, the much too frequently occurring iodoform poisoning. Afterwards he mentions a case where a patient died with indubitable evidence of acute yellow atrophy of the liver, following the introduction of an iodoform gauze tampon in a case of resection of the liver. You can understand why I am not won over to the cause of iodoform by these facts.

When, in 1886, my predecessor, Oscar Bloch, assumed the position of head surgeon in Ward C, just such a mingling of aseptics with iodoform and sublimate antiseptics was in general use. In the beginning he remained faithful to the Lister methods of antisepsis such as he had succeeded to after Saxtorph. Later its impractical side quickly evinced itself under his critical regard.

That incorrigible rogue-carbolic spray-liad first to take its departure, for further investigation showed that atmospheric infection,-i.e., infection of the wound during operation with the bacteria floating in the air, which was so dreaded by Lister,-played quite an insignificant rôle in comparison with infection caused by touching the wound with unclean fingers and utensils. Thus the importance of

${ }^{1}$ Heile: "Ueber die antiseptische Wirkung des Iodoform," Langenbeks Archiv, Bd. lxxi, p. 787 (1902). 
the spray in antiseptic surgery diminished so that its value by no means counterbalanced the inconvenience it caused by enveloping the area of operation in a dense mist of carbolic vapor, or the confusion it caused when the spirit-lamp either went out or caused conflagration because an ether tampon or a dry wadding or a jute bandage approached it too closely. With a sigh of relief the spray was set aside with other historic objects. Soon Bloch abolished the typical Lister dressing, and in place of the damp antiseptic bandage of eight layers of Scotch carbolic gauze covered with mackintosh, he fashioned a dry aseptic bandage of high value. In place of the bandage material, impregnated with carbolic, and for that reason quite untrustworthy, he substituted vapor sterilized gauze and wadding. Bloch's bandage differed from that used by the Germans in that he used non-absorbent cotton for the outer covering, while in other parts of the world men used, as they still do, absorbent wadding, which has the unfortunate result that the dressing becomes quickly saturated directly over the wound, and this drenched material forms a connection between the wound and the outer world, quickly conveying infection from one to the other. With Bloch's dressing, on the other hand, the wound secretions are compelled to expand in ratio to the surface. Saturation begins when the entire mass of gauze is sucked full, and always at the edge of the bandage, away from the wound, so that, if one shifts the former rapidly the infection from outside has no time to diffuse itself in the wound. The non-absorbent wadding, as we even then knew from bacteriological experiments with the re-agent glass, is absolutely impenetrable to bacteria, and at the same time possesses the great advantage, in full measure, of allowing the filtered air to pass, which causes the secretion in the interior of the bandage to dry up, and thereby delays and prevents saturation. It should, however, be noted that Bloch is not the first who has used this non-absorbent wad- 
ding as a dressing material: the honor of this is due to the French surgeon Guérin, who, even before the introduction of Lister's antiseptic method, had applied this dressing with great success. A peculiarity of Bloch's dressing was that he packed it between two

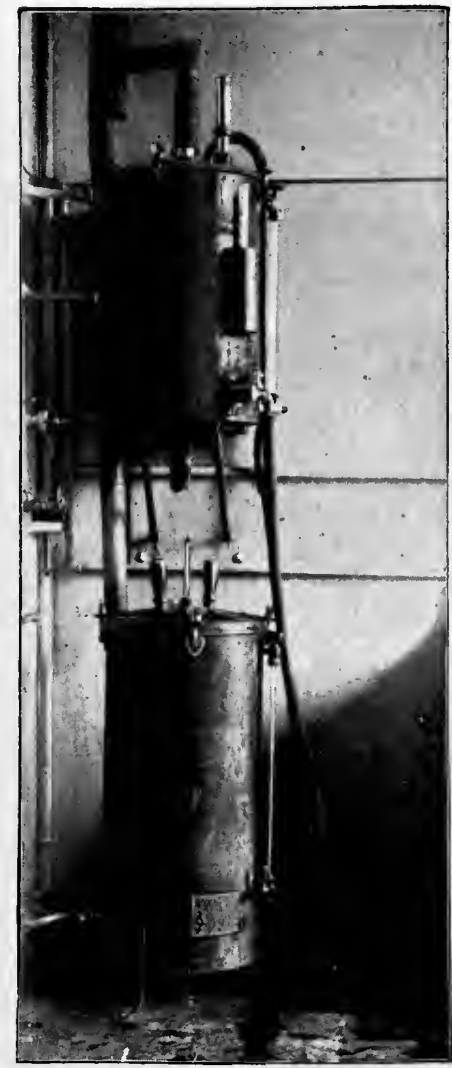

FIg. 1.-Water sterilizer which has an internal spiral copper pipe, through which cold water can be conducted, in order that the boiling water may quickly be reduced to the required temperature $-40^{\circ} \mathrm{C}$. layers of filter-paper, so that it was possible to move and preserve the dressing without incurring any breach of the aseptic technic, thus the surgeon who was to use the dressing allowed some other person to open the outer and unclean paper, while he himself, with sterilized hands, seized the inner parcel, which was aseptic. This excellent dressing, certainly the best in the world, and one which you see in constant use in this ward, is highly antiseptic, insomuch as the non-absorbent wadding renders entrance to the wound impossible for microbes. But in reality this was an off-shoot of the German's aseptic method. In order to make it an antiseptic dressing, in Lister's sense, Bloch used to moisten the innermost gauze with 3 per cent. carbolic water.

Antiseptic surgery is indebted to Blocl for still another contribution, namely, the first reliable sterile catgut-the so-called carbolic alcoholic catgut. Moreover, Bloch improved antiseptic methods first and foremost by absolute cleanliness in every direction; 
among other things, by introducing operating aprons, etc. If we add further that Bloch continued to use iodoform, in the form of sterilized iodoform gauze for tampons, in the vagina and rectum and throat, and as méche in infected, and especially in tubercular wounds, you have, on the whole, the main features of the antiseptic methods as they existed in this ward when I took cliarge of it.

We now arrive at the third stage-the development of antiseptics in this ward since I have been in charge. Firstly, there is the extraordinary increase in our operative activity, especially with regard to laparotomy, which re-

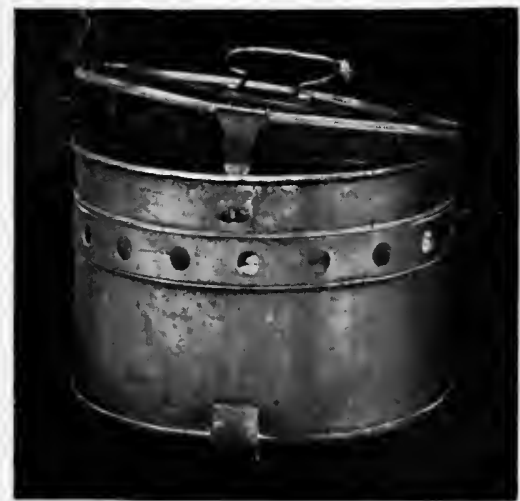

Frg. 2.-Pail for bandages.

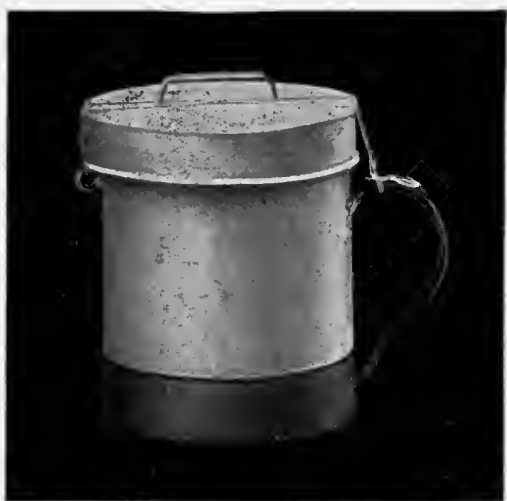

Fra. 3.-Pail for gauze and wadding tampons.

stricts me to improvements of a quantitative nature. In two particulars especially I encountered difficulties. It was impossible, figuratively speaking, to obtain large quantities of warm sterilized water quickly, and at night it was quite impossible to do so, as all our warm water was conveyed to us in barrels from the laundry, which, of course, was closed at night; and, further, it was impossible to have a sufficiency of sterilized bandages, aprons, sheets, etc. I therefore had an apparatus installed in the lobby of the laparotomy room, which apparatus, in the course of a short time, sterilized 70 litres of water (Fig. 1), together with an - autoclave for sterilizing bandage material with vapor. 
When visiting the wards, we no longer use the packed bandages, but, for changing the dressings during operations, we use large nickelled metal pails, such as you see in Fig. 2, three of which pails can be contained in the autoclave at the same time. Each pail contains layers of material for a large number of bandages, and these are stowed away together in such a manner that the non-absorbent wadding forms the boundary between the layers, and thus undoubtedly protects each layer from any infection which might arise from the layer previously removed. We use only packed bandages when a dressing has to be removed independently of ward visits, and therefore the nurse in each ward has a certain number of packed dressings in case of emergency : in the same way that the medical practitioner should always have at hand a certain number of Bloch's dressing packages.

But in addition to these improvements, which have been made essentially necessary on account of increased activity, the antiseptic methods here in this ward have also developed in qualitative fundamental tendency, of which you will hear later on. 


\section{LECTURE III}

\section{ANTISEPTIC METHODS-PART II}

\section{Present Antiseptic Methods Employed in the Ward}

Is spite of all the advances made in perfecting the antiseptic methods which I have described to you in my last lecture, these antiseptic methods cannot be termed ideal. Even in the so-called "aseptic" operations, where one has operated in a perfectly clean field, in a case where no inflammatory or infectious disease existed, one saw-and by no means rarely-that the course of the wound healing was not always so ideal as might have been expected; that it was interfered with, to a greater or less extent, by a more or less extended and deeply-seated suppuration. The cause of this was discussed with exceptional eagerness, and, as the suppuration generally appeared first in the suture stitch holes, one was inclined, for a long time, to lay the blame on the material used for the suture, and the catgut thread, which, as you know, is prepared from sheep's intestines, was strongly suspected. Yes, at once and as a matter of course, one termed these cases "catgut suppurations." Therefore, we directed our efforts, for a long time, toward finding more perfect methods for sterilization. Some replaced the catgut with silk, which could be safely sterilized by boiling. But these efforts did not lead to the desired result, and this is not so strange, because, later on, it appeared that the cause lay in quite another direction, viz., previously overlooked difficulties in absolutely cleansing the skin against microbes.

In the early days of antiseptics we had a blind faith in the efficacy of carbolic. We believed that a light application of 3 to 5 per cent. carbolic acid solution would most surely 
sterilize the skin. The merit of proving that the problem was more complicated is due to Kümmel, who, in 1886, by a series of rational bacteriological experiments, was the first to prove that a most energetic and persevering mechanical cleansing with soap, water, nail brush and nail cleaner, etc., was the first and most important step toward making the skin assuredly sterile by means of the subsequent application of chemical antiseptics. This fact was made clear when cultures from skin treated in this manner gave negative results in nutritive media. Then when Fürbringer, in 1888, had taught us to treat the skin with alcohol after the washing with soap, we really thought that we had reached the solution of this question. It is quite certain that there is no better method of disinfecting than Fürbringer's, especially when modified, as I use it,-i.e., dilution of the proof alcohol with equal parts of 1 per cent. sublimate solution; for alcohol evinces a far stronger antiseptic ability when diluted with water-50 per cent. for preference--and when other antiseptics are mixed with it, it increases the germicidal quality of these to a great extent. In addition to the effect the alcolol has in dissolving the fat and hardening the skin, it appears to be a powerful germicide. Numerous cultural experiments have proved that by these means the. skin becomes completely sterile-on the surface, but, unfortunately, only on the surface; and the skin does not, as one had imagined, become entirely and thoroughly free of microbes. Very clever experiments, especially those from Mikulicz's school, ${ }^{1}$ have taught us this. The antiseptic effect does not thoroughly penetrate the hair follicles and sebaceous glands, and microbes may hide in these recesses, even when the surface is perfectly sterile, and these bacteria, after a time, are brought to the surface by the perspiration and fat secretions. The period required for this to take place is quite indefinite, varying, according to circum-

${ }^{3}$ Gottstein: Beitr. z. Klin. Chir., 1899, B. 24-25. 
stances, from a few minutes to a quarter of an hour; but with every extensive operation the surgeon's hands will become again infected.

Just the same thing will occur with the skin of the patient. It is perfectly microbe-free at the commencement of the operation, but will seldom be so at the conclusion; and beneath one of the hitherto mentioned dressings the perspiration and fat secretions will, in any case, soon convey the bacteria to the surface, and bring them into contact with the wound. Therefore it is not so strange that Bloch, by his interesting investigations into the so-called clean aseptic operation wounds, always found microbes present. At that time these investigations and their result caused great surprise. Even greater surprise was caused when it was found that these wounds healed, nevertheless, without suppuration. Now, however, we understand this also. It is due simply to the circumstance that, as a rule, the germs that are present are innocuous or non-pathogenic. Therefore the question of infection does not play a prominent rôle where the patient's skin is healthy; the danger lies solely in the operation itself. But it is a question of great importance when a patient's skin is, or has been, the seat of an infectious disease-furunculus, acne, etc.,--from which the glands, even in apparently healthy parts of the skin, may be infected.

A great danger also exists because the surgeon's hands cannot be safely sterilized, for every day, in the course of his official duties, he is compelled to touch highly infectious substances. In opening large abscesses, especially abdominal abscesses, he often has to hold his hand for quite a long time in pus which swarms with pyrogenic microbes; and, shortly afterward, perhaps, he may have to perform laparotomy or some other big operation: amputation, resection, trephining, etc. You will easily understand how great is the danger incurred by the clean wound from contact 
with the surgeon's hands, even if he has washed and disinfected these according to the best methods and with the greatest care.

Since we learned how to eliminate the surgeon's fingers as an infectious factor, we have removed the main source of many mysterious wound infections following clean operations. This fact is strongly borne out by the results which we have obtained in this old, thoroughly-infected ward, where all cases, even the most serious and highly virulent infections and aseptic abdominal cases, lie side by side.

The means of elimination consist in the use of rubber gloves, the introduction of which I consider to be one of the most important links in the development of antisepsis in this ward since I have been in charge. Important not only for those among you who in the future may hold important surgical appointments, such as that of hospital surgeon, but for every medical man who has to perform sporadic operative work, and who, a few minutes after operating for phlegmon or panaritium, may be summoned to a confinement necessitating the introduction of his hand into the patient's uterus in order to deliver a retained placenta, etc. In short, it is of the utmost importance that you all know of the advantage which exists in the use of rubber gloves. Let me, then, in a few words precisely define for you the indications for their use. First of all, you must cover your hands with rubber gloves in every examination or operation in infected areas; in explorations in the rectum and vagina; with the opening of phlegmons, abscesses, peritonitis, etc. Thereby you will not only insure yourself against infections, specific and non-specific, but you will secure greater safety from the danger of infecting your patients in clean operations, even though you perform these without rubber gloves. There are surgeons who use rubber gloves only in septic cases and in examinations, 
giving two reasons for not using them in clean operations: (1) That they decidedly impede the more complicated operations by reducing the sense of touch, and by their smoothness make manipulation uncertain, especially the handling of instruments; and (2) that if, as may easily happen, the glove is pricked by a needle or torn by tweezers, then there is relatively greater danger of infection from the accumulated perspiration inside the glove. It is quite certain that the difficulties here mentioned exist, but they are so easily overcome that they need not be considered, when compared with the great-yes, perfect-safety which the systematic use of rubber gloves affords the patient in every operation. The difficulties connected with a delicate touch disappear with a few days' practice; but more serious is the objection that smooth instruments and smooth organs are not easily held with smooth rubber gloves; that these latter are easily damaged by a slip of the needle or by some other instrument. We easily avoid both these drawbacks by using cotton gloves over the rubber ones (Fig. 4). With thread gloves one gets a perfectly safe grip of the instruments and smooth organs-yes, a much safer hold than with the bare hand-and they afford provision against lesions of the rubber gloves. On the other hand, one cannot palpate so delicately with the doubly-gloved hand; but, then, we quite simply pull off the thread glove whenever we wish to palpate finely. The great advantage of this systematic use of gloves lies in this; that even the busiest surgeon's hands are kept perfectly smooth and free from those furrows and fissures which make it so difficult, even impossible, to really disinfect the skin. The surgeons who work without gloves must frequently scrub their hands with a brush, not only before but during the operation, whereby they develop many little abrasions of the skin. The skin becomes rough and peels off the whole hand. Still 
more dangerous are the deep fissures which develop on the radial side of the forefinger, which is used so mueh for tying ligatures and sutures. These fissures, often very deep, easily become the seat of inflammation, and the pathogenie

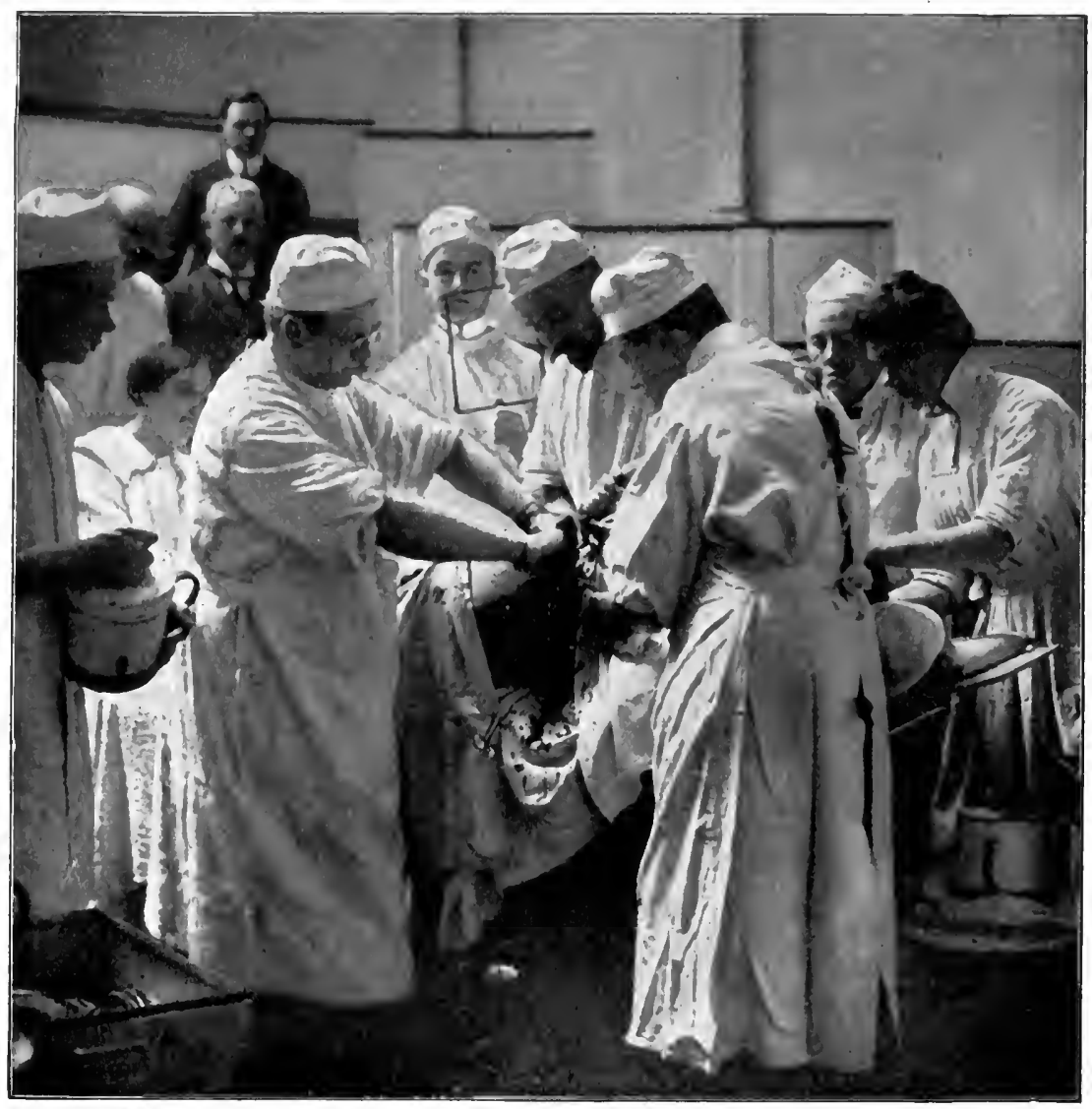

Frg. 4.-Operation in the large operating theatre. The operator and assistants are seen wearing sterile eaps and linen gowns, rubber and cotton gloves.

microbes with which the physician eomes in touch are here caught and leld. As such a skin is far less adaptable to complete disinfection, one will easily understand the danger these fissures involve for patient and physieian. I have no doubt that most of the so-called catgut infections are 
not due to deficient sterilization, but that the catgut became infected by the surgeon's hands while being manipulated.

Of far lesser importance, though worthy of notice as a source of infection during operations, is the surgeon's hair and the perspiration and particles of saliva which, while he is speaking, may enter the wound. Of these factors I consider the hair to be the most essential, because often, in their eagerness to see, the surgeon and his assistant approach one another too closely, so that the hairs of their heads touch, and thus dust may be disturbed and fall down. Therefore we always wear sterilized linen caps (Fig. 4), while the assisting nurses, in order to avoid the possibility of infection from perspiration and saliva particles, tie a piece of sterile gauze round their hair. Many surgeons abroad and a few at home use a kind of nose-bag, or better, perhaps, a Turkish veil which covers the face completely, with the exception of. the eyes (Fig. 5). This, however, I find somewhat of an exaggeration and too inconvenient compared with the danger from these two sources of infection, which can be counteracted by always

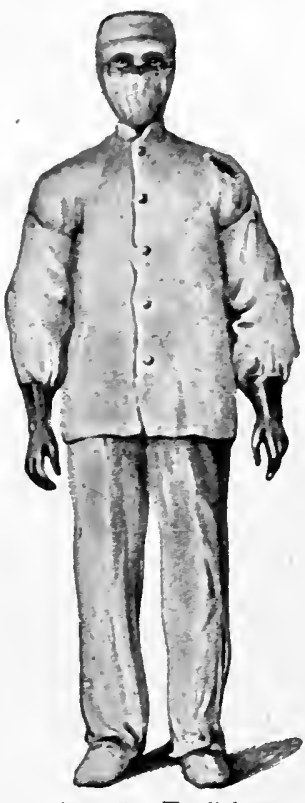

Fig. 5.-English surgeon with rubber gloves, sterile cap and spectacle mask fixcd at the top to a spectacle frame. (Moynihan.)

having a nurse ready to wipe away the perspiration with sterile gauze, and, with regard to the saliva, by speaking as little and as softly as possible during the operation. This is also greatly to be recommended in other respects.

Now, with regard to the patient's skin, we are far from likely to find pathogenic bacteria hiding beneath the skin. Every now and again, however, this happens, and there is no doubt that a good many unexpected wound suppurations 
are due to this cause. Therefore methods have been devised to cover the patient's skin during the operation; for instance, by coating the skin with a solution of paraffin or with a solution of gutta-percha, which, by the evaporation of the solvent, remains as a fixed rubber coating and closes all the pores of the skin. I do not believe that these beautifully devised methods are of any real importance, because, however carefully we cover the skin before the operation, yet we cannot avoid opening up the deeper lying gland ducts by our incision, at which time

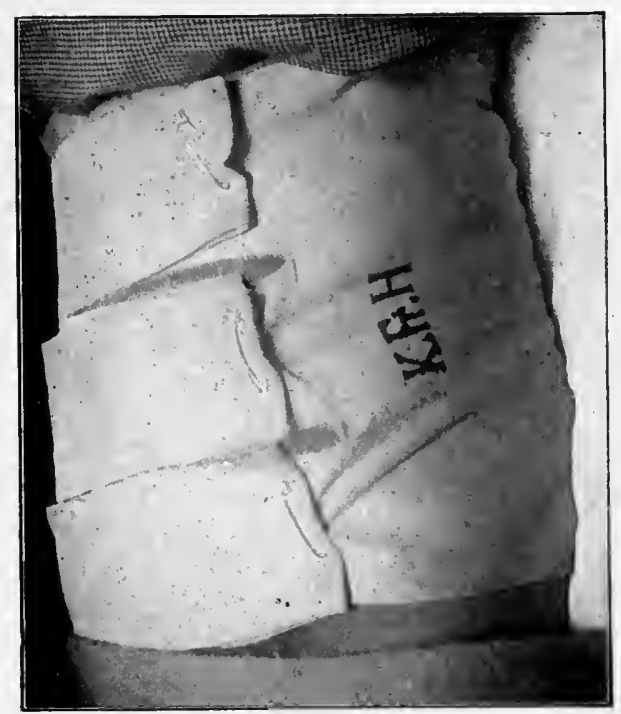

FIG. 6.-Cingulum dressing after abdominal operation. the microbes therein contained have free access to the wound.

On the other hand, we can protect the sutured wound against subsequent infection from the surface, partly from those microbes which advance by degrees to the surface, and partly from those -and this is of much greater importancewhich are spread over the skin through the drainage openings, which occurs whenever we make our way through the healthy skin to a deep-lying infected organ, in order to drain it. As an example, let me mention nephrotomy or nephrolithotomy of an infected kidney. Here, if you simply stitch up the wound in front of and behind the drainage opening, you will find, to your chagrin, that in a great number of cases suppuration sets in, after a few days, in all the stitch holes; and if you have sutured with catgut, the entire suture line breaks down, and you have a large suppurat- 
ing wound, the healing of which under the most favorable circumstances takes a long time, and which, with virulent infections, may involve serious danger to the patient's life. Such suppurations, formerly rather frequent, I seldom see now, because for fifteen years I have sealed the suture line hermetically with collodion and cotton wool. When the suturing is completed, wash the suture line with pure alcohol, and by wiping this with cotton wool the line of the wound and its surroundings become so dry that the collodion adheres. Then place a thin strip of sterile cotton wool over the line of the wound and coat the edges of this strip so that it adheres to the skin, using a small sterilized camel's-hair brush for the purpose. Next coat over the centre, when the collodion and cotton wool form a combination $\mathrm{wh} \mathrm{i} \mathrm{ch}$ everywhere adheres to the wound and its surroundings. It is ab-

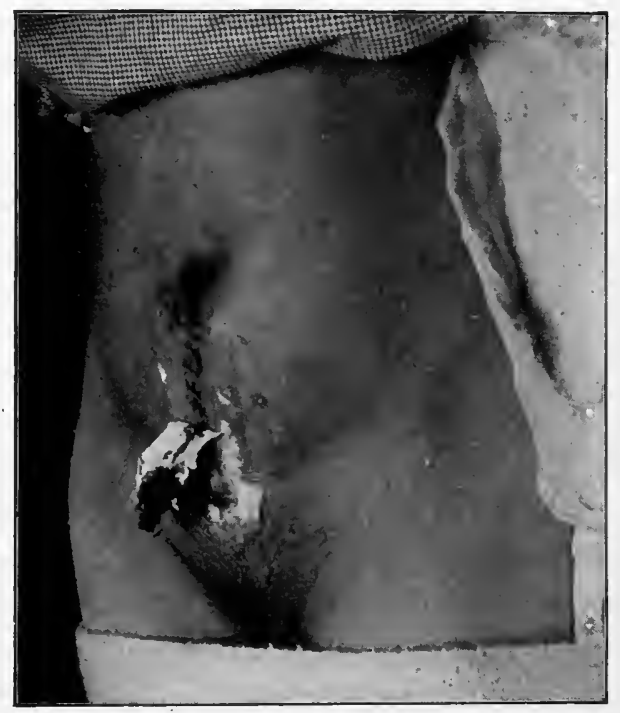

FIG. 7. The collodion-cotton wool covering is being removed

solutely impermeable to air and water, and, on the other hand, closes all the pores of the skin and prevents the bacteria contained in the glands from appearing on the surface.

I now wish to show you some patients treated with this collodion-cotton wool dressing. Here you see a patient on whom, a fortnight ago, I performed a complete extirpation of the uterus. You will notice that the patient's entire dressing consists of a cingulum which is fastened with three safety pins round a piece of non-absorbent cotton wool 
(Fig. 6), and on removing this you will notice in the centre the collodion-cotton wool strip, $16 \mathrm{~cm}$. long, which has covered the line of the wound for fourteen days. This dressing is so simple in all those cases where we can join the wound in its entirety that you will easily understand how advantageous it is, since you can inspect and palpate the abdomen and the immediate environs of the wound in particular without imperiling the wound by infection, when one

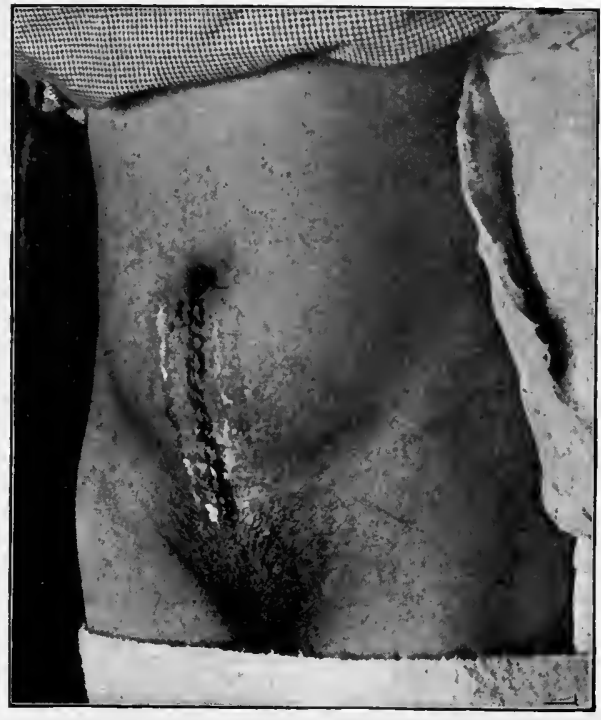

F1a. 8.-After removal of the covering, aluminum bronze threads are seen in the wound healed without reaction.

ed (Fig. 8) without any reaction. symptom or another gives cause for alarm. You will notice that the skin at the side of the collodion-cotton wool covering is quite natural (Fig. 7). I now loosen the covering at the upper end of the wound with the aid of a pair of tweezers until I can take hold of it with my fingers, when with a slight pull I am able to remove it entirely without causing the patient any pain. You see that the wound is quite healsutures, which consist of aluminum bronze thread, and, as every suture opening must be regarded as a small and still open wound, the line of wound is once more covered with collodion and cotton wool (Fig. 9), which may be removed after a few days or left till it falls off of its own accord. In those cases where the skin is sutured with catgut the remains of the thread usually come away with the collodioncotton wool. 
In most cases it will be quite sufficient to cover the wound with collodion-cotton wool, but with important operations I further cover the entire wound area with sterilized, nonabsorbent cotton wool, in order to insure the wound against infection in those rare cases where a hæmatoma may form and loosen the collodion at some point. The collodion-cotton wool covering is an excellent and practical dressing, especially on the hands and feet, as it allows the free use of these together with the ordinary coverings of gloves and boots, while providing the $\mathrm{g}$ r e a t e s $t$ safety against infection.

I shall now show you two cases where I have been unable to unite and cover the wound throughout its entire extent, because the infectious nature of the disease has necessitated drainage. Here the collodion-cotton wool

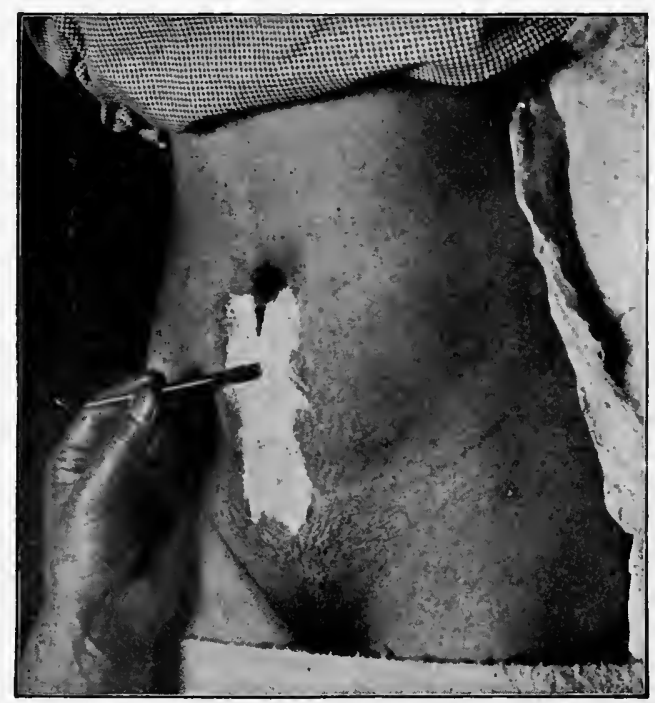

FIG. 9.-Line of wound covered with collodion-cotton wool.

covering has rendered excellent service by protecting the joined portion of the wound against infection.

One patient, a man forty years of age, was operated upon by me twelve days ago for a calculus pyonephrosis with a great accumulation of pus containing staphylococci. After the removal of the stones I inserted a large drain in the kidney cavity and sewed the kidney wound tightly around the drain, which was led out through the lumbar wound and down into a glass at the side of the bed. You will see that I have sutured the remainder of the lumbar wound above 
and below the drain and covered it with collodion-cotton wool. The suturing is performed in three layers: fascia transversa being joined by silverized catgut, musculus obliquus ext. by aluminum bronze which is buried, while the aluminum bronze of the skin is now removed. After I have removed the bandage and the non-absorbent cotton wool flap, you will see that the neighborhood of the drainage opening is covered with dark-brownish gauze. This is silver nitrate (lapis) and has to intercept the secretion which oozes along the drain. . You will notice that a fair quantity has oozed into the gauze; but, after the collodion-cotton wool has been removed, you will see that the entire lumbar wound has healed per primam intentionem.

The other patient whom I am about to show to you was operated upon seven days ago for a very serious gangrenous appendicitis with diffuse peritonitis. I made a long, slanting incision in the right iliac fossa and removed the appendix, but by reason of the extent of the peritonitis it was necessary to drain thoroughly. In this instance the form of drainage differs greatly from that which you saw in the former case. It consists of the so-called Mikulicz's bag drainage, with a rather important modification introduced by me. The clever part of the Mikulicz's method is that through a small opening in the abdominal or the chest wall an enormous tampon can be used and removed, and, furthermore, that this can be removed gradually, so that the cavity which we are draining is allowed to contract by degrees. Mikulicz accomplished this by using a large, square gauze handkerchief, in the centre of which was attached a thread; he introduced this into the cavity which he proposed to drain in such a manner that the corners and edges of the handkerchief lay outside the skin, thus forming a bag. Long strips of gauze were then packed into the bag thus formed, around the central silk thread, the ends of the strips leading out of the wound through the thin neck of the bag. The illustration 
(Fig. 10) will best explain this. The wound is now sutured around the neck of the sack and the numerous strips, acting as wicks, drain the secretions into the outer dressing. Later on one removes the large mass of tampon either strip by strip, little by little, or all at once, through the relatively small opening. Finally, the bag itself is removed. Iodoform gauze or dry sterile gauze was formerly used for this; but these, which are excellent when the question is one of stopping bleeding, have displayed several unfortunate defects where drainage is concerned, and especially so in the peritoneal cavity. The fact is that the gauze quickly adheres to the walls of the wound cavity and produces adhesions between the adjacent intestines and peritoneum whereby the peritoneal cavity is shut off from the wound cavity. This may be fortunate enough in some cases where the inflammation has been previously en-

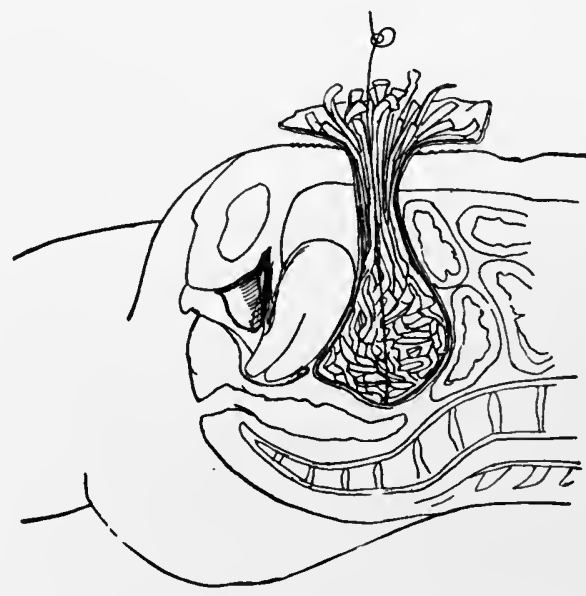

Frg.10.-Sack drainage (after Mikulicz) used in the fossa of Douglass in a case of suppurative peritonitis. capsulated, but it is, naturally, very unfortunate where ${ }^{\bullet}$ we want to drain the peritoneal cavity and to hold the way open to this. Added to this is the fact that the bag often becomes adherent to the peritoneum and the intestines, which causes difficulty in its removal. Often serious bleeding takes place and adhesions are broken up. At times the removal becomes so difficult that the weakened patient has to be placed under an anæsthetic once more. I have corrected the unpleasant features of the method by the simple modification you notice here (Fig. 11). I have covered the bag, on the outside, with a layer of thin mackintosh provided with a number of longitudinal slits. The water- 
proof side faces the peritoneum and thereby the adhesions are avoided, while the secretion from the peritoneal cavity is absorbed through the slits into the bag, which-and here is my: second modification-is filled with strips of silver nitrate gauze. By these means a strong antiseptic influence is exerted over the wound secretion and the microbes in the walls of the wound cavity, so that the inflammation is often eliminated at once, and in any case the secretion is always

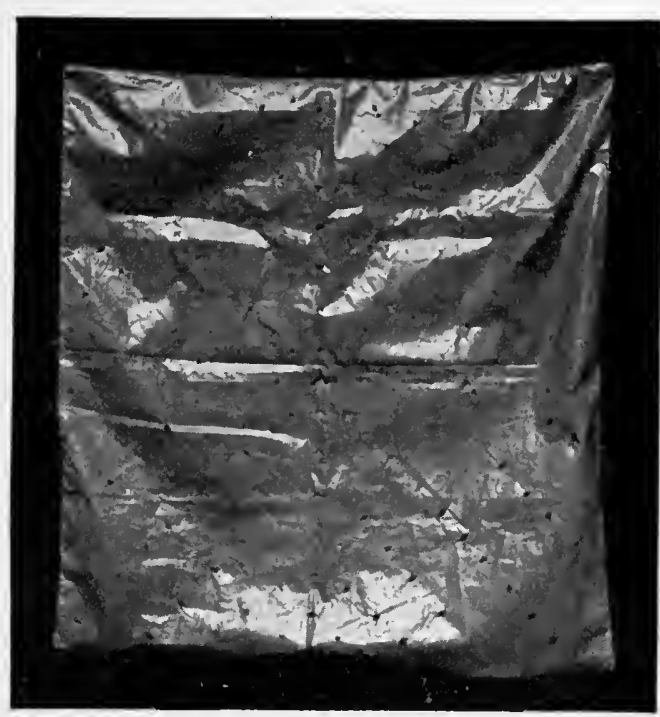

Fig. 11.-Mikulicz's bag covered with slit mackintosh. less in amount and less odorous. You now see how the sutured part of the wound lies perfectly protected by the dry collodion-c o $t \mathrm{t}$ o $\mathrm{n}$ wool covering, which I leave untouched until the threads have to be $\mathrm{r}$ e moved on the fourteenth $\mathrm{d}$ a $\mathrm{y}$. Meanwhile I will remove the wicks and the bag to let you see how easily this is done. I have now removed the seven gauze strips, which are free from odor and, as you notice, have absorbed splendidly, for not a drop of fluid is retained in the bottom of the bag. I now remove this, and you see that, with a gentle pull here and there at the edge of the bag, it becomes loosened and slips out with the greatest ease and without causing the patient the least pain.

I shall return to the drainage question later on, but let me now conclude my remarks on the collodion-cotton wool dressing. You have seen how excellently it protects clean wounds against infection, but in addition to this it is most 
beneficial in confining the source of an infection (furunculus, infected wound) anywhere contiguous to the area of operation, and in preventing it from spreading along the healthy skin. I also apply the method with furunculosis, which often is not a morbid predisposition to furunculous formation, but simply a spreading of infection caused by incorrect treatment of furuncle, which is a purely local infection. Even now you will often see physicians treat furuncles, which most often occur in the occipital region, with poultices and water fomentations. This, of course, eases the pain and accelerates the perforation of the boil, but the moist warmth greatly favors the growth and spreading of the microbes in the surrounding skin, which has been macerated and made susceptible by the fomentation. The result is that for each furuncle cured in this manner fresh ones are produced, and if the treatment is continued the disease may be carried on through years in a most painful manner. For many years I have successfully availed myself of the simple procedure of separating the furuncle from the surrounding parts with collodion-cotton wool, which is renewed after each treatment until the furuncle is healed. With punctures, also, the collodion-cotton wool dressing is the simplest and surest method of protecting the small wound until it is healed.

While the collodion-cotton wool dressing is our sovereign remedy for the protection of a clean wound joined by suture until healing per primam has supervened, it is, on the other hand, not adapted for certain clean wounds, which, for some reason or other, cannot be joined by suture. Here, first and foremost, I refer to burns. The great extent of burns often interdicts the collodion-cotton wool covering. It would also have a painful and irritating effect on the uncovered nerves, besides increasing the already vigorous serous secretion from the surface of the wound, and this secretion would loosen the collodion. The application 
of dry sterile gauze, and more especially of antiseptic gauzes, of which iodoform gauze is to a great extent dangerous on account of its toxicity, has proved just as unfortunate and irritating to these wounds. This is one of the reasons why many physicians have completely renounced aseptic treatment with these wounds, and aspire to heal per primam, without suppuration, and, in defiance of all antiseptic rules for the treatment of clean wounds, cover them with egg-oil, ointments, and such things. As a result of such treatment many burns which in the beginning are, above all things, aseptic, by reason of their having been caused by fire, steam, or boiling water, are converted into infected suppurating wounds, which then became a source of danger to the patient. In reality, most burns are very responsive to a thorough aseptic treatment, though this requires special technic. For this character of wound I have devised a method which seems to me to satisfy all the special indications and to solve all the special difficulties which burns present. This is as follows: After that portion of the skin which adjoins the burn has been carefully cleansed with soap, water, ether, and alcohol, the burned area is washed with a physiological salt solution, the blebs are opened and the surface is covered with a layer of sterile oilsilk provided with numerous slits. This allows the serous exudate to percolate into the dressings which are placed outside the oilsilk: firstly, silver nitrate gauze; secondly, sterile gauze; and on the outside, non-absorbent cotton-wool. The serum coagulates under the oilsilk like a vitreous cover over the wound and level with the surrounding skin, and from this the epidermis grows out over the cover with astonishing rapidity, favored by the moist heat and the smooth surface of the oilsilk, which keeps away all irritation. It is of marked advantage, too, when the dressings need changing, an ordeal so dreaded by patients suffering from burns, on account of the great pain caused by the 
adherence of the dressing to the wound; with this dressing the procedure is quite painless, since the smooth oilsilk, no matter how long it has been in position, can be removed without the least resistance. The same dressing is the only rational one for Thiersch's transplantation, as it greatly encourages growth and nutrition in the skin grafts, and can be replaced without any risk of displacing them. Furthermore, oilsilk is of great value when, by an operation, we expose or uncover some vital or sensitive organ, such as the brain, the intestines, etc. Finally, we use oilsilk as a dressing in cases where we wish to fill a cavity or to replace a loss of tissue ad modum Schede. You allow the cavity or fissure (as with Phelps's operation for club-foot) to be filled with blood and then place the oilsilk over this, level with the skin. The blood now coagulates under the oilsilk, and the epidermis quickly grows from the edge of the skin across the surface of the coagulated blood, which becomes organized as fibrous tissue, and thus the deficient tissue is supplemented.

Not all aseptic wounds, however, can be sutured and covered with collodion-cotton wool in their entirety. This can be done only where the wound can be closely joined in all its various layers; but where the operation leaves a space, such as occurs after the removal of tumors and entire organs, or with deep cavities, as with resections of the hip-joint, we are compelled to drain. Opinions on this question are at variance, and were especially so in former days, when one believed in the absolute disinfection of the skin, for many surgeons maintained that the drainage itself opened a way for infection from the outside into the wound cavity, whereas a complete closing, performed in the course of a properly-performed aseptic operation, would, to a certainty, exclude infection. We have now learned, however, that an absolute disinfection of the skin is impossible. Furthermore, we have learned that even if Lister's 
fear of atmospheric infection was exaggerated, and the danger of contact infection the essential, yet atmospheric infection is a factor to be reckoned with, especially when operating in an amphitheatre or in operating rooms where many spectators are present.

I shall now allow two Petri dishes filled with gelatin to pass round the room, in order that you may be convinced of the correctness of my remarks. I left one of these dishes in the large auditorium during the operation which was performed in the course of my last lecture. It was a sectio alta which lasted twenty minutes, and you will now notice the number of the atmospheric microbes which, in the course of twenty minutes, have settled on the gelatinous surface (Fig. 12), and how each has here formed its own bacterial colony. You will notice that these colonies can be counted by the hundred and that they vary considerably in appearance. Most of them are whitish or grayish, and I feel convinced that many of these are harmless and not pathogenic microbes. But we are sure to find pathogenic species among them; for instance, we have found several colonies of Staphylococcus pyogenes aureus, and, here and there on the surface, you will find small, bright-yellow groups of this dangerous microbe. The other gelatin plate was left uncovered for twenty minutes during an operation in our laparotomy room, which, previous to every operation, is disinfected from ceiling to floor, and to which only a few spectators, attired in sterile coats, are admitted. You will notice that the number of colonies on this plate (Fig. 13) is certainly far smaller than that on the plate from the amplitheatre, but yet the number is so large that we must perforce assume that some atmospheric pathogenic microbes may by chance be deposited in the wound. This is, of course, of no importance with a closely-joined wound or where the resorbent conditions are favorable; but if we have an enclosed cavity with stagnating blood, then the means are provided 
for a single pathogenic microbe to propagate and to change the hæmatoma into a dangerous accumulation of pus which, if it does not break the suture in time, may give rise to pyæmia and serious intoxication. One should always, therefore, drain such cavities.

The drainage may be performed in different ways. Sometimes tube drainage is most adaptable, for instance with cavities, the walls of which may quickly and easily fall in if the blood merely is drained away, as happens with most superficial operations,-e.g., amputation of the breast, ex-

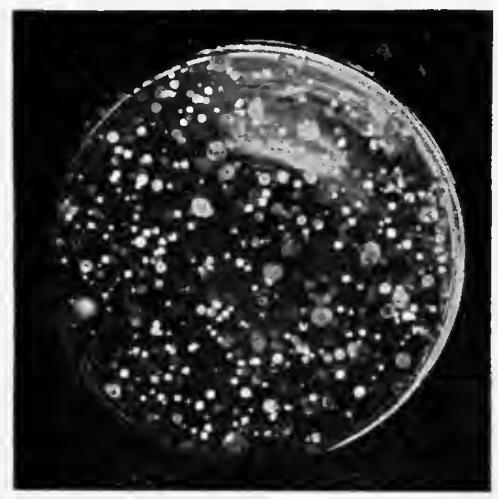

Fia. 12.-Colonies of bacteria on gelatine surface, exposed to the air for 20 minutes during operation in auditorium, about 100 persons being present.

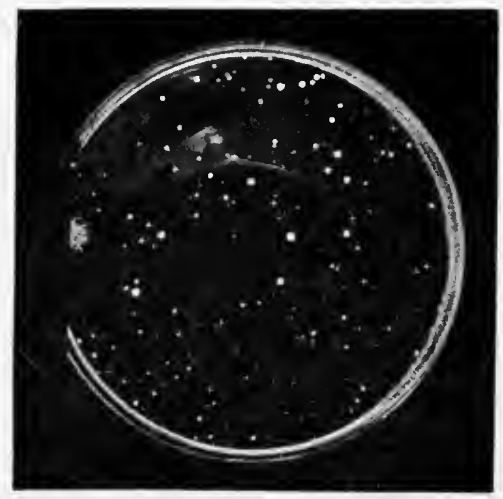

Fra. 13.-Colonies of bacteria on gelatine plate which has been exposed to the air for 20 minutes during operation in laparotomy room, about 20 persons being present.

tirpation of tumors of the neck, etc. In the most dependent part of the wound we introduce a drain tube which, as a rule, is made of india-rubber, as this causes no pressure; though, on the other hand, there is the risk that the tube itself may become compressed and cease to drain. For this reason many surgeons prefer to use glass tubes such as you see here: slightly curved glass tubes fitted with scattered perforations on the side and with two projecting arms at the end to prevent the tube from being drawn in. In many cases these are really practicable, and I would advise you to have them on hand. It is an advantage that they can be easily 
inserted between the sutures, and after the entire wound has been stitched together you can, here and there, in suitable places, insert a small glass drain. If you have to drain large suppurating cavities with stiff walls, such as an empyema of the pleural cavities, large congestive abscesses, or the gall-ducts, the kidney, the brain in hydrocephalus, etc., then you should not cut the drain short and so allow the secretion to ooze into the dressing, but, on the contrary, you should conduct a long drain, fitting, closely into the drainage opening, out through the dressing and down into a glass or, when the patient is able to be up, into a little bottle fastened to the body, as with this patient (Fig. 14) whose pleura we are draining in this manner. The dressing can then remain for weeks without becoming saturated, and we avoid not only the great trouble and expense of constant changes but also the danger of mixed infection from the outside. In patients suffering from spondylitis with congestive abscess we form an opening in the plaster bandage, through which the drain is led out.

A second method is the méche or wick drainage, so called because an absorbing gauze or a wick of some other absorbing material is introduced. But I would strongly advise you against the use of this kind of méche when draining through small apertures, for here the gauze often has a blocking, instead of a draining effect. It is adaptable only when we have to deal with a deep cavity, the walls of which cannot fall together as per example after a resection of the hip, or where the walls are temporarily prevented from falling in, as with certain hemorrhages in the abdomen. In such cases the drainage takes the form of a tampon and acts to control hemorrhage at the same time that it drains. Midway between these two types of drainage stands the cigarette drain, a combination of the tube and méche drains, inasmuch as the absorbing wick is introduced through a glass or rubber tube. I have simplified and modified this 
method by merely rolling the méche in oilsilk, just as one rolls a cigarette. The advantage of this form of drainage is that retention can never occur, because, if the méche is ineffective, the secretion works out along the outer side of

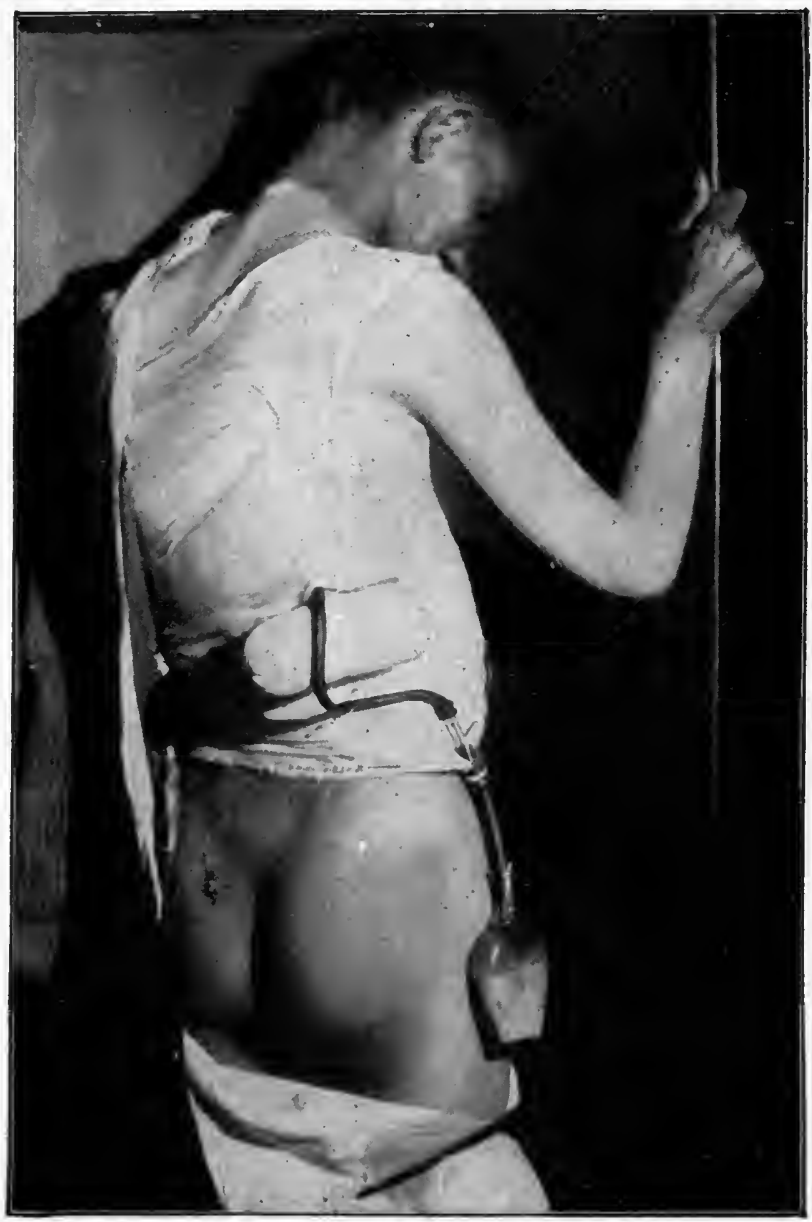

FIG. 14.-Drainage of empyema by drain conducted through the dressing down into a bottlo.

the smooth oilsilk; and it has the added advantage that the removal of the méche drain is painless. With restricted wound tracts, or where the drain opening is very much contracted, I often drain with oilsilk only, as this is preferable 
to the ordinary rubber tube drain, because the escape of the secretions along the side of the drain never ceases, while with the rubber tube drain it often becomes blocked by fibrinous matter and thereby is converted into a plug which causes retention instead of assisting drainage.

We must not forget that the grievance of those people who oppose drainage because the drain may prove a road for infection from the outside into the clean wound is by no means groundless, and this you will often see for yourselves if you use a purely aseptic dressing, because saturation of the dressing opens a way for microbes from the outside to enter the wound. To insure against this you must transgress the aseptic regulations by introducing some antiseptic material as an immediate cover for the wound and drain; but this material must possess the qualifications of being not only very powerful but also of being permanent and non-injurious to the patient. For this reason $I$ do not generally moisten the innermost gauze with carbolic water, because the carbolic is volatile and quickly disappears; nor do I advise dressing material impregnated with sublimate, because this is poisonous.

Here, as so often elsewhere, I make use of nitrate of silver, which is, according to my experience, the best of all the antiseptics, because it combines in itself three supreme qualifications: (1) It quickly and surely destroys bacteria; (2) it is non-poisonous to the organism, even in its most highly concentrated form, as here mentioned; (3) it is more permanent than any other antiseptic, not only because it does not volatilize but because it straightway enters into a new chemical combination, which is also antiseptic.

In the case which we are now discussing it is utilized in the form of nitrate of silver (lapis) gauze,-e.g., gauze thoroughly moistened with 1 per cent. or 2 per cent. solution of nitrate of silver. If this gauze is saturated with blood or serous fluid, some part of the salt of silver is certainly 
transformed into albuminate of silver; but this combination is also antiseptic, and any infection which penetrates the moist layer of a saturated dressing meets an impenetrable barrier in the nitrate of silver gauze.

If, as you see, we cannot dispense with antiseptic remedies, in the original meaning of the word, as a prophylaxis against infection with perfectly clean wounds, you will understand that these remedies are indispensable in an even greater degree where we have to deal with infected wounds; or with operations where the infection cannot be excluded, as in the vagina, the anal region, and the rectum; with operations in the mouth, nose, and throat. In these the point is partly to have antiseptic fluids to wash out abscess cavities, inflamed joints, and serous cavities; for the uterine cavity, vagina, bladder, rectum, and, finally, for the nose, throat, and mouth; partly to have antiseptic dressing material adapted for use as tampons in the cavities mentioned and for the dressing of infected wounds. Bloch has done good service by warning us against the use of sublimate in this matter, and I can only endorse his warning while I impress upon you that sublimate acts as a powerful poison to the human organism, and we therefore only dare to make use of it when the skin is intact, and never for wounds, and least of all for the washing out of cavities. A number of deaths are due to the use of sublimate in this manner, though the late Professor Stadfeldt was the first to reveal and draw attention to the danger.

Those materials which formerly held sway in this ward were carbolic acid and iodoform gauze. The change which has taken place here since I have been in charge of the ward is that carbolic water is now used only for certain special infections, especially with tuberculosis, the treatment of tuberculous cystitis, tuberculosis of the joints and bursæ, and also with congestive abscesses; but the details of these modes of treatment find place in the lectures on 
these diseases. As a general antiseptic carbolic has been replaced by phenosalyl and nitrate of silver.

Phenosalyl belongs to the so-called "compound antiseptics," and for it we are indebted to our fellow countryman, Dr. J. Christmas, ${ }^{2}$ of Paris.

Compound antiseptics aim at avoiding any poisonous effect-local or general-of any individual antiseptic by allowing it to enter in only as a component part with a number of other antiseptics, and in such proportion that any poisonous effect is excluded. The most important component of phenosalyl is carbolic acid, but the proportion of this, when combined with a whole series of other antiseptics, is so small that its poisonous and irritating qualities prove ineffective.

Christmas has indeed been singularly fortunate with his compound of phenosalyl, for he has provided us with a 1 per cent. solution which is as destructive to bacteria as is a 3 per cent. solution of carbolic acid, and one which, adversely, is non-poisonous, does not irritate the skin or the tissues, has a pleasant odor and taste, and can therefore be used for rinsing the mouth and throat. For these reasons I can warmly recommend this antiseptic for your use. If prepared in dispensaries. according to the recipé which I will now give, it will not be costly.

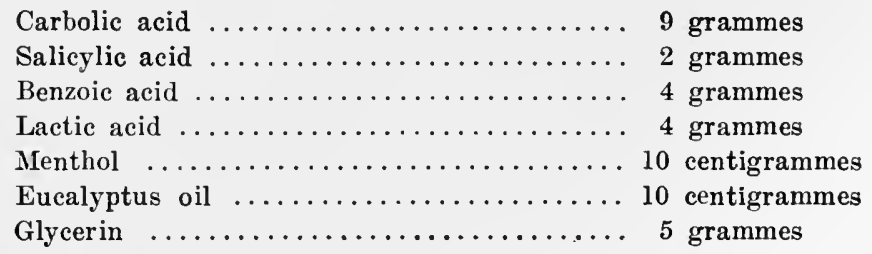

The first three are mixed and then dissolved in the lactic

${ }^{2}$ Christmas: "Phenosalyl, a New Compound Antiseptic," Hospital Journal, 1893, No. 43 . 
acid; finally the menthol, eucalyptus oil, and glycerine are added in the order mentioned.

The phenosalyl forms a clear fluid which dissolves easily bcth in water and alcohol. A 1 per cent. water solution is equivalent in strength to a 3 per cent. solution of carbolic water; but at the same time the toxicity of the phenosalyl is from three to four times less than that contained in carbolic acid, and thus you will see that a 1 per cent. phenosalyl solution is from nine to ten times less poisonous than a 3 per cent. carbolic water solution. Therefore we can tranquilly employ phenosalyl for antiseptic irrigation of large cavities, serous cavities, the uterus, and the intestines,-in short, everywhere where a large resorbent surface would make the use of carbolic a dangerous procedure. Therefore, here in this ward, it is in daily use as an irrigating solution and, in view of the investigations which $I$ have just mentioned regarding the relatively large number of bacteria present in the circulating air of our rooms, we always wash and rinse out the wound with a 1 per cent. phenosalyl before closing it by suture.

But a solution of phenosalyl is only effective on the surface, and its essential elements are volatile. When I require a deeply-penetrating, active, and a lasting effect I use a solution of nitrate of silver, the so-called lapis infernalis.

Even in pre-antiseptic days inflammation of the bladder had been successfully treated; the optic membrane and gonorrhœal urethritis were controlled with lapis solution, though without suspecting that here the question revolved around its germicidal qualities. In my monograph upon the pathology and treatment of cystitis, published in the latter eighties, I concluded that silver nitrate was the only medium by which one could quickly and surely cure a cystitis; the reasons being as follows: (1) Lapis in a solution of from 
1 per cent. to 2 per cent. destroys pathogenic microbes more quickly and surely than any hitherto known antiseptic material. (2) Its effect penetrates deeply; the nitrate of silver penetrating the cells and the intercellular substance. (3) That the chloride of silver formed by its mixing with urine also has a strong antiseptic effect, and that the same effect is obtained from those silver albuminates which arise from its contact with blood, pus, and other albuminous fluids. (4) No possibility of poisoning exists where adequate surgical methods of using the remedy are employed. No other method possesses this happy combination of good qualities.

Immediately, therefore, when faced by a serious, hazardous wound infection, virulent suppurations in a cavity such as the pleural cavities, the maxillary sinus, or the frontal, I fill the cavity with a 1 per cent. or 2 per cent. solution of nitrate of silver, according to the virulence of the infection, and allow the solution to remain for from five to ten minutes, when, as with cystitis and ophthalmoblennorrhœa, we often see the infection overcome.

With regard to the use of antiseptic gauze for dressings and tampons, iodoform is completely abolished from this ward and is replaced by silver nitrate gauze. This has many advantages over the iodoform gauze, the most important being that it is non-poisonous, whereas iodoform, as previously stated, is decidedly poisonous. Also, it has a powerful antiseptic effect, while iodoform has no effect at all upon the bacteria, but only on some of the end products of the infection. Furthermore, lapis gauze is odorless, while iodoform has a strong, penetrating odor which, though agreeable to some, is, I think, objectionable to most normal olfactory organs. Lastly, the preparation of lapis gauze is simple to such a degree that the physician himself can prepare it in a few moments. The method is as follows: A 1 per cent. to 2 per cent. solution of nitrate of silver is 
poured into a brown glass cylinder and pieces of sterile gauze of convenient size and shape are immersed in the fluid until it is absorbed by the gauze, which should be thoroughly moistened without being soaked. With the more virulent infections the gauze is impregnated with a 2 per cent. lapis solution. The prepared lapis gauze can be packed in sterile brown oilskin, which retains the moisture and excludes the light, and can thus be easily sent away or carried with one on his rounds. This lapis gauze has entirely replaced the iodoform gauze in our clinic for use as tampons in the nasopharyngeal cavity and for wounds and cavities contiguous to the mouth, for the uterus, vagina, and rectum. It can also be used with excellent results for facilitating drainage from infected wounds elsewhere,-e.g., in cases of osteomyelitis, where, after chiselling the bone away and removing the infected marrow with a sharp spoon, I first fill the hollow with a solution of lapis and then tamponade it with lapis gauze; in eases of mastoiditis, infections of the antrum and middle ear, with phlegmons, abscesses, etc. A wide field for the usefulness for lapis gauze presents itself in the cases of intraperitoneal suppuration, including peri-appendiceal abscesses and abscesses in the fossa douglassi, etc., where it is used as a tampon. It is quite astonishing to see how rapidly the suppurative cases improve, and how long a time a tampon will remain odorless even when intestinal bacteria are present.

Antiseptics in powder form, still extensively used in many cases, are, so to speak, not employed in this ward. They are superfluous with clean wounds and are dangerous with secreting wounds, because with secretion they often form a crust, behind which there is retention. Finally, the method of their application, by powder bellows, etc., makes it difficult to keep them free from atmospheric infection. There is only one powder which is used to any extent in this 
ward, and that is powdered boracic acid, not so much on account of its antiseptic properties but more on account of the stimulus it gives the formation of the epidermis, and therefore I apply it to all superficial skin lesions: excoriations, burns, abrasions, sudor pedum, etc.

We formerly believed that the majority of unexpected suppurations after aseptic operations were due to "catgut infection,"- that is to say, that the catgut utilized had not been thoroughly sterile, and that the infection came from microbes within the catgut being freed by the resorption of the catgut. This point of view was strengthened by the fact that these suppurations generally appeared around the skin sutures and about the catgut sutures and the ligatures. Generally speaking, however, the suture material is sterile, but becomes infected with microbes from the patient's or doctor's skin. It may, however, still be termed a catgut suppuration, as the catgut plays an important rôle in originating and favoring suppuration, inasmuch as it is an excellent, nutritive submedium for bacteria and consequently stimulates their increase.

This circumstance has consequently led to a further reform here in this ward, and we have to a great extent eliminated the use of catgut as a suture material in wounds of the skin and fascia. Here catgut is superseded by aluminum bronze thread, an excellent, pliable, and strong metal thread with which we can employ intricate or continuous sutures as easily as with ordinary thread. I venture to say that, generally speaking, since the introduction of aluminum bronze for skin suture we no longer see suppuration in the stitch holes in aseptic operations, the explanation being that this alloy possesses distinct antiseptic properties and thus directly prevents bacteria, although they may be present, from developing. We are indebted to the renowned German-American dentist, Miller; for our knowledge with regard to metals, and especially in the case of copper and 
its antiseptic properties. Not until Miller demonstrated the germicidal qualities of copper and its amalgams could we understand how dentists could so often and without penalty plug up decayed teeth, thus confining infectious material. Miller took a series of gelatine and agar plates and distributed small fragments of metal over their surface. He then infected the plates with various bacteria. $\mathrm{He}$ observed that a wide zone remained absolutely sterile around the pieces of metal, while the remaining portions of the plate were found to be richly overgrown with colonies of bacteria.

In using aluminum bronze, even in infected areas where suppuration is unavoidable, one has the added advantage that the suture remains after the suppuration has subsided. With catgut, on the other hand, the thread is absorbed or sloughs out and the wound breaks open. Silk thread, which many people use in place of catgut, is not absorbed, but becomes impregnated with the pus and remains as a foreign body until the thread is either removed or sloughs out.

The importance of using aluminum bronze in suturing the fascia for the prevention of hernia after abdominal operations, and for a radical cure of umbilical hernia, we will speak of when lecturing on hernia.

There is, however, a large field of usefulness for catgut. In the first place, we always use catgut for ligatures, and in my opinion no other material can replace it for this purpose. Secondly, it is employed for sutures in the peritoneal eavity and in the intraperitoneal organs, and for sutures in the kidneys, bladder, pleura, and lungs. Lastly, we use it for the mucous membrane of the mouth, the tongue, and many other places where metal thread is unsuitable.

As already emphasized, there is no real difficulty in producing a bacteria-free catgut, and this ward has perhaps been especially fortunate in providing three separate, first-class methods for disinfecting catgut. To Professor 
Bloch is due the announcement of the excellent carbolicalcoholic catgut, a thoroughly sterile and excessively strong catgut, the preparation of which was described by Bloch in 1889, in the "Nordisk Medicinsk Arkiv," as follows: "The catgut threads are gathered together and rolled tightly round a glass cylinder which remains submerged in a closed glass cylinder containing 5 per cent. carbolic water for forty-eight hours. The catgut threads are unrolled in a dish containing fresh 5 per cent. carbolic water, and are then wound tightly round a porcelain cylinder which is placed in proof alcohol containing 5 per cent. carbolic acid." In 1902, Claudius, who was then clinical assistant in this ward, brought forward the iodine catgut, which, on account of its extremely simple and safe manner of preparation, has gained great favor and been extensively used. The raw threads are simply placed in a water solution of 1 per cent. iodine in iodide of potassium, and after a week's immersion in this fluid the catgut is quite bacteria-free and ready for use. A very good modification is, subsequently, to keep the iodide catgut in alcohol, whereby it becomes considerably stronger and is more slowly absorbed by the tissues.

If, in this ward, we no longer use these two preparations of catgut, in themselves so excellent, it is for the following reasons. For use in aseptic operations, especially intraperitoneal ones, I require an aseptic catgut which does not irritate the tissues. We have obtained one catgut which is ideal in that respect since Hofmeister has made it possible for us to boil the catgut, just as we boil the instruments before operations. If you merely place a piece of catgut thread in boiling water or steam, you will find that the thread becomes quite unfit for use, as it curls up, frays, and becomes quite soft. But when, in accordance with Hofmeister's directions, we first treat the catgut with formalin, it becomes not only thoroughly disinfected but will then 
withstand boiling. The formalin treatment is as follows: After the catgut has been tightly wound up in a single layer on glass plates, it is placed for from twelve to forty-eight hours in a 4 per cent. solution of formalin,-i.e., a 10 per cent solution of the trade 40 per cent. formalin. Great care must be taken to tie the ends of the thread firmly together, as the catgut contracts so greatly under the influence of the formalin that a loose knot is easily broken. The catgut as thus treated is then placed in running water for twelve hours, to remove the free formalin, and is finally boiled for a period varying from ten to thirty minutes. To preserve it, the catgut is placed in absolute alcohol; as an added precaution in many major operations we boil the catgut immediately before the operation. As Heerfordt has pointed out, it of the greatest importance to have the threads tightly stretched or wound while they are

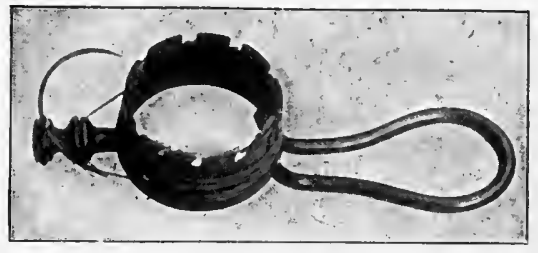

FIG. 15.-Heerfordt's cylinder for long catgut thread provided with needlc.

boiling; therefore the long suture threads, provided with a needle, are rolled up on one of Heerfordt's brass cylinders (see Fig. 15), while the ligature threads are stretched on brass frames, as seen in the accompanying illustrations (Figs. 16 and 17), which we call the catgut harp. On one side of the frame are two side rods provided with a row of small pegs, over which the thread is stretched continuously from one side of the frame to the other. When the thread has passed all the pegs, it is held in place by two brass rods perforated to correspond with the pegs and fastened to the frame with screws. There are now as many ligatures as there are pegs, stretched like strings on a harp. The whole apparatus is boiled immediately before the operation, and each time a ligature is required, an assistant extends the harp toward the operator, 
taking the handle in his left hand, while with his right he severs one end of the thread, which falls so that the operator can grasp it, whereupon the assistant cuts the other end. In this manner no one save the operator himself touches the thread after it has been sterilized. This boiled catgut is exceedingly strong and durable,-i.e., resorbed slowly,and seems to me ideal both for ligatures and sutures in areas which are absolutely aseptic.

On the other hand, where a really antiseptic catgut is needed, I do not find that either carbolic or iodide is satis-

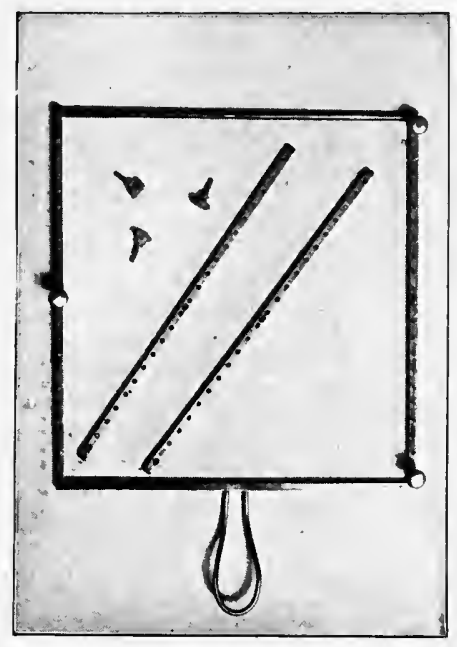

Fia.16.-Component parts of catgut harp.

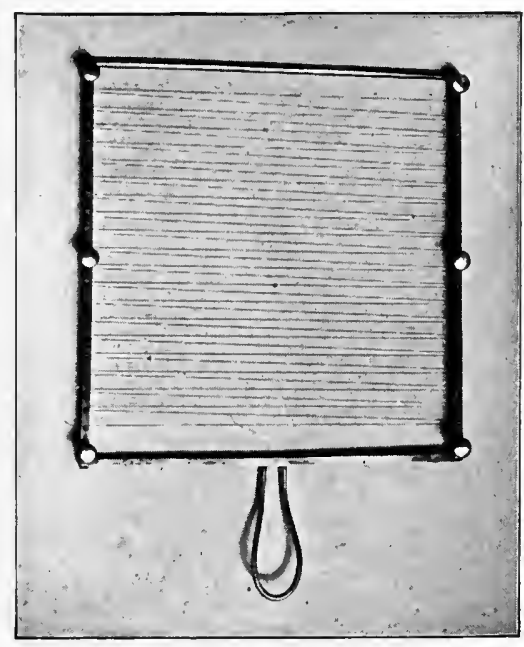

FIG. 17.-Catgut harp ready for use.

factory, because their antiseptic qualities are quite evanescent. The carbolic evaporates quickly and the iodide immediately combines with the blood salts and is converted into iodide of potassium and iodide of sodium and disappears, leaving the catgut an easy prey for infection. You will remember the excellent antiseptic qualities of nitrate of silver, already mentioned, and the added advantage of the new silver combinations brought about by disintegration of the salt within the wound. These are germicidal and 
maintain their effect in loco. You will understand that I, as a link in the chain of our lapis antiseptic method, have endeavored to prepare a serviceable lapis catgut. The endeavor succeeded beyond all expectation by means of the following mode of procedure, which is even simpler than the preparation of iodide catgut: You place the spools, with the scoured catgut rolled round them, in single layers at the bottom of a glass cylinder, and then pour over them a 4 per cent. watery solution of nitrate of silver. Here the catgut is allowed to remain for four days and nights. Then you merely add three times as much absolute alcohol, and in this the 1 per cent. "lapis alcohol catgut" is preserved for use. You here see such a silver nitrate catgut thread, which is quite black where severed, a sure token that the silver nitrate has permeated the thread, which is extraordinarily strong and supple-yes, stronger than any other catgut thread which a series of investigations at the government testing institution has produced; investigations, too, made with apparatus constructed for the purpose of testing the strength of threads. The longer the catgut remains in the fluid the stronger it becomes. Try a piece of this thread which was prepared a year and a half ago; it will take a strong man to tear it apart. In my opinion, the chief advantage of "lapis catgut" is this: that the antiseptic matter does not leave the thread during resorption, and thus takes the place of the thread.

I use silk only for suturing tendons, and in the peritoneal cavity in cases of resections, and when doing an anastomosis operation where I wish to make sure of a juncture by some non-absorbable material. With regard to silk, I use boiled silk in aseptic operations, and silver nitrate silk, prepared and preserved in the same manner as silver nitrate catgut, in infected areas (Fig. 18).

Sterilization of Instruments.-This we do by simply boiling all glass and metal instruments immediately before 
operating. In order to prevent rust, 7 per cent. bicarbonate of soda is added to the water. For the office where small operations are done the instruments should be kept ready for use standing in a glass cylinder filled with absolute alcohol. Before use and just after removing the instrument from the glass you ignite the spirit attaching to the instru-

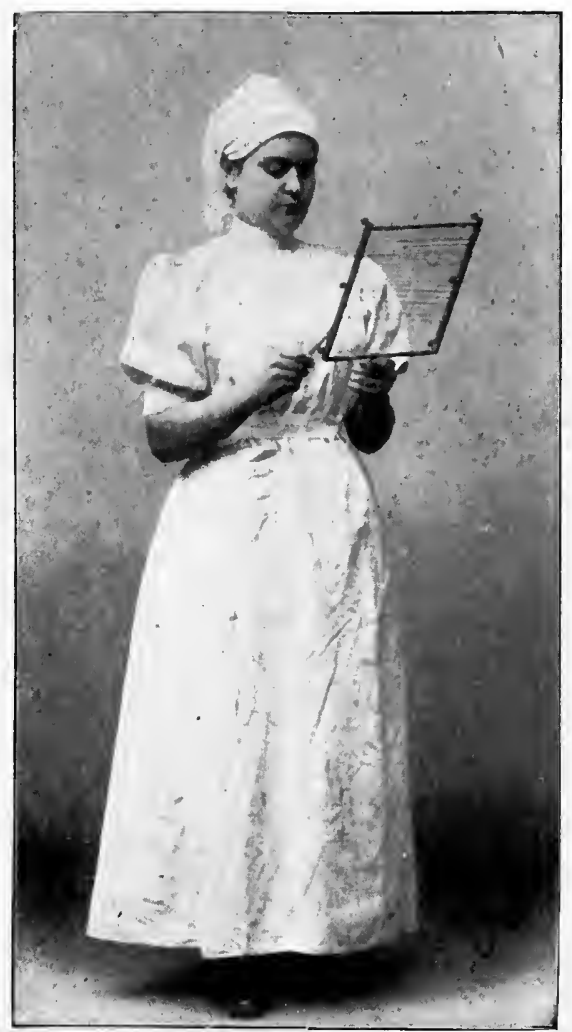

Fig. 18.-Author's catgut harp in use. ment with a match, and thus obtain sterilization by heat. Special metal bougies and catheters can remain in alcohol for years without harm.

The so-called silk rubber catheters and bougies occupy a unique position, inasmuch as they can withstand boiling only a few times and for a short period and they are quickly destroyed by repeated boilings. For these instruments sterilization by means of formalin vapors is the best method, because it not only preserves the instruments but increases their strength and durability to a great extent. Here the instruments are placed in a long glass cylindrical tube which is closed at both ends with non-absorbent, sterile cotton wool and then put into a formalin sterilizing apparatus designed by me, a description of which is to be found in my "Surgery of the Urinary Organs" (see Fig. 19). For the rest, you can easily improvise a 
simple and cheap formalin sterilizer in your own homes by using an ordinary biscuit tin, at the bottom of which you place some formalin in a little bowl covered with wire netting. In order to destroy the pathogenic germs with certainty, the formalin must act for thirty-six hours and, before the instruments are used, the glass tubes should have been removed from the apparatus for two hours, in order that the formalin may evaporate. We place the glass tubes in a simple, transportable stand (see Fig. 20).

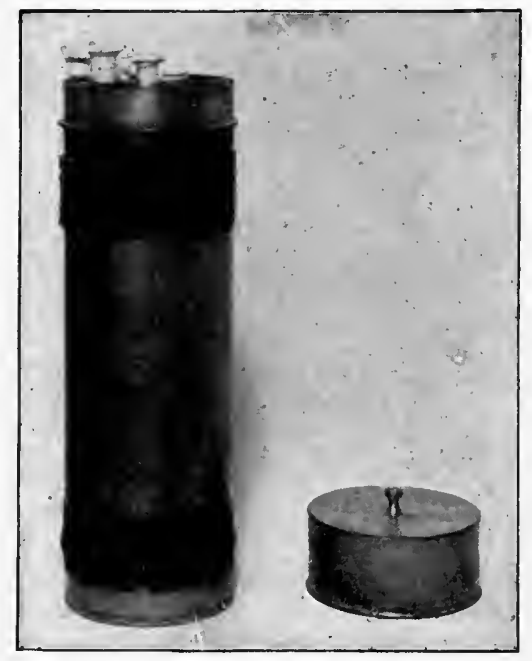

Fig. 19.-Forf's formalin sterilizator for rubber catheters.

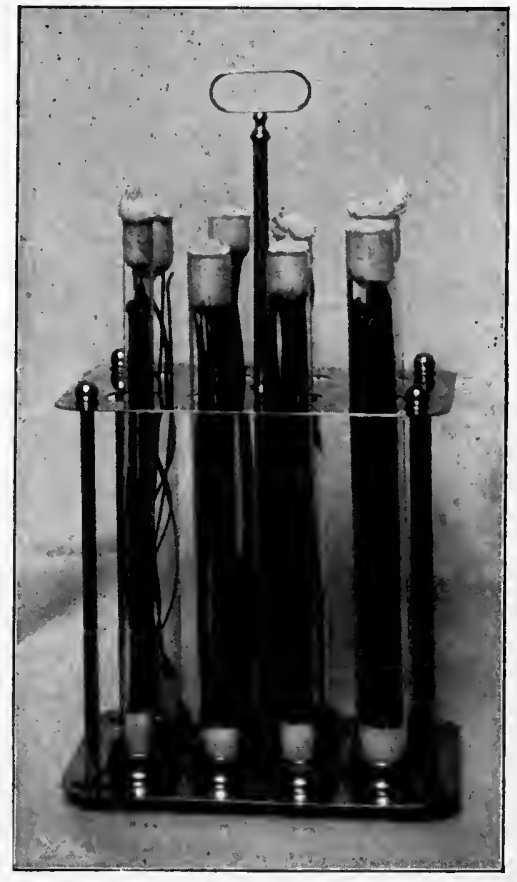

Fig. 20.-Catheters and bougies in cotton-wool-stopped tubes, in which they are sterilized. Transportable stand. (Nyrop.)

Rubber goods may be sterilized by steam. This applies to rubber aprons as well as rubber gloves. If rubber does not lie next to rubber and the layers are separated by gauze or linen, no trouble will result. Formerly we used to cut five-finger-shaped pieces of linen, which were placed inside the glove, but it has since proved to be sufficient to sprinkle the inside of the glove with French chalk. Rubber gloves 
can also be sterilized by being immersed in sublimate solution. Oilsilk is sterilized and preserved in this way because it cannot withstand heat.

In this lecture I have tried to describe to you the history of antiseptic methods as it has developed in this ward. I have tried to describe to you particularly those methods which enable us to keep pace with the foreign up-to-date surgical clinics and obtain equally good results from operations. I hope that I have told you sufficient to enable you to adopt for yourselves some really reliable antiseptic method along these lines when you enter upon your practical work. As a future help in life I will give to each of you these printed regulations regarding the disinfection and treatment of wounds in the surgical clinic of the University.

\section{RULES FOR DISINFECTION AND WOUND TREATMENT AT THE ROYAL FREDERIK'S HOSPITAL, WARD C}

I. DISINFECTION OF THE SURGEON'S HANDS AND ARMS

Every one who has to perform an operation or arrange a dressing or assist with either must disinfect his hands and arms in the following manner:

(1) Cleanse with soft soap and brush, with two changes of hot water at least; and subsequent use of nail-cleaner.

(2) Cleanse hands and arms energetically with $1 / 2$ per cent. sublimate alcohol-i.e., $1 / 2$ gramme sublimate dissolved in 1000 c.c. 50 per cent. alcohol. ${ }^{3}$ Special attention to be paid to the interstices of the fingers and nails.

\section{CLEANSING OF AREA OF OPERATION}

(1) Shaving.

(2) Hot water, soft soap, brush.

(3) Energetic rubbing of the skin with ether by means of gauze or cotton wool tampons.

(4) Cleansing with $1 / 2$ per cent. sublimate alcohol.

\section{WOUND TREATMENT}

(1) Sublimate is never used to rinse and scour wounds or wound cavities. For use in the peritoneal cavity and in aseptic wounds, sterile physio-

${ }^{3}$ Before laparotomy and other important operations, beard and face to be washed with sterile gauze moistened with 50 per cent. alcohol. 
logical salt solution is used. With infected wounds a $1 / 2$ to 1 per cent. solution of phenosalyl or $1 / 2$ to 1 per cent. solution of nitrate of silver is employed, according to the severity of the infection.

(2) All recently-incurred, dirty wounds resulting from an accident are drained with rubber drains or oilsilk, even when it looks as if all the dirt had been removed.

(3) All aseptic wounds completely closed by suture, unless special conditions, such as a dead space or cavity, demand drainage.

(4) All bleeding vessels are ligated with silver nitrate catgut or formalin boiled catgut, using as fine gut as the size of the vessel will permit.

(5) Suture material: Catgut for peritoneum, joint capsules and facial skin; otherwise, aluminum bronze, sterilized by boiling immediately before use, is the ordinary material employed for bone, fascia, and skin sutures. Silk is used only in exceptional cases (intestinal and stomach sutures; suture of tendons and skin under great tension).

(6) The suture line is covered with collodion and cotton wool-a thin layer of sterile non-absorbent cotton wool being spread over the wound and then painted over with collodion, the wound being thus hermetically sealed. Often, with slight, well-joined wounds no other dressing is necessary. If drains are placed diagonally in the wound, a further treatment with silver gauze and sterile non-absorbent wadding attached with gauze bondage is required.

(7) Burned areas and the surrounding parts must be cleaned and disinfected with the utmost care-under light ether narcosis, if necessary. Bullæ are cut open and removed. The wound is then covered with sterile oilsilk which has been rinsed out in salt water, and which should protrude only a few millimetres beyond the edge of the wound. This covering should be provided with numerous small slits to enable the secretion to ooze out into the outer dressing, consisting of silver nitrate gauze, layers of sterile gauze, and non-absorbent cotton wool.

(8) Suppurating wounds are tamponed with silverized gauze after they have been washed out, using sterile gauze moistened with a 1 per cent. solution of nitrate of silver. Sterile gauze compresses wrung out in sterile water are then applied, over which are placed rubber tissue, cotton wool, and bandaging.

With the treatment of infected wounds rubber gloves are always used. 


\section{LECTURE IV}

\section{ANÆSTHETICS}

I will speak to you to-day about the second of the two great discoveries which led to the enormous advance of surgery during the last century-anæsthetics and narcotization. I hope I shall be fortunate enough in this and the following lesson to find time to demonstrate to you those methods of narcotization which are employed in this ward and which I can recommend to you; and also to give you the main features in the development of the question of anæsthetics. We discriminate between (1) general narcotization (narcosis-total anæsthesia), by which we produce a state of insensibility and more or less complete abolition of the reflexes, and (2) local ancesthesia, by which we produce insensibility in some restricted area of the body of the otherwise wideawake and fully conscious individual. To-day we will only occupy ourselves with general anæsthesia.

GENERAL ANESTHESIA

The efforts to procure freedom from pain for patients under operation can be traced to the most remote days of surgery. We know that the ancient Egyptians, before and during an operation, gave the patient an intoxicating draught composed chiefly of cannabis indica and opium, and that the Chinese employed the same method in the Third Century A.D.

Dioscorides and Pliny relate that mandragora wine, a spirituous extract of the mandragora root, was in their time given with good results before operations. But not until the Middle Ages do we find any attempt to bring about anæsthesia by inhalation, narcotization by respiration. The Italian, Dominican Theodoricus da Cervia, who lived about 
A.D. 1250, prepared a highly stupefying extract from narcotic herbs,-opium, hyoscyamus, lactuca, mandragora, and others,-in which he dipped sponges which were dried in the sun. Before the operation such a sleeping-sponge was dipped in hot water and then placed before the patient's nostrils, who thus inhaled the narcotic fumes.

These methods, however, scarcely proved satisfactory, inasmuch as they were not lasting; and it was reserved for the nineteenth century to solve the problem of general narcosis. At the very end of the eighteenth and the beginning of the nineteenth century the thought of anæsthesia by the inhalation of volatile chemical matter began to take form. Humphry Davy, in the course of his investigations into the physiological effects of protoxides, discovered that they had the effect of stupefying a person, and in the year 1800 first announced that protoxides, which he designated "laughing gas" on account of the lively delirium brought about by them, might possibly be used with advantage to produce anæsthesia during operations. Some years later, in 1818, Faraday propounded the theory that ether fumes mixed with the atmosphere possessed a similar power. As you know; it was not until the forties that Davy's and Faraday's propositions were carried to their conclusion by two American dentists. One of these, Horace Wells, in the year 1844, allowed himself to be anæsthetized with protoxides during the extraction of a tooth. The anæsthesia was completely successful, and Wells, with great enthusiasm, now tried to propagate this method of anæsthesia generally among dentists. He encountered such mistrust, however, from the whole medical profession that he committed suicide from grief and disappointment. After his death, protoxides, little by little, gained ground, until at the beginning of the sixties they came into general use for the extraction of teetl.

The path of ether grew more thorny. The chemist Jackson, who by personal experiment had studied the anæs- 
thetizing effects of ether, induced the dentist Morton to try the effect of ether anæsthesia on his patients. As this proved a complete success, they appealed to the surgeon at the Massachusetts General Hospital, Dr. Warren, who, on the 17th of October, 1846, performed the first important operation with the patient under ether narcosis. A series of successful operations then followed without intermission, and the important news spread rapidly, not only in America but also in Europe, after Jackson, on November 13, 1846, had imparted his discovery to the Académie de Médicine in Paris.

But even in the next year, 1847, the progress of ether was checked by the introduction of a new narcotic which quickly eclipsed ether; it was chloroform. This substance, which was discovered by Soubeyran in Paris in 1837, had, like ether, been used a good deal as a soothing inhalation remedy for affections of the respiratory organs; but not until March, 1847, did Flourens, by experimenting on animals, prove its valuable narcotizing effects. Nevertheless, this announcement caused no great sensation; but there was a most remarkable effect when the then famous Edinburgh gynæcologist Simpson, on November 10, 1847, before the Medical and Chirurgical Society at Edinburgh, presented the results of eighty cases of narcosis by means of chloroform, the first subjects having been himself and his relations. He represented chloroform as being a quicker and much more agreeable narcotic than ether; and chloroform, with astounding rapidity, found favor and deposed ether from its recently-acquired position throughout the greater part of the civilized world. One essential reason why ether was at first so easily dominated was, undoubtedly, the sinister appearance which ether narcosis presented when undertaken in accordance with the suffocation method then prevailing. It was thought necessary to overpower the patient immediately with ether, obstructing all the air passages. 
This not only caused a feeling of suffocation, with the result that the patients, especially nervous people and inebriates, became tremendously excited and offered energetic resistance, requiring considerable compulsion to keep them quiet, but caused an appearance of real suffocation, inasmuch as their faces became dark blue, their eyes projected, and they foamed at the mouth, as a result of the increased production of mucus by the mucous membrane in the mouth and throat, irritated by the ether fumes. Although no real danger worthy of the name was concealed behind this sinister appearance, one can well understand that it might intimidate those who for the first time witnessed such an anæsthesia, and that they would prefer chloroform narcosis, which, as a rule, offers the semblance of a far easier and more natural state of somnolence. However, it was not very long before the great enthusiasm for chloroform cooled down, partly on account of the reports concerning deaths which had occurred during anæsthesia. At first it was said that these deaths were due to a misuse of the chloroform, which led to greater care being taken with anæsthetics, both with regard to the quantity administered and the method of administering it. Instead of at once placing a cap, with plenty of chloroform poured upon it, in front of the patient's mouth and nose, there was a return to the slow and careful induction of narcosis by dropping chloroform onto an Esmarch's flannel mask, which allowed a free admixture of air with the chloroform. But the belief that the deaths were due solely to the misuse and careless administration of chloroform was responsible for many deaths from chloroform, and for a long time retarded a real apprehension of the truth. Not until systematic narcosis statistics were taken in hand, as was the case in Germany with Gurlt's indefatigable and most praiseworthy labors, lasting over many years, did it become évident to all unprejudiced people how much more dangerous chloroform is in comparison 
with ether, even when administered with every precaution by experienced physicians. I will now give you the conclusions arrived at from a study of the four most extensive and comprehensive statistical records.

Statistics of Narcosis.

\begin{tabular}{|c|c|c|c|c|c|c|}
\hline & \multicolumn{3}{|c|}{ Chloroform. } & \multicolumn{3}{|c|}{ Ether. } \\
\hline & $\begin{array}{c}\text { Number of } \\
\text { cases of } \\
\text { narcosis. }\end{array}$ & Deaths. & Mortality. & $\begin{array}{c}\text { Number of } \\
\text { cates of } \\
\text { narcosis. }\end{array}$ & Deaths. & Mortality. \\
\hline 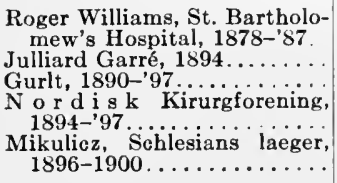 & $\begin{array}{r}12,368 \\
524,507 \\
240,806 \\
25,305 \\
87,530\end{array}$ & $\begin{array}{r}10 \\
161 \\
116 \\
15\end{array}$ & $\begin{array}{l}1: 1236 \\
1: 3258 \\
1: 2075 \\
1: 1687\end{array}$ & $\begin{array}{r}14,581 \\
345,873 \\
56,233 \\
2,926\end{array}$ & $\begin{array}{r}3 \\
23 \\
11 \\
0\end{array}$ & $\begin{array}{c}1: 4860 \\
1: 15038 \\
1: 5112 \\
0 \\
1: 1029\end{array}$ \\
\hline Surnmary .... & 878,148 & 344 & $1: 2552$ & 409,149 & 38 & $1: 10767$ \\
\hline
\end{tabular}

When we consider these figures we find that, whereas one death occurs from narcosis by chloroform in 2550 cases, only one death occurs from narcosis by ether in 10,767 cases; but I must warn you against drawing conclusions from statistics without due criticism, because nothing is more dangerous; and it is not without some justification that the advocates of chloroform have objected to the figures of some of these statistics as being of doubtful value, for one cannot rightly say whether death is due to the narcosis itself or to the disease for which the operation was undertaken. In reality, these figures represent only those deaths which occurred during the course of narcosis, and have, of course, in a certain number of cases no bearing upon the cause of the death. For instance, where the patient is brought to the operating table in a desperate condition, brought about by extensive hemorrhage, diffuse peritonitis, brain injury, etc. Naturally, one should not designate every death which takes place during narcosis as a narcosis death, but only those which are due to the poisonous effect of the narcotic. In order, therefore, that we may better appreciate the 
figures contained in these statistics, it will be necessary to obtain more detailed information regarding the circumstances under which each individual death has occurred. Most statistical records are supplemented by a text dealing with just this phase of the subject. Gurlt's comprehensive statistical record is of especial value because it gives in addition a detailed account of the patient's illness, the course of the narcosis, and the result of the autopsy. If one studies these records it will be found that the figures give an entirely wrong impression. In going over the records of the eleven deaths which occurred under ether narcosis, as found in Gurlt's statistics, it becomes apparent that ether must be acquitted of blame in ten instances, because in these cases there existed quite sufficient cause for death in the patient's deplorable condition. And there remains only one instance where no other cause for death can be adduced. This case was that of a nine-month-old child who, during an operation for the removal of a large angioma of the chest wall, by Madelung, became asphyxiated. With the aid of artificial respiration the child revived, but shortly afterward again became asphyxiated and died without a further administration of ether. The autopsy did not explain the cause of death, so this cannot with certainty be regarded as a death due to ether, but, as the possibility cannot be excluded, it must be put down as a death due to ether. But, even then, you will notice that the number of deaths from ether narcosis is reduced to one in 56,233 cases. The mortality records are similarly reduced by further investigation of the other ether statistics. Thus, of the three deaths reported by Williams, two should be excluded, and the same remark applies to Mikulicz's four deaths, three of which should not be considered as due to ether.

If, now, we examine Gurlt's chloroform statistics in the same manner, we find eight cases where the deplorable condition of the patient should be considered as the cause of 
death, and these cases must therefore be deducted. Then there remain 108 deaths which are undoubtedly due to collapse under chloroform. These deaths naturally fall into two very different categories: the primary and the secondary collapse. (1) By the primary collapse under chloroform we mean those most extraordinary cases where, as frequently happens, the apparently strong patient, after inhaling choloroform for a few moments, has a brief stage of excitement, followed by a sudden collapse, and dies, apparently, from an acute and, more is the pity, well-defined cessation of the heart's action. The sinister nature of this primary collapse will be best made clear to you by my telling you that in spite of its being far less frequent than the secondary collapse, it is, nevertheless, the cause of the majority of all the deatlis from choloroform. In Gurlt's statistics, 65 of the 108 cases come under the heading of primary death from chloroform, in 60 per cent. of which deatl came suddenly without warning, like lightning out of a clear sky, striking down a strong, vigorous patient suffering from some minor surgical ailment or who required an anæsthetic for purposes of more thorough examination. Death occurred in the majority of cases after the patient had inhaled only a few cubic centimetres of chloroform. These deaths are to be accounted for solely as the result of some incalculable idiosyncrasy.

(2) The secondary collapse under chloroform which occurs after complete anæsthesia is established we understand better, because the margin of safety between the quantity required for the narcosis and the quantity which will prove fatal-Paul Bert's so-called zone maniable-is so narrow that a relatively small amount added to the quantity necessary for narcosis may prove fatal. A slight neglect can lead to an overdose, and the patient passes from narcosis to collapse. This secondary collapse presents a more hopeful condition than the primary collapse, for in most 
cases the patient can be brought back to life by the rapid application of artificial respiration, heart massage, tongue traction, electricity, etc., but in a certain percentage of these cases all efforts prove fruitless. When reading the accounts of the deaths pertaining to this group, one is astonished how frequently here also death strikes young, strong people who have come under the surgeon's care on account of some trivial or minor affliction.

The fact is that, according to the best statistics, one death occurs in every 2000 cases of chloroform anæsthesia. This percentage is probably too small, because these statistics are compiled entirely from the records of the larger hospitals, where, as a rule, the patients are carefully examined and prepared before the narcosis, and are anæsthetized by careful and experienced assistants, while, in addition, every expedient is at hand in order to bring a collapsed patient quickly back to life again. It can scarcely be doubted, therefore, that the average of mortality would rise considerably if we were to include all the cases of narcosis induced outside the hospitals, under primitive conditions, by inexperienced practitioners upon unprepared and fully-clothed patients, without the expedients necessary for assistance and attempts to resuscitate.

Every attempt to eliminate or reduce the mortality from chloroform by demanding purer chloroform, a combination of chloroform inhalation with inhalation of oxygen, etc., has proved unavailing. My predecessor in this ward, Professor Bloch, endeavored to avoid the danger of collapse by the use of the so-called primary chloroform narcosis in conjunction with local anæsthesia by means of ethyl chloride. The patient is not fully anæsthetized, only receiving a few cubic centimetres of chloroform. It is indisputable that one hereby diminishes the risk of a secondary collapse from an overdose; but the danger of primary collapse under chloroform, which is the cause of more than 
one-half of the deaths from chloroform, is, of course, not avoided by this means; and Professor Bloch himself reported two cases of death primarily caused by chloroform since the introduction of this method.

Notwithstanding all efforts to lessen the danger of chloroform narcosis, the mortality records still show one death in every 2000 cases, though it should be added that for each case of collapse which terminates fatally chloroform is the cause of hundreds of cases of collapse which are satisfactorily overcome, though the operation is thereby interfered with and affects the operator and his staff by reason of their anxiety for the patient.

Why, therefore, has ether narcosis, which according to statistics only causes one authentic death from narcosis in 50,000 cases, and under which collapse is an event of the greatest rarity, not been able to dislodge chloroform in every clinic and to reoccupy its sovereign position among the methods of narcosis? I believe the first and foremost reason to be human vanity, more is the pity: the difficulty men have in abandoning an opinion which they have maintained in good faith for a long time.

In this respect it was certainly a calamity that from early days the question of chloroform versus ether was an acute point in debate between Lyons and Paris. The more vehemently the Lyons surgeon Ollier and his pupils sang the praises of ether and emphasized its harmlessness as compared with chloroform, so much the more passionate and blind became the Paris surgeons' defence of chloroform against the "provincial" hospital's attack. Even to this day it is a point of honor with the Parisian surgeons to make no concession in this matter; and when one considers how many doctors there are, both from the Old World and the New, who take their cue from the city on the Seine, one will understand how fatal it has been for ether that its true value should have been first revealed in Lyons and not in 
Paris. This is also the real reason why chloroform is still preferred in certain American centres. Of course the champions of chloroform adduce certain grievances against ether in support of their hostile attitude, the most important of which is that ether has a distinctly irritating effect upon the air passages, and is the cause of a very considerable number of cases of pneumonia, producing a series of postoperative deaths, almost as numerous as the cases of fatal collapse from chloroform. Ether should, therefore, be contra-indicated in those cases where the organs of respiration are affected; for example, in those who have bronchitis, emphysema, etc. Next, it is asserted that ether causes a violent and protracted stage of excitement and that it does not produce a complete relaxation of the muscles, and therefore is not the best anæsthetic to use for children and inebriates.

Those of you who have followed the course of our work in this ward for some time past will scarcely be able to credit these contentions, because here you see all our patients, without exception, children, adults, inebriates, and those suffering from affections of the respiratory organs, anæsthetized quickly and easily by means of ether. You would understand this question better if you had ever seen ether administered in accordance with those older methods which are still employed in many places. I have already mentioned the old method of overpowering or suffocation which causes violent excitement and often active salivation and mucus wheezing. In order to avoid this weird result many physicians, Germans in particular, went to the other extreme and have employed the method of administering ether by the drop method, providing a good admixture of air. Some have used Esmarch's chloroform mask and others have used Sudeck's metal mask, which is here illustrated (Fig. 21). The ether is dropped onto a gauze compress which is placed in the dome-shaped space in the mask. This 
space has a large opening above and below, and is separated from the lower portion by a perforated diaphragm with inspiratory valve. On one side of the mask there is a ventilator through which the patient exhales, and which rattles so long as the breathing continues. Both these methods possess the great disadvantage that it takes a long while to anæsthetize the patient, and that they occasionally result in failure. This has led many people to think that one should start with chloroform and continue the anæsthesia with ether.

But here, naturally, the danger of primary collapse still exists. In addition, both methods have the disadvantage

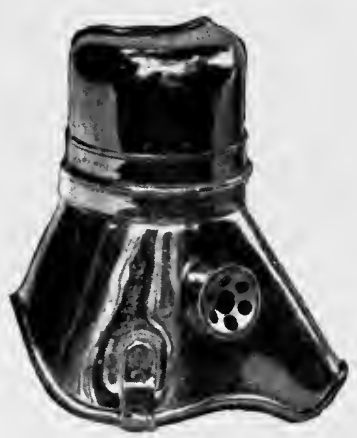

Fia. 21.-Sudeck's ether mask. that the operating room is filled with ether fumes, and that a large quantity of ether is consumed. Finally, Sudeck's mask has the drawback, in common with the old ether cone, that it occasions a refrigeration of the apparatus itself and of the air which is inhaled, often so marked that ice crystals are deposited on the apparatus and in the gauze. There is no doubt that such a refrigeration is in jurious to the respiratory organs, and especially so when these are below par.

If ether narcosis appears to be an ideal anæsthetic to all those who see it here in this clinic, then the reason is that we are so fortunate as to possess a method which has overcome all the above disadvantages. Primarily, our method is adapted from Wanscher's, inasmuch as we make use of his excellent narcotizing apparatus, which is here illustrated (Fig. 22). It consists of a soft rubber mask which is connected by a metal thread frame with a roomy rubber bag, into which the ether is poured. But latterly I have modified this method, and the alterations which this method has 
undergone are best expressed by my terming our method the morphine-ether narcosis with intermittent inhalation. Half an hour before starting the anæsthesia with adults I inject $11 / 2$ centigrammes of morphia subcutaneously, while with children of proper age I inject a proportionately smaller dose, and with quite small children I inject a little opium or nothing at all. The effect of this is most satisfactory, inasmuch as the patient's uneasiness and anxiety disappear and with most patients the stage of excitement is eliminated;

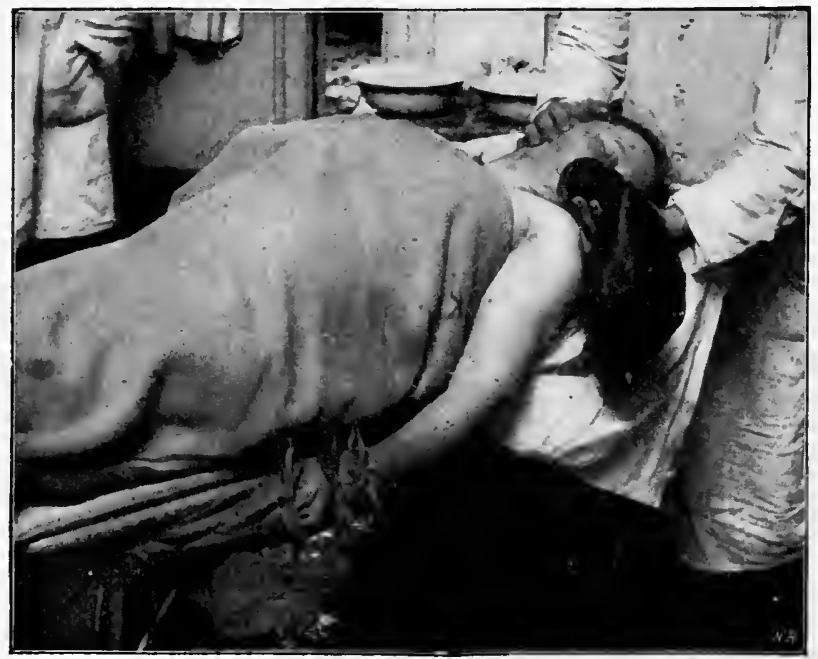

FIG. 22.-Ether narcosis with Wanscher's apparatus. The arm fixed to the table with author's device.

while even with confirmed inebriates and very nervous individuals, it becomes quite insignificant and transitory. Whereas Wanscher's method was a form of suffocation, inasmuch as he held the mask close to the patient's face until the narcosis was well advanced, I allow the mask to approach the patient's face little by little, and not until the patient has accustomed himself to the scent of the ether and is quietly inhaling it is the mask held close. Whenever the color of the face indicates a beginning cyanosis, the mask is momentarily removed, to be re-applied as soon as the color 
is normal. Therefore, as a rule, you see our patients with a good color during the whole course of the narcosis, and often free from the uncomfortable accumulation of mucus and rattling in the throat. You will find the mode of procedure detailed in this guide to ether narcosis, which I am now about to distribute among the older students who will start life as physicians at the end of this term (see page 94).

One great advantage with Wanscher's apparatus is, moreover, that the ether fumes are warm when the patient inhales them, because they mingle with the warm respiratory air already in the bag. Thus one avoids that cooling of the patient's air passages which resulted from the other methods, and may possibly have been the cause of some of the bronchial affections and pulmonary complaints which some surgeons have encountered after ether narcosis. Finally, we have the great advantage that no fumes can escape into space, poison the atmosphere, and be wasted. The method has the further advantage that the consumption of ether is far less than with all other methods. With major operations lasting from one to two hours we, in this way, do not consume more than from 60 to 70 cubic centimetres of ether, whereas with other methods one consumes from 300 to 400 cubic centimetres.

With regard to the conditions consequent to this form of ether narcosis, it must first be mentioned that the patients quickly recover consciousness and, generally speaking, feel well and in good spirits. Vomiting after the narcosis is of rare occurrence; indeed, we seldom see the prolonged and most unpleasant vomiting which so often follows chloroform narcosis.

Now with regard to the question of postoperative pneumonia, the recognized frequency of which is constantly advanced by the advocates of chloroform as an argument against the use of ether: a glance through our yearly register will soon convince you that, with the form of ether 
narcosis used by us, pneumonia rarely follows. Only three instances in 552 cases of ether narcosis are mentioned in last year's records where pneumonia was the cause of death, and two of these instances refer to patients who were greatly reduced by disease or malnutrition when they were operated on: one, a five-month-old, rhachitic, hare-lipped child suffering from bronchitis, and the other a woman who was greatly weakened by an infectious icterus of long standing.

It is astonishing, moreover, that the misconception that ether has a harmful influence on the pulmonary passages still exists, because in reality the correctness of this view has long since been refuted, both clinically and experimentally. From a clinical point of view it was Mikulicz's report in 1898 which drove the nail home. Mikulicz, on account of the somewhat frequent occurrence of postoperative pneumonia, had deserted ether and taken up chloroform, in the belief that the pneumonia was due to the irritative effect of ether. To his surprise, however, it appeared that the cases of chloroform narcosis were followed by a still greater percentage of postoperative pneumonia. He therefore decided to give up narcosis by inhalation entirely, and thereafter employed local anæsthesia in all operations, even the major ones. But, to his yet greater surprise, the result was that the lung complications, far from decreasing, increased to a considerable extent: with 114 laparotomies he had no less than twenty-seven lung complications! Naturally, this experience overthrew the old conception that postoperative cases of pneumonia were "narcosis pneumonia." One curious fact should long ago have aroused the surgeons' suspicions; namely, that almost every "narcosis pneumonia" manifested itself after laparotomy, while it is extremely rare to find pneumonia following operations on the extremities, thorax, and head. To what was this strange occurrence due? Surely, in the main, to two circumstances: (1) That 
peritoneal infection is conveyed to the lungs partly by way of the lymph vessels and venous blood, and partly by embolism; and (2) that a patient with laparotomy wound dares not cough or breathe freely, inasmuch as this involves pain in the wound. If, therefore, there is a previous bronchitis, or if an infection of the lung sets in, the development of pneumonia is favored by the deficiency in expectoration and lung ventilation.

It has been proved experimentally with animals-and I myself have substantiated the fact by experiments-that

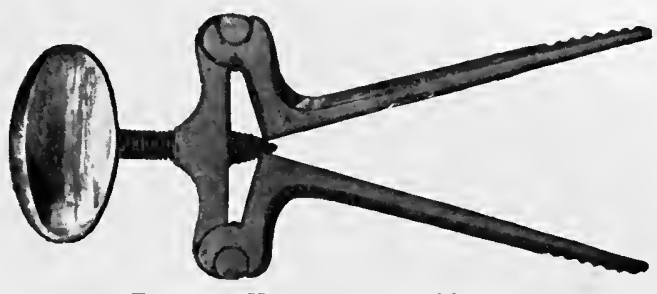

Fra. 23.-Ireister's mouth dilator. ether does indeed occasion increased salivation in the salivary glands of the mouth, but that the air passages-larynx, trachea, and bronchiare not irritated at all, even when the animals are killed by administering ether through a tracheotomy tube until they are dead. Therefore the only way in which ether narcosis

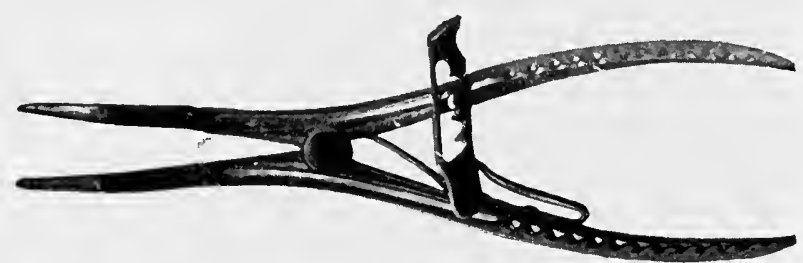

Fra. 24.-Koenig's mouth dilator.

per se can cause pneumonia is by aspiration of accumulated saliva in the throat. This, however, is always due to some technical error in the narcosis, for saliva should not be allowed to accumulate in the throat to any extent. One great advantage of the administration of morphia previous to the ether narcosis is that it prevents salivation, as a rule; but if it does occur it is quite easy to remove the saliva at the corner of the mouth by placing a strip of gauze or a drainage tube in sulcus alveolo buccalis. To obtain access 
to the throat during the spastic stage also, when the patient clinches his teeth firmly, the anæsthetizer should always have at hand a mouth-gag or dilator, whether it be the old classical Heister's (Fig. 23) or Koenig's apparatus (Fig. 24).

With some patients, especially inebriates and smokers, the salivation may be so considerable that at certain intervals a large quantity of mucus collects in the throat, which one recognizes by the mucus rattling during inhalation and exhalation. In such cases I introduce a drainage tube into the throat and connect it with a glass receiver in which is a suction ball which draws the fluid downward whenever it begins to inconvenience the respiration (Fig. 25). This practical little apparatus was devised by my former assistant, Holger Strandgaard.

If, therefore, it is proved that the ether fumes do not in any way irritate the main air passages, one should admit that the other assertion must also be wrong. I mean the assertion that ether is contra-indicated in patients suffering from lung diseases: emphysema, bronchitis, bronchiecta-

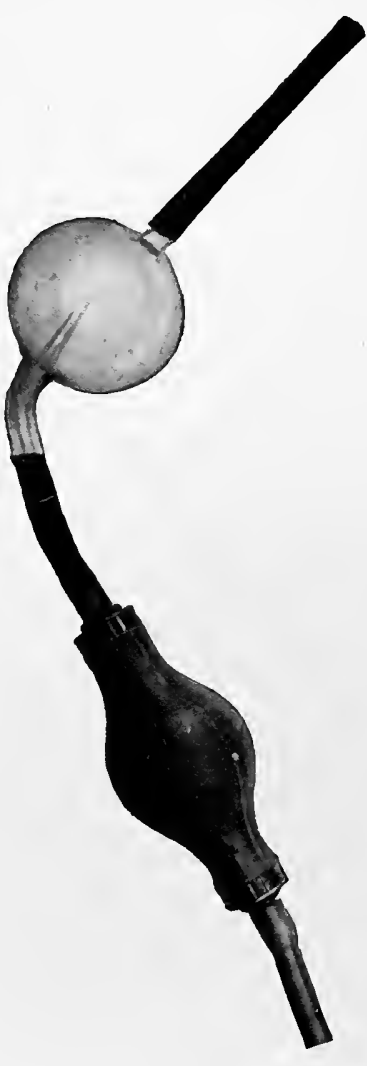

Fig. 25.-Apparatus to draw mucus from the throat during the narcosis. sis, abscess of the lung, etc. As was to be expected, this fact has been evidenced in our employment of ether narcosis with lung patients, an application which, at first, was most carefully employed, but which was undertaken with more and more confidence as time went by. It has, however, been a matter of greater surprise to me that with 
certain badly-afflicted lung patients ether is not only tolerated but appears to have a quite specific, beneficial effect. I discovered this about five years ago, when a patient was admitted to this ward with a view to an operation for a cancerous tumor of one breast. She was an extremely stout woman, sixty years of age, who was suffering from emphysema and bronchitis to such an extent that for the previous ten years she had been obliged to sit upright day and night in order that she might breathe at all. The color of her face was almost indigo, owing to cyanosis, and her breathing so troubled, being accompanied by mucus rattling and coughing, that a narcosis did not seem to me to be in any way possible. This I explained to her, and I stated that I did not think I could operate on her unless she was sufficiently courageous to have the operation done under local anæsthesia. To this she agreed, and under Schleich's local anæsthesia the operation was commenced. However, on account of the pain and the manipulations of the wall of the thorax the patient developed such acute attacks of dyspnœa and heart weakness that the operation could not possibly be continued. With fear and trembling I then decided to venture an attempt with ether narcosis, but it is difficult to describe the astonishment of all those who were present when they saw the change caused by the inhalation of ether in the course of a few minutes. The respiration became deep, calm, and regular, the cyanosis gave place to a healthy, natural complexion, and the pulse grew strong, full, and regular; so the operation could be completed without the least difficulty, while the patient was placed in a quite natural, recumbent position. The wonderful part of this experience was, however, that this change in the patient's condition lasted for thirty-six hours after the cessation of the narcosis, so that the patient imagined herself cured of her lung affection; but then the attacks recommenced and quickly resumed their former character. Since then we 
have had this experience confirmed time and again, so that we deem it not only permissible to use ether narcosis with lung patients, but we consider it advisable, as being specially adapted to such patients. I wish to call to your attention also, in this connection, that ether was first employed in medicine exclusively as an inhalation remedy for lung diseases. It is a question whether a new era is not perhaps awaiting ether as a remedy for asthma, emphysema, and bronchitis. It would be a fitting rebuke to those surgeons who have unjustly charged that ether has an injurious effect on the air passages.

For operations on the lungs themselves I use the socalled negative pressure apparatus, which prevents collapse of the lung and the dangerous phenomena which are incident to a pneumothorax when it occurs suddenly during an operation. For this purpose I employ an apparatus constructed by our Danish colleague, the physiologist Holger Moellgaard, which is not only less expensive but also, in my opinion, considerably better than Brauer's original "Ueberdrukapparat." A complete description of this complicated apparatus is to be found in "The Surgery of the Lung," and in "Hospital's Tidende," penned by Moellgaard. Here I only wish to say that the lung is prevented from collapsing by pumping in air which passes through the ether fumes; and it appears that this liberal supply of the air has the effect of preventing salivation, nausea, and cyanosis. The patients recline, the skin is of good color, and they inhale without any rattling in the throat. Therefore, the employment of this method of narcosis should be extended beyond the limited domain of lung surgery to include all operations which have to be performed on patients who have weak hearts or are suffering from diseases of the lungs.

For operations which demand preliminary tracheotomy (operations on the larynx, resections of the upper jaw, etc.) the etherization is best and most conveniently carried on by 
means of a long, tightly-fitting drain inserted through the tracheal cannula and connected with the ether-bag. On the side of this tube a small opening is found which can easily be closed with one's finger, or left open to admit air if desired.

One of the great and decided advantages of ether over chloroform is that with ether we need never fear heart collapse; for there is a much wider margin of safety between the dose of ether necessary for a complete narcosis and that which results in death, and therefore collapse, as a result of ether poisoning, need not be seriously considered. In addition to this, the dose of ether necessary for narcosis does not, like chloroform, diminish the blood-pressure and the strength of the heart, but, on the contrary, enhances them. This fact has been recognized for a long time by even the keenest votaries of chloroform, inasmuch as they employ ether in place of chloroform when operating upon patients suffering from enfeebled hearts, or upon deeply anæmic, debilitated patients (internal hemorrhage; for example, ruptured extra-uterine gestation). And it is indeed quite indisputable that the majority of such operations could not be performed at all without the aid of ether and the cardiac stimulation which ether provides. For these reasons, also, it is far easier, even for the inexperienced, to administer ether than chloroform. Thus, when operating in the country, if you have personally induced the narcosis, you can safely allow some unskilled person to continue it, because the only thing to be dreaded is difficulty of respiration, and an excellent feature with this narcosis is the audibly hoarse breathing, which enables you to control the course of the narcosis even at a little distance. When you no longer hear this sound of breathing, or when there are symptoms of mucus rattling, you know at once that it is time to take further measures. Although here in this ward, with a view to the training of physicians we are frequently 
obliged to change our anæsthetists, I venture to say that ether narcosis has never for a moment caused me any anxiety-a wonderful contrast to the days of chloroform narcosis, when scarcely a day passed without one's being alarmed by a threatened or real collapse.

Hitherto we have only occupied ourselves with the full ether narcosis, as used in combination with morphia in major operations, but ether narcosis has a further characteristic which makes it exceedingly adaptable for short or minor operations, such as tooth extraction, incision of abscesses and furuncles; for if you allow a patient to inhale ether a complete condition of unconsciousness sets in after the lapse of a few moments, and lasts for several minutes, when, without causing the patient any pain, you can perform slight operations before the stage of excitement is reached. We call this stage the primary ether narcosis as opposed to the ether intoxication (etherrausch) described by Sudeck and employed for the same purpose. But his method differs from ours, inasmuch as it is a suffocation narcotization, during which the patients become quite blue in the face and, though indeed unconscious, kick and offer violent resistance. We, on the other hand, do not allow our patients to become cyanotic, and they present an appearance of calm sleep, as I am about to demonstrate with this little girl whom we desire to anæsthetize, in order to excise a tuberculous lesion in the region of the hip. To begin with, and to familiarize her with the odor, I allow her to smell the ether at a distance, and so soon as she is accustomed to the odor I fasten the apparatus firmly on her face. Her breathing at once becomes deep and regular, so with her the result will be quickly effected. Even now, after a lapse of scarcely three minutes, I can nip her skin without her feeling it. Now I am operating! You perceive that I have had ample time in which to perform the operation; and now she is conscious, having no notion of what has taken place. 
P. Kuhn Faber ${ }^{1}$ has proved that this primary ether narcosis is very useful for the multiple extraction of teeth, and in this respect ought entirely to supersede chloroform narcosis, which has also claimed not a few victims from the dentists' chair; and I should imagine that it has already succeeded in winning the support of dentists to some extent. But in my opinion, so far as dentistry is concerned, ether should be preferred not only to chloroform but also to protoxide (nitrous oxide gas), because protoxide, in part, is by no means so innocuous as dentists assert. By careful search of recent literature I have discovered thirteen deaths from this anæsthetic, the reports of which in some instances have been suppressed for obvious reasons. But to this must be added the fact that protoxide narcosis is of so short duration that it allows no time for the more complicated tooth extractions; as, for instance, successive extractions such as dentists have to undertake preparatory to the adjustment of artificial teeth. If the extraction does not progress favorably the physician or dentist becomes nervous, by reason of the short time which the narcosis leaves at his disposal, and many instances of broken teeth, unextracted roots, and extraction of the wrong tooth are due to this cause. By this I mean that primary ether narcosis is greatly to be preferred to protoxide; not only because one runs no risk with it, but also because the duration of the anæsthetic lasts longer and allows an uninterrupted execution of even difficult and multiple extractions.

With obstetric narcosis, also, I have tried ether as a substitute for chloroform in a small way and with excellent effect. You seat yourself at the head of the bed where the patient is about to be confined, holding Wanscher's apparatus filled and ready, and constricting the neck of the bag in order to prevent the ether from evaporating into the

\footnotetext{
"Paul Kuhn Faber: "Primary Ether Narcosis with Dental Operations," Hospitalste, 1904, p. 243.
} 
room between the birth-pains. Whenever the labor-pains are renewed you advance the mask quite close to the patient's face, so as to be able to press the mask tightly to the face as soon as the pains become severe. The deep, powerful breathing gives such an ample supply of ether that uterine contractions become painless, so to speak. One great advantage which ether has over chloroform is that it strengthens rather than diminishes the contractions. When the mask is removed, the patient not only becomes immediately conscious but is in unusually good spirits, which phase usually con. tinues until the labor-pains appear again; and thus the whole of this otherwise painful process of confinement is brought to a conclusion in a bright and cheerful spirit, both as regards the patient and her sur-

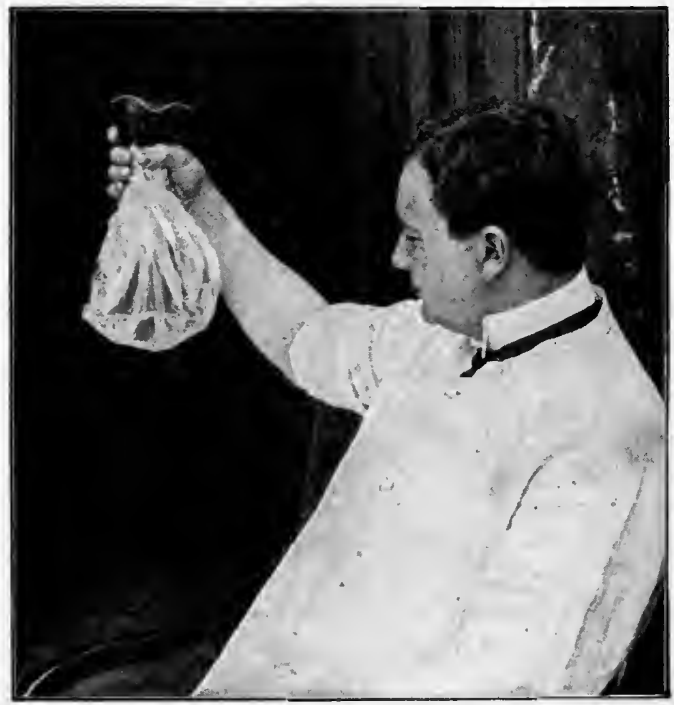

Fig. 26.-Ether bag of Gaudafil.

roundings. Those obstetricians whom I have persuaded to try ether have confirmed my somewhat limited experience.

Ether narcosis, as I have described it to you and as you have seen it performed here, is, in my opinion, an almost ideal means of anæsthesia; and I can safely recommend you to employ primary and total ether narcotization in your practice wherever general narcosis is indicated. As an objection to this method it has been stated that it is very difficult for a physician to keep the mackintosh bag in a fit condition; that is, for a physician who only occasionally has 
need of narcosis. It is quite true that this difficulty exists, but it is not insurmountable. The secret is to keep Wanscher's apparatus, like all rubber materials, in a warm place, because cold always harms it, causing it to stiffen and crack. If one wishes to make quite sure, one can keep the bag in glycerine.

Lately I have been searching for a practical and inexpensive material from which the practitioner himself can make an ether-bag at a small cost, and I think I have discovered it in the material which I show you here. The material, the name of which is gaudafil, I discovered during my last trip to Germany. It mostly resembles thin oilsilk in form and consistency. It differs in that it is white and transparent, will withstand boiling, and is unaffected by ether. It takes only a couple of minutes to make a bag with this material and attach it to the neck of the mask with elastic. Furthermore, it has the great advantage over Wanscher's of being as transparent as glass, so that you can at all times see how much ether the bag contains (see Fig. 26).

\section{INSTRUCTIONS FOR INDUCING ETHER NARCOSIS WITH WANSCHER'S APPARATUS}

(1) Place the patient's head low, so that the chin is the most elevated feature.

(2) Address the patient in a reassuring tone, and make clear to him how necessary it is that he breathe quietly and regularly, and preferably through his nose.

(3) Pour from 40 to 50 grammes of ether into the bag (about 25 grammes are sufficient for minor operations).

(4) When the ether which, during the process of filling the bag, has moistened the inside of the mouth-piece, has evaporated, the mouth-piece is advanced to within one or two centimetres of the patient's face, and is kept at this distance until the patient has taken from five to ten quiet inhalations.

(5) Now the mouth-piece is brought so near the face that its edge touches it. (At this stage it is important that no sudden movement of the ether takes place in the bag.)

(6) If there is strong secretion of mucus from the mouth, the patient's head is turned on one side, and a gauze meche is placed in sulcus alveolo buccalis. With spastic stridulous respiration the jaws are kept' apart by means of a dilator or mouth-gag, while the narcosis is continued. 
(7) If the patient does not respond when spoken to, and if the respiration is regular, the edge of the mouth-piece is pressed firmly on the patient's face, so that the air is excluded. As soon as cyanosis appears the mask is removed from the face for a few moments until its natural color is regained.

(8) Full narcosis is established when the cornea reflex disappears. From that moment until the operation is completed, the mouth-piece must rest, lightly pressed, on the patient's face, so that the air can obtain entrance here and there beneath the rim. If the patient shows signs of a return to consciousness, the rim of the mouth-piece is again pressed tightly on the face for a few moments. It is not advisable to shake the bag in order to increase the dose of ether.

(9) If there is any tendency toward vomiting before the narcosis has set in, it is useless to try to prevent it, and when it is finished the mucous membrane of the mouth should be quickly wiped with gauze, after which the mask is again closed tightly on the face.

A tendency to vomit after the narcosis has set in can usually be checked, either by removing the mask completely for a few moments, or by pressing with the finger-tips on the phrenic nerve above the left, clavicle.

(10) The best position for the anæsthetist is, as a rule, on the left side of the patient, holding the mouth-piece in his left hand, while the patient's left arm rests in the crook of his own left arm. With laparotomy in the Trendelenburg position, his best position is on a low stool behind the head of the bed or table.

(11) With operations on the front of the body, the patient's arms are laid alongside the trunk and fastened to the table or to the thigh, either with a broad bandage which is easily unfastened, or with a broad linen strap which fits firmly on the wrist, being fixed to the table or the thigh by bands (see Fig. 22).

For operations with the patient lying on his side, the lower arm can be fastened to the trunk, while the upper arm is brought over the chest.

(12) The so-called narcosis paralysis is most frequently due to pressure of the caput humeri on the plexus brachialis, owing to the protracted elevation of the arm during the narcosis. Therefore the arm must never be fixed in a high, elevated position,-i.e., vertical with the neck and head.

(13) In adults I inject $1 \frac{1}{2}$ centigrammes of morphine subcutaneously half an hour before the narcosis. With inebriates $I$ give 50 centigrammes of veronal in addition. With patients who cannot take morphine $I$ administer 1 gramme of veronal one hour in advance. 


\section{LECTURE V}

\section{AN 死STHETICS}

\section{Local Anæesthesia}

In my last lecture I had only sufficient time to present what I considered the most essential points concerning general anæsthesia. In connection with that I wish to lecture to-day on local anæsthesia, the importance of which depends so much upon the extent to which one finds narcosis serviceable. I will demonstrate on patients the methods which we use in this ward and which I can recommend to you for use in your practice.

The history of local anæsthesia takes us back to ancient days. First, stupefying drugs, such as opium, cannabis indica, mandragora, lactuca, and hyoscyamus, were tried in order to produce a local anæsthetic effect. They were used also as fomentations in the treatment of painful wounds, but nothing worthy of notice as an anæsthetic for surgical purposes was obtained until Alexander Wood, of Edinburgh, showed us how to use anodynes by subcutaneous injection in 1853. Wood injected morphine and opium in the neighborhood of nerve-centres in order to produce local anæsthesia in cases of neuralgia. The local effect of such injections, liowever, was soon overshadowed by the generally soothing effect of the morphine, owing to its rapid absorption. A local anæsthesia by means of vegetable alkaloids suitable for surgical work was first produced by Corning and Roberts, who recommended the use of cocaine injected into the tissues. This became of practical value in 1885 , although the wonderful effect which cocaine produced on the mucous membrane was proved by Koller in 1884, and since that time has caused a revolution in operative ophthalmology and laryngology. 
Another method of local anæsthesia which was employed in olden days, and perhaps with equal success, was compression: partly of the nerve-centres only, and partly of the fleshy portions generally; that is to say, by binding an extremity above that part where the operation has to be performed. Our experience with Esmarch's bandages, which showed that the binding not only keeps the tissues free of blood but also reduces sensibility to a great extent, was already known and made use of in olden days. Nowadays, indeed, compression is not used as an independent means for producing local anæsthesia, but as an important adjuvant to various methods. For instance, Schleich's cocaine anæsthesia and Bier's latest cocaine anæsthesia can now be attained with a very dilute and relatively nonpoisonous solution of cocaine, inasmuch as compression reduces the sensibility to such a great extent. Of later date, yet not uninteresting to us from a historic point of view, is refrigeration ancesthesia, a form of local anæsthesia, which-long before anæsthesia by chemical agents-attained such perfection that it obtained real and widely spread importance. In literature we get our first information regarding the application of refrigeration for the production of local anæsthesia from our famous fellow countryman, Thomas Bartholome, who relates that when in Naples with his instructor, Marcus Aurelius Severinus, he learned to make the skin insensible by a somewhat lengthy application of snow or ice, previous to the incision. Bartholome's experience, however, does not seem to have attracted much attention, and not until the commencement of the last century did the idea of refrigeration anæsthesia crop up again, J. Hunter proving by vivisection that freezing mixtures can make the skin quite insensible. Moreover, Napoleon's great army surgeon, Larrey, compelled by necessity, made the same discovery during the unfortunate campaign in Russia, inasmuch as the amputations which 
had to perform on the wounded, in a temperature of 17 to 19 degrees of frost, took place quite painlessly. Anæsthesia by freezing did not attain general popularity before 1866 , when Richardson taught us to apply the ether spray in order to produce the refrigeration of a limited skin area. Richardson's apparatus, which I have brought here to show to you (Fig. 27), is quite simple: A bottle of ether closed by a pierced cork, through which a long metal tube touches the bottom of the bottle, and extends upward in a thin terminating tube, within which, and tight over the cork, another tube

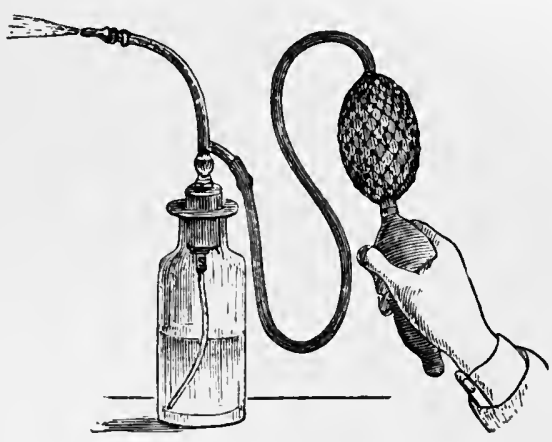

FIG. 27.-Richardson's ether spray for local anæsthesia.

is seen, connected with a powerful rubber air ball. A strong current of air being driven out through the tube, the ether is absorbed and finely distributed in the air, and forced through the point of the tube in a strong spray, which, when it touches the skin, causes the ether to evaporate quickly, producing an intense degree of cold. This method, which has considerable merit, has been compelled to make way for the simpler and quicker ethylchloride local anasthesia, which I will now demonstrate with this patient who has to have a large furuncle incised at the back of his head.

Here you see a clear fluid enclosed in a small, closed glass tube with a metal screw stopper (see Fig. 28). This is ethyl chloride, also called kelene, the chemical formula for which $\left(\mathrm{C}_{2} \mathrm{H}_{5} \mathrm{Cl}\right)$ is, of course, known to you: A substance which is remarkable because it boils and evaporates at a temperature of 11 degrees of warmth; that is to say, at a temperature considerably lower than the warmth of one's hand. It is this quality that is made use of. I now 
unscrew the cover and take hold of the glass tube with my whole hand. The fluid immediately boils and escapes in a strong spray through the very fine opening. I direct this spray downward toward the furuncle, and by moving the tube backward and forward in a set line I shall, in the course of a few moments, produce a shining, white strip on the red, inflamed tissue. Here it is already! You see how sharply the line is defined, and you saw that it whitened rather suddenly. The skin corresponding to the strip is frozen and quite insensitive; so, without causing the patient any pain, I now incise the furuncle along this line.

Ethyl chloride local anæsthesia is extremely useful and handy for minor operations, and I advise all of you to provide yourselves with a box of these small receivers when you start in practice. But it may happen that you will drive out some day without this apparatus, and, when out in the country and far from home, you will be faced with a phlegmon, abscess, ingrown nail, etc., which demands prompt treatment. Then it will be useful for you to know that a freezing mixture prepared from one part kitchen salt and two parts finelycrushed ice will serve your purpose. You know that this mixture produces a temperature of $11^{\circ} \mathrm{C}$. of cold, quite sufficient for a rapid

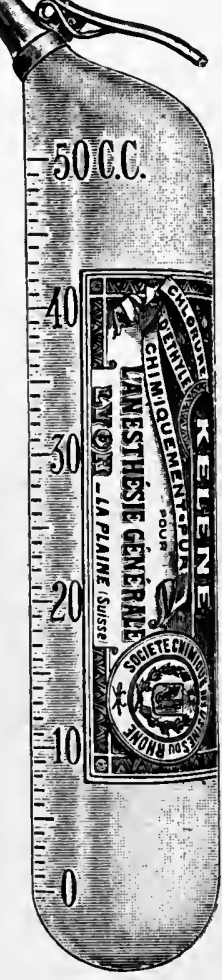

Frg. 28.-Tube with ethyl chloride. production of intense local anæsthesia. Kitchen salt, of course, can be obtained in every house; and ice can, as a rule, be easily procured in the country, even in the summer, where it is to be found in every dairy.

In order to demonstrate how sound and simple this method is, I will now apply it to a patient from whom we have to remove an ingrowing toe-nail, a very painful 
operation, as you know. Over this glass bowl I spread a double layer of gauze, and on this I place finely-crushed ice, which I mix well with kitchen salt. Now I fold the four corners together so that we have the freezing mixture in this little gauze bag, which, with its contents, is so soft and pliable that, as you see, I can bury the toe in it. The sure sign that anæsthesia has commenced is here; also, that the skin becomes snow-white and hard-it is frozen. Even now, in the course of three minutes, you see the alteration in color. I now hasten to thrust the pointed scissor blade up under the nail, and you will notice that this otherwise very painful operation is as little heeded by the patient as are the incision along the nail groove and the evulsion.

You will observe that a few minutes after I have completed the operation-without loss of blood and without pain to the patient-the whitened area changes to a vivid red; the blood pours forth from all sides, and the patient begins to complain loudly. Here we have the limitation of, and the serious drawback to, this method. Its limitation consists in its being applicable only with quite small, transitory operations, which can be completed before the ice is melted by contact with the warm blood of the body. Its drawback is that the sensation of pain returns in an intensified form so soon as the freezing effect ceases. Every one who has suffered from a touch of frost-bite of the toes, fingers, nose, or ear-lobes, whereby these become quite insensitive, knows how, and for how long, the blood throbs and hurts in these members when one enters the warmth and "thaws them." It is the same here, and the patient is, as it were, duped by reason of the subsequent pain and smarting; but the operation and the dread of it are over, and at the thought of this the patient can better tolerate the pain. From the physician's point of view everything is satisfactory, because he has been enabled to undertake the operation quite tranquilly and without hindrance. Un- 
fortunately, the range of the method is restricted to quite small, short operations, punctures and simple incisions, because, more is the pity, the method has the great advantage of being absolutely harmless to the organism and thus differs from those methods incidental to lengthy and more complicated operations, all of which are modifications of one principal method: cocaine ancesthesia.

The previously-mentioned experiments of Corning and Roberts in 1885 proved surely enough that injection of solution of cocaine in the tissues was, to some extent, an ideal means of local anæsthesia, because, in the course of a few minutes, a complete insensibility was attained, which lasted for hours. On the other hand, however, it soon appeared that its application incurred the danger of collapse and death by poisoning; a most characteristic and sinister poisoning, which, when once witnessed, is not easily forgotten. A violent state of excitation suddenly sets in, and the patient, with burning cheeks, glistening, protuberant eyes, breaks forth into loud laughter and delirium, followed by epileptic cramps and then-sudden collapse as the result of heart-failure.

Such fatal, acute cocaine poisonings are sometimes caused by accidentally injecting the cocaine directly into a blood-vessel, so that it is carried in a relatively large and concentrated quantity to the heart and central nervous system. But fatal poisoning can also arise from mere absorption through the tissues; yes, even from the mucous membrane itself. There is authentic evidence of nearly a score of instances of fatal cocaine poisoning from the mucous membrane of the urethra. Therefore, I never risk the otherwise so tempting cocaine anæsthetization of the urethra previous to cystoscopies or other painful insertions of instruments, and here I will strongly advise you against the use of cocaine. There does not appear to be any danger worth mentioning from using it on the conjunctiva and in 
the throat, but when laryngologists paint the larynx and œsophagus with 20 per cent. cocaine in order to introduce broncho- and œsophagoscopes, without general narcosis, I consider it hazardous, and actually prefer the narcosis as being less dangerous. That some people die in this manner, after a mere painting of the mucous membrane, which so many have endured, proves that here, as with so many poisonings (chloroform, iodoform, etc.), an indiosyncrasy asserts itself,-i.e., certain people, without any ostensible reason, are wanting in a normal ability to tolerate the drug, and are poisoned by quite infinitesimal doses. We should, of course, never forget this incalculable and dismal factor, and it should induce us to be generally careful with cocaine. The numerous clinical experiences, however, and no less the long series of experiments with animals-among which I may mention Mosso's (1887), Albertoni's (1890), and Maurel's (1892) - have proved that the risk from the application of cocaine depends chiefly on the concentration of the substance used. In this way Maurel has proved that a quantity of cocaine, quadruple in bulk to that which if injected in a 10 per cent. solution produces violent poisoning, is endured without reaction when injected in a 1 per cent. solution. Therefore endeavors have been directed chiefly toward reducing, as far as possible, the concentration of the cocaine solutions employed for local anæsthesia.

As early as 1885, Corning thought of combining the old, well-known method of compression for producing insensibility with cocaine anæsthesia, and pointed out that, if Esmarch's sling was previously applied to an extremity or to a part of it, a $1 / 2$ per cent. solution of cocaine would be sufficient to obtain local anæsthesia-which would otherwise require a 25 per cent. solution. This solution has become very valuable for operations on fingers and toes since Oberst has shown us that in order to make the entire finger insensible in the course of a few minutes it is suffi- 
cient to make a linear, dorsal, volant, subcutaneous injection of a $1 / 2$ per cent. solution of cocaine at the root of and crossing the longitudinal axis of the finger; the reason being that here the sensitive nerves of the finger lie subcutaneously and, in consequence, are locally affected by the cocaine, resulting in interruption of pain impulses along the nerve: what the Germans call conductive anæsthesia (nerve blocking). It is most important that you should know this method, and be in a position to make use of it in those frequent cases where injuries from machinery or other causes require suturing, resections, or amputations under circumstances where deficient assistance makes narcosis impossible or difficult; also, with phlegmons on the ends of the phalanges, paronychia, etc., this method, which does not require injection into the diseased tissue, is excellent.

To-day, therefore, I will demonstrate the application of the method on this young workman from the docks, who was injured this morning by the falling of an iron plate, which crushed the terminal joint of his left forefinger to such an extent that amputation of the phalanx or disarticulation between the first and second is necessary. You see the patient prepared, carefully disinfected, and with a thin drainage tube laced around the finger-tip, by means of which the finger is depleted of blood, and is so dulled that a $1 / 2$ per cent. solution of cocaine will be sufficient for the injection. Now you will see me do this. I draw up the freshlyboiled cocaine into a sterilized Pravaz syringe which is fitted with a fine cannula, and insert this under the skin of the radial edge of the finger, just below the band. After I have injected a little of the solution, I press the cannula forward transversely, and inject continuously, thereby preparing a way for the cannula right over to the ulnar side. I then draw the cannula back and proceed in exactly the same manner on the other side of the finger. In a few moments 
the anæsthesia will be complete, and my assistant will perform the operation, while we proceed with our subject.

Where the hand and foot are concerned, we can also obtain regional ancesthesia by Oberst's method, by injecting cocaine across or at right angles to the course of the nerves, and above the wrist or ankle. In Fig. 29, for instance, you see how the entire radial area is rendered insensible by injection at the line indicated.

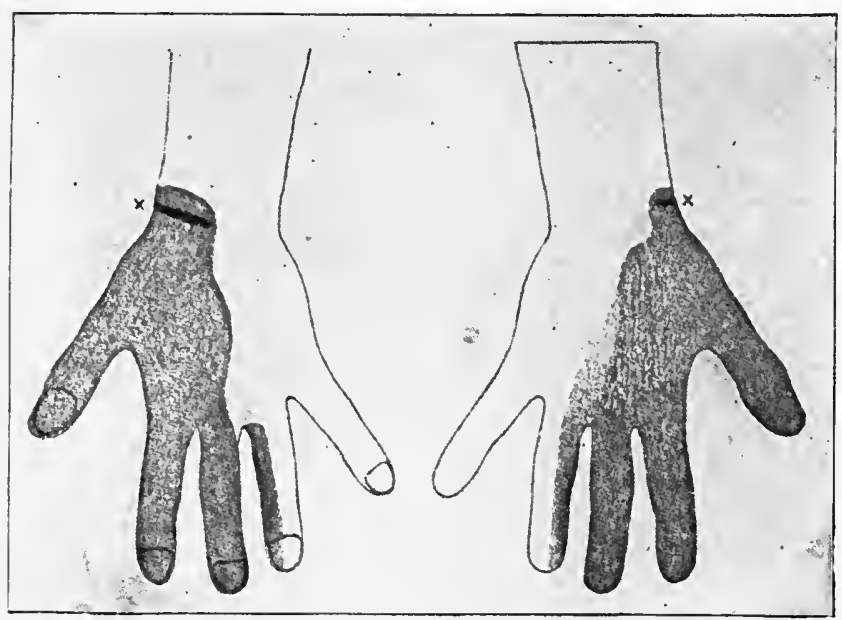

FıG. 29.-Cocaine anæsthesia after Oberst's method.

Where an entire extremity is concerned Oberst's method cannot be employed, because here the nerves lie too deeply and too close to large blood-vessels. Amputations and resections cannot, therefore, be performed with this method, but, even if they could be, they should, in my opinion, never be done under local anæsthesia, but under ether narcosis, because it is really inhuman to allow a fully conscious patient to undergo such dangerous and physically overwhelming operations when we are in possession of such a safe general anæsthetic. Bier, however, has lately attempted to make such operations possible under local anæsthesia by the following procedure: Having depleted the ex- 
tremity of blood, and having applied an elastic band both above and below the field of operation, he exposes a large vein by incision, and through this injects the entire limited area which the vein drains with a solution of cocaine; the solution then soaks through the vessel wall to the tissues and affects the nerves. In this way he has succeeded in obtaining anæsthesia in some instances, so one has to admit that the interesting experiment can be successful; but we must question its fitness to be classed as a method of local anæsthesia for use among human beings: In the first place, because it is quite superfluous and, as I said before,inhuman; and, secondly, because one can safely foretell that it will prove very dangerous. To begin with, such a large quantity of cocaine is required to fill the empty net-work of vessels, and this, when the Esmarch's band is removed after the operation, suddenly enters the circulation, so that poisoning is only avoided with difficulty. But the danger from phlebitis and embolism seems to me to be just as dangerous. As it is, we are sufficiently afraid of this complication during the healing of the wound, and to vulnerate and fill the whole net-work of veins above the wound with a poisonous, alien fluid, seems to me to be tempting fate!

The assistant surgeon has now completed the amputation of the finger without the patient's feeling any pain whatever. Anotler patient is now being brought in : an old man with complete retention of urine, owing to hypertrophy of the prostate, on whom I will demonstrate for you cocaine anæsthesia as employed in other regions of the body.

Here we must dispense with the great advantage offered us by Oberst's method with the fingers and toes,-i.e., that the injection can be made outside the immediate area of operation, and we are compelled to use a cocaine solution of greater concentration, because we cannot, here as there, benefit by the constriction of the afferent nerves and vessels. However, we are able to obtain sufficient anæs- 
thesia with a weaker solution of cocaine by producing local tension, and thereby compression and anæmia of the tissues.

Where the skin is concerned we have two methods for producing cocaine anæsthesia: the endermatic, by which we inject the fluid into the cutis itself, and the subdermatic, by by which we inject it under the skin, and here act upon

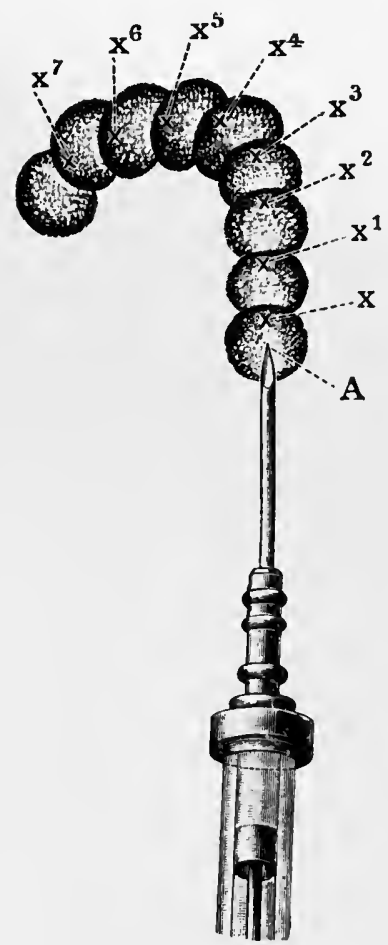

FIs. 30.-Formation of bullæ by endermatic cocaine injection. the nerves leading to the skin, consequently conductive anasthesia.

With the endermatic method, which I am about to demonstrate with this patient, I begin by thrusting the cannula into the skin, just where I judge that the top of the incision should be-in this case almost midway between the umbilicus and symphysis. Now, while I am injecting, you see (Fig. 30) that a circular, distended, whitish wheal arises. When I now advance the cannula to the foremost limit of the wheal, and re-inject, you see that a new round wheal forms; and thus they form themselves side by side, producing a broad, white, œdematous strip right down to the symphysis. You will understand that here, in order thus to thrust apart the layers of cutis, a strong pressure is involved, which assists the anæsthesia; and here, also, we can do with a solution of $1 / 2$ per cent. to 1 per cent. cocaine in quite a small quantity. On the other hand, if we wish to make use of the subdermatic method, which Reclus and Schleich partly employ, you will understand that there can be no talk at all about such a pressure in the soft, yielding, subcutaneous, fibrous tissues, and for this reason 
one must also use the stronger concentration of 1 per cent. to 2 per cent. under such circumstances, whereby the danger from poisoning is increased; which danger is further increased if the operator soaks each succeeding layer of tissue as it is exposed with cocaine, as Reclus recommends. True enough, Schleich, in his so-called infiltration ancsthesia, has given us a modification of Reclus's method which will diminish the danger of poison, inasmuch as the concentration of the cocaine solution is reduced to 1 per cent. or 2 per cent. On the other hand, the quantity of fluid injected is so much greater, owing to the importance of distending the tissues and thereby compressing the vessels. It is the anæsthetizing effect of the compression and the anæmia which Schleich would once more make use of to reduce the cocaine concentration. I shall not further dwell upon the question as to whether Schleich attains his object or not, because the method has one objection which induces me to warn you against its employment: It produces an artificial cedema, extending in circumference far beyond the area of the operation itself, whereby it not only obliterates the anatomical feature and confuses the operator, but also damages the tissues and thus extends the avenues for present or future infection. Now, fortunately, this infiltration of all the tissue layers with cocaine is, as a rule, quite unnecessary, owing to the fact, which Oscar Bloch ${ }^{1}$ demonstrated by a series of clinical experiments, that all tissues save the skin are insensitive to all operative manipulations, except when traction of sensitive nerve-roots is produced. With all operations where one can avoid traction of the tissues and organs, one can operate painlessly, if only the skin has been completely anæsthetized. This question of the sensitiveness of the various nerves and organs, especially as regards the abdominal organs, has been studied later

'Oscar Bloch: "On the Restriction of General Anæsthesia," Bibl. f. laeger, 1898, p. 69. 
and with great zeal by Lennander, who, from his investigations, has deduced the main conclusion that all tissues which are supplied by cerebral-spinal nerves may cause pain; while all organs supplied by sympathetic nerves are insensible. This conclusion is not universally accepted. It is true enough that the intraperitoneal organs are quite insensitive to operative attack-a fact which most of you have had the opportunity to substantiate during our clinical lessons; as, for instance, when I open an exposed intestine or stomach with a knife or with Pacquelin's cautery without causing the patient any pain. I do not, however, agree with Lennander that these organs must be quite incapable of pain sensation. I need only mention the pain of stomach ulcer, gall-stones, intestinal colic, and endo-appendicitis to make clear to you the incorrectness of Lennander's assertion. To me there seems no doubt that wounds, inflammation, and violent contractions of the muscles, especially if caused by obstruction in the passages and ducts, can call forth most acute pain even in the organs supplied by the sympathetic nerves. Nor is Lennander correct in stating that tissues and organs supplied by cerebral-spinal nerves are all sensitive to pain from operative invasion. These tissues react painfully to certain irritants, especially inflammation, but Bloch on the whole is right in saying that, apart from the skin, these tissues also are quite insensitive in a normal state.

My contribution to this question is voiced in a small treatise, "Eleven Sectiones Altæ without Narcosis," dated $1898 .{ }^{2}$ To avoid the danger of chloroform narcosis with very old people, who had to undergo a sectio alta on account of retention of the urine from hypertrophy of the prostate or a calculus in the bladder, I endeavored to perform the operation under cocaine local anæsthesia, and I found that it was quite sufficient to make a linear, intercutaneous in-

${ }^{2}$ Hospitalstidende, 1898, p. 1173 (Eleven Suprapubic Cystostomies, etc.). 
jection of $1 / 2$ per cent. cocaine or 1 per cent. eucaine conformable with the incision, because the subcutaneous tissues and the facial, muscular, and prevesical tissues permitted painless incision. The only thing which involves pain on the way to the bladder is the drawing aside of musculi recti just at the moment when this takes place; while they are afterward held apart by forceps without causing the patient any pain at all. The vesical wall, remarkably enough, is insensible to pricks, incisions, and sutures-provided it is not the seat of ulcerative cystitis. When ulcerative cystitis exists, a similar manipulation causes acute pain. These interesting observations opened my eyes to the untenability of Lennander's conclusion; because, in the bladder, as we all know, we have an organ which frequently reacts painfully to a whole series of irritants-distention, foreign bodies, and inflammation-although the normal bladder is quite insensible to the knife and to Pacquelin's cautery, precisely as the intraperitoneal organs are.

Since the introduction of morphine-ether narcosis I rarely have occasion to perform the sectio alta under local anæsthesia; still, I have in all performed the operation about fifty times in this manner. With this elderly man whom you now see me operate upon I avoid narcosis because he is somewhat uræmic. His kidneys, also, are considerably the worse for wear, and you know that ether-though to a lesser extent than chloroform-is capable of producing irritation of the kidney; which irritation is shown during the first forty-eight hours by cylindruria and albuminuria. With previously sound kidneys these remarks have no application, but with such debilitated kidneys as this man possesses narcosis might prove fatal.

You noticed that the patient winced slightly when I bluntly and forcibly separated the muscles, but for the rest we have succeeded in laying bare the bladder without causing 
pain, and in placing the two silk sutures, between which I am about to incise the bladder.

The incision is now completed without the patient perceiving it, and what you see pouring forth is the injected phenosalyl water. I now insert my forefinger in the incision and palpate the entire inside of the bladder, and feel a diffuse, slightly lumpish hypertrophy of the prostate, though without any noticeable protuberances, and no stone. Pezzer's catheter, No. 26, is now introduced, and the vesical wall is joined closely about this with catgut suture. I now join the aponeurosis centrally with a continuous aluminum bronze thread, and finally the wound in the skin, without the patient noticing it at all, because, in the meantime, the cocaine has rendered the skin anæsthetic. You understand that this is the main distinction from refrigeration anæsthesia, inasmuch as the latter lasts for only a few moments, just long enough for an incision, while cocaine anæsthesia allows of operations lasting for hours.

Ever since the equally harmless and more humane morphine-ether narcosis has been at our disposal I rarely find indications for cocaine local anæsthesia for the essentially important operations in this hospital; but every now and again there are cases where it is of the greatest importance. Let me just mention the operation necessary for empyema: pleuro- or costotomy with a completely debilitated dyspnœic patient, or tumors on the neck which cause violent dyspnœa, threatening suffocation. See, for example, this illustration (Fig. 31) of a lady with an enormous struma, which produced constant suffocation, making every attempt at narcosis impossible. It was removed without any pain to speak of by a simple streak-like, intercutaneous, cocaine injection in the line of incision, such as you see on Fig. 32. where the patient is depicted freed of her tumor. Also with ileus, and especially with incarcerated hernia with greatly debilitated patients, it can prove of great importance. 
But, besides these indications, cocaine anæsthesia will avail you with many others in your general practice when assistance with minor operations is not easily available: the removal of tumors, such as atheroma, lipoma, angioma, etc., glandular extirpations and the like.

So long as you maintain the intercutaneous injection of $1 / 2$ per cent. to 1 per cent. solution, the risk is very slight; but if you wish to insure yourself further, you can use

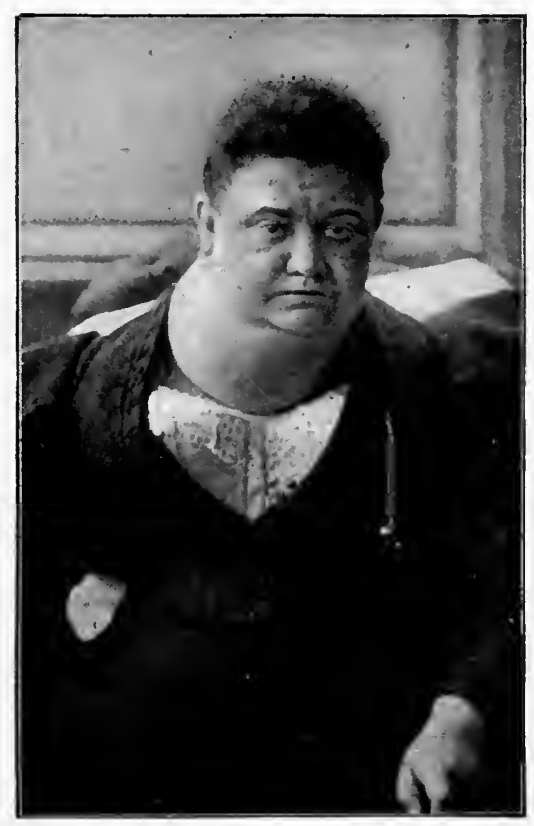

FIG. 31.-Before the strumectomy.

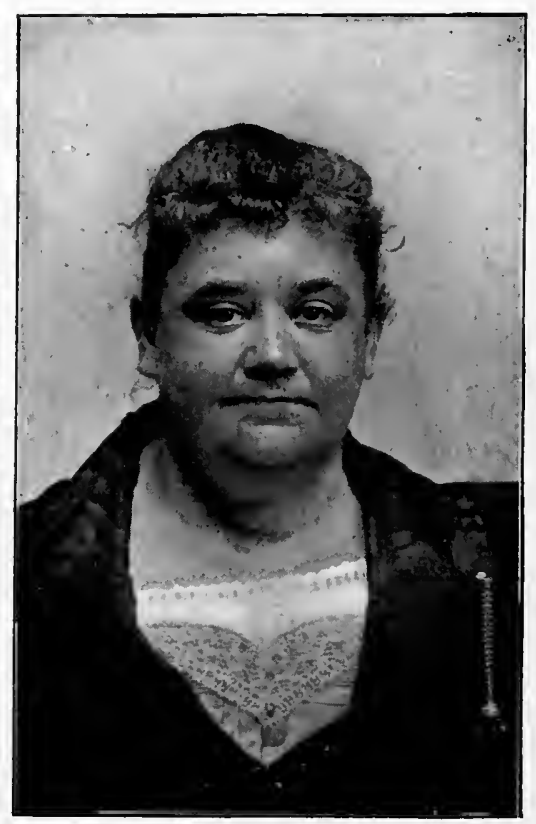

Fig. 32.-After the strumectomy.

encaine $B$, or, rather, the muriate of eucaine $B$, in place of cocaine. This is, as you know from pharmacology, an artificially-produced alkaloid, which in its chemical composition and effect closely resembles cocaine, though it has the advantage of being far less poisonous, and of being able to withstand prolonged or repeated boiling, whereas cocaine will not, and, indeed, is not uncommonly destroyed by boiling. From the antiseptic point of view this latter 
point is very important. It is said that eucaine $\mathrm{B}$ is about one-half as dangerous as cocaine, so that you can safely apply a 2 per cent. solution of this for injection where a 1 per cent. solution of cocaine would have been indicated.

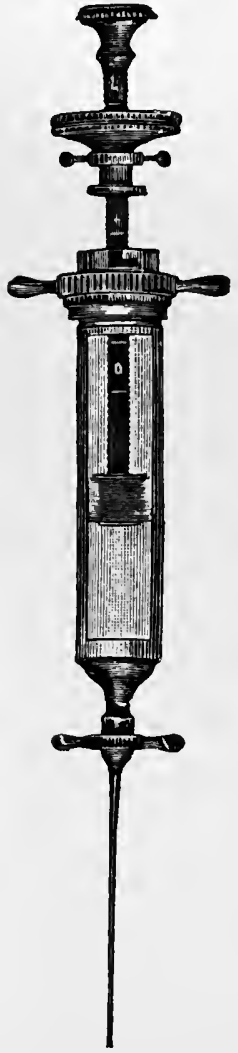

Fig. 33.-Braun's syringe for local anæsthesia.

Eucaine, however, is a purer and safer preparation, while the plant-extracted cocaine is more capriciously variable, whereby it may give rise to un-

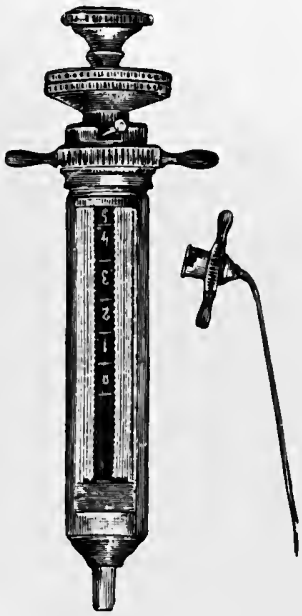
expected poisoning.

With these injections it is a matter of course that careful asepsis be observed. Both the injection fluid and the syringe must be freshly boiled. You can use an ordinary Pravaz syringe, provided the piston stands boiling (asbestos, metal), but with larger incisions, larger s y ringes with longer needles are, of course, far preferable, in order to obviate repeated punctures and the refilling of the syringe. Where the area of operation is prominent, as with very stout people, and with superficial tumors, you must have an angular cannula to enable you to apply the injection. The syringe which you saw me use can, I think, be recommended to you (see Fig. 33). It is constructed by Heinrich Braun, ${ }^{3}$ who has written a book on local anæsthesia, which book is well worth reading. His

${ }^{3}$ Heinrich Braun: "Die Localanæsthesia, ihre wissenschaftlichen Grundlagen u. praktische Anwendwug: Ein Hand- und Lehrbuch,” Leipzig, Ambrosius Barth. 
syringe consists of glass in a metal case with an asbestos piston, and has one advantage which is of great practical importance; namely, that the cannula is screwed on; while with the ordinary Pravaz syringe one is continually annoyed by the cannula and the syringe being forced apart owing to the strong resistance.

For very extensive infiltrations Alfred Madsen's syringe, which draws up the injection fluid from the flask like a suction pump through the drainage tube placed at right angles to the syringe, is very practical, though chiefly in hospitals and nursing homes.

I have already told you that for some years past I have restricted the use of local anæsthesia to a small minority of our operations; whereas other nations still endeavor to extend its field so far as possible with all operations. Naturally, you will ask me to explain the variation in its employment in the one case and the other. To answer you is easy. In every case where one still contends with the disasters which may arise from chloroform, and where one does not yet realize the true application of ether, one naturally endeavors to avoid narcosis collapse and pneumonia by means of local anæsthesia. This is perfectly natural, even sound and intelligible to us, for whom this is a thing of the past. On the other hand, it is quite incomprehensible to me that in many places, with the same justification, the so-called lumbar anasthesia, or, to use a better term, spinal-cord anasthesia, is made use of. I never have used and Inever will use this for operative purposes; but, as you will see it recommended in foreign text-books, and may perhaps have seen spinal-cord anæsthesia used and recommended elsewhere in this country, I must mention the method to you and explain my reasons for utterly condemning it.

The first person to perform a spinal-cord cocaine anæsthesia was the American doctor, Leonard Corning, who, as 
early as 1885, injected cocaine into the lumbar cavity around filium terminale, in order to relieve pain accompanying spinal-cord afflictions-a very sensible remedy and the only form of this anæsthesia which I, for my part, will always recognize as justifiable.

Not until 1899, fourteen years later, did Bier, independently of Corning's little-known experiment, think of applying a cocaine injection into the spinal canal in the lumbar region, the so-called lumbar cavity, in order to

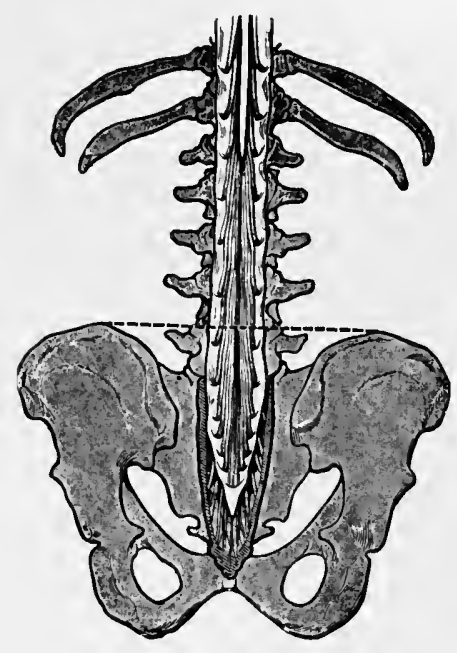

FIG. 34 . produce anæsthesia of the lower extremities during operations. Bier's communication caused a tremendous sensation, especially because he had experimented on himself and a friend before doing so with others. The attempt was successful. Their shin-bones became so insensitive to pain that they could be beaten with a metal hammer, though it was quickly apparent that the method could be attended with unpleasant results, for eight days after the experiment Bier suffered from headache and

vomiting. But he enthusiastically explained that these were not the result of the cocaine, but were due to his friend and himself having celebrated their success with a "WeinAbend" instead of with a "Bier-Abend" such as they were accustomed to.

The operation is quite an easy one. As you all know, the spinal-cord terminates on a level with the second lumbar vertebra, whence it sends forth filium terminale and numerous nerve-fibres down into the very wide, roomy lumbar cavity formed by arachnoid and pia; and, as the 
object is undoubtedly to avoid medulla while penetrating the cavity, one rightly chooses the centre of the three intervertebral spaces at one's disposal and punctures between the third and forth lumbar vertebra. This third intervertebral space is generally detected in that line which connects the top portions of crystæ ilei (Fig. 34). The puncture should not be made in the central line-the interspinal ligaments being thick enough to offer considerable resistance to the needle-but $1 / 2$ to 1 centimetre to the right or left of this, from which point the needle is introduced slantwise (see Fig. 35) and the dura is encountered almost in the central line. The only certain and, therefore,

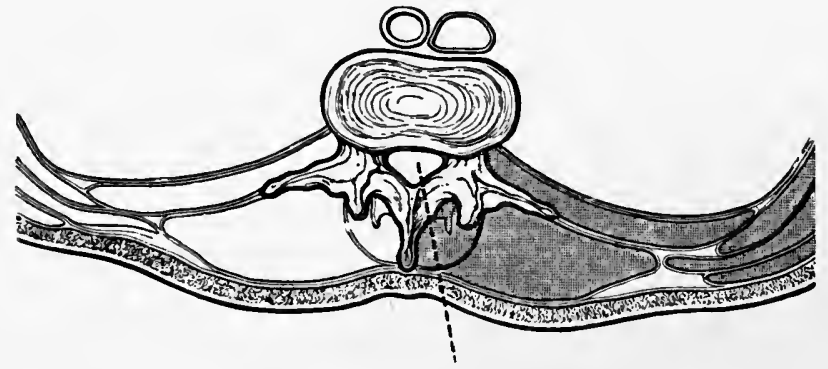

Fig. 35.-The cannula is introduced in the direction indicated by the dotted line.

necessary indication that the needle has entered the dura cavity is that cerebrospinal fluid issues forth; but it is important to know at what depth one should expect to encounter the cavity. This varies from 4 to 6 or 7 centimetres, according to the corpulency, obesity, and muscularity of the patient. If no fluid escapes when you have penetrated to this depth, you have gone either too far or in a wrong direction.

Undoubtedly, it is most convenient to proceed as Tuffier suggests, and let the patient sit in an inclined position during the operation; but in many instances the patient's condition does not allow of this, and then the recumbent, side position, with bent back and updrawn knees, as recommended by Bier and Quincke, is preferable. 
When one is assured as regards the penetration, one injects the $1 / 2$ to $11 / 2$ centigramme cocaine solution. With successful cases, complete anæsthesia of the lower extremities and the pelvis takes place in the course of five to ten minutes, thus enabling one to perform amputations and rectal and hernia operations, etc., during which the patient is conscious and capable of conversing with the operator, and following the course of events.

As a physiological experiment this is very pleasant and agreeable, so it is little wonder that it attracted the attention of many surgeons, and at first sight appeared to be an ingenious solution of the anæsthesia problem, which at that moment essentially was how to avoid chloroform with its attendant dangers.

Nevertheless, when from the very first I deemed it indefensible to attempt this process at all, my reason was that a puncture, and especially an injection of fluid in the spinal-cord canal, is in itself perilous for the patient. I feel as justified in making a lumbar puncture for a diagnostic or therapeutic purpose with an existent, perilous, cerebrospinal affection as I feel unjustified in exposing a paitient's sound, healthy, central nerve system to an infectious meningitis.

You will know, from my lectures on antiseptics, that a complete disinfection of the skin is unobtainable, however careful our efforts-daily baths, thorough cleansings, poultices, etc., lasting over several days, such as we institute previously to the more important abdominal operations. We never know whether pathogenic microbes exist deep down in the pores and glands of the skin. When we consider that the lumbar puncture must be performed in a particularly unclean region, which is difficult of disinfection by reason of its proximity to the anus and rima natium, and that the disinfection previous to a lumbar puncture-if performed in the ordinary and every-day fashion-is often 
of a very rapid and superficial nature, then it must also be clear to us that the risk of infection is not quite insignificant, even with such a minor encroachment. Experience also proves this. For instance, Sonnenburg reports no less than three deaths from infectious cerebrospinal meningitis among 800 cases of lumbar anæsthesia; a fourth death occurred in 1907, at the University Clinique in Berlin. And this in surgical institutions of the first rank, where the antiseptic apparatus acts like clockwork! Now imagine this method in the medical practitioner's hand, away, somewhere, in a foul home! But only one such death should condemn this mode of anæsthesia, even if it possessed no other potentialities of danger.

But it appears to possess these in full measure, for shortly after Bier's announcement, and when the method was being tried out, some most serious cases of poisoning were reported, several of which were fatal. The first case of lumbar anæsthesia which I witnessed made an ineffaceable impression on me. This was at Kocher's, in Berne; the patient, a twelve-year-old boy, suffering from tuberculosis, on whom a resection of the knee was performed. The operation passed quite painlessly, but it was painful to witness the boy's anxiety and excitement while watching the preparations for the operation, and while following the course of this. Even during the operation distressing vomiting and violent headache commenced, increasing during the following twenty-four hours. Next day came convulsions, followed by paralysis of respiration and heart; and, finally, death, undoubtedly due to cocaine poisoning. Additional reports of collapse during operations and of paralysis of respiration and the heart were announced, whereby the method was discredited, until the Paris surgeons, led by Tuffier, enthusiastically adopted lumbar anæsthesia, hoping thereby to dispense with the discomforts and dangers arising from chloroform anæsthesia-without con- 
ceding anything to the adherents of ether in Lyons. Whien, in addition, Frenchmen asserted that they had obtained a perfectly innocuous substitute for cocaine in stovain, as expounded by Fourneau, which is a fanciful appellation for the hazardous $a$-dimethylamine and $b$-benzoyl-pentanolchlorhydrate, then medullary anæsthesia made further progress, and not least in Germany, where Bier is regarded as the father of this method, a fact of which Germans are justly proud. Since that time nothing has been able to check the advance of lumbar anæsthesia. This is very deplorable, and will some time be surely recognized as a slur on the surgical knowledge of our period: not only because the non-perniciousness of stovain and other substitutes (alypin, novocaine, tropacocaine, etc., for example) has proved to be a tradition, but because the drawbacks and dangers from this method are so immeasurably greater than those from chloroform narcosis that the method should have been long ago doomed and consigned to oblivion.

I need only tell you that the latest and most complete statistics produced by Kohler at the German Congress in April, 1909, showed twenty-two deaths in 7884 cases of lumbar anæsthesia,- - that is, one in three hundred and fifty,-in order to make clear to you the absurdity of any endeavor to avoid the danger of narcosis by these means, which cause ten times as many deaths as chloroform causes, without mentioning ether. But, over and beyond this mortality, lumbar anæsthesia is responsible for a whole series of calamities, the most important of which I shall now relate.

Firstly, because in 4 to 10 per cent. of the cases the effect fails to materialize, even with such thoroughly experienced practitioners as Bier, combined with perfect skill. Secondly, because sudden collapse during the anæsthesia, together with a suspension of the heart's action and of the respiration-which often refuse to resume their 
functions without one-fourth to one-half hour's artificial respiration and heart-massage-seems almost to be quite as frequent as with chloroform narcosis. Thirdly, because of the serious after-effects: (a) The recurrent vomitings, enduring for days, and sometimes for weeks after the operation. (b) Very frequent and persistent headaches, which may continue for months, even for six months after the operation. (c) Paralysis-of brain nerves; for example, abducency frequently manifests itself, and, what is even worse, paralysis of the bladder muscles, involving a total retention of the urine, necessitating continual catheterization, whereby the patient is somewhat exposed to the danger of urinal infection. (d) Myelitis, partly compression myelitis, the result of extradural bleeding from a lesion of veneplexus (Borchardt), and to some extent intoxication myelitis (Kœnig), and, finally, infectious meningo myelitis (Sonnenburg and others).

Rehn has explained to us by means of a series of experimental investigations the reason of these peculiar and unpleasant after-effects. These investigations have proved that Bier's presuppositions-(1) that the cerebrospinal fluid shifts only in accordance with the laws of gravitation, and (2) that the resorption from the cerebrospinal fluid, together with the secretion of the poison, should take place rapidly-are quite misleading. As a matter of fact, one cannot confine the effects to the lumbar region, as one would wish. The cerebrospinal fluid current quickly (Rehn thought in ten to twenty-four minutes) diffuses the poison over the brain ventricles, whereas the resorption, where stovain is concerned, takes forty-eight hours. In the spinal cord itself and in the brain it partly causes transitory and partly chronic deterioration of the cells. From these investigations we understand how headaches and paralysis of the brain nerves often occur after lumbar anæsthesia.

With ether narcosis, as you see, such mishaps and draw- 
backs do not exist; and when we add to this the fact that postoperative cases of pneumonia are at least as frequent after lumbar anæsthesia as after narcosis, you will understand that the former contains all the same dangers as the latter does, together with a series of other and even more deterrent ones. The fact has been emphasized as an advantage that the patient retains consciousness during the operation, but this is a very doubtful argument indeed; because one cannot dispute the point that with all important operations it is far more agreeable for the patient, and far more convenient for the operator, and better for the progress of the operation, that the patient is unconscious; while with the few important operations where one might wish for the coöperation of the patient, such as the removal of cartilaginous concrements and operations upon foreign bodies, ordinary local anæsthesia can be easily employed.

On the whole I cannot discover any possible justification for the application of spinal-cord anæsthesia in operative surgery. The only complaint for which the use of it seems to be indicated is that for which Corning first employed it: insufferable and otherwise indubitable pains with spinalcord afflictions, such as cerebrospinal meningitis, tetanus, etc. Just as lumbar anæsthesia has been interesting as a physiological experiment-permissible as it perhaps was to experiment with it on human beings while one believed it to be relatively safe-so, with the results now before us, has this perilous experiment upon mankind become unpardonable! It is to be hoped that this will soon occur to the surgeons throughout the world, though at present there are but slight indications that it will be so. Such an indication may be deduced from Bier's own admission at the congress lately held in Berlin, that the method was precarious and dangerous, while at the same time he considerably narrowed the sphere of its utility. In this discussion, 
which seemed to me so strange, and where most people appraised lumbar anæsthesia as the method of the future, though almost all agreed that the most important factor was to avoid the peril arising from narcosis, whether it were by lumbar anæsthesia or by local anæsthesia, the famous gynæcologist, Pfannenstiel, who unfortunately died shortly afterward, sounded these saving words, which I myself had had at the tip of my tongue. He said: "The whole explanation of this disputation merely is that you are unacquainted with the Danish ether narcosis by means of Wanscher's mask; it makes everything else superfluous."

Yes, and he who is so fortunate as to have learned the morphine-ether narcosis with the help of Wanscher's mask, as you have now done, will never have any occasion for lumbar anæsthesia, and very rarely for local anæsthesia. 


\section{LECTURE VI}

\section{FOREIGN SUBSTANCES CONTAINED IN THE ESOPHAGUS, THE STOMACH AND INTESTINES}

A patient has just been hurriedly admitted in a state of suffocation, resulting from the presence of some foreign substance in the œsophagus, and since we have another patient who has also swallowed some foreign substance, I will seize the opportunity to discuss this important question. In the first place, I must ask you to take note of the patient who is now being brought in. He is twenty-one years of age; slight, but in other respects an apparently healthy-looking young man, who only half an hour ago was eating his hasty lunch, which seems to have consisted of a rather tough veal cutlet. Having a large morsel of this in his mouth, and finding some difficulty in masticating it, he swallowed it whole, but at once realized that the mouthful had stuck, so far as he knew, in the upper part of the csophagus. He immediately felt a disagreeable sensation of choking, and, becoming frightened, first tried to reach and pull out the obstruction with his fingers, failing which he made energetic efforts to swallow it, though without any noticeable effects. As the dyspnoea became more and more apparent, and as those present noticed that his countenance became more indigo in hue, they had sufficient presence of mind to take him to the hospital at once, instead of sending for a local practitioner. On the way here he had several somewhat unpleasant attacks of suffocation, during which his countenance became dark blue, while he gasped for breath with stridulous respiration. Following a brief alleviation, the fear and the dyspnœa recommenced. You see how he gasps for breath. His lips, complexion, and conjunctivæ are distinctly cyanotic. His eyes are somewhat protuberant. His 
pulse, though powerful, is rather accelerated. Here one must act quickly. While opening the jaws by means of Kœnig's dilator, I insert my forefinger into the throat to ascertain whether $I$ can reach the obstruction. I cannot feel it, and therefore at once introduce this probe, which as you see, consists of an oval ivory knob screwed onto a stiff whale-bone handle (Fig. 36, A), corresponding with No. 40 Char. rière. With this I will first ascertain the locality of the foreign substance, which, according to the symptoms, I expect to find just behind cartilago cricoida-the first confined place in the œsophagus, and just where the respiration is most liable to be interfered with. As you see, the bougie is arrested exactly in this spot. The quickest and best method in such cases is to try, with the aid of the probe, to push the substance away from this dangerous neighborhood, and farther down into the œsophagus, preferably down into the stomach. You notice that there is considerable resistance, which now yields suddenly. You see low the bougie glides downward, following the foreign substance continuously, and you now notice that it is once more entirely arrested. In order to ascertain where the stoppage is, we remove the bougie, so as to estimate by measurement the exact location of the foreign substance in the œsophagus. We are no longer hurried,

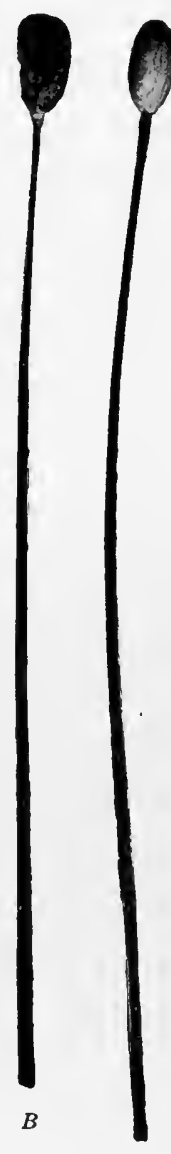

A

FIG. 36. because you can see from the patient's pleased and relieved expression, as from his deep, unrestrained breathing, and from the almost entire absence of cyanosis, that the immediate danger is past. You see that I have marked with my finger on the handle of the bougie the spot where this touched the teeth when it was arrested. It appears that the probe 
entered about 37 centimetres, and as we know from experience that, in an individual of medium height, the cardiac end of the stomach lies at an average depth of 40 centimetres below the teeth line, we know that the piece of veal is now arrested above the lowest contraction of the œsophagus, which lies just over the passage through the diaphragm. We again insert the bougie, and by its means we now try to push the obstacle down into the stomach-a procedure which is not difficult. Now it slips down, and the piece of veal is in the stomach, delivered over to the gastric juices, and we need worry about it no more.

You saw that the very first thing I did was to insert the forefinger of my left hand deep down in the pharynx to ascertain if any part of the obstruction lay across the entrance to it, in which case one could at once grasp it with curved forceps and draw it out-quite the best thing to do in such a case. Suffocation is frequently caused by pieces of meat because the victim has been on the point of swallowing two morsels of unequal size, connected by a bit of fascia, of which the first and larger places itself like a cover over aditus laryngis, while the œsophagus, in attempting to swallow the other, only pulls the first more firmly toward aditus and increases the suffocation. In such a case any attempt to thrust the obstruction down would be quite improper. Here one must grip the topmost portion, and by means of this draw the entire obstruction out through the mouth. For this purpose one can make use of a pair of corn extractors, if one does not possess œsophagus forceps with fixed or movable curvature specially constructed for the purpose (see Fig. 37). But when, as in this case, the obstruction has slipped so far down, you should immediately try to push it farther down, for otherwise you might injure the mucous membrane in the pharynx and œsophagus, resulting in disagreeable consequences; whereas, to force it down, as in this case, is the most temperate and harmless 
mode of procedure. I expressly say in this case, because this by no means applies to all foreign bodies, but only to the soft, pliable ones, such as meat, fruit, and potatoes, which cannot injure the mucous membrane by its forcible and often somewhat violent passage. If the question concerns solid bodies, or if your information regarding the nature of the substance swallowed is indefinite, you must refrain from any attempt to push it down. You must do all you can to ascertain the nature of and the seat of the foreign body.

Undoubtedly, the most common foreign bodies which people swallow accidently are fish-bones. This happens to a far greater extent than we imagine. The frequent discovery of large accumulations of fish-bones in the colon and rectum has enlightened us on this subject, and, according to Faber's investigations, these accumulations occur more often in persons who lack hydrochloric acid in their stomach contents, from which we conclude that if other persons are exempt it is because their gastric juice is able to digest the fish-bones, and not because they do not swallow so many. There is scarcely anyone, how

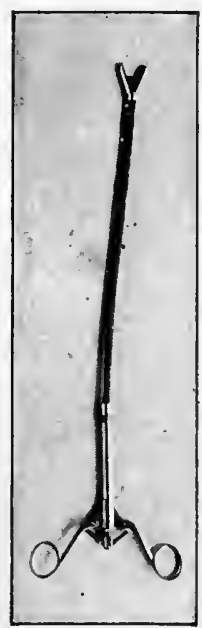

Fra. 37.-Pliable œsophagus
forceps. ever, who has not at some time been interrupted in the enjoyment of his fish by the sensation of a fish-bone impaling the mucous membrane in the pharynx or œsophagus, thereby experiencing the fright and pain involved in this slight mishap, which, irritating as it is, is mostly to be feared from the enjoyment of the most delicate fish, such as garfish, pike, and, above all, carp. In ratio to the frequency of this occurrence is the fact that everybody knows of some panacea for it, of which the best and most common is to swallow a large mouthful of soft bread or masticated potato, in the hope that the fish-bone may become involved 
in, and drawn down with, the doughy conglomeration. Often, also, this seems to suffice, but every now and again your advice will be sought on account of pains and sensations which continue for hours, sometimes for days. In such cases you should use a bougie from which the knob has been unscrewed, and fasten a piece of sponge (Fig. 36, $B)$ to the end of the whale-bone handle, large enough so that it will come in contact with the entire circumference of the cesophagus as it passes through it. As a rule, the bone will be caught as the sponge passes downward, but if the sponge slips past it, it will generally be caught on the return as the sponge is withdrawn, and it will be held by it. At times the instrument has to be moved up and down, more than once, before the fish-bone is loosened. As a rule, the pain ceases at once; but if the bone has been fixed for any length of time, and has thereby caused irritation, or if infection has lodged in the small wound, then a smarting pain may endure for a considerable period, and give rise to considerable anxiety, especially in nervous people. You should then apply a 2 per cent. solution of cocaine by means of the sponge, and afterward prescribe food of a soft, pulpy, and liquid nature, which must not be taken hot or in an irritating form. Fish-bones very rarely cause trouble during their further passage through the stomach and intestinal tubes, yet it sometimes happens with patients whose gastric and pancreatic juice is faulty. Two years ago I operated on a patient who was admitted to the ward suffering from an irregular, diffuse tumor situated just above the unbilicus; there was some fever and a circumscribed peritonitis. Here I diagnosed a perforating cancer coli, but a pleasant surprise awaited me at the operation, inasmuch as I found an abscess caused by a fish-bone between colon transversum and the abdominal wall. The bone had pierced the wall of the colon, and was protruding centrally through the bottom of the abscess cavity. The hard 
tumor which I had encountered was the swollen omentum, which had adhered to the abdominal wall round the abscess, thus preventing diffuse peritonitis. In two instances I have removed large masses of fish-bones twisted into strange, porcupine-like bodies and impaled in the rectum. These two patients were brought to me with the diagnosis cancer recti and had suffered from alternating attacks of constipation and mucosanguineous diarrhœe. Both were completely cured, though in a third instance an inoperable carcinoma recti had developed around the impacted bone.

So much for fish-bones! If, however, one is concerned with other solid, unyielding bodies, and especially with pointed, sharp-edged, irregular ones, the greatest care should be taken in conducting an examination; because here the slightest pressure forward may cause the sharp, firmly attached body to perforate or lacerate the wall of the œsophagus. For the practitioner, the ivory-knobbed bougies are always best for the preliminary investigations. The rubber bougies, which at first you would perhaps regard as the best and most useful, are in reality not so adaptable; chiefly because the rubber bougie is softened by the warmth, often bending over and doubling up, so that one not only receives a wrong impression as regards the seat of the obstruction, but may also cause irritation. Still worse is the possibility of the bougie gliding past the substance, if this does not fill the lumen of the œsophagus, without the operator perceiving its presence. And this may occur either where there is some irregularity in the shape of the foreign substance, or where this consists of some circular, level object, like a coin which has become fixed edgewise. You avoid this when you use a thick ivory knob, and you do no harm, if you only use it with care and gentleness. Then you may feel, and sometimes even hear, it strike the surface of the obstruction, and you can measure with a fair degree of accuracy the depth at which the foreign 
substance lies, which is of considerable importance in view of a possible operation.

A more serious type of foreign bodies, which we frequently meet with in adults, is dental fixtures. According to Eyloff's reckoning, they account for 62 per cent. of such bodies. Sometimes from forgetfulness, sometimes from the

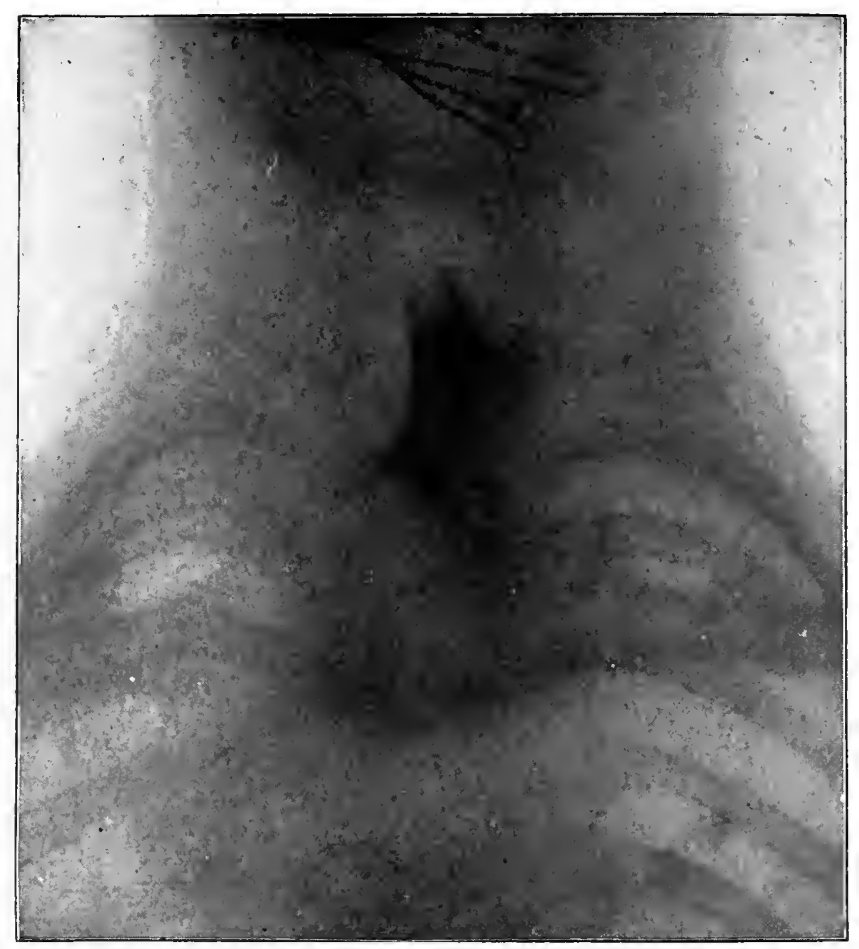

FIG. 38.-Gold plate with four teeth in the cesophagus of a lady 74 years old; removed by œsophagotomy.

effect of alcohol, but no doubt most often from vanity, it happens that those concerned have omitted to remove their false teeth before retiring to rest, and have consequently swallowed the set of teeth while asleep. Even worse are the smaller plates or fixtures, because the metal hooks by which these are fastened to the remaining sound teeth are especially dangerous in the œsophagus. They may even perforate 
though the œsophagus, if allowed to remain for any length of time. Here in Denmark such cases have been observed and successfully treated by Schmiegelow (see Fig. 38).

On rarer occasions one has to deal with sharply-pointed and sharply-edged pieces of bone, while with mentally afflicted people one meets with a most varied assortment of articles: pieces of glass, keys, nails, etc.

Children, during their earlier years, feel an unfortunate inclination to put all possible kinds of things into their

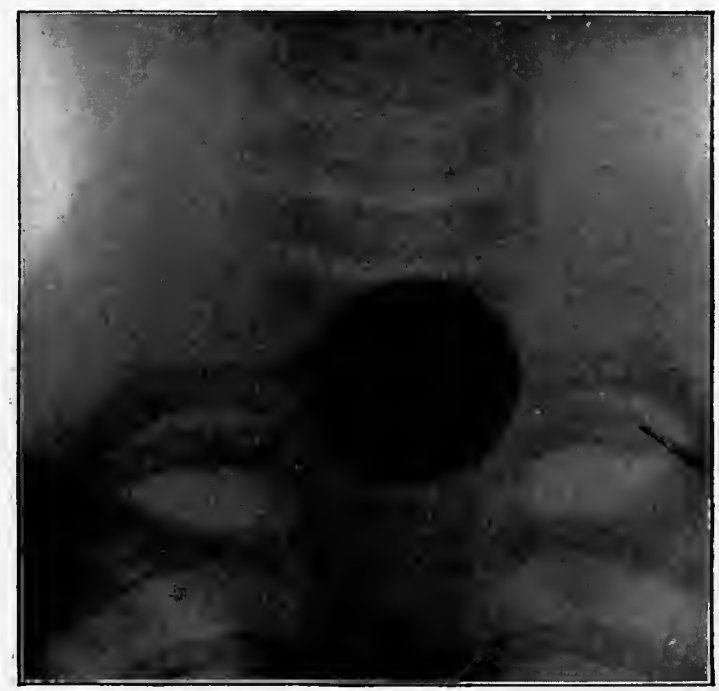
FIG. 39.-An English penny in the cesophagus of a 6-year-old
girl removed by cesophagotomy (Schmiegelow).

mouths, and it frequently happens that, during a fit of laughing or crying, and sometimes in order to cover the evidence of their offence, they swallow the foreign substance. If the children are so young that they cannot articulate, or if they will not speak the truth, one feels painfully incompetent to define the shape and nature of the substance. In all such cases the Röntgen ray is a diagnostic aid of the greatest value; because, so to speak, all solid foreign substances, such as may be relative to the point at issue, will produce a dis- 
tinct shadow with the Röntgen ray, especially if one conducts the examination in the manner that Wilms has recommended, -i.e., obliquely, in order to avoid the shadow of the foreign substance being overshadowed by the spinal column or the heart.

Here you see a Röntgen picture (see Fig. 39) which shows a coin jammed in the central part of the œsophagus and near the uppermost chest aperture; and here another

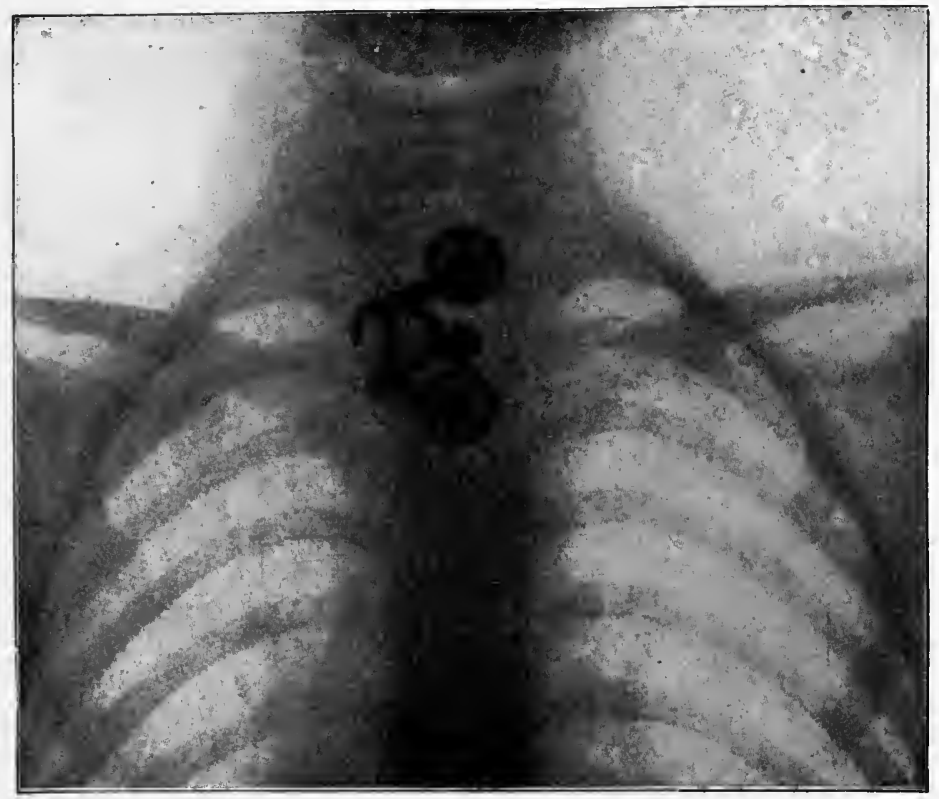

FIG. 40.-Radiograph of a toy bicycle tightly wedged in the œsophagus of a 4-year-old girl (Rigby).

even more interesting picture, which shows how a little fouryear-old girl swallowed a toy bicycle, which was arrested in its course through the œsophagus by the steering handle (see Fig. 40) penetrating the mucous membrane. The child was operated on at the London Hospital, where Dr. Hugh Rigby removed the article by means of œsophagotomy. With children, however, one must expect to be confronted with the most various foreign substances, such as pieces of glass, 
stones, etc.; while with soldiers' children regimental buttons and metal numbers are very frequently encountered. The latter are especially dangerous, because certain numbers, such as 4,7 , and 11, have several sharp protuberances, in addition to the pin by which they are attached to the jacket or cap. For many years an effort has been made to develop direct œsophagoscopy, by which I mean an inspection of the mucous membrane of the œsophagus by means of a long metal tube, through which the area to be inspected is illuminated, either by means of a reflected light from without, or else by means of a

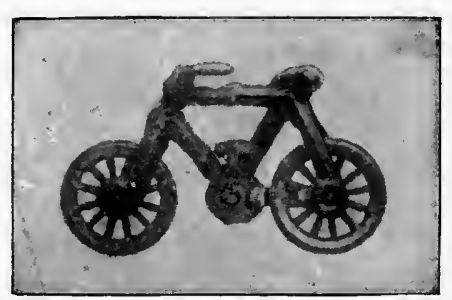

Fra. 41.-The toy bicycle removed by csophagotomy. small electric lamp attached to the end of the tube. It is due to the famous surgeon, the late Professor Mikulicz, and to his persevering endeavors to perfect œsophagoscopy, that we have now succeeded in simplifying the method to such an extent that it is available for every surgeon, and not only for the specialist in that limited region. Here you see the apparatus. It is merely a metal tube corresponding in length and width to a normal œsophagus (there are other sizes suitable for children of various ages), and provided at the top with a handle and a small fork to facilitate the application of Casper's panelectroscope (see Fig. $42, A$ ), a very practicable apparatus, which can be utilized to provide light with oto-, laryngo-, and endoscopes, etc. The œsophagoscope, when inserted, is provided with an obturator (see Fig. 42, $B$ ), which prevents the mucous membrane being injured by the edge of the tube. When one first sees this instrument, one says, "It is surely impossible to pass this metal tube through the mouth and down into the œsophagus, when these two in conjunction almost form a right angle." One forgets that this angle, though present when the head is carried naturally, is completely straight- 
ened out when the head is stretched backward, so that the insertion is accomplished with surprising ease (see Fig. 43). It must be remembered, however, that the throat has previously been made insensitive to pain by means of general or local anæsthesia. But here we have one of the defects of the method: because, naturally, it is very difficult to maintain general narcosis during the insertion of such an instru-

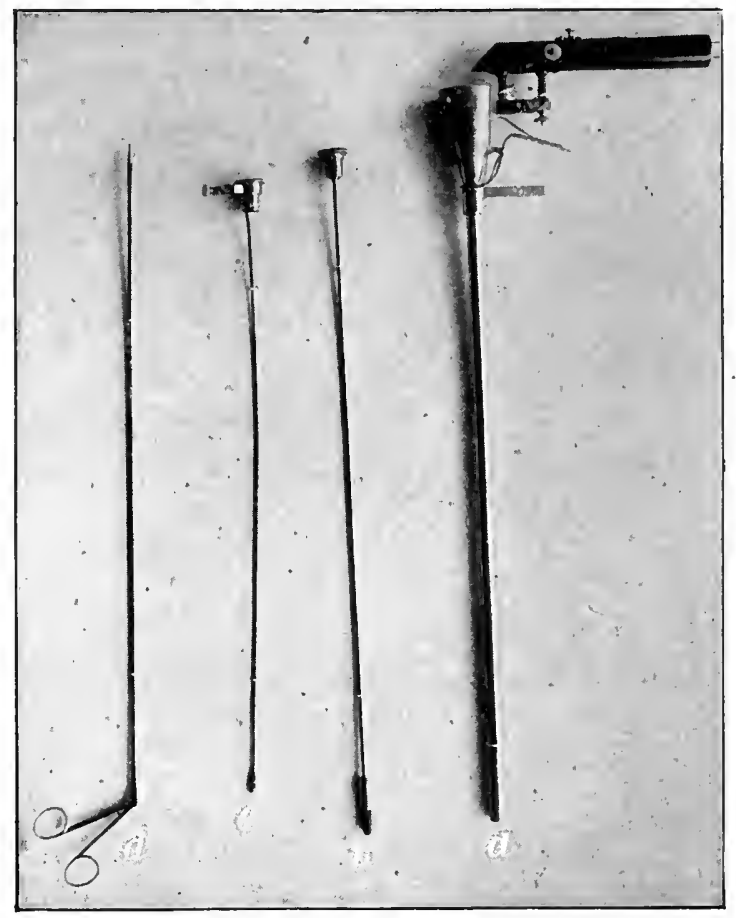

Fio. 42.-Esophagoscope with Casper's panelectroscope.

ment, while the act of vomiting is most inconvenient during such an examination, which has to be conducted most carefully and demands considerable skill. Therefore, as a rule, one prefers local anæsthesia by means of cocaine, though this is unpleasant enough; because, in order to produce suf: ficient anæsthesia, it is necessary to use a 20 per cent. solution, which may give rise to serious cocaine poisoning. Also, 
a careless or rough insertion of the tube may force the foreign substance farther down and thus increase the injury.

Now that we have the Röntgen ray as a means of diagnosis, which is so certain and so harmless, and so agreeable to the patient, the œsophagoscope appears to be of secondary importance. The Röntgen ray has the great advantage, also, that if one does not find the foreign body in the œsophagus, one can immediately continue the search for it, and can often ascertain how far it has progressed into the

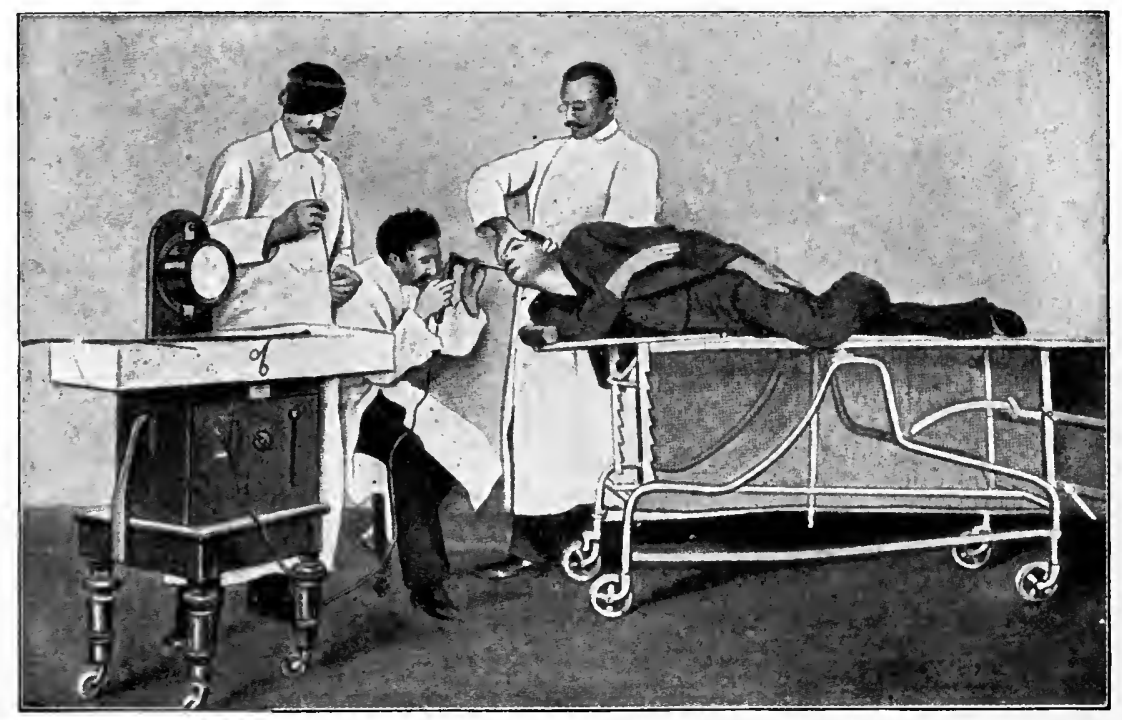

FIG. 43.-Esophagoscopy (Mikulicz).

stomach or into the intestinal canal, with a view to following its course during the ensuing days. This is well illustrated by a case which we have in the ward at the present moment.

A few days ago some terrified parents brought us their little girl, who, according to their statement, had been playing with a three-inch nail on the previous day. Suddenly the child began to scream and her face turned blue, while she beat the air with her arms; meanwhile the nail had disappeared. However, the child's complexion and respiration 
became normal, though she continually complained of pain whenever she was given something to drink. By means of an $\mathrm{X}$-ray screen it was discovered that no nail was to be found anywhere in the cosophagus; but by moving the screen down in front of the abdomen, and by examining this, we ascertained that the nail had already reached the ileocreal region (Fig. 44), and the following day we located it in the left fossa iliaca in flexura sigmoidea, and it soon passed out with purgation.

Although the Röntgen-ray examination has made cesophagoscopy superfluous in most cases as a purely diag-

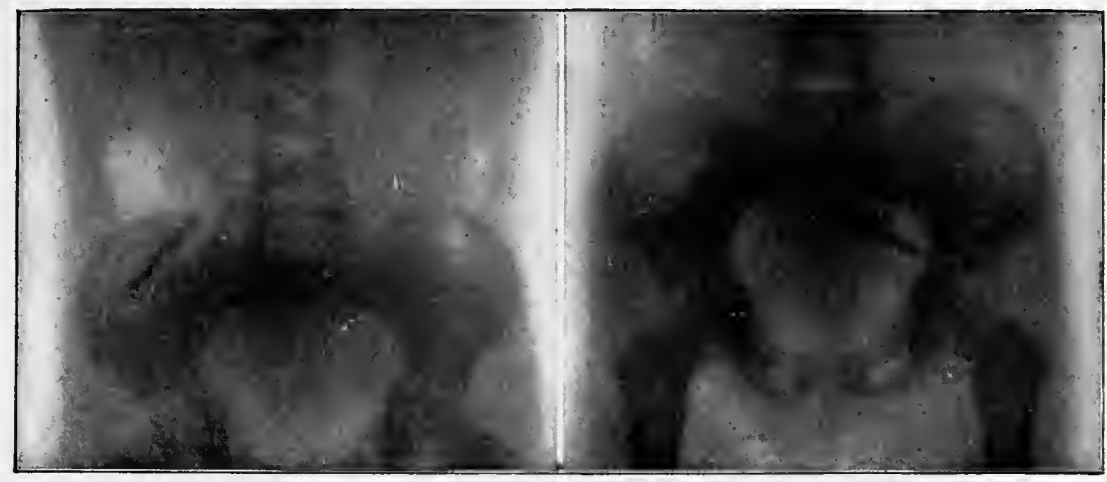

FIa. 44.-Three-inch nail followed day by day by Röntgen illumination. First seen in the cæcum, and next day in the rectum. Personal observation.

nostic expedient, yet esoplagoscopy has attained increasing importance as a means of removing certain foreign substances. Long, slender tongs and hooks have been constructed (Fig. 42, D), which can be introduced through the csophagoscope and by means of these the foreign substance can be removed under the guidance of the eye. In this manner coins, buttons, nails, needles, and, in fact, all small and relatively simple foreign substances, may be removed far more surely and far less unpleasantly than by a coin-catcher or a pair of tongs. I wish to warn you especially as regards the coin-catcher, that ingeniously contrived but very dangerous instrument of Graefe's which you see illustrated here 
(Fig. 45). It consists of a little flat metallic basket with a sharp edge, and is very mobile on the bougie to which it is attached, so that it can avoid the coin on its way downward and intercept it on its return. With this instrument the danger lies in the facility with which the edge of the basket fixes itself in cartilago cricoidea. This may lead us to the quite incorrect conclusion that we have intercepted the coin, and our consequent forcible effort to extract it may lacerate the wall of the œsophagus, and may even cause a wide, transverse gash in the trachea.

I myself once witnessed a case of this kind. A medical practitioner of this town, in the course of his consultation hour, had endeavored to extract a half-penny from the csophagus of a six-year-old boy. He gripped something solid, which he imagined to be the coin, and in consequence pulled all the harder. This hurt, and, the boy jumping suddenly backward, a violent jerk ensued. The somewhat exhausted boy was immediately brought into this ward, but a septic mediastinitis, which developed with astounding rapidity, led to the boy's death within twenty-four hours.

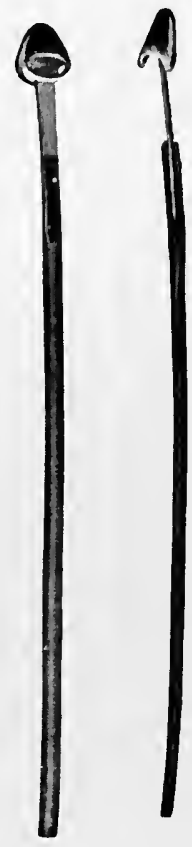

With tongs, also, there is danger. One may Fra. 45.-The injure the mucous membrane, and frequently coin catcher. the trial ends in failure. Therefore, removal by means of the œsophagoscope is preferable, though in the presence of large, wedged-in, irregular foreign substances this does not suffice. Indeed, any attempt to extract such substances involves considerable risk of injury to the parts; so here the removal must be accomplished by manipulation. In order, therefore, to employ the correct method of operating, it is necessary to know with certainty. where the substance is 
lodged in the œsophagus; because only when it lies in the upper two-thirds of the csophagus can it be removed by csophagotomy through an incision in the gullet, which is exposed by an incision which follows the anterior margin of

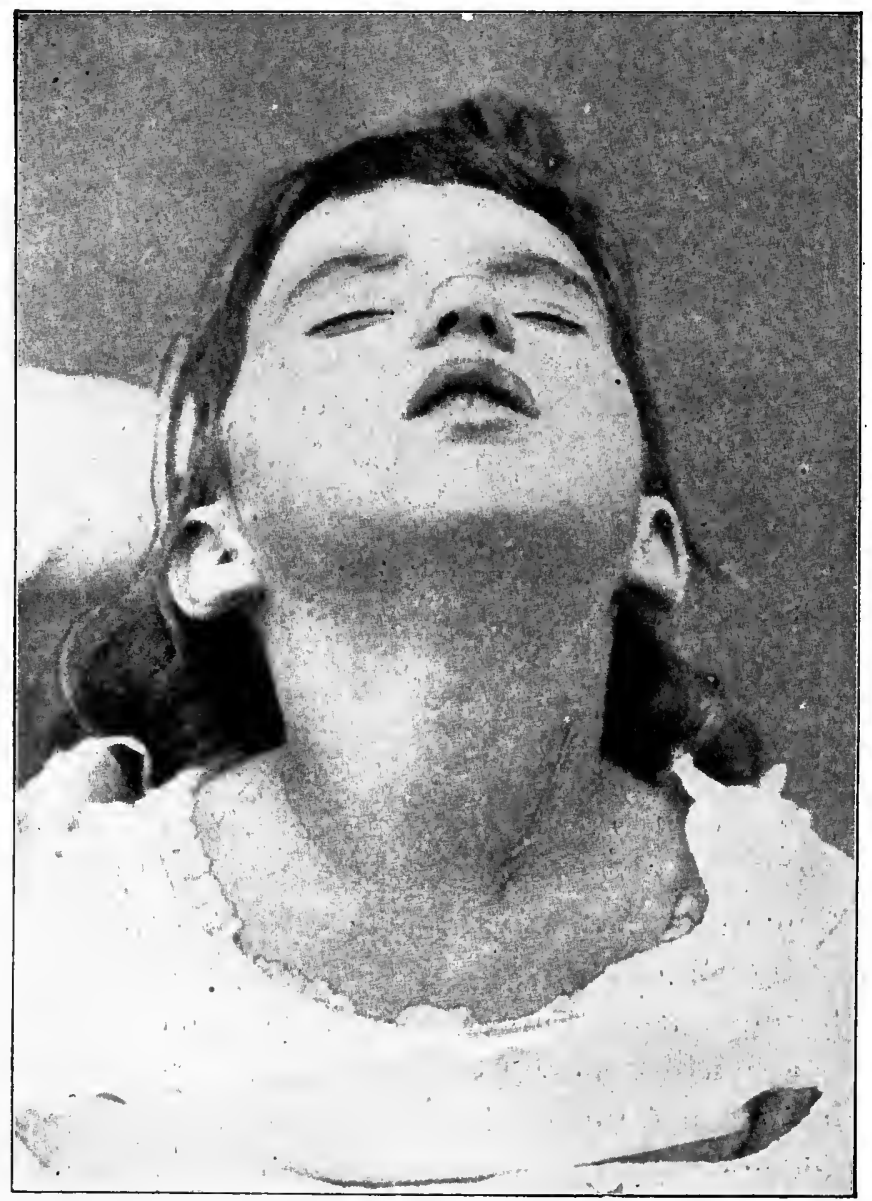

Fia. 46.-Shows healed line of incision after æesophagotomy for toy bicycle (Rigby).

the left sternocleidomastoideus (Fig. 46); the dissection passes between the trachea and the large vessels which are drawn to one side. The operation itself is not particularly difficult. The osophagus is easily recognized because of its 
outer muscular coat, which runs longitudinally and is easily identified when the bougie is inserted. The foreign body frequently presents itself conspicuously, and, when it does not, it is easily located by inserting a finger or bougie in the opened csophagus. If the substance should lie at any considerable depth below the point of the incision, and if it is firmly wedged, the release may often cause great trouble and bring about considerable laceration both at the point where the body is lodged and in the edges of the wound itself. This, perhaps, is one of the reasons why œsophagotomy wounds so rarely heal per primam intentionem. Judging from Balacescus and Kohn's large collection of statistics, fistula formation takes place some days after the operation in no less than 95 per cent. of the cases. Possibly this is due to the general practice of providing nutrition by means of a tube after the operation. It seems evident that the insertion of a tube several times in the course of a day incurs a risk of weakening the suture line and infecting the wound. Therefore the patient should be fed entirely per rectum until the wound is healed.

If, however, the foreign body in the course of its passage through the opening in the diaphragm becomes fixed in the lower third of the csophagus, above its third and lowest contraction, then it can be removed from below only by gastrotomy. This operation presents two difficulties, -i.e., the prevention of infection and the inaccessibility of an object far up in the œsophagus. To avoid infection it is preferable to draw the stomach outside of the abdomen if possible; but here, to enable one to reach the foreign substance, a very large incision is necessary in order to permit the introduction of the entire hand into the stomach, and so large an incision of the stomach naturally increases the danger of the operation in every respect. Wilms ${ }^{1}$ has endeavored to surmount both difficulties with a delicately

${ }^{1}$ Deutsche Zeitschrift f. Chir., Bd. 60, 1901. 
devised modus operandi. He draws the stomach forth, makes a quite small incision, just sufficient to allow of the passage of his rubber-covered forefinger, and, by aid of a tobacco-pouch suture, closes the small incision closely round his finger. This and the stomach are then inserted in the abdomen, and the entire hand follows. The finger can then be inserted into the œsophagus without hindrance, where it palpates the foreign substance, carefully loosens it, and, grasping it firmly, draws it down into the stomach. The hand is withdrawn from the abdominal wound, accompanied by the stomach and the sewed-in finger. When the peritoneum has been protected with napkins, the suture is cut and the wound in the stomach is enlarged sufficiently to allow the substance to be extracted. If this is successful, the stomach is sutured with two rows of sutures and replaced. But this extremely attractive method is scarcely sufficient in all cases. Thus, with very large and hard foreign bodies it may be necessary to crush these with strong forceps in order to extract them. Here, then, one is compelled to work through a large incision in the stomach. This applies also to the relatively common cases where the retention of the foreign body in the gullet is due to a previously-existing stricture. Here the contracted area must first be gradually dilated by the introduction of bougies, and if it cannot be sufficiently expanded at one séance, then the stomach must be sewed up and the gastrotomy wound kept open for a longer period.

If the foreign substance has reached the stomach without artificial aid, or been forced down by the probe, the treatment must, of course, adjust itself to the nature and size of the substance; if the foreign body is of such a nature that it might cause perforation or could only pass through the intestinal canal with great difficulty, then gastrotomy should be undertaken as quickly as possible. With proper asepsis and employing the highly-developed operative tech- 
nic of the present day, this encroachment is not a dangerous operation. Through an incision in the median line of the epigastrium the stomach is drawn forth and enveloped in sterile gauze, so that the peritoneal cavity is absolutely guarded against any influx of the stomach contents. The foreign substance is then removed through an incision in the anterior stomach wall, and the wound quickly closed in two layers, before the stomach is replaced.

On the other hand, it is most astonishing that, even as early as the beginning of the seventeenth century (1602 and 1635), we have evidence of two successful gastrotomies performed on "contortionists:" the one performed by Florian Mathis (1602) in Brandenburg, the other by the surgeon Daniel Schwabe in Königsberg (1635).

Jugglers and sword-swallowers on the one hand, and mentally afflicted persons on the other, have provided the greater proportion of the patients on whom gastrotomy has had to be performed on account of foreign substances. Incredible quantities of assorted rubbish have been extracted from the stomachs of mentally diseased persons: forks, knives, keys, tools, and whole stores of nails, bits of glass, stones, etc.

Among the foreign substances which may compel us to practise gastrotomy, "hair-balls" occupy a unique position. They are enormous, chignon-like agglomerations, which form themselves inside those people who eat hair, especially young, chlorotic or hysterical women, who are addicted to chewing their plaits or to biting their hair ends; or who roll up the combings, biting and swallowing them: a bad habit, which is associated with biting the finger-nails and eating slate pencils, etc., and is sometimes contagious, appearing epidemically in girls' schools. When these hairballs become large, they may give rise to very serious symptoms; may cause gastritis, ulceration, and stenosis of the pylorus; may even resemble huge abdominal tumors. 
Wölfler and Lieblein have collated twenty such instances of hair-balls removed by means of gastrotomy. All of the patients recovered.

It is more difficult to decide the indications for operation with smaller, but pointed, foreign substances which are not dissolved in the gastric juice: artificial teeth, nails, needles, splinters of glass. Experience shows that such bodies often pass through the alimentary canal without causing any harm; though, on the other hand, instances are not lacking where such substances have perforated the intestinal wall and caused peritonitis, or have entered other organs-the bladder, for instance. What action, then, shall we take here? We must make a point of observing and watching these patients most carefully until the substance has passed away. We must put the patient to bed, and supply him with copious quantities of soft food, bread, porridge, potatoes. We must note the temperature accurately, also any symptom of pain, and, if possible, by daily Röntgen illumination, follow the course of the foreign substance, as we did with the patient with the nail, a picture of which $I$ have shown you. If any signs of inflammation or peritonitis then appear, or if the substance remains immovable in some one place or another, we must attend to it operatively, and remove it by enterotomy, being guided by the Röntgen picture.

Before I close, I wish to draw your attention to the fact that too much medicine may at times give rise to the formation of foreign substances, which may eventually cause obstruction of the bowels: ileus. Aage Kiaer has removed a bismuth stone, weighing nine grammes, caused by bismuth treatment of a cxcal hernia. The cæcum is specially adapted for the accumulation of such ingesta, which then have time to form concrement, and especially so when mucus and pus, resulting from inflammation, supply a cohering material. 


\section{LECTURE VII}

\section{STRICTURE OF THE CESOPHAGUS}

We will occupy ourselves to-day with two patients, both of whom have arrived here in a very critical condition with contraction of the œsophagus, and who will serve as a basis for a lecture on contraction of the œsophagus, inasmuch as they represent the two most important groups, the benign and the malignant types. But you will see, however, that the appellation "benign," by which we understand "noncancerous, must not be taken too literally; because, unfortunately, it is a great question whether the prognosis of the former is more favorable than that of the latter.

The first patient is a young girl, sixteen years of age, nevertheless an old acquaintance of mine. Eleven years ago, when a surgeon at Queen Louise's Hospital for Children, I treated her for the same complaint which now brings her here. In a few words I will give you the history of her disease, which, by the bye, in its time was reported in "'Medicinsk Selskab," November 27, 1894 (see Hospitalstidende, 1895, p. 108).

Hilda $\mathrm{K}-$, who then was five years old, entered the children's hospital on January 24, 1894, on account of an increasing difficulty in swallowing. The mother informed us that the child had become suddenly ill one month previously in a grocer's shop, at which time she was suddenly seized with severe pains in the throat and with trouble when swallowing and breathing, and to such an extent that her face became bluish-red, while she stamped on the ground with her feet. She ran home and said that the grocer had treated her to a piece of apple, which she had "choked upon" while eating. From that very moment she had great and increasing difficulty with swallowing, was unable 
to swallow solid food at all, and liquid food only with pain and inconvenience. The child, who formerly had been strong and healthy, now became pale and greatly emaciated, with a sunken abdomen. I found the mucous membrane of the mouth and gullet red and distinctly irritated by small, very red sores on the tongue and lips. She was able to swallow small mouthfuls of water and milk, but everything else-even fine crumbs of white bread-immediately came up again. The œsophagus probes-even the thinneststopped at about 18 centimetres from the jaw, although one could not feel distinctly any foreign substance.

It was, of course, somewhat extraordinary that a morsel of apple should remain wedged for a whole month without dissolving, but according to the mother's and the child's explanation we were compelled to believe that a wedged-in foreign substance was involved, and the instantaneous blockage of the passage strengthened our belief in this explanation, and I thought it possible that the child had swallowed some solid substance, the nature of which she would not divulge for fear of punishment.

On January 31, therefore, I attempted an œsophagotomy through an incision along the anterior edge of sternocleidomastoideus, from the level of the hyoid bone to the sternal insertion of the muscle, and you can still see the fine cicatrix on the neck resulting from that operation. Partly with my finger and partly with a probe I then palpated the interior of the œsophagus through the incision, but no foreign substance could be felt. Nevertheless, commencing almost level with the upper aperture of the chest, I felt a granulated, slightly bleeding surface, which, funnelshaped to begin with, ended in the course of a few centimetres in a contraction which would not allow of the passage of a quite thin silver probe: not even of one so small as the finest rubber bougie. It was then evident that we were dealing with a rather elongated stricture, which could 
PLATE I

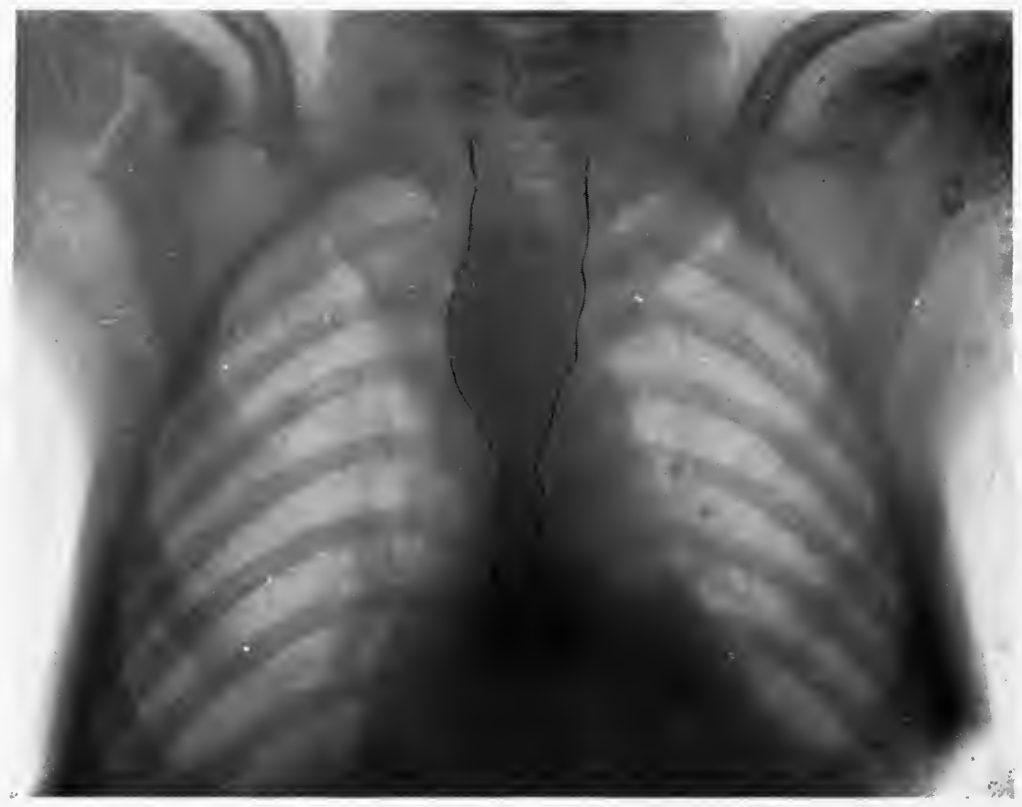

Non-malignant stricture of thoracic portion of oesophagus with dilatation above caused by the swallowing of lye. 

scarcely be due to anything but caustics, while the forementioned mouth affection, the bright color of the mucous membrane and the sores thereon, which had hitherto been regarded as a secondary stomatitis, now supported this theory. I then introduced a thick drain in the œsophagus, through the incision, and fixed it to the csophagus and the skin with "Fils de Florence," and, having joined the upper and lower ends of the wound, temporarily desisted from further encroachment in order that the anamnesia might be entirely cleared up.

Upon applying to the forementioned grocer, we were informed that the tale of a piece of apple was a white lie which the small daughter of Eve had told to her mother. The truth was that, during an unwatched moment when the grocer's back was turned, the child had seized a bottle standing on the counter, and swallowed a large draught of the contents, which proved, unfortunately, to consist of caustic soda, and not the refreshing beverage which she anticipated.

This history of this illness should be very instructive to you. It shows you how difficult it may be to induce children to speak the truth when the question concerns the swallowing of forbidden articles, such as I showed you when lecturing on foreign bodies; but it also presents a very typical and serious case of caustic stricture of the œsophagus. Soda, potash lye, spirits of ammonia, nitric acid, popularly called "aqua fortes" or "hydrochloric acid," are the corrosive fluids which most frequently cause the disaster; because either, as in this case, the fluid is swallowed by mistake, the bottle often being mistaken for a beer bottle, which often happens in workshops, or purposely, with more or less serious suicidal intention. This is almost typical of the way servant girls behave when confronted with the results of fruitful intercourse. Some years ago "nitric acid" and "caustic soda" were quite superseded by 
carbolic acid (acid. carbol. liq.) with this kind of attempt at suicide, thanks to a strange ordinance by which carbolic acid could be purchased from the chemists ad lib. across the counter, while a prescription was necessary to obtain a little carbolic water for dressing wounds or for the purpose of "sprinkling." A real epidemic of "carbolic acid dramas" broke out, and lasted for some years, until, finally, one thought of the clever expedient of selling carbolic water across the counter, and carbolic acid only with a prescription. The reason why, both before and after this period, carbolic acid dramas became so frequent in proportion to "nitric acid dramas" was, surely, that the servants soon ascertained that carbolic acid as a corrosive was far weaker in its effect and milder in its consequences than the other. Carbolic acid rarely left serious consequences in the way of ulceration and stricture, if the attempted suicide was unsuccessful.

The case is quite different with inorganic acids and alkaline caustics, the effects of which are illustrated in a very striking manner in this case.

You noticed that, from the very moment of the accident, she was unable to swallow even finely apportioned solid food. This, of course, is not due to a so quickly developed cicatricial, fibrous contraction, but is the immediate consequence of the corrosive, and is what we might term an inflammatory stricture due to extensive swelling of the mucous membrane. Yet, you see how quickly a cicatricial, fibrous stricture-even one of great seriousness-develops from this; for with this case even a month after the occurrence we were unable to insert the very finest probe. If we had seen the case immediately after the lesion, the diagnosis would surely have been easy enough; because the cauterization of the mouth and mucous membrane of the gullet could not have been misunderstood, and would have made the story about the apple quite improbable. But when, 
a month later, we find only the remains of the cauterization, which so greatly resembles stomatitis caused by the stagnation of food behind a stricture in the csophagus, the affair is not so simple.

If I had at once had the real anamnesia, instead of the fabricated story of a foreign substance, I should not have done the csophagotomy, which, of course, was suitable only for the removal of a wedged-in foreign substance, because, if there were no possibility of conducting even the finest bougie through the mouth, there was no"probability of its being successfully conducted through the œsophagus opening. Is not œsophagotomy, then, indicated for œsophagus strictures, just as urethrotomy is with stricturea urethræ? Here the reply must be: Yes, if we are faced with a limited, single stricture in that part of the cosophagus directly accessible from the neck, an œesophagotomia externa is clearly indicated; but this does not hold good with caustic strictures, which are characterized by being multiple and diffusely extended. In this case, where the stricture begins just below the accessible part of the œsophagus, the operation was especially useless. We would then have immediately resorted to the operation of gastrostomy, which has now been delayed for some days, during which the patient's condition has become further reduced. In such cases this operation has a twofold object: firstly, to remedy the existing state of inanition, because as soon as the fistula is consolidated one can supply the patient ad lib. with the most nourishing liquid food; and, secondly, to admit of a retrograde dilatation of the stricture, because it is the same here as with strictures of the urethra, even though they be ever so impermeable to the introduction of bougies from in front through the urethra and œsophagus, it is very easy to find a way through from behind-from the bladder or stomachsimply because here we have the healthy orificium internum and cardia respectively. 
You must not misunderstand me when I say very easy. It is easy enough when one has the cardia lying before one on the anatomist's table, but in the clinical hospital there are indeed many dangers and difficulties to overcome before one can insert one's bougie through this passage. When, in 1894, I had to face this problem with this patient, there had been only five cases reported where retrograde dilatation by gastrostomy had been successful. At that time, from fear of peritonitis, one did not venture to make a wide incision in the stomach in order to pass the bougie straightway up through the cardia with the hand introduced into the stomach. One considered it necessary to perform gastrostomy in two séances: the first stage consisted of suturing the serosa of the stomach to peritoneum parietale in such a way that a small portion of the stomach was extraperitoneal. This usually was close to the greater curvature. When the surfaces had coalesced, the stomach was opened sufficiently to allow of a drain being inserted. But, even then, there were fresh dangers and difficulties, because the gastric juice and the contents of the stomach oozed out alongside the drain and percolated through the dressings and, what was the worst of all, "digested" the edges of the wound. Added to this came the great difficulty of finding one's way up to the cardia with one's bougie through this small opening; because, when this reached the lesser curvature, or the posterior wall of the stomach, it bent, slid along this to the pylorus and into the duodenum. In fact, in only two cases of permeable stricture had any one succeeded in entering the œsophagus in this way, though Socin and Kraske achieved their object by an ingenious artifice, inducing the patient to swallow a shot to which a long thread was fastened. After protracted attempts to swallow, they succeeded in causing the shot to enter the stomach, whence the thread was then drawn forth through the gastrostomy opening and attached to a bougie in place of the shot. By pulling the other end of 
the thread, which was hanging from the mouth, the bougie was drawn up through the œsophagus and the stricture.

In this case I succeeded in circumventing the forementioned principal difficulties by means of the following procedure: I placed the incision very high, parallel with the left costal curvature, and then drew down the small, contracted stomach, lying high up under the diaphragm, until I was able to fix a very elevated part of the fundus to peritoneum parietale. That part of the wall of the stomachabout the size of a sixpence-which was reserved for a later incision, was marked by two threads of silk, which were led through the serosa and muscularis only, and by aid of which the conical end of the stomach was lifted forth through the opening in the abdominal wall, while the stomach was attached to peritoneum parietale, at a distance of three centimetres from the conical point, with a row of silk sutures. The abdominal wound was then closed around the small, exposed portion of the stomach and, on the following day, alter tempo was undertaken by my opening the stomach, layer by layer, between the two silk sutures, and in such a manner that the opening in mucosa became quite small: only one-half centimetre in extent. Owing to the high position of the opening and to its limited dimension, I obtained complete command of the contents of the stomach, and was at once able to undertake a vigorous nourishment of the child without allowing the progress of the wound to be disturbed by the juices of the stomach. The introduction of bougies proved to be impossible, as they continually yielded with a slight curvature, slipping out through the pylorus instead of up through the cardia. I conceived the idea that, with the aid of Hegar's dilatators, I could perform a temporary, progressive dilatation of the fistula of the stomach until such time as I could insert my forefinger and therewith safely and easily guide the bougie up through the cardia. A bougie finally was 
passed through and up into the mouth, and from here was drawn forth between the rows of teeth; then I tied a long, thin silk thread to the point of the bougie. With the withdrawal of the bougie through the osophagus and the fistula of the stomach this silk thread was also drawn out, and then left with the ends fastened with American adhesive plaster on the cheek and abdomen respectively. By means of this silk thread an increasing number of bougies were then daily drawn through the stricture until this could be kept open from above, and without difficulty, by the introduction of bougies. On March 14 the drainage tube was removed from the stomach. The stomach fistula quickly contracted, and on April 6. the wound was entirely healed. You should notice this, also, as being of great advantage with my method: that formerly, when one made a considerable opening in the neighborhood of the large curvature, one encountered the greatest diffculty in closing the fistula, on account of the strong outflow from the stomach.

You perceive that the result of the operation, as thus contrived, answers in every respect the purpose and expectations; while, so far as I know, no better method has since been discovered.

(Postscript.-Now, moreover, dilatation with Hegar's dilatators has become superfluous, seeing that by means of my gastroscope-specially constructed for the purpose-it is feasible, under the control of one's own eye, to conduct a thin bougie up through the cardia into the œsophagus, as will be described in my lecture on the examination of the stomach, pages 151, 205.)

But, as is the case with so many of these cautery strictures, the immediate satisfaction with the result achieved was somewhat eclipsed, both from the standpoint of the physician and the patient, by a tendency to retraction, which necessitates, if not a daily, yet a very frequent and 
systematic introduction of bougies. Should this be neglected for any length of time, the stricture contracts, and may some day become impermeable, and the trouble begins all over again. Notwithstanding our most careful instructions, this girl has not had sufficient strength of character to carry out the needful bougie treatment. During the first six months, and so long as she was under my weekly control, all went well; but shortly thereafter she had been left to her own and her mother's devices, and as soon as she was able to swallow whatever she pleased she and her mother decided that the bougie was unnecessary, with the result that in the course of a few months she again began to throw up her food, thereby becoming emaciated, and when once more she applied to us for assistance it was only with the utmost difficulty that I could find a way through from above with the very thinnest bougie. One would think that, after this lesson, both mother and child would take future care to preserve the recovered, free passage. But it seems as if more perseverance and character are demanded than one would think, certainly more than this patient possesses, for time after time she has put aside the bougies, and again and again reappeared with relapses which have proved more and more difficult to overcome. When we now admit her in the extremely exhausted condition in which you see her, not only excessively emaciated, but also so deeply anæmic, that her blood shows only 19. per cent. of hæmoglobin, the reason is that a most serious complication has set in, viz., sudden, profuse vomiting of blood from the œsophagus and melæna. Whether these hemorrhages are due to a direct lesion of a larger vessel, or to an attempt to pass the bougie, or to ulcerations on the old, cauterized parts, or, finally, to an ulcus ventriculi being added to the other misfortune, cannot be definitely decided; but their effect has been such that for a long time now one has not dared to attempt the introduction of bougies for fear of causing hemor- 
rhage, in consequence of which the stricture has assumed such dimensions that liquid food also has great difficulty in passing.

Also, the patient is so debilitated and anæmic that a moderate repetition of the hemorrhage would certainly prove fatal. Therefore I shall not venture any attempt to insert the bougie from above, nor dare I attempt dilatation of the stricture from below, at present. For the moment, the œsophagus should remain perfectly at rest, while our chief aim will be to relieve the inanition by a course of strong nourishment. I will, therefore, again make a gastrostomy opening through which I shall nourish the patient, and which I shall utilize for a retrograde insertion of the bougie though the stricture into the œsophagus so soon as the patient has recovered sufficient strength, and when the injured part of the œsophagus may be considered as presenting no further risk of hemorrhage. Of late years I have introduced our improved method of controlling the escape of the stomach contents, by employing Pezzer's rubber catheters for insertion in the stomach in place of drainage tubes. For in this way, the button of the catheter being placed close against the stomach-wall, the oozing of the contents of the stomach along the catheter-formerly so dreaded from the very commencement of the operation-is prevented.

I consider the operation itself to be almost free from danger, despite the patient's rundown condition. But in this case the prognosis is doubtful on account of the danger of a recurrence of hemorrhage; because, if there is a question of a perforation or an erosion of the wall of a large artery-and you know how dangerous the vicinity of the œsophagus is in that respect throughout the entire corresponding course of the stricture-then even if the œsophagus be quiescent, the wound may at any moment burst open and a fatal hemorrhage ensue. 
Of what great assistance gastrostomy may be, if performed in the manner thus described-using Pezzer's drainage tube-I am, as it happens, able to demonstrate to-day in the person of another patient who has returned, one year after the operation. In his case the stricture in the œsophagus was due to cauterization by nitric acid, but here it was not an accident, but an attempt to commit suicide, which induced him to swallow the corrosive draught. It is now four years since he endeavored to free himself from military service in this way. He succeeded in this object, though not in that of taking his life; because at the garrison hospital he was successfully tided over the state of collapse. An adhesion took place between the tongue and the forward arch of the palate even at the hospital. This coalescence was easily loosened; but later he suffered from increasing difficulty in swallowing, so that in a year's time he had to be admitted to this ward, where a stricture somewhat low down in the œsophagus was verified: 24 centimetres from the teeth. With the aid of bougies the stricture was so successfully expanded that he himself could insert bougie No. 22 Charrière without difficulty. During the two years following he was able to insert this bougie daily, though,

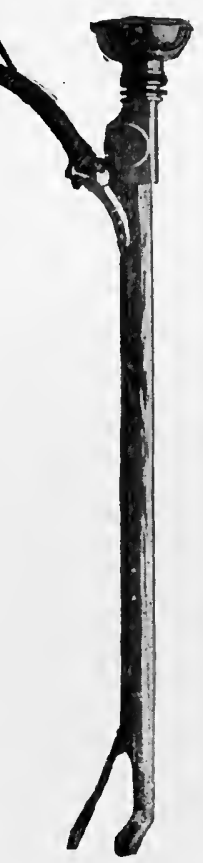

FIG. $47 .-$ Author's gastroscope for introduction of bougie into the œsophagus. curiously enough, this did not affect his nourishment, because the food could not pass, first the solid and then the liquid food being vomited. The only possible explanation of this is, that his stricture was what we term elastic; that it would easily allow of the passing of the bougie, though only to contract anew. He was then again admitted to this 
ward-shortly after I had taken charge of it-in an utterly wretched condition, being not only emaciated to the last degree, but suffering also from nervous excitement and anxiety, brought about by every attempt to swallow water and saliva, which resulted in violent dyspnœa and distinct cyanosis. You will understand that in this case also I was compelled to establish a gastric fistula, notwithstanding the stricture was passable for bougies. I performed this operation at the beginning of November, 1904, and as the man has arrived this very day to present himself for examination, after the lapse of twelve months, I take advantage of the opportunity to show him to you. He now looks so flourishing that you will have difficulty in realizing how ill and wretched he was previous to the operation. In this instance we are obliged to allow the fistula to remain permanently; this does not seem to have inconvenienced the man, who expresses himself as extremely contented with the arrangement. As you see, he carries the Pezzer catheter hanging freely from the fistula, which closes tightly round the tube, so that neither bandage, adhesive plaster nor other fixture is necessary. The catheter is led through stitched openings in the undergarments and fastened loop-wise beneath the waistcoat. When ready for a meal, he draws it forth, removes the cork, and pours the food through a funnel which fits tightly onto the opening of the catheter. Should he wish to gratify his sense of taste, he may do so by first rolling it round in his mouth, and then letting it descend the funnel. ${ }^{1}$ You see how perfectly healthy and free from reaction the skin round the fistular opening is, a sure sign that the catheter has fitted quite tightly, thus preventing the calamity which mars the result with other treatments,-i.e., oozing of the gastric juice along the drain,

${ }^{1}$ Even in 1909 the patient reports that he is very well satisfied with the stomach fistula and Pezzer catheter. 
which unavoidably leads to excoriation, eczema, and ulceration.

The other greatly-afflicted patient, who is now being brought in, and of whom I wish to speak to you to-day, is a man fifty-four years of age, who, little by little, has developed dysphagia, without any ostensible reason whatever. It began fully six months ago with solid food, and especially meat, refusing to pass the œsophagus. He was conscious of the point at which it lodged, situated on a level with the centre of the sternum, whence it was disgorged a few minutes later. These difficulties increased to such an extent that, when admitted here two months ago, he could swallow only soft fish-cakes and bread-pap, in addition to liquid food. He had lost about twenty pounds in weight, but at that time was still in fairly good condition. By an examination with an ivory-knobbed œsophagus probe, No. 40 Charrière, a stricture was discovered at a distance of 35 centimetres from the jaw. In other words, the stricture began at a good distance-quite 5 centimetres-above the cardia. Thereupon we endeavored, by means of ever thinning bougies, to pass the stricture, and eventually were fortunate enough to do this with a rubber bougie, No. 13 Charrière, without hemorrhage.

The stricture could be distinctly felt as a hard, firm infiltration, so that a spastic stricture, which may at times give similar symptoms, as for instance with nervous persons, or with a fissure at the cardia, could be immediately excluded; because here, when one has pressed the bougie gently against the stricture for some time, one suddenly feels this yield as if it were opening entirely.

Notwithstanding the man's fairly fresh appearance, the diagnosis was probable cancer œsophagi, because experience teaches us that in ninety-nine out of one hundred such cases, where a stricture has developed gradually, it is a question of cancer. You must, however, never fail to 
bear syphilis in mind, because now and again you will experience the pleasant surprise of seeing all symptoms disappear with an antisyphilitic treatment. Consequently you must always question the patient as to syphilis, and, with the objective examination, have your attention keenly directed toward possible stigmata, cicatrices on the penis or elsewhere, exostosis, general glandular swellings, etc.; but do not imagine that a negative result precludes the presence of syphilis. Here the question to be considered is a tertiary syphilis, a gummatous infiltration, though this is especially frequent with individuals whose syphilis has been neither diagnosed nor treated. ${ }^{2}$ Therefore, with most cases of tertiary syphilitic affections, you get no enlightenment from the patient, nor can you count on finding stigmata by the objective examination. But where the diagnosis cancer is not absolutely certain you must not fail to try an antisyphilitic treatment.

In this case we found no symptoms of syphilis with the anamnesia, and with the objective examination only an abnormity pointing to previous syphilis; this was a pronounced pupillary difference, inasmuch as the right pupil contracted incompletely under the influence of light. Therefore I at once instituted an antisyphilitic treatment with iodide of potassium and mercury ointment externally. Under this treatment a striking improvement in the obstructive signs set in, and in the course of a fortnight he was able to swallow bread, fish, and even meat, and I discharged him, expecting great improvement, and in the belief that the point in question had been a syphilitic stricture. But the improvement lasted only a short time, for in a week the difficulty with swallowing increased once more, and during the last month he has not been able to swallow solid food at

'We have since obtained in Wassermann's reaction a highly valuable expedient, which often provides a diagnosis when all other standards have failed. 
all, and has had increasing difficulty in swallowing liquid food; so that, when he was admitted here some days agothree and a half months after his discharge-he could not swallow even water. The consequent lack of moisture in his body resulted in a diuresis so insignificant that a danger of complete anuria arose. By subcutaneous infusions of salt water -4000 c.c. in twenty-four hours -and by nourishing the intestine three to four times a day with injections of a physiological solution of kitchen salt in which are dissolved 20 grammes of glucose, we have succeeded in improving his condition to a very considerable extent, but, as you see, the appearance of the patient is seriously cachectic, and, to those who saw him when he was last here, the emaciation and alteration which he has undergone appear to be strikingly rapid. Under these circumstances there can be no doubt that something malignant is in question, such as a cancerous stricture. How, then, shall we explain the strange improvement which immediately succeeded the antisyphilitic treatment? Of course, one cannot deny the possibility that lues has played a part, that a gummatous infiltration, perhaps, has been the prime cause, and that cancerous degeneration has set in secondarily. To me it seems more likely that the temorary improvement in the attacks of stricture are due to pure accident, being caused, perhaps, by a decay in the bulk of the tumor, due, perhaps, to the disappearance of an œdematous or inflammatory swelling which had increased the difficulty.

While the general condition of the patient has deteriorated so strikingly, we find by examination with œsophagus bougies no noticeable alteration in the local affection. The ivory-tipped bougie which we employ in our examination stops, as before, 35 centimetres from the jaw; and, as before, we have succeeded in passing through a quite thin rubber bougie, No. 8, and, later, bougies of continually in- 
creasing size, up to No. 15. The insertion of bougies is performed with great care; no blood has appeared on the bougies, and the patient has neither vomited nor disgorged blood.

When the patient has been removed, we will discuss the prognosis and treatment of this case, as it is one which concerns you closely, because, in your general practice, you will frequently have to face similar cases, and perhaps be in doubt as to what you shall tell the patient and how to advise him. In this instance the patient has been sent here by his physician certified operationis causa, it having been explained to him that a cancerous tumor was in question, and that an operation was, therefore, quite necessary. Here I will impress upon you, above all, never to mention the word cancer to your patients as a diagnosis. For the layman it holds not only a certain sentence of death, but also some undefinable horror and idea of being eaten and gnawed away, with terrible pain. In short, you increase the bodily torment ordained, by a great mental torture, without benefiting the patient in any way whatever. Now I know very well that, when saying this, I am at issue with several highly-esteemed colleagues. Yes, there are even countries like Norway where the majority of doctors, influenced by some agitating, eminent doctors; communicate the diagnosis to the patients on principle. In justice to these gentlemen I must state their vindication of their view of the question, so admirable in itself, but, according to my views, so untenable. They say that not to face death consciously, being fully aware of when and why and wherefore one has to die, is not consistent with the dignity of mankind. That sounds very fine, manly, and brave, but it must be borne well in mind that this is a courage which one thrusts upon others, and when one has again and again seen how the most determined and cultivated people become transformed and possessed of a fear bordering on madness 
when they receive this information, one does not understand it to be part of a physician's duty to act so inhumanly. Here, by way of enlightenment, I will give you an example. An elderly lady, known for her strength of character, has been operated upon several times by one of our leading surgeons for a constantly-recurring cancerous tumor. One day when he visited her she demanded to be informed whether it was cancer, declaring 'superciliously that the information would not affect her, and, as she maintained her demand, the surgeon considered himself obliged to comply with it. But when, a week later, he attended his patient again he was not a little surprised to be overwhelmed with reproaches for having yielded to her request. "You, as a physician, should have known," said she, "that the strongest-minded person cannot bear such knowledge. I have not slept since you were here, and shall have no peace before I die." But there is another circumstance which should remove all ethical scruples, viz., our diagnostic imperfections. I could mention a number of instances where, even after having laid bare the diseased organ by operating and when convinced that we were face to face with an incurable disease, further development has shown us that we were wrong; and among these are instances where our most experienced microscopists have arrived at the same diagnosis with the extirpated portions of the tumor. How disastrous to have given these people cause to dread cancer for the rest of their lives, and how compromising for yourselyes to await in vain the fatal termination you have foretold!. Finally, it must be remembered that, in a certain number of cases, we succeed in curing cancer patients, though if you have informed these patients that their complaint is cancerous their joy of recovery is spoilt by the fear of a recurrence. With our patient, also, the fact that his physician informed him that his complaint was cancer 
has had a very depressing effect, and I have vainly endeavored to distract his attention from the thought.

After this discussion we are confronted with the question as to whether we shall operate or not. The operation which the physician had in mind, and the only one which the symptoms can be thought to indicate, is gastrostomy. The operation is not of vital importance, is rather easy to perform, but has, nevertheless, a very high mortality in cases like this, where the virulence of the cancer is so far advanced and the patient's power of resistance so slight. We must, therefore, carefully consider what the real gain would be with such an encroachment before we take the risk. Of course, if all goes well, we succeed by means of our drain-Pezzer's catheter-in supplying the patient with liquid nourishment, but this we have already achieved by a daily introduction of bougies. For the moment, therefore, I can see no advantage whatever, but a great danger accruing to such an encroachment. Even if the introduction of a bougie were difficult, impossible, or ineffective, as is sometimes the case, yet I would not perform gastrostomy in such a case as this, because the man's rapid emaciation and cachexia are not due to inanition only, but, in a very essential degree, to the unseen malignancy and growth of the cancer. Therefore I do not think that gastrostomy here would prolong life to any appreciable extent. I do not mean to infer by this that I never find gastrostomy indicated with cancer œsophagi, but my opinion is that it should be reserved for cases where the patient still has considerable strength, and where, at the same time, the stricture entirely, or to a great extent, hinders the passage of liquid food, and where dilatation by bougie cannot be performed. 


\section{LECTURE VIII \\ DILATATION OF THE ESOPHAGUS}

I sHaLl show you to-day two male patients whose symptom of disease has developed in quite a homogeneous and characteristic manner. One is seventy years of age, the other barely fifty: Considering their age, both appear to be fairly healthy and strong, and if one did not know that a few months ago they both weighed some thirty pounds more than they do at present, one would scarcely believe that they were suffering from a serious disease, though this is, nevertheless, the case.

This illness began some two months ago when the patients found a difficulty in swallowing solid food. The seventy-year-old patient relates that this occurred quite suddenly, while he was swallowing a mouthful of meat; that he then felt a severe pain on a level with processus ensiformis, and experienced a sensation as if the piece of meat were firmly wedged at that point. His opinion is, however, that it passed down into the stomach-although ever since that day he has been unable to swallow solid food-it would go down part way and then come up again. In the case of the other and younger patient, the difficulty with swallowing developed more gradually in the earlier stage; possibly because he had been better able to masticate his food. But it is common to them both, that in the course of three to four weeks the difficulty increased to such an extent that even liquid food, even water, could not pass, so that during the last month a most precarious state of affairs has developed, which causes the patients great anxiety. Not only do they immediately eject what they eat, but, after taking a few mouthfuls of liquid, coughing 
fits ensue. Hereby a quantity of clear mucus is first ejected, the cough grows more and more strained, and is accompanied by a sensation of suffocation, until, suddenly, a mass of fetid, grayish-red fluid with a considerable sediment of grayish, slightly blood-colored detritus is dis-

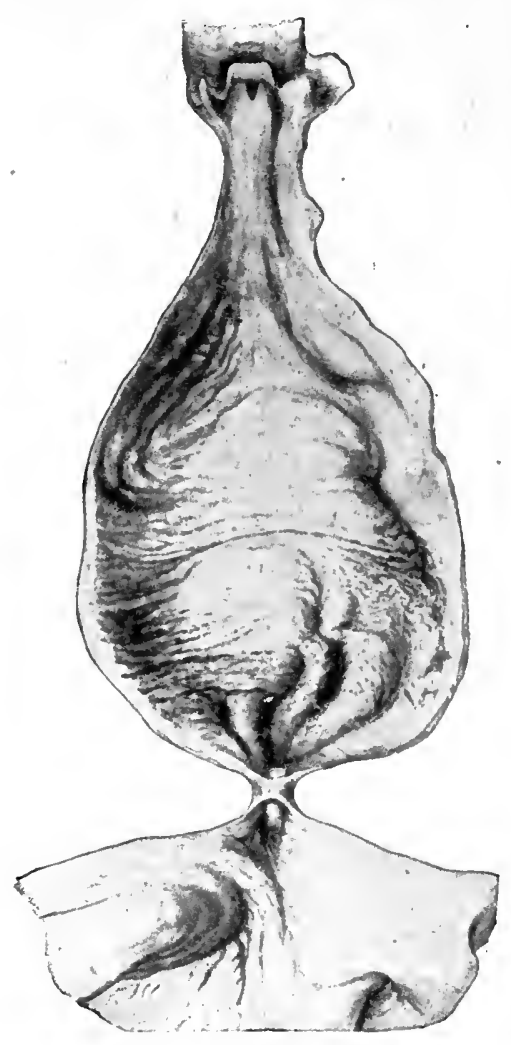

FIG. 48.- "Idiopathic" dilatation of the cesophagus. gorged. Not until an expectoration glass is half full of this fluid does the coughing fit diminish. These attacks can also occur without the patients' having eaten anything, and what has so alarmed them during the past fortnight is that the attacks occur when they lie down. This has necessitated their sleeping in a sitting posture, but every now and again they sink back into a recumbent position during their sleep. They then awake abruptly with acute sensations of suffocation, and the fear of this has caused their rest to be greatly interrupted, and, in addition, insufficient.

There can be only one explanation of these serious cases: a quantity of fluid must be stagnating in the œsophagus, which, when the patient lies down, pours upward, and, if aditus laryngis is not closed, threatens to drown him. That, now and again, a little oozes into the larynx cannot, of course, be avoided, and in this we have the explanation of the cough and respiratory difficulties. 
Such an accumulation and stagnation of fluid in the œsophagus may be due partly to a partial and partly to a total dilatation of this organ. The partial expansion is owing to the so-called diverticulum formation, an inflation of the œsophagus wall in such a manner that either all its layers participate in the diverticulum formation, or the mucous membrane protrudes like a hernia through the muscular layer. Such a diverticulum formation, or the disposition to such a one, is, as a rule, congenital, but in most instances it is a long time before it occasions sy m ptoms worthy of considerationsometimes not till late in life. This first happens when the diverticulum has become gradually dilated and its walls so atrophied that it is unable to discharge its contents spontaneously into the esopha-

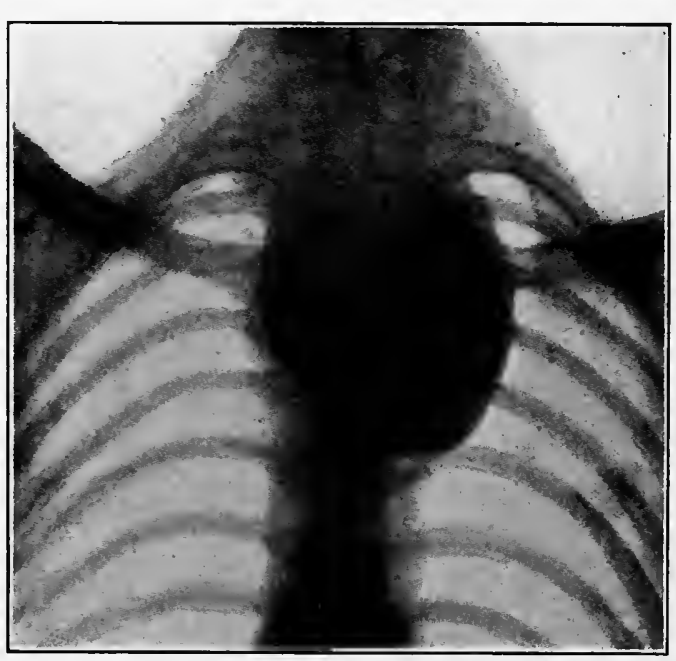

Fıo. 49.-Röntgen picture of œsophagus diverticulum filled with bismuth pulp (Keen).

gus (Fig. 48). Here I shall show you a Röntgen picture (Fig. 49) of such a diverticulum. You will understand that when this is distended by firm or liquid food it will be in a position to compress the œsophagus from without, and to impede or hinder the passing of food through this, and if, at the same time, the opening to the diverticulum has become much expanded, you will also understand that it might give rise to symptoms resembling in their main features the two cases here present.

A diffuse or total dilatation of the œsophagus may also 
be congenital, or may be due to a congenital contraction; but such cases are so rare that they have more pathologicanatomical than clinical interest. With regard to the congenital strictures of the œsophagus, I can refer you to an excellent Danish work on this subject, inasmuch as Hirschsprung's medical treatise deals extensively with this matter. The effects of this stricture, however, will generally appear very shortly after birth. On the other hand, we may often come across the so-called idiopathic, diffuse dilatations in a later stage of life. Here in Denmark, Gregersen ${ }^{1}$ has described a typical instance of this disease, in which we find a highly characteristic hypertrophy of the wall of the esophagus, the pathogeny of which is just as obscure as the so-called Hirschsprung disease, which is a quite similar congenital "idiopathic" dilatation of the large intestine. "Idiopathic" is an euphonious word, which only means that we know nothing about its pathology.

The most frequent cause of diffuse expansion of the œsophagus is stricture, and it is especially the cancerous stricture which is here concerned. Per se, one would expect dilatation always to develop above a contraction in a passage into which a quantity of liquid and some solid food are introduced daily. It is, therefore, somewhat surprising and incomprehensible that, in the majority of cases of stricture, no dilatation at all, or only slight dilatation, develops, while in a small minority of cases we find a surprisingly distinct dilatation.

If we now consider what is the cause of the dilatation with our two patients, we surely have every reason to disregard congenital abnormities in the case of the seventyyear-old man. Here we have reason to consider scarcely anything but the effect of an acquired strictura osophagi; and here again, in view of the man's age, it is most natural to think of a cancerous stricture. There are two points,

${ }^{1}$ Gregersen, Hospitalstidende, 1902, p. 1139. 


\section{PLATE II}

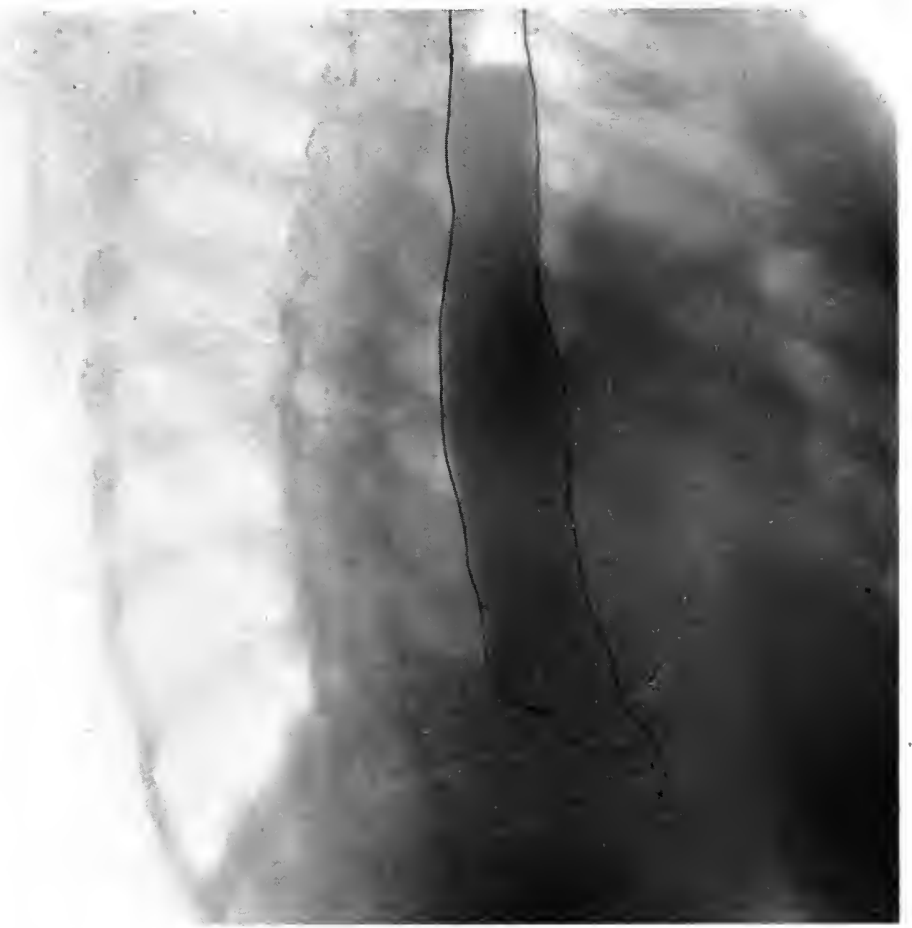

Typical cardio-spasm with the general diffuse dilatation of esophagus proximal to the constriction.

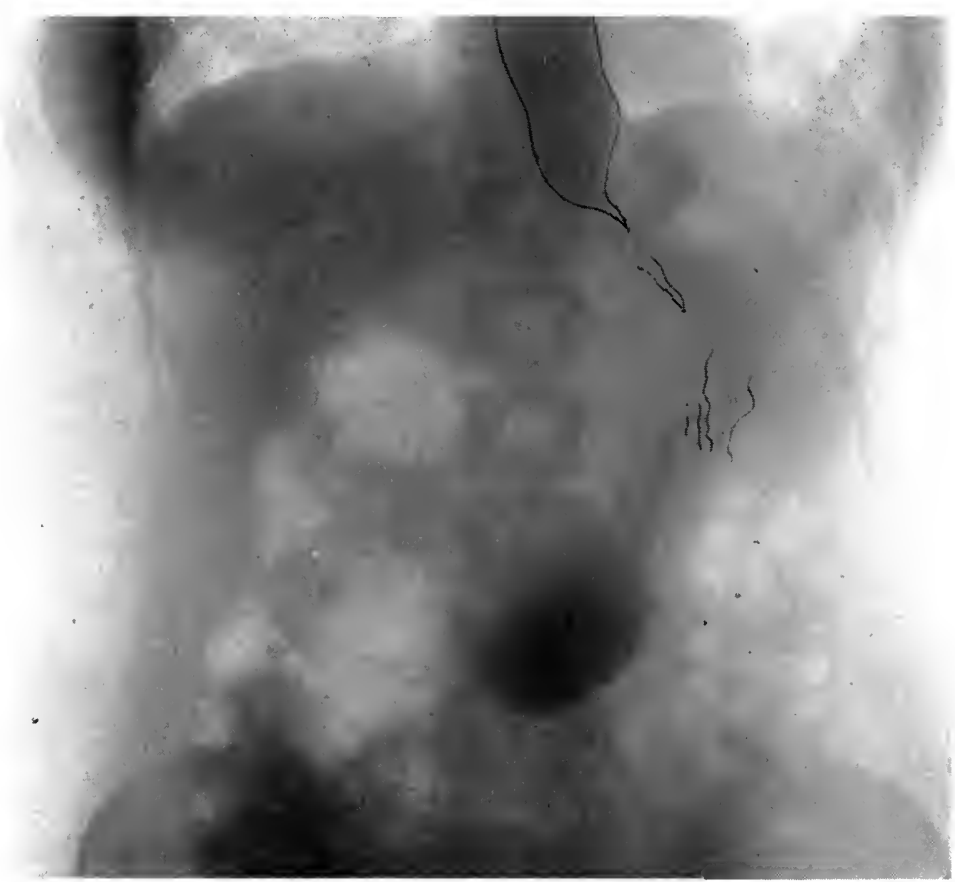

Cardio-spasm of the cesophagus as superimposed upon a carcinoma of the cardiae end of the stomaeh. 

however, which cause one to hesitate before certifying this diagnosis. Firstly, the astonishingly healthy and vigorous appearance which the patient presents, which induces us to consider the possibility of a benign stricture. If so, syphilis must be considered; and the other point to which I alluded, and which most of you must surely have already noticed, viz., the numerous scars covering the patient's face-this, especially, should attract our attention. In this case one immediately contemplates the possibility of a syphiliticulcerative process being the origin of these scars. They have not, however, the typical arched form, and on questioning the patient upon the subject we get an entirely different explanation, because the scars prove to be the result merely of the numerous lesions which the man's occupation as a stone-mason explains. We then again fall back upon the cancer diagnosis, and all the more so because the nature of the disgorged, malodorous, blood-colored, ragged sediment points directly to a process of decaying ulceration.

With the other, the fifty-year-old patient, one has more cause for doubt. His appearance is far healthier than is usual with patients in advanced stages of cancer, and his age, which certainly in no way precludes anything malignant, might, on the other hand, be quite consistent with the idea of a diverticulum or idiopathic dilatation. You will notice, however, that there is a sinister similarity between his and the old man's expectoration, which shows us that, also, a process of ulceration probably exists here. This entirely agrees with the external clinical estimate, and, despite the healthy appearance of the patient, I shall regard the diagnosis strictura cancerosa as very certain. In order to allay all doubts as to the nature and position of the obstruction, there still remains at our disposal the examination by probe and Röntgen illumination, both of which are very valuable. 
Examination by bougies is at the disposal of every physician, and is naturally that to. which he always resorts. Therefore, there is all the more reason to emphasize the dangers which this examination involves, and the precaution which it calls for; none the less so because it takes place in the physician's consulting room. The fact is that a moderately forcible attempt to pass a thin, tapering bougie through a cancerous stricture may easily lead to perforation of the mediastinal tissue; while a thick, firm bougie (ivoryor steel-knobbed) may lead to a laceration, with fissure formation of the mediastinal tissue. The consequences of this are most serious,-i.e., an acute, and often progressingly fatal, mediastinitis. In this way I remember seeing one of our best surgeons, when endeavoring to find a passage through such a stricture, surprised by feeling the point of the bougie protruding beneath the skin of the neck. A phlegmon of the tissue with glottis-œdema necessitating tracheotomy quickly developed. Recently I was called to see an elderly man, the record of whose disease resembled in its main features that of these two patients. He was suffering from difficulty in swallowing which had developed rapidly, and was treated for this by a specialist by the insertion of rubber bougies, with a view to progressive dilatation. While attempting to force through a large-sized bougie, the patient suddenly experienced violent pains and collapsed. Mediastinitis, pleuritis, and a septic condition with severe albuminuria immediately set in. Only after energetic treatment with salt-water infusions and stimulants, lasting over several days, did I succeed in pulling the patient through. Unfortunately, the stricture, which I had hoped would prove benign, appeared to be of a cancerous nature.

Even though it seems justifiable to say that it is no great misfortune for a patient with cancer œsophagi to pass away in this manner, yet it is always a very awkward situation for the physician when death supervenes in this 
way as a result of a simple examination; especially so if he has failed to inform either the patient or the relatives that any danger was involved, or even pointed out to the family the seriousness of the disease. Moreover, when a man is unexpectedly removed without having had time to arrange his affairs, it often means great loss and misfortune for the survivors. You are just as little justified in telling a patient that he is suffering from cancer as you are in failing to inform him that his condition is critical, and that, in case of emergencies, he should arrange his affairs.

If, on the whole, the introduction of bougies in cases of cancerous stricture calls for great circumspection, it is especially necessary with such cases as these to-day, where the œsophagus is dilated by and filled with malodorous matter; because we must be prepared for the infection having extended, affecting resolution not only of the cancer but also the adjoining wall of the œsophagus.

You will notice that I do not use a pointed rubber bougie for my investigation, but a round, ivory-knobbed bougie, No. 20 Charrière. It cannot penetrate anywhere, and if one guides it with a light hand one can feel without fear of causing harm.

You saw that the result of the examination was the same with both patients: the probe glided unhindered down to a point lying 39 to 40 centimetres from the jaw. Here it was stopped by a contraction, which is consequently situated far down in the œosophagus, and so close to the cardia that it is reasonable to assume that a cancer cardiæ is involved, which has spread slightly upward into the œsophagus.

If we have hitherto considered the possibility of a diverticulum, we must now dismiss the idea, because in that case we should have to imagine that this heavily-tipped probe had entered the diverticulum, which would be difficult enough, and also-which is decisive-that the bottom of the 
diverticulum reached down to the level of the cardia. Sometimes, naturally, one may be in doubt, but then the Röntgen illumination is an excellent resource if one first allows the patient to swallow an emulsion of bismuth. With a diverticulum, one then sees this revealed as a blind sac at the side of the œsophagus, and like a pear, stalk upward, joining the œsophagus (Fig. 49). With a stricture, however, one sees the bismuth-filled œsophagus as a fusiform extension above the stricture, as you see in this illustration, taken from

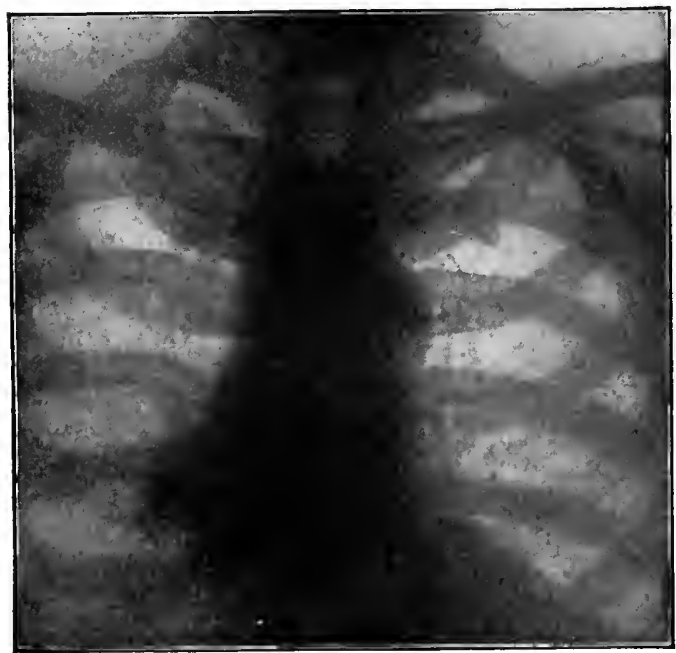

FIG. 50.-Cancer cardiæ with dilatation of the osophagus. Author's observation. a third patient, whom I am at present treating for cancer cardiæ (Fig. 50). There is another point in the diagnosis upon which we may expect enlightenment from the Röntgen illumination, and to which we have had our attention directed, viz., the question whether the stricture is quite impermeable, as the dilatation and the stagnation of the fluid might suggest, or whether it is still permeable, as the relatively healthy appearance, tolerable strength, and somewhat abundant diuresis indicate.

Never forget to examine the quantity and quality of the urine! It is an index of the extent of the inanition. In the course of a previous lecture I showed you a patient who came here with almost absolute anuria, resulting from impermeable stricture of the œsophagus. Here you see the quantity of urine discharged by our two patients during 
twenty-four hours, and you will notice that it still aggregates 300 to 400 cubic centimetres, but is quite thick with urates, which suggests that the reabsorption of

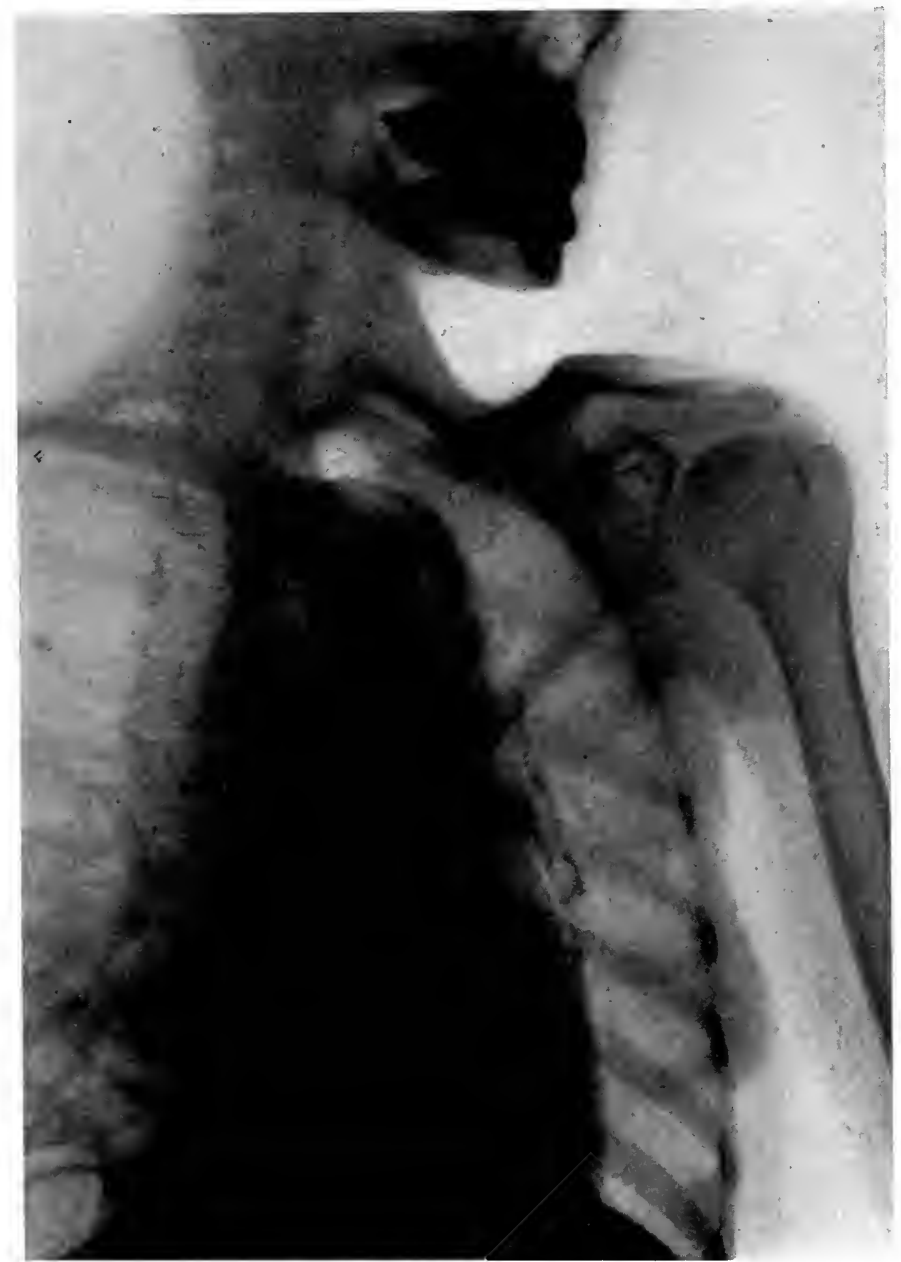

Fig. 51.-Extreme gastroptosis with marked cachexia. The illustration is a radiograph of the greatly dilated œsophagus which has resulted from a dragging down of the cosophagus into the abdomen and a kinking of the œsophagus where it passes through the diaphragm. The stomach is seen in Fig. 52.

fluid is greatly deficient, while the body tissues still have a quantity of fluid of which to dispose. Correspondingly, the Röntgen examination has also shown 
us that the stricture is not quite impermeable, because we find bismuth shadows in the stomach and intestine. Therefore, some of the emulsion of bismuth given to the patient has passed. I think this resembles an areaway in autumn which, after heavy rains, is filled with water, only a little of which can ooze away, because of the sodden, withered leaves which fill the drains. Here it is detritus, sodden,

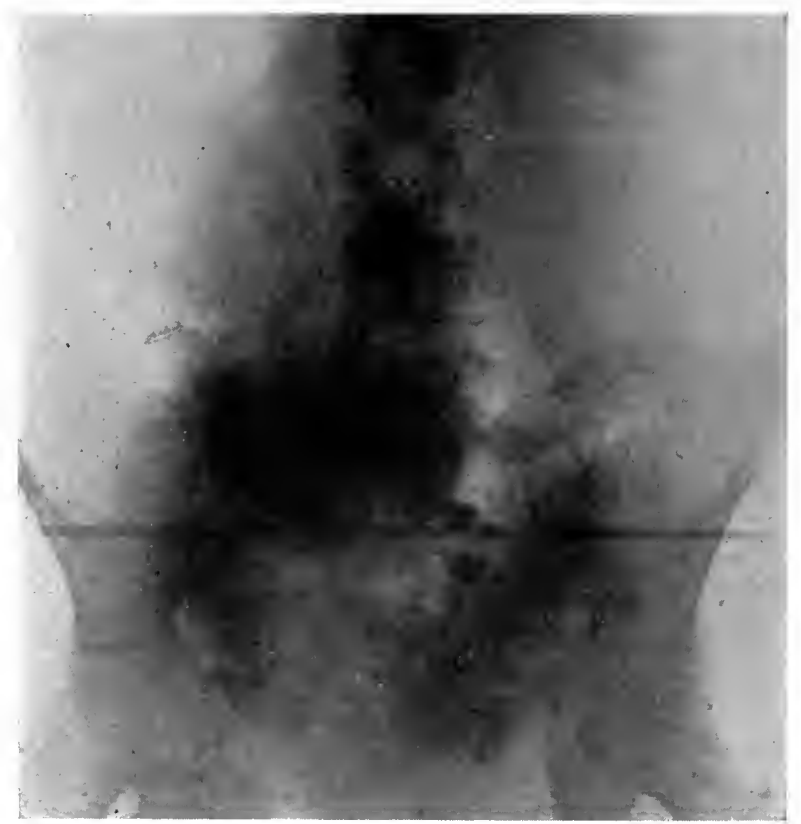

FIG. 52.-Radiograph showing the position of the stomach lying down below the umbilicus in the pelvis. Some bismuth paste retained in the lower segment of the œesophagus is seen in the picture. In this case gastropexy was performed together with gastrostomy. Wound closed after two months, the cesophagus having entirely regained its contractibility. Patient made a good recovery. Companion picture to Fig. 51 .

slimy remains of food which accumulate above the opening of the stricture, and, though allowing of the constant percolation of small quantities of fluid, cause a great disproportion between supply and discharge.

Our diagnosis, then, with both these cases is stricture in the lower region of the cesophagus, probably caused by cancer cardix, and the question now is how to deal with 
this disease. In a previous lecture I advised you, when confronted with cancer œsophagi, generally to adopt a passive attitude. So long as liquid food can pass more or less unhindered, I see no need for surgical treatment. Should the passage of even liquid food be impeded, a careful dilatation with œsophageal bougies is indicated wherever the introduction of the bougie encounters no obstruction. Should the stricture prove to be impermeable, the condition of the patient will, as a rule, be so advanced that an operative encroachment is senseless, and one must then restrict one's self to merely palliative measures : rectal nutrition, etc. But I told you also that there are exceptions to this rule, where a more active treatment is indicated, and in this respect the two patients with whom we are concerned to-day are very instructive examples. Here we have a menace of inanition with two otherwise vigorous persons, also the extremely disagreeable condition due to stagnation of liquid in the csophagus, with the danger of being drowned thereby when recumbent, and the cough, attacks of suffocation, etc. I have already relieved these patients to a great extent by emptying the œsophagus of its stagnant contents once or twice daily, and by carefully rinsing it out with antiseptic fluid: a treatment which I greatly recommend to you when confronted with such cases. If you empty it only once in the twenty-four hours, it should be in the evening, because you thereby insure a perfectly restful night for your patient; he, to his delight, being once more able to lie down and sleep, instead of, as formerly, being compelled to sit upright and cough throughout the night. Moreover, you relieve him of the evil flavor and odor of the stagnant fluid, and reduce the absorption and the intoxication, together with its virus, whereby the general condition of the patient is improved. I accomplish the depletion in the same way as I deplete the stomach, but instead of Nelaton's soft stomach tubes I use the firm rubber bougie, 
or a rubber catheter with a rounded nozzle. Such a one is passed much more easily and surely through the gullet, which is so irritated and inflamed in such cases, and it does not give rise to retching, does not double up, etc. You see how easy it is. I now connect the tube with this glass, from which I exhaust the air by means of the suction pump, when you see how the stagnant fluid from the œsophagus pours down into the bottle, which is capable of holding 500 cubic centimetres, and almost fills it (Fig. 53). You are surely

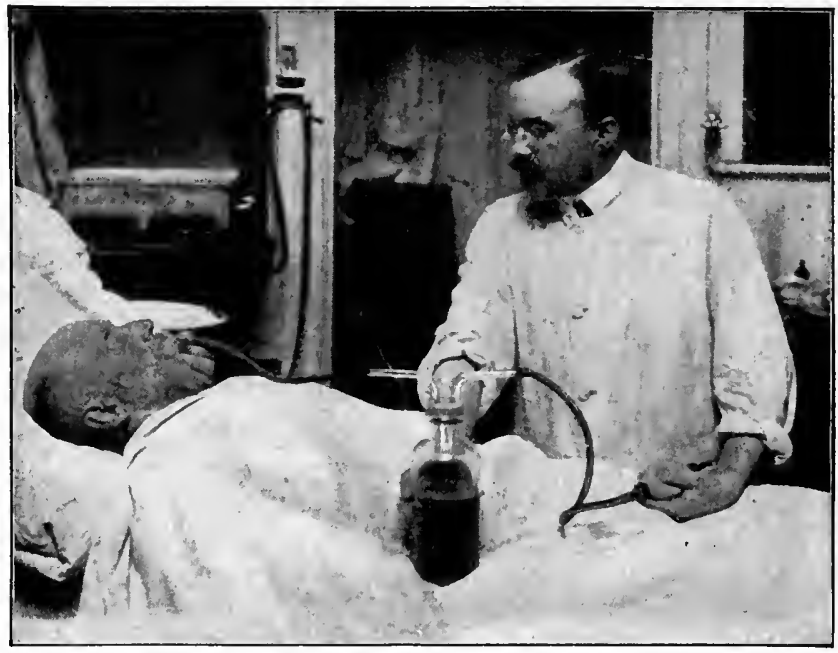

Fig. 53.-Author's apparatus for exhaustion of stagnant contents of the cesophagus and stomach.

surprised at the volume, but when the patient was admitted the quantity was 800 cubic centimetres, so that the retention has already been considerably diminished. Now, with a piston syringe, I flush the bottom of the œsophagus somewhat forcibly with a 1 per cent. solution of phenosalyl, and thereby whirl up the detritus, pus, and mucus, which you now see discharged together with the solution. But you will easily understand that this is work for the Danaides so long as the patient continues to drink and so long as the contraction continues. Therefore we must endeavor either 
to remove the stricture or to circumvent it by nourishing the patient.

The thought of radically removing the affected part of the œsophagus in order to cure the patient has greatly exercised the daring pioneers in surgery during the last decade, and, although they have not so far succeeded in curing anyone, in spite of all their persevering endeavors, we must yet accord them our homage- I allude first and foremost to Mikulicz and his pupils-for advancing so far as they have in the vanquishing of technical difficulties. You need only recall to memory the anatomical position of the lower portion of the œsophagus in order to realize immediately the technical difficulties which an access to this presents. Close to the left of the œsophagus lies the aorta, to the right the vena azygos, and close to the wall of the gullet run the two nervi vagi with their anastomoses, almost forming a net around the œsophagus. Above the passage through the diaphragm

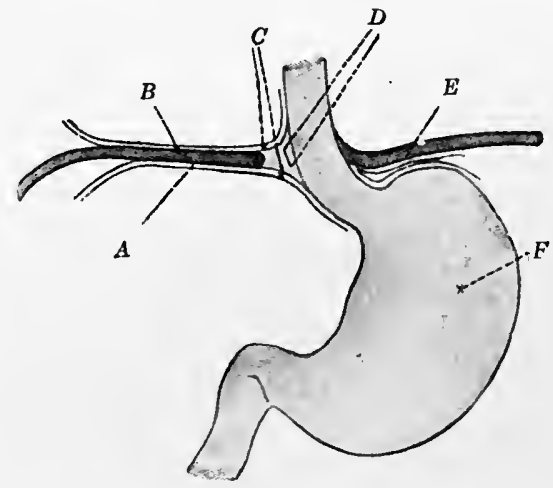

Frg. 54.- Relation of pleura and peritoneum to cardia. A. Peritoneum; B. Pleura; $C$. At tachment; $D$. Esophagus; $E$. Diaphragm; $F$. Stomach. the œsophagus has become attached to the pleura, and below to the peritoneum (Fig. 54). As a lesion of nervi vagi spells death, and as even a small lesion or a unilateral cut may prove very dangerous, one must be in a position to free them under the control of one's eyes from their attachment to the œsophagus, and to preserve them by resection. Therefore, a resection of the superdiaphragmatic section of the œsophagus is not attainable by laparotomy, which might otherwise seem in many ways advisable, and especially so as a means of avoiding injury to the pleura with the consequent pneumothorax. One might consider 
removing quite small tumors from the cardiac end of the stomach and the small subdiaphragmatic fragment of the œsophagus in this way (Heidenhain, Völcher) (Fig. 54); this does not apply where the superdiaphragmatic portion is in question, which, practically, will always be the case with cancer cardiæ and œsophagi, because you must always remember that if there is to be a possibility-generally speaking-of a radical recovery, our resection must extend for several centimetres into healthy tissues. The way to the œsophagus is, then, through the chest-wall and pleural cavity, as Mikulicz long ago pointed out, while his talented pupil, Sauerbruch, has succeeded in overcoming the technical

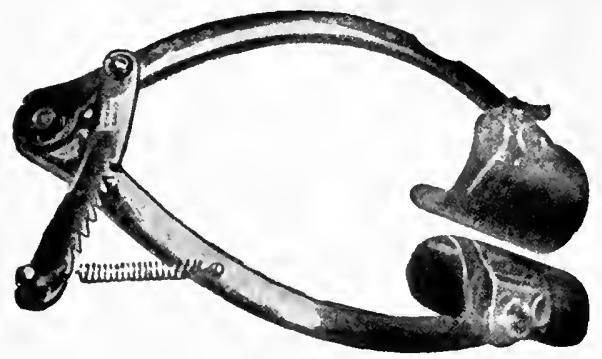

Fis. 55.-Mikulicz's rib separator. difficulties, so that a resection of the œsophagus comes within the realm of possibilities. The great danger involved in the opening of the pleural cavity, which, owing to pneumothorax, collapse of the lung, and, in consequence, insufficient ventilation, so often resulted in death on the operation table, has been eliminated by Sauerbruch's so-called Unterdruckverfahren, by which it is understood that, previous to the operation, the patient is so arranged that his chest area is environed in a rarefied atmosphere, together with the operator, while his head and the narcotizer are excluded. The rarity of the air prevents a collapse of the lung after the opening of the pleural cavity. The installation of Sauerbruch's kammer (chamber) with these so rare operations is very costly, and somewhat inconvenient for the operator. Brauer's überdrukverfahren is more satisfactory. His device aims at achieving the same result conversely, and has proved equally effective, because 
in this case it is the patient's head only which is partly enclosed in an atmosphere of increased atmospheric pressure, while the entire field of operation and the surgeon are quite free. Access to the œsophagus is gained through a long intercostal incision, which is made on the right side in the third intercostal space, while on the left side it must be placed lower in the fifth or sixth space. The rightsided incision is best adapted for a cancer situated higher up in the thorax, in the neighborhood of the bifurcation

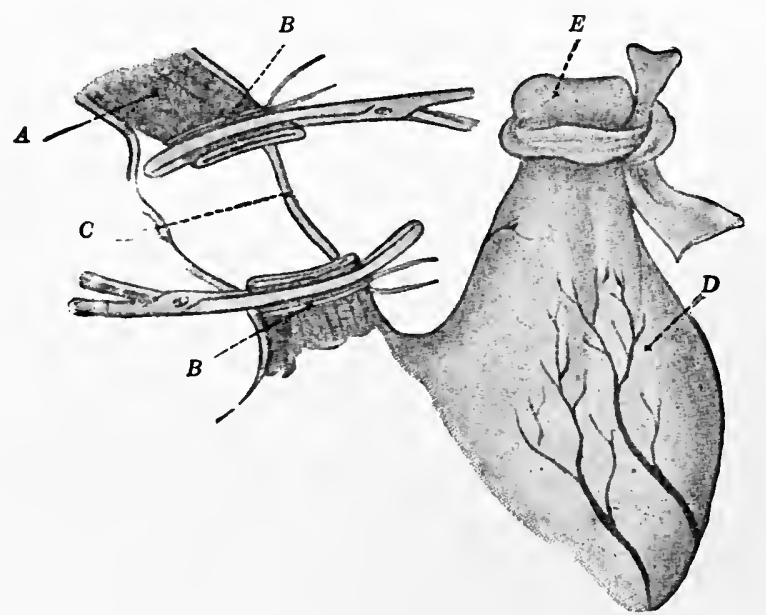

FIG. 56.-Resection of cesophagus performed between two clamps. End of stomach drawn up through diaphragm (Sauerbruch). A. Esophagus. $B$. Purse string sutures. $C$ Vagus nerves. $D$. Stomach. $E$. Portion of stomach wall drawn up.

and the bend of the vena cava; while the supradiaphragmatic region and cardia are more easily approached from the left side. With Mikulicz's rib separator (Fig. 55) the ribs are now forced apart as far as possible, then the pleural cavity is incised and the lung pushed aside with tampons until one sees the esophagus. Nervi vagi are now carefully loosened with anatomical forceps, and the œsophagus is drawn out by a loop of gauze or rubber which is placed round it. The cesophagus is then loosened with tweezers and curved scissors from the edge of the hiatus in 
the diaphragm, the pleura and peritoneum being first separated. One now divides the diaphragm in front to the extent of about one centimetre, so that one can draw up the cardiac portion and the fundus of the stomach through the diaphragm without difficulty. The affected portion of the cesophagus is then removed, between two compression forceps (Fig. 56). Now remains the difficulty of joining the stomach and the esophagus. Difficult for two reasons: because, on the whole, the limitation of space does not per-

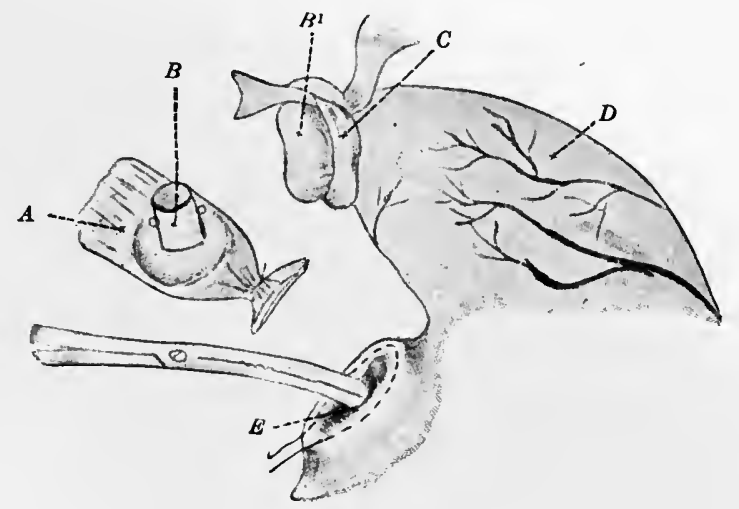

Fro. 57.-Halves of the Murphy button in place. Cardial portion of cesophagus closed. $A$. Esophagus. $B$ and $B^{1}$. Halves of Murphy button. C. Ligature. D. Stomach, E. Closing cardial orifice of stomach.

mit the easy manipulation of the instruments necessary for suturing, and also because the suture, when one has finally succeeded in inserting it, so easily cuts through the fragile tissue of the osophagus. Sauerbruch has therefore arrived at the conclusion that here is the sphere of the Murphy button, which we have otherwise rejected in intestinal surgery, in favor of suture. From a technical point of view (Figs. 57 and 58), this operation has been successful; not only with animals, but also with a few human beings, though none of these have recovered, having succumbed to mediastinal suppuration and heart-weakness. It is still an open question in cases of cancer whether we dare in any way 
hope for a real recovery from a resection of the œsophagus; because, quite apart from the technical difficulties and the difficulties of obtaining asepsis, the question arises whether we ever have an opportunity to treat cancer at such an early stage that it is restrainable, and while the patient's strength is sufficient for our purpose. In cases such as these two of ours, where the œsophagus is so greatly dilated and the wall distinctly attenuated, fragile, and infected, any attempt at a radical operation would be quite hopeless. Can we, then, remove the stricture in any other way? Here we have only bougie dilatation at our disposal, but with refer. ence to what I previously told you of the danger of perforating the delicate cancer tissue in such cases I must absolutely dissuade you from this. We must therefore resort to another treatment, which aims to avoid the stricture, and enables us to provide the patient with sufficient nourishment from another direction. The easiest

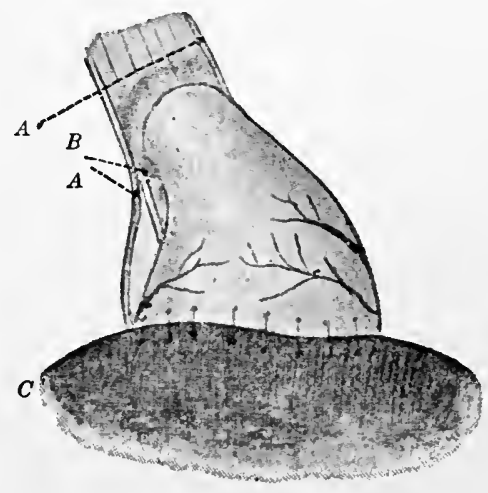

FIG. 58.-Anastomosis formed by joining two portions of the button. Diaphragm sutured around the opening through which the stomach has been drawn. A. Vagus nerves. B. Anastomosis. C. Diaphragm.

and least intrusive method would be per rectum, but you know that, valuable as this method is when it is a question of a patient's retaining his vitality for a few days after some operation or another while one deprives him of nourishment through his mouth, it is just as inadequate in the long run, because the only thing we can expect with certainty from the rectum is resorption of water with, possibly, a solution of salt and glucose. A veritable nourishment during long periods can only be obtained by conducting the food into the stomach itself, or into the upper part of the small intestine. And, in reality, it is only gastrostomy and jejunostomy which can 
here be considered. The oldest of these, and the one in most general use, is gastrostomy, - that is, the employment of a stomach fistula through which the patient is then fed,-an operation which has a special interest for us Danes, because it was first performed here at Frederik's Hospital by the eminent physician, afterwards politician, Fenger. It has given much trouble, however, to place the fistula in a satisfactory manner,-e.g., so that the gastric juice and the

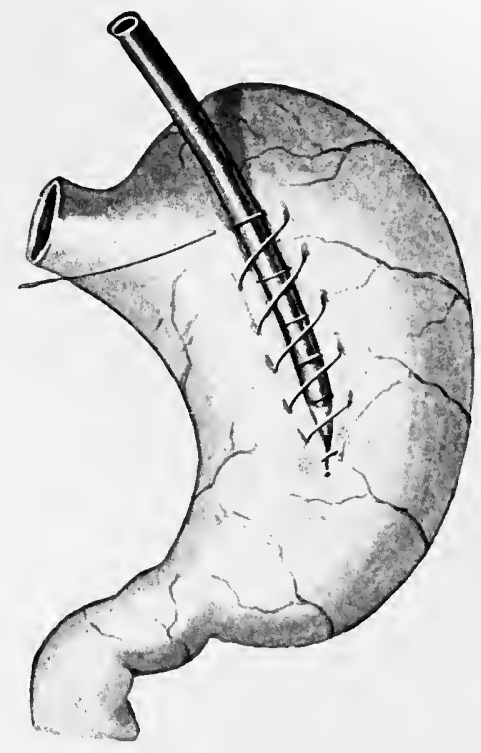

FIG. 59.-Witzel's oblique fistula. injected food do not ooze out through the fistula, whereby ensue not only percolation and evil odors from the dressing and clothes, but also eczema and ulceration of the edges of the skin surrounding the fistula. In our text-books you will find several rather complicated methods of operation which have been devised for preventing these complications. The best known are Witzel's and Kocher's. Witzel's method (Fig. 59) consists in the placing of the so-called " oblique fistula." After having drawn the stomach forth, he folds up the stomach-wall around a drainage tube to an extent of five to six centimetres, and then stitches these folds together around the tube, from above downward; but before closing the canal thus formed he incises the stomach at the end of this, and inserts the drain through the little hole. When, later, he completes the joining of the aforementioned folds, the drain lies in a long canal which perforates the stomach only at the end. The purpose is to prevent the contents of the stomach from oozing out. The oper- 
ation has this drawback, however, that one submerges the lowest part of the canal, where the opening into the stomach is, and if the sutures here do not hold, then the contents of the stomach flow over the peritoneum, and one has peritonitis. Finding the way into the stomach when shifting the drain may also give rise to difficulty.

Kocher's method is based on the use of musculus rectus as a deterrent. After the stomach has been brought forth through an incision in the centre line, a long strip of it is drawn under the paunch of the left musculus rectus and led out through a small incision to the left of musculus rectus, where it is attached to the skin with silk threads, just as fixation sutures are placed at the right edge of the muscle from the serosa to the rectus sheath. In other words, the approach to the stomach is bayonet-shaped, $\boldsymbol{r}$, the intention being not to employ the drain $a$ demeure, but to remove the bandage and insert a drain whenever the patient requires nourishment: as you see, very complicated; while the fistula rarely holds tight.

The method which I employ is not so ingenious, but is extremely certain, simple, and effective. I performed it first in 1894, in a case of impermeable, caustic stricture (see med. Selskabs Forhdl., 1894, og Hospitalstidende 1895), and have since employed it with equal success in a series of gastrostomies in cancer patients. The method, which has undergone some slight alterations, is now as follows: An incision is made in the central line from processus ensiformis ten centimetres downward; the stomach, which as a rule lies compressed high up under the diaphragm, is drawn down, and a part of the anterior wall, the size of a half dollar and as high up as possible toward the cardia, is drawn forth into the abdominal wound, and when two silk thread loops have been attached to that spot where the opening will be made later on, and while by the aid of these one lifts the conically-shaped portion of the stomach 
up, the serosa of the stomach is sewn to peritoneum parietale with a continuous silk suture. The remaining portion of the abdominal wound is joined in three layers, and, if the patient's condition does not necessitate immediate nourishment, I wait twenty-four hours before I open the stomach: until absolute peritoneal adherence has taken place. The exposed portion of the stomach is covered around the silk loops with a small mèche, while the joined parts of the abdominal wound are hermetically sealed with collodion and cotton wool. When I open the stomach-which I can do immediately and without danger-I sear through the stomach-wall, layer by layer, between the two silk loops, with

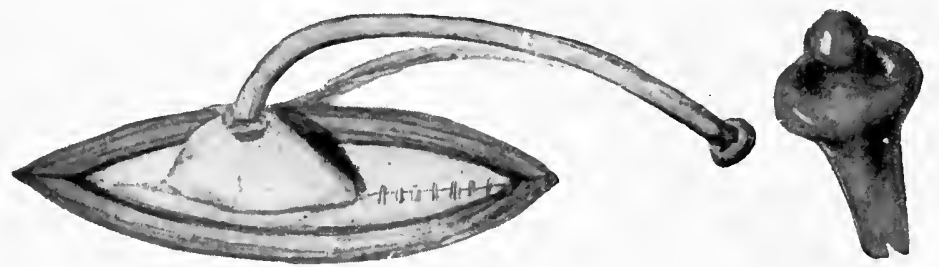

FIG. 60.-Gastrostomy with employment of Pezzer's drain after author's method.

Paquelin's knife, raising the mucous membrane between two péans before puncturing it, and in the meantime insertin a Pezzer catheter No. 26 by aid of a conductor. The use of a Pezzer catheter makes all artificial methods of operation superfluous; if only one is careful that it is drawn close up to the stomach-wall (Fig. 60), then the fistula holds perfectly tight from the very first. The drain is surrounded by a small strip of nitrate of silver gauze and is led out through a small dressing of gauze and cotton wool. It is provided with a stopper, and is attached to the outside of the clothing, so that one can at any time remove the stopper and pour food into the stomach. Every hour, or every alternate hour, 200 cubic centimetres of custard, peptonized meat, cream, cocoa, etc., are poured in.

We will proceed in this manner with our two patients 
to-day, and I hope that, when no further food is introduced into the œsophagus, we shall succeed in causing the dilatation and accumulation of fluid to disappear by a continuous emptying and antiseptic rinsing of the csophagus. But this is not quite certain, because you must remember that saliva is being constantly swallowed, and that the inflamed and ulcerated surfaces give forth a serous ooze-an admixture of pus and blood. Should the accumulation of secretion above the stricture continue to an inconvenient extent, I shall take advantage of the further benefit offered by my simple method of gastrostomy, and shall insert my gastroscope, which is constructed for the retrograde introduction of the bougie into the œsophagus. Then, from the cardia, I shall cautiously try to conduct the bougie up through the stricture, thereby opening a way downward for the stagnating fluid.

Gastrostomy performed in the manner I have indicated presents so many advantages, and is so easily and quickly performed, that it ought to be the regular operation in such cases. Only in exceptional cases would I prefer jejunostomy, namely, those in which the cancer has already spread from the cardia to a considerable portion of the stomach, and one perceives that the gastrostomy opening will soon be involved in the process.

Through an incision to the left of musculus rectus, two fingers' width above the umbilicus, an elevated coil of the jejunostomy, namely, those in which the cancer has already difficulty of preventing oozing, especially of gall and pancreatic juices, which, as you know, come from the afferent loop of the intestine. Here most people employ Witzel's "Oblique fistula"; but in this instance it commends itself even less, because the lumen of the intestine easily becomes too straitened by the folding and pressure of the drain. On the other hand, I have assisted myself by the following method, which I carried out for the first time in September, 
1904, and described in a lecture here. I draw forth a coil twenty centimetres in length, and first make an anastomosis between the loops of the intestine, ten centimetres from the top of where I intend to place the fistula (Fig. 61). You will notice that the top of the coil, independently of the contents of the intestine which pass through the anastomosis, serves exclusively for the reception of food through the drain, for which I employ one of Pezzer's small

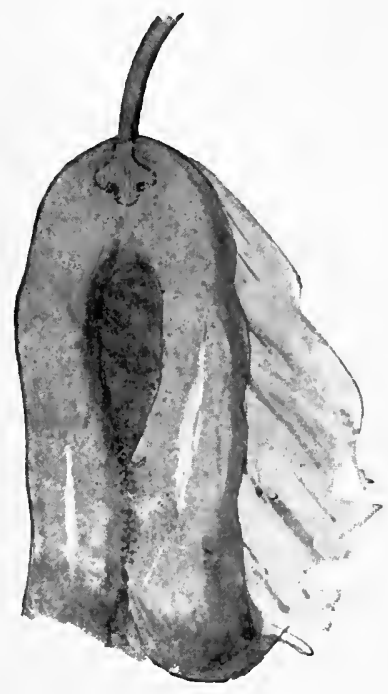

Fig. 61.-Author's jejunostomy. eatheters. Mayo Robson has later made use of a similar method for jejunostomy.

These two operations - gastroand jejunostomy-it seems to me, accomplish all that can be demanded in those cases where it is a question only of alleviating the sufferings and preventing death from starvation. The patient's longing to taste food can easily be satisfied by allowing him to masticate it before placing it in the funnel. There are surgeons, however, who are not satisfied with this, but want to form a connection between the topmost part of the œsophagus and the jejunum. Wullstein is, I think, the first who has formulated the idea of forming, by means of a skin-plastic, a tube-formed connection outside the thorax, between a jejunostomy and an œsophagostomy on the neck, but Roux of Lausanne has the honor of having partly carried out this idea in a far more daring manner, which can be best understood from these four illustrations (Fig. 62). Roux excises and sets free a suitably long piece of the jejunum in such a way that it remains connected with the mesentery and a nourishing artery, but can otherwise be easily drawn onto the outside of the thorax and up to the neck (Fig. 62, $A$ ). 

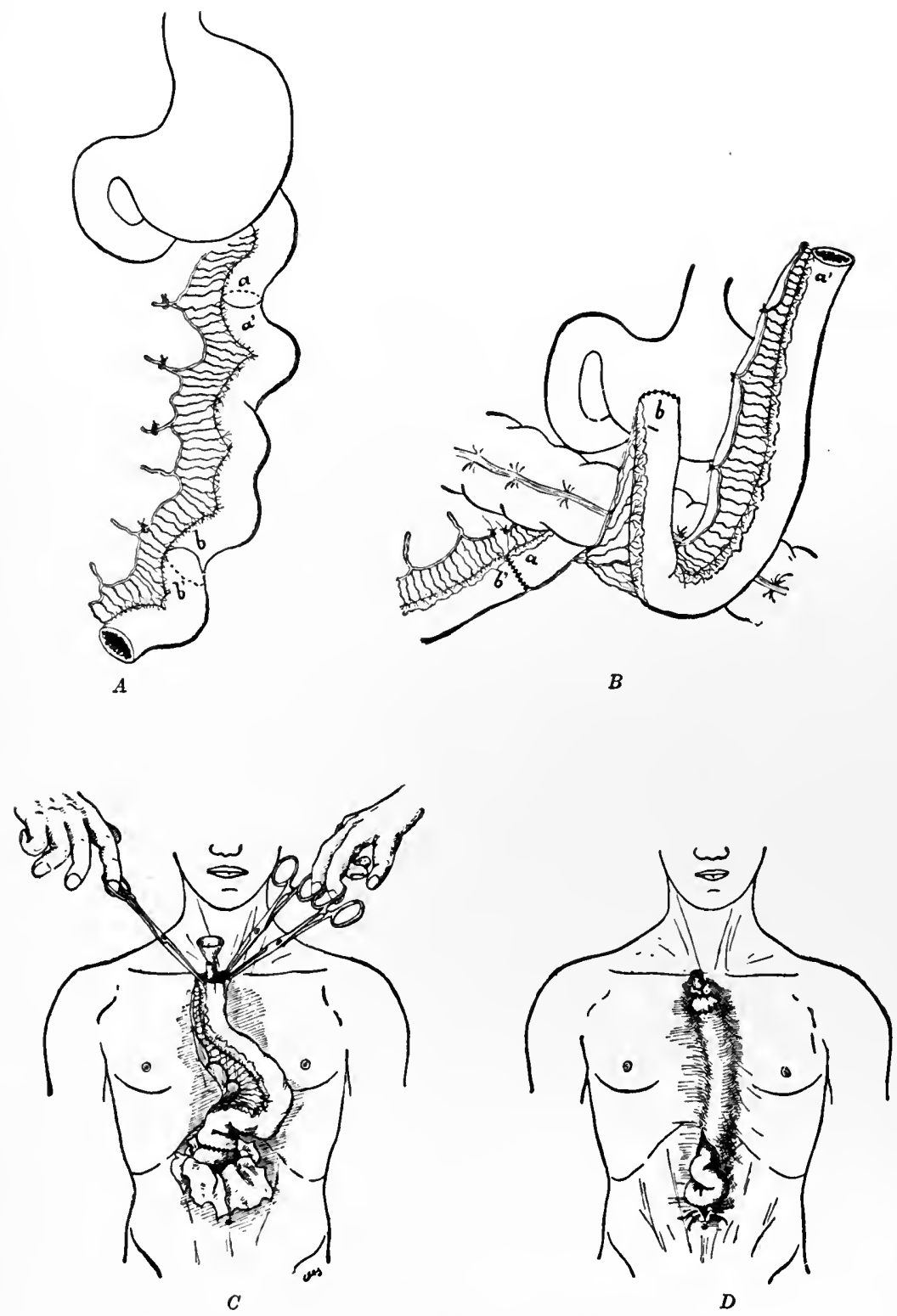

Fig. 62.-Roux's gastro-jejuno-cesophagostomy. 
Thereupon the central and peripheral ends of the jejunum are joined (Fig. 62, $B, a, b$ ), and the peripheral end of the loosened piece of the intestine is implanted in the stomach $(B, b)$. He then undermines the skin of the thorax and the top part of the abdomen with a blunt instrument, and draws the loosened piece of the jejunum through this tunnel in front of the thorax up to its opening in the neck (Fig. 62, $C, D)$, where he intends to place it in connection with the œsophagus by a second operation. It is of extreme interest that Roux has succeeded in pulling the patient through this operation alive and in getting the jejunum to heal inoffensively beneath the skin; but, just as it is worthy of admiration as a technical work of art, so it is unimportant, not to say senseless, as a means to soothe and prolong life for patients with stricture of the œsophagus. With benign strictures, as was the case with the boy operated upon by Roux, the encroachment seems unnecessarily great and dangerous, especially as one can probably avail one's self of a retrograde dilatation by gastrostomy. In the case of a patient with cancerous stricture the encroachment would hardly ever be survived by the patient, and would in any case be senseless, considering the short span of life left to the patient. Even if Roux should succeed in carrying through the second stage, the joining of the jejunum with the osophagus-and it is only then that anything is achieved-he never disposes of the cul-de-sac which the dilated œsophagus forms from the stricture up to the anastomosis, and whatever the patient swallows runs down into this immediately. It is an experiment which one admires more for its daring than for its prudence. 


\section{LECTURE IX}

\section{THE DIAGNOSIS OF DISEASES OF THE STOMACH}

IF one of my predecessors could now pay a visit to this ward, he would be amazed at the alteration which has taken place in the character of the patients. He would first wonder at the overcrowding of the patients and still more so to find as a reason for this, that according to the diagnosis on the slate, almost one-half of the patients would in his time have belonged to the medical ward, and not the least would he be surprised at the number of patients with affections of the stomach. When, in 1898, I reported the first cases of resection of the stomach, gastro-enterostomy and gastropexy, only four instances in all, of an operation for a benign affection of the stomach, had been performed here in Denmark. But since then circumstances have altered apace, so that the stomach patients now constitute a large percentage of those in the surgical wards, and the practising physicians find occasion to consign them to the surgical wards in increasing numbers. It is not to be wondered at that this development has taken place with great reluctance on the part of the genuine medical man, who sees with mixed feelings this domain, where medical therapeutics formerly held sole sway, glide within the lines of the so-called "'border land." But, nevertheless, they begin to regard the surgeon's share in the work more and more favorably even in this territory, and they will surely discover more and more that, if we deprive them of anything, we always provide a quid pro quo. Here I allude not only to the fact that we can cure a number of their patients whose ailments they have been unable to prevail againstwhich they must, of course, greatly appreciate-but especially to the mass of experience and instructive facta-at 
times highly surprising-which our operations bring to light. It is with regard to enlightenment on the difficulties attending the diagnosis of the various diseases of the stomach in particular, though also with regard to the effect and scope of the internal treatment, that the operative autopsy has already given most valuable information. We surgeons should in nowise object to the medical men taking their revenge by drawing within the domain of medicine some of those cases which have hitherto been purely surgical. If, for example, they could cure our locally tuberculous and cancerous patients by a serum treatment-which we may well hope-we should be very grateful, because the border land has added so enormously to our labors that we could certainly do with relief in other directions. Not the least of the additions to our work is that we must now master completely the medical methods of investigation with diseases on the border land, and must spend much time in accomplishing this, because a conscientious surgeon can only exceptionally perform an operation on another's diagnosis and investigation. Therefore we must occupy ourselves, in the surgical clinic also, in examining and diagnosing diseases of the stomach, and to-day I intend to employ the lecture hour in examining in your presence three patients who have been admitted with the diagnosis of stomach affection. The first patient is a butcher, thirty-three years of age, and, as you see, a big, burly man with a fairly fresh color and slightly obese, though he states that he has lost a good thirty pounds in weight during his illness. This began about twelve months ago with gastric symptoms, inasmuch as he had distaste for his food, while oppression, eructation, and occasional vomiting ensued. He then applied to his physician, who, from these symptoms, diagnosed catarrh of the stomach, which he considered due to the patient's somewhat abundant indulgence in beer. He therefore put him on a strict 
diet of milk-food, gruel, and fish, and interdicted all spirits. For a while the patient felt that he was better, but later the attacks became rapidly worse, and during the last few months he has vomited all food, and has become rapidly emaciated, while his strength has decreased so greatly that he is now quite incapable of work. When, a few days ago, the patient was at the physician's office for the last time, the latter became so alarmed at his appearance that he decided to examine him unclothed, which he had not previously done. He then found a large tumor in the abdomen, which he took to be an enormously expanded stomach resulting from a cancerous stricture of the pylorus. During all this time the bowel movements have been normal, and the passing of urine somewhat frequent, though this has been clear, and without albumin or blood.

If we now examine the patient, we find that he is rather pale, perspires freely, and is nervous and greatly apprehensive regarding his illness. While the extremities above and below are somewhat flaccid and emaciated, you see that the abdomen, on the contrary, is very full and protuberant between the epigastrium and the symphysis, is capped by the umbilicus, and falls thence in all directions. On the central part of the tumor the percussion gives a completely dulled sound, which continues up to the epigastrium, where it is replaced by a tympanitic percussion in the triangular portion between the curvatures. The dulled resonance extends in the centre line right down to the symphysis, while at the sides there is a tympanitic tone. The tumor is fairly tense, smooth all over, and undoubtedly fluctuating. We are assured by this that there can be no question of ascites, which gives inversely a dulled resonance in the flanks, and a clear tone in the centre where the airfilled intestines float in the fluid. It was reasonable enough, however, to think of ascites, because cancer in the stomach so often causes this. Here we have an absolute conviction 
that we are faced by a fluctuating tumor, which-has forced the intestines aside, and our conviction is further strengthened by our ability to move the growth from the one side to the other. If the patient were a female we should immediately say: large, one-celled ovarian cyst. But the patient is a male, and when I felt his abdomen I immediately thought this can only be an enormously dilated urinary bladder, because the swelling resembles so greatly what one might encounter in patients with hypertrophy of the prostate or contraction of the urethra. The position is, however, that we are dealing with a young man, thirtythree years of age, with whom hypertrophy of the prostate is excluded, who has never had urethritis, and with whom the investigation with bougie à boule has shown a normal calibre of the urethra. Moreover, the patient has never suffered pain or difficulty when passing water; this only having been rather more frequent and scantier than formerly, which is not to be wondered at during a state of inanition, and one must again ask one's self, therefore, if this tumor may not be an enormously distended stomach, or a cyst of the pancreas or mesentery, with which the dominant gastric symptoms can be brought into better accord. Well, it is not impossible that it may be a distended stomach, because there are instances in literature where a surgeon has exposed just such a tumor as this, in the belief that he had to do with an ovarian cyst, but where he has been astounded when, after incising the presumed ovarian cyst, carrots, plum-stones, and other contents of the stomach streamed forth in large quantities. Tscherning ${ }^{1}$ has reported just such a case. On the other hand, seeing that the dulled resonance continues right down to the symphysis, I consider that a cyst in the pancreas is out of the question, because this usually has a belt of tympanitic intestines beneath it, as it forces itself forth between the stomach and

${ }^{1}$ See Nordisk Kirurgforenings Forhandlinger, 1898. 
the colon or right above the stomach. Nor do the attacks fit in with a cyst in the mesentery, which might well explain the vomiting as a chronic condition of ileus. But cysts in the mesentery are usually globular and extremely mobile, while this tumor is more pear-shaped, with the narrow portion down toward the symphysis, and I must therefore hold to my first diagnosis as being the most probable one, viz., that it is a distended bladder, though we have not yet been able to assign any cause for this. Moreover, I see no objection to connecting the stomach symptoms with a retention of urine; because, if this is a distended bladder, the retention is of such long duration that there must necessarily be a strong reaction on the kidneys; and the stomach symptoms can be explained quite naturally as uræmic symptoms. The first thing I shall do here, then, will be to introduce a catheter into the bladder. A Mercier's rubber catheter No. 17 glides in quite unhindered, and you now see the urine stream forth, while the swelling gradually diminishes: Its uppermost boundary has already passed the umbilicus; thus all doubt with regard to the diagnosis is removed. It is a very interesting question as to what this retention is due. Is it due to a congenital, valvular formation, or to an affection of the central nervous system? But this question does not concern us to-day. I have produced this patient only in order that you shall never forget how necessary it is, when a patient complains of stomach symptoms, to remember that these are often only reflex and secondary to affections in other organs, and always to undertake a thorough examination of the patient unclothed. No diagnosis is given so light-mindedly as that of "stomach catarrh." It is a diagnosis which is frequently given "from the outside of an overcoat," in accordance with the patient's own description of his symptoms, and a more or less strict diet is prescribed. If the patient is a wealthy man, he may perhaps be ordered off to Carlsbad or Kissinger. 
Not long ago it was fairly good Latin to diagnose cardialgia, eructation, and vomiting as diseases of the stomach (catarrhus or ulcus ventriculi), and, with the same certainty, to diagnose constipation and diarrhœa as enteritis or colitis, commencing a systematic, dietetic treatment of these diseases, without any thorough examination. The energetic and clever work of the interne pathologists in diagnosing digestive diseases during the last decennary, and the surgeons' constantly increasing experiences, have shown us the surprising frequency of such a deceptive conclusion. According to my own experience, I am almost tempted to say that constipation is quite as frequent a symptom with stomach affections as with intestinal affections, because it always accompanies gastroptosis and most cases of ulcus ventriculi (ulcer of the stomach), while, inversely, cardialgia, eructation, or vomiting can-without the stomach itself being affected-be the predominant symptom with gall-stone and intestinal affections, such as ulcus duodeni, appendicitis, intestinal tuberculosis, cancer coli et recti, not to mention pregnancy and diseases of the female generative organs.

You will understand, then, that you must always look well ahead, in order to avoid going astray.

When you have questioned the patient regarding the anamnesia, you begin by examining him, or her, unclothed: first in an erect posture, if the condition of the patient will allow of it, and then in a recumbent one. The examination in the erect position often gives excellent results. Firstly, you see if there is a sagging belly, and how the muscles liewhether relaxed or vigorous; if there is a hernia; if there is venous dilatation, pulsation, or protuberance of one part or another of the abdominal wall. Secondly, while allowing the patient to relax his muscles, or-if he is unable to do so-by palpating downward with your hand during expiration, you feel whether there is any subsidence or 
swelling of the fixed organs: liver, spleen, kidney; or any tenderness when pressing the cardia; and whether this is in the centre line or to the right or left of it-which may prove of extreme importance. If the pain is on the right side, there is more reason to consider the gall-bladder or the duodenum as the seat of affection; if it is in the centre or on the left side, the suspicion of a stomach affection is confirmed. With tumor of the stomach, inversely, you must palpate during full inspiration, as only then is the tumor pressed so far down that it can be felt. Examination in an erect position is often of great importance, because a tumor, which is imperceptible in a recumbent position. then sinks down and becomes palpable.

You must perform the so-called scraping ausculation also in the erect position: a valuable method of examination, for which we are indebted to the Finnish physician, Runeberg. The object is to define the limits of the hollow organs, especially of the stomach and colon. I shall demonstrate this method for you with this patient, a spinster, thirty-four years of age, who has been admitted under the diagnosis ulcer of the stomach, but where, as there has never been hæmatemesis, while the quality of the food seems to play a small rôle as compared with the quantity where the development of pain is concerned, I have a suspicion that the morbid attacks are due to a ptosis.

You see that I now place a stethoscope at the top of the cardia, just within the curvature of the left rib. While, now, I lay my ear to the stethoscope, which I hold in my left hand, I make little scraping movements with the nail and tip of the right forefinger, at first just below the stethoscope. Through this I hear a very strong, scratching, hollow sound. So long as I hear this, while my scratching nail moves in all directions from the stethoscope, I am still over the stomach, because directly I leave it the sound ceases suddenly, or suddenly becomes quite distant, weak, and sub- 
dued. I now make pencil marks to correspond with the outlines, and you see that the greater curvature is situated six centimetres below the umbilicus. By this simple examination you can often, in your consulting rooms, arrive at the diagnosis: gastro- and colo-ptosis, though you must remember that, at times, this method may deceive you, viz., where a colon transversum distended with air covers the stomach which is prolapsed behind it. You will easily understand that in such cases you can ascertain the normal boundaries of the stomach with the aid of Runeberg's method, even where the stomach has prolapsed considerably.

It is, therefore, very fortunate that we have another and far surer method for defining the location of the stomach,-i.e., the Röntgen illumination of the body, after the patient has consumed a plateful of bismuth porridge, 25 grammes carbonas bismuticus apportioned to 200 grammes oatmeal porridge. You then see the bismuth porridge depicted as a sharp shadow, which determines the position of the stomach, because the lowest edge of the shadow will correspond with the greater curvature, and the upper edge, as a rule, with the lesser curvature because the stomach contracts around the contents. If you observe the stomach through the phosphorescing screen you very often see it in lively peristaltic motion and can study it. Sometimes, from the violence of this motion, one can diagnose a stenosis of the pylorus with hypertrophy of the stomach-wall, caused by hard work. If you wish also to have a record of the shape and position of the colon, you merely repeat the operation twenty-four hours after administering the porridge. If you wish contemporaneously to delineate the stomach and the large intestine, you must fill this by injecting, through the anus, two pints of bismuth solution-100 grammes bismuth to 1000 grammes milk or water. You can trace with colored chalk, directly on the screen, the outline of the 


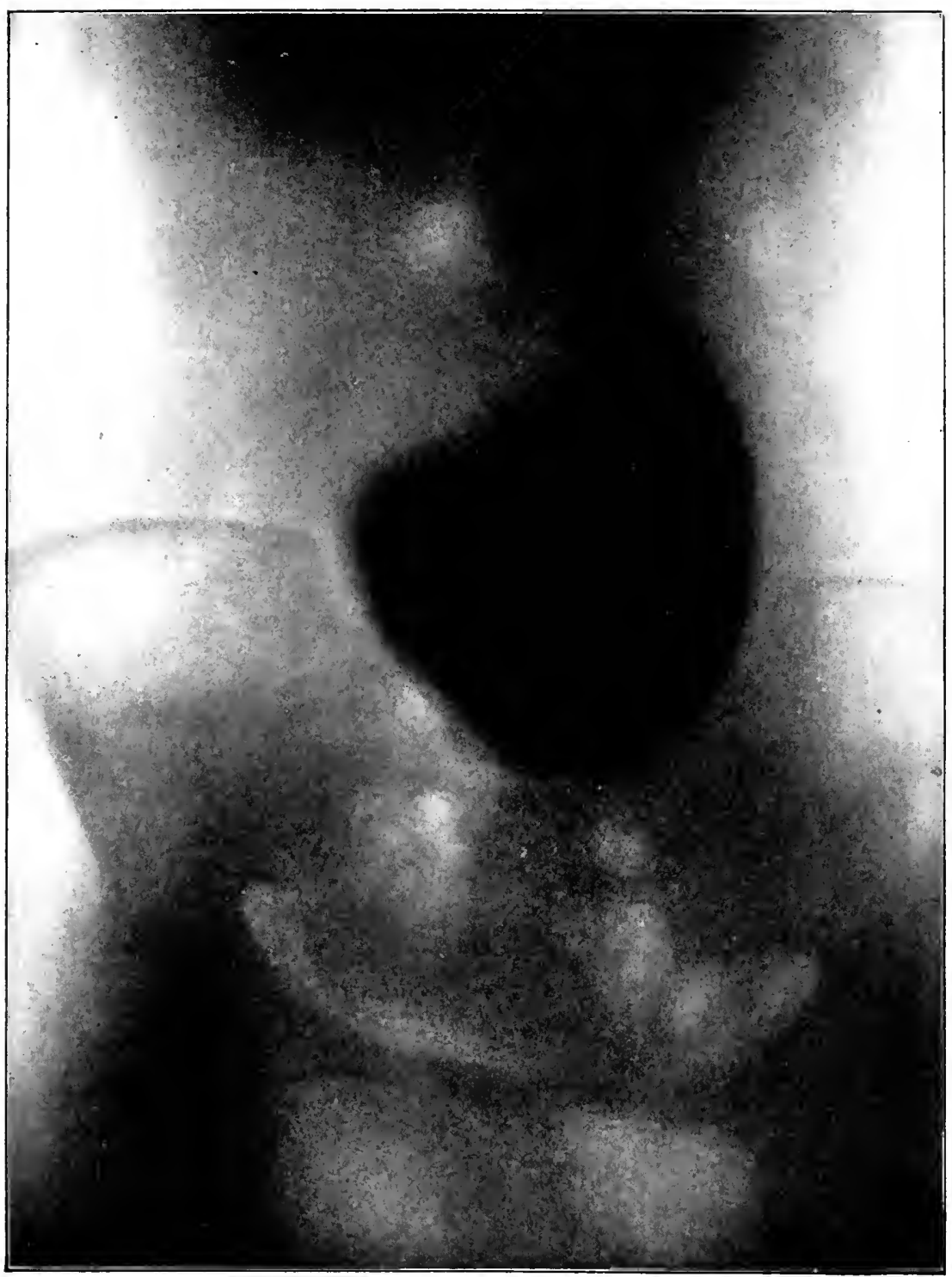

FIG. 63.--Radiograph of stomach filled with bismuth and greatly prolapsed.

stomach and intestine then presented, and from there can trace it on paper for preservation in the history. But you can also fix the picture on a photographic plate, and so have an exact record of it. In this way I will show you these 


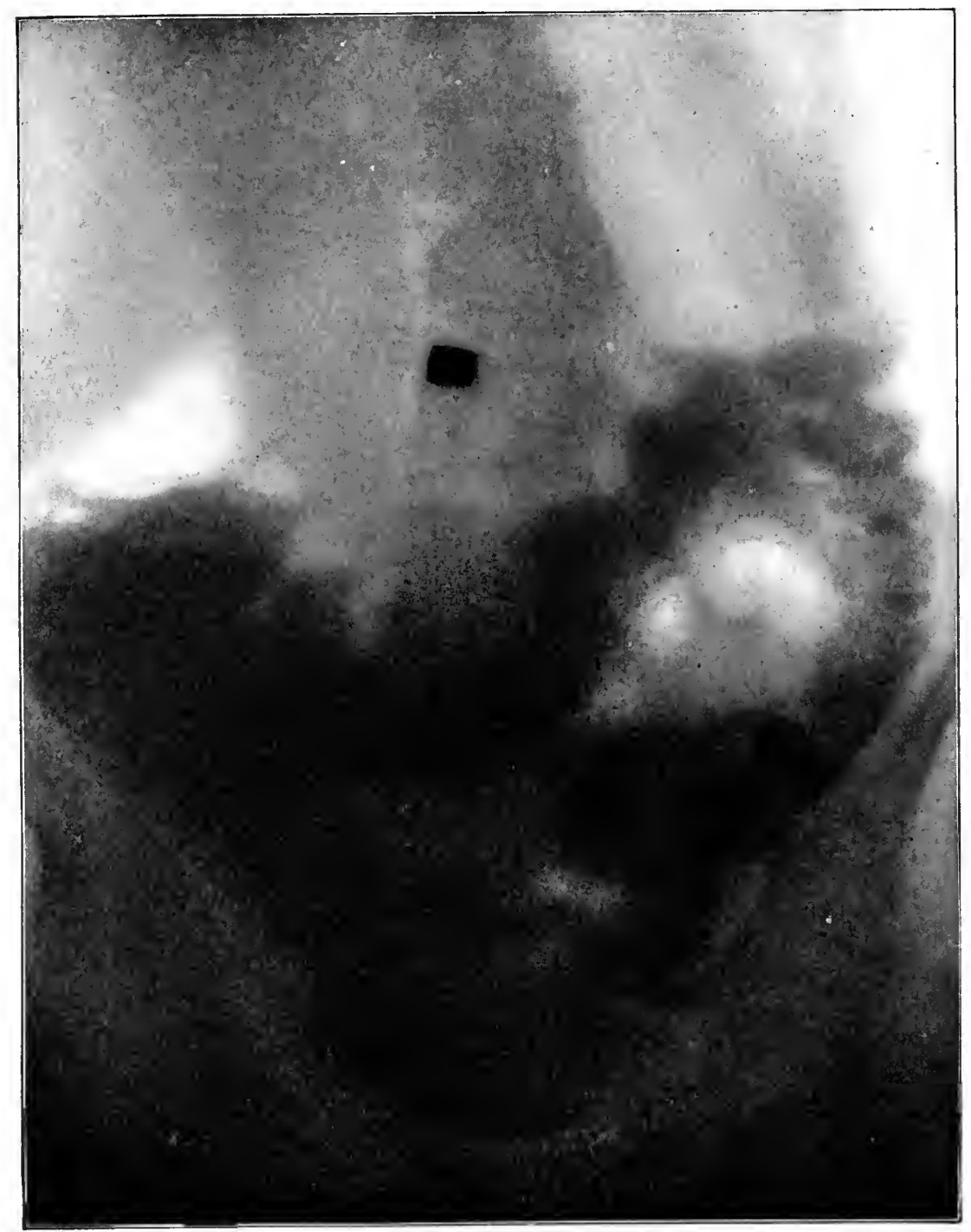

FIG. 61.-Radiograph from same patient 24 hours later, showing abnormal position of colon.

two pictures (Figs. 63 and 64) which have just been taken of the patient whom we are examining. The one shows you clearly the position of the stomach, corresponding exactly with the tracing of our scraping ausculation; while on the 
plate taken twenty-four hours later you see the colon filled with bismuth throughout. You see, then, how the colon transversum hangs down like a festoon from the two flexures which are sharply angulated, so that the lower portion of the festoon reaches down almost to the symplysis. When you see this picture you understand also why constipation is the most constant symptom with gastro- and colo-ptosis.

The Röntgen illustration, however, may also deceive one at times, because the bismuth can cause the muscles of the stomach to contract so greatly that this is drawn up to a normal, or almost normal, position, and the view of the ptosis is effaced.

But the fact that we have now found a gastroptosis by no means precludes the possibility of the stomach symptoms being due, entirely or in part, to some other affection of the stomach-a gastritis or an ulcer. We should always, therefore, make a careful investigation regarding the stomach's function; I should say, rather, a series of investigations having as its object an examination, partly of the chemical function of the stomach, and partly of its motility.

We examine the chemical function by emptying the stomach of its contents with a stomach tube, partly when the stomach is empty, and partly after experimental meals which are withdrawn at varying periods after they have been eaten. Where an examination of the secretion and chemical condition of the gastric juice is in question, we employ Ewald's so-called test-meal, consisting of an unbuttered roll and a cup of tea; this is withdrawn an hour afterward and examined. Where, on the other hand, the point is to judge the ability of the stomach to discharge its contents, and to ascertain whether any retention of the contents of the stomach takes place-a phenomenon of great diagnostic importance-we provide a larger,more compound, and less digestive meal. We make use of a modified Bourget's test-meal, consisting of 250 cubic contimetres gruel, 
75 grammes minced meat, 50 grammes bread, one tablespoonful cowberry, and ten boiled prunes: the latter because experience teaches us that cowberry seeds and pruneskins do not easily pass out from the stomach, and often adhere to ulcerated surfaces, and are, moreover, easily traced in the stomach centents when it is withdrawn. If you still find remnants of such a meal in the stomach eight to twelve hours after administering it, there is undoubtedly an abnormally slow discharge, due to a morbid condition. To-day we shall not consider in detail the importance of such a retention, which may represent the meal in its entirety, or merely a small remnant of it, because my object today is essentially to give you a comprehensive view of the methods of examination and of the technic and manner of carrying out these methods. On the contrary, I will now demonstrate our manner of withdrawing the meal with this patient, who, at 11 P.M. last night-fully ten hours ago-took one of Bourget's experimental meals. In the first place, we pass a properly-lubricated stomach tube of red vulcanized rubber, with rounded ends and side opening, through the œsophagus down to the stomach. I will warn you against the sharp-edged stomach tubes with the opening at the end, because they may cause a lesion of the mucous membrane of the stomach if it adheres to the opening during suction. The tube should have only one side opening, as those with two openings easily bend and double up above the cardia, so that they cannot pass. As soon as the tube has passed the cardia, one generally hears a bubbling sound, as of rising air. You then place your open hand over the epigastrium and see whether you can press the contents of the stomach out through the tube. Then the stomach tube is connected with the suction apparatus which you see here: a rubber-corked glass bottle (Fig. 65) pierced by two angular glass tubes, of which one is connected with the peripheral end of this little exhausting clyster-pump, while the other is connected with 
the stomach tube. By compressing the exhaust bulb we now draw the air from the bottle and, after a few compressions, you see the contents of the stomach beginning to be sucked down into the bottle, and can observe and judge of them at your leisure. You see that in this case the contents of the stomach are quite clear, no remains of food can be seen macroscopically; there is an insignificant quantity of mucus, but no blood; and all that we can withdraw amounts to barely 20 cubic centimetres. If we wish to convince our-

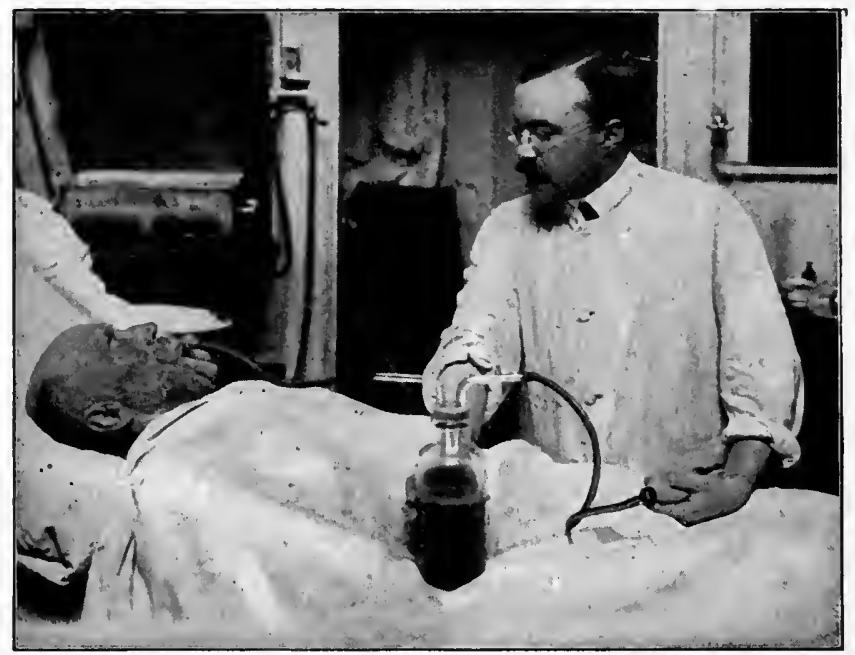

FIG. 65.-Withdrawal of retained meal by aspiration.

selves that no fragment of prune-skin is concealed in a fold, we can rinse out the stomach. We take away the bottle and connect the tube with this large glass funnel, through which we then allow warm water to run quickly down into the stomach (Fig. 66). By lifting the funnel higher, and then suddenly lowering it as far as possible, we obtain strong suction. The result is, as you see, that this water returns perfectly clear: therefore there is no retention. It often is instructive, with patients who can bear the examination in an erect position, to distend the stomach with air, partly to determine its dimensions, and partly to ascertain if there is 
gastroptosis; because the stomach is drawn downward by distention, and its outline is often depicted sharply through a thin abdominal wall, and, in any case, you can then define its limits by scraping auscultation. Such an operation is now performed very simply by my turning the clyster-pump and connecting its point with the stomach probe. When I compress the bulb I pump air down into the stomach through

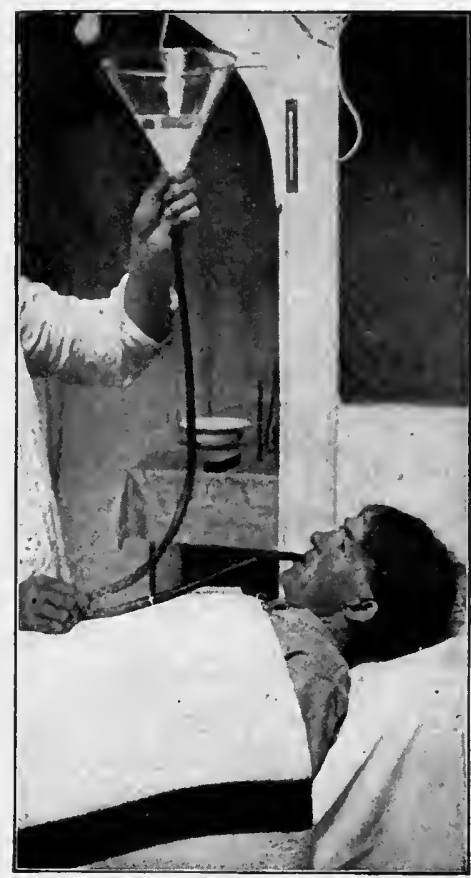

Fra. 66.-Rinsing out of the stomach. the tube, and, if you observe the patient's abdomen while I am pumping, you will see the stomach depicted through the abdominal wall, inasmuch as a sausage-shaped distention first raises itself in the previously so sunken epigastrium to the left of the median line, and afterward an upward concave, downward convex complement raises the abdominal wall between the umbilicus and the symphysis. With abnormalities in the shape of the stomach, such as the so-called hour-glass stomach, we can often prove their presence by this method of examination alone, just as a tumor first betrays itself during this examination when it becomes plainly perceptible. After the examination we exhaust the air through the tube before removing it. This very simple apparatus, which I have used for ten years for examining the stomach, I can recommend as being very practical. I think it is far preferable to the apparatus recommended by Alfred Madsen and which is in general use in the medical wards and special clinics, where the bulb 
is so placed that the contents of the stomach are sucked up into it. It has the drawback that the bulb is cleaned only with great difficulty, and has to be boiled and sterilized, whereby the bulb suffers and time is wasted. Sk. Kemp has pointed out that even with very energetic boiling and rinsing one cannot be sure of removing all the food remnants, so that one risks finding prune-skins, cowberry seeds, and grains of starch originating from a previous examination, which must naturally lead one astray in one's diagnosis. With my apparatus you need only a clean stomach tube and bottle for each patient, and can use the same clyster-pump, which has the double advantage that it can be used both for exhausting the stomach of its contents, and for inflating it with air.

You see that the assistant surgeon has followed the same procedure with our third patient, also a woman in the thirties, who for many years has presented similar symptoms: cardialgia, eructation, somewhat frequent vomitings, and constipation. But you must note with this patient, in contrast with the last, that the quality rather than the quantity of the food plays the more important rôle as regards the symptoms. This patient suffers from pain immediately after eating rich or spicy foods, while she can take milk and milk food without discomfort.

In her case you see a very considerable quantity of the contents of the stomach streaming down into the bottle, which is soon filled with a brownish fluid, on the surface of which splotches of mucus are floating, while soft substances sink to the bottom. If we whirl these about you see distinct flakes of prune-skin. You see how different it is from that of the last patient. In this case we cannot doubt that a serious affection retards the motility of the stomach. One may surmise a stenosis of the pylorus caused by an ulcercicatrix or a cancerous tumor; but it may also be caused by a diffuse inflammatory or cancerous infiltration in the wall 
of the stomach. The stomach contents withdrawn in both instances will now be sent to the laboratory, where a microscopic and exact chemical examination will be made. We always test the specimen immediately to determine its reaction by dipping a piece of Congo paper into the fluid. You see, then, that, whereas the stomach contents of the last patient do not in any way alter the red color of the paper, this paper becomes bluish under the influence of the stomach contents of the previous patient, which here shows that there is free lydrochloric acid, while the last patient was quite deficient in this-achylia. This in turn demonstrates that the glands which secrete hydrochloric acid in the stomach have been destroyed, or are incapable of performing their function, which is most frequently due to a diffuse inflammation of the mucous membrane of the stomach, less often to a gastritis or to a carcinomatous infiltration.

You see that, after washing out our last patient, a quantity of sediment is still stirred up, even after the discharge by suction is completed. I think we can rinse for a long time before we entirely cleanse the stomach. Note that with the withdrawal the wash is distinctly blood-colored, a sure token that the sediment has been screening an ulcerated portion of the mucous membrane.

While we inflate the stomach with air-very carefully here where we have to consider the possibility of an ulcer on the point of perforation-you see the region beneath the umbilicus arch itself, and by scratching auscultation we find the lower boundary of the stomach quite as low down as in the case of our last patient. But the difference is this: that while with her the upper boundary-the lesser curvature-was also found depressed, almost down to the umbilicus, here the stomach resonance is continuous over the entire epigastrium. I beg you to pay particular attention to this important difference, which here shows us that there is no gastroptosis as with the former patient, but a dilated 
stomach: here only the greater curvature is lower. I emphasize this because one often sees the error committed of diagnosing ptosis from the altered position of the greater curvature only.

If, by examining the stomach, you find nothing which can explain the dyspeptic attacks, emaciation, etc., then never forget to explore the rectum and examine the feces. Last year I was consulted by a patient who for three years had suffered from cardialgia, eructations, vomiting, constipation, and occasional diarrhœa. Two and a half years ago he came over from Jutland to consult a specialist in diseases of the stomach, who proceeded to make functional tests of his stomach. The patient was comforted by the information that nothing was amiss; he was placed on a diet. He returned every fourth month for examination, and went home each time with the consolation that the "function of his stomach" continued satisfactory. But, as he became more and more emaciated and felt extremely weak, he decided to seek further advice, and consulted me. When, finally, I inserted my finger in the rectum, I at once felt a firmly-rooted, circular, and inoperable cancer recti, which had caused the stomach symptoms. Had the specialist only thought of the rectum, the tumor would have been discovered and might possibly have been removed radically. With obscure affections of the stomach the examination must be conducted with special reference to the possible presence of blood, as the admixture of blood to the feces may often guide us to the diagnosis ulcus ventriculi or ulcus duodeni in an otherwise obscure disease.

Even with a thorough application of all these methods of examination, there yet remain a number of cases where the diagnosis is quite indefinable. In order, if possible, to arrive at a diagnosis in such cases without operating, one has worked for many years to develop a method which will bring the affected part within range of one's vision. The oldest of these methods is the so-called diaphanoscopy: 
the illumination of the stomach and abdominal wall by means of a glow-lamp introduced into the stomach through the osophagus. When the patient is placed in the dark, the illuminated stomach is seen shining through the abdominal wall, and any large tumor or area of infiltration should be revealed as a shadow against the light. This method, the idea of which originated with the French physician, Milland, in 1867, as a result of his experiments on dead bodies, was first attempted by Einhorn in 1889, and was employed in his clinic, but has never had any great significance, for reasons which are easily understood. Firstly, it shows only those gross abnormalities which can easily be diagnosed in other ways; and, secondly, only the very small part of the stomach which is not covered by the liver and chest-wall can be seen. Our hopes centred on a gastroscope to be constructed on the same lines as the cystoscope for introduction throngh the œsophagus. Since 1881 Mikulicz and his pupils have worked energetically to solve this problem, and have indeed succeeded in constructing instruments whereby one can observe through a long tube a portion of the mucous membrane illuminated by an electric lamp, but they have never succeeded in making these really practical. Partly because it is very difficult to insert this large instrument, for much practice on the part of the physician and gradual habituation on the part of the patient are required, notwithstanding the use of very strong solutions of cocaine; partly because the field of vision is too small, and a survey in all directions is impossible. Where the diagnosis is uncertain and medical treatment ineffective, we must resort to exploratory laparotomy. As a rule, the inspection and palpation of the exposed stomach give us at once the diagnosis, so far as this is needful to define our therapentic encroachment; but it cannot be denied that a relatively large number of cases remain where this examination leaves us just as much in doubt as we were before: cases where we do not know whether an ulcer is 
present or not; others where we know that there mușt be an ulcer, because of symptoms of hæmatemesis and melæna, though we can neither feel nor see it; and others, again, where irregularities of the serosa or adhesions cause us to suspect the presence of an ulcer, though this might be due to other causes; and cases in which we find an infiltration of the stomach-wall, but do not know whether this is a new growth or an inflammatory infiltration. You understand how important, how absolutely necessary, indeed, it is to have all possible information, in order to avoid a fatal mistake in the treatment and in the choice of operation: whether a simple procedure like gastropexy is sufficient, or whether a gastro-enterostomy, or possibly a resection of the stomach, is called for. A mistake in the choice of operative methods, founded on a doubtful diagnosis, is responsible for the bad results of many operations.

In order to avoid these mistakes, I have devised a method of examination, which is most helpful in many cases and which is so simple and easy in its application that every surgeon can easily avail himself of it. I have called this method direct duodeno-gastroscopy and diaphanoscopy, a descriptive title. Here you see my gastroscope, an instrument constructed like Nitze's cystoscope, though much larger, as it corresponds with Charrière's Nos. 39 and 40, while the cystoscope corresponds with No. 21. Thus we obtain a far better illumination, and, what is of more importance, an enlarged field of vision. Along the front of the tube runs a flat canal, which terminates at the fenestra with a transverse fissure, and opens upward, and is connected with an insufflator by a rubber tube (Fig. 67). The entire apparatus is sterilized in formalin vapor and held in readiness at the operation. If the diagnosis is doubtful, 1 make a small incision midway in the stomach, two centimetres above the greater curvature, and just large enough to permit the instrument to pass. In this way it is not necessary to make a tobacco-pouch suture to close the 
stomach-wall tightly. As soon as the instrument is inserted, an assistant begins to inflate the stomach with air-by compressing the inflator-until all creases are effaced. The lamp is lighted and the large, sterilized blind lowered, so that the room is in darkness. One sees the stomach shining like a large electric lamp, and one sees all anatomical details revealed in its wall with wonderful accuracy.

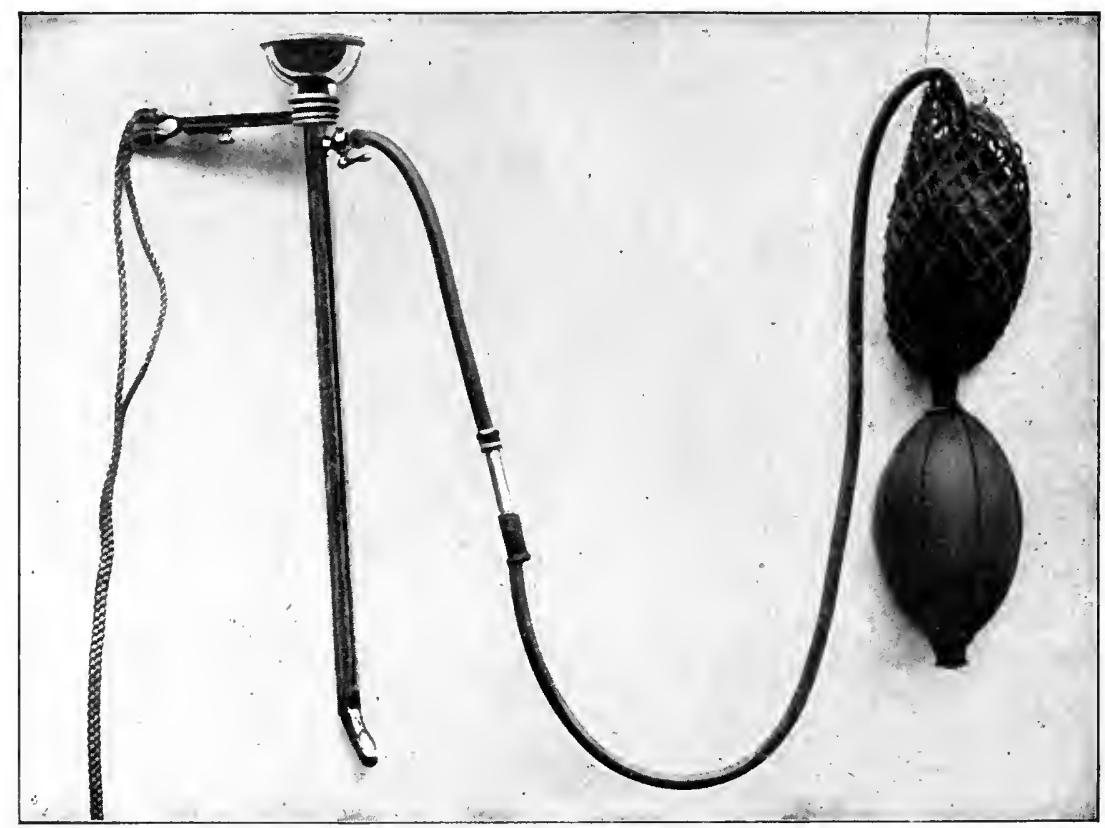

Fig. 67.-Author's gastroscope.

With a normal stomach one sees the larger vessels of the greater and lesser curvatures running toward each other with continuously dichotomous ramifications in the clear, somewhat rose-colored stomach-wall, but one does not see them meet. The course of the muscle-fibres in the various layers is seen with the greatest distinctness, while the crypts are seen as small, whitish dots. A disease which is at once revealed by diaphanoscopy is gastritis in its various stages (Plate III, Figs. 1, 2, and 3); because the stomach-wall is then diffusely red, from strong rose to deep bluish-red; and, while with the normal stomach you only see the 

Figl

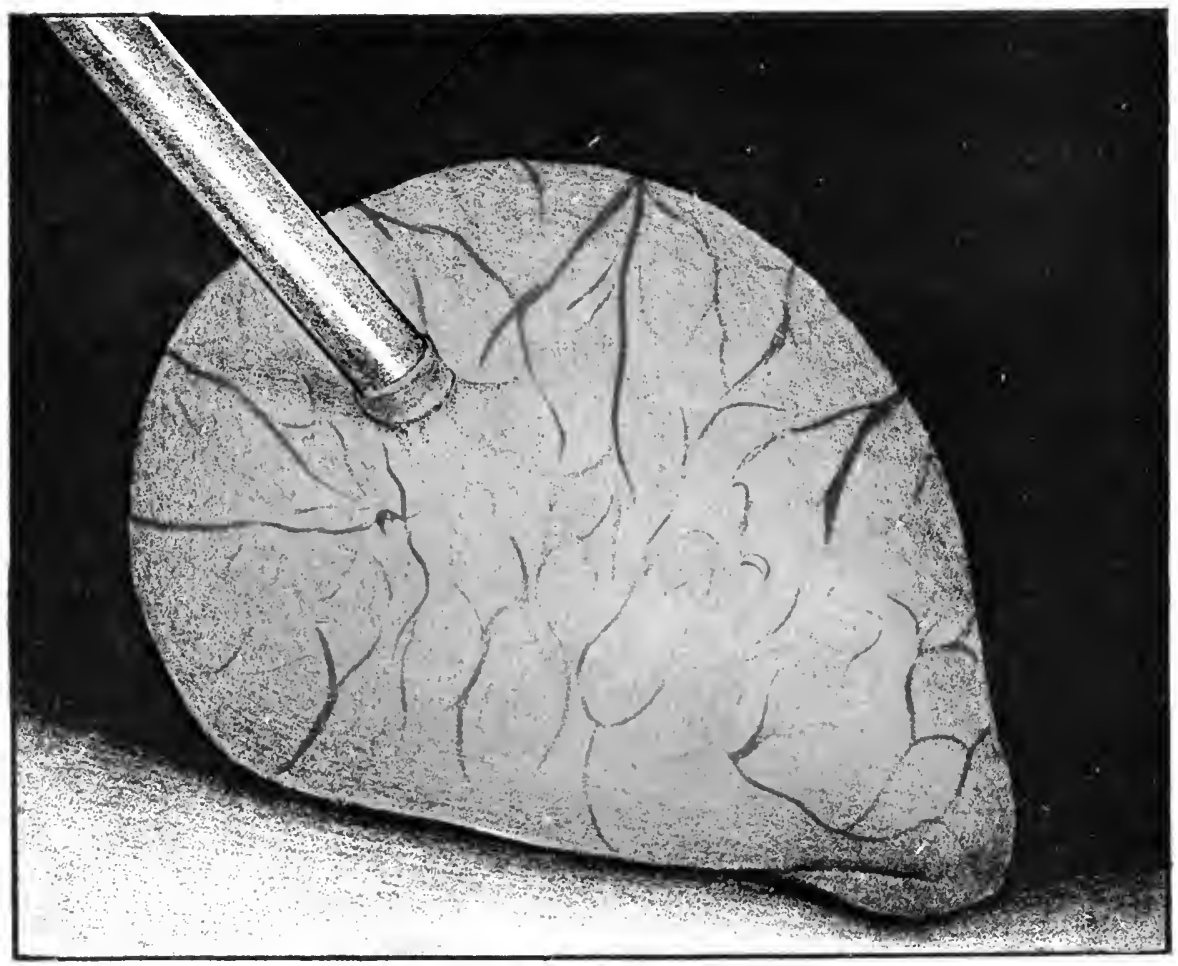

Fig. 3

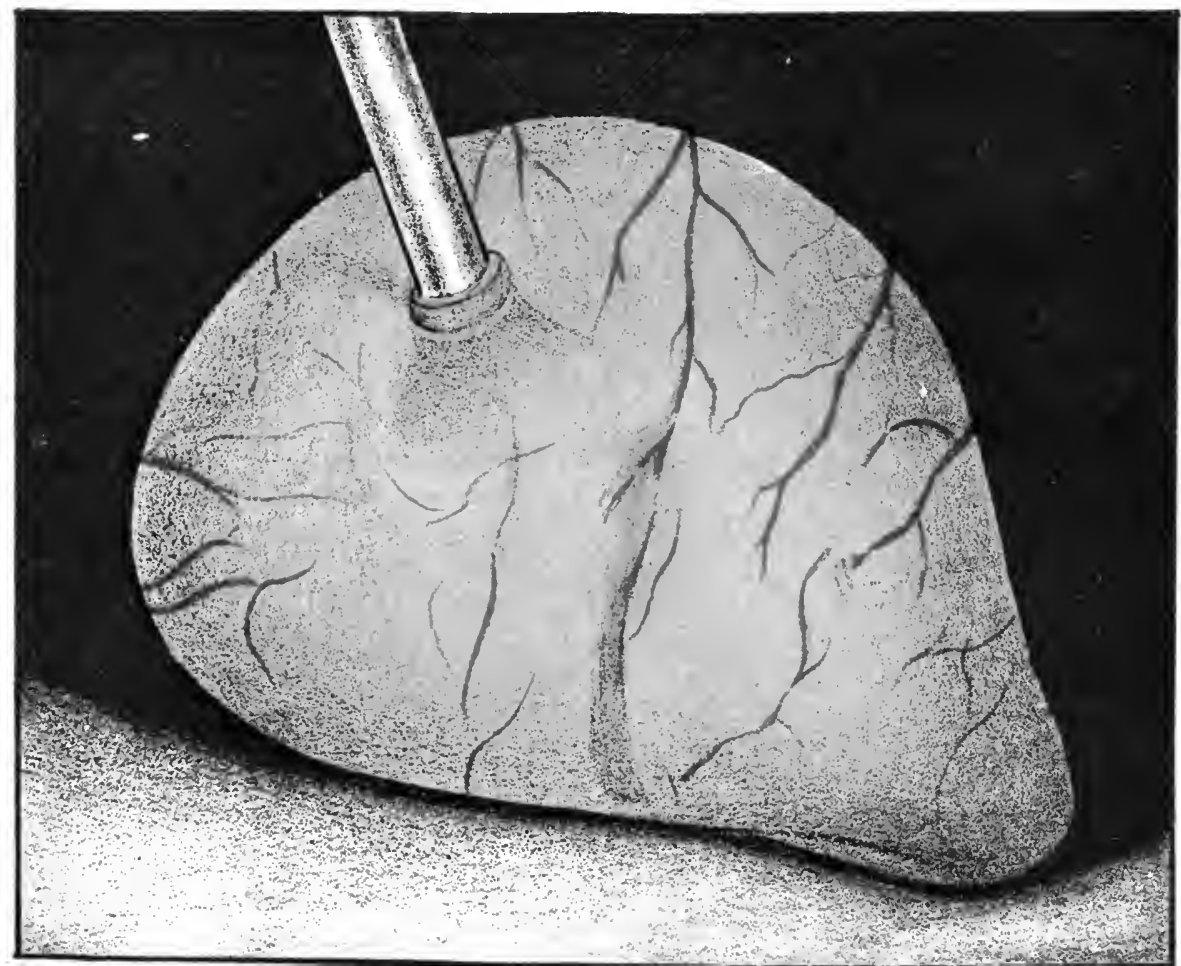






Fia. 4

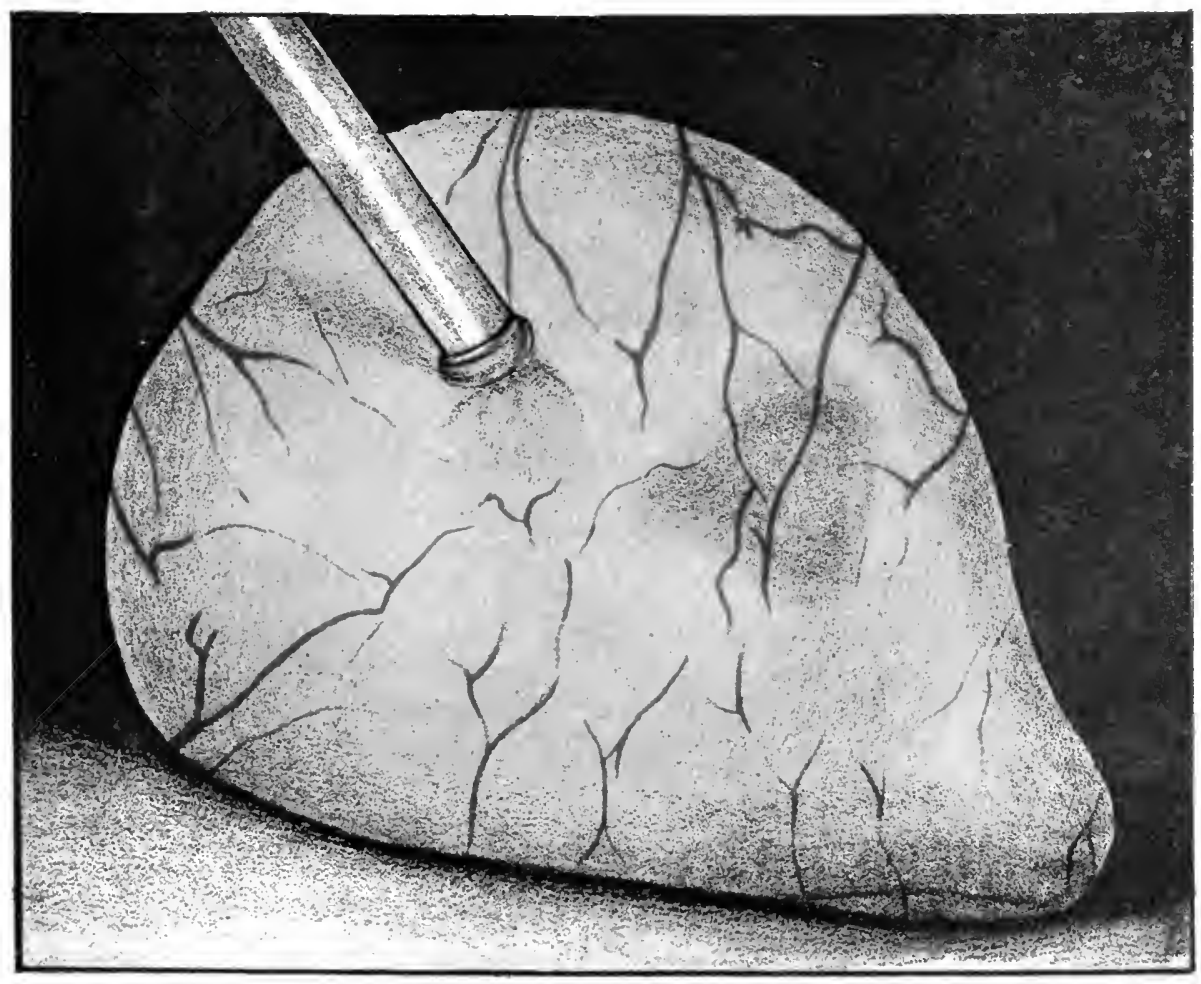



larger vessels terminating near the centre, here we see them connected by a more or less profuse plexus.

When there is no ulceration or gastritis, tumors are seen as dark, diffuse shadows on the otherwise clear stomachwall. This is well illustrated in Plate III, Fig. 4, which shows a tumor which had not betrayed itself clinically by any other symptom than achylia. At the operation, which was undertaken for a small hernia linea alba, a flat infiltration of the anterior wall of the stomach was discovered, the nature of which could not be decided until diaphanoscopy and gastroscopy proved it to be a flat, non-ulcerating tumor. Immediate resection of the stomach was performed and the patient recovered. The tumor was an adenocarcinoma.

In strong contrast to this is the picture of the deep, chronic ulcer, which, as Plate III, Fig. 2, shows, is distinguished by its cicatrix with its decayed centre, its cicatrized part showing itself as a porcelain-like, whitish spot with a paucity of vessels, contrasting sharply with the surrounding red, hyperæmic part. If, on the other hand, small superficial ulcers of the mucous membrane are concerned, these are not revealed by diaphanoscopy, but are only discovered when viewing the mucous membrane through the gastroscope. Only when they are hemorrhagic, bleeding, or covered with coagula does one see them depicted as dark spots on the wall, and, when such a sore bleeds, one sees the stream of blood as a dark stripe pursue its way toward the greater curvature, as in the colored Plate III, Fig. 3.

In order to inspect the posterior surface of the stomach thoroughly, the omentum and colon transversum must be raised. When the stomach has been examined, the gastroscope is passed through the pylorus into the duodenum. Should there be the slightest difficulty in passing the gastroscope through the pylorus, one knows that it is contracted. The wall of the duodenum, which is far thinner than that of the stomach, can be illuminated more strongly and more satisfactorily, and a tumor or ulcer can be easily discerned. 
You see what a surprising amount of information we can obtain from this illumination of the stomach. Often we obtain the complete diagnosis: gastritis, ulcer, or tumor. In most cases the illumination also helps the subsequent gastroscopy, because one immediately turns one's attention to that feature which the illumination shows to be abnormal. And, if one is then in doubt as to whether a shadow is due to an ulcer, a tumor, or an extravasation of blood, a direct observation through the gastroscope will provide a complete elucidation. The view obtained through the gastroscope is far better, sharper, and more certain than that presented to the bare eye by an examination of the opened stomach, not only because the view is considerably enlarged and the illumination greatly intensified, but also because, when the stomach is inflated, all the folds, between which a small abnormality is so easily concealed, are smoothed out and made freely accessible to the investigator. With this gastroscope, therefore, one can make a systematic inspection of the entire mucous membrane of the stomach and of the first portion of the duodenum in the course of a few moments. The normal mucous membrane of the stomach shows itself in the gastroscope as a smooth, rose-colored surface, which merges at the cardia and pylorus into radially placed folds, which diverge, funnel-shaped, toward the circular opening. Any abnormality in these two openings is shown in a very characteristic manner. If the pylorus has an annular infiltration or contraction, one sees a stiff, yawning circle, through which one often obtains a glimpse of the duodenum; if only half of the pyloric circle is infiltrated, this becomes crescent-shaped, because the soft mucous membrane on the sound part caves in to the rigid half. Ulcer formations at the pylorus itself are easily detected, but the folds of the mucous membrane around the pylorus require more exact attention. In those cases where the cardia was abnormal the gastroscope showed an extraordinarily satisfactory and distinct view of the tap- 
shaped, prominent cancer of the cardia and of its extensions to the neighboring portions of the mucous membrane of the stomach.

With cases of ulcer and cancer, gastroscopy is of value when the main features of the diagnosis are assured, because it defines precisely the limits of the affected mucous membrane. It is of even more importance because it so often clears up doubtful points which the diaphanoscopy has left unsolved. In this way the gastroscope picture has in many instances shown rugged, though new growths in the mucous membrane, which left no doubt as to the malignant nature of the infiltration, when we imagined that a simple ulcer was present. What seems, however, to be of the greatest importance is that in a series of cases gastroscopy has proved the presence of ulcers which could not have been diagnosed in any other way. This is particularly applicable to the duodenal ulcers and to the small, bleeding ulcers in the mucous membrane of the stomach.

This method, which is still in a developmental stage and which undoubtedly will be improved upon when better lamps and better optical apparatus are invented, has already stood its test in a long series of cases, and has proved itself a very rich addition to our diagnostic expedients for diagnosing diseases of the stomach and duodenum.

My gastroscopic method attains a special value with the treatment of impermeable contractions of the œsophagus by retrograde dilatation, because one can now insert a thin œsophageal bougie backward through the cardia with the greatest ease and under the control of the eye. For this purpose a special gastroscope is employed (illustrated on page 151, Fig. 47), provided with a canal in which the bougie glides. When the point of the bougie reaches the mouthcavity, one attaches a long silk thread to it, by means of which other bougies of continually-increasing dimension are led through. 


\section{LECTURE $X$}

\section{GASTROPTOSIS}

I wILL speak to you to-day about a common disease of the stomach, the clinical importance of which was quite unappreciated until a few years ago, and even now-especially abroad-is greatly underestimated. It is the so-called gastroptosis, by which is understood an abnormal laxity and weakness in the fixation of the stomach to the diaphragm, and therewith, an abnormal mobility of the stomach, so that this, when filled, and the patient being in an erect position even when empty, sinks by its own weight more or less low down in the abdomen. As the stomach generally rises again to its normal position when the patient assumes a horizontal position, the term ventriculus mobilis would perhaps be even more appropriate than gastroptosis. This expression, however, is so generally used as an outgrowth of Glénard's enteroptosis that it cannot be easily changed, and it is suitable enough if you only know the limits within which it is here employed. Strictly speaking, the word enteroptosis mer'ely means prolapse of the intestines, but Glénard comprises under this title a prolapse of one and all of the organs attached to the dome and walls of the abdominal cavity: the liver, spleen, and kidney, as well as the stomach and intestine. Glénard considered enteroptosis to be a disease due to lack of nutrition, the result of a somewhat enigmatical diathêse hépatique, the first result of which ought to be a prolapse and shrinkage of the intestine and a diminution of the intra-abdominal pressure; and, secondarily, gastroptosis, nephroptosis, hepatoptosis, and splenoptosis should follow. He considers even the relaxed abdominal wall as secondary to the enteroptosis. You will easily understand that, if this theory were correct, enteroptosis 
would be quite without surgical interest. That the theory, however, is incorrect is already evident from the fact which has been substantiated many times, both on the dissecting and operating tables, that a gastroptosis may exist without a nephro- or hepatoptosis being present, while, conversely, we can have an isolated nephroptosis. In the next place, clinical observation has taught us that the disease of the nutritive organs, which, truly enough, is the predominant feature with advanced cases, is, as a rule, secondary, and therefore, a result of the subsidence of the organs, and not, as Glénard asserts, the cause; because, if one succeeds in refixing the organs in their normal positions, the patients quickly recover their normal condition.

Another theory, advocated by Stiller, is that the enteroptosis is the result of a congenital, often hereditary, disposition: a general asthenia. Yes, several authors actually regard the disease simply as a degenerative phenomenon. Just as certain as it now is that this theory is quite inadmissible as regards the great majority of cases of ptosis which occur with previously quite healthy women, when their abdominal walls become relaxed and pendent as the result of repeated pregnancies, so certain it is that, with the group of enteroptosis patients who have not produced offspring and with whom the disease develops shortly after the age of puberty, such a typical abnormality of the bodily structure is found, that one is certainly justified in talking of a congenital predisposition to the disease. You all know this type of woman: thin, atrophied, gracile bodies with long, thin, "rickety" waists, extremely mobile in comparison with the relatively broad pelvis. But this theory explains as little as the previous one the origin of the enteroptosis, and, at the outside, presents only momenta toward its solution. We only arrive at this by mechanical theories: the theory of the corset and of the derangement of the intra-abdominal pressure. 
It is characteristic of enteroptosis that it is a distinctly feminine complaint. Therefore, the cause was first sought in the conditions which are peculiar to women. When one considers how the misuse of corsets and tight lacing. give rise to not only lasting changes in the shape of the chestboth of the skin and the flesh-but also to liver and stomach ulcers, it is natural to think of it as a possible cause for prolapse of the sub-diaphragmatic organs. Dr. Hertz, in his excellent thesis for his doctoral degree treating of the change in position of the abdominal organs, points out ingeniously and clearly how the liver, owing to the pressure of the corset, acts as an interposing factor in dislocation of the kidney. This statement seems confirmed by the overwhelming frequency of the right-sided nephroptosis, which forms 85 per cent. of all cases of nephroptosis. But that corset pressure and tight lacing play a part also in the origin of other and more rare instances of ptosis, gastroptosis, and hepatoptosis there can scarcely be any reason to doubt. Yes, we must search for the explanation of this virginal enteroptosis occurring in quite young females mainly in this pressure, often favored, perhaps, by congenital predisposition, the delicate, slender body-structure offering no resistance worth mentioning to the pressure.

In the majority of cases, those in which the enteroptosis develops later in life, with women whose abdominal walls are relaxed owing to pregnancy and childbirth, the pathogeny is best explained as the result of a diminished, inner, abdominal pressure: a theory which is promulgated in its most attractive form by the Russian physicians Wolkow and Delitzin. These men consider that the subdiaphragmatic organs owe their position not so much to the weakened ligaments by which they are attached to the diaphragm and neighboring organs, but far more to the support afforded by the inflated intestines. They pertinently describe the kidneys as being supported by a sort of bandage, con- 
PLATE IV

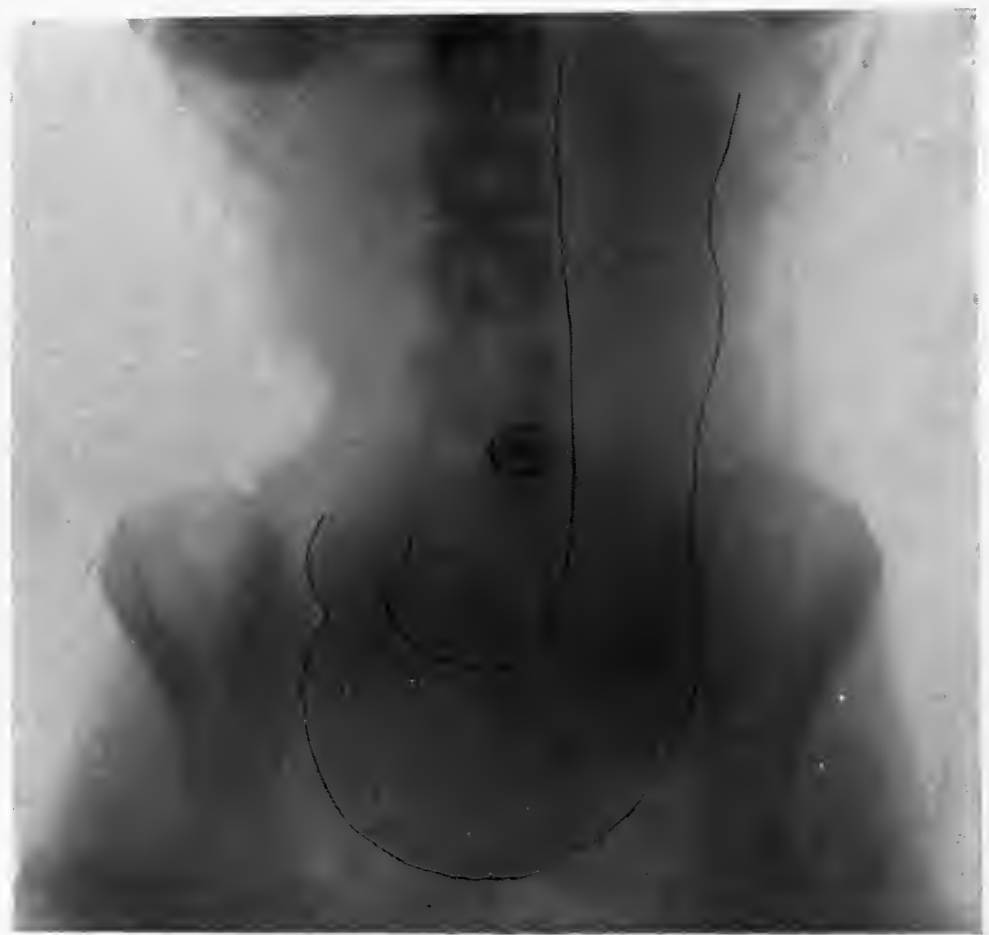

Radiogram of a typical, marked prolapsus of the stomach with extraordinary elongation of the cardiac end of the fundus. 

sisting of an inflated pelotte-the intestines-and an elastic girth-the abdominal wall. When this loses its strength and elasticity, as happens with women after frequent childbirth, and with patients who have undergone an illness involving considerable dilatation of the abdomen, with the subsequent collapse, the liver, stomach, and kidney are deprived of their support and drop down.

Just as there have been much confusion and difference of opinion regarding the pathogeny and nature of enteroptosis, of which you can read in extenso in Blad's clever doctoral treatise, so, also, the most divergent views have been held as to its symptomatology, diagnosis, and treatment. What has specially caused confusion is the fact that many patients with ptosis, apart from a slight constipation, enjoy good health, and often do not seek medical assistance. From this one is often led to believe that the serious affections which other enteroptosis patients present cannot be due to enteroptosis, as such. Another reason is that the morbid attacks caused by enteroptosis, and especially by gastroptosis, may resemble the symptoms caused by other diseases, especially ulcer of the stomach and colitis. But when, by a more careful study, one is able to recognize the characteristic and typical symptoms of ptosis, the differential diagnosis is, as a rule, not difficult to define, if only one is on the lookout for a gastroptosis as the cause of such symptoms. Since I, in 1897, had my eyes opened by just such a misconceived case-which I succeeded in curing by an operation-to the great importance of disassociating these attacks from other gastric and intestinal diseases, I have endeavored to the best of my ability to develop the differential diagnosis and make clear the clinical aspects. At first my efforts in this direction met with the greatest distrust on the part of the medical men, many of whom insisted that they had never known gastroptosis to cause symptoms worth mentioning. But I am glad to be able to 
say that here in Denmark, even if a divergence of opinion still exists, the medical men have, in the main, gradually admitted that my contention that gastroptosis frequently causes morbid symptoms which are mistaken for other diseases in the stomach and intestine is correct, and that many of these cases require surgical treatment. Abroad, however, you will still find but slight indications that gastroptosis is regarded as a serious disease.

As I have already intimated, I distinguish between the two types of gastroptosis: the virginal-maidens who have never borne children-and the maternal-mothers who have frequently borne children-and I attach great weight to this classification, because these two types differ so much, both as regards the pathogeny and as regards the symptoms, diagnosis, and treatment.

I shall now further elucidate this by presenting two patients representing the two groups of gastroptosis.

We will first consider this young unmarried woman, a book-binder, twenty-seven years of age, whose history is as follows: As a child she was always slender, but otherwise healthy, her trouble beginning at the age of puberty with the commencement of menstruation, which first appeared at the age of sixteen. From the very commencement the menstruation was accompanied by great suffering, which was not confined to the small pelvis, the pains radiating from the pelvis to the flanks. These molimena have recurred with each menstruation, while the discharge of blood has never been particularly severe or protracted. Shortly after the menstruation commenced she began to suffer from cardialgia, nausea, frequent vomiting, and constipation, which in the course of a few years became extremely persistent. She became emaciated, languid, and listless, and at the age of twenty-two she was quite unable to work, and was, therefore, admitted to the medical ward. Here it was at first thought that the symptoms originated 
in an ulcer of the stomach; she was therefore put on an ulcer diet-milk and milk food. As, however, this diet did not have the desired effect, and as the very careful investigation did not show any positive symptoms of an ulcer, or, on the whole, of any organic gastric disease-no retention, no hyperacidity, no blood either in the feces or in the romit-the diagnosis gastric ulcer was abandoned in favor of the diagnosis intestinal dyspepsia, which is, in Faber's opinion, colitis with dyspepsia. Therefore she was compelled to undergo the treatment specified by Faber for this complaint, viz., milk was eliminated and the socalled anti-constipation diet ordered: purée, stewed fruit, graham bread, etc. After being treated in this manner for four months, the attacks disappeared and she was discharged. But, shortly afterwards, the symptoms recommenced, and six months later she was again admitted to the medical ward, with exactly the same symptoms as before. On this occasion, again, no indication of an ulcer could be proved. For three months she was again confined to bed and ordered an anti-constipation diet. Once more the patient improved, but, whenever allowed to get up, relapsed. It is just this circumstance which I have emphasized as being quite typical of those cases of dyspepsia and constipation which are due to ptosis: that the patients are apparently cured by a suitable diet while confined to bed, because the organs then resume their normal position; whereas they relapse as soon as they get up, because the organs then prolapse. This attracted the attention of the physicians, who investigated the local condition of the stomach by distending it with air and by subsequent scraping ausculation. It then appeared that curvatura minor ventriculi lay four fingers' width below the umbilicus, while curvatura major lay four fingers' width above the symphysis: a very considerable gastroptosis, therefore. She was then supplied with one of Vermehren's abdominal 
belts, which I shall shortly show you. This belt immediately produced good results, and for six months she enjoyed good health. Then the morbid symptoms began gradually to develop again, and for the third time she was admitted to the medical ward, in an even worse condition than before, complaining also of a sinking sensation and general pains, and giving one the impression that she was extremely nervous. The diagnosis nervosismus was added. So it has continued, backward and forward, during the past five years, eighteen months of which she has spent, all in all, confined to bed in the medical ward. Of late some have considered her complaint to be a purely nervous one, but, as the therapeutic treatment applied for this has not improved her condition, she has been removed from the medical polyclinic of this hospital to this ward with the diagnosis gastroptosis, with possible operation in view.

We will now observe the patient, who, on the whole, offers a somewhatcharacteristic picture of a virginal ptosis patient. We notice that she is pale, with a nervous, tired, and rather pained expression. She is a little over medium height, slenderly built and rather thin, the lower extremities being especially thin, with relaxed, somewhat pendent muscles. Even more characteristic is the view presented by the chest and abdomen. You find the meagre chest frame unusually long, and you see that this is essentially due to the condition of the lower aperture, because the rib curvatures, instead of at once receding from each other and the processus ensiformis in a broad, downward, convex curve, descend sharply, receding quite gradually from the central line, and in such a manner that the epigastrium, instead of retaining its normal shape as an inverted $V$, assumes the shape of an inverted $\mathrm{Y}$, the tail of which forms the long, narrow fissure between the rib curvatures. At the top of this long fissure one feels the point of processus ensiformis, and it seems as though the abnormal length of the chest 
frame has been brought about at the expense of its breadth by the compression of the rib curvatures. Perhaps this is really a permanent result of the strait-waistcoat-like effect of the corset on the delicate, soft chest-wall during the years of puberty. You will notice that this narrow, long waist contrasts in a singular manner with the broad pelvis. If we now ob. serve the abdomen in its bony frame (Fig. 68), we notice that the portion above the umbilicus regi o epigastrica sags concavely. One perceives a distinct pulsation in the epigastric region. When I place my hand on the epigastrium, I feel the aorta $\mathrm{puls}$ ating quite close to my fingers; you can see, in fact, how my fingers rise and fall simul. taneously with the puls e-wave. You

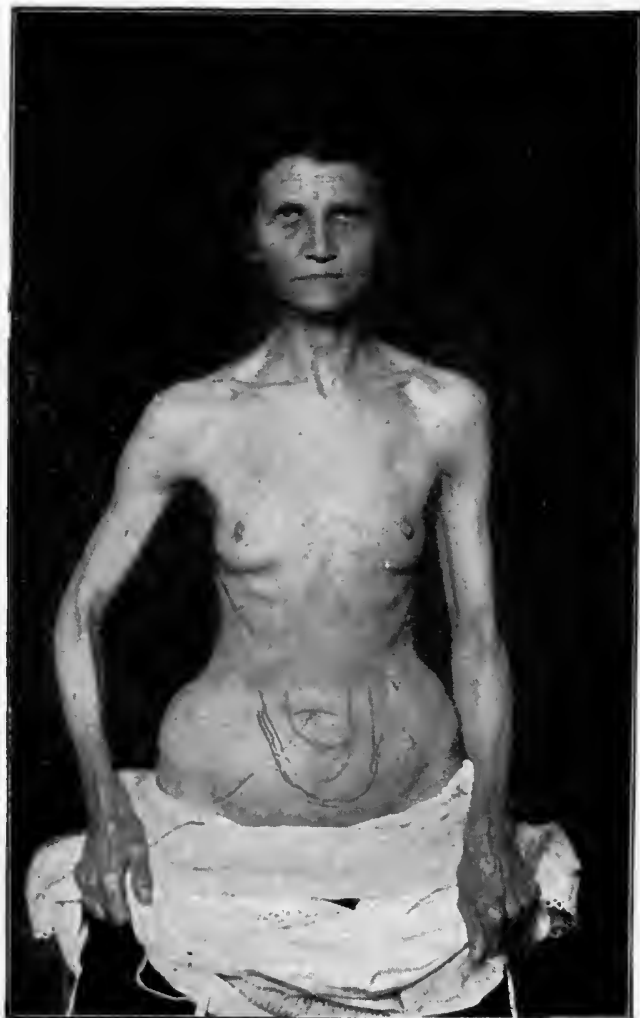

Fsa. 68.-Virginal ptosis, tense abdominal wall. Position of stomach depicted in accordance with scraping auscultation.

will never find this with individuals whose stomachs occupy their normal position. It is merely due to the fact that the stomach, which usually covers the vertebral column like an air or water cushion, has glided down from its position into the abdomen. It is, therefore, a pathognomonic symptom of gastroptosis. 
In contrast to this and to the generally meagre condition of the patient is the plumpness of the remaining portion of the abdomen situated below the umbilicus. As a matter of fact, the impression of good condition which this part presents is very deceptive, and is only due to the subsidence of the stomach and colon transversum, which, normally, should not be here at all. That this is really the

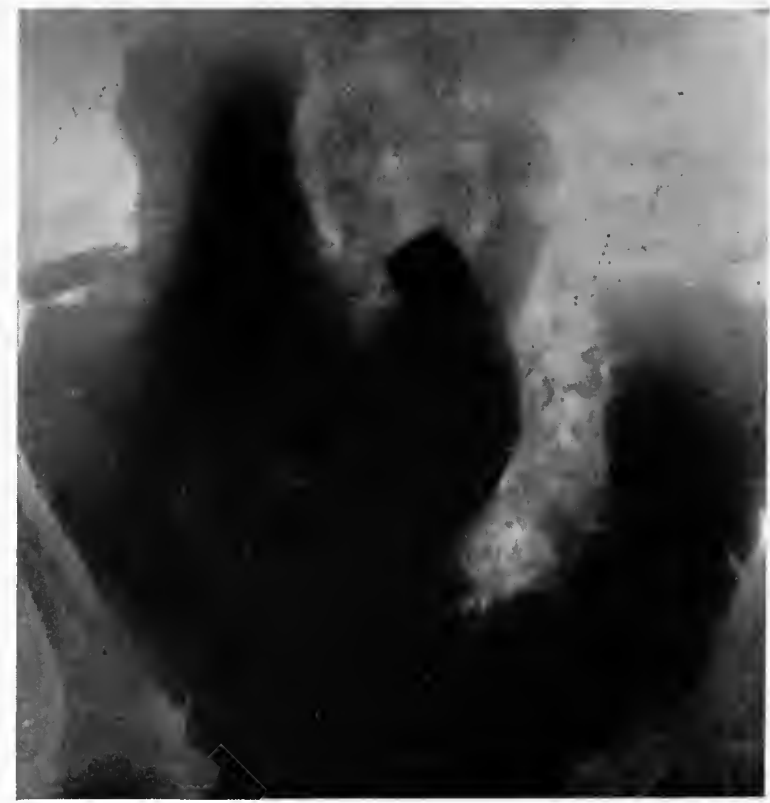

FIg. 69.-Gastro-coloptosis. Radiograph showing the greatly prolapsed stomach and transverse colon.

case, we have substantiated by scraping auscultation, which shows us that the lesser curvature of the stomach lies four centimetres below the umbilicus, and nineteen centimetres below processus ensiformis, behind which it usually lies. The greater curvature is found four centimetres above the symphysis. Furthermore, we have obtained a radiograph of the stomach (Fig. 69) immediately after a meal of bismuth-porridge, and, twenty-four hours later, one of the colon. The plates show you that the 
stomach lies just where the scraping auscultation indicated; though, of course, you have a clearer perception of its. form. You see how the stomach, like a long, narrow sausage, runs precipitously down the left side, and low down turns upward in a concave curve, and then, rising sharply to the right, passes into the duodenum at a somewhat sharp angle-the "fish-hook" shape. It is as though the cardiac area were drawn out to three times its length, and directly you see this picture you understand, also, that it is an invariable symptom with those patients that they always feel pains dragging and gravitating in the left side of the epigastrium after meals and after standing or walking for any length of time. It can be easily understood that the strain on the peritoneal attachment to the diaphragm must cause pain, which is in accordance with what Bloch's and Lenander's investigations regarding the manifestation of pain in the abdominal organs have taught us. It is just as interesting, perhaps, to see the picture of the colon. Instead of the horseshoe-shaped figure where colon transversum crosses the epigastrium transversely from the hepatic flexure and makes its way across obliquely under the free border of the ribs in the left side to the splenic flexure, you see colon transversion like a festoon suspended at the flexures, the lowest, convex part of which lies close above the symphysis, encircling the greater curvature of the stomach at a distance conformable with the normal length of ligamentum gastrocolicum. When you see the pointed angle under which colon ascendens passes into colon transversum, and this again into.colon descendens, you will at once understand the second, invariable symptom of ptosis: constipation.

If, now, we finally add that with this patient the abdominal wall, far from being soft and relaxed, is, rather, somewhat tight and tense, then we have in this instance a typical example of virginal gastroptosis, without prolapse 
of the other abdominal organs, because we do not feel any prolapse of the kidney, liver, or spleen.

Quite typical, also, is the history of the patient's disease as regards the diagnostic difficulties which even this uncomplicated case presents, and it shows us in typical succession the diagnostic errors which gastroptosis may so easily give rise to. Firstly, on account of cardialgia and constipation, the diagnosis ulcer of the stomach is given, and the patient is ordered a strict milk diet. The excellent clinician who treated this case quickly realized that this diagnosis was wrong, because the cardialgia remained quite unaffected by the diet, and a thorough examination of the function of the stomach revealed no sign of an ulcer.

The next diagnosis was intestinal dyspepsia: constipation with colitis as the primary cause of the dyspepsia. As the regular therapeutic treatment, consisting of aperients and anti-constipation diet, only gave slow and temporary relief, and as the patient relapsed as soon as she left the hospital, to return time after time more debilitated and more nervous and more psychically changed than before, her complaint was diagnosed as nervosismus: nervous disorder of the digestion.

Virtually all the simple, virginal cases of gastroptosis which I have had under treatment have been classified under these three diagnoses, and it is my conviction that a great number of female patients who really suffer from ptosis are finally given up by the physicians and allowed to sail for the rest of their lives under the dismal flag of hysteria or nervousness.

One cannot, however, reproach the physicians for this, because many of the best known interne pathologists maintain with great decision the actuality of the conception "nervous affection of the stomach": a very convenient term, inasmuch as they include under this head all the gastric affections which they cannot cure and the cause 
of which they can neither define nor understand, certainly a conception which greatly needs to be critically revised. And it is my opinion that, when the day for revision arrives, ptosis will include nearly all the nervous affections of the stomach.

When such an influential authority as Strümpell can with general approbation fling forth the statement: "It is not the stomach affections which cause the nervousness with these patients, but the nervousness which causes the stomach affections," then we are face to face with an extensive and dangerous superstition, which cannot be too sharply contested. This much I venture to tell you: that if you find ptosis with patients suffering from constipation and gastric symptoms for which you can assign no reason, and whom you have treated in vain with the prevalent methods, you are not justified in relegating these patients to the retiring room reserved for hysteric or nervous affections of the stomach before you have attempted a judicious treatment of the ptosis.

The other patient whom I wish to show you to-day is a peasant woman, forty years of age, whose history, in a few words, is as follows: In her childhood and youth she was healthy and strong; she was married at the age of twentytwo years, and subsequently gave birth to eight healthy children. She recovered from her confinements easily and naturally, and only after the seventh confinement, five years ago, did she commence to suffer from indigestion and constipation, varied at times by diarrhœa and a sensation of oppression in the abdomen: as if the bowels were dropping down. Of late she has had pains in the epigastrium immediately after partaking of food, and once or twice has vomited. When we question her as to the relations of the pains to the various hours of the day, she tells us that, as a rule, the pains do not begin until a couple of hours after she has gotten up in the morning and begun her work, but 
increase steadily with standing or sedentary work. On the other hand, she tells us definitely that all pains and sensations cease so soon as she lies down. This information is of vital importance, and informs us almost certainly that all her sensations are due to ptosis. I cannot sufficiently impress upon you the importance of always questioning patients with chronic abdominal complaints whether these disappear with confinement to bed. With the majority of ptosis cases you will at once get onto the right track by means of this simple question. It is, nevertheless, most important to remember that only when lying on their backs the great majority of ptosis patients are relieved of pain. When lying on their sides, a great number complain, on the contrary, of pains and disagreeable sensations, generally in the side opposite to that on which they are resting. This is particularly the case with patients who suffer also from floating kidneys; but even patients with gastroptosis pure and simple often complain of pains when lying on their sides. Finally, it must be remembered that there are a number of atypical cases where the symptoms are quite lacking, and where the patients feel the same pains, whether lying down or standing. But it would take us too far afield to enter upon such complications to-day, especially as we have two really typical cases before us.

If, now, by way of comparison with our first patient, we make an objective examination of this peasant woman, you at once see how greatly her appearance and build differ from what we found with our virginal ptosis patient. Here we have a strongly-built, fairly well nourished, and healthylooking woman, and, in contrast with our first patient, a thorax of the normal type, inasmuch as the rib curvatures recede normally one from the other. And, whereas with the former patient we found the skin smooth and the abdominal wall everywhere compact and tightly fitting, here we find the skin so greatly relaxed that it can be gathered together 
PLATE V

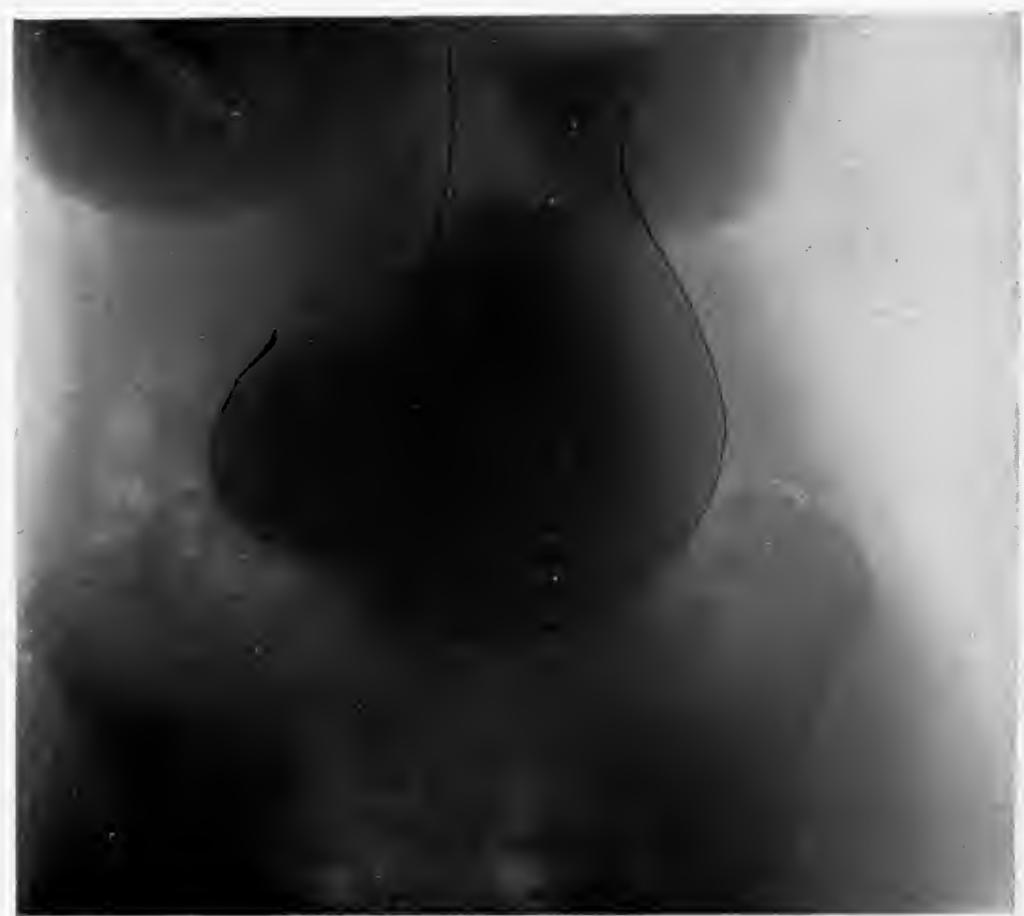

Radiogram of a dilated, markedly atonic and prolapsed stomach. 

in large folds, which gives one the idea that there is a great deal more skin than is necessary. But the fibromuscular portion of the abdominal wall is also puckered and relaxed, attenuated by the distention of many pregnancies. In the centre line we feel a gaping, broad furrow between the two musculi recti. Here, by invaginating the thin abdominal wall, I can pass my hand between the musculi recti into the abdomen, and palpate the vertebral column, etc. When the patient lies down, the abdomen expands, so to speak, on every side; becoming slightly concave at the centre and convex at the sides. In a recumbent position the epigastrium appears normally flat or slightly curved, without apparent pulsation; but so soon as we permit the patient to stand on her feet the whole aspect is completely altered. The entire abdomen now drops down like a half-filled sack, while the epigastrium becomes empty and concave; and we now see and feel the aorta pulsating here. The flanks also are now relatively sunken, because the prolapsing bowels now collect at the bottom and forefront of the pendulous pouch here formed by the abdominal wall.

As with the former patient, so here, scraping auscultation and radiography reveal the stomach with the colon transversum greatly prolapsed, so that the lesser curvature is five centimetres below the umbilicus. With this patient, also, an examination of the functions of the stomach has shown quite normal conditions. Here we find that both kidneys have also prolapsed, and we have, on the whole, a general impression of a more important and more extensive prolapsus than was the case with our former patient. How comes it, then, that the peasant woman's sufferings are of little account when compared with those of our first patient, or that the nervous system and general health are so slightly affected, while our first patient is not only quite incapable of work, but also in a somewhat alarming state of digestive disorder and psychical depres- 
sion? The explanation which I have to give of this very typical difference between the virginal and the maternal enteroptosis is simply this: that, while the virginal abdomen with its tight abdominal wall makes it impossible for the other organs to make way for and adapt themselves

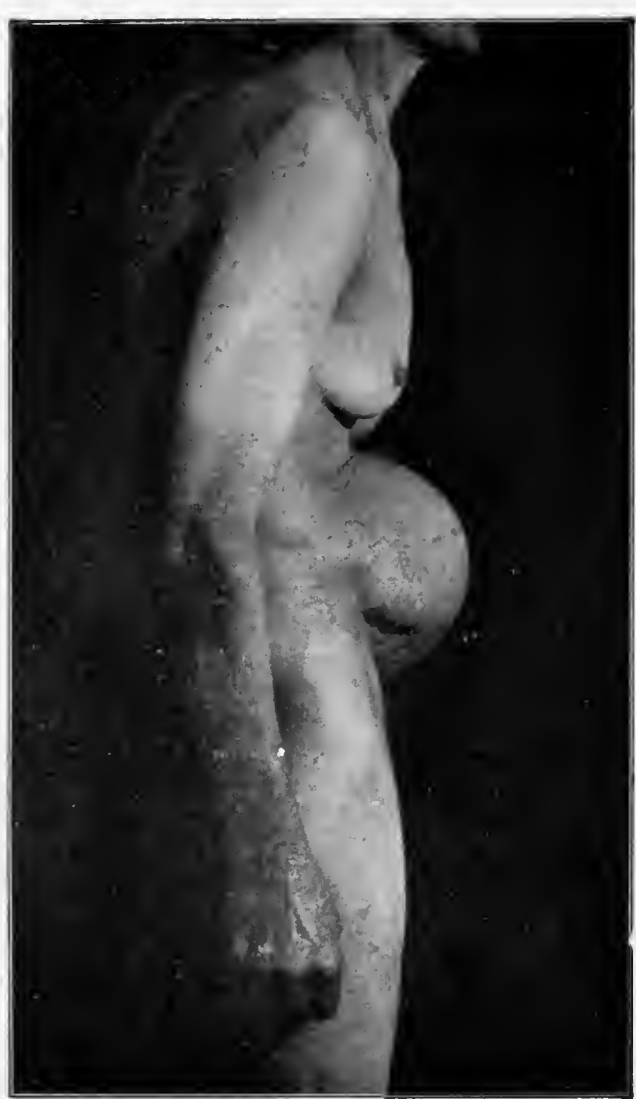

Fig. 70.-Maternal ptosis. to the prolapse, the organs in the large, r e laxed abdomens of women who have borne children have ample scope for it. It goes without saying that many pains and sensations must result with virginal ptosis patients, but, on the other hand, the large, relaxed, pendent abdomen may sometimes in $\mathrm{v}$ o l $\mathrm{v}$ e acute and dangerous attacks which we seldom find with virginal ptosis : attacks of ileus resulting from volvulus of the too mobile intestinal coils. To what an extent the relaxation and inflation may increase witl maternal ptosis patients you see in the picture which I here show you of a patient whom we had for treatment in this ward some time ago; when not only the stomach, intestines, and kidneys, but also the liver, had prolapsed to the bottom of the abdomen (Fig. 70). The great difference between the two types asserts itself also when we come to the question of 
treatment. We have two surgical methods for the treatment of enteroptosis: bandage-treatment and the operative fixation of the sunken organs as nearly as possible in their normal positions. Experience shows that, with the virginal ptosis patient, bandage-treatment does not give any relief at all, or only temporary relief; whereas with the majority of maternal ptosis cases it gives satisfactory assistance. The explanation of this simply is that it is very difficult, often impossible, for a bandage to have any effect through a tight, vigorous, virginal abdominal wall. To effect this a pressure is required, which can neither be easily endured nor procured, not to mention maintained. Quite different is the case with the maternal, relaxed abdominal wall. Here the ptosis is mainly due to the changed conditions of pressure in the abdomen, because -making use once more of Wolkow and Delitzen's striking illustration-the "pelotte" of air-filled intestines, which should support the subdiaphragmatic organs, is no longer held in position by a tense, elastic abdominal wall. This has become relaxed, attenuated, and pendent. Any one can understand here that there is use for a bandage, which can easily have effect through such an abdominal wall.

It has, however, given greater difficulty than one could have believed, a priori, to construct an effective, workable bandage, which could replace the compression of the intestines caused by a normal, vigorous abdominal wall.

The abdominal belts which were formerly in general use here in Denmark and are still greatly used abroad are formed to resemble as nearly as possible a more or less tightly fitting bag, in which the patient carries her pendent abdomen. Such a belt can, perhaps, offer an agreeable support for the abdominal wall, but it cannot in any way replace the sunken organs. Moreover, all these belts must be laced behind, which necessitates the patient's applying it when in an erect position, and, therefore, after the intes- 
tines have fallen. An effective belt must undoubtedly be constructed so that it fastens in front, when the patient can apply it in a recumbent position, and while the organs are lying in their normal position.

So far as I know. the best belt hitherto constructed is of Danish origin: it is Vermehren's abdominal belt, which is
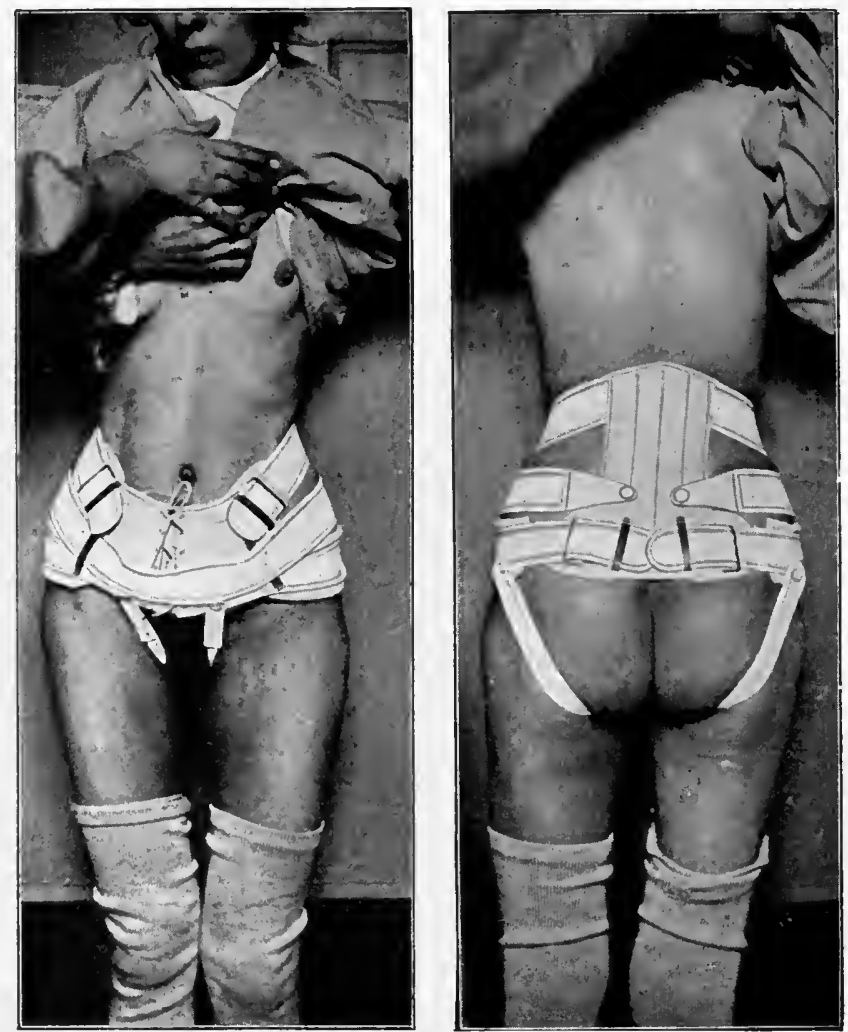

Fig. 71.-Vermehren's abdominal belt.

generally used here, and is beginning to be recognized abroad. I am all the better able to demonstrate this to you because we have had one constructed for the patient with maternal ptosis. You see that it consists of a high back-piece stiffened with a few pieces of whale-bone, thereby replacing in some degree the corset. This is connected with 
the lower front-piece: below by two broad elastic straps which on each side run beneath the crista ilei, and above by two straps directed toward the top corners of the frontpiece, which should not be tightened, as they only serve to keep the top of the bandage from shifting; the front and back pieces are finally joined below by two thigh-straps. The front-piece is provided on the inner side with a pélotte, which, according to circumstances, is made more
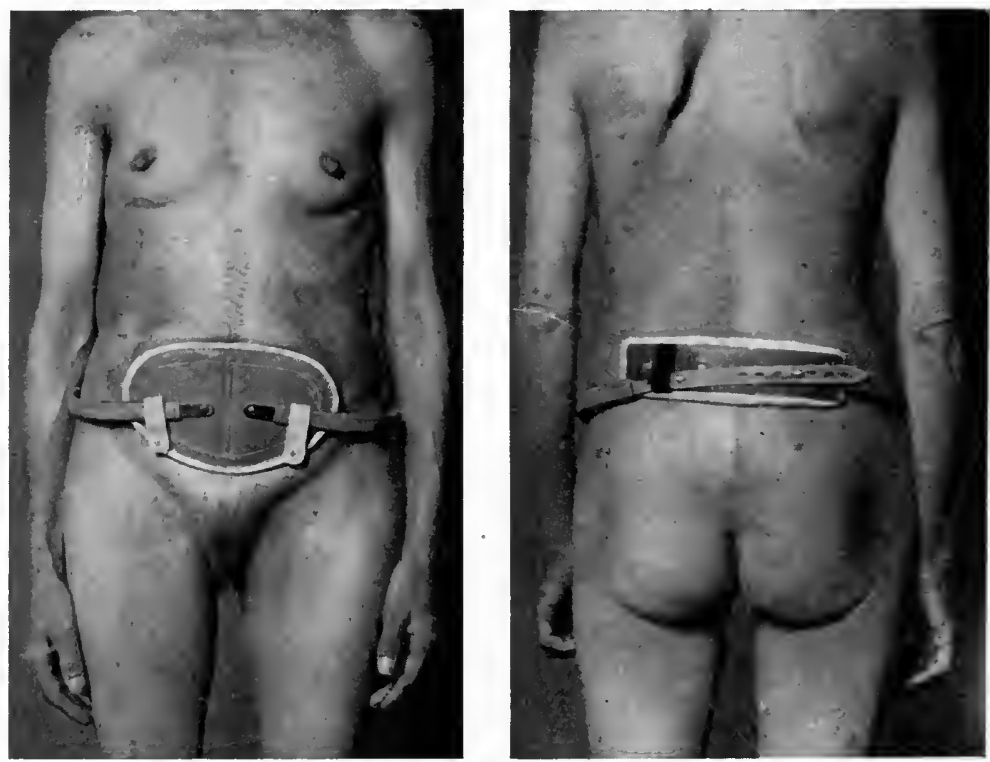

FIG. 72.-Curtis's abdominal support.

or less full, and by tightening the elastic side straps the pélotte is pressed into the hypograstrium, and thus lifts the intestines by forcing the abdominal wall beneath them. To obtain an even greater pressure I have introduced yet another broad elastic strap, which passes across the front-piece and is buttoned on the back-piece (Fig. 71). We now adjust the belt while the patient is lying down, and when the intestines have assumed a more or less normal position. You see that it works well, because we lace 
it in front. When we now allow the patient to rise, you see how the abdomen is borne up, and how the epigastrium, formerly so shrunken, is now filled.

I think that this belt will suffice to relieve the patient of her pains and to improve her constipation, which we will simultaneously counteract by a judicious diet, and, if necessary, by aperients.

With the other patient, on the contrary, we have heard that Vermehren's belt, which seemed to help for a short while, is now quite incapable of giving relief. The only hope of recovery with this patient lies in an operative encroachment which will refix her stomach and colon in their normal positions: a gastropexy, to which must possibly be added a colopexy. In another lecture I will tell you about the technic, difficulties, and prospects of this operation. To-day time compels us to stop. 


\section{LECTURE XI}

\section{GASTROPTOSIS-CONTINUED}

THe two patients with whom we shall occupy ourselves to-day have both been admitted-operationis causa-for serious diseases of the stomach; one being transferred to us from the medical ward with the diagnosis ulcer of the stomach, and the other being sent by a general practitioner on account of a cancer of the stomach.

The first is an unmarried woman, twenty-two years of age, who has borne no children. She tells us that she enjoyed good health till her seventeenth year, when she began to suffer from cardialgia, dyspeptic symptoms, and a rather persistent constipation. These attacks have grown gradually worse in the course of time, and, during the past two years, vomiting after meals has been added, which has by degrees assumed such a serious nature that, as she says, she has vomited all she has eaten during the last twelve months; in any case, every meal has been followed by vomiting. Simultaneously her constipation has become more and more persistent, weeks passing without her being able to induce her bowels to work, in spite of powerful aperients, and her pains in the cardia, which commence as soon as she begins to eat, have become more and more tormenting. As she had gradually become incapable of work, and as her condition seemed, on the whole, alarming, she was admitted to the medical ward of this hospital five months ago. Notwithstanding the strictest ulcer diet, her cardialgia and vomiting have not been controlled, as she has continued to vomit everything after her meals.

For the constipation, also, the physician has employed all his art in vain, as it defies all aperients and all dietetic influence, because the patient vomits all that is given to her. 
You see that the patient is of medium height and pale, with a worried expression, but, in spite of a most inefficient nourishment, by no means thin. The abdominal wall, especially, is somewhat full, muscular, and rather difficult to feel through. When we ask whereabouts in the cardia the pains are, she tells us most decidedly that they are on the left of the centre line, where, also, she is sensitive to pressure. These symptoms fit in very well with the symptoms of an ulcer of the stomach, and in former times, in With's period, one would at once have given the diagnosis from them. Nowadays we demand for the confirmation of this a series of examinations. Firstly, we demand an examination of the stomach contents taken during a fasting period; next, a further investigation as regards the chemical and motory function of the stomach, and the administration of certain experimental meals, the preparation of which by the stomach is then investigated by withdrawing them at a fixed period after they have been partaken of. It is very important to know whether food remains in the stomach longer than it normally should do, because such a retention of food suggests the presence of an ulcer, which either contracts the exit-the pylorus-or weakens the muscles of the stomach by infiltration, and thereby diminishes its expulsive power. In this case there is absolutely no delay with the evacuation. This symptom of ulcer is, therefore, wanting, but we must bear in mind that its absence in no way precludes the presence of an ulcer. If, on the other hand, we turn to the examination of the chemical condition of the stomach, we find an abnormality which is frequently found with ulcers, and is considered by some to be a sure sign of it. It is the presence of a greatly-increased amount of hydrochloric acid during a period of fasting. The abundant hypersecretion, the so-called gastrosuccorrhœa or Reichmann's disease, was formerly considered an independent gastric disease, which 
often caused the development of an ulcer by a hydrochloric acid erosion of the mucous membrane. Now, one is inclined to reverse the condition, and regard the gastrosuccorrhœa as the result of an ulcer. Some people, indeed, like Rubow, who has written an interesting treatise on this feature of the disease, regard it as an almost certain sign of ulcer. It is undoubtedly this symptom, then, which in conjunction with the vomiting and constipation has formed the basis for the diagnosis ulcer in this case. I do not, however, accept this diagnosis without question, because it seems to me suspicious that an ulcer which causes such violent vomiting and has existed so long causes no retention of food particles nor any bleeding; furthermore, that this patient has never vomited blood, nor has any trace of blood been found in the feces.

In spite of the lack of these decisive ulcer symptoms, I should scarcely have doubted the correctness of this diagnosis had we not by our examination been able to substantiate an abnormality of the stomach, which, as it seems to me, explains all the symptoms in a natural manner. Both by inflation of the stomach and by the radiographic plate of this abdomen after the patient has partaken of bismuth porridge, it appears that the stomach occupies an abnormal position, because the greater curvature is depicted seven centimetres below the umbilicus, and the lesser curvature one to two centimetres below this. In other words, there exists a gastroptosis of very considerable extent, the more so when we consider that our patient is a virgin with vigorous abdominal muscles.

Can such a gastroptosis, then, give us an explanation of all the morbid symptoms from which the patient is suffering? Yes, in my opinion it not only can but very often does present the appearance of exactly such a disease as this. Even in 1898, by reporting my first gastropexy, I drew attention to the fact that gastroptosis-especially 
with virgins-often presents an appearance which is easily mistaken for ulcer of the stomach by anyone who has not carefully elicited the history and weighed the evidence.

Clinically, I have classified gastroptosis cases in two. divisions: the virginal, which we find in women with a virginal, intact abdominal wall and the maternal, which we find in women who have borne children, the result merely of the altered conditions of space in the abdomen and of the relaxation of the abdominal wall caused by pregnancy and confinement. While the latter form is very frequent, though giving morbid symptoms other than constipation in only a limited number of instances, the virginal is frequently accompanied by extremely painful symptoms: pains in the cardia, vomiting, persistent constipation, irregularity of menstruation, dysuria, and, often, innumerable nervous symptoms, which accounts for these cases being so often recorded under the diagnosis hysteria. It seems to me that the reason for this is not difficult to understand. In the virginal abdomen there is no room for the subsiding organ, which in consequence collides with the organs upon which it subsides, weighing and pulling them down; while in the case of a ptosis arising after childbirth there is ample space wherein the organ can subside from its normal position. It must be added that pains and abdominal sensations react far more actively with these young persons.

Cardialgia arises with ptosis patients, just as with ulcer patients, so soon as the food descends into the stomach, but I have drawn attention to this great difference: that while the quality of the food is the deciding factor with ulcer, with ptosis it is the quantity. With an ulcer patient the cardialgia occurs with vomiting after eating rich, irritating food, but not after drinking milk and gruel. With a ptosis patient, however, it is quite immaterial whether one thing or another is eaten, and only the quantity of the food 
partaken of is of importance. It is in conformity with this that one never succeeds in curing ptosis cardialgia with an ulcer diet, which consists merely of a qualitative change of food. When the medical men are beguiledmore is the pity! - to believe that they have cured a presumed ulcer when a ptosis is really in question, one reason is that they have confined the patient to bed, under which circumstances the stomach resumes its normal position, and the symptoms, especially the cardialgia, in many cases cease quite naturally. Here it is not the diet but the confinement to bed which has had the desired effect, which is best proved by the whole trouble recommencing so soon as the patient gets up again. With our patient you will notice that the strict ulcer diet pursued for many months has not in any way influenced the cardialgia or the vomiting. This must immediately cause one to mistrust the diagnosis ulcer. You may, however, answer: Yes, but at the same time she has been confined to bed, so why has not this affected the ptosis? To this I reply that although it is a rule that ptosis symptoms disappear with confinement to bed, yet in a minority of cases this treatment fails to give relief. In my opinion the reason is that, in most cases, the deeply-sunken colon transversum, filled with hard, heavy feces, prevents the stomach from freely gliding back to its normal position, and by its weight, and assisted by ligamentum gastrocolicum, drags it downward, which drag gives rise to the pains.

What favors our belief that in this case the cardialgia is due to ptosis is that the pains are located on the left side of the epigastrium. This is quite typical of gastroptosis and, in my opinion, accords with the pains being due to the pull exercised upon the cardia by the prolapse, whereby the peritoneal covering of the diaphragm is distended. It is quite true, of course, that the ulcer pains also may be localized to the left of the centre line, but that is only in 
the rare cases where the ulcer is in close proximity to the cardia. In the great majority of cases the pain is in the centre of the cardia, and radiates therefrom on both sides, which accords with the frequent localization of ulcer in the vicinity of the pylorus. The fact that the vomitings are not affected in the slightest degree by the quality of the food also speaks volumes in favor of gastroptosis.

Constipation is certainly a very frequent symptom with ulcers, but is a far more frequent-indeed, an invariablesymptom with gastroptosis. The simple explanation of this is that a prolapse of the stomach necessarily involves a prolapse of colon transversum, and occasions a sharp bend at both flexures, and seriously hinders thereby the passing of the stomach contents.

The only thing, then, which might seem to speak in favor of ulcer and against ptosis is Reichmann's aforementioned symptom, gastrosuccorrhœa, whereby we understand a constant and abnormally large secretion of gastric juice. Opinions have been greatly divided as to what this symptom is due. One is inclined to agree that it is a question of a reflexive hypersecretion. As I have previously mentioned, Dr. Rubow, in an interesting little treatise, has recently championed the point of view that the irritant which produces this reflex must always be an ulcer. A circulus vitiosus, so to speak, should appear, because the ulcer causes an increased secretion of sour gastric juice, which, again, by its corrosive effect serves to nourish and irritate the ulcer. There are many, however, who consider that, with nervously disposed individuals, other irritants also may produce gastrosuccorrhœa, and, to the best of my understanding, it seems highly probable that the prolapse of the stomach, and the consequent bending of the pylorus, and the puckering of the abdominal wall may be capable of producing this symptom. I need not, however, limit myself to conjecture in this respect, but dare ven- 
ture a more decided assertion, because in two former cases of quite a similar nature I have seen the gastrosuccorrhœa, and, indeed, all the gastric symptoms, disappear without leaving any trace, in immediate response to a simple gastropexy. Without, then, actually denying the possibility of there being a small mucous membrane ulcer, I still feel convinced that all the symptoms are here due to gastroptosis; that I will restrict myself to a gastropexy, unless, by inspecting and palpating the stomach, I unexpectedly find unequivocal signs of an ulcer. We must remember that it is not rare to find an ulcer and ptosis in the same case. The ptosis may then be secondary, resulting from a contracting ulcer at the pylorus; but there is a considerable dilatation also, owing to the weight of the great quantity of liquid and solid food, which, accumulated and retained in the stomach, weighs this down. But, conversely, I have no doubt that a primary ptosis may favor the development of an ulcer. Yes, I have at hand a whole series of observations which are fairly convincing as regards this assertion. The characteristic of these ulcers is that they develop just in those places where the wall of the stomach suffers from a permanent bend or crease as a result of ptosis. Such bends are typically found in the passage between the cardia and corpus ventriculi and near the pylorus, either on the stomach side or on the duodenal side, while the ordinary ulcus rotundum rather has its seat on the lesser curvature, between these two. Often these mucous membrane ulcers are superficial, and at times multiple, as on this preparation which you see here (Fig. 73), where three pea-sized ulcers are located in a transverse fold of the mucous membrane, which was attached by the coalescence of the peritoneal covering. We must discuss this matter further when we some day occupy ourselves with the pathology of gastric ulcer. With this patient, as I have already stated, I do not believe that there is an ulcer, despite the 
fact that the symptoms are of many years' standing, because neither motory insufficiency nor the least sign of blood in the contents of the stomach or feces is found.

I now wish you to observe the other patient, who has been admitted to this ward with the diagnosis cancer of the stomach. The correctness of this diagnosis will seem very probable to you when you glance at her; I had no doubt when I first saw her. You see not only a thin but also

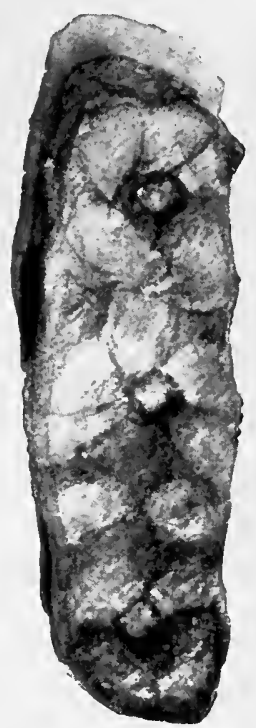

Fig. 73.-Ulcers on a ptosis crease of the mucous membrane of the stomach.

a greatly emaciated person, with sunken, worried features and a sallow complexion. Your impresion is strengthened when you hear that this woman is only fifty years of age, when you must surely have thought that she was well along in the sixties. As regards the history of her disease, this is briefly as follows: As a child and in her youth she was healthy, marrying early and giving birth to four children quite naturally. Since the third birth, fifteen years ago, she dates the commencement of her present sufferings, because she then began to experience oppression and discomfort after meals, which often increased to rather serious pain in the region of the cardia. Simultaneously she began to suffer from constipation, which, during all these years, has grown more persistent, so that a daily use of aperients has become necessary. During all these years, also, she has suffered, on and off, from vomiting and increased cardialgia, but not until last year did the vomiting become continuous, when it occurred after each meal and after each attempt at a meal. By degrees she has become greatly emaciated, and six weeks ago she was so weak that she could no longer work or be up and about.

She tells us that formerly the symptoms disappeared 
or were ameliorated when she retired to bed, but of late such has not been the case, as notwithstanding continual confinement to bed, every attempt to partake of food has resulted in pain and vomiting.

You will easily understand the view of the physician who admitted this patient that, after suffering for many years from a chronic ulcer and with this as a starting point, she was then suffering from cancer of the stomach. The more evident clinical symptoms may, perhaps, agree very well with such a supposition; but, as you now see, a more thorough objective examination has produced far more surprising results, and has justified a much lighter view of the nature and prognosis of the disease.

While an examination of the head, neck, and chest organs does not present any abnormality, you at once notice that the abdomen is greatly relaxed and somewhat peculiarly shaped, as we see the upper portion of the epigastrium rather sunken, and divided from the remaining slightly balloon-shaped and rounded part of the abdomen by a transversely running furrow slightly above the umbilicus. Furthermore, when one palpates the upper abdomen in search of a tumor, the first impression seems to be confirmed, because one really does feel a firm mass with a free edge crossing the epigastrium just above the umbilicus. You will quickly discover, however, that this edge is sharp and thin, quite resembling the normal edge of the liver, and you will then ask yourselves: Can this be an enlarged liver due to a cancer metastasis secondary to a stomach cancer concealed beneath the liver? To decide this, we must first decide the extent and limit of the liver by means of percussion. While I now percuss the chest from above, in order to define the upper margin of the liver, we find that this is not lying in its normal position at the level of the sixth rib, but is considerably lower. We at once know, then, that the liver is displaced in a downward direction, 
and that here, in any case, we have a hepatoptosis, and the question remains: Is the displaced liver of normal size, or has it, perhaps, prolapsed because it is the seat of a tumor? To settle this, we now allow the patient to lie down. I at once feel the liver glide back to an almost normal position, and, by taking the liver between two fingers, I can now push it beneath the free border of the ribs. There is absolutely no tumor to be felt in the abdomen, but, on the other hand, in the epigastrium I feel the aorta pulsate close under my fingers, and feel the vertebral column protruding distinctly. I am sure that those who are nearest to me can also see a distinct pulsation in the epigastrium. I must ask you to make a note of this symptom, because it signifies that the stomach has deserted its position, and no longer covers the aorta with its cushion as it normally should, and no longer lifts the abdominal wall away from this.

The question now is whether this is a simple ptosis, a prolapse of the otherwise normal stomach, or, as the serious symptoms might suggest, a greatly dilated stomach which has prolapsed on account of the weight of its stagnating contents. In order to account for the dilatation it is natural to. suspect a cancer in the pylorus, which has developed, perhaps, at the base of a chronic ulcus ventriculi. To throw light upon this matter, we have naturally resorted to an examination, by means of one of Bourget's retentionexperimental meals, in order to determine the ability of the stomach to empty itself. It is evident, however, that not only after the lapse of twelve hours but even after six to eight hours the stomach is found to be quite empty, and neither by aspiration nor by washing out have we been able to withdraw food remnants. So it is no contraction which has hindered the onward passage of the stomach contents. Now, as a cause of the cachexia, one might have a cancer so situated that it would not affect the normal mo- 
tility of the stomach, and quite lately I have had occasion to demonstrate such a case. The patient was a man, who was admitted for a small hernia in the epigastrium, though he had never shown symptoms of a stomach disorder. His cachetic appearance led us to make an examination of the motory and chemical functions of the stomach, the result being that, while the ability of the stomach to empty itself proved quite normal, the gastric juice was quite deficient in free hydrochloric acid. On the strength of this symptom, which is so frequently found with benign gastric diseases, and in conjunction with the anæmic appearance of the patient, I nevertheless suspected cancer, and was induced to perform an exploratory laparotomy. I drew forth and examined the stomach. I then found and removed a rather large, flat adenocarcinoma situated in the centre of the anterior wall of the stomach, which had not ulcerated the mucous membrane. As a matter of fact, achylia is an extremely frequent symptom with cancer, and of great importance where the diagnosis is doubtful.

But if we now examine our present case by means of Ewald's experimental meal, it appears that the conditions of acidity are nearly normal, because in a total of fiftyeight we have thirty-one of free hydrochloric acid. By the examination of the stomach, then, we obtain no support for the diagnosis cancer of the stomach,' which,. with the rougher clinical observation, immediately appeared to be the most natural one. All that we have been able to demonstrate is a very considerable enteroptosis, prolapse of the liver, stomach, and colon. The kidneys are in their normal positions; the urine is normal; and exploratio rectovaginalis shows no abnormality. All the dyspeptic symptoms,-cardialgia, vomiting, and constipation,-can, as it were, be explained by the gastroptosis. But is this also the case with regard to the cachexia and extreme exhaustion? Yes, and some of you may know that, in a former work 
published in 1899, I enumerated the conception of gastroptosis-cachexia, which was based on the observation of even more miserable and emaciated patients. You will certainly admit that the worst of these, whose picture you here see (Figs. 74 and 75), resembles a patient in the last stage of cancer, and will remind you mostly of pictures of the famines in India and China. This case, an account of which I published in 1899, concerned a twenty-two-year-

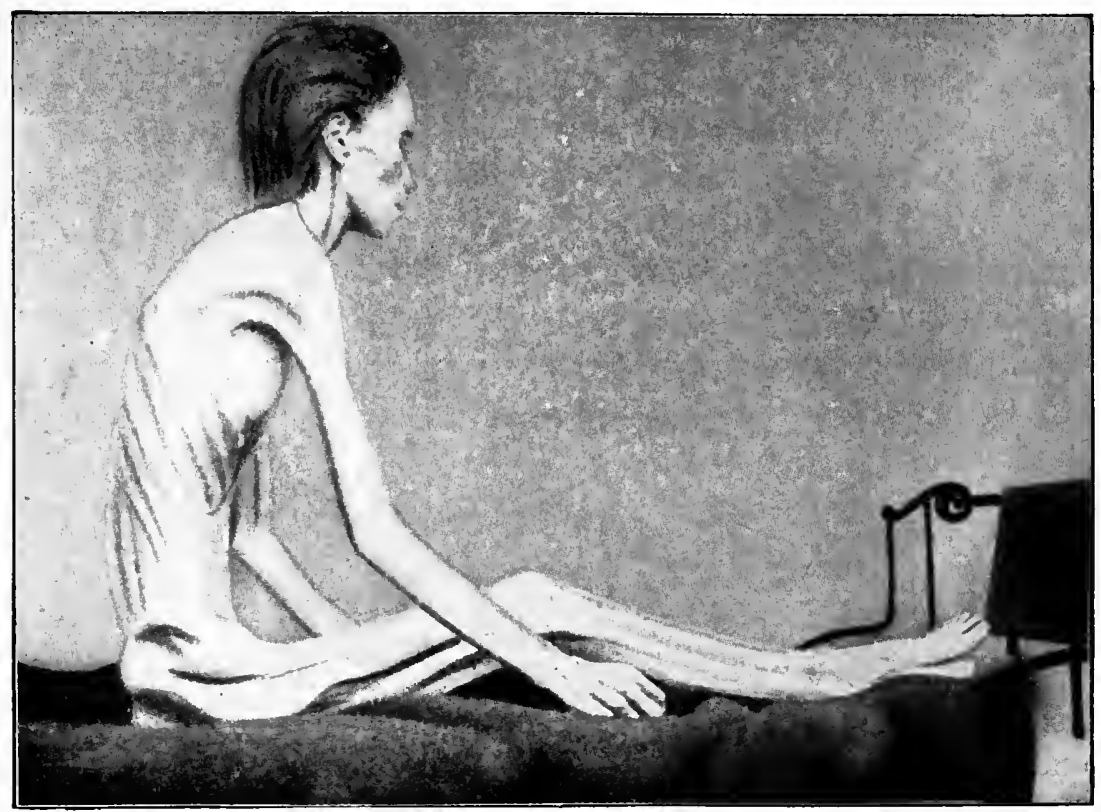

Frg. 74.-Gastroptosis cachexia. Personal observation.

old servant girl, whose history is very typical. Only once in her life had she menstruated, while from her seventeenth year she had suffered from increasing cardialgia, vomiting, and constipation. During the last eighteen months she vomited after each meal, and a month might pass without her bowels moving. Her weight had fallen from 140 pounds to 56 pounds when she was admitted to my clinic at the Frederik's Hospital with the diagnosis stenosis pylori (cancer?). At the operation only a gastro- and 
colo-ptosis were found. Unfortunately, I felt so sure that a cancer or stenosis must be present that I performed gastro-enterostomy instead of gastropexy. Only a slight improvement followed this operation, and the patient died six weeks later. At the postmortem examination, performed by Professor Fibiger, it appeared that, apart from a quite recent, small tuberculous infiltration in the inferior lobe of the left lung, she presented no othermorbidity than gastroptosis. $P$ er $s e$, there is nothing strange in the idea of such a condition. developing with patients who vomit everything they eat, and with whom every attempt to partake of food produces pain, so that,

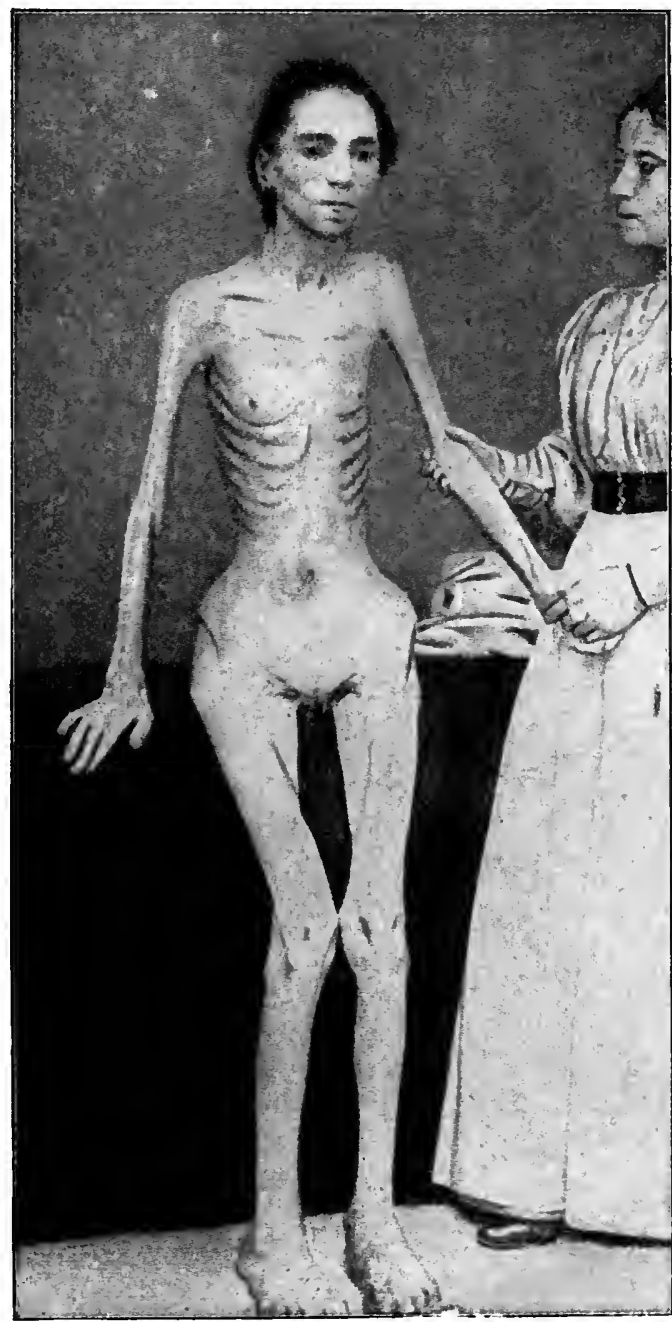

Fig. 75.-Gastroptosis cachexia. Personal observation. finally, they dare not eat at all. It is really very important to be aware of this state of affairs, so as not to confound such a gastroptosis-cachexia with cancer-cachexia, because then one of two things will happen: either you give up all thought of an operation, or, if attempting such, relinquish all hope 
when you open the abdomen and find the stomach normal, and close the wound in the belief that a malignant tumor exists in some other and inaccessible spot. In either case the patient's fate is hopeless, as a result of a faulty diagnosis; whereas there would have been a possibility of complete recovery by means of a comparatively safe operation.

The cachetic condition of our patient can be very well explained, therefore, by the gastroptosis alone, which is very considerable, as is shown by the Röntgenoscopy, after a meal of bismuth-porridge. You may still ask me, however, why these attacks do not disappear with confinement to bed if this is a simple ptosis. Here, also, I think the Röntgen picture supplies the answer, as it shows that the colon transversum lies low down in the small pelvis, far from the greater curvature of the stomach. In other words, ligamentum gastrocolicum is considerably elongated. In such a case the colon remains prolapsed, even though the stomach glides up to its normal position, and all the symptoms caused by the coloptosis and the bend in the colon remain unaffected by confinement to bed. I think, also, that both the patients who have to-day been the subject of our reflections are suffering from gastroptosis and coloptosis, and the latter from a prolapse of the liver in addition, and that all the morbid symptoms arise from the ptosis. Whether I am correct in my diagnosis will appear at the operation which I shall perform in the laparotomy room after this lecture, the result of which $\mathrm{I}$ shall report at our next lecture. To begin with, the operation will naturally consist of an exploratory laparotomy. If, contrary to my expectations, it should turn out that, with the first patient we are faced by an ulcer and with the second by a cancer, the choice of operation must rest between resection of the stomach and gastro-entero-anastomosis, two rather serious encroachments; while, if my diagnosis is correct, I can restrict myself to sewing up the sunken organs in a normal, or as nearly as possibly normal, position. 


\section{LECTURE XII}

\section{GASTROPTOSIS-CONTINUED}

\section{Treatment}

To-DAY I shall commence my lecture by reporting the findings at the operations performed on the two patients who occupied our attention during the last lecture.

I first operated on the young girl admitted for ulcer of the stomach, a diagnosis founded on the very severe gastrosuccorrhœa. I made an incision from processus ensiformis to the umbilicus; as soon as the peritoneum was opened, the stomach literally sprang out through the wound, and lay on the outside of the abdominal wall, so that the greater curvature reached about half-way between the umbilicus and the symphysis. When the stomach springs forth in this way it is very characteristic of pronounced cases of virginal gastroptosis, and at the same time is suggestive of an abnormally narrow space in the abdomen and of a great slackness of the stomach-walls. The suddenness and vigor with which it springs forth gives one a most vivid impression of how the elastic, vigorous abdominal wall-like a strait-jacket-has held it suppressed in the narrow and-as regards its movements-quite insufficient space, and it seems to me that one should immediately realize that, just by reason of this disproportion of space and by reason of the addition of food, which weighs down and dilates the stomach, the ptosis must occasion pain, vomiting, etc.

Here, as in so many other cases, we noticed another typical result of the too narrow lower chest aperture; namely, that the whole length of the edge of the liver was curly or wavy, instead of forming a sharp, homogeneous convex line, as it should do. Nowhere was any trace of an ulcer seen or felt. The pylorus, which, with six centimetres 
of the duodenum, could be drawn outside the abdominal wall, was open and allowed the passage of a finger invaginating the wall of the stomach. This was of about normal thickness, if anything rather thicker, and the stomach was not dilated. We then drew out the colon transversum together with the heavy omentum majus. Ligamentum gastrocolicum was likewise thick, fatty and of normal breadth, and not, as is so often the case with enteroptosis, stretched to more than twice its breadth, as thin as silk paper, and often perforated. It seemed, therefore, that in this instance the colon had prolapsed with the stomach, and had not prolapsed primarily, drawing the stomach with it.

Owing to this finding I concluded that all her symptoms must be due to gastroptosis, and, therefore, performed only gastropexy, after a method which I shall describe to you presently. Let me for the present inform you only of the convincing effect which this operation has had pro tem. as to the correctness of the diagnosis. This patient, who for many months has vomited everything that she swallowed, has been quite free from vomiting since the operation, and this although I put her on a light, full diet the day after the operation. The other remarkable point is that afterward, during the last two days, the patient's bowels have acted naturally and spontaneously, while formerly all the aperients in the world had proved ineffective. I think one can safely conclude from this that the diagnosis ulcer may be excluded, and at the same time it seems to me clinically-ex juvantibus-proved that her pains, vomitings, and constipation have been caused by gastroptosis; because, how otherwise are we to explain the fact that the fixation of the stomach in an approximately normal position immediately, so to speak, relieves all the symptoms?

Possibly the fanatics who oppose the notion that gastroptosis is the cause of the disease, and who prefer to 
term all those cases where they fail to verify an apparent ulcer "nervous disease of the stomach," the result of a functional neurosis, will try to explain the success of this operation as being due to suggestion. If one wished to be amiable one might reply: "Yes, call it suggestion if you will, but one, nevertheless, which you have not succeeded in producing by any other method, and, therefore, all the same, an effect of great value." I do not think, however, that one is too daring when one denies that it is a question of effect by suggestion, because there are plenty of proofs wherewith to controvert the supposition: partly in the many cases where a gastro-entero-anastomosis has been performed on the supposition that the disease was due to ulcer, and partly in those cases where gastropexy has been performed in a faulty or inadequate manner; because in such cases the patients generally deteriorate steadily, while if only a suggestive effect were concerned, the one operation would be as effective as the other.

Another strange objection which has been raised again and again by the opponents of gastropexy and gastroptosis is this: that they cannot understand how, while so many people actually go about with a gastroptosis without suffering pains or other morbid symptoms, others should have pains, vomitings, etc. To this objection it is only necessary to reply with the contra-question: Will you deny that, because so many people can actually go about for years with a large, deep ulcer of the stomach without feeling pains, an ulcer can cause pain? Or that gall-stones can cause pain, because so many people go about with gallstones without feeling pain?

There is, however, no difficulty whatever in explaining the symptoms in cases of gastroptosis, and their variations with different individuals. One symptom, indeed, is always found with these patients: it is constipation; and on account of the frequent occurrence of gastroptosis 


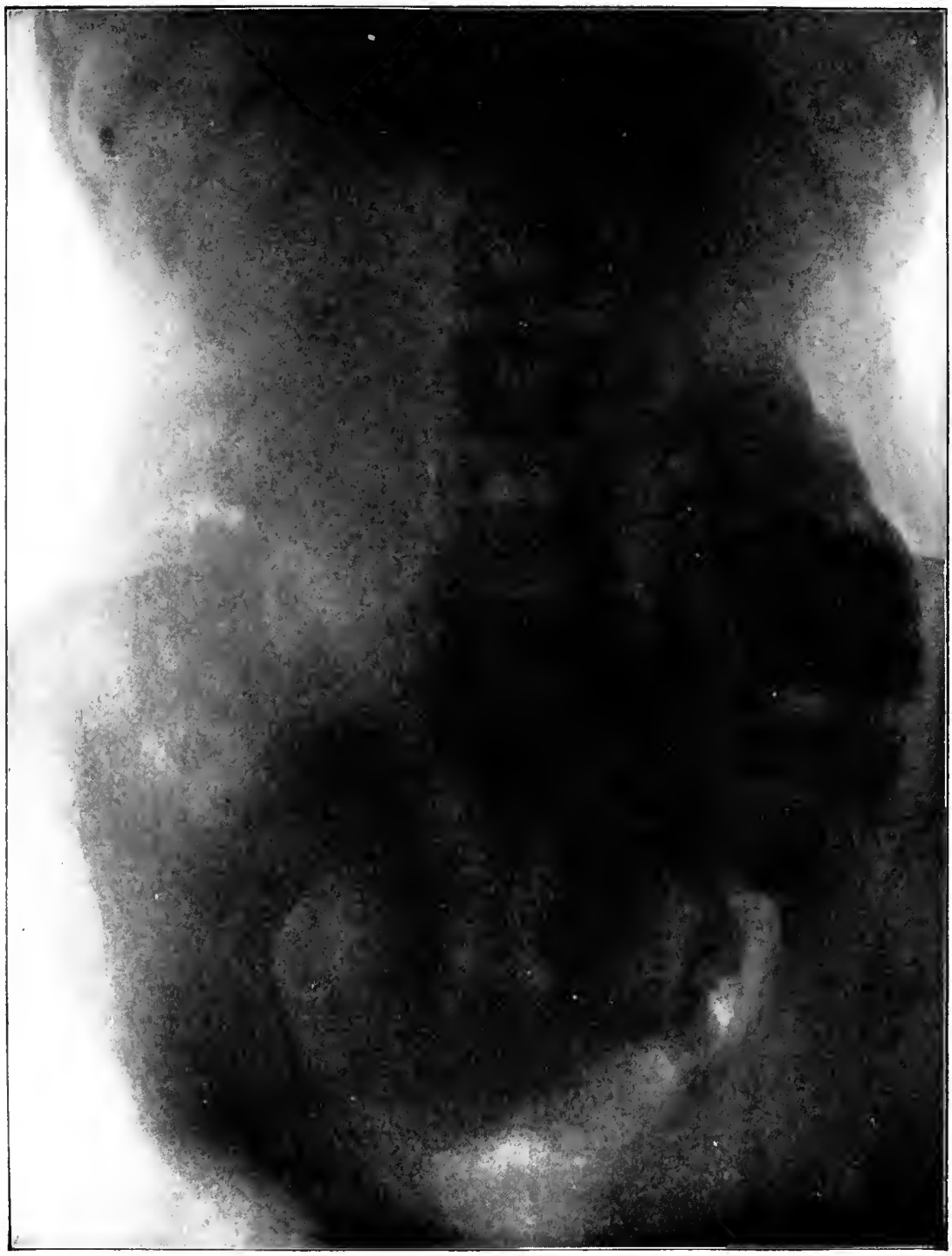

FIG. 76.-Rontgen picture of the bismuth-filled large intestine. Colon tranversum lies prolapsed in the small pelvis like a festoon. The acute-angled left flexure is seen lying much higher than the right flexure.

among women we have also, I think, the explanation of the far greater frequency of constipation among females than among males. The explanation of constipation being such 
an invariable symptom with gastroptosis is undoubtedly the obstruction caused in the passage of the solid feces by the sharp bends between the prolapsed colon transversum and the two flexures, of which the left one, in particular, lies very high and firmly fixed, so that the colon transversum -as you see in this radiograph of the bismuth-filled intestine (Fig. 76), rises steeply from the right side of the pelvis up to the dome of the diaphragm, to join the colon ascendens at an acute angle. One understands how it becomes day by day more difficult for the muscles of the intestines to force the ever heavier fecal matter up along the steep passage and round the sharp corner. Therefore, after a protracted ptosis we find the wall of the colon also relaxed and thin, as a result of the atroplyy of the muscles. Many of the symptoms may be attributed to the constipation. You all know the picture of disease described by Faber; and known as intestinal dyspepsia. You know that there are a number of gastric symptoms, such as oppression, nausea, and vomiting, without any ostensible disease of the stomach itself, but, on the contrary, an affection of the intestine characterized by constipation. You know, further, that these dyspeptic symptoms often disappear when the patients are confined to bed, with a diet suited to the intestinal condition. We have, therefore, an analogy recognized by the medical men themselves in which the gastric symptoms appear in intestinal disorders. I am inclined to think that it is not an analogy only, but that the intestinal affections in many of Faber's intestinal dyspepsia cases are due to ptosis. This conviction is strengthened when we learn that these cases need both rest in bed and careful diet before they recover, and that relapses occur when the patient gets up, notwithstanding a continuation of the diet, probably due to the effect of the ptosis.

Another symptom common to all cases of active gastroptosis is pain in the left side of the epigastrium, which 
appears immediately after the patient has partaken of food, and sometimes lasts for the whole day and sometimes during twenty-four hours. I think that these pains are due to the heavy drag on the attachment of the cardia to the diaphragm. The peritoneal covering of the diaphragm is drawn down by the stomach, and a shift of position takes place between the peritoneum and the subperitoneal tissues, a pull on the here overlapping sensitive nerves, that quite naturally causes the pains. Both Bloch and Lennander have shown us by their personal experiments bearing upon the pain sensation in the stomach that, while the peritoneum itself is insensible to traumatic influence, every drag on the peritoneum which involves a shifting toward the subperitoneal tissues causes pain.

The violence and extent of these symptoms must naturally be influenced by the extent of the ptosis, by the nervous temperament of the patient, and the extent of the space, which varies so greatly in virgins and with women who have borne children.

When it is thus seen that the symptoms may one and all be explained quite naturally by the changes caused by the ptosis, and when it appears that the collective symptoms in a whole series of cases disappear directly the organs are raised and fixed in a more or less normal position, then it does not seem necessary to resort to hysteria as the cause of the disease, or to suggestion as its cure when operated on. Now it must undoubtedly be admitted that a number of these patients present innumerable nervous symptoms, and that several of them are pronouncedly hysterical, a phenomenon which in some cases, perhaps, is a phenomenon of degeneration coördinate with the ptosis; but in most cases it seems to me more natural to interpret this nervous condition as the result of the ptosis. Even the insufficient nourishment, the constipation, and the consequent poisoning of the organism by reason of the stagnating intestinal contents, are in themselves sufficient to 
explain the strong diminution of the resisting power of the nervous system. But there is, per se, nothing at all remarkable in the prolapse itself producing nervous symptoms and psychical changes. For my part, I think I have arrived nearer the solution of the incontrovertible nervous and psychical changes by my clinical observation that nearly all virginal ptosis patients have highly peculiar derangements in the functions of the genital organs. We very often see that, simultaneously with the other ptosis symptoms, a complete cessation of the menstruation supervenes, or that this becomes painful, causing pain in the prolapsed organ especially. This last is invariable with floating kidney. Furthermore, I have substantiated the very interesting phenomenon that a nephropexy absolutely, and a gastro- and colopexy generally, cause menstruation to recommence in the course of a few days: even when one has purposely performed the operation immediately after the regular menstrual period. Among the cases which I have treated by gastropexy were three patients who had never menstruated, though they were thirty years of age or more, but who had their menses for the first time on the day following the gastropexy.

When one knows the extent to which morbid changes in the function of the genital organs affect the psychical condition of women, one at once understands that ptosis can produce abnormal psychical conditions only by the forementioned effect on the genital organs. But, apart from this, I have no doubt that the subsidence itself, and the sensation of a subsidence, may involve disturbed equanimity. In addition to the anxiety which the various pains may cause the patient is the faint nondescript sensation that something is wrong, that something is loose, which begets a feeling of uncertainty and a psychical uneasiness which by degrees upsets the equilibrium of their nervous systems.

With the other patient, also, gastropexy has demolished 
all the symptoms at one blow. Here there was no reason to take nervousness into consideration, but you remember that her cachetic appearance and intense emaciation greatly encouraged the thought of a malignant new growth. On opening the peritoneum we saw, through the entire incision and right down to the umbilicus, the prolapse liver revealed, smooth and shining, without any signs of tumormetastasis or cirrhosis. Not until we lifted the edge of the liver did we see the deeply-sunken stomach, which, in contrast with the previous case, where the stomach sprang forth througl the incision, had to be drawn forth from the depth of the abdomen, where it was held down by the weight of the colon and omentum. After the stomach had been drawn forth and placed on the outside of the abdominal wall, the greater curvature reached down to within a few centimetres above the symphysis, and eight centimetres of the duodenum accompanied the pylorus out through the wound. As was expected, ligamentum gastrocolicum was found drawn out and very attenuated; in places as thin as tissue paper, so that the distance between the greater curvature and the colon was three to four times as great as it normally should be. I at once felt for the pylorus, to ascertain if it was contracted, infiltrated, or uneven, but it appeared to be quite normal, and easily admitted my forefinger when, while invaginating the anterior stomach-wall, I passed it througl the ring of the pylorus. The stomach did not appear to be dilated, and, on the whole, impressed me as being perfectly normal, with the exception of a single spot on the lesser curvature, close to the cardia, where I felt a small induration, which I did not dare to define with certainty as simply a fold of the stomach-wall or as an ulcer. On account of the serious aspect of the disease, it seemed to me necessary to arrive at a decisive diagnosis, and I then, for the first time, put into effect a thought which $\mathrm{I}$ had been turning over in my mind for 
some time: to introduce through a small incision a gastroscope constructed on the same lines as a cystoscope, but provided with a pipe to inflate the stomach with air, in order thereby to smooth out the folds of the mucous membrane of the stomach. For this proposed experiment I arranged my'ureter cystoscope in such a manner that the canal through which the catheter otherwise runs was connected with an air-bulb by a hermetically closed drainage tube. The entire instrument, together with the conducting wires, was disinfected in formalin vapor. The cystoscope was introduced through quite a small incision in the middle of the anterior stomach-wall, and by the aid of a small tobacco-pouch suture the wall of the stomach was hermetically closed round it. The stomach was then inflated like a balloon, and on lighting the lamp I was surprised at the fine view which the illumined and everywhere transparent stomach-wall presented-not unlike an electric arc-lamp, and in the rose-colored wall of which I could observe all anatomical details, and especially the course of the vessels. Through the optical apparatus I could observe with the greatest distinction the smoothed-out mucous membrane, and could examine it fairly quickly in its entirety from the cardia to the pylorus, and substantiate my opinion that it was normal. Finally, I passed the cystoscope through the pylorus into the duodenum, and, finding the mucous membrane quite normal, all doubt seemed excluded: I liad a simple, uncomplicated gastroptosis before me. I therefore closed the small incision in the stomach wound, after having removed the cystoscope, and decided on gastropexy.

The difficulty with the gastropexy in this case, as in so many others, was that the sunken liver prevented the replacing of the stomach in a more or less normal position. It was first necessary, therefore, to raise the liver to a normal position, and here fix it to the peritoneal covering of the diaphragm. I carry out such a hepatopexy by first scarify- 
ing the peritoneal covering of the liver and diaphragm with many needle-scratches, and afterward apply a greater or lesser number of rectangular sutures of nitrate of silver catgut or silk. As you see in this illustration (Fig. 77), the thread is led in and out through the capsule and peritoneal covering of the liver on the dorsal surface, beginning transversely and parallel with and close to the edge, to be led in and out through the peritoneum longitudinally up to the highest attainable point of the diaphragm. From here the needle is passed transversely through the diaphragm with the same latitude as was allowed at the edge of the liver, then descending again through the peritoneal covering of the liver until one meets the

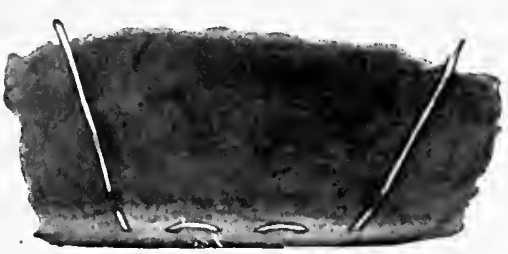

Fig. 77.-Hepatopexy. The liver fixed to the diaphram by angular sutures.

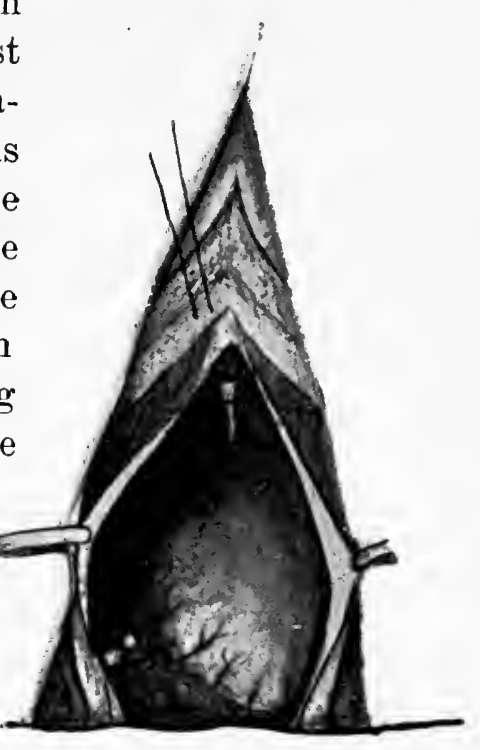

Fig. 78.-Heratopexy by aid of the severed ligamentum teres.

starting point of the thread. When the surfaces are exactly adjusted, the thread is carefully tightened and tied. From one to four or five such rectangular sutures are necessary for the attachment of the liver, according to the extent of its prolapse. Latterly I have, with great advantage, made use of ligamentum teres, because after a double ligature I have severed this midway, and stitched the upper end to the diaphragm, so that the liver is suspended by this solid, round band (Fig. 78).

In some instances it is more difficult to accomplish a 
hepatopexy. This is when the liver is deformed and hypertrophied, as, for instance, when the left lobe of the liver is elongated in a tongue-shaped form, and reaches down into the abdomen and overlaps the lesser curvature, thereby hindering the replacement of the stomach. In such cases it then becomes necessary to remove the lobe of the liver by resection, an operation which $\mathrm{I}$ have been obliged to perform in four instances, as a preliminary to the gastropexy. Now you know, perhaps, that resection of the liver is considered a very difficult and dangerous affair: partly on account of hemorrhage, and partly because of the difficulties encountered in suturing the fragile tissues. Hemorrhage is feared in a double sense: the parenchymatous bleeding from the many small vessels which cannot be caught, and the bleeding from the larger

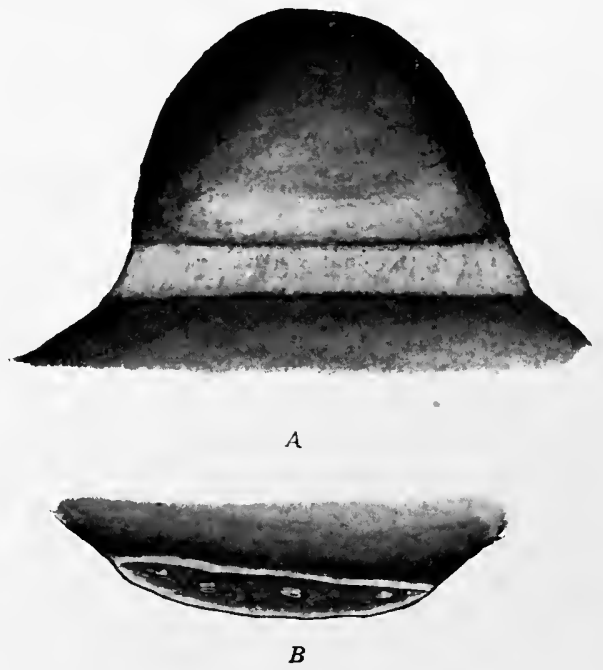

Fig. 79.-Liver resection with pressure forceps. $A$, after application of the angiotribe the tab of liver is suspended from the liver only by a paper-thin layer of the peritoneum; $B$, vessels project from the surface of the fracture without bleeding, and can here be caught and tied.

vessels which cannot contract owing to the stiff liver tissue. The difficulties in suturing are due to the fragility of the liver tissue and to the difficulty in finding a covering for the resected surface. I have, however, devised a technic which quite changes the unpleasant character of a resection of the liver. I begin by pressing the liver tissue together along the whole line of resection with Roux's angiotribe. The result is as follows: the fragile liver tissue bursts, while the fibrous, elastic parts-the peritoneum and vessels- 


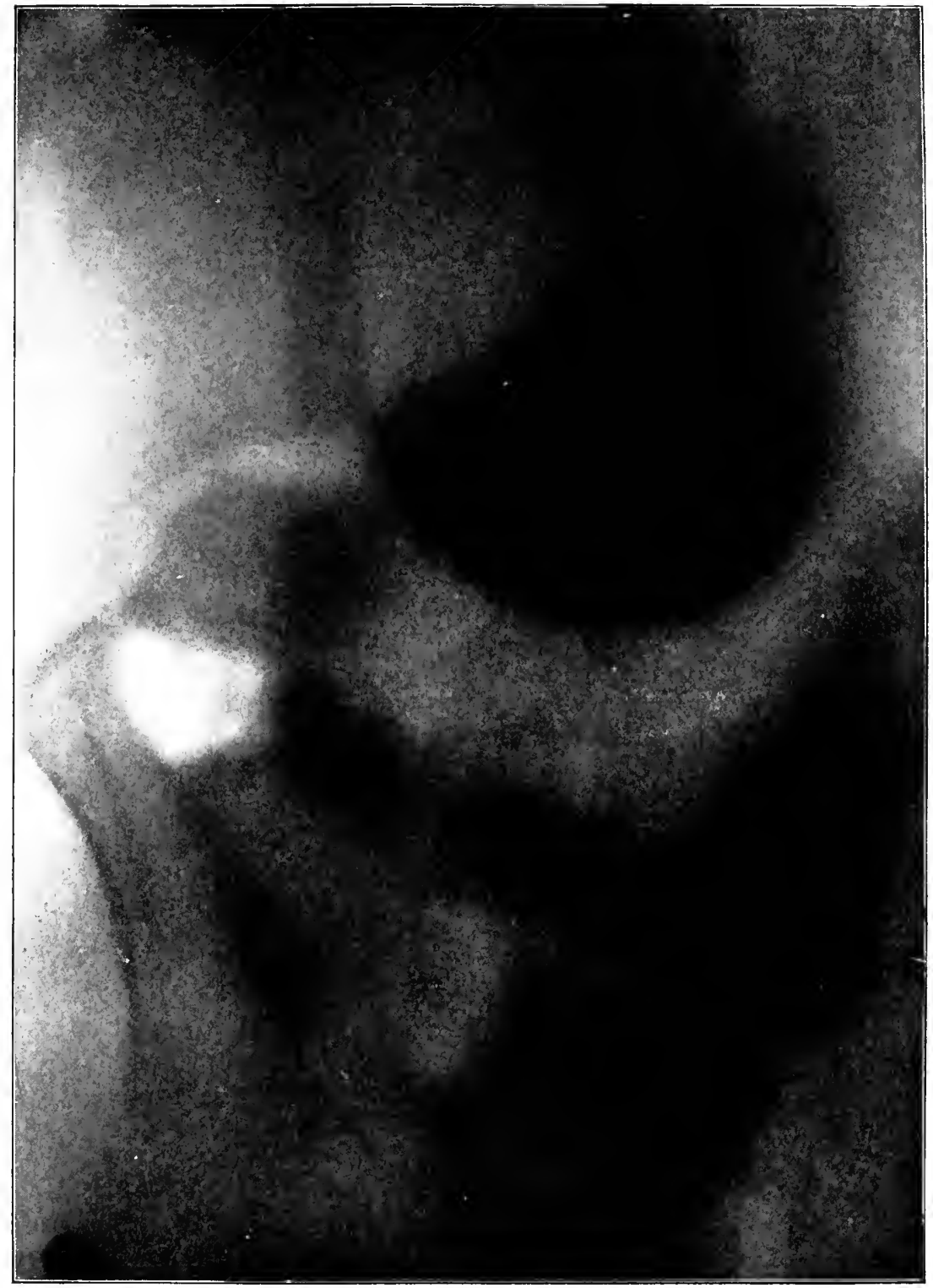

Fig. 80.-Case of primary coloptosis with secondary gastroptosis. Stomach and large intestine, filled with bismuth, photographed simultaneously. The great distance between curvatura major of the stonach and the colon well illustrates the abnormal length of ligamentum gastrocolicum. 
hold, and are held in the paper-thin tissue which joins the tongue-shaped formation to the liver like a bridge, after the angiotribe is removed (Fig. 79, $A$ ). This bridge is then severed and the lap of liver removed without any trace of bleeding. If one has confidence in the effect of the angiotribe on the larger vessels, one can content one's self with merely making a suture through the adhering leaves of the peritoneum. But one can also quite easily tie the larger vessels, because they project like small compressed tubes from the surface of the resection (Fig. 79, B). Sometimes, when very fragile, the peritoneal covering bursts, and $I$ have remedied this in such cases by sewing a piece of the omentum over the defect.

Before proceeding with the gastropexy itself, I first had to bring the colon into its normal position toward the stomach by pleating together the thin, perforated ligamentum gastrocolicum, drawn out to a length of about fourteen centimetres. You will understand this operation and the need of it when I show you this radiograph (Fig. 80), which I have taken of another patient with primary coloptosis and secondary gastroptosis, after I had simultaneously filled the stomach and the large intestine with a preparation of bismuth. You will notice the quite abnormal length of ligamentum gastrocolicum, and you will see that here, even if we fix the stomach at its normal height, the colon remains hanging like a festoon deep down in the abdomen, with acute bends of the flexures. If one wishes to correct the constipation and coloptosis, ligamentum gastrocolicum must be shortened and the colon attached to the greater curvature of the stomach. I do this by means of a row of strong catgut threads, which, beginning in the serosa coating of the colon, are passed in and out through all the layers of ligamentum gastrocolicum, whereby the omentum majus as well as the mesocolon is caught, and end finally in 
the serosa of the stomach along the greater curvature (Fig. 81). In order not to injure the large and numerous vessels which course through the omentum and ligament, I let an assistant hold the colon transversum well up, so that the daylight passes through ligamentum gastrocolicum,

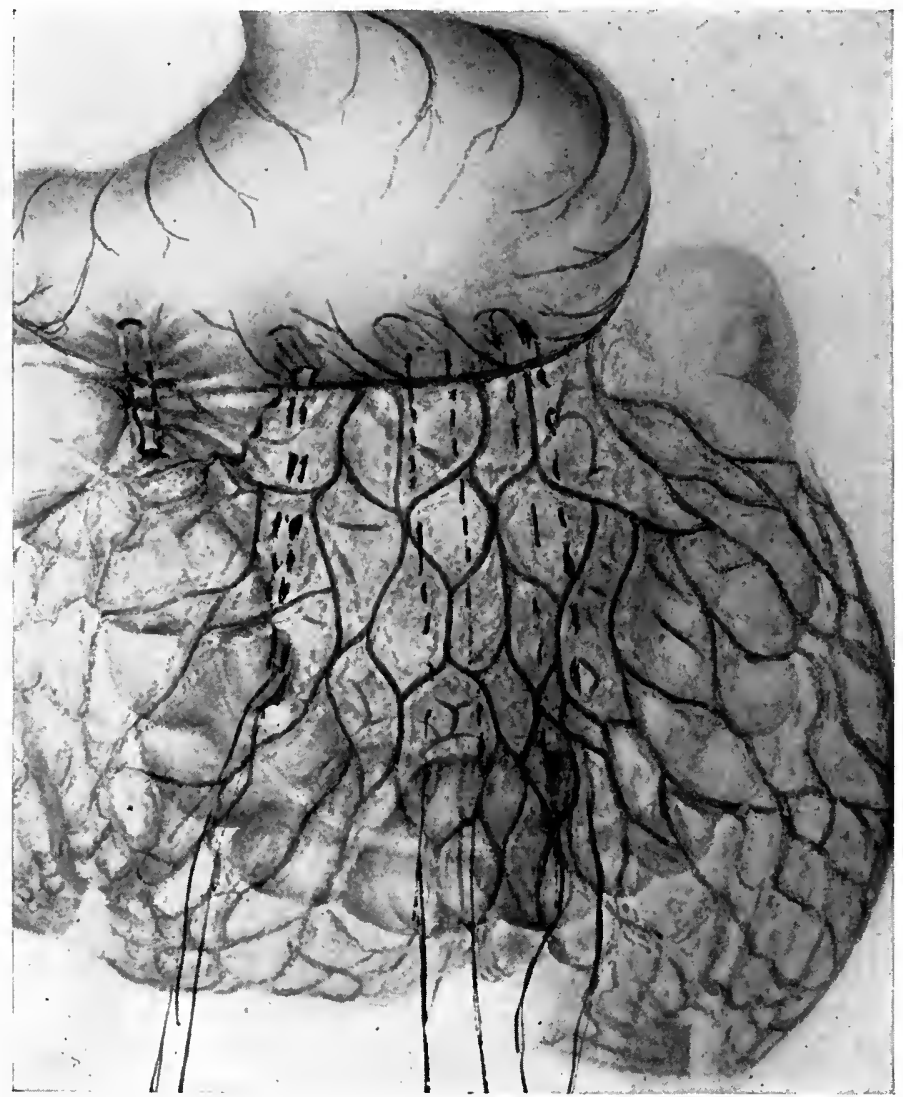

Fig. 81.-Author's method of shortening the gastrocolic ligament.

and I can clearly see every vessel, and can easily avoid injuring them by passing the needle through the clear interstices. When we tie these four or five sutures, of which that farthest to the left connects the fundus of the stomach with the portion of the colon transversum lying nearest to the left flexure, while that farthest 
to the right connects the pyloric end with the portion of the colon transversum lying close to the right flexure, you will understand that ligamentum gastrocolicum has become a thick ligament a few centimetres in length, which holds the colon transversum close up to the greater curvature of the stomach. This must be performed before the gastropexy and while the stomach and intestine are lying

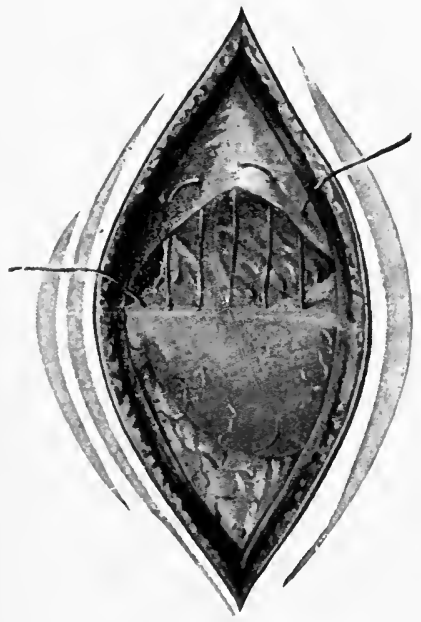

FIG. 82.-Duret's gastropexy.

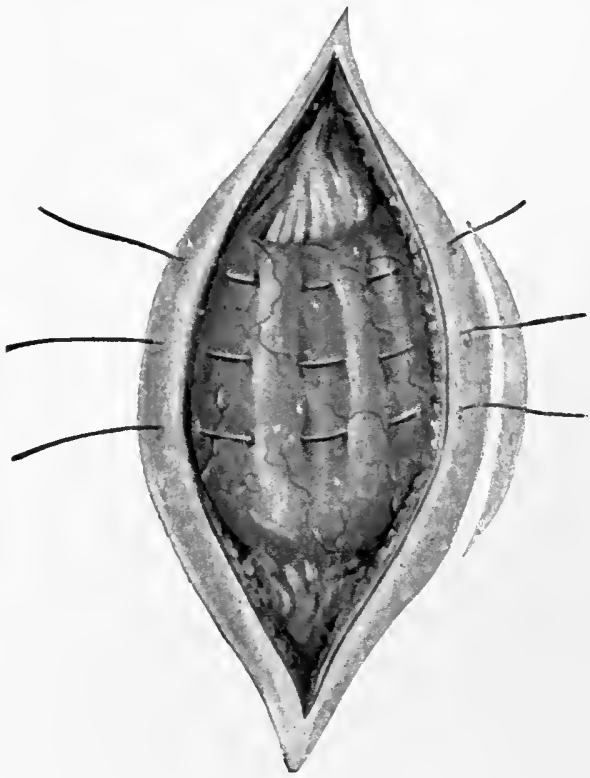

FIG. 83.-Rovsing's first method.

conveniently outside the abdominal wound. The colon is then replaced and one proceeds with the gastropexy.

Before I describe to you the technic of my operation in the case of the two patients here in question, I shall give you a short historical survey of how, for my part, this operation has developed. When, in 1897, I for the first time thought of performing gastropexy on a patient, who, by-the-bye, was admitted like our last patient with a diagnosis cancer ventriculi, it appeared, after searching the literature, that such an operation had been performed only 
once before; namely, by Professor Duret of Lille, and after the following method (Fig. 82) : He made an incision through the skin and muscle from processus ensiformis to the umbilicus, but incised the peritoneum only at the lowest angle of the wound, and, as you see in the illustration, then fixed the lesser curvature of the stomach to the untouched peritoneal surface high up with a single silk thread,

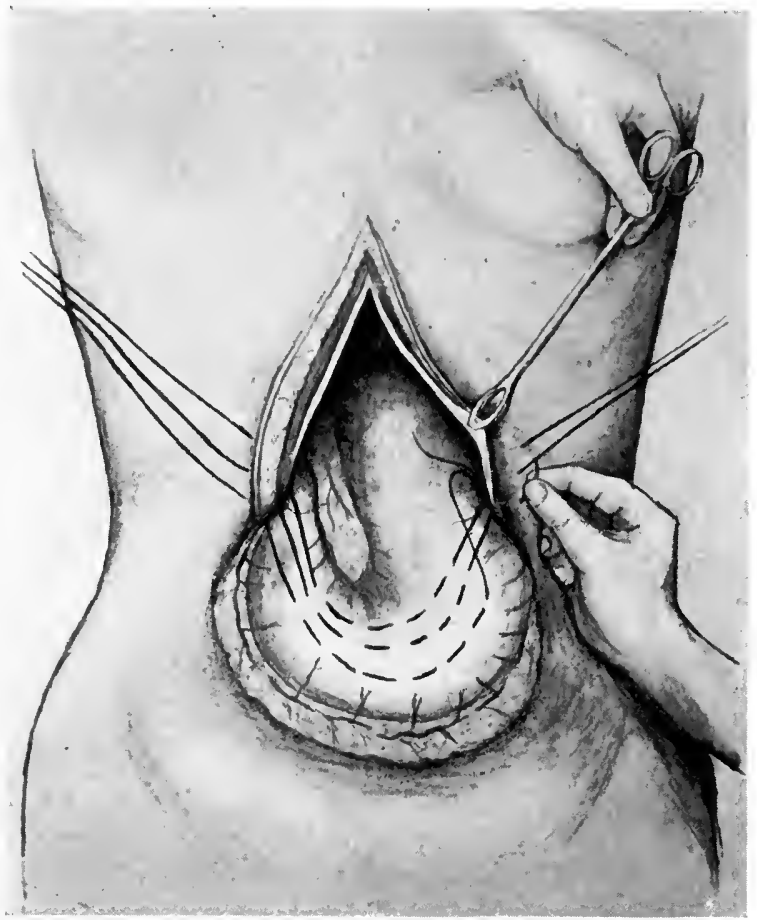

Fig. 84.-Author's method of gastropexy.

which was led alternately in and out through the serosa covering of the stomach and peritoneum parietale. The thread was finally led out through musculi recti, and its ends tied together subcutaneously above this. For several reasons this mode of procedure did not appear to me quite satisfactory. Firstly, I thought that a single thin silk thread was an unsafe means of suspension, and, secondly, 
I thought it a precarious plan to fix the pylorus-which should normally lie deep and be mobile-to the anterior abdominal wall; and, finally, I perceived that the stomach must unavoidably suffer a serious shrinkage and folding by the tying of the silk thread in the centre line. With my first case I made use of the following method-a method which I have employed, essentially unchanged, with my first

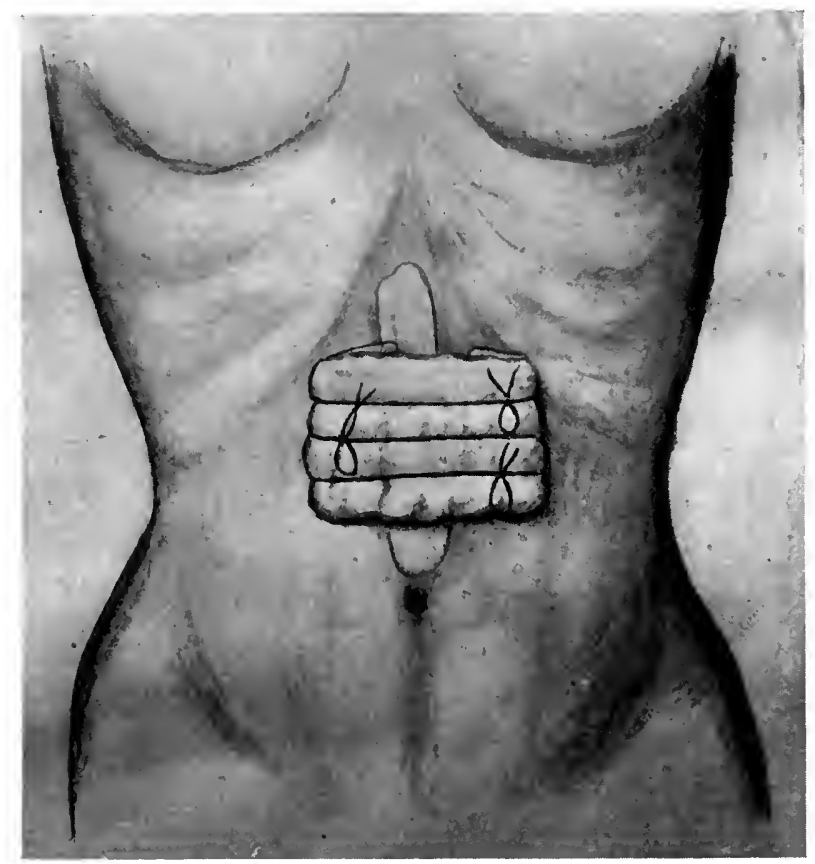

Fia. 85.-Author's method of gastropexy. The silk sutures are held over a glass plate.

eighty cases (Fig. 83) : I passed three strong silk threads in and out through the serosa covering of the anterior surface of the stomach, each thread being parallel with the axis of the stomach, though at a different level, pars pylorica being left alone (Fig. 84). The uppermost silk thread was placed just under curvatura minor, the centre one two centimetres below this, and the lowest three centimetres above the greater curvature. The ends of the 
threads were led out through the abdominal wall on either side at a distance of three to four centimetres from the central line. To promote adhesions, a series of small scarifications was made with a needle on the serosa of those surfaces of peritoneum parietale and viscerale which it was desired should adhere. Then the peritoneum was joined with catgut, and the muscles and skin with aluminum bronze. When the wound had been covered with collodion and cotton wool, I applied a glass plate wrapped in sterile

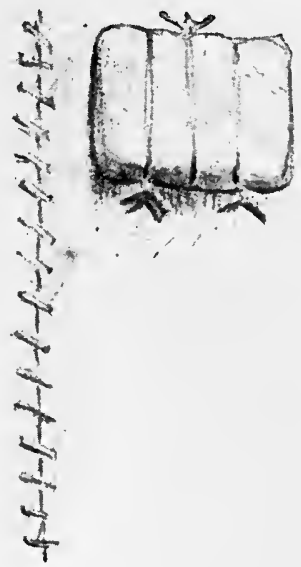

Fig. 86.-Author's seeond method. The three gastropexy threads are seen tied over the gauze-wrapped glass plate to the left of the centre line. gauze, and of somewhat larger dimension than the part of the stomach which had to be fixed, and over this glass plate were tied the three silk sutures, which had been previously drawn to enable the anterior surface of the stomach to lie flat and close to the abdominal wall (Fig. 85). These threads were allowed to remain for four weeks, and were then removed, as a complete coalescence had taken place.

Although the results of this method had been particularly satisfactory in my first ninety cases, especially as regards the durability of the fixation, yet, for reasons which $I$ shall state presently, I employed the following modification with the next sixty-nine cases (Fig. 86): I place the three threads across the longitudinal axis of the stomach so that each thread runs from the lesser to the greater curvature in and out through the serosa, and in this way it follows that only the fundus portion is broadly fixed to the left of the centre line, as all three threads are led out through the abdominal wall to the left of the line of the wound, and here tied over a glass plate in the same manner 
as with my first method. In this way I succeed in leaving the entire antrum pyloricum free, and it is essentially the great importance ascribed to this portion of the stomach, as regards digestion, by Cannon and others through their later experiments, that has decided me to adopt this modification. As you perhaps know, it ought to be possible for this pre-pyloric part to be shut off entirely from the fundus part by circular contraction, when the solid food thus shut up is subjected to a process of crushing and kneading by the extremely powerful muscular contraction of this part. Provided this is so, I thought it of great importance to preserve a perfectly free contraction of this part of the stomach. Thus it is only theoretical reflection which has led to this modification, which now further experience has proved to be far inferior to the first method. The systematic after-examination of all my cases up to January, 1911, shows that, while the first method gave sixty complete cures in ninety-four cases, the latter gave only twentynine cures in sixty-nine cases. For this reason $\mathrm{I}$ have returned to the first method and employed it in my last thirty cases, the results so far being excellent.

Many theoretical objections have been raised to this operation, which essentially are that the broad fixation of the stomach must involve such restriction and disturbance of its motility that stagnation, dilatation, etc., must ensue.

This theoretical fear, which, a priori, did not seem unjustified, has been proved quite unfounded by practical experience. My former assistant, Dr. M. T. Krag, who unfortunately was interrupted by a fatal illness in an extensive and promising work on the results and indications of gastropexy, examined with great care all those patients who had been operated on by me, and whom he was able to trace, and found that even the first patients-those operated on nine to ten years ago-showed the stomach completely empty eight hours after a test-meal. 
Another thing is that the formation of adhesions in the abdomen should always - so far as possible-be avoided, because they may give rise to pains; but if any one can suggest an effective operation for gastroptosis which avoids the formation of adhesions, I shall be the first to discard my method in favor of such a one; but those methods hitherto proposed to supersede mine have not stood the test.

Independently of each other, as it appears, three surgeons-Stengel, Bier, and Beyea-have proposed to raise the stomach by shortening the ligaments by which it is suspended: omentum minus and ligamentum gastrohepaticum. This ought to be achieved by passing three silk threads transversely in and out through the ligament, so that this is shortened when the threads are tied. This operation, which theoretically is so simple, I have found to be quite useless in practice, because that duplicature of the peritoneum in which the stomach is suspended is, in the case of gastroptosis patients in particular, as fine as the thinnest tissue paper, and so fragile and perforated that it is quite unadapted for sutures. As gastroptosis is mainly due to the inability of this ligament to carry the stomach, it seems far from rational to shorten it when one cannot simultaneously strengthen it. Even if the operation has a temporary result, the same power-the weight of the replete stomach, etc.-which originally caused the trouble will again stretch out the ligament, and the stomach will sink down once more. Sometimes omentum minus is quite loose, and hangs down from the lesser curvature like a small, fatty, tongue-shaped apron. In such cases I have attached this to the diaphragm with a silk thread, and have used this suspension of the lesser curvature as an auxiliary operation to my usual gastropexy, or with Coffey's operation.

The latter operation, which you will easily understand 
by looking at this sketch (Fig. 87), consists in attaching omentum majus to the anterior abdominal wall, level with the greater curvature of the stomach. This operation, whereby one in some manner forms a second diaphragm in the abdomen consisting of the omentum, colon transversum, and mesocolon, on which the stomach might be expected to rest and be supported, greatly recommended itself to me a priori, and I therefore attempted it in a series of cases. Unfortunately, it has not fulfilled my expectations, as, after a transitory improvement, the old symptoms have reappeared. This is due to two facts: (1) the omentum is not strong enough to withstand the weight of the replete stomach, and (2) the impossibility of attaching it firmly throughout its entire width. On both sides, and especially on the left, an opening remains through which the fundus of the stomach can prolapse.

Finally, in a relatively large number of instances, gastroenterostomy has been performed in cases of true gastroptosis,

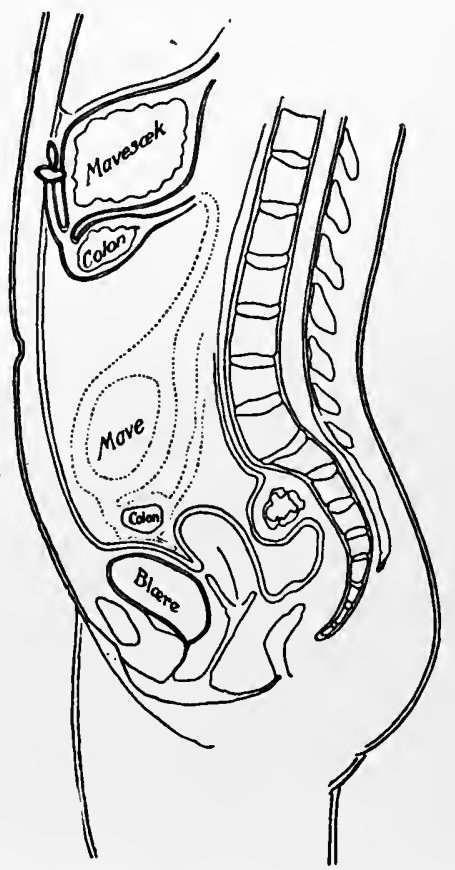

Fig. 87.-Coffey's operation for gastroptosis: omentopexy. either by mistake because the symptoms have been misinterpreted, or because one has erroneously assumed that the symptoms of gastroptosis are due to delayed depletion,-i.e., retention. Kemp, in his clever work on the conditions of the discharge of the stomach contents, has certainly shown that in some cases of gastroptosis the discharge proceeds more slowly than it normally should; that is to say, it takes five to six hours; but this has scarcely 
any significance as regards the symptoms-if one can call such an insignificant delay pathological: firstly, because the cardialgia appears immediately after meals; and, secondly, because all the symptoms generally disappear when the patient is confined to bed, with the exception of the "atony" - as this delayed discharge is called-which seems quite unaffected by the stomach gliding up to its normal position.

As a rule, however, there is no delay-not to mention retention-with even the most severe cases, and gastroenterostomy must, therefore, a priori, seem useless. Moreover, it has proved to be not only useless but generally responsible for a considerable deterioration in the patient's condition, bringing him "out of the frying pan into the fire." To the hitherto painful symptoms is now added a series of quite new ones, originating in a more or less pronounced circulus vitiosus! It is easy enough to understand that the coil of the jejunum which forms the gastroentero-anastomosis is drawn down by the subsiding. stomach as soon as the patient gets up, and that tension and bending of the coil must result therefrom, which involve a difficulty in the outflow from the stomach and duodenum, and often an influx of gall to the stomach.

I venture to say that the injurious effect of gastroenterostomy on the gastroptosis patients is so invariable that I must warn you most strongly against its use as a serious fault, unless an undoubted condition of retention or a complication with an ulcer provides the indication; if undertaken, it should be combined with a gastropexy.

I have in this ward just now two patients who illustrate this in a sad way. They have both suffered from severe ptosis, for which they were treated with gastropexy, respectively, three and four years ago, at two different provincial hospitals, with the result that their condition 
improved greatly. Twelve months ago they both suffered from pain in the cardia after working hard, probably the result of adhesions, though not nearly so much as formerly. They were admitted to two different hospitals in this town, where the old gastropexy adhesions were loosened and gastro-enterostomy performed. The patients have been perfectly miserable ever since, because not only have the pains increased, but nausea and vomiting of gall have been added to the old symptoms. You see here the patients, and will notice how emaciated and exhausted they appear. With both of them we have tried a dietetic treatment, daily washing of the stomach, and rest in bed, but in vain. We brought about an improvement in one of the patients by removing the gastro-entero-anastomosis which had been previously done. After having located the anastomosis, which here was on the posterior surface of the stomach, I divided the anastomosis between two clamps, after which I closed the stomach and intestine separately with a double row of silk sutures. I loosened as far as was possible the numerous and extensive adhesions which had formed between the coils of the small intestines, and thereby straightened out the resultant bends. Unfortunately, the adhesions will re-form to a great extent, but by the operation we have succeeded in stopping the nausea and vomiting, and the gall and intestinal contents no longer enter the stomach. In this way we have remedied the essential harm caused by the gastro-enterostomy. I have proposed the same treatment to the other patient, but she refuses it for the present, as she feels too weak to undergo another operation.

You have now seen that none of the methods hitherto proposed can show results which are even fairly satisfactory. However much we may wish to avoid fixing an organ like the stomach to the abdominal wall by a broad, firm surface of adhesions, this has, nevertheless, proved 
quite necessary in order to obtain a real and lasting result. And, if you will now glance at my statistics of the gastropexies performed in accordance with the methods which I have described, you will see that we have in this operation a fairly safe and very valuable remedy for the removal or relief of the severe sufferings present in the advanced cases of gastroptosis, which are not susceptible to medical treatment.

Since 1897, when I performed my first gastropexy, up to January 1, 1911, I have personally performed the operation 163 times, and have received information from other Scandinavian surgeons of 93 operations performed in accordance with my method. All of these 256 patients have been traced and their condition since the operation carefully examined, with the following result:

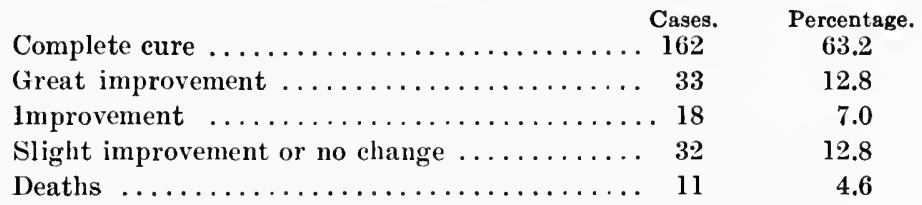

In my personal series of 163 cases, the statistics are as follows :

Statistics of

Personal statistics. Scandinavian surgeons.

No. of cases. Percentage. No. of cases. Percentage.

\begin{tabular}{|c|c|c|}
\hline Cure $\ldots \ldots \ldots \ldots \ldots \ldots . . . . .$. & 92 & $56.4\}$ \\
\hline Great improvement $\ldots \ldots \ldots \ldots$ & 24 & 14.7 \\
\hline Improvement $\ldots \ldots \ldots \ldots \ldots$ & 18 & 11.0 \\
\hline Slight improvement or none .. & 21 & 12.8 \\
\hline Deaths $\ldots \ldots \ldots \ldots \ldots . . . . . .$. & 8 & 4.9 \\
\hline & $\overline{163}$ & \\
\hline
\end{tabular}

Firstly, with regard to the mortality, it may well be said that a mortality of 4.6 per cent. is, per se, small. But on analyzing the cause of death in the individual cases the real mortality from gastropexy proves to be far smaller. As concerns my own patients, two died a fairly long 
time after the operation from tuberculosis of the lungs, while four extremely exhausted patients died from bronchopneumonia during the week following the operation, but without any morbidity at all in the peritoneal cavity.

On the other hand, two died from ileus; the one from a gastro-duodenal ileus due to the strangulation of the duodenum over an old adhesion, the other from ileus of the small intestine due to an accidental strangulation of a coil of the bowels over an old adherence due to an old hysteropexy. With regard to the three deaths mentioned in the statistics of the other Scandinavian surgeons, two of them had absolutely nothing to do with gastropexy as such; the one was due to a casual perforative appendicitis, the other to bleeding from a gastro-enterostomy wound performed simultaneously with gastropexy. In the third case, however, death must be ascribed to gastropexy, inasmuch as it was due to ileus of the stomach, the result of neglecting to do a hepatopexy; the liver prolapsed across the pyloric part of the fixed stomach and caused obstruction. The mortality with gastropexy proper is, then, three in $256=1.17$ per cent.

With regard to the recoveries, 71 to 75 per cent. of the patients were cured to the extent of being relieved of their sufferings, of regaining their health and strength, and, from being incapable, depressed, miserable wrecks, have become able-bodied, healthy, and happy people.

I wish to draw your special attention to this: that gastropexy in 76 of my 163 cases has relieved the patients of their constipation of many years' standing which was not influenced by any other treatment! All these cases were equally severe, and some were more serious than those in which Sir Arbuthnot Lane advised extirpation of the colon or ileosigmoideostomy: operations the dangers of which are extremely great as compared with those of gastropexy. 
There then remain about 25 per cent. of the cases where the effect of the operation has not been satisfactory; in 11 per cent. the condition was improved considerably, but in 12.8 per cent. the improvement was quite insignificant or nil. Concerning the cause of the bad results, an analysis of these cases warrants the hope that still better results may be attained in the future.

Here I shall first point to the circumstance, to which I have already drawn your attention, that in a series of 73 cases I employed, from fear of fixing the prepyloric portion, a method which a revision of the results has proved to be greatly inferior to my original method, inasmuch as the cures were 20 per cent. less in number. It is obvious that a future systematic carrying out of the original method of operation by a broad adherence of the stomach to the abdominal wall will improve the results considerably. On other points, also, a change in the method of operation will involve improvement in the results.

In all cases where ligamentum gastrocolicum is considerably elongated one does not obtain by gastropexy alone sufficient elevation of the colon to relieve the constipation. In order to obtain this a special operation is required, and some of my less successful cases in earlier days are surely due to my non-appreciation of this fact and to later experiments with various inferior methods. Here the correct operation is the shortening of the omentum and the mesocolon by basting this with the aid of a row of thick catgut threads, which commence in the serous coating of the colon and end at the greater curvature (see Fig. 81). For me this has proved to be a more ideal method than colopexy and omentopexy, using the anterior abdominal wall, because, while these restrict the mobility of the large intestine and in addition often cause adhesions in some cases, still, shortening of mesocolon and the omentum raises the intestine without restricting its mobility. The result seems to be per- 
manent. You here see the radiographs of one of the first cases I operated on. On the first you see clearly the great distance between the greater curvature and the colon, and on the last, which was taken three years after the operation, you see how beautifully the colon has remained in the position where it was placed at the operation. I think, therefore, that a systematic employment of this operation as an accession to gastropexy in all cases with elongated ligamentum gastrocolicum will increase the number of the complete cures.

In some cases an imperfect diagnosis has been the cause of the bad result, as, for instance, the overlooking of an ulcer in the stomach or in the duodenum, or of cancer in another organ. The improved diagnostic methods, and especially the gastroduodenoscopy introduced by me, will probably decrease the number of such mistakes in the future.

Finally, there is a very important point which explains the difficulty of obtaining in many cases a complete cure for these patients, viz., the many consequences of enteroptosis and lacing which are coördinate with gastro- and coloptosis. Firstly, the narrowing of the lower aperture of the thorax may be so considerable that there is no longer any room for the liver and the stomach, and therefore it is impossible to fix the stomach in a satisfactory manner without jamming it, just as it is impossible to get the colon sufficiently raised up to straighten out its folds and bands.

Even if the condition of the patient improves somewhat, the pains and the constipation in particular will nevertheless continue after the gastropexy.

Where the stomach is concerned I think that one may obtain better results at times by abandoning any idea of its reposition, and be content partly by fixing it lower down in the abdomen where there is room for it, and partly by making the abdominal cavity more capacious with a plas- 
tic enlargement of the abdominal wall. In many cases of gastroptosis one finds the space above the umbilicus so contracted that it is difficult to insert even a finger between the vertebral column and musculi recti; if one fixes the stomach up here where it ought normally to lie, it is jammed.

In such cases I have obtained excellent results by widening the space between the rib borders by splitting the

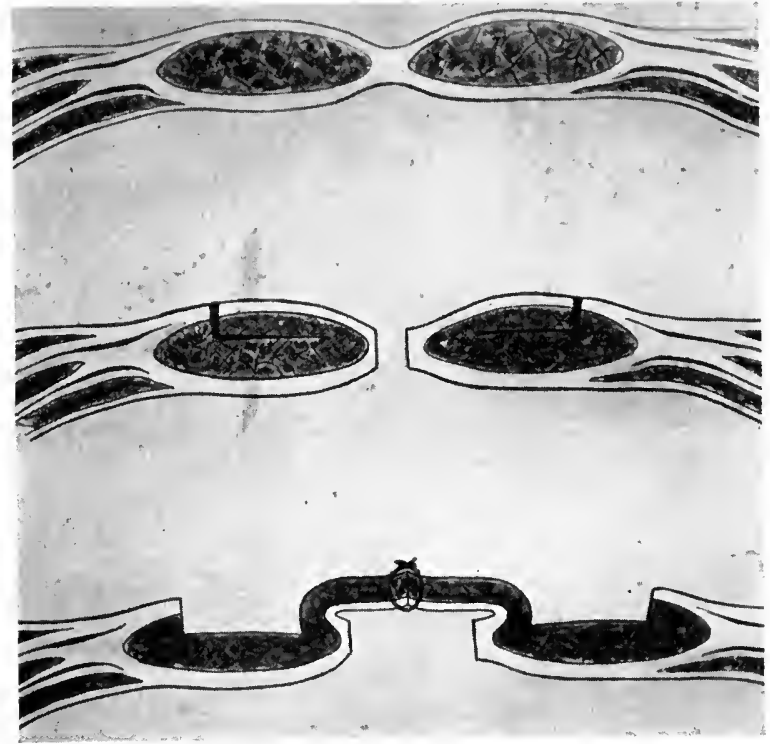

Fig. 88.-Author's method to enlarge the abdominal wall in cases of virgunal ptosis.

musculi recti and proceeding as follows: The anterior half of the rectus muscle and sheath are dissected up and from each side are folded toward the centre and then joined in the median line. This is shown in Fig. 88.

In those cases where the colon is prolapsed it is always difficult to make room for the stomach. The bends at the flexures become sharper and sharper, and become fixed by adhesions and bands which form between the peritoneal surfaces at the angles. The constipation becomes more and 
more obstinate, the auto-intoxication increases, and the pains render these patients utterly miserable.

Here, in this fortunately small group of cases, we find the real indication for Arbuthnot Lane's operation of ileo-sigmoideostomy, which I have employed with excellent results in two otherwise quite desperate cases. It was most interesting to notice low the distaste for food and the malaise of many years' standing gave place, after the operation, to a ravenous, almost immoderate, appetite. In one case the purgation following the operation showed a tendency to turn into diarrhœe, which, however, I was able to keep in check by dieting.

The coexisting ptosis of the other abdominal organs, especially the liver and kidneys, greatly influences the results of gastropexy. An extensive hepatoptosis would endanger the result from a gastropexy unless the ptosis of the liver were corrected, inasmuch as the liver would prolapse after the operation and lie across the stomach, producing pain and the possibility of ileus of the stomach, as was the case in one patient reported by Hertel.

Therefore, in cases of hepatoptosis, hepatopexy should always be performed previous to the gastropexy. Personally, I have had to perform the combined operation in sixtyeight of my patients. In performing the operation I generally use silk sutures (Fig. 78) to attach the peritoneal covering of the liver to the under surface of the diaphragm and, in addition, suture the ligamentum teres (Fig. 79). This ligament is first divided between ligatures and resutured to the diaphragm with strong silk threads. In addition, it is important to scarify the opposing surfaces of the diaphragm and liver in order to promote adhesions.

Sometimes, as the result of tight lacing, a large hypertrophied left lobe of the liver results, which pushes down the lesser curvature and makes the replacement of the stomach quite difficult. In four cases I have been obliged 
to resect such an hypertrophied left lobe in order to perform gastropexy. As previously described, the resection of the liver is accomplished by use of Roux's angiotribe.

In a few cases where the gastropexy has failed to relieve the pain and constipation I have found that one or both kidneys were prolapsed, and the patients did not recover entirely until after a nephropexy. This was so in ten of my cases. A nephroptosis on the right side often involves prolapse of the colon ascendens and pressure on the crecum whereby the pain and constipation persist.

In closing the lecture I wish to impress upon you that the operation of gastropexy has been employed generally in those cases which have been treated for years by medical men and have been given up as hopeless, and the results which we have obtained have been reached after medical treatment has failed. You can, then, readily understand how important the surgical treatment of gastroptosis is as a supplement to the medical treatment. 


\section{LECTURE XIII}

\section{CHRONIC ULCER OF THE STOMACH}

We will take up to-day the consideration of a case the seriousness of which becomes evident when one views the patient. He is an unmarried teacher, fifty-three years of age, whose body is emaciated and his wax-white complexion and anxious expression give you the impression of a man who is seriously afflicted. His history is very typical and will suggest to you the probable diagnosis. In childhood and youth he was perfectly healthy. At eighteen years of age he began to suffer from repeated attacks of dyspepsia, evidenced by severe pain in the epigastrium, nausea, and occasional vomiting. By living carefully, the spells passed without medical assistance, and frequently several years intervened between the attacks. They caused him no alarm until four years ago, when one day, after suffering for some time from increasing nervousness and languor which he himself could not explain, and without previous symptoms referable to his gastro-intestinal tract, he suddenly vomited considerable quantities of blood. Some time afterward it was noticed that the feces were distinctly blood-stained, but no special attention was paid to these attacks, and the patient felt fairly well until six months ago, when he again began to suffer from piercing pains in the epigastrium, accompanied by nausea and pyrosis. The attacks were especially apparent in the morning, and improved after eating something. Only once has he had a slight vomiting of blood-tinged mucus, but he has noticed that since then the feces have been tar-colored. He went about and did not diet himself, although his condition steadily deteriorated; and not until he was so languid and tired that he could work no longer was he admitted to the 
medical ward of this hospital on October 22, 1908. When admitted, the patient was found to be somewhat aged, though not particularly thin or pale, while the examination of his blood showed a hæmoglobin estimation of 95 per cent. An examination of the stomach contents made by the patient's physician slortly before he was admitted showed 210 c.c. of coffee-colored; blood-stained, thin fluid an hour after Ewald's experimental meal. It contained free hydrochloric acid of 67 with a total acidity of 90 . It was found that the excretions were continuously tar-colored and contained blood.

After these typical symptoms the diagnosis ulcer of the stomach was made, and the patient was put on a strict milk diet. In spite of this, which has now been carried out for three weeks, the patient has steadily and rather rapidly deteriorated. The cardialgia, indeed, has become somewhat less, but the patient's strength has decreased to an alarming degree, his skin and mucous membranes have become paler and paler, a second examination of his blood has shown that the quantity of hæmoglobin has decreased from 95 per cent. to 65 per cent., and one finds the red blood-corpuscles correspondingly diminished to 2,550,000. Therefore Professor Faber has not dared to continue the medical treatment any longer, but has moved the patient to this ward with a view to operative treatment.

Here what we must essentially discuss to-day is the possibility of saving this patient by operative measures but, although the case seems so typical and the diagnosis so certain, yet we must necessarily occupy ourselves first with the question of diagnosis, which, for the surgeon who has to base his plan of operation on it, requires a far more detailed test than that which the medical man, strictly speaking, requires to direct his treatment. In this case one can have no doubt that there is an ulcer; in his previous history we have a hæmatemesis, and at present we have a severe 
melæna, while blood has been evideneed in the stomach contents. To the medical man this in itself is sufficient for the institution of a correct medical treatment, but for the surgeon, who is compelled to work operatively, the question as to the seat, extent, and nature of this ulceration forcibly obtrudes itself. After all, is this an ulcer of the stomach, as sounds so certain from the history? To this my answer is, that I can well consider it highly probable that we are faced with an ulcer, though I am far from being sure of this, because again and again it has happened to me, when operating on cases with symptoms of ulcer as typical as this and even more so, that I have found the stomach free and the ulcer situated in the duodenum, and in this case especially there is one point which makes me cautious with the diagnosis; namely, the severe melæna without hæmatemesis during the later stage of the patient's illness. It is easy to understand that duodenal ulcer, as a rule, reveals itself only through melæna. Only by severe bleeding from an ulcer which is situated close to the pylorus, or which has narrowed the passage through the duodenum, do large quantities of blood stream into the stomach, irritating this and causing vomiting. So far this case might quite well be duodenal ulcer, but we must not forget that the stomach ulcer also often causes melæna without causing hæmatemesis. Another symptom which might suggest ulcer of the duodenum rather than ulcer of the stomach is the localization of the patient's pains, especially his pain on direct pressure; when I make this pressure you can easily see from the man's expression that the pain is to the right of the centre line. If the pain had been localized exclusively to the left of the centre line, then it would have spoken very decidedly in favor of stomach ulcer, while the pain to the right of the centre line, as we now find it, speaks as much in favor of duodenal ulcer as of stomach ulcer in the vicinity of the pylorus. Were the pain and the tenderness on 
pressure farther away, about in the mammary line, it would speak strongly in favor of duodenal ulcer. You will easily understand how important it would be to the surgeon to be able to decide in advance not only if the ulcer had its seat in the duodenum or the stomach, but, if in the latter, to be able to decide also whereabouts in the stomach. Great efforts have, therefore, been made to attain this object.

Even in 1881 Mikulicz constructed a gastroscope for introduction through the œsophagus, but, though he and his pupils have worked ardently during the past twenty-seven years to improve this bloodless gastroscopy, they liave not yet succeeded in making the method of practical value. One has next tried to find a base from which to ascertain not only the presence but the localization of the ulcer, through the results of the modern examinations of the chemical and motory function of the stomach. For a long time, also, it was thought that in this way the problem could be solved, because we naturally thought that the stagnation of food remains in the stomach and the dilatation must be a sure sign that the ulcer had its seat in, or close to, the pylorus, and had produced a cicatricial or spastic stenosis; while the lack of retention in otherwise typical cases of ulcer must be synonymous with the ulcer having its seat a certain distance from the pylorus. Unfortunately, our increasing operative experience has shown us how untenable these conclusions are. It has been proved that the conditions of greatest retention can be owing to ulcers which lie rather far from the pylorus, and that they are then due either to extensive infiltration of the wall of the stomach, with ensuing paralysis of its motility, or to formations, cohesions, or folds in the wall of the stomach. Conversely, we can have a somewhat considerable contraction of the pylorus, so considerable that a slate-pencil can scarcely pass, and yet no retention. This is what we term compensatory pyloric stenosis, inasmuch as the obstruction is counter- 
balanced by the hypertrophy of the muscles of the stomach and the thereby increased motility. Finally, a retention may also be due to a contracting ulcer in the duodenum. You see that, however valuable the proof of retention of the stomach contents may be as regards the diagnosis of ulcer, it affords us no certainty as to the location of the wound.

The circumstance that the stomach-tube, though introduced with the greatest care and without any difficulty, causes severe bleeding by coming into contact with some part or another of the wall of the stomach, can sometimes reveal not only the presence of an ulcer, but also the approximate position-especially so with ulcers just below the cardia or in the region of the fundus. But this is by no means a safe manner of proving the presence of an ulcer, and in cases where there is, or has been, severe hæmatemesis or melæna the introduction of a tube is altogether improper. With this patient, therefore, we, like the physicians in the medical ward, have refrained from examinations which demand the introduction of tubes, and are thus quite debarred from any guidance in our decision as regards the seat of the ulcer.

I take the opportunity to impress upon you, as an invariable maxim, never to carry out the usual methods of examinations to determine the function of the stomach, which demand the introduction of tubes, in patients who have had severe melæna and hæmatemesis in the immediate past. You can thereby cause irreparable harm, partly by bringing about a perforation and peritonitis, partly by loosening that thrombus which, perhaps, for the moment ensures the patient's life, and thereby bring about a fatal bleeding of the coronary artery. It is necessary to impress this upon you, because nowadays the practitioners start work trained to perform all these examinations with test-meals and inflation of the stomach, and the filling of this with large quantities of water to test its capacity, and 
prepared with each case they encounter to let the patient undergo the whole curriculum of diagnostic examinations. Although it should be evident how dangerous this is where there has been severe hemorrhage, yet one often sees that the patients, without regard to this, have been subjected to the whole series of experimental meals, etc. But I will go a step farther and ask you to remember that many ulcers which have never bled may be just on the verge of perforating. I will, therefore, counsel the greatest caution with these examinations: caution with the introduction of the tube and caution with the aspiration, but, above all, care with the dilatation of the stomach, particularly with fluid, which adds greatly to the weight and pull on the stomach, and this can easily loosen the adhesions and cause a deeply-rooted ulcer to perforate.

To return to our patient, here we have to be content with the rougher clinical facts, which only allow of the conclusion that a continuously bleeding ulcer exists, which most probably has its seat in the stomach, but possibly in the duodenum. The anamnesia, the course protracted during many years, tells us that we have before us a typical chronic ulcer, but we cannot with certainty exclude the possibility that this ulcer, as so often happens, may be on the point of malignant cancerous degeneration. I do not think so, but the probability is there.

Have we indication here for a surgical intervention? Undoubtedly! Every chronic stomach ulcer ought to be operated on, provided that the patient is not too much run down. This patient should have been operated on long ago to obviate the four great dangers which exist in the presence of chronic stomach ulcer-i.e., inanition, hemorrhage, perforation, and cancerous degeneration. This patient has waited so long he is now threatened by three of these dangers. For years past a very insufficient nutrition has consumed his strength, and the new danger, the con- 
tinued hemorrhage from the ulcer, becomes twice as great by attacking such a debilitated individual. Finally, such an incessant and severe hemorrhage as this makes it most probable that it is due to erosion of a large vessel at the base of an ulcer, which is on the verge of perforating, and perhaps has perforated, the wall of the stomach. Here, then, it is not so much a question of whether there is indication for an operation, but rather whether the general condition of the patient will permit an operation-whether he is not too debilitated to stand the necessary encroachment. What we must here particularly turn our attention to is the condition of the blood. The percentage of hæmoglobin as well as the. number of the red blood-corpuscles has, as you heard, sunk to half of what is normal. This may be serious, but does not yet contra-indicate an operation. I have successfully operated on patients whose hæmoglobin has fallen as low as 16 per cent., due to hemorrhage from an ulcer. But, if it is under thirty, one must always hesitate before proceeding to an operation by which loss of blood cannot entirely be avoided, and where there is a risk of great loss of blood. As our patient still has 60 per cent. hæmoglobin, and as the examination of lung, heart, and kidney shows no special complications-one must always reckon on an incipient degeneration of the myocardia in such cases-it is my opinion that the condition of the patient still allows of an attempt to help him by an operation.

The question now is: What shall this operation consist of? A few days ago every surgeon would, and even to-day most surgeons will, answer: Naturally, a gastro-enterostomy must here be performed, an operation by which, as you all know, we form a new connection between the stomach and the intestine, because (1) we either, like the Swiss surgeon Wölfler, the father of this important operation, join a coil of the jejunum, taken about fifty centimetres from its 
commencement in fossa duodenojejunalis, with the anterior wall of the stomach, by leading the jejunum up in front of colon transversum and fastening it close above the greater curvature; or (2), ad modum von Hacker, join the jejunum to the posterior surface of the stomach, for which one takes a much shorter coil of ten to fifteen centimetres. The mainpurpose of this operation was to procure a free outlet for the contents of the stomach, in the event of a contraction of the natural passage - the pylorus-which so often results from an ulcer in regio pylorica. It soon appeared that the idea was perfectly correct: it was remarkable to see how quickly and surely this operation relieved the serious and morbid attacks common to pyloric stenosis. It was then tried in case of ulcers located elsewhere in the stomach, and here also, in many cases, the morbid symptoms disappeared shortly after the operation. It was thought that the rapid emptying of the stomach had the effect that the contents of the stomach, and especially the sour gastric juice, no longer came in contact with the ulcer, and that this then healed. Subsequently there came a time when gastroenterostomy dominated gastric surgery, and was absolutely the only correct operation in all cases of stomach ulcer, even if there was no sign of retention or of obstruction to the passing of food along the natural passage.

Doubting voices were quickly raised against this panegyric, and I think I was one of the first to raise a note of warning against such a general and systematic use of this operation, which in itself was so excellent. A priori, it seemed to me quite obvious that the real domain and indication for this operation must be the state of food retention in the stomach. It seemed inconceivable to me that a gastro-enterostomy could have a hæmostatic effect in cases of acute and severe ulcer hemorrhages, and, finally, it seemed to me very daring to restrict one's self to a gastroenterostomy in cases of deeply-rooted, perforating ulcers. 
By my increasing personal experiences these doubts became, on the whole, confirmed, and by the study of the rapidlygrowing literature, especially of the large, carefully-compiled statistics, were further strengthened.

A perusal of the statistics compiled from my own material, which you see summed up here on the table to January, 1909, and which comprises 125 cases operated on for ulcer, is very well adapted for placing the pros and cons of gastroenterostomy in the right light, and for the demarcation of its legitimate domain. It appears, first, that the operation is not especially dangerous, so far as the patient's life is concerned, though it is incorrect to characterize the operation as safe, as some surgeons have done--Kocher, for example. Such an operation naturally always involves a certain danger to life. In my 95 cases of gastro-enterostomy I had six deaths, but when we further examine these, it appears that only one can be assigned directly to the operation itself. In this case a man fifty-three years old developed an ulcus pepticum in the region of the healed suture line of the gastro-enterostomy, which gradually extended, destroying all the coats, and gave the patient perforative peritonitis; a very anæmic and debilitated man, seventy-two years of age, passed quietly away in his sleep the day following the operation, for no other ostensible reason than old age and infirmity. If, on the other hand, we consider the other deaths, we must conclude that they were not due to the operation itself; however, they are no less interesting on that account, because they cast some doubt on the curative effect of the operation. In this way I have had three cases where the patients, a fortnight, a month, and three weeks respectively after the operation, have suddenly had violent hemorrhages from their ulcer, so violent that every hope of salvation was excluded. In two of the cases it was stomach ulcer on the lesser curvature; in the one duodenal ulcer, at the base of which we 
found, on autopsy, a large, gaping arterial lumen. The sixth patient died from an infectious icterus, which was due to a duodenal ulcer, which had involved the papilla and blocked the gall-duct.

AUTHOR'S OPERATIONS FOR GASTRIC AND DUODENAL ULCER.

\begin{tabular}{|c|c|c|c|c|c|}
\hline Class of operation. & $\begin{array}{c}\text { Total } \\
\text { number. }\end{array}$ & Deaths. & $\begin{array}{l}\text { Good } \\
\text { result. }\end{array}$ & Medium. & Bad result. \\
\hline 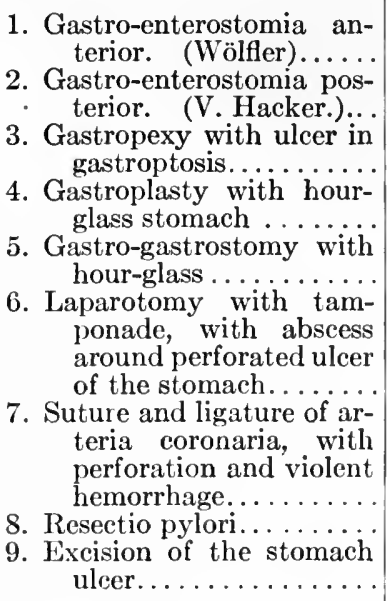 & $\begin{array}{r}1 \\
8 \\
13\end{array}$ & $\begin{array}{c}6 \\
\ldots \\
\ldots \\
\ldots \\
\ldots\end{array}$ & $\begin{array}{r}58 \\
\ldots \\
1 \\
\ldots \\
1\end{array}$ & $\begin{array}{r}2 \\
\cdots \\
1 \\
\cdots\end{array}$ & $\begin{array}{r}12 \\
2 \\
\ldots \\
\ldots \\
\ldots\end{array}$ \\
\hline Total... & 125 & 11 & 77 & 23 & 14 \\
\hline
\end{tabular}

If we now consider the survivor's, we will find that, while about two-thirds appear to be completely cured and free from all symptoms, the rest have either remained free from their former attacks, or have, after an apparent recovery, lasting a longer or shorter period, suffered a relapse, or become burdened with an entirely new complaint, which undoubtedly is due to the gastro-enterostomy opening. On closer investigation it appears that the completely successful cases are in reality all cases of stenosis of the pylorus, or ulcer in the pyloric portion of the stomach. The first group of the less successful cases-those who liave shown no signs of their old attacks after the operation-is comprised essentially of ulcers, which have not given rise 
to retention, and which have been located in the cardiac portion of the stomach, especially at the point of connection between the cardiac part and corpus ventriculi. As regards one of these patients, we know that she died after a violent hæmatemesis some months after she returned to her home.

The second group-those who have suffered a relapseconsists essentially of cases of retention, whether this be due to an ulcus duodeni, a stenosis of the pylorus, or to a large ulcer on the lesser curvature which was thought to be completely healed, because of the cessation of all morbid symptoms, but where, sooner or later, one receives a sad reminder that this is by no means the case. As serving to exemplify this group, I shall mention the case of a young man who was operated on in this ward in October, 1905. He was profoundly anæmic, having had a series of violent hemorrhages from an ulcer which was situated in the duodenum. After the gastro-enterostomy the bleeding ceased, and by degrees the patient completely regained his health, and after his discharge lived for over two years, free from all symptoms, and fully able to do his work. This case seemed to prove that the gastro-enterostomy could bring about the cessation of even a violent hemorrhage, thereby giving the wound the chance to heal; but two years and three months afterward the patient was again brought to this ward with a serious hemorrhage from his ulcer. In two other cases, where I also thought that I had cured the patients completely of a typical ulcus rotundum, I have had the disappointment of seeing them both, respectively two and three years afterward, with incurable cancer. The ulcer which I thought was healed had, without giving rise to any symptoms, continued to grow, and, like so many ulcers, had undergone a malignant degeneration.

Finally, we have the last group, where, as a result of gastro-enterostomy, a new and painful condition has de- 
veloped. This group constitutes a much more serious objection to gastro-enterstomy, because all the cases in this group consisted of small superficial or even doubtful ulcers with patients with more or less pronounced gastroptosis. The symptoms in these patients consisted of nausea and vomiting of bile-colored material, which occurred especially in the morning, after they had been lying in a horizontal position. It is in reality a certain measure of circulus vitiosus we are faced with here, which may appear in lighter and more endurable degrees with free, comparatively good intervals, but which can also attain quite unendurable and serious dimensions, which may cause the patients to languish little by little, enervated by inanition, till they finally succumb either to renewed attempts to relieve them by an operation, or to an intercurrent disease. These patients would have been much better off if they had not undergone gastro-enterostomy. The reason for this fact, as I have been able to call attention to in the statistics of other surgeons, does not seem to me to be difficult to explain, because it goes without saying that the enteroptosis, with its sagging of the organs, may cause great derangement in the conditions of the circulation of the food; but with these patients especially adhesions also develop which may fix the coils of the intestines in awkward positions. The world over one has, little by little, had one's eyes opened to these rather deficient results from gastro-enterostomy, and by experimenting on animals one has tried to ascertain the manner in which gastro-enterostomy affects some ulcers and fails to affect others. These attempts are, however, in the main stranded on this: that one has not at all succeeded by any means in producing a chronic gastric ulcer with animals. No matter how one produces the sores, they quickly heal by the aid of nature (Rörte, Clairmont). On the other hand, most interesting information has been obtained concerning the effect of the gastro-entero- 
anastomosis on stomachs which are still in possession of their normal motility, information which completely subverts the theory on which this treatment was hitherto based; namely, that the food always left the stomach quickly through the anastomosis, did not touch the pylorus at all, and quickly passed over ulcers on other parts of the stomach.

It is the American surgeons to whom credit is due especially in this matter. Blake and Cannon ${ }^{1}$ studied this subject with cats, by means of Röntgen rays. After having performed gastro-enterostomy, they fed the animals with food containing bismuth, and then observed on the fluorescent screen the passage of the food from the stomach into the intestine; it was found that, irrespective of the method of gastro-enterostomy employed, the food never passed through the anastomosis, but wandered unopposed along the ordinary way through the pylorus. In another series of experiments they produced an artificial stricture of the pylorus, but even then, to their astonishment, they saw the food traverse the natural passage; and not until they had entirely closed the pylorus by ligature did the food allow itself to be forced through the anastomosis. Tro other American surgeons, Leggett and Maury, ${ }^{2}$ employed another ingenious device: they allowed dogs, on whom gastro-enterostomy had been performed, to swallow a piece of meat in which a small ball was imbedded. This ball was attached to a long thread, the free end of which was fixed in the animal's mouth. After some time the animal was killed, and at the autopsy the ball and the thread indicated most clearly the route which the food had pursued. From this experiment, also, it appeared that the ball, in the ma-

${ }^{1}$ IV. B. Cannon and J. B. Blake: Gastro-enterostomy with Pyloroplasty: an Experimental Study by Means of the Röntgen Rays," Annals of Surgery, 1905, vol. xli, p. 686 .

${ }^{2}$ Studies upon the Function of the Pylorus and Stoma after Gastro-enterostomy has been Performed," Annals of Surgery, Oct., 1907, vol. xlvi. 
jority of cases, had taken the natural course through the pylorus. In one instance it had passed through the anastomosis, but had then taken a course back through the duodenum and pylorus into the stomach, whence it had made a second journcy in the same manner. These experiments explain, in the clearest way, why we have, in so many cases, bad results following a gastro-enterostomy in cases in which the stomach is capable of emptying itself.

On the other hand, we must look for the cause of the many good results which indisputably follow this operation. Yes, if I must give my opinion, I can really only imagine that these are due to an influx of gall into the stomach from the intestine, whereby the sour gastric juice becomes neutralized and loses its corrosive effect. But you will understand that all these experiences and observations have quite overthrown the theory that gastro-enterostomy is a universal remedy for all chronic gastric ulcers. It is altogether doubtful whether it has any curative effect. We can only say with certainty that in certain cases it acts as a palliative, and particularly so in all cases where the stomach's ability to empty itself normally is destroyed or greatly weakened. Therefore it is not strange that one has sought for more radical means of curing this most serious discase. Seven years ago, with bleeding ulcers, I began to advocate a direct attack on the ulcer itself, in order if possible to extirpate it, or to tie the bleeding vessel with ligature. Since then Professor Krogius, of Helsingfors, has energetically advocated the excision of the ulcer as a normal method; but so far he has raised his voice in vain. Nor can one deny that hitherto great difficulties have stood in the way of a general application of the excision, especially one's inability in so many cases to locate the ulcer by inspecting and palpating the exposed stomach, and even in those cases where there was clear evidence of an ulcer it was difficult to tell whether the lesion was single or multiple. 
This state of affairs, however, is altered since we have had direct gastroscopy. We are now in a position to decide quickly and accurately the number and location of the ulcerations; and in this ward this method has already given us great and important information. It is thus evident that the typical symptoms of gastric ulcer are due, far oftener than was previously supposed, to an ulcer in the duodenum, whereby we have the explanation of a number of the negative discoveries resulting from the examination of the exposed stomach in apparently certain and serious cases of gastric ulcer.

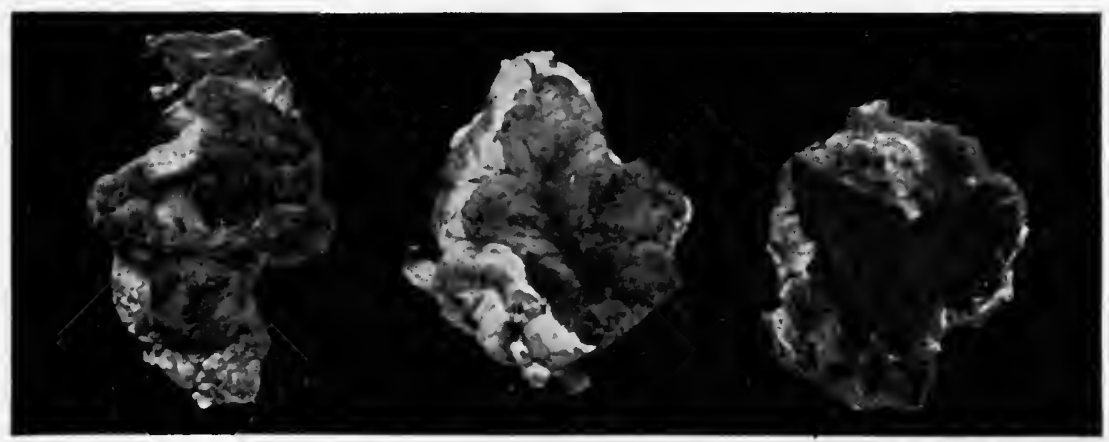

FIG. 89.-Perforated stomach ulcers removed by excision.

Another difficulty frequently exists in the unexpected extent of the ulcerations, which calls for a rather large resection of the stomach. The magnitude of the necessary operation has had a deterrent effect. I have no doubt, however, that the radical removal of ulcers will gain ground more and more, especially with well-defined ulcera rotunda, which have caused perilous hemorrhages or threaten perforation. In twenty-one cases I have thus adopted a radical treatment of the ulcer. In thirteen of these it was a question of a simple excision of one or more ulcers which had not affected the pylorus. (See Figs. 89 and 90.) Only one of these cases terminated fatally. It concerned a man who was admitted with diffuse peritonitis sixteen hours after 
the perforation of the stomach ulcer. His death was the result of his peritonitis, because he came too late for the operation, and cannot be charged to the method. All the others have recovered, and are radically cured, and I therefore do not hesitate to declare the radical extirpation of such ulcers to be not only the most rational but also the least dangerous treatment. In the other eight cases it was necessary to resect the pylorus, together with greater or smaller portions of the duodenum; naturally, this is a far more serious operation for these enfeebled patients; three of these eight have also died. A lady sixty-seven years of age

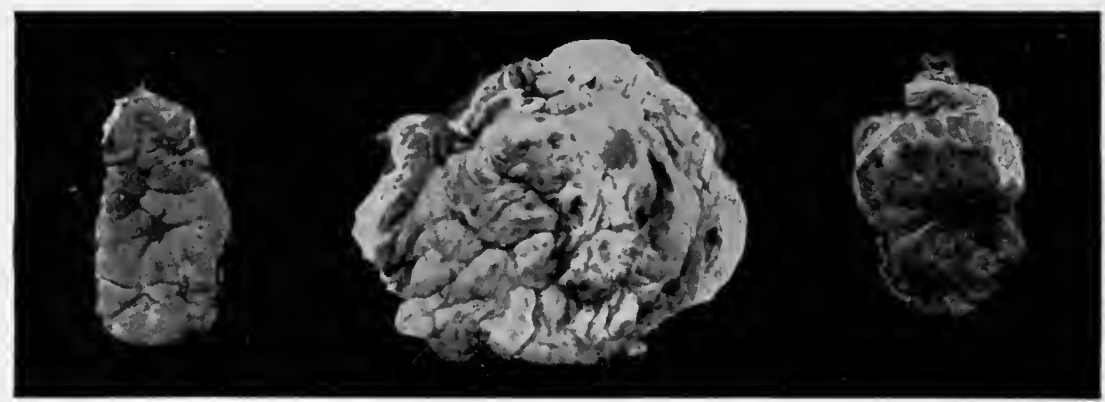

FIG. 90.-Extirpated stomach ulcers. Cases with no perforation.

died from embolism of the pulmonary artery three weeks after the operation, and after the patient was allowed up and the wound had healed. The two others were old men of nearly seventy, with arteriosclerosis and degeneration of the heart; they died from heart-failure and hypostatic pneumonia. If one considers the very serious nature of this and the other cases, the result is, in reality, rather encouraging.

If, after these reflections, we now return to our patient to discuss which operation he should undergo, it will be clear to you that this cannot be decided until we have ascertained, by gastroscopy conducted during the course of an exploratory laparotomy, the exact seat and the character of the ulceration. If I find an ulcus rotundum on the lesser 
curvature, as one would be led to expect from the history and symptoms of the patient, then we would simply excise the ulcer together with a small amount of sound tissue, and then join the wound in the stomach by suture in two layers. If the pylorus is also affected, then we are obliged to resect this.

We are in worse plight if it should happen that we are faced with a duodenal ulcer, because here an excision may easily be impossible, unless the ulcer is situated at a considerable distance from the papilla. You will easily under-

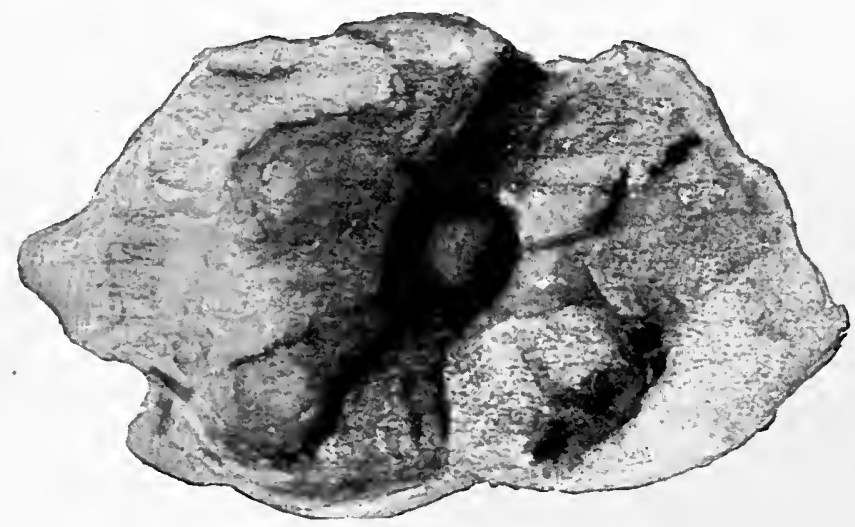

FIG. 91.-Ulcer removed by extirpation.

stand that an operation which occludes the biliary and pancreatic ducts would be fatal for the patient. Quite recently I have shown you such a case, where violent hemorrhages from an ulcer had repeatedly endangered the patient's life. In this case I did as follows: I extirpated the pylorus, and after I had loosened the adhesions which cemented the duodenum to the surrounding organs I found the artery, which was eroded at the base of the ulcer, and which had caused the recurrent, perilous hemorrhages. It was distended by a thrombus, and, therefore, easily recognizable. I tied it off above the thrombus, cut through it, invaginated the duodenum, and closed it with a double row of sutures, 
so that the spot which threatened perforation was entirely covered. Then the stomach wound was closed and the operation completed by performing a gastro-enterostomy and entero-anastomosis. The result was excellent, and if we are here so unfortunate as to have an ulcus duodeni I shall act in a similar manner.

The operation showed it to be an ulcus rotundum, which had already perforated, but was still shut off from the large peritoneal cavity by new peritoneal adherences to the liver. You see here the fairly large ulcer (Fig. 91), which was removed by extirpation. The stomach wound was at once joined in two layers, and the abdomen closed without drainage. The subsequent course of the wound has been satisfactory, and the patient is now up, takes light, ordinary food, and will shortly be discharged. 


\section{LECTURE XIV}

\section{THE DIAGNOSIS OF ULCER OF THE STOMACH}

To-DAY I will speak to you concerning the diagnosis of ulcer of the stomach, and present two patients who have just been admitted to this ward, suffering from this condition. An exact diagnosis of these cases is of the greatest importance, especially when we consider operative interference, inasmuch as the operations are always of a serious character and vary according to the nature and the situation of the ulcer. We must always strive before operation to make as exact a diagnosis as possible in order to facilitate the operation. You may think that when the case is presented to the surgeon there is very little left to be done to establish the diagnosis, because, as a rule, only those cases of ulcer of the stomach reach him which have defied medical treatment for a long time, and which have been under observation for a long period, and the diagnosis of which one would have considered to be assured beforehand. The fact is, however, that the diagnosis of stomach ulcer often presents far greater difficulties than most physicians suspect, difficulties the presence of which has been brought to light by the rich surgical experience of later years. I refer to the frequent cases where an apparently certain diagnosis of ulcer has been proved, at operation, to be incorrect. I feel it all the more important to lay these difficulties before you because they are little appreciated, and are not sufficiently emphasized in the text-books.

The last generation recognized two cardinal symptoms in the diagnosis of stomach ulcer,-i.e., pains in the cardia and hemorrhage, partly in the form of hamatemesis, partly as melana. While all agreed that these two symptoms together assured the diagnosis, there was considerable dis- 
cussion as to whether it was permissible to base it on cardialgia alone. You know, perhaps, that Carl With, Professor Faber's predecessor as teacher of clinical medicine, adopted the standpoint that every patient who showed signs of cardialgia at the conclusion of meals was treated as if suffering from ulcer of the stomach, while others strongly contested this standpoint, and claimed that hemorrhage was the decisive factor. Since then we have obtained a most valuable auxiliary in the examinations of the function of the stomach, which permits us in a certain number of cases to establish the diagnosis with great certainty; namely, where we can prove a delayed emptying of the stomach, a condition of retention, coexisting with the old familiar clinical symptoms of ulcer. It is in those cases where the ulceration either has its seat at the pylorus and causes this to contract, or where the ulcer has largely infiltrated the muscles of the wall of the stomach. But there are many ulcers where no state of retention is to be found, and where we are once more reduced to the old rough clinical symptoms.

I have already, at different times during this term, had the opportunity of pointing out that, even if With were undoubtedly right in stating that hemorrhage is completely lacking in many cases of stornach ulcer, still you expose yourselves to the risk of many bad mistakes if you depend solely on the presence of cardialgia and vomiting after meals to substantiate the diagnosis of stomach ulcer.

I recently demonstrated to you a case of inactive gall stone, which, on account of the constant gnawing and piercing pains in the epigastrium and the frequent vomitings after meals, was erroneously interpreted, and for a long time treated, as ulcer of the stomach.

As a kind of analogy to this, I have been able to show you a case of firmly-fixed, right-sided kidney. stone, where the nature of the pains had occasioned a similar confusion, and 
caused the patient to spend years in different medical wards on a severe ulcer diet. During her last sojourn at the hospital albuminuria was discovered, and nephritis diagnosed, and the diet, therefore, made even more rigorous. She came to me in an utterly exhausted condition. By examining the urine I found red corpuscles, and ureter catheterism showed me that the albuminuria had its source in the right kidney, while the urine from the left kidney was quite clear. By nephrotomy I then removed a jagged concretion, following which all the ulcer symptoms disappeared.

I have been able to show you in a small series of cases that appendicitis also is frequently confused with stomach ulcer. In many cases of appendicitis, especially in children, the pain is not always found at the typical, so-called MacBurney's point, but in the centre of the epigastrium or close to the umbilicus. In the case of children, the reason for this often is that the ileocecal junction lies abnormally high, and that the appendix often extends in a bow upward and inward, so that the point lies in the middle of the epigastrium. A short time ago I operated on a young man who had been treated for many months by one of our most eminent medical men for a supposed gastric ulcer, on account of violent cardialgic pains. As the medical treatment was quite ineffectual, I was consulted. The seat and character of the pains quite resembled those of an ulcer, but my suspicions of appendicitis were roused when I elicited pain on compressing colon descendens from below and upward, and by there being an occasional rise in temperature. At the subsequent operation it appeared that the appendix was the seat of a chronic inflammation, and lay with its point in the middle of the epigastrium, adhering slightly to peritoneum parietale. After the removal of the appendix the cardialgic pains disappeared entirely. The picture can be even more confusing when, with a chronic appendicitis, peritoneal adherences have fixed omentum 
majus to the pelvis and the abdominal wall in the right fossa iliaca. The omentum drags on the stomach, just as the stomach, in its peristaltic movements and in its various degrees of repletion, drags on the omentum. In this way both pain and vomiting after meals are produced; indeed, a state of retention and dilatation of the stomach may easily develop. Those among you who followed my lectures during last term will remember just such a case where all the gastric symptoms vanished after loosening the omental adherences and removing the appendix.

But gastroptosis is far more frequently confused with gastric ulcer than the here-mentioned afflictions, because gastroptosis, when it does give rise to distinctive morbid symptoms, invariably appears with cardialgia and constipation-the two symptoms which, as a rule, we always find with gastric ulcer. In a previous lecture I have taught you how to make the differential diagnosis when once your suspicion is aroused; and you must always suspect gastroptosis when the cardialgia is localized to the left of the median line. It seems obvious, then, that the single symptom of pain after eating is an unsafe foundation on which to build the diagnosis of stomach ulcer, and this ought, indeed, to be generally known by now. On the other hand, the majority of physicians consider the diagnosis stomach ulcer as quite safe when hæmatemesis has appeared in addition to the cardialgia. One should, however, be very careful when deducing an ulcer from hæmatemesis. I shall not dwell on the fact that hemorrhages from the air-passages, the gullet, and varices in the œsophagus (cirrhosis of the liver) are from time to time confused with hæmatemesis, because these really play only an inferior rôle, inasmuch as other ulcer symptoms are, as a rule, lacking in such cases. Far more serious is it that real hæmatemesis hemorrhages from the stomach itself not infrequently appear without any gastric ulcer being present. With a true hemorrhage 
in the stomach, a solutio continui is naturally always found. But when we speak of the disease as gastric ulcer we mean a lesion of unknown origin, a more or less chronic wound or lesion attended with discharge, and do not include sores caused by pointed articles or other small abrasions or ruptured blood-vessels which are quite temporary; for in reality such often give rise to hæmatemesis. That fishbones and other foreign substances can produce abrasions and produce hæmatemesis goes without saying, and from time to time such a hæmatemesis occasions confusion with ulcer. Here, as a rule, only a single temporary hæmatemesis is in question, occurring in individuals having no other ulcer symptoms. It is of far greater significance that there are patients in whom cardialgia and vomitings together with frequent hæmatemesis occur and simulate the symptoms of ulcer, without any trace of ulcer formation being found.

In this category, in my opinion, belongs the first of those patients whom I wish to show you to-day. The patient, an unmarried woman, twenty-six years of age, is a shop girl who has suffered since her eighteenth year from cardialgia following meals, constipation, and periodical vomitings. Six years ago she had hæmatemesis for the first time, and since then, generally at intervals of some months, she has suffered from hæmatemesis. These attacks have not been very serious, but, on the other hand, she has undoubtedly vomited blood, although small in amount. Under the diagnosis stomach ulcer she has repeatedly been treated at medical hospitals for ulcer of the stomach, with confinement to bed and strict ulcer diet. On each occasion her condition quickly improved under this treatment, but as soon as she returned home and began to work the old attacks quickly reappeared. A week ago a hæmatemesis recurred, and her physician sent her to the surgical ward, because he considered that these stomach bleedings, lasting over a 
long series of years, and the constant cardialgia must be due to a chronic ulcer which could not be cured by medical dietetic means. On the basis of the rough clinical observation this reasoning is in every respect correct and plausible. As a matter of fact, at the back of such complex symptoms as these and such a course as this there will be, as a rule, a gastric ulcer of long standing and of most dubious prognosis ; inasmuch as nobody can tell when the ulcer may gnaw a hole in arteria coronaria and thereby cause a fatal hemorrhage. There is also a constant danger from perforation and cancerous degeneration in such cases. It is always best to refer such a case to the surgeon, so that he may perform a radical removal of the ulcer if a more exact examination confirms the suspicion of ulcer or, where this is impossible, can perform a gastro-enterostomy. In this case, however, the further examination causes me to doubt the diagnosis of ulcer. Those points which make me doubtful are the following: That, notwithstanding the fact that the patient was admitted on the same day that the hæmatemesis occurred, we liave been unable to find any trace of blood in the feces. These examinations have extended over a period of ten days. From a further study of the patient's history while in the medical wards, where she was formerly treated, it appears that no blood was found in the feces at that time. This appears to me highly suspicious, for in my own experience a chronic stomach ulcer which causes hæmatemesis always presents traces of blood in the excrement, not only at the time of the attacks of hæmatemesis, but also during the intervals between these; yes, in a great number of cases hemorrhage from a stomach ulcer does not reveal itself by hæmatemesis at all, but only by an admixture of blood in the feces, whether it be as a macroscopically apparent melæna or only as a microscopically or chemically demonstrable admixture. On the other hand, one frequently sees that cases of hæmatemesis of other 
origin, occasional abrasion or rupture of blood-vessels in an otherwise healthy stomach, are not followed by melæna. I think this is partly because the sound stomach completely disposes of the blood by the vomiting, and partly because the bleeding is quite temporary, so that the small quantity of blood which might remain in the stomach is digested, and disappears here and in the small intestine; while with a chronic stomach ulcer where we have constant oozing of blood, the stomach, just as with hæmatemesis, far from disposes of all accumulated blood because of its reduced motil. ity. What has made us more suspicious in this case is that six to twelve hours after administering an experimental test-meal we have not been able to prove the slightest retention of food in the stomach! With a chronic stomach ulcer which had existed seven years and caused attacks of hæmatemesis this would be rather strange.

Finally, our investigation has shown that our patient has an affliction which makes the theory of a stomach ulcer unnecessary, in so far as it can explain to us all her symptoms. Scraping auscultation and Röntgenoscopy after bismuth porridge have shown us that she has a very considerable gastroptosis. This explains to us her pains in the cardia, which are just to the left of the median line and certainly occur just after meals, but do not seem to depend on the quality of the food, and have, therefore, the characteristics of ptosis cardialgia. Constipation is an even more constant symptom with cases of ptosis than with ulcer. Hæmatemesis alone remains to be explained! Can gastroptosis cause hæmatemesis? Yes, unquestionably; and it occurs in quite a number of cases of ptosis where no ulcer is present. This circumstance, which I have demonstrated in an exceptionally large number of cases of ptosis on which I have operated, has hitherto been unknown, and quite naturally so, because it was taken for granted that hæmatemesis combined with cardialgia signified ulcer, an 
error which only the operative autopsia could prove and rectify.

I therefore consider it doubly important to call your attention to this, and to dwell somewhat upon the point. That hæmatemesis which occurs in cases of ptosis is not so difficult to explain: Many of the patients have, indeed, violent and incessant vomitings, and these might, per se, cause bleeding of the mucous membrane; but added to this is the fact that the vessels of the stomach-by reason of the sharp bends and folds in the stomach-wall caused by the ptosisare themselves bent, dilated by stasis, and thus disposed to rupture easily. We know that hemorrhages of this kind occur in other dislocated organs. For example, hæmaturia with floating kidney is well known, and is considered to be due sometimes to torsion or bending of the ureter, sometimes to torsion of the pedicle. However one may explain these hemorrhages with ptosis, they are, nevertheless, a rather frequent phenomenon, which always misleads one, and make us suspect gastric ulcer, because these patients also suffer from cardialgia and constipation, which well deserve to be called "ulcer symptoms."

In my opinion, we have in this the key to a hitherto enigmatical incongruity between the clinicians' and the pathological anatomists' statements of the frequency of stomach ulcer in the two sexes. While all clinical statisticians report that stomach ulcers occur far more frequently among women than among men, the pathological anatomists find on the dissecting table that the chronic, open stomach ulcer is absolutely more frequent with men than with women. Harslöf has collected data from the clinics which show 144 cases in women, as opposed to 78 cases in men, while his data collected from the dissecting room show that the chronic ulcer with all its consequences is frequently found in men. On the dissecting table, however, one finds small cicatrices on the mucous membrane of 
the stomach far more frequent with women than with men after small lesions, which in my opinion are due to ptosis, and the pressure from corsets or laces, and probably correspond with the temporary lesions which cause hæmatemesis in ptosis patients.

I think, then, that with our patient here we have a simple ptosis, and that her affliction can be removed or greatly improved by a gastropexy. I shall, of course, carefully examine the wall of the stomach for an ulcer. If, contrary to all expectation, I should find this, I shall excise it, but take good care not to perform a gastro-enterostomy, which, as I have previously emphasized in a former lecture, is only indicated with ulcers which involve an obstruction of the outlet.

Hæmatemesis and melæna seldom occur with gastritis, but when they do, confusion with stomach ulcer may more easily occur, because the pains here, as with ulcer, are proportionate to the quality of the food, and because the motility may be considerably reduced.

In this way you see that hæmatemesis, which so many are inclined to consider a decisive criterion of the presence of a stomach ulcer, may not infrequently lead you astray. In any case I will advise you always to be suspicious with cases of hæmatemesis where you cannot simultaneously prove obvious or occult melæna. You know that we understand by melæna that the excrement by an intimate admixture of blood has become as dark as tar; and by occult melæna-really a contradictio in adjectowe understand an admixture of blood which cannot be perceived by the naked eye, and only demonstrable by chemical or microscopical examination. On the whole, I consider the proof of an intimate admixture of blood with feces, when it occurs together with cardialgia, as a surer symptom of gastric ulcer than hæmatemesis, and so much more valuable because this symptom occurs far more 
frequently than hæmatemesis, which is found in scarcely one-fourth of the ulcer cases. We must, of course, remember that melæna may also be due to ulcers in the upper portion of the intestinal canal, especially duodenal ulcer, but here, as a rule, we are able to make a differential diagnosis: firstly, from the location of the pain, which with duodenal ulcer is nearly always found to the right of the median line, while the pain with gastric ulcer is, as a rule, situated in the centre of the epigastrium or to the left of the median: this is especially so with regard to pain produced by pressure; secondly, the examination of the function of the stomach with duodenal ulcer will, as a rule, present quite normal conditions, both with regard to the stomach contents and the motor ability, while with melæna due to gastric ulcer you will also find blood in the stomach. Yet you may be deceived here if a duodenal ulcer is concerned in which a considerable contraction in the duodenum has been produced, because the part of the duodenum and the pylorus lying above this is then distended, and blood from the duodenal ulcer may then run into the stomach, and in the presence of an active hemorrhage may fill it and sometimes cause hæmatemesis.

At this moment we have lying in this ward two young men, both in the early thirties, who illustrate what I say. They have both been admitted to this ward with the diagnosis gastric ulcer. One of them, as you see, is a stronglybuilt man, somewhat thin, but otherwise of a healthy appearance. For four years he has suffered from cardialgia and sour eructations after meals, especially after partaking of rich and heavy food, but he has had no vomiting. As he has frequently submitted to a course of medical treatment including strict ulcer diet, he decided to seek possible surgical aid. On palpating the abdomen of this young man we immediately found marked tenderness in the centre of the epigastrium, confined to an area the size of a half- 
crown. We also found a darkish discoloration of the feces, which a chemical examination proved to be due to blood. On further examination relative to the chemical and motory condition of the stomach we found a marked hypersecretion and hyperacidity, and, in addition, retention of food remnants, even ten hours after the experimental meal, which contained blood in the residue. I feel quite sure that here I am confronted with an ulcer, and I think that we shall, by and by, have the opportunity to verify the diagnosis on the operating table.

The other young man, who is now brought in, was the subject of our lecture fully two years ago, and some of you will perhaps remember him. At that time he was admitted with the diagnosis bleeding stomach ulcer, and in such an utterly anæmic condition that the hæmoglobin examination of his blood showed only 18 per cent. He was reduced to this condition by a series of serious attacks of hæmatemesis and protracted melæna. In spite of the at. tacks of hæmatemesis I here diagnosed duodenal ulcer, for three reasons: (1) Because his pains were regularly reported as being to the right of the centre line, occurring especially in the night, and never as a direct result of a meal. It is natural that the pains from duodenal ulcer, as opposed to the pains from gastric ulcer, do not appear till long after the meals, when the food passes the duodenum. I will ask you particularly to pay attention to the very characteristic occurrence of the pains at night, which, in my opinion, are caused by the strong influx of bile into the duodenum during this period of the twenty-four hours. (2) Because examination here shows that the stomach is normal. (3) Because of the severe melæna discharge of great quantities of blood with the feces. My line of reasoning was that, if this strong bleeding were due to gastric ulcer, we ought also to find blood in the stomach with reduced motility. At the operation it was found that the 
stomach was perfectly normal, and that the ulcer was situated in the duodenum, but, unfortunately, so close to the entrance to ductus choledochus that a radical operation was impossible. I therefore had to content myself with performing a gastro-enterostomy, in the hope that by diverting the stomach contents in this manner, I should be able to protect the ulcer in the duodenum from the corrosive effect of the hydrochloric acid and the irritation of the food, and thereby allow it to heal. It really looked as if we had attained our object. The bleeding and the pains ceased, the young man recovered by degrees, and after the lapse of three months he had regained his strength. As this was maintained for two years after the operation, we thought the ulcer completely healed, until a few days ago he was readmitted in the state in which you now see him, deeply anæmic and exhausted by reason of hemorrhage from the ulcer, which this time has not revealed itself by hæmatemesis, but only by severe melæna.

I have now illustrated for you the significance of the "typical" symptoms of stomach ulcer, and the diagnostic mistakes which an overestimation or an erroneous estimation of these may lead to.

I will not conclude these remarks on the diagnosis of stomach ulcer without emphasizing the other great source of error in diagnosing this condition,-i.e., that "ulcer symptoms" may be completely wanting even with fairly large ulcers. It is in reality by no means seldom that large chronic ulcers are discovered by chance on the dissecting table in the person of patients who have never had pains, inconvenience, or vomitings after meals. Here in this ward we have repeatedly received patients with diffuse peritonitis, which was due to the perforation of a great stomach ulcer, which had never caused the patient pains or other symptoms. Perforative peritonitis was the first symptom! 
In conclusion, I will show you this patient, before he is discharged, as a fine example of a "symptomless" ulcer. $\mathrm{He}$ is a man sixty years of age, who was admitted here with the diagnosis cancer coli. He has never suffered from cardialgia or vomiting, but from an increasing constipation with oppression and pains in the lower part of the abdomen, a feeling of distention, abhorrence of food, and consequent emaciation. His shallow, somewhat cachectic appearance immediately directed one's thoughts to cancer. The examination of the stomach showed quite normal chemical and motory conditions. No tumor could be felt per rectum, but I thought I felt a tumor in flexura sigmoidea. I made an exploratory incision in the left fossa iliaca, with the intention of making a resection or colostomy, as the circumstances required. It appeared, however, that the supposed tumor was only hard scybala at the flexure. I now inserted my full hand into the abdomen, and palpated systematically throughout the peritoneal cavity, searching for the cancer in one place or another. I did not find anything till I reached the stomach, when, on the lesser curvature, I found a firm infiltration about the size of a half-crown. I now closed the first incision and made an opening in the centre line between processus ensiformis and the umbilicus. It then appeared, to my great surprise, that the patient's affliction consisted of an ulcus rotundum with callous edges, which was situated on the lesser curvature and was just on the point of perforating. I excised the ulcer and the entire infiltrated portion of the stomach, and joined the edges of the wound in two layers, after which I closed the abdominal wound.

The course of the wound has been ideal, and you here see the ulcer which was removed, in which miscroscopy has not been able to prove any signs of malignant degeneration. After its removal all his morbid symptoms vanished, the constipation was relieved, and at present he has added twelve pounds to his weight. 
You will see that it was quite impossible to give this diagnosis without an exploratory operation. Here there was only one pronounced symptom: constipation, and certainly this is a very frequent symptom with gastric ulcer; but to build the diagnosis on this one symptom is naturally.impossible. On the contrary, it will then be natural to seek the cause in the large intestine itself. It was my good fortune that the cachectic appearance of the man should induce me to fall in with the supposed colon-cancer, and I am glad that, when this proved to be incorrect, I performed the thorough examination of the abdominal contents which led to the surprising discovery of an ulcer.

In addition to the difficulty of a clinical diagnosis, this case will also teach you, in case of doubt, never to forget to consider the possibility of a stomach ulcer, even though all the "typical ulcer symptoms" are wanting. 


\section{LECTURE XV \\ CHARACTERISTIC TYPES OF ULCER OF THE STOMACH}

To-DAY I can again show you three patients admitted to this ward with a diagnosis of stomach ulcer. I am all the more pleased to make these patients the subject of my lecture to-day because they represent two different types of stomach ulcer, which present distinctly different clinical pictures, the symptoms of which we had no opportunity of bringing out at our last lecture. The typical cases of stomach ulcer mentioned hitherto have been situated on the lesser curvature of corpus ventriculi, the most common site of these lesions. But the patients whom I wish to show you to-day present, in my opinion, typical examples of ulcer formation at the two extremes of the stomach: the cardia and the pylorus.

The first patient whom I will show you is forty-six years of age, and keeps a restaurant; he enjoyed good health in childhood and youth, and was able to serve his time in the army. He denies genital affections, but admits that he had consumed a quantity of alcolol until about ten years ago, when his present illness forced him to desist. His complaint commenced about sixteen years ago with sour eructations, bad taste in the mouth, oppression and pungent pain in the epigastrium after meals, and vomitings as the direct result of these, there has never been fresh blood in his vomitus, but some five or six years ago, this is reported to have been of a chocolate-brown color. His evacuations have been sluggish, with occasional intervals of four days; tar-like coloring has not been noticed. It is these symptoms which have troubled the patient on and off during the last sixteen years, and have prevented his eating ordinary food, thereby involving serious emaciation, languidness, and a 
diminution of his ability to work. He has frequently been in the hands of specialists for considerable periods, obtaining thereby quite insignificant and temporary relief. When we now analyze the symptoms more particularly it appears that his cardialgia is always situated to the left of the median line, where he is also sensitive to pressure. The pains commence as soon as he eats and the food passes down into the stomach, and the patient informs us that he often has a sensation of obstruction when the food passes through the first part of the stomach. When he vomits this comes immediately after the partaking of food. Sometimes the pains occur when he is hungry, and then become worse when he eats something, but, as a rule, they appear as an immediate result of the meals, most frequently of the evening meal. That food which he can endure least is rye bread, rich food, cabbage soup, and coarse food generally. When the attacks are especially bad he has to go to bed with hot fomentations, and has a sensation of inflation and great discomfort in the abdomen. From the objective examination we find the patient somewhat pale and thin, though his appearance is otherwise natural. No tumor is felt on palpating the abdomen, and, apart from the attacks, there is no sensitiveness to pressure in the epigastrium. The introduction of a stomach-tube meets with no opposition, either in the œophagus or in the cardia, in the same way that the examination with the œsophagoscope has shown normal conditions. Here, therefore, we can exclude some form of stricture, which the symptoms really suggest. It appears, further, that the stomach's ability to empty itself is normal; at any rate, even eight hours after administering one of Bourget's experimental meals we find no microscopic retention of food. As regards the chemical function, we find, by withdrawing one of Ewald's experimental meals, a complete lack of hydrochloric acid, whereas an examination made six months ago showed the 
proportions of acids to be rather high, free hydrochloric acid 41, with a total acidity of 76 . A normal motility in the presence of a stomach ulcer which had existed for sixteen years is unusual, and therefore one cannot fail to think of the possibility that all the attacks may be due to a gastroptosis, and all the more so because this would fit in well with the localization of the cardialgia to the left of the median line. Partly by scraping auscultation, partly by Röntgen illumination of the bismuth-filled stomach, we have defined the position of the stomach, but have found this to be quite normal, because curvature major lies six centimetres above the umbilicus. If we can exclude ptosis as well as disease in the lowest part of the œsophagus, then we are forced to assume the presence of a chronic stomach ulcer in the vicinity of the cardia. Such can, in reality, explain all the symptoms - the pains in the left side, the presence of these immediately after the arrival of the food in the stomach, and the hyperacidity - but at the same time only such a one, because an ulcer of sixteen years' standing at or near the pylorus would undoubtedly have caused motory difficulty and retention.

Let us now regard our other male patient, a chemist, forty-two years of age, whose affliction is almost of the same duration, inasmuch as it began seventeen years ago with cardialgia, which had its seat in the centre of the epigastrium, and had the peculiarity, as opposed to the pains in the other patient, that they did not occur after meals, but, on the contrary, always when the stomach was empty and the patient hungry - the patient even declaring that they were alleviated by his taking food. This patient has never had vomiting, but rather frequent sour-tasting eructation. Melæna has never been observed. During the first thirteen years these attacks did not alarm him at all: he hastened to eat something when the pains commenced, and then they passed. Altogether, he thought merely that it 
was a question of what is generally termed "pain from hunger." Four years ago severe attacks of pain began to occur; these he describes as stomach cramp, and even if these are not very frequent, yet they are steadily increasing in frequency and severity, so that the patient, alarmed thereby, has sought medical advice. As you see, the attacks from which he has suffered, although just as protracted, may be said to be comparatively much lighter than those of the first patient, and when you now see the patients side by side you will also get the impression that this man looks less exhausted, healthier, and stronger, even if he is rather thin. You will be all the more surprised to hear the result of the examination of the stomach in this case. By inspection we have already discovered a distention of the stomach which extends from the middle of the epigastrium far down into the abdomen, where it ends in a downward convex line midway between the umbilicus and the symphysis. Now we allow the patient to breathe deeply, and you can see this distention move up and down under the thin abdominal wall. On palpation no tumor or ascites can be demonstrated, and by scraping auscultation we easily substantiate the fact that the distention is in reality the distended stomach.

By the introduction of a stomach-tube on an empty stomach, we have extracted 2000 cubic centimetres of thinly flowing stomach contents which give a strong reaction for free hydrochloric acid, the last part of which contains food remnants which have been in the stomach some time. There is no blood in the contents of the stomach, and no blood could be found in the feces. Herewith we have the diagnosis. This man is suffering from a far-advanced stenosis of the pylorus; this we can assume with a considerable degree of certainty to be due to a stomach ulcer at the pylorus, partly or completely cicatrized. There is not much reason to discuss the differential diagnosis here. The only other cause one could imagine for such a stenosis 
-a malignant tumor, a cancer pylori--I think I may exclude on account of the long-protracted course of the illness and the hyperacidity of the contents of the stomach, and the absence of cachexia. It is important, if not conclusive, that we do not feel any tumor in the abdomen of this patient, either, where the palpation does not offer special difficulties. Here, then, you see an instance where gastric ulcer has existed for seventeen years and led to a severe contraction of the pylorus, with enormous dilatation of the stomach without the patient having presented any other symptoms than cardialgia, on an empty stomach. This cardialgia is, however, very characteristic of stomach uleer, and is often of great differential diagnostic importance in this way: that it contrasts with that cardialgia which is due to simple gastroptosis, and always grows worse with eating, while it is never relieved thereby. How, then, shall we explain to ourselves the fact of an ulcer pain being relieved by food entering the stomach? It can only be explained by the fact that when the stomach is empty the gastric juice attacks the ulcer, causing pain by its corrosion; but as soon as food enters the stomach the gastric juice is not only diluted but also enters into combination with the food. Why, then, in cases such as our first, does pain occur just when food arrives in the stomach? This difference, like all the differences in the two aspects of the two diseases which I have here placed before you, is quite simply explained by the diametrically opposed localization of the ulcers - the one by the cardia, the other by the pylorus. The cardial ulcer causes pain to the left of the centre line, and the pains are necessarily produced when the crude food passes the ulcer on its way down to the fundus, and such an ulcer is less exposed to the eroding effect of the gastric juice than an ulcer in pars pylorica. An ulcer in the pars cardia gives, en ipso, no retention, unless it has formed in an hour-glass shaped stomach.

Our third patient is forty-three years of age, and the 
widow of a farmer on whom we operated in this ward a couple of years ago for a malignant growth of the kidney. This woman has suffered for many years from pains to the left of the median line in the espigastrium after her meals. She has not paid much attention to these pains, which vanish when she lies down, inasmuch as she has been able to attend to her work and her house, and has been tolerably well, on the whole. But shortly after her last confinement, four years ago, she began to suffer from vomiting and severe pains, which were situated to the right of the median line, and radiated in all directions throughout the epigastrium, and upward into the right side of the chest and back. There has never been any hæmatemesis, nor has melæna or jaundice ever been observed. These pains differ greatly from the earlier ones, which were on the left side: firstly, because they never occur in connection with or following her meals, but, on the contrary, are worse at night, and when the patient is hungry; whereas they are relieved when the patient takes something to eat. Her evacuations are very sluggish, often occurring with an interval of several days, despite the use of various aperients. As you see, she is greatly emaciated and pale, and on examining her blood we find the quantity of hæmoglobin reduced to 60 per cent. While the pains in the left side, from which she has suffered since the age of puberty, are most probably due to gastroptosis, we must, a priori, think of two things for the explanation of the pains in the right side,-i.e., gall-stones and ulcer in the stomach, or just as probably in the duodenum. The beginning of these pains in somewhat direct response to a confinement might indicate gall-stone, because, as you know, the increased pressure in the abdomen during the latter part of pregnancy, and especially during childbirth itself, often forces hitherto symptomless gall-stones into ductus cysticus, and thereby produces attacks of colic. Furthermore, the pains radiating into the right side of the 
chest, and their appearance at night, favor the idea of gall-stone; but we must remember that the pains with ulcus duodeni have exactly the same characteristics, and this is quite natural, because the strong secretion of bile which occurs during the night will irritate the duodenal ulcer by its influx to and distention of the duodenum, just as it can produce gall-stone colic by the pressure it exercises on the wedged-in stones. Finally, we must also consider the possibility of cancer, on account of the severe emaciation and anæmia of the patient. When, with these questions in our mind's eye, we now examine the patient, you see that the epigastrium is sunken, and you see, furthermore, a very strong pulsation in the median line of the epigastrium-a symptom, as I have previously told you, which suggests that the stomach has prolapsed, and no longer lies like a cushion in frout of the vertebral column and the aorta. By scraping auscultation we then map out a very marked gastroptosis, because we find the lesser curvature lying three centimetres below the umbilicus, and the greater curvature nine centimetres below the same. One feels and sees no tumor, even when the patient inhales deeply, which speaks volumes against the presence of cancer, as here it is so easy to feel through the attenuated, thin abdominal wall. On the other hand, you will notice that the patient winces distinctly when pressed slightly under the right border of the ribs, and at the tip of the ninth rib; one does not feel the gall-bladder, whereas the edge of the liver is felt distinctly, rather sharp and thin. Here, naturally, the determination of the chemical and motory function of the stomach is of great significance. This has shown us that there is a considerable delay in the passage of the food, for twelve hours after the partaking of one of Bourget's retention meals we find numerous prune-skins and cowberry seeds in the relatively small amount of liquid gastric contents (about 60 cubic centimetres). One hour after Ewald's 
experimental meal, 145 cubic centimetres are withdrawn, which, with a total acidity of 29 , show 14 free hydrochloric acid. Here, then, we have before us a patient with considerable gastroptosis and with a retention of food. The question now is, where and of what nature is this hindrance? I feel convinced, by reason of the symptoms before us-the pains in the right side-that the obstruction is situated in the duodenum, not, as with the previous patient, in the pylorus itself, and I think there is a causal connection between the patient's gastroptosis and her affliction in the duodenum; but I am not quite sure whether this is a real ulcer, or whether it is only due to the sharp bend which so often appears at that spot where the fixed part of the duodenum comes into contact with the part dragged down by the gastroptosis. As I have previously mentioned, such a bend alone can form an obstruction to the normal passage of the food,-in other words, cause a state of retention in the stomach, especially when the folded peritoneal surfaces gradually coalesce and fix the bend. But in such a case specially favorable conditions for the development of an ulcer duodeni are created, just on the protruding fold of the mucous membrane thus fixed. In such a case the part of the duodenum lying above becomes in a way part of the stomach cavity, because the pylorus becomes dilated on account of the contraction in the duodenum, and thus the duodenal mucous membrane is exposed to the constant action of the gastric juice. This, as it seems to me, best explains why we so often find duodenal ulcers in patients who have ptoses. To what extent it has here advanced toward ulcer formation I dare not say, because the patient has never had hæmatemesis, and has never noticed tar-colored stools; no blood has been found in the stools, either by Weber's or Schröder's method.

At the exploratory laparotomy which we shall undertake after this lecture we shall naturally have our attention 
directed toward the possibility of a stone-filled gall-bladder, which one might think would cause an obstruction of the passage by its pressure on the duodenum; but I do not believe in this eventuality.

So far, then, we have defined the probable seat of the lesion by taking into account both the location, and character of the pains, together with the examination of the function of the stomach. The question now is: What is the prognosis in these cases and how shall we plan the operative treatment, which now seems the only possible help in these two cases? I must at once tell you, then, that I have the smallest hope with the first case, where we have diagnosed an ulcer in the cardial part of the stomach; in fact, far smaller than I dare tell the patient, who has just been carried out. I say this, although the examination of the function of the stomach, apart from the lack of hydrochloric acid, showed no functional disturbance at all. I view this case gloomily ; firstly, because I greatly fear that this ulcer has degenerated into a cancer, of which the man's exhausted appearance and the achylia which has succeeded the hyperacidity found by former examinations are so ominously suggestive. But, even if it should be only a large callous ulcer, it is very doubtful if we can effect anything here by surgery, because you easily understand that, in this case, only one operation can help,- that is, the radical excision of the ulcer; but if the ulcer extends up close to the cardia, this operation is impossible; and even if a narrow border of sound tissue is found under the cardia, it will be a very difficult and serious operation. A gastro-enterostomy, to which we can otherwise resort so successfully with ulcers on other parts of the stomach, when by coalescing with the pancreas or other large vessels they cannot be removed radically, is of no avail in this case, where the food must pass the ulcer. In my opinion, then, the chances are ten to one that we must close the laparotomy wound 
without accomplishing our object. However, on account of the patient's miserable suffering, I feel in duty bound to offer him this small chance.

I am far more hopeful concerning the other two patients, because, even if we dare not promise a safe issue from a radical operation, yet we can always achieve something where we have a condition of retention before us, inasmuch as we can procure better conditions of depletion by a gastroenterostomy. This is generally very effective in cases of stenosis of the pylorus, and our operation with patient No. 2 will probably be confined to this.

In the third case, however, I prefer to avoid a gastroenterostomy, which, as I have formerly told you, gives very unsatisfactory results when gastroptosis is present, and in any case it would then have to be combined with a gastropexy.

In the next lecture I shall give you further particulars with regard to the course and the result of the operations. 


\section{LECTURE XVI}

\section{CHARACTERISTIC TYPES OF ULCER OF THE STOMACH-CONTINUED}

I will show you to-day the three patients who were presented at the previous lecture two weeks ago with supposed ulcer of the stomach. The first patient, who was a restaurant keeper, forty-six years of age, in whom I had diagnosed an ulcer of the stomach in the neighborhood of the cardia, presented a malignant degeneration of an old ulcer in the neighborhood of the cardia, a condition which I greatly feared on account of the advanced emaciation and cachexia which were present in the patient. We found an ulcer with a deeply infiltrated growth beginning near the cardia and forming a half circle, with its centre raised about five centimetres. It was a hard, scirrhous, glandular tumor which showed all of the characteristics of malignancy. After satisfying ourselves of this condition I at once closed the abdominal wound, as any attempt at radical removal would have been useless, and a gastrostomy did not seem in-. dicated, inasmuch as liquid food could pass the obstruction without difficulty or pain. The operation did not affect the patient unpleasantly, and to-day he looks better than before the exploration. This undoubtedly is due to the satisfying effect of the operation, for he has gained new hope and seems to feel a decided relief from the operation. This effect, which we frequently see under similar conditions, often lasts for a long time and is fortunate both for the patient and for the surgeon, for in the case of the latter it helps to lessen the disappointment and depression which always follow the finding of a cancer which is inoperable. This case should be a lesson to you, emphasizing the fact that it is dangerous to keep a patient who has symptoms 
of pronounced ulcer of the stomach from year to year without operation; that the presence of free hydrochloric acid is by no means a guarantee that cancer is not present. If this patient had been operated upon some years ago, it might have been possible to remove the ulcer, or, in any event, by a gastrostomy and forced feeding, he might have been relieved of his pain and perhaps obtained sufficient good health to promote the healing of the ulcer.

In the second patient, a chemist, forty-two years old, whose stomach was enormously dilated and contained, after fasting, two litres of fluid, I found, as expected, a considerable stenosis of the pylorus. The pyloric portion was changed into a hard fibrous canal three centimetres long and the size of one's little finger, running between the normal-looking duodenum and the large, relaxed, but yet fairly thick-walled, stomach. The peritoneal covering, especially the superior surface, showed a distinct cicatricial change, and scattered over the peritoneum covering the pylorus some small whitish grains were found, which caused a suspicion of a possible commencing cancerous degeneration. I did not venture, therefore, to restrict myself to a gastroenterostomy, as I had intended doing, because it is the least dangerous encroachment and is best indicated in cases of simple cicatricial stenosis of the pylorus, but at once decided to perform a pylorectomy-I prefer this term to that generally but incorrectly employed "pylorus resection."

I performed the operation in this way. After having loosened ligamentum gastro-hepaticum upward and ligamentum gastro-colicum downward from their attachments to the pars pylorica, I clamped off the duodenum with a pair of rubber-covered forceps at a distance of three centimetres from the pylorus, and applied a similar pair to the stomach, four centimetres from the stricture; then I removed the pars pylorica between these clamps. It then appeared that 
the opening of the stomach was somewhat larger than that of the duodenum, though not so much so that a direct connection could not be made. First I sutured together the peritoneal surfaces on the posterior side of the duodenum and the stomach with a continuous silk thread; then I joined the two lumina, using a continuous suture of catgut, including all the layers; next I removed the clamps which had prevented all escape of intestinal or stomach contents; the next step consisted of completing the closure of the peritoneal covering by continuing the original silk suture around the anterior half. The suture line was further fortified by bringing together the omentum minus and a piece of the omentum majus in such a manner that the suture line was entirely covered in. These portions of omentum were attached to the peritoneal covering of the duodenum and stomach by catgut sutures. The excised portion of the pylorus is shown in Fig. 92. You will notice that the mucous membrane is normal, excepting a small portion on the anterior

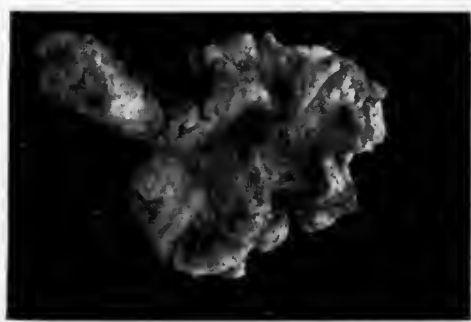

Fig. 92.-The resected pylorus with the adjacent stomach-wall, showing the stomach side of the stenosis and the small, deep ulcer which has caused the stenosis. superior surface where a small, crater-like ulcer is seen, which penetrates deeply and is accompanied by infiltration of the stomach. The muscularis and the peritoneal coats of the duodenum, which are somewhat odematous and partly fibrous, are the result of the chronic inflammation. Microscopic examination of this ulcer base shows that there is no cancerous degeneration. The course of the wound in this case and the effects of the operation have been ideal, as you will see from the chart (see Fig. 93). The temperature, after having been about $38.6^{\circ}$ for the first two days, rose on the morning of the fourth day to $40.1^{\circ}$, although the abdominal wound was quite reaction- 
less and the abdomen quite soft and normal, just as the general condition of the patient was remarkably good.

It must be remembered that the line of gastro-intestinal suture is exposed to the action of the gastric juice and to infective micro-organisms contained in the intestinal tract. I concluded that infection alone in the suture line of the gut had occurred, and therefore ordered nitras argenticus in the form of pills, two centigrammes three times daily, to disinfect the stomach contents. I have often in similar cases attained excellent results from this medication. You

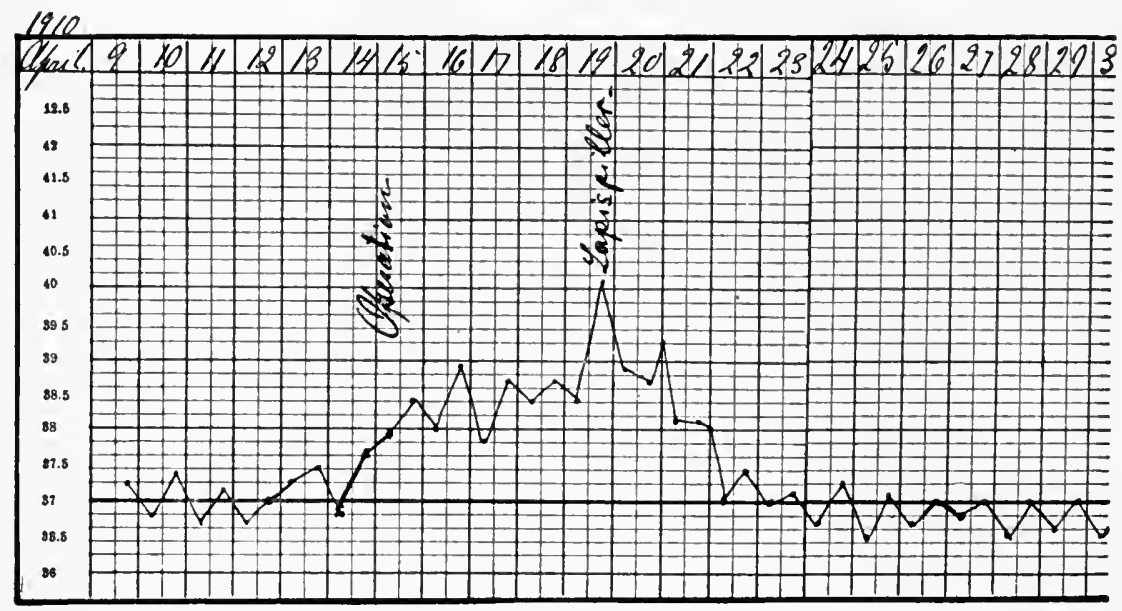

Fig. 93.-Temperature curve after pylorectomy, showing the result of silver nitrate pills.

see how the temperature fell quickly in this case and reached normal in the course of two days.

With regard to the after-treatment, which plays such an important part in the successful result, I administered ten centigrammes opium three times in the twenty-four hours, to insure a cessation of peristalsis. This is best given in the form of suppositories. I consider it a mistake to begin with aperients, eserine injections, and other remedies, immediately after the operation, in order to rouse and incite the peristalsis, as so many surgeons do in order to prevent ileus with paralysis of the intestines. This treat- 
ment is suitable only after operations for diffuse peritonitis or ileus, where there is already a paralytic or paretic condition of the intestine, and it might easily cause injury in other cases. On the fourth day, as a rule, I give no opium; on the morning of the fifth day a tablespoonful of castor oil as an aperient.

The method of nourishing is of the greatest importance during the first days following a pylorectomy, especially in cases like this, where the stricture had caused enormous stagnation and dilatation of the stomach. You must remember that all the food has to pass the point of resection, a point where the swollen edges of the wound protrude into the lumen, as a result of œedematous swelling of the mucous membrane. At first, therefore, the passage is rather obstructed, and as it is important not to irritate the wound, but to allow it to heal in peace, all solid food should be restricted for eight to ten days, until we can reasonably expect the healing to be well advanced. At first one should also be very careful with liquid nourishment, because it is important to allow the dilated stomach to contract undisturbed. If one allows a large amount of fluid to accumulate in the stomach, this is weighed down and drags on the area of the resection, thus producing a bend between the new pylorus and the stomach, whereby the obstruction of the passage recurs. During the first days, therefore, the patient should be allowed to drink only small quantities of boiled fluid. During the first twenty-four hours I give water only - a teaspoonful at a time, in all one-half pint; during the next twenty-four hours gruel and tea are also given, in all one pint. The nourishment and the supply of fluid, therefore, first take place essentially per rectum. Three to four times during the first twenty-four hours, 300 to 400 cubic centimetres of physiological salt solution are injected, into which fifteen grammes of glucose are dissolved, and sometimes raw eggs and a little milk. If the patient, by 
reason of his inanition, anæmia, and toxic albuminuria, requires a rapid and abundant supply of fluid, this is given in the form of a subcutaneous or intravenous salt-water infusion-one to three litres in the twenty-four hours. In the present case I found it necessary to use an infusion of salt water for only one day; on the fifth day of the illness, when the temperature rose so high, I gave him two litres of salt solution subcutaneously. On the seventh day I dispensed with the food injections, and gave him custard, broth with eggs and cream, in addition to the gruel and tea. As he stood this well, I gave him pea and potato puree on the next day, and on the tenth day fish-cake and apple compôte, and now he eats fish and bread in addition.

In order to determine how much good was obtained from this operation relative to the function of the stomach, we gave the patient one of Bourget's test-meals with prunes and cowberries. At the examination, next morning-ten hours later-we were agreeably surprised to find the stomach quite empty; nothing could be extracted by pressure or aspiration from the stomach, which a fortnight ago retained 2000 cubic centimetres residuum.

The healing of the laparotomy wound has, so far as we know, been quite reactionless. I now remove the cottonwool dressing, and you see that the wound has healed completely. I now remove the sutures, and again cover the wound with collodion-cotton wool. To-morrow this patient will be allowed to sit up in bed, and next day he will be permitted to get up, and we hope to discharge him within a week. You see to what extent his looks have already improved, especially his complexion, and he is also beginning to put on flesh.

It is, then, quite an ideal result which we have obtained by this pylorectomy with a direct joining of the stomach and the duodenum. How ideal this method is in comparison with the gastro-enterostomy generally employed in such a 
case as this is sufficiently obvious: by the latter operation one leaves the ulcer with the possibility of cancerous degeneration, perforation, etc., forming an artificial outlet from the stomach, the function of which is often very deficient and sometimes causes an influx of gall and vomiting,-i.e., circulus vitosus in a more or less pronounced degree; by our pylorectomy we have not only radically removed the disease, but have even reëstablished the natural passage for the food! Why, then, is pylorectomy so seldom performed in cases of ulcer-stenosis? One reason is that pylorectomy, as compared with gastro-enterostomy, is a rather difficult operation, because the pylorus generally lies fixed in its position, while gastro-entero-anastomosis is performed on the most mobile part of the stomach, which, together with the intestinal coil, which has to be employed, can be easily drawn forth from the abdominal wound. A second reason is because the immediate result of gastroenterostomy is, as a rule, excellent in cases of stenosis of the pylorus, inasmuch as it removes with one blow the stagnation which was the cause of all the conspicuous symptoms. Finally, experience has shown that the direct joining of the duodenum and the stomach after the extirpation of the pylorus for cancer or ulcer is dangerous on account of the greatly differing dimensions of their lumens.

The disproportionate sizes of the opening in the severed ends of the duodenum and stomach necessitate a partial closure of the stomach wound and the insertion of the duodenum into the lower end of the incompletely closed stomach wound. This method was devised by Billroth (see Fig. 94). This method, however, was not satisfactory on account of the meeting of three suture lines, which left a decidedly weakened spot, and, therefore, it was thought best either to close both the duodenal and stomach openings and complete the operation by performing a gastro-enterostomy, or else proceed as suggested by Kocher, by first 
closing the opening of the stomach and then implanting the duodenum into the posterior surface of the stomach. It must be understood that such an operation is more of an undertaking than was necessary in the present case. In benign cases, especially those due to ulcer, the gastro-

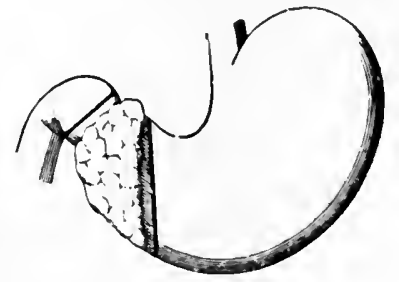

A

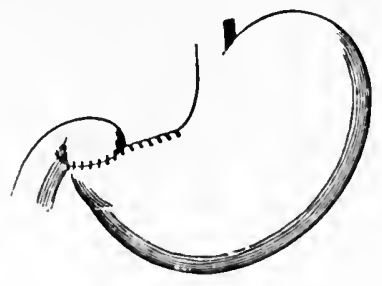

B

Fig. 94.-Pylorectomy after Billroth's method; $B$ shows the weak point where the two sutures join.

enterostomy recommended by Wölfler and von Hacker are still to be preferred, especially when the ulcer is situated on the lesser curvature.

Other methods have been devised in order to avoid the dangerous operation of extirpation of the pylorus and partly to avoid the drawbacks of the gastrojejunostomies
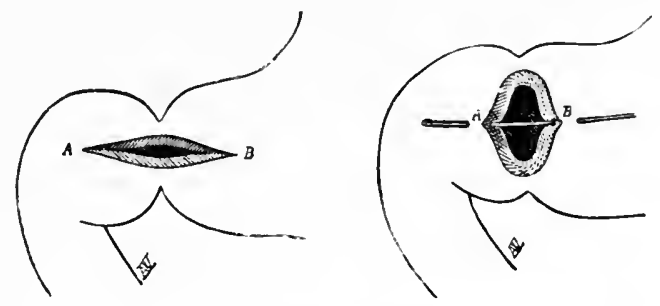

Fig. 95.-Heinicke-Mikulicz pyloroplasty.

devised by Wölfler and von Hacker. The oldest method is that of Loreta, in which a small incision is made in the stomach, and the pylorus is dilated by introducing a finger, a bougie, or a dilator of some kind. This operation, however, is only of temporary value, and may even be dangerous in the presence of fibrous strictures which may cause a rupture of the viscus and give rise to peritonitis. 
Another operation, however, the so-called pyloroplasty, also called the Heineke-Mikulicz operation after its two progenitors, still has its adherents among Mikulicz's pupils (Kausch, Küttner). It consists in cutting the pylorus from outside with a longitudinal incision, which is then joined transversely, so that the terminating points of the incision $A$ and $B$ meet in the centre of the suture line (see Fig. 95); in this way the contraction is changed to a dilatation. This operation, however, has been abandoned by most surgeons, because the cicatrized and inflamed tissue is so unsound, and so insufficiently nourished for operative purposes, that relapses easily occur. The method of pyloroplasty devised by Finney is really a combination of this and duodeno-gastrostomy, inasmuch as he prolongs Mikulicz's incision through the pylorus downward, both on the duodenum and stom. ach, so that the inci-

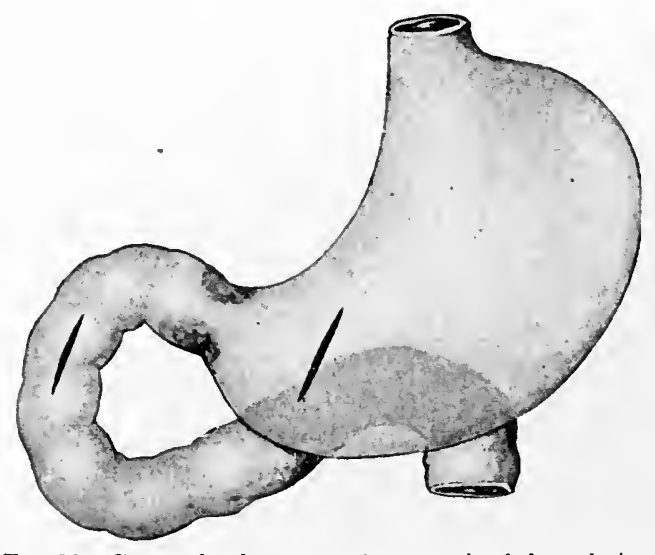

Fig. 96.-Gastro-duodenostomy for stenosis of the pyloris. After Jaboulay and Villard. sion acquires the shape of a reversed $U$ or of a horseshoe.

The anastomosis between the duodenum and the dilated stomach (see Fig. 96), which was first performed by Jaboulay and Villard, has many more adherents, especially since Kocher has facilitated the union between the stomach and the duodenum by his technic. He mobilized the duodenum by means of a longitudinal incision through the peritoneum where the visceral layer passes over into the peritoneum parietale from the duodenum. I myself have no doubt that this anastomosis is far more natural than those generally employed, but when applying. it one meets with 
the same difficulty as one does with pylorectomy: a deeplying and less accessible field of operation. Under these circumstances it seems to me far more natural to extirpate the pylorus itself. In the presence of a genuine cicatricial stenosis, when only the pylorus itself is removed, the direct union of the duodenum and the stomach presents no difficulties, and, besides being a radical operation, is no more difficult or complicated than Kocher's gastro-duodenostomy; and where a large ulcerated tumor is present, which calls for the removal of a large portion of the stomach, the duodeno-gastrostomy will be attended with great difficulty, partly in the execution and partly because its effect may so easily prove insufficient, inasmuch as it leaves such a large wound. In such a case one would therefore rather remove the pylorus and the diseased portion of the stomach, completely close the large opening in the stomach, and, if possible, implant the duodenum into the posterior side of the stomach. If this is not feasible, I prefer a Wölfler's anastomosis. I should think, therefore, that pylorectomy, in cases of ulcer stenosis, will be the operation of choice in future. If, however, it is a question of pylorospasm, with a small superficial ulcer, then gastro-enterostomy is indicated.

Our third patient was a widow, forty-three years of age, who, as you remember, had suffered from pain to the left as well as to the right of the centre line. You remember, we construed the left-sided pains, from which she had suffered for many years, as genuine gastroptosis pains, while I interpreted the very acute pains in the right hypochondrium, which developed only during the last four years, together with vomiting and retention in the stomach, as being a stenosis on the duodenal side of the pylorus, arising from a fixed bend between the sunken stomach and the fixed duodenum, with a possible ulcus duodeni developed 
thereon; because, as I described to you, gastroptosis sometimes leads to ulcer formation. Finally, you will remember that I entertained a certain fear of a commencing cancerous degeneration, on account of the considerable emaciation and miserable appearance of the patient. When now you look at the patient, who has just been brought in, you will find difficulty in recognizing her, looking, as she does, so well, happy and contented. This is because, in her case also, we have succeeded in entirely removing the stenosis and the retention, so that she can now eat and digest all kinds of food; but we have attained this with a far less extensive operation than with the last patient. At the operation, in fact, we made the following characteristic discovery: As soon as the peritoneum was opened the dilated and greatly folded stomach leaped forth through the wound, and deposited itself on the outside of the abdominal wall, accompanied by the pylorus and about five centimetres of the duodenum. As I had expected, the pylorus itself did not appear contracted, but, on the contrary, dilated, because the obstruction was situated in the duodenum, and was partly due to the sharp bend between the fixed portion and that portion which had subsided with the stomach, and partly to a strong, whitish cord which extended downward from the hilum of the kidney, over the duodenum, to the pyloric part of the stomach, drawing a deep furrow across the duodenum, which was bent sagittally over this cord. On further examination the cord appeared to be the anterior leaf of ligamentum hepatico-gastro-duodenale, which by the prolapse of the stomach had been drawn into a long band-like cord. It was tied with a double ligature, and as soon as it was snipped between the ligatures, the entire contraction was removed. No ulcer of the duodenum could be found.

When I examined the stomach itself, I found 
a thickened area which appeared at first to be an ulcer on the point of perforation, situated on the lesser curvature in the angle between pars cardia and corpus. In the centre of the infiltrated part the exploring finger slipped down into a funnel-shaped hole, which at the first glance I interpreted as a loss of substance in the wall of the stomach, but on further examination, by palpating the side of the mucous membrane through the anterior wall of the stomach, it appeared that, corresponding with the depression felt on the outside, the wall of the stomach projected globularly or conically into the lumen. It now appeared that the condition was due to a fixation of the deep folds of the stomach wall between pars cardia and corpus by extensive adhesions which had formed between the serosa surfaces.

As I have previously told you, an ulcer may develop on a fold of the stomach-wall, which is present in the so-called hour-glass stomach. In the present case I introduced a gastroscope into the stomach and examined the very prominent fold which was present, and found the projecting mucous membrane swollen and red, but without any loss of substance. After examining the remainder of the stomach, I found no other abnormalities. I passed the gastroscope into the duodenum and found the mucous membrane quite normal. I then closed the small incision in the stomach and performed a gastropexy, believing that the ptosis was the real cause of the stenosis and the retention of food in the stomach. That this was correct has been strikingly proved by the immediate relief to the patient. The pains entirely disappeared, the vomiting ceased, and the appetite has returned since the operation. Here you see the patient two weeks after the operation looking fresh and happy and already well on the way to recovery, instead of a depressed and emaciated and almost cachectic patient.

You see, therefore, that our diagnosis in this case has 
not been entirely correct. There was a stenosis of the duodenum resulting from ptosis, but it had not reached the ulcer formation, which would certainly have been the case if the patient had continued under her former treatment. In many cases like this it is impossible to make an exact diagnosis or to tell beforehand whether the ptosis has led to an ulcer or not, but it is very fortunate when, as in this case, one forestalls the formation of ulcer and removes the predisposition by a gastropexy.

In a case where an ulcer had already formed on the fold between pars' cardia and corpus a simple gastropexy was followed by recovery, but if the stenosis here had resulted from a cicatrizing ulcer, there would have been indication for a gastro-enterostomy. 


\section{LECTURE XVII}

\section{TUBERCULAR ULCER OF THE STOMACH}

Lex coincidencia, there were brought into this ward at the same time two patients whose symptoms are very similar in many points and, if my diagnosis is correct, are rather rare and of a serious nature. The first of these patients whom you see lying here is a woman thirty-one years of age, who comes of a fairly healthy family and who had enjoyed good health until about five years ago, when she had an attack of pleuritis, for which she was treated by puncture. A large quantity of clear fluid was withdrawn from the chest cavity. Since that time she has had no further pulmonary symptoms, and had felt quite well until eighteen months ago, when she began to suffer from cardialgia, accompanied by sour eructations and a sense of oppression and the presence of a swelling in the abdomen, which became quite prominent after meals. This was followed by constipation, which, on and off, gave place to diarrhœa. After these attacks had existed for some months she began to have attacks of vomiting, which would come on two hours after meals. She vomited some fluid and particles of food, some of which she had taken into the stomach on previous days. There was no evidence of blood or blood-clots in the vomitus. Twelve months ago she was admitted to the Kolding Infirmary on account of these attacks and she was placed upon a light diet, was given bismuth, eastor oil, and her stomach was washed out. In this way her condition was greatly improved; and, after she had been shown how to wash out her own stomach unaided, she was discharged. She continued the lavage every three or four days, and felt fairly well until three months ago, when the vomiting again appeared. She thinks she 
vomited as much as two to four pints at a time, at intervals of two to three days. There was no blood in the vomitus.

One month ago a tumor appeared in the abdomen which when seen showed signs of fluctuation. This was incised by a physician and the wound quickly healed, with the exception of a small fistula, which persisted. A short time afterward another tumor appeared in the fossa supraclavicularis. This was also incised and the fluid withdrawn. These operations were accompanied by considerable exhaustion, and the patient states that she lost twenty pounds in weight during the year. At all events, she is very thin and on admission weighed only eighty-eight pounds. Her complexion is rosy and she does not show any cachexia. Examination of the lungs shows them to be normal. On the right side of the neck are several enlarged lymph-glands which are seen above the cicatrix of the last operation. The functional examination of the stomach shows a considerable retention of foodstuffs, which accords well with the patient's history, and the stomach seems to be considerably dilated, inasmuch as it holds two litres without difficulty. After giving the patient a plateful of bismuth porridge, a Röntgenray examination shows the stomach to be greatly contracted, almost in a state of spasm in an attempt to drive the porridge through the pylorus; but as the peristaltic wave reaches the pylorus it is repelled and a counter antiperistaltic movement takes place, so that the bismuth remains in the stomach. This gives us very valuable information concerning the motility of the stomach, which is demonstrated to be unimpaired and possibly even increased by hypertrophy of the muscles of the stomach wall. It is important to know this in giving a prognosis of such a case, for it tells us that as soon as we succeed in removing the contraction or providing another exit from the stomach that organ will be able to empty itself.

The question now is, what sort of a stenosis is this, and 
and is it due to an ulcer or a new formation? To decide this may be very difficult. With regard to a new formation, we have three cardinal symptoms on which to found our diagnosis: cachexia, achylia, and ostensible tumor, but you must remember that none of these symptoms is really decisive, and that they may all be present without there being any question of cancer, just as there may be cancer without the presence of these symptoms. In this instance one is justified in mentioning cachexia, because the patient is very emaciated, though a genuine cachectic complexion is lacking. By examining the chemical function of the stomach, assisted by one of Ewald's experimental meals, we find a proportion of 15 free hydrochloric acid in a total acidity of 52, which is, if not a normal acidity, yet far from achylia. But if this speaks mostly in favor of ulcus, yet it in no way precludes cancer, because even with cancer we may at times find a considerable quantity of hydrochloric acid present. Conversely, we may frequently have achylia with a simple gastritis, or with a gastritis arising from an ulcer.

Finally, where a palpable tumor is encountered, it must always influence our judgment. If I palpate the epigastrium while the patient lies as she is now doing, and respires naturally, absolutely no tumor can be felt; but if I allow the patient to inspire deeply while my hand rests on the epigastrium, I at once feel a tumor, resembling in size and shape a small melon-shaped apple and lying crosswise, thrusting itself forth from under the right curvature toward the median line. To the touch the tumor is rather hard and smooth and fairly mobile, especially in an upward and downward direction. If one does not obtain a firm hold on it, it glides back beneath the liver and under the border of the ribs during expiration. The presence of such a tumor must naturally give rise to a serious suspicion of cancer, but in itself it constitutes no proof of this, because an ulcer 
near the pylorus can eause such an inflammatory infiltration around the pylorus that it may resemble a tumor, which, even when the abdomen is opened, is not macroscopically distinguishable from a new growth. Sometimes a swelling of the lymphatic glands lying near the pylorus may contribute greatly to the impression that there is a tumor. Even microscopically it is sometimes difficult to decide whether we are faced by a simple ulcerous tumor or a combination of this and cancer, because you must remember that most stomach cancers originate in an ulcer base. If the cancerous degeneration or infection-call it what you will-is limited to a single small area, you will understand that you must trust to chance to find the cancer by microscopy; that is, if you do not make systematic serial section of the whole tumor.

What diagnosis shall I give you, then, in this case, when I regard it from a purely clinical point of view, and compare the symptoms as they have developed with the results of the objective examination? Well, I must say that I feel assured, despite the presence of a tumor, that we are face to face with a stenosis of the pylorus with dilatation of the stomach, due to ulcus pylori, and, without venturing to maintain it with certainty, I think there is a great probability that this ulcer is of tuberculous origin. We have before us a patient whose complaint commenced with pleuritis of an indubitably tuberculous nature. Three years later her ulcer symptoms began, and, after these had lasted for some time, a suppurating tuberculous adenitis appeared in the right regio supraclavicularis, which spread to the glands of the neck. The patient's appearance is absolutely non-cachectic, and reminds one far rather of the erethistic form of scrofula; moreover, the gastric juice contains no small quantity of free hydrochloric acid.

Perhaps I incline all the more toward this diagnosis when I observe the second patient, who is now being brought 
in. He is a laborer, forty-one years of age, whose attacks greatly resemble those of the patient examined last. This patient is said to have enjoyed ordinarily good health until twelve months ago, when he began to suffer from dyspeptic attacks similar to those of the last patient: pains and oppressive sensations in the epigastrium after meals, and vomiting some time after these. These vomitings, which have become more and more copious, have never contained blood, but, on the other hand, have often contained remnants of previous days' meals. With this patient, also, a glandular tumor developed in the right supraclavicular space, and a similar tumor developed shortly afterward on the neck, but both healed quickly, after being treated by incision and erasion, though leaving small tuberculous fistulæ.

On examination you find a strongly-built but greatlyemaciated man with a bad, pale, but not distinctly cachectic complexion. Here the examination of the stomach-quite as with the previous patient-indicates a very considerable retention. The chemical examination shows free hydrochloric acid (12 with a total acidity of 70 ). With this patient the Röntgen illumination of the stomach after bismuth porridge shows no peristaltic movements, in which it differs from the last case: one sees the bismuth porridge lying immovable at the bottom of the greatly-dilated stomach. This means that in this case the process is more advanced and serious, because the stomach has ceased to contend with the obstruction, and we must, therefore, be prepared for a severe atrophy of the muscles of the stomach.

Finally, this case is distinct from the last because here we do not feel a mobile, well-defined tumor in the region of the pylorus, but an apparently more diffuse distention deep down to the right of the centre line. Without venturing to deny the possibility of cancer in this case, I am 
mostly inclined to believe in a pylorus stenosis ex ulcere; and in this, as in the previous remarkably similar case, I consider it highly probable that it is a tuberculous pyloric stenosis, caused by infiltration, and formation of fibrous tissue, and processes of shrinkage at the circumference of a tuberculous stomach ulcer.

Perhaps you will ask me: Do tuberculous stomach ulcers exist at all? And with some justice, because the occurrence of stomach ulcer is, indeed, so little known that it is not mentioned in many of the text-books in general use.

When in December, 1902, I was confronted with this question for the first time by finding, when operating on an hour-glass stomach, that the perforated stomach ulcer which had caused the deformity was greatly suggestive of tuberculosis, not only was its appearance suggestive, but we also found a large, cheesy-like lymphatic gland situated in front of the lesser curvature, which showed under the microscope typical tuberculosis. There was then practically no surgical literature, and very little obtainable from the pathological anatomists concerning the occurrence of tuberculous stomach ulcer, so that I published my interpretation of the case with great reservation in Hospitalstidende (No. 15, 1903) under the title "Ulcus Ventriculi Perforatum Tuberculosum?" I was all the more reserved because the cases were quite devoid of surgical interest from a pathologic-anatomical point of view, inasmuch as they essentially concerned small multiple ulcerations of the mucous membrane which had arisen $s u b$ finem vito with tubercular patients. But since then other surgeons as well have shown that the "surgical" stomach ulcers are not uncommonly of tuberculous origin. Yes, it is not improbable that, when the attention of surgeons is more generally roused to this possibility, it will appear that a 
no small percentage of the cases of pyloric stenosis and ulcer is in reality of tuberculosis origin.

Ricard and Chévrièr in particular have pointed out, in a treatise in the Revue de Chir., $1905,{ }^{1}$ that tuberculous ulcers of the stomach are of considerable surgical interest, and not, as formerly supposed, only found in the last and hopeless stages of general tuberculosis. Besides those cases described by the pathological anatomists, where small mucous membrane ulcerations are found in tubercular patients scattered on the stomach-wall, there are quite distinct forms of tuberculosis of the stomach, which, like the surgical tuberculosis in other organs, occur especially in patients who are otherwise free from tuberculosis; and, while the older known forms of tubercular ulcerations of the stomach are due to direct infection of the mucous membrane of the stomach by the bacilli swallowed with the expectoration, these forms of gastric tuberculosis seem to be due to embolism, and this again is in conformity with the other forms of local surgical tuberculosis in other parts of the organism: bones, kidneys, etc.

What one might then call surgical tuberculosis of the stomach rarely occurs, as in my first case, in the form of a large, deep penetrating ulcer on the lesser curvature, but far more frequently in or near the pylorus, thus causing stenosis of this. It is this form in particular which Ricard and Chévrièr have studied, basing their observations on sixteen cases, of which four are their own, and the rest collected from the literature. To these, as early as 1907, Conrad Brunner ${ }^{2}$ can add eleven cases of tubercular stenosis of the pylorus: one personally operated on by him, and ten collected from the literature. Since then I myself have operated on three patients with tuberculous stenosis of

1 "De la Tuberculose et des stenoses tuberculeuses de Pylore," R. de Chir., B. 31 , p. 559 .

2"Tuberkulose, Aktinomykose, Syphilis des Magen-darmkanals," Deutsche Chirurgie Sieferung, 46e, 1907. 
the pylorus, and if the two patients I have shown you to-day should also prove to be tuberculous, my personal contribution will be no less than six cases. That so many cases have been collected from the records of so few surgeons seems to me to indicate that it is a rather frequently occurring affliction, the nature of which it is often impossible to diagnose clinically. Even at the operating table it can easily be misinterpreted, because it resembles cancerous stenosis in some cases and simple ulcer stenosis in others. If we analyze further the recorded cases, it seems that tubercular inflammation appears in two very different forms, just as is found in other parts of the body: the fibrous and the hypertrophied or infiltrating. The fibrous form, which I believe to be represented in the first of these patients whom I have shown you to-day, is distinguishable by this, that generally from a small ulceration a sclerotic inflammation spreads diffusely into all the layers of the pars pylorica, so that it feels like a hard, cylindrical tumor with a smooth surface, and is comparatively sharply defined. If you find large, hard glands along the lower edge of the pylorus, and small, seed-like nodules on the peritoneum overlying this, you can readily understand that it is very suggestive of cancer; and if you are then content with a gastro-enterostomy, and do not, as I have done in two cases, extirpate either the pyloric portion or the swollen glands for examination, you may easily remain in ignorance of the true diagnosis.

The other form, which I believe to be represented in our second case, often gives the impression of deep infiltration due to an ulcer or cancerous infiltration. That these cases also may easily be misjudged even during an operation is, therefore, obvious.

It is, then, not unreasonable to assume that the attention of surgeons, when directed to the possibility of contracting, callous, even perforating stomach ulcers being of a tuberculous nature, will greatly increase the casuistry, 
and make the tuberculous stomach ulcer an important chapter in gastric surgery.

The fact that a certain number-be it large or small-of stomach ulcers regarded hitherto as ulcus rotundum or simplex are tuberculous has great significance, not only as regards diagnosis and treatment, but also, and not least, with regard to the question of the pathogeny of the stomach ulcer. This remains, as you know, in strikingly profound obscurity when one considers the frequency of the disease and the ardent research which has for so long been devoted to it. By means of numerous experiments on animals one has endeavored to disperse the obscurity which surrounds these cases.

A priori, one would imagine that the whole question was quite clear and the origin of the wound easily explained as a result of the corrosive, digesting effect of the gastric juice on small, accidental, traumatic lesions of the mucous membrane. It is certain that the mucous membrane of the stomach is frequently abraded by the hard, pointed objects conveyed to it in the food,-i.e., fish-bones, meat-bones, wood splinters, etc.; also, it is certain that "autodigestion," the eroding and digesting effect of the gastric juice, must play a part, since we only find ulcers of this description in those places to which the effect of the gastric juice has access; namely, in the stomach, the upper part of the duodenum, and the lower part of the œsophagus-never in the remaining part of the intestinal canal, except when an anastamosis has been formed, artificially or by disease, between the intestine and the stomach. The so-called ulcus pepticum, the development of which forms a distressing and dangerous complication in a certain number of cases of gastro-enterostomy, is, therefore, only another proof of the correctness of the theory.

This simple and plausible explanation met with various objections. Firstly, because in the majority of cases the typical stomach ulcer is situated on, or close to, the lesser 
curvature, and most frequently in the neighborhood of the pylorus, though one would expect all the other parts of the mucous membrane to be quite as exposed to traumatic lesions, and even more so to the influence of the gastric juice, which one would expect to be even more constant and intense at the bottom of the stomach. There are actually two Danish theories in explanation of this state of affairs. The one was propounded in 1886 by Anker Frode Rasmussen (now Halk), and was to the effect that the frequent occurrence of ulcers on the lesser curvature was due to the pressure of corsets and laces, because at numerous dissections of women at the General Hospital he had found cicatrices corresponding exactly with the furrow of the lace. This theory, which caused a great sensation and found many supporters, is untenable, however, because it rests on the generally-accepted but incorrect idea that it is essentially women who suffer from gastric ulcer. I have shown you in a previous lecture that this is a clinical delusion due to confusing gastric ulcer with gastroptosis, which is so general with women; the fact of the matter being, as shown both on the dissecting and on the operating table, that the chronic stomach ulcer is, if anything, more frequent with men than with women. But, as a man does not lace himself or wear stays, Rasmussen's theory falls to the ground.

For the other Danish theory we are indebted to C. E. Bloch (Hospitalstidende, 1905), who founds it on the observations made by Max Matthes, Griffini, and Vassale when experimenting on animals, that defects produced experimentally in the mucous membrane always heal quickly, because the muscularis contracts, and, from the sides, the folds of the mucous membrane completely cover the wound and prevent any erosion by the gastric juices. Bloch has now proved, by examining a number of stomachs which were fixed by formalin injection immediately after death, that the mucous membrane of the lesser curvature, and 
especially toward the pylorus, cannot be folded like the rest of the mucous membrane of the stomach, and finds in this the explanation of the stomach ulcer being so often located in the vicinity of the lesser curvature and pylorus. Here Bloch has undoubtedly indicated a factor of great importance, especially with regard to certain ulcerations. I formerly had opportunity to draw your attention to an analogous state of affairs which exists among ptosis patients, inasmuch as I have shown you that, when an ulcer develops in a prolapsed stomach, as it rarely does, it does so in places where a fold of the stomach has become fixed by peritoneal adhesions, so that an immovable crest of the mucous membrane projects into the stomach, and is then especially exposed to traumatic abuse, and is incapable of avoiding the action of the gastric juice. But Bloch's theory does not quite suffice to explain to us the origin of the typical round stomach ulcers; because, with numerous experiments on animals, even large pieces of the mucous membrane of the lesser curvature have been extirpated without a chronic ulcer having been successfully produced. In the course of a sliort time, also, such a loss of substance often heals without leaving a cicatrix. In order to produce a chronic ulcer at all in this manner, it has been necessary to resect nervus vagus (Talma, van Ijzeren; and Zironi). One can then produce typical chronic stomach ulcers; but, at the same time, it las been produced under conditions which do not exist in patients in whom we find stomach ulcers. It is necessary to search elsewhere for an explanation of the stomach ulcer. It would be easy to lay the blame on chlorosis and anæmia, because these are supposed to involve fatty degeneration of the small vessels with subsequent thrombosis. The theory of infection has had many adherents (Mayo, Robson, Moynihan), and according to them the ulcers were supposed to be due to pathogenic bacteria from carious teeth or other foci of infection in the mouth cavity, and they have succeeded in supporting this 
theory by experimenting on dogs. For instance, Turk insists that he has produced a stomach ulcer by administering a pure culture of colon bacilli during several months; while Gibelli is said to have achieved the same result by injecting the animals with strepto- and staphylococcus after he had injured the mucous membrane of the stomach. The ulcerations thus produced have no resemblance whatever to the typical stomach ulcer, but resemble far more closely the small, superficial, scattered ulcers, which we also see develop acutely with people during some severe infectious disease, especially with septicæmia and pyæmia. A stomach ulcer which might correspond better with the true conditions has been produced in a different way. It consists in producing, aseptically or septically, an embolism in the vessels of the stomach. In 1869, Godivier reports a case of duodenal ulcer with embolism of arteria pancreatico duodenalis; but, experimentally, it is Panum who deserves credit for producing a gastric ulcer (1862) by injecting small wax balls into the circulation. It is easy to understand that the necrosis produced in this way must be a far better object of attack for the gastric juice than a fresh small wound; and I will not deny that I feel strongly convinced that, as a rule, if not always, there is a process of embolism at the foundation of most chronic stomach ulcers.

If now we return to our discussion of tuberculous ulcers and ask ourselves whether it is more reasonable to suppose that these are due to an embolic process, or to a direct infection of the mucous membrane by tuberculous bacilli which have accidentally entered the stomach with the food, I must express myself as being absolutely in favor of the embolic origin. When we realize how seldom it is that tubercular patients, who swallow large quantities of sputum, have gastro-intestinal tuberculosis, it is per se not very probable that patients such as our two, who have no demonstrable phthisis, and with whom, therefore, tuberculous bacilli could only be supposed to have been accidentally mixed in 
the food, should have been infected in this way. At one time we believed that the gastric juice was absolutely destructive to tuberculous bacilli, but this has been disproved (Strauss and Würtz), for Fibiger ${ }^{3}$ has been able to demonstrate five cases of indubitable primary tuberculosis of the intestines and the mesentery; in one of these cases he found a tuberculous ulcer of the stomach five centimetres in length. Therefore, one cannot entirely deny the possibility of infection through the mucous membrane, though the presumption of an embolic origin appears to be far more probable, especially when one considers that surgical tuberculosis always arises in just this manner in other organs. Arloing's experiments on animals also favor this view, though he succeeded in producing a tuberculous stomach ulcer only by embolism. You will understand that, if many typical stomach ulcers appear to be of tuberculous origin, this ar'gues much in favor of their being contingent on processes of embolism with necrosis, which are easily attacked by the gastric juice. Therefore, Bloch's theory may well retain a certain importance, because it is possible that necrosis in the movable part of the intestinal mucous membrane is, as a rule, healed by the surrounding folds of the mucous membrane covering the ulcer.

P. S.-You will remember that, in our last lecture, I rather audaciously diagnosed tuberculosis as being the most likely complaint with both the patients then present; and to-day I am so fortunate as to be able to inform you that the diagnosis proved correct in both cases. I will relate to you quite briefly what we discovered at the operation, and it will interest you to compare this with the account of the disease given in the last lecture. With the first patient we found the stomach greatly dilated, but also greatly infiltrated, which, as you remember, we had expected from

${ }^{3}$ Fibiger and Jensen: "Transfer of Human Tuberculosis to Cattle," Hospitalstidende, 1904, pp. 6-9. 
the strung contractions under the Röntgen illummation. I at once turned my attention to the pylorus, and here found a cylindrical, smooth, and hard tumor, which had grown fast to the pancreas, and along the lower edge of this tumor we found some large, very hard lymphatic glands, which directed my suspicions to cancer. I extirpated these lymphatic glands, and at once sent them to the laboratory for immediate microscopic examination, which was done while I performed gastroscopy. The gastroscope showed the mucous membrane round the pylorus to be hyperæmic, while the pylorus itself appeared to be contracted in the shape of a funnel; on passing the gastroscope into the funnel I discovered in a downward direction an ulcer the size of a three-penny bit, and having a grayish base, from the upper edge of which masses of granules invaded and completely closed the opening. The aspect greatly resembled tuberculosis-not carcinoma-and when a message from the laboratory informed me that the glands showed typical tuberculosis, but no signs of cancer, I decided to dispense with the resection of the pylorus, which must be considered a difficult and dangerous operation on account of the relation of the tumor to the pancreas. I simply performed a gastro-entero-anastomosis in accordance with Wölfler's method, making use of the incision which had been made in the stomach wall for the gastroscopy. To this I added, as usual, an anastomosis between the two arms of the loop of the jejunum, ten centimetres from the anastamosis in the stomach, to insure the patient against circulus vitiosus.

The other patient was, as you may remember, more open to suspicion on account of her cachectic appearance and the insignificant quantity of hydrochloric acid. Here also we found a cylindrically-shaped pyloric tumor the size of a small apple, which was not adherent to the pancreas. On the other hand, we found it adherent to the liver; further, I found that the omentum was infiltrated and fixed low down in the pelvis by old peritoneal adhesions. After I 
had mobilized and drawn out the pylorus, I found a walnutsized, cheesy gland. This was immediately taken to the laboratory for examination, and it showed tuberculosis. It then seemed reasonable to assume that the peritonitic adherences between the liver and the pylorus, and between the omentum and the abdominal wall, were the remains of an old tuberculous peritonitis resulting from the pyloric ulcer. As the under surface of the pylorus was not attached, I considered it proper to remove the entire diseased portion by resection, and all the more so because there was the possibility of a secondary malignant degeneration. I removed the entire diseased portion, together with two centimetres of the healthy duodenum and wall of the stomach, closed both wounds, depressed the duodenum, and performed a Wölfler anastomosis of the stomach with the jejunum. Finally, entero-anastomosis was performed between the coils of the jejunum.

The resected portion of the pylorus, which you see here, was so markedly contracted that only a thin knitting needle would pass through. You see how enormously infiltrated the wall is, essentially the seat of fibrous changes; but at the lower level of the infiltrated wall you see a cystic cavity filled with bodies resembling rice grains. This part shows typical tuberculosis, but $\mathrm{Dr}$. Permin-the clinical assistant-has discovered, on further examination, other parts which exhibit features of commencing adeno-carcinoma. On this point also, then, my suspicion seems to have been confirmed, and I feel greatly satisfied with having performed a radical resection of the diseased part.

P. S.-Both patients have presented themselves in this ward seven months after the operation completely cured of all symptoms as regards the stomach. The first patient has increased twenty-eight pounds in weight, and the other thirty-six pounds. The only complaint of both is that the broken-down glands of the neck have not yet healed. 


\section{LECTURE XVIII \\ HOUR-GLASS STOMACH}

IT is not long since we studied the signs and symptoms presented by a series of patients who were suffering from contraction of the pylorus. To-day I am able to show you a patient who, on the whole, presents the same clinical aspect, but where a single symptom shows us that something more complicated than a simple pyloric stenosis is concerned.

The pale, markedly emaciated patient whom you see here is a married woman, thirty-two years of age, who, ever since her earliest youth, has suffered from stomach trouble. With the commencement of puberty, about sixteen years ago, she began to feel oppressive pains in the epigastrium immediately after meals, to which were added nausea, eructations, and vomiting, but never any hæmatemesis, just as she had never observed melæna. Formerly the quality of the food seems to have played no part, but she could enjoy very little at a time. Periods of severe pain, together with severe spells of nausea and vomiting, have alternated with periods of relatively good health. She has often had to remain in bed for long periods, two to three months, which at first resulted in complete recovery. During the last two years the symptoms have not only grown much worse, but she has suffered all the time. As the patient is so emaciated and so exhausted that she is quite incapable of work, she has been admitted to this ward with a view to eventual operative treatment.

You see that the slenderly-built patient is extremely thin and pale, but without a really cachectic complexion. There are no enlarged glands of the neck, and the stethoscope shows normal conditions. You at once see, however, 
that the chest is long and narrow, and that the abdomen has to a pronounced degree the form characteristic of a ptosis patient; the epigastrium is drawn in and boat-shaped, while the region below the umbilicus is dilated convexly and is rather balloon-shaped, though not tense. You see, also, that the soft abdominal wall can easily be compressed. Furthermore, one quickly notices two very important peculiarities with this abdomen: a strong pulsation in the epigastrium, which you can all see with your naked eye, and which the palpating finger can feel as if the aorta lay quite close under the thin abdominal wall, and an audible splash with intermittent palpation of the dilated portion below the umbilicus. What do these two symptoms tell us? The pulsation in the epigastrium tells us that here we have a gastroptosis, that the aorta is no longer covered by the stomach; and the distant sound of splashing makes us suspect that the fallen stomach is also dilated. This is only a suspicion, because you must know that, if the patient has just drunk plenty of fluid, a prolapsed stomach can produce this distinct sound of splashing without there being any trace of dilatation. In this case, however, examination with an experimental meal and the stomach-tube have proved the suspicion to be correct, and that even a considerable state of retention is present, because, ten hours after administering one of Bourget's experimental meals we find an abundant quantity of stagnating fluid with numerous prune-skins and cowberries, which appear especially at the end of the suction process, and with the ensuing rinsing of the stomach. This is difficult, because whenever one imagines the stomach to be clean on account of the water returning clean, repeatedly a new admixture of food remains suddenly appears.

At the chemical examination of the contents of the stomach, which are withdrawn an hour after one of Ewald's experimental meals, rather high figures of acidity are found: 
45 free hydrochloric acid with a total acidity of 77 . From this it is natural to suppose that we are faced with a pyloric stenosis, resulting from ulcer with cicatricial formation, or from a ptosis bend; but a rather simple examination, which I shall perform in your presence, shows a most curious state of affairs, which alters our diagnosis considerably. I now pass a rubber tube down into the stomach and inflate this slowly by means of our usual little air-bulb. If you observe the patient's abdomen carefully during the inflation, you will first see depicted under the left rib curvature a sausage-shaped tumor, which extends downward to a point which lies mid-way between the umbilicus and ligamentum poupartii. This distention extends vertically downward from the left rib curvature, and all the way keeps slightly to the left of the centre line, and has the shape of a long sausage or banana. But if we continue the inflation, you will see that, from the centre of the epigastrium, a new tumor protrudes downward in the abdomen, parallel with the first, and separated from this by a furrow. It has a somewhat different shape from the first, inasmuch as it broadens upward, aiming toward the right rib curvature with its outer edge. This phenomenon, which is here typical in quite an unusual degree and is clearly defined, informs us with certainty that a case of hour-glass stomach - a ventriculus bilocularis-lies before us, and we now understand why food remains constantly return with the rinsing, when we thought the stomach perfectly clean. It is the food remains from the pyloric stomach, which, partly owing to pressure on the abdomen, partly owing to vigorous rinsing, are driven over into the cardial part.

In order that you may be able to understand what effect this discovery can have on the treatment, it must first be made clear what we mean by an hour-glass stomach, and how the different forms of this arise. From the designation hour-glass itself you will understand the characteristic 
of the affiction is, as a rule, that the stomach is in some way or another divided into two, and sometimes three, spaces, which communicate with each other by a more or less narrow opening or passage. The title hour-glass is at the first glance apparently not so appropriate, because the contraction is not circular, but seems to appear only when curvature major is drawn up (see Figs. 97 and 98). In several respects, however, this is a deceptive impression, which is due to the fact that the stomach is a soft pouch, fixed above and free below. In reality, the wall formation,

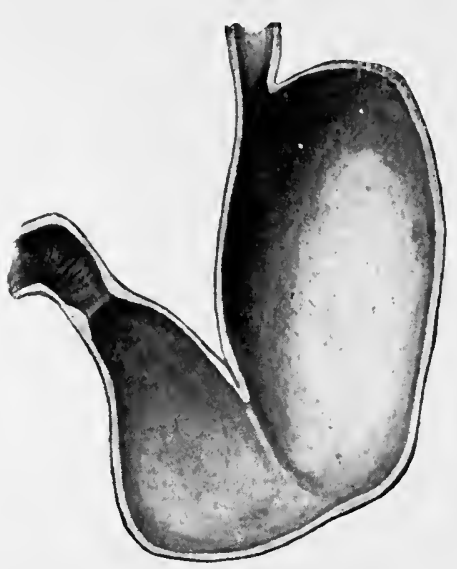

FIG. 97.-Commencing hour-glass folding. Ptosis bend in the duodenum.

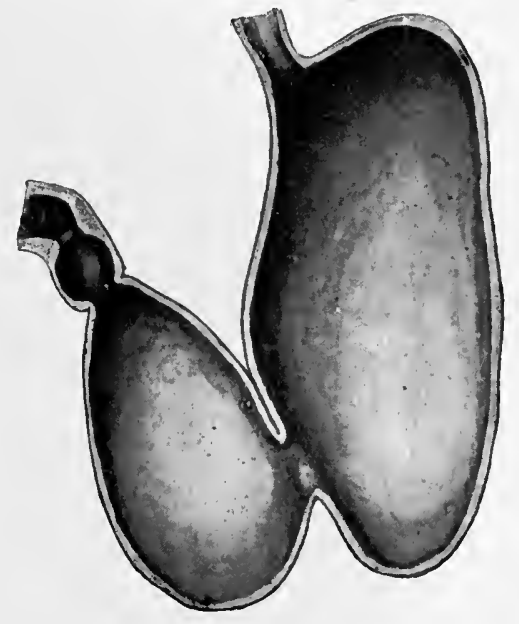

Fia. 98.-Developed hour-glass stomach with three spaces. Pyloric stenosis.

the pathologic process, starts almost always from the lesser curvature, but, as this is fixed, the contracting process expresses itself in the freely movable greater curvature being drawn upward. In this way a concave curve is produced, which is further marked out and deepened by the stagnating contents of the stomach, because both sides of it weigh down the stomach, so that the greater curvature bags strongly out at the sides of the part which is drawn up.

If we now inquire what is the cause and pathogenesis of this peculiar deformity, we plunge into the midst of a con- 
troversy which for many years has agitated the mind and has not yet reached its solution. Morgagni, who described the first case, and Monroe, who in 1813 was able to report a whole series of cases observed by Lorry, Walter, Ludwig, Hufeland and van der Kolk, found ulcer in all these cases, and took it for granted that the deformity was due to cicatricial retraction of the wall of the stomach. In the middle of the last century (1851), Struther made the assertion that there are two forms of hour-glass stomach, the one congenital and the other acquired-the latter founded on cicatrices following ulcer. In the following decades a rather large series of cases belonging to both these classes was reported, especially from England and America. By a critical perusal of all the cases then published (Virchow's Archiv, Bd. 140, p. 459), Hirsch, by the following reasoning, arrived at the conclusion that virtually all the cases must in reality be considered congenital, and were due to a congenital deformity. In a number of cases no ulcer was found at all, and in the majority of the cases where an ulcer was found it had the characteristics of a secondary ulcer, arising as the result of the effect of the stagnating contents of the stomach on certain parts of the partition wall, while the remainder was found covered with normal mucous membrane. In some cases, the ulcer was situated on the pyloric side of the partition wall. After this some went so far as to assert that, even in cases where the entire partition wall seemed to be made up of cicatricial tissue, this was due to an ulcer which had originated in a congenital hour-glass stomach, and had extended and finally destroyed the tissue in such a way that the original conditions were quite effaced. This theory predominated for a long time, and was, from a clinical point of view, rather plausible; because, with many of these cases, it was proved from the history that the symptoms of obstruction of the passage had existed even as far back as the years of 
puberty, and even of childhood! while the ulcer symptoms were of far later, relatively recent, date. By way of supporting this theory, I myself, in 1889, published an account of such a case of hour-glass stomach (Hospitalstidende, 1899, No. 32), where, at the operation, I found no ulcer at all on the partition wall, which was covered everywhere with normal mucous membrane, with the exception of a section the size of a florin toward the lower part of the cardial side, where a cicatrix was found in the mucous membrane. That the condition cannot in reality be the result of an ulcer, of a simple cicatricial contraction of the stomach, in the majority of the cases, seems to me incontrovertibly indicated by the aforementioned conditions. Nevertheless, during the last few years the tide has turned tremendously in favor of the ulcer theory, due in particular to the eminent English surgeon Moynihan's contribution to the dispute. He does not, in fact, undertake to invalidate the proofs against the ulcer theory, but he defends them indirectly by his explanation of the evidence that the disease should be congenital. He reviews the cases reported in the literature, and points out that, in reality, no evidence is forthcoming that in these cases the hour-glass stomach has been congenital. He also emphasizes very justly that, if the relatively numerous cases of hour-glass stomach were congenital, one ought to meet with this deformity rather frequently during the dissection of newlyborn children, but, in his opinion, only one certain observation of this kind exists in all literature. To an unbiased mind it seems reasonable that the hour-glass stomach may sometimes be congenital, and sometimes may be due to an ulcer, but that neither of these explanations applies to the large majority of the cases. It seems that authors have hitherto not been at all able to imagine any third possibility, but it is becoming necessary to look around for this, as the validity of the two other explanations has, in fact, 
been refuted, and with good reasons. Any such attempt at explanation would have to be attended by the consideration of a peculiarity of the hour-glass stomach which has not been dragged into the arena by either of the contending parties, perhaps, because it is equally unfavorable to both old theories. It is the factum that the disease occurs, so to speak-with very few exceptions-only in women. This is destructive to the ulcer theory, because the chronic, round stomach ulcer is at least as frequently, and the more severe forms absolutely more frequently found in men than in women, and can be just as easily reconciled with the thought of a congenital origin of the deformity.

The one person whose eyes have been opened to the fact that this disease must have some cause peculiar to women is Anker Frode Rasmussen ${ }^{1}$ (now Halk), who in his well-known work (1886) on lacing furrows in the stomach and tight-lacing as the causation of gastric ulcer also advances the theory that the hour-glass stomach is due to the same cause, because the pressure ought to produce a cicatricial shrinkage of the serosa. I think that here Rasmussen has put his finger on the right spot, inasmuch as he blames the misuse of corset and laces for the frequency of this disease among women, but I do not think he is right in his manner of explaining the effect of lacing as atrophy due to direct pressure, occasioned by the persistent pressure of the left rib curvature on the stomach, in a line reaching from the lesser curvature, close to the pylorus, down on the left toward the great curvature. For various reasons this does not fit in with the facts; because, firstly, we most frequently see the partition wall issue from the juncture of the cardia and the small curvature, dividing the stomach into a small cardial portion and a large pyloric portion, and the latter often becomes particularly large and distended

${ }^{1}$ A. Rasmussen: "A New Theory for the Origin of Ulcus Ventr.," Hosp. Tid., 1886, No. 17. 
by the presence of a stenosis in the region of the pylorus. Sometimes, as mentioned, we find two contractions of the stomach, so that this is divided into three portions. All this does not fit in with Rasmussen's lacing furrow, which should stretch obliquely from the pylorus to the fundus portion. It must be remembered, also, that he has demonstrated these lacing furrows with very old, emaciated women, while the serious cases, which surgeons get for treatment, concern, as a rule, middle-aged individuals, who for many years, often from the age of puberty, have shown symptoms of the existence of the disease. In fact, everything suggests the development of a deformity in the early years, and I have good reason to believe that, with predisposed individuals, the disease is due to the indirect effect of the corset pressure during these years.

I have emphasized for you, as being the two essential effects of corset pressure, the gastroptosis-sinking of the stomach-and the folding of the stomach from side to side, which we might term gastroplicatio. You remember that, when mentioning gastroptosis, I described to you how, with virgins having a tight abdominal wall which prevents the stomach from escaping freely down into the abdomen, the corset pressure forces the stomach to form folds, which run rectangularly on the longitudinal axis. The folds are most general at the angle of juncture between the fundus and corpus ventriculi; more rarely at the point of juncture between the pylorus portion and the duodenum, because adhesions form partly between these and partly between the stomach, gall-bladder, and liver. In a series of cases we have observed that these folds have led to an agglutination of the peritoneal surfaces lying against each other (Fig. 99), from quite fresh and fine, fibrinous adherences to broad, firm adhesions, by which the folds have become fixed. Under these circumstances you will understand that, on the boundary between the cardial part and corpus ven- 
triculi, there is formed a protruding crest or partition wall, which divides the stomach more or less completely into tro chambers, according to the depth and extent of the folds (see Figs. 97 and 98). Further, I have shown you cases

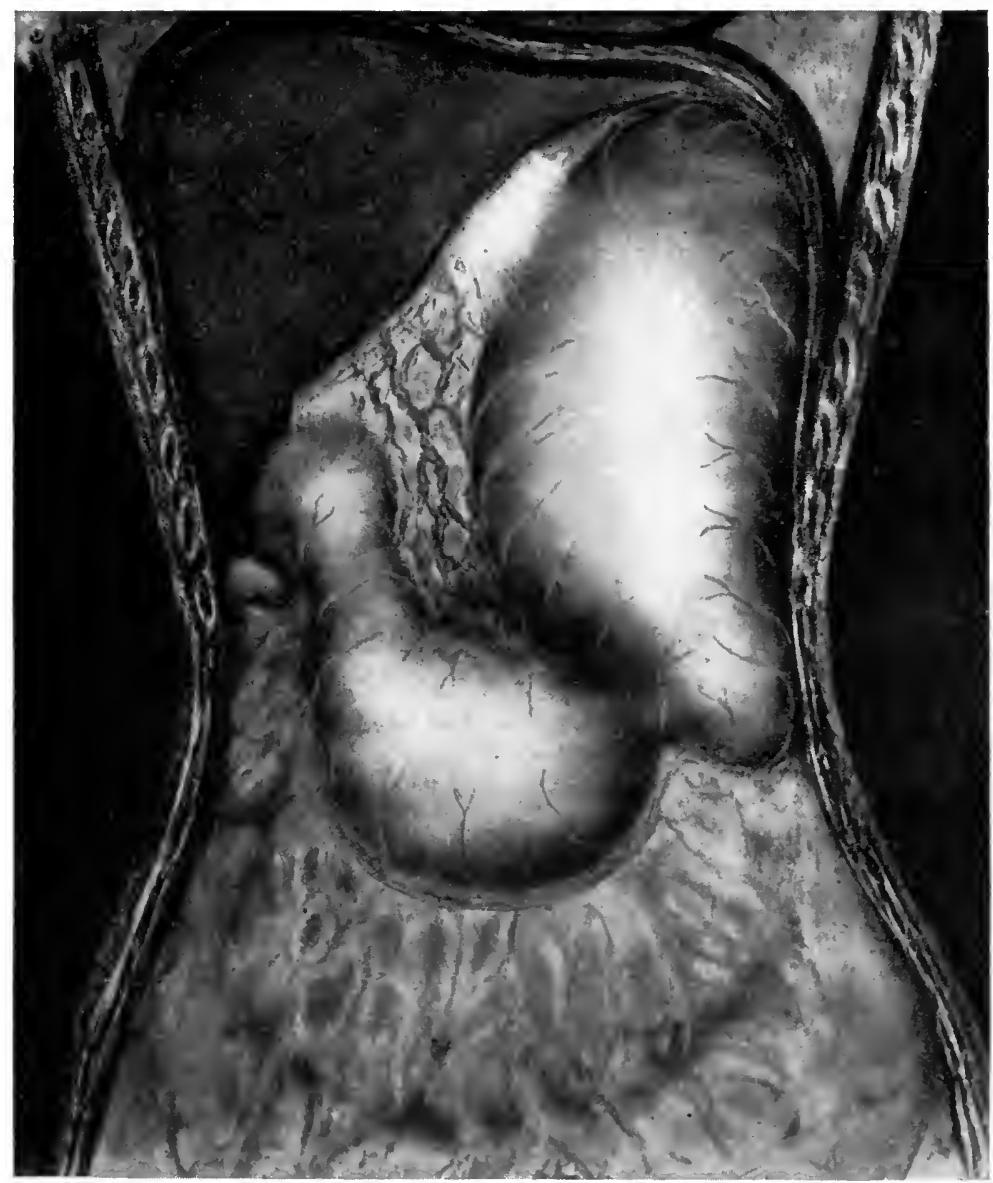

F1G. 99.-Hour-glass stomach, resulting from the folds growing together with their peritoneal surfaces.

where ulcerations have formed on this crest. In other words, we have seen a whole series of the phases of the development of the hour-glass stomach, which explains in the most natural way the origin of this strange disease. 
We now understand not only that this is a female disease par excellence, but also that it may be relatively symptomless for many years, and, further, that we sometimes find ulcer and sometimes do not, and that the greater portion of the partition wall is, as a rule, covered with normal mucous membrane. Finally, we understand the hitherto so inexplicable factum that the pyloric part of the stomach lying on the other side of the partition wall is so often the

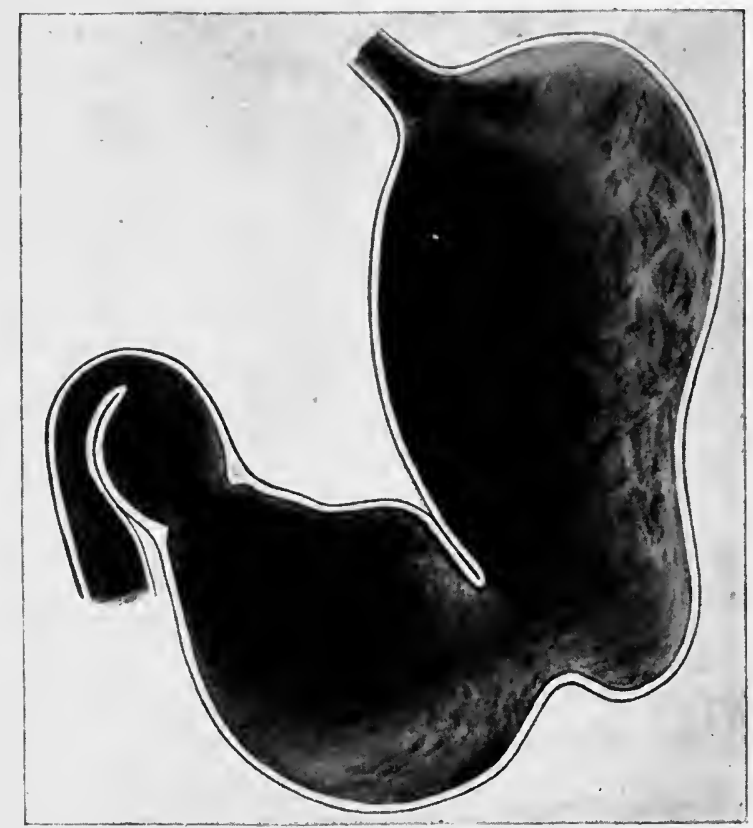

Fig. 100.-Hour-glass stomach cut through.

seat of dilatation and retention; because, between the fixed part of the duodenum and the stomach, a bend of the ptosis or of the folds is, so to speak, constantly found, which often becomes fixed by adhesions between the peritoneal surfaces, and in this manner causes a hindrance of the passage, even if no ulcer or cicatricial stenosis forms on the surface of the mucous membrane (Fig. 100).

When I now regard the case, of which I published an 
account in 1899, in support of the theory of the hour-glass stomach being a congenital abnormity, and consider it in the light of the experience of the past eleven years, it is quite clear to me that it was in reality a typical example of folds in the stomach resulting from corset pressure. Among the ten patients whom I have operated on since then, there is only one instance where the pathogenesis seems to me doubtful; this was a case of perforating, tuberculous ulcer (published in Hospitalstidende, 1903), and here it is impossible to say whether the ulceration has been the primary cause, or whether the hour-glass stomach has been present from youth, resulting from the fold in the stomach wall. I am most inclined to believe in the latter view, as the patient, for thirty-one years and ever since the age of puberty, had suffered from stomach disorders. In all the other cases there has been no doubt about the pathogenesis as indicated by me.

If, therefore, I dare not deny that the hour-glass stomach may occasionally be caused by ulcer formation, and rarely be congenital, I venture to assert that, as a rule, it is due to folds and bends of the stomach resulting from a restriction of the conditions of space in the lowest chest aperture, produced by corset pressure or lacing, and from a shifting of the stomach.

Now, to return to our patient, you will see that the whole of her history, dating back as it does sixteen years, to her seventeenth year, points to the same pathogenesis, and all the more so because the view given us by the inflation not only indicates an hour-glass stomach but also is suggestive of an obstruction or stenosis of the pylorus. In cases where the passage through the pylorus is free, we generally see the pyloric portion depict itself as a small distention, which, with a slightly convex bow, swings up toward the pyloric area. Here the illumination with Röntgen rays, after the partaking of bismuth porridge, does not give such a satis- 
factory picture as the inflation does, probably because the bismuth porridge remains lying too long in the cardial part of the stomach.

For the rest, I will warn you against relying on the Röntgen picture alone when diagnosing hour-glass stomach, because at times the bismuth produces a protracted, tetanic, circular contraction of the muscles of the wall of the

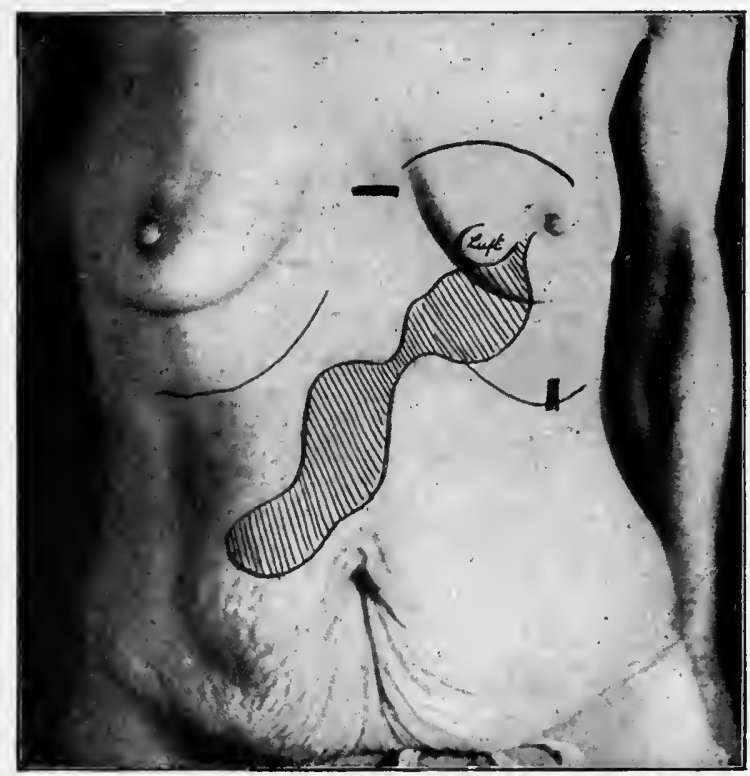

Fig. 101.-Tetanic contraction of normal ptosis stomach. Radiograph after partaking of bismuth porridge. Counterfeit hour-glass stomach.

stomach, so that an entirely deceptive picture of an hourglass stomach may appear in a quite normal stomach. In respect to this, I shall show you a diagram (see Fig. 101) which was returned to us with a patient with violent stomachic symptoms, whom we had sent down to the Röntgen ward for examination.

The reading of the diagnosis was undoubted hour-glass stomach, and you will understand that the general appear- 
ance fitted in well with this, but the operation revealed a simple gastroptosis.

The hour-glass stomach is a far more frequent affliction than had formerly been supposed, and the increasing intervention of surgery is quite natural with diseases of the

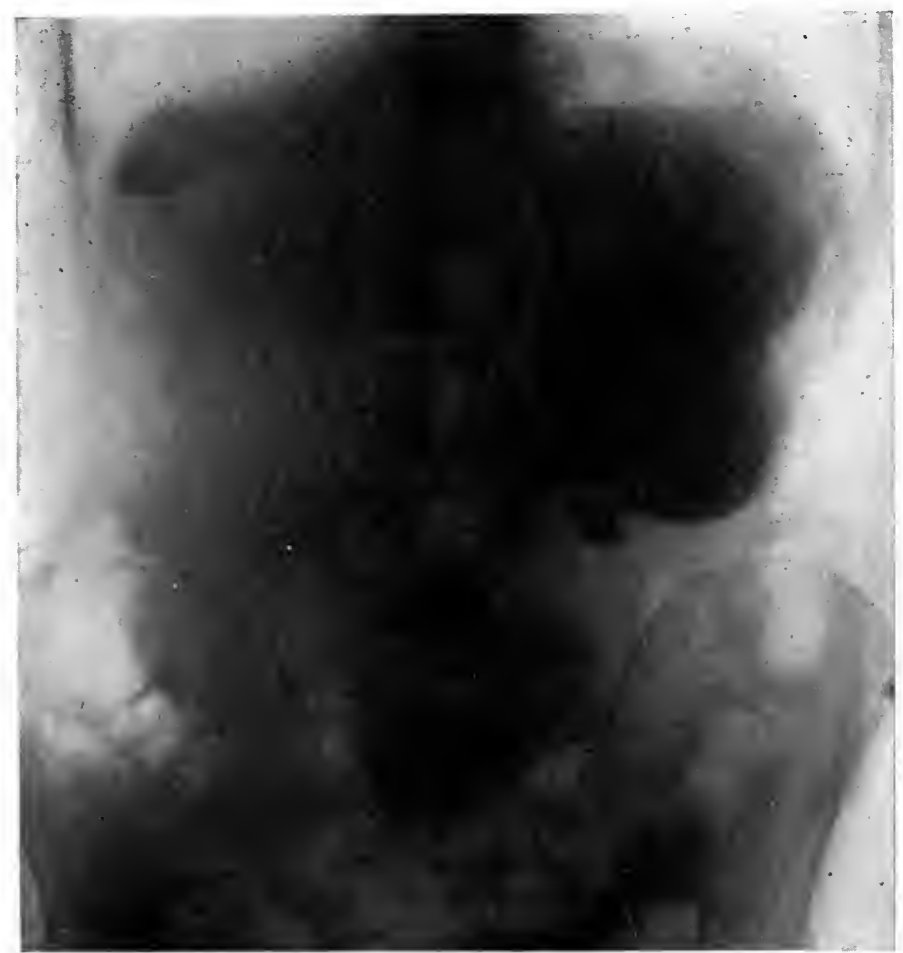

FIG. 102.-Radiograph of an hour-glass stomach demonstrating that the Röentgen picture alone may be incorrect, for in the photograph the two segments of the stomach seem to be a great distance apart, though at the time of the operation they were found to be close together. This is due to the fact that the lower segment of the stomach is not entirely filled with bismuth. In this case a resection of the stomach was done and the result shows in Fig. 103.

stomach, and particularly with those which, by causing difficulty in the passage of the food, have brought this factum to light. You thus see that, in a total of three to four hundred operations of the stomach, I have found ten hour-glass stomachs, and Harslöf, in his aforementioned treatise, states having found no fewer than sixty-one hour- 
glass stomachs in 5196 dissections-including 206 cases of ulcer from the dissecting room of the General Hospital, of which fifty-eight were females; moreover, with a clinical material of 222 cases, he has found twenty-eight with hourglass stomach-twenty-seven females and one male. It is, then, a comparatively frequent affliction, and an abnormality

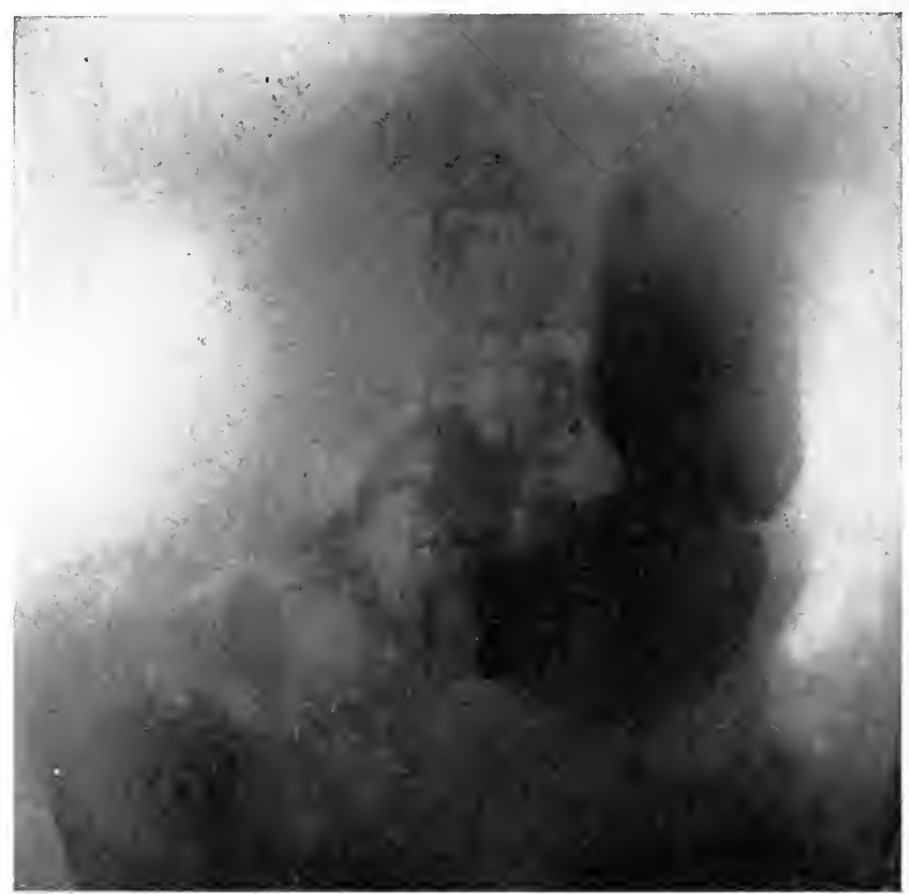

Frg. 103.-Radiograph showing the result of resection of the stomach in a case of hour-glass stomach. This case represents the writer's views as to the relation between hour-glass stomach and gastroptosis virginalis, in which folds of the stomach become fixed by adhesions. The patient was admitted with a diagnosis of cancer of the stomach, was extremely emaciated, had a total achylia, and could not retain anything on her stomach. Operation showed a marked degree of gastroptosis, a typical hour-glass stomach with a very small isthmus, but no trace of ulcer or any inflammatory process. Resection of the stomach was done and rapid recovery followed.

which one must be prepared to meet when one starts to work operatively on a presumed gastric ulcer. It is also of great importance to be clear in one's own mind as to the prognosis of this affliction, and the treatment which should be indicated in the various cases.

With regard to the prognosis, the examinations on the 
dissecting table show, indeed, that moderately-developed hour-glass stomachs do not shorten the life of the patient, and often have in no way caused distinctive symptoms, appearing as a casual discovery on the dissecting table; but, again, the more advanced cases with marked deformity are very serious, partly because they produce inanition resulting from obstruction in the passage of the food, and partly because of the certainty of ulcer formation, with all its ensuing dangers,-i.e., perforation, hemorrhage, and cancerous degeneration. And when these cases come to us for treatment at all they are such as have betrayed their presence by stenosis or ulcer symptoms. Then, in trutl, there is always danger afoot, and an operative encroachment strongly indicated, because a mechanical hindrance in the passage can be treated only surgically.

What, then, shall this treatment consist of? A priori, and considered superficially, the answer may seem quite simple, viz., it is only necessary to provide for a new outlet or passage between the two sections of the stomach or between the stomach and intestine. Unpleasant experiences, however, have taught surgeons that the matter is not so simple; because in a very large percentage of the cases there is also obstruction at the pylorus, and if one overlooks this, the operations will be only partially successful. Even a gastro-enterostomy from the cardial part to the jejunum may deceive one, because, although it relieves the retention in the cardial part and thus procures passage for the food, it does not influence the stagnation in the pyloric section, in which ulcerations frequently develop, which have caused fatal perforation or hemorrhage, some time after a seeming good recovery following gastro-enterostomy.

The operations which have hitherto been employed in cases of hour-glass stomach we can divide into four groups : (1) Gastroplasty, (2) gastro-gastrostomy, (3) resection, and (4) gastro-entero-anastomosis. 
(1) Gastroplasty is the operation which was first employed successfully in cases of hour-glass stomach. It was Schmid-Mounard who, in 1892, performed the first operation, inasmuch as he employed Heineke-Mikulicz's method in cases of pyloric stenosis. These pictures (Figs. 104 and 105) show you, in outline, the purpose of this operation:

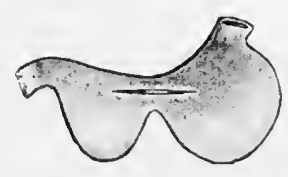

FIG. 104.

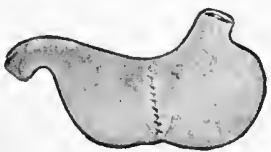

FIg. 105.

how, by joining the longitudinal incision transversely, one will increase the dimensions of the narrow portion. As a rule, however, this operation is far from being so ideal in its effect as appears from the pictures. In cases where ulceration has been present the incision is made in cicatricial tissue, which is badly adapted for healing; in cases where there is no ulceration the incision does not include the partition wall which projects from above and behind, but only involves the normal stomach wall. One may succeed in lengthening the anterior wall by this procedure, though

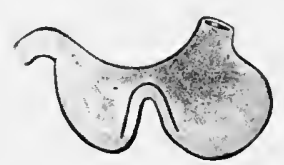

FIG. 106.-Kammerer's operation.

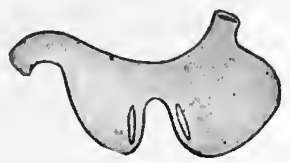

FIG. 107.-Wölflers gastro-gastro-anastomosis.

at the same time the space between this and the projecting shelf becomes smaller and the passage of food past the projection even more difficult than before. Thus it happened with me, as with others, in my first case. Shortly afterward I had to re-operate on the patient for stenosis, and then performed gastro-entero-anastomosis with permanent good results. 
These drawbacks induced Kammerer to modify gastroplasty, in the same spirit as Finney modified the pyloroplasty, by turning the arms of the incision downward on both portions of the stomach in the shape of a horse-shoe (see Fig. 106); this operation, however, belongs naturally in the next group.

FIG. 108.

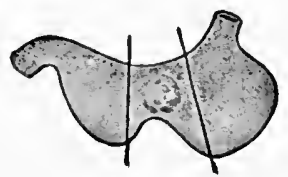

FIG. 109.

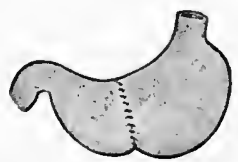

FIGs. 108, 109.-Resection of stomach with direct joining of the two segments of stomach.

(2) Gastro-gastro-anastomosis, as Wölfler first performed it (see Fig. 107), is to be preferred. He united the two parts of the stomach by a broad anastomosis, leaving the narrow canal untouched. I have performed this operation in one case with excellent results.

(3) Resection of the stomach (see Figs. 108 and 109). Complete or partial resection of the contracted portion of the stomach and subsequent union of the cardial and pyloric pouches is an operation of such magnitude that it should

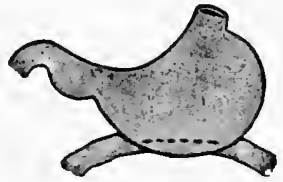

FIa. 110.-A simple ǵastroanastomosis.

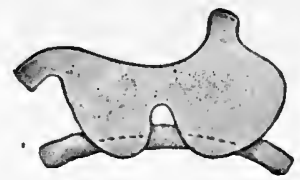

Frg. 111.-Weir's double gastro-enterostomia posterior.

only be undertaken when compelled by necessity. But one may be driven to it when perforation or hemorrhage threatens the patient's life, or when the contraction ring is situated so high up, and the cardial portion is consequently so small, that it is impossible to perform an anastomosis with the pylorus part or the intestine.

(4) Gastro-entero-anastomosis has been displaced by 
gastro-gastro-anastomosis, in cases of hour-glass stomach, even by Wölfler. If the entero-anastomosis is made in connection with the cardial portion of the stomach, the retention continues in the pyloric stomach, and if one performs it on the pyloric stomach the retention continues unchanged in the cardial stomach. This in itself is true enough, if

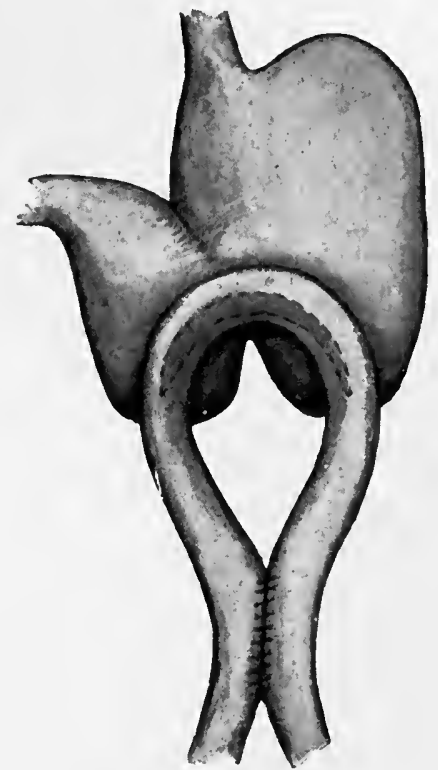

F1G. 112.-Author's method for gastro-entero-anastomosis. there is retention in the pyloric part; but in many cases this part is small and empties itself completely. Here, naturally, a simple gastro-enterostomy, as illustrated in Fig. 110, will be of great advantage. In those cases where the pars pylorica is also dilated and retention results, both gastro-gastrostomy and gastroplasty are, in my opinion, equally inadequate and are inferior to gastro-enterostomy, because a connection between the cardial pouch and the jejunum always ensures the nourishment of the patient, while a gastrogastro-anastomosis with a contraction of the pylorus does not benefit the patient from the stand-point of nourishment.

The whole question hinges, therefcre, on whether the passage through the pylorus is normal, because, if this is the case, a gastro-gastrostomy, if technically possible, is the natural and best operation, as involving the most physiologically normal conditions. But if the passage in the pylorus is impeded at the pylorus, then the gastro-gastroanastomosis is absolutely improper, and the gastro-enterostomy the correct operation. In such a case it does not suffice to connect only one of the pouches with the intestine. 
Wier solved this problem in a simple manner by forming two anastomoses on the same intestinal coil, one with each section of the stomach (see Fig. 111). There is this objection to the method, that the piece of intestine lying between the anastomoses can easily become kinked and obstruct the passage; therefore it is quite necessary to add to the anastomoses an entero-anastomosis between the two legs of the intestinal coil, lower down. This involves three operations, and so is somewhat more serious.

I have therefore devised another procedure, shown in the illustration (see Fig. 112). I make an incision through the isthmus on the anterior wall of the stomach as with gastroplasty, but then prolong the incision on both sides, and slightly curved, down toward the greater curvature, and then form a broad anastomosis between a coil of the jejunum and the whole incision, so that at one stroke I obtain a connection between both portions of the stomach, and between each of them and the intestine. In the case where I applied this method I also, for safety's sake, performed entero-anastomosis. The result was excellent, for which reason, if the case before us is also an hour-glass stomach with stenosis of the pylorus, I shall act in the same way.

P. S.-The diagnosis proved to be correct: a typical hour-glass stomach with almost uniform dilatation of the cardial and the pyloric portions. No sign of ulcer on the partition wall. Marked folding stenosis of the pylorus. The projected operation, a broad jejuno-anastomosis communicating with both pouches, was performed with excellent effect. 


\section{LECTURE XIX}

\section{PERILOUS ULCER HEMORRHAGE}

I wILL lecture to-day on a most important and most difficult subject: the indications for operative intervention in cases of active hemorrhage from gastric and duodenal ulcer. My doing so is occasioned by the patient, a cottager, thirtyeight years of age, who lies before us, and has been sent here from "Tyen" by his physician on account of repeated and dangerous hæmatemeses, which, in his opinion, are due to gastric ulcer. I will briefly relate his history to you.

His family is supposed to be healthy, and he limself had been fairly well until his present illness. I must particuiarly emplasize the fact that there is no personal or family history of hæmophilia. He dates the beginning of his illness to a period eleven years ago, when, after he had suffered for some time from nausea and sour eructations after eating, he repeatedly vomited small quantities of blood (estimated one or two tablespoonfuls on each occasion). He was confined to bed for three weeks, and placed on a strict milk diet. Thereafter he felt quite well, and was free from dyspeptic symptoms and was not constipated. Three years ago he woke up one night and was nauseated, and shortly afterward vomited blood-about a wash-basinful. He suffered no pain, and the next day could enjoy ordinary food. Since then he had felt quite well until November of last year, when suddenly, one morning, he felt dizzy and had violent hæmatemesis. He fainted, and remained in bed for four or five days, and was on a strict diet. For several months he was very anæmic and weak and quite incapable of work, but without the slightest dyspeptic symptoms. After this he recovered his health until three weeks ago, when, one evening, he suffered from dizziness and nausea. 
He went to bed early, therefore, and slept well; but next morning when he wished to get up he felt so weak that he was unable to stand on his feet, and about midday vomited an enormous quantity of blood-several basinfuls. $\mathrm{He}$ fainted. Later in the day smaller hæmatemeses occurred. Numerous fainting spells occurred on this day and on the following days. The physician attending him describes his condition as extremely anæmic and critical. At first it was quite out of the question to think of transferring him to a hospital for an operation. He let the patient lie quite still, without nourishment for the first day or two, afterward allowing a very rigorous diet; but at once wrote to me to ask if he might send the patient over to Copenhagen if he recovered sufficiently to endure the journey. Fortunately the hemorrhage did not recur, and by degrees he improved sufficiently to justify the journey, arriving liere a few days ago. You notice that he is a strongly-built man, though looking pale and exhausted. His paleness would be even more noticeable if his occupation as a farmer had not caused him to be so weather-beaten. The quantity of hæmoglobin in the blood is scarcely 50 per cent. The pulse is 120, regular but small and soft. Stethoscopy shows normal conditions, apart from an anæmic systolic murmur. The abdomen is natural in form and appearance, and he is nowhere sensitive to pressure, while I can nowhere point to any distention. The urine is normal.

Here, then, we have before us a man still young, who for eleven years, with certain intervals, has vomited blood; at first little and seldom, but during the last years with shorter and shorter intervals, and simultaneously with the blood increasing in amount each time, and on the last two occasions becoming so perilous that the physician doubted whether the patient would get over it. . When the hemorrhage manifested itself the patient immediately became so relaxed and weak that the physician had to resign all 
thought of transferring him even to the nearest infirmary. He was therefore forced to an expectative treatment, and was fortunate in that the hemorrhage stopped this time before it caused a catastrophy. Realizing that this would not do if a similar hemorrhage should recur, he sent the patient here as soon as he could undertake the journey, in order that liere, by an operation, we might find and render harmless the source of the hemorrhage. Hitherto, fortunately, no fresh hemorrliage has occurred, and, as things now are, we can consider in comparative peace and quiet how and to what extent we can assist the man by an operation. Our assumption for thinking that we can help him operatively-insure him against menacing hemorrhage in the future-is this: that the hemorrhage comes from a gastric or duodenal ulcer, and so situated that we can find it and render it harmless. Now, is the diagnosis gastric ulcer under which the patient has been admitted certain? Yes, if we consider the patient's anamnesia and history we can really substantiate only one symptom: vomiting of blood. Some nausea and slight eructations have occurred just before the romitings, but no cardialgia, no dyspeptic symptoms, no constipation! All these "classical" ulcer symptoms are quite wanting here. The question is, then, is himatemesis an infallible symptom of ulcer? Formerly, when mentioning the diagnosis of stomach ulcer, I have warned you against this theory. Then it was especially \& question of small hæmatemeses, even if these are attended by ulcer-like pains and dyspeptic symptoms. On the other hand, you can, as a rule, take it for granted that extensive hæmatemeses occurring in patients who have presented these symptoms for a long time are due to an eroded vessel in an old chronic ulcer. But what about violent hæmatemeses which suddenly befall patients with no other gastric symptoms? Here we must be careful, because, as I have had the opportunity to show you, an ulcer can cer- 
tainly exist for many years and erode all the layers of the stomach, and therefore injure large vessels, without the patient having felt ill for one moment. But this is the rare exception; while, on the other hand, we must not forget that we may have enormous hemorrhages from quite other sources. Let me first call your attention to hemorrhages from varices in the lower part of the œsophagus, as they present themselves in cases of cirrhosis of the liver, especially the syphilitic cirrhosis. Here the hemorrhage occurs as an enormous hæmatemesis without forewarning of any kind, and these patients are often admitted as peremptory operationis causa, under the diagnosis-bleeding stomach ulcer. You will understand that, in such a case, an operation is not only useless but naturally diminishes the patient's chances of getting over the anæmia. In such cases you should think, therefore, of this possibility; go over the anamnesia again, keeping in mind syphilis; examine the liver and spleen, which are usually enlarged; and search for ascites, the presence of which is a valuable sign of cirrhosis.

In the second place, you must not forget that there is a condition called hæmophilia! A short time ago we had a patient here who had incurred a fracture of the right crus while intoxicated. He was ebrious when he arrived, and, during the following days, on the verge of obvious delirium, so that it was impossible to extract a proper anamnesia from him. Then he suddenly had violent hæmatemesis and melæna, which were repeated time and again, so that the patient's condition seemed extremely perilous.

At a further examination, it was seen that petechiæ and ecchymosis had appeared under the skin in different parts of the body and the extremities, while it was ascertained that typical hæmophilia existed in the patient's family. For this reason, naturally, we dismissed every thought of an operation, which would only have caused fresh hemor. 
rhages, and would have led to certain death. As it was, despite the fact that the patient was almost moribund, we tried an intravenous injection of ten cubic centimetres of antidiphtheritic serum encouraged by a report which had just appeared in "Mittheilungen a. d. Grenzgebieten der Medicin und Chirurgie," by E. Baum, ${ }^{1}$ dealing with the arrest of hemorrhages in cases of hæmophilia by injecting a foreign blood-serum. The effect was astounding. The hemorrhage ceased at once and the patient recovered. Since then we have again had the opportunity to substantiate the effect of the injection of serum in cases of hæmophilia, and I earnestly advise you to try this with threatening cases. Is a matter of fact, there is nothing in this patient's anamnesia or in the objective findings which might point toward the two above-mentioned possibilities; but it must be remembered that there are certain cases where patients who present no other trace of hæmophilia bleed to death from continuously repeated hæmatemeses and melæna, in which no demonstrable ulcer is present, or any other source of the hemorrhage! I do not mention this because I think that anything of the sort is the case with our patient, but because, although he has never presented any other symptom of ulcer than his hemorrhages, he may yet cause us surprises, even though I consider it most probable that here we have before us a chronic ulcus rotundum, which, quietly and without causing pain, has gnawed a hole in a large artery, the lumen of which is, under ordinary circumstances, blocked by a thrombus. I imagine that, when this is occasionally loosened, sudden and violent hemorrhage takes place, to be stopped once more by the formation of a new thrombus, until finally the patient bleeds to death.

But where, then, is this ulcer situated? In regard to this question, the symptoms leave us quite undecided, be-

${ }^{1}$ Ernst Baum: "Der Wert d. Serumbehandl. bei Hämophilie auf. gr. Experiment. u. klin. Untersuchungen," Bd. 20, p. 1. 
cause, as you have heard, this patient had never suffered from pain, and it is just the position of the pain-to the left or to the right of the centre line-and the time when it occurs relative to the meals, which should essentially be our guide, when deciding the locality of the ulcer.' If I should draw any conclusion from this lack of pain, I should say that it speaks more in favor of duodenal ulcer than of gastric ulcer, because, although this may run its course quite painlessly, yet it does so more rarely than the duodenal ulcer. Another circumstance seems also to speak in favor of a duodenal ulcer, viz., it appears from the history that the patient bled long before the hæmatemesis commenced. On each occasion that the hæmatemesis has occurred the weakness and dizziness have lasted for a longer periodsometimes even for twelve hours. This seems to me to fit in better, with the idea that the stomach has gradually filled with blood, which has oozed from the duodenum into the stomach, which, only when quite filled with blood, reacts by vomiting. If, on the other hand, the blood came from an ulcer in the stomach itself, one would expect it to react far more quickly by contraction. I am, therefore, most inclined to think that the hemorrhage is due to ulcus duodeni, but dare by no means deny the possibility of its being a stomach ulcer, and therefore dare not enter upon the usual stomach investigation, although one might expect this to give valuable enlightenment, partly concerning the question of retention, and partly concerning the chemical conditions of the gastric juice.

With regard to the operative treatment, it is of great importance to know where the ulcer is situated, and to carefully consider beforehand the various possibilities, and to contrive which method one shall employ in the various cases. Not a few operations have failed, or have been without result, because the surgeon has deduced a stomach ulcer from the hæmatemesis, and, not finding this, has simply 
closed the wound again, or been content with gastro-enterostomy. The hemorrhage has reappeared, and has then proved to be due to ulcus duodeni. Therefore, if one fails to find the source of the hemorrhage in the stomach, one should carefully investigate the duodenum also. Ulcus duodeni is far more frequent than was formerly supposed, and how frequently its symptoms are confused with those of the stomach ulcer first became clear to me when I began to employ my duodeno-gastroscope for the disclosure of ulcer during the operation. In a whole series of cases where the diagnosis was stomach ulcer, and where inspection or palpation of the stomach and the intestine gave a negative result, by gastroscopy we found the ulcer situated in the duodenum, and most frequently on the posterior wall of this, one or two centimetres from the pylorus. Should this patient's hemorrhages, relapsing and ever increasing during eleven years, be due to a stomach ulcer, it is most probable to expect a typical chronic ulcus rotundum on the lesser curvature, in which arteria coronaria lies eroded, closed for the present by thrombosis. If this is so, the right treatment consists in excising the ulcer, tying the vessels, and suturing the stomach walls in two layers. In exceptional cases such an extirpation is made more difficult because the portion of the wall containing the ulcer has grown fast to the pancreas or the large vessels in front of the vertebral column. In such a case one must either be content with tying off the coronary artery on each side of the wound, adding thereto a gastro-enterostomy, or perform a resection of the stomach, leaving the adherent part of the ulcer, and destroy it with Paquelin's thermocautery. The problem becomes far more difficult if it is an ulcer in the duodenum, because, as you will easily understand, an excision of this is accompanied by far greater difficulties, because the duodenum lies so deep and is so difficult of access, especially from an incision in the median line. But, in ad- 
PLATE VI

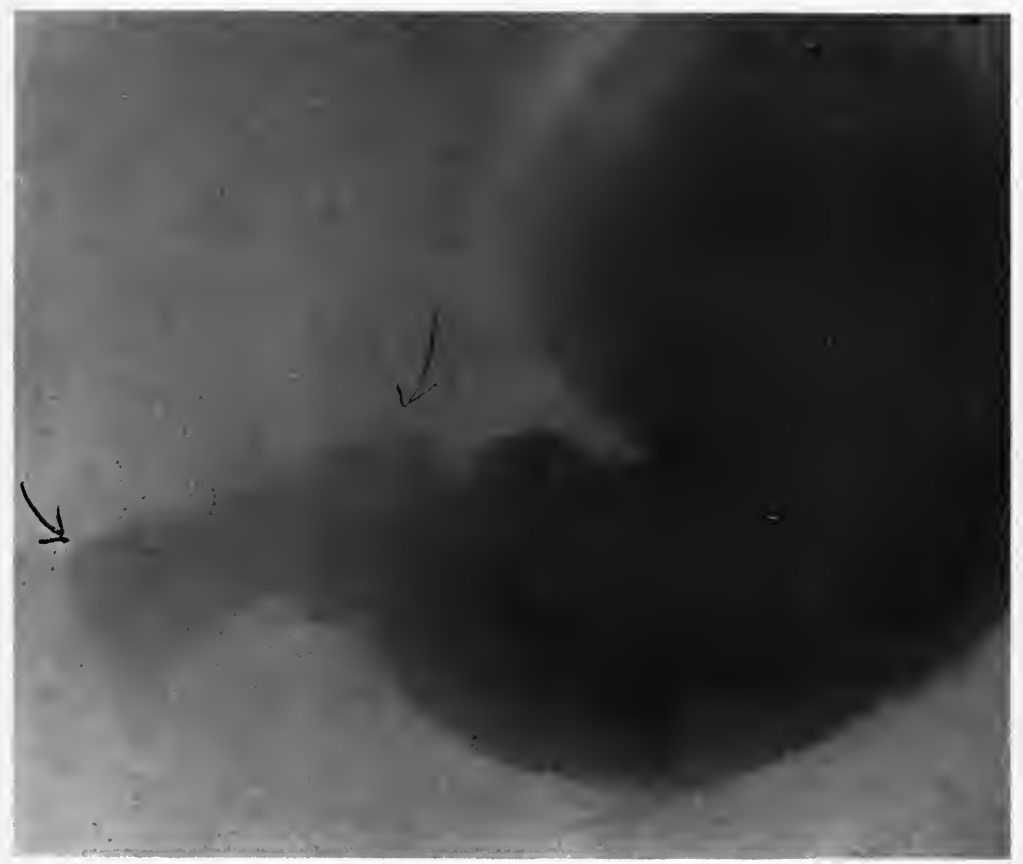

Radiogram of case with perilous ulcer hemorghage, showing flattening of duodenum due to adhesions, and stellate filling defect due to duodenal ulcer. 

dition to this, an extirpation is only possible where the ulcer is situated at a certain distance from the biliary duct, so that one runs no risk of closing the aperture. I hope that we shall obtain a solution of these doubtful questions at the operation to-day, but I trust that you will be impressed by the difficulty and uncertainty which attend the surgeon's encroachment in such a case, even when he has the opportunity to operate during an interval between two hemorrhages, and at the moment when the patient has somewhat recovered.

Far more complicated and difficult is the question of surgical intervention during the acute, menacing hemorrhage itself, a question which has been keenly discussed by surgeons during the last decade, though so far no reconeiliation has been attained between the dissenting views. Many otherwise daring and eminent surgeons-as, for example, Körte of Berlin-liave been led by experience and reflection to decide to set aside all thoughts of operation during profuse hemorrhage from the stomach. They reason as follows: with most patients with profuse hemorrhage from the stomach this ceases under medical treatment, viz., by keeping the patient absolutely quiet, by depriving him of all food by mouth, and by nourishing him by injections per rectum. It is impossible under such treatment to decide which patients will recover and which will not. The additional loss of blood and shock which accompany the operation might prove fatal to a patient who would otherwise have recovered without operation.

Even Mikulicz himself, who in 1887 was the first to recommend and perform gastrostomy and direct search of the ulcer in order then to stop the hemorrhage by suture and cauterization, expressed himself in 1902, when this question was the subject of an interesting discussion at the Surgical Congress at Berlin, almost in favor of abandoning every surgical encroachment. His reason for this point 
of view was that the search for the ulceration is often extremely difficult and not rarely unsuccessful, because even if one has opened the stomach by a very long incision, almost from the pylorus to the cardia, the numerous folds of the mucous membrane of the stomach may easily conceal a small ulcer, and all the more so because blood and blood-clots obscure the examination. During the examination the bleeding continues, and the shock is increased by the unavoidable hemorrhage caused by the operation itself, and the whole situation is rather a desperate one. Formerly those who held that an expectant treatment was better than an operation were certainly justified, for it was clear that with these patients every drop of blood was precious, and no operation was justified unless it could be done rapidly and surely. Czerny of Heidelberg proposed a simple gastro-enterostomy. He had performed gastrostomy in three cases of acute hemorrhage of the stomach in which he found the ulcer and extirpated it, but all of these cases died. In four other cases he performed a simple gastro-enterostomy without touching the ulcer, and all of his patients recovered. From this he concluded that the gastro-enterostomy itself stops the hemorrhage. This conclusion was not, however, quite justifiable, and at once met with protest. In reality there was nothing to explain why this encroachment should stop the hemorrhage from an ulcer. The most probable explanation was this: that the hemorrhage in these, as in so many other cases, had stopped spontaneously, and at the cessation had quite accidentally coincided with the gastro-enterostomy.

One might suppose that when the obstruction was removed by providing the gastro-enterostomy opening the stomach ulcer would heal, being favored also by the neutralizing effect of bile upon the hydrochloric acid. Unfortunately this is not so, for in a number of cases in the course of a year after a gastro-enterostomy the pain and dyspepsia 
had somemhat lessened, when suddenly a violent and sometimes fatal hemorrhage occurred, showing that the ulcer had still remained unchanged or grown worse, and the patient had only been relieved of his symptoms and not of his disease.

Among my patients I have a number of examples of this. Therefore the gastro-enterostomy is not rational, even in the interval between the hemorrlages. The source of the hemorrhage here, as with the acute hemorrhage, must be sought for in a direct manner and invalidated, if one wishes to operate at all on this indication.

Since that discussion in 1902 , however, the chances for an effective and rational, surgical encroachment during acute, profuse hemorrhage from the stomach have improved greatly. Even in the same year Witzel reported that, in the presence of a menacing hemorrhage from an ulcer situated on curvatura minor, he had quickly and completely stopped the bleeding by tieing arteria coronaria, superior dextra, and sinistra, on eacl side of the ulcer, without opening the stomach. This operation, which can be performed in the course of a few moments, and which does not involve any danger to the patient worth mentioning, especially when performed under ether narcosis, I have employed with success in two cases, and there is no doubt that this is the only rational operation in cases of hemorrhage from. old, callous ulcers on the lesser curvature-but, at the same time, only in these! If the severe hemorrhages always, or even in the majority of cases, were due to such ulcers, the question would be solved and all difficulties surmounted. Unfortunately this is far from being the case. Even if the bleeding ulcer is situated on the lesser curvature, in many cases it cannot be substantiated by inspection and palpation of the exposed stomach, but in numerous instances the ulcer is situated far from the lesser curvature, in one part or another of the anterior or posterior wall, and the ulcer: 
is then often so small that it has proved difficult, even impossible, to find it on the dissecting table. Dieulafoy, in particular, in 1898, proved that the most violent hemorrhages may arise from quite small ulcerations in the mucous membrane: he terms such "exulceratio simplex." The explanation of this is anatomically simple: from the coronary arteries which run along the greater and lesser curvature branches are distributed transversely to both sides, to meet midway on the anterior and posterior walls; these branches pass in under the mucosa, and extend along beneath it even when the vessels are large, so that a lesion of the mucous membrane alone may strike such a vessel and involve a perilous hemorrhage. It was in such cases that one formerly sought for hours for the source of the hemorrhage and with such cases you will easily understand that Witzel's operation is quite inapplicable.

But even in this type of concealed hemorrhage we are no longer helpless, for by employing the diaphano-gastroscopy it is possible to discover the bleeding point. The bleeding spot would betray its presence as a dark point, interrupting the course of an artery, and from this a dark stripe down toward the great curvature would indicate the current of the blood, such as I have seen with an accidentally arisen hemorrhage (see Plate III, Fig. 3). I imagined that one might stop the bleeding by surrounding the bleeding point with a purse-string suture direct from the outside during the illumination, an operation very easy to perform in a few moments.

My prediction was verified more rapidly than I had dared to hope, because, only a few days after I had given my lecture to the Medical Society, a woman suffering from an acute, profuse hemorrhage from the stomach was admitted to the ward of the senior physician, Dr. Vermehren, at Frederiksberg Hospital, and as the hemorrhage could not be stopped by rigid medical treatment, and as the con- 
dition of the patient was becoming perilous, Dr. Vermehren transferred the patient to the head surgeon, Dr. Kraft, who then immediately operated on her. As soon as the gastroscope was introduced and the stomach illuminated, Dr. Kraft noticed a dark spot on the anterior wall and an artery running straight up to this spot, when it was interrupted abruptly. Through the gastroscope he verified the result of the diaphanoscopy, and demonstrated a small ulceration of the mucous membrane exactly on this spot. He then divided the stomach wall down from the gastroscopy wound as far as the ulcer and inserted a purse-string suture from the inside, after which the incision was rejoined. The patient recovered. In addition to this case, five similar operations owe their success to the coöperation of these two eminent physicians. On each occasion Dr. Kraft has succeeded, by means of gastro-diaphanoscopy, in quickly and surely demonstrating the small bleeding ulcer, and in these five cases he employed the far quicker and more simple mode of procedure which I have just mentioned, making straight for the bleeding spot during the illumination, and surrounding it from the outside with a silk thread going through all the layers. On the outside of this a serosa suture was made for safety's sake. Only one of these patients died but this was due to a sad accident during the gastroscopy, by reason of a failure in the town current supplied to the electric apparatus, which led to combustion and gangrene of twothirds of the stomach-an accident which can never occur if one employs an accumulator as a source of light. If this accident had not occurred, Dr. Kraft would probably have had the unique result of a percentage of 100 recoveries in this series of extremely serious cases. But even the five recoveries speak highly in favor of the method and in honor of Dr. Kraft, not forgetting Dr. Vermehren! Because the art of judging such a case correctly,-i.e., to choose the right moment at which to call upon the surgeon for assistance,- 
is as difficult and just as important as the art of performing the operation. As only a few of you will have to operate in such cases, though all of you will find yourselves, as medical practitioners, in the responsible position of having to decide the treatment, I feel it right to linger somewhat over this difficult question, and endeavor to give you some certain, invariable rules for navigating these dangerous reefs.

Immediately on arriving at the patient's bedside it is most important to estimate how serious the hemorrhage is, and how threatening the immediate situation. This is by no means always easy. If there has been vomiting of blook, you will naturally request to see the vomit at once, and, if this has been thrown away, endeavor to ascertain exactly how much blood has been vomited. This may be difficult enough, because the relatives, in their excitement, often exaggerate this greatly, confusing blood-colored aliments with pure blood, etc. If, by some means or other, you have verified the patient's having vomited one-half to one litre of blood, then you always know that the affair is very serious; but you must not conversely conclude a less serious affair because of a small vomiting of blood, because, as you see exemplified in our patient to-day, the stomach may be full of blood without the occurrence of vomiting, or the blood may pass into the intestine, which can hold enormous quantities.

It now seems obvious that the surest road to information is through an estimation of the hæmoglobin, which can be performed in the course of a few minutes, wherever you are in practice, after Tallquist's excellent method for rough use, if only you have the test-book with you. You should always perform this examination at once, and if the quantity of liæmoglobin is mucl lowered, to 50 per cent. or under, then it is a very serious sign, especially with first bleeding. But here again the point is, that you must not 
conversely allow yourself to be reassured by a high percentage of hæmoglobin, because the fall in this does not come in direct response to the loss of blood, but only when the loss of fluid is replaced. At first there is only a small quantity of blood, but of the same concentration. Therefore we often witness the circumstance, which may easily mislead one who does not know it, that the hæmoglobin percentage continues to fall after the hemorrhage has ceased. The most pronounced example of this which $I$ have seen was presented by one of Kraft's cases, where the percentage of hæmoglobin when the patient was admitted to the hospital with enormous hemorrhage was about 49 per cent., fell to 30 per cent. after the operation, when the source of the hemorrhage was definitely stopped. This circumstance is especially deceiving to the physician if he wishes to infer from the percentage of hæmoglobin from hour to hour and from day to day, whether the hemorrhage is continuing or not, just the capital question when deciding in favor of operation or expectant treatment.

I will, therefore, warn you against inferring too much from, and from having too much confidence in, these apparently convincing examinations. The decisive factor is the impression of the patient's general condition, which the ordinary clinical examination provides: the psychical condition, strength, color, extent of pallidness, warmth of the skin, whether the extremities are cool or perspiring, and, finally, but above all, the state of the pulse. If the patient is restless, agitated, hands and feet cool, covered with cold perspiration, the pulse rapid, small, and soft, then the situation is very serious, and the subsequent judgment rests on the improvement or not in these symptoms, in spite of the treatment which you immediately institute, and which should consist essentially in complete quiet, entire avoidance of food by the mouth, hot-water bottles, stimulants such as camphor and caffeine injected subdermatically, 
nourishing elyomata, and subcutaneous injections of salt water. There are, however, two things highly recommended in medical handbooks which I must warn you against. The one is an ice-bag placed on the abdomen, a contrivance which can never diminish the bleeding in the stomach, but would rather tend to increase it, because the blood is driven from the skin. It is always disagreeable. The other is washing out the stomach with styptic solution, especially with a thin solution of chloride of iron, or with hot water. I cannot deny that you could sometimes stop bleeding by such washing, but the danger of producing renewed or increased hemorrhage by loosening the thrombuses is so great that you ought to banish these rinsings as being too hazardous.

On the whole, one can say that if the general condition, pulse, etc., does not improve under this treatment, or if evident signs of fresh hemorrhage (renewed hæmatemesis) appear, the patient should then be taken as quickly as possible to an infirmary or clinic where operative assistance is available. But it is of great importance, with regard to your general action, whether you are faced with an old, chronic ulcer, or the hemorrhage from a more superficial, acute lesion or ulceration of the mucous membrane. It is not always possible to give the differential diagnosis, but if profuse hemorrhage occurs in a patient who has presented typical ulcer symptoms for many years, and especially when there has been a previous hemorrhage, there is overwhelming probability that the hemorrhage comes from a large artery, which is eroded at the base of the ulcer, and then, if the patient's condition in any way permits it, he should undoubtedly be handed over for surgical treatment. If one succeeds in stopping the hemorrhage by rest and diet, the patient should be operated on as soon as his strength permits, because recovery is only possible through operation.

If, on the other hand, we have a patient before us who has presented stomach symptoms for only a short period, then the probability of an ulceration and the possibility of 
recovery under medical treatment are far greater. Only in the rare cases where the condition deteriorates steadily, in spite of the treatment, or where the hemorrhage is recurrent, ought one to interfere.

As you see, it is a difficult and very responsible part that the physician plays in these cases of acute hemorrhage from the stomach. Especially does it often demand great tact and a sure clinical eye to decide whether the patient will have the better chance from expectant treatment or not.

The surgeon to whom these patients are entrusted must naturally consider seriously the same questions before he decides on the encroachment. But, if he once makes up his mind, he must have his plan of campaign and his technic laid out; because here, if anywhere, the point is to act quickly and surely with these extremely weak patients. If he at once finds a typical callous ulcer on the lesser curvature, then he should not hesitate to tie the coronary artery ad modum Witzel. If he does not find it, he should perform gastro-diaphanoscopy. If he finds the ulcer in the stomach, he ought, as Dr. Kraft did, to surround it by a circumsuture; if he finds nothing in the stomach, he should not forget to examine the duodenum, where, as a rule, he will then find the ulcer-the source of the hemorrhage.

When operating I found the stomach distinctly dilated, but found no signs of an ulcer anywhere. On examining the pylorus-the seat of the disease-I found it dilated instead of contracted, as might have been expected. On examining the duodenum, this was found apparently normal, except toward the back, where I felt a string-shaped adhesion, a few centimetres from the pylorus, stretching from the base of the liver to the posterior side of the duodenum. On lifting and inspecting the edge of the liver, I saw at the edge of this adhesion a tortuous artery running toward the posterior surface of the duodenum, and near the duodenum it was distended by a thrombus. Along curvatura major- 
especially in the vicinity of the pylorus-a number of swollen lymphatic glands were found. By the gastrodiaphanoscopy I verified the presence of a severe gastritis, because, by the illumination, the wall of the distended stomach was everywhere seen diffusely dark red, though no shadows were seen. By the gastro-duodenoscopy I found the mucous membrane red, but no ulcer, and not until the instrument was passed into the duodenum did I find an ulcer. Here was an ulcer the size of a three-penny bit, situated on the posterior wall of the duodenum, a few centimetres from the pylorus. The ulcer was round, descending in the shape of a funnel. I easily substantiated my belief that the aforementioned thrombus-stopped artery led into the base of the ulcer, and was evidently the source of the terrific hemorrhages. It was thus clear that the patient's life was dependent on this thrombus, and that a renewed loosening of this would involve a new and probably fatal hemorrhage. The first thing I did, therefore, was to tie this artery above the thrombus-to exclude every possibility of bleeding to death from this. Then I performed a gastro-enterostomia anterior and entero-anastomosis ad modum Braun. As the duodenal ulcer was evidently on the point of perforation, I did not dare to let it remain untouched, but, on the other hand, an excision was impossible on account of nearness of the ulcer to the mouth of the choledochus. Then I hit upon the idea of making it harmless in the following manner: I excised the pylorus, and, after closing the stomach wound with a double row of sutures, I invaginated the duodenum into itself in such a way that the duodenal ulcer was covered by the invaginated portion, which was fixed in this position by the aid of two rows of silk sutures.

P. S.-The patient was discharged four weeks after the operation, and has enjoyed good health during the two years which have since passed. 


\section{LECTURE XX}

\section{PERFORATING ULCERS OF THE STOMACH}

I HAVE had an opportunity of showing you during this term several cases of chronic stomach ulcer which were admitted on account of the late results of the ulcer, either due to stenosis of the pylorus with retention of food in the stomach, or on account of hemorrhage which threatened the patient's life. To-day I will show you a patient who was admitted to this ward three weeks ago in a very critical condition, suffering from diffuse peritonitis which was caused by the sudden perforation of an ulcer of the stomach. She is now out of danger as the result of the operation performed at that time; but it may be of interest for you to see her and hear her history in order that the dangers of perforation and the lack of warning symptoms in these cases may be impressed upon you. The patient is a servant, thirtythree years of age, who has never suffered from any serious or acute illness, but since the age of puberty she has had attacks of cardialgia pyrosis and sour eructations with slight vomiting from time to time. There has never been any blood in the vomit, nor any blood in the stools. For the last two years these attacks had entirely disappeared, so that the patient thought herself entirely recovered from her stomach disease. Eight days ago she had a slight attack of vomiting after eating some apple pudding. During the two days previous to operation she felt a little soreness in the epigastrium, more pronounced when bending over. Aside from this, she had not felt ill until eight o'clock in the morning of the day she was admitted, when she was suddenly attacked with violent pain in the abdomen, being simultaneously seized with such a sensation of fatigue and faintness that she sank into a chair. That morning she had only eaten an 
apple. The pains, which, according to her statement, were at first located mostly in the left fossa iliaca, and later on spread throughout the entire abdomen, had again localized in the right fossa iliaca at the time of admission. A few drops of morphia and a little soda-water ordered by the physician were followed by slight eructations and hiccoughs. She was admitted here at 7 A.M.- -eleven hours, therefore, after the commencement of the illness.

On examination the patient was found to be well nourished, but in a very exhausted condition, pale, and complaining continually of pains in the abdomen, which was everywhere very sensitive to the touch. The respiration was rapid and superficial; on deep inspiration she felt pain in the cardia. The pulse was 108, regular and somewhat soft; the abdomen was found greatly distended, especially from the umbilicus downward, on both sides. The abdomen was very tender everwhere, most markedly in both fossæ iliace and across the mesogastrium. By exploratio rectovaginalis no swelling of the adnexæ or any distention was felt in the pelvis. The urine gave a slight albuminous reaction. I made a positive diagnosis of perforative peritonitis, certainly, but wavered with regard to the seat of the perforation. With perforative peritonitis in women we have four organs to consider as the source of the trouble; they are, in order of their occurrence, the fallopian tube (rupture of a gonorrhœal pyosalpinx), appendix vermiformis, the stomach (ulcer), and gall-bladder (cholelithiasis with empyæma).

As a source of infection it seemed to me that the fallopian tube could be safely excluded, and that the gall bladder was most improbable, but I hesitated between perforationappendicitis and stomach ulcer. Because the pain had begun on the left side first, and no soreness was found at McBurney's point, it was at variance with the first, and it was also at variance with the latter, because the pains began 
far down in the abdomen, and the history showed that the patient had been free from symptoms referable to the stomach for over two years. The soreness which was present in the mesogastrium was more suggestive of ulcer than of appendicitis. Inasmuch as appendicitis is the more frequent of the two cases of perforative peritonitis, an incision was made in the right iliac fossa. The operation was done thirteen hours after the beginning of the peritonitis. As soon as the abdomen was opened a quantity of gas escaped, which was of very foul odor. This strengthened our suspicion of a perforated stomach ulcer. On examining the appendix it was found to be healthy although its peritoneum was inflamed. On examining the pelvis a quantity of odorless purulent fluid was found which resembled weak gruel. A specimen of this was taken and tested with Congo paper to detect any free hydrochloric acid. The result was negative. I then made an incision from the umbilicus to the ensiform in order to examine the stomach. I immediately found the peritoneal covering of the stomach markedly inflamed, and on pressing the stomach lightly I saw mucus ejected through a perforation in the lesser curvature close to the angle of the corpus and the cardial part. On searching further I found that the stomach was attached on either side of the perforation to the under surface of the liver, and at this point there were evidences of an old abscess cavity which had evidently ruptured. I separated the adhesions and came upon a large accumulation of pus which was contained in the saccus omentalis minor. This purulent matter was swabbed out with gauze, and the question arose as to how to treat the perforation. It has been the rule in such cases to draw together the edges of the perforation with sutures, for the chief indication in such debilitated patients is to conclude the operation as quickly as possible with a minimum amount of operative work. The ulcer itself was about the size of a half-crown, situated on 
the curvature, with a perforation about the size of a pea in front of the vasa coronaria. The tissue surrounding the perforation was so fragile and inflamed that it was impossible to secure it properly by suture. I then decided to excise the diseased areas and perform a partial resection of the stomach, employing fairly sound tissue. This was done, and the edges of the wound were secured by two rows of sutures, the inner one catgut, including all the sutures, and the outer one silk for the peritoneum. During the examination I examined the interior of the stomach and could find no other ulcer. The lesser pouch was drained by inserting a rubber-covered Mikulicz bag. This was filled with silver nitrate gauze. The median abdominal incision was closed in the usual way in three layers. While the assistant surgeon was suturing this wound I made a third incision, beginning a little above the symphysis, reaching obliquely upward and outward to the left along the edge of the left musculus rectus. Here also was found an accumulation of pus and gas. Pus was found everywhere in the abdominal cavity, and especially in the pelvis. The small intestines were only slightly inflamed and distended. The pelvis and the entire abdominal cavity were washed out with physiological salt solution until the solution returned clear. A large rubber-covered Mikulicz bag was introduced through the last incision, and.numerous split rubber tubes were used to drain the pelvis. These tubes were filled with strips of silver nitrate gauze. The incision on the right side was drained with a small rubber bag. Dressings of silver nitrate gauze and cotton wool were applied. A Pezzer catheter was introduced into the bladder in order to facilitate the exit of the urine; following this, an infusion of salt water into the subcutaneous tissue in order to control the diuresis, which is of the greatest importance as an index to the prognosis of the condition and the further conduct of the treatment. The patient was naturally very much shocked by this extensive 
operation, which occupied one hour and a half. She was placed in a warm bed in the so-called Fowler's position (see Fig. 113). By this we understand that the patient was placed in an almost upright sitting posture with the knees sharply fixed in order that all fluid in the abdomen, according to the laws of gravitation, may sink into the pelvis, where suction drainage is applied.

By this simple device one can often prevent a local free peritonitis from becoming general and often cure a diffuse

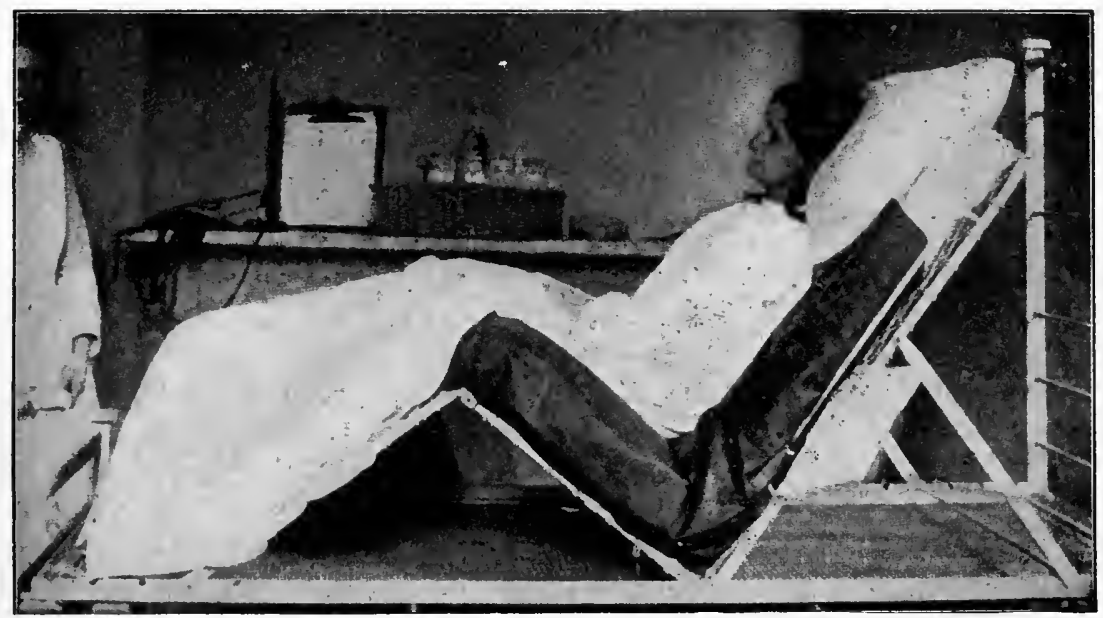

Fig. 113.-Fowler's bed.

peritonitis. The horizontal position would favor a diffusion of the peritonitis and would surely make recovery impossible. Finally, after the patient had been placed in bed, a subcutaneous infusion of salt water was immediately administered, 1500 c.c. being slowly introduced during a period of six hours. This precaution should never be neglected in cases where one has operated and found unlimited peritonitis. It is important for three reasons: (1) In order to dilute the toxins which are already in the blood and promote their rapid excretion by the kidneys; (2) to prevent further absorption by increasing the blood-pressure, and 
(3) to effect a transudation in the peritoneal cavity and thereby help to remove the products of inflammation by means of the drains. That such a transudation does take place into the peritoneal cavity after an infusion can be easily demonstrated in the clinic, because it has been shown that pieces of gauze inserted in the wound soon become drenched with a thin, clear fluid sooner or later after the infusion. It may be asked, why these measures to hinder resorption and dilute the toxins just after we have completed an operation by which we have removed the pus and toxins whicl we feared? The answer is that, in the first place, the operation itself accomplished the removal of a large amount of the toxin partly through the manipulation in the peritoneal cavity, but more especially by the pus coming into contact with the fresh wound surfaces presented in the operative field. As a proof of this, we find the urine for some time after such an operation giving evidences of a marked reaction for albumin where it was formerly normal, or increased reaction for albumin where the reaction was formerly only slight. Albuminuria is a very important and valuable danger signal in these conditions showing a degree of toxæmia, and therefore we should always test the urine in abdominal operations, and especially in cases of peritonitis, or where we fear peritonitis as the result of the operation. First and foremost, we should examine the urine before the operation to make sure whether at this stage it is free of albumin, or, in case albuminuria is present, its extent should be determined. Following the operation, the urine should be examined frequently for the first few days, and, if the patient passes no urine for twenty-four hours, he should be catheterized in order to determine whether the urine is present in the bladder or not. Albuminuria appearing in a patient in whom none has been known previous to operation is a sign of danger, even where all other threatening symptoms are 
wanting. You should at once counteract this condition by giving the patient infusions of physiological salt solution intravenously if the situation appears serious; subcutaneously or by rectum if the patient can hold fluid. Three hundred to four hundred cubic centimetres should be given at a time, repeated three or four times during twenty-four hours. It may be also given drop by drop, according to the method of Katzenstein. These infusions are always beneficial and often are very rapid in their action, so that the impression is given that the life of the patient has been saved by their use. In the case of the patient under consideration there was a weak but distinct reaction for albumin present on admission, and the urine passed after the operation showed a great increase in the amount of albumin present. We immediately gave her slight infusions subcutaneously, and it was absorbed very slowly in her case, taking five to six hours, where usually it takes only from one-half to one hour. What is the reason for this? Usually it is due to the cannula being introduced into an unsuitable spot where no loose tissue is found; but if such cause is not present, it is, as a rule, due to cardiac weakness and is, therefore, an indication for stimulation of the heart.

We do this also in rare cases with excellent results. In my opinion, the best drugs to give are oil of camphor 1-10 and caffeine (benzoas natric. caff. 3-10) 1/2-1 Pravazsyringeful four to five times in twenty-four hours. By using these stimulants in connection with two to three thousand cubic centimetres of infusions of salt water daily we succeeded, in the course of five days, in bringing the patient through this eritical state of collapse, which gradually yielded; the rapid, irregular pulse improved by degrees. It became fuller, stronger, and more regular. The high temperature slowly fell to 38 degrees, where it remained for a long time, and finally, on the sixth day, the urine was free from albuminuria. 
The bag drainage with strips of silver nitrate gauze which absorbs the secretions from the peritoneal cavity through the slits in the rubber acted very satisfactorily. On the third day I removed half of the strips, on the fifth day the remainder, and on the seventh day the bags themselves. As usual, there was no retention as these were removed. They were replaced by thick cigarette drains made of oilsilk and nitrate of silver gauze.

As you view the patient you see that the two large incisions are entirely healed, while a small fistula remains at the upper end of the median incision corresponding with the point at which the bag drains were inserted into the sub-diaphragmatic space and into the lesser omental cavity. It is not strange that this drainage has been less effective than the others, because the Fowler's bed, which favored the drainage of the larger peritoneal cavity, is less suitable for the drainage of an abscess contained in the lesser cavity, which would naturally be served better by the Trendelenburg couch; but to employ both at the same time was quite impossible.

The suction drainage has been quite satisfactory. The suturing of the stomach has entirely healed. For the first week following the operation the patient was fed entirely per rectum, and the second week her diet was gradually commenced by the stomach. She has rapidly regained her strength and is now in a rosy condition, a rather remarkable change for three weeks.

The history of this patient is typical of this serious complication of stomach ulcer perforation with diffuse peritonitis. Unfortunately the conditions are so acute that they cannot be presented at a lecture, because the indications are for immediate operation, but at the same time it is of greatest importance for you to know the symptoms of this disease and to understand the treatment which should be given them. 
Perforation is rather a frequent complication of stomach ulcer; the exact percentage in which it occurs it is impossible to say. From the standpoint of the internist the percentage is very low, because the diagnosis of stomach ulcers is far more frequent than is justifiable, and partly because perforating ulcers as such are rarely taken to the medical ward. The surgeons, on the other hand, will give too high a percentage because most of the serious cases are sent to them. For example, Leube and Ewald give 1.2 per cent. for perforating ulcers, while Musser, in a surgical series of 1871 cases, reports the percentage of perforation as being 28 per cent. The post-mortem records probably are more accurate, and they show from 13 to 18 per cent. In Denmark, Harsloef's statistics from the General Hospital taken at the autopsy table show 341 perforations in 206 cases. These perforations occur more frequently near the lesser curvature, more often on the anterior than on the posterior surface; more rarely near the pylorus in the neighborhood of the greater curvature, and very seldom at the fundus or at the cardial end.

The perforation may occur in different ways, depending upon the degree of perigastritis and the adhesive peritonitis which occur during the perforation, and also the extent and the firmness these safeguards have attained before the moment of perforation. This depends to some extent upon the seat of the operation and partly on its nature, especially if it is complicated by a suppurative gastritis and perigastritis.

Ceteris paribus, it is clear that a perforation of the anterior stomach wall leads directly into the greater peritoneal cavity, increasing the possibility of peritonitis, while perforations on the posterior wall open into the lesser peritoneal sac, where the inflammation may be easily defined by adhesions between the peritoneal walls. It is not surprising that most of the cases of perforating stomach 
ulcer with diffuse peritonitis are due to ulcers on the anterior wall, in spite of the fact that most stomach ulcers are confined to the posterior wall, for the perforating ulcer on the posterior wall usually meets previously-formed adhesions of the peritoneal folds of the lesser pouch, and, if the inflammation extends farther, we have adhesions to the pancreas or even ulcerations extending into the pancreas itself.

If, as 'is often the case, we have a stomach ulcer occupying the saddle of the lesser curvature, we may have various results, including a perforation into the general peritoneal cavity, or a perforation solidly confined on all sides by adhesions to the anterior abdominal wall-as a rule, to the left of the median line at the edge of the free border of the ribs. The inflammation may often extend into the abdominal wall and cause a phlegmon, abscess, or even osteitis, or chondritis of the ribs, as was one of Jolın Berg's cases. In such cases the symptoms referable to the stomach are rather vague and usually consist simply of mild signs of local peritonitis. Sometimes, indeed, there is an exquisite tenderness high up above the space occupied by the abscess, or a fluctuating mass may be felt. But if the area is restricted by adhesions,-that is, closed off from the peritoneal cavity only in front, while the space beneath the liver and diaphragm is free,-it will lead to the formation of a subphrenic abscess. This type of abscess is by no means rare, and may be either retroperitoneal when it originates from the kidneys, or from the colon and appendix, or intra-peritoneal when it is secondary to inflammations of the intestines, sometimes appendicitis, but more frequently secondary to inflammations of the liver, gallbladder, and stomach. The perforating stomach ulcer plays a particularly prominent part in these subphrenic abscesses, and they vary according to the seat of the perforating ulcer, as I will show you in this rough diagram. The 
figures represent the sagittal section through the body to the left of the median line, and I indicate the extent of the abscess by sketching it in. Fig. 114 shows you a type which we find where the perforating stomach ulcer is situated on the anterior wall of the stomach and is confined by adhesions to the anterior abdominal wall and posteriorly by the ligaments between the stomach and the under surface

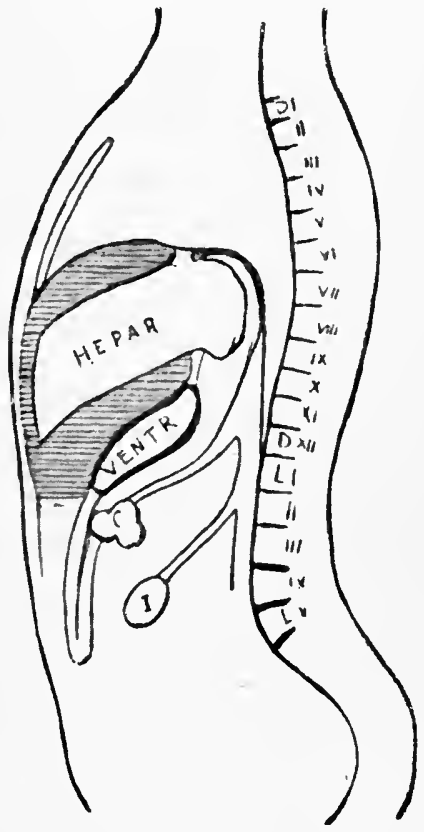

Fig. 114.-Subphrenic abscess secondary to perforating ulcer of the stomach on the anterior surface.

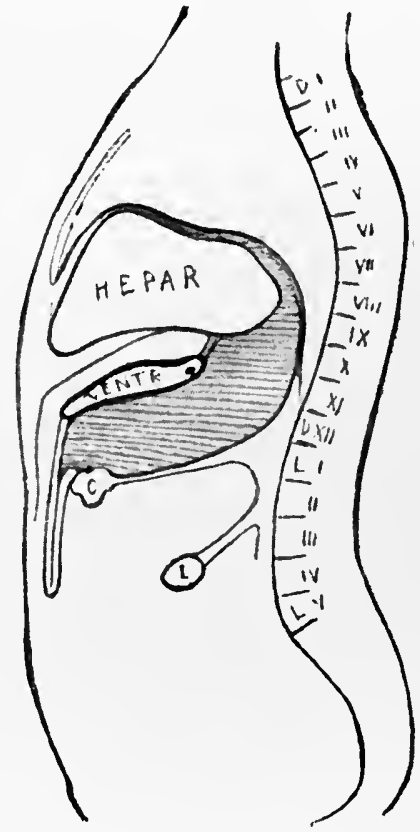

FIG. 115.-Subphrenic abscess secondary to perforating ulcer of the stomach situated on the posterior wall.

of the liver. You see here that the abscess curves beneath the edge of the liver in the shape of a bow and rises up between the convex surface of the liver and the diaphragm, being limited by the ligamentum suspensorium hepatis. Fig. 115 shows you a different type, which is of special interest to us to-day because it represents just what we found in the patient whose history I have given you as the motif for our lecture to-day. Here you see that adhesions between 
the stomach and the under side of the liver form the anterior partition wall toward the large peritoneal cavity, and behind this there is an abscess, which is confined above by the under side of the liver, and below shows a large, bag-shaped extension down on the posterior surface of the stomach. In reality this bag is only saccus omentalis minor distended with pus. Such an appearance occurs, as a rule, with a stomach ulcer, which perforates the posterior wall, but it can also arise, as in our case, with a perforation of the anterior wall close to the curvature, when adhesions between the liver and the stomach shut off the ulcer from in front. The forming of such an abscess is naturally a piece of good fortune, provided the abscess is discovered and operated upon before it has broken forth into the peritoneal cavity; but in the opposite case, if the abscess is not discovered until it suddenly breaks into the peritoneal cavity, then you understand that the situation is far more serious than with a simple free perforation through the anterior stomach-wall. Because in the latter we only have oozing of the contents of the stomach, which is often aseptic, and, when the stomach is empty, only a small volume of secretion, here we have a sudden outpouring of pus into general peritoneal cavity-pus which may be sterile, but most often contains bacteria, and always inflammatory toxins. You will understard that the resorption of these toxins alone from the powerful, fresh, and resorbing surface of the peritoneum may involve, in the course of a few moments, a perilous condition of intoxication. Therefore one frequently sees a complete state of collapse develop very quickly, almost instantaneously, which may be partly due to the sudden pain and peritoneal irritation, but certainly, to a greater extent, is due to the rapid resorption of toxins by the blood, and to the effect of these on the heart, the central nervous system, and the kidneys. There is no doubt that in our case the question has eoncerned the 
breaking of just such a large sub-diaphragmatic abscess, which had developed relatively without symptoms, after the stomach ulcer had perforated behind the adherences, the remains of which we plainly saw at the operation. This accounts for the thick, old abscess membrane, and above all for the large abscess in saccus omentalis minor, which could not possibly have developed in the course of a few hours as the result of a perforation of the anterior wall.

We have been rarely fortunate in saving such a patient by operation thirteen hours after the rupture. You will appreciate this when you hear that the latest statistics published in literature and collected by Bamberger, concerning the 513 cases of perforated stomach ulcer operated on from 1906 to 1909, show a joint mortality of 42.1 per cent. (298 cured, 215 dead). Of these, the operations performed within twelve hours after the perforation gave 30 per cent.; those performed twelve to twenty-four hours after, 45 per cent., and those still later, 60.9 per cent. of deaths. You must now further consider that in these statistics the good and the bad cases were mixed, and were one to sort out those where the peritonitis, as in our case, was diffuse and total, one would find scarcely 10 per cent. cured. by operation thirteen hours afterward.

The point is that one should operate as early as possible. Leube has proposed that one should defer the operation until the patient has recovered from the shock of the perforation. Lennander has justly and most forcibly opposed this suggestion. Certain it is, that in a number of cases there is a genuine condition of shock after the perforation, but it passes quickly - as a rule, before the physician arrives. If, even then, the patient is still suffering. from shock, it is probably a collapse resulting from intoxication, and infection from the peritoneal cavity, and no remedy is better for this sort of shock than a rapid removal of the source of the poisoning. 
In reality, it is clear, judging from statistics and the combined experiences of the surgical and medical clinics, that the only correct treatment in cases of perforated stomach ulcer is immediately to search out the perforation by opening the abdomen, to close the perforation and to render it harmless, and to remove the contents of the stomach or pus found in the peritoneal cavity. A few hours only are available, as a rule, for saving the lives of these patients. We have seen from the statistics that surgical aid can save about 70 per cent. of the patients when it is provided within the course of twelve hours; but a closer investigation teaches us that all, so to speak, could be cured if it were possible to convey them to the operating table in the course of two or three hours. Is this, then, attainable? There are still parts of the country where, owing to the distance between the patient and the nearest surgeon, it is quite unattainable. But in most places, thanks to modern conveyances and the ever-increasing number of clever surgeons all over the country, a patient can be in the surgeon's hands in the course of a few hours, provided that the physician called in is capable of making the diagnosis and knows what has to be done in such cases. In this day of specialists one is often inclined to undervalue the importance of the general practitioner. This is a huge mistake. It is more than ever necessary to have clever practitioners, and the position of a clever practitioner is just as full of difficulty and honor as that of a clever specialist, because much and comprehensive knowledge is required to determine which are the serious cases, and to realize when to call for the right assistance at the right moment. As I have told you so often, the patient's life and well-being depend, in the first instance, on the practitioner's examination, and this is more than ever the case when there is a question of perforating stomach ulcer; because, what avails it that the surgeons are ever so competent, when the physi- 
cian first called in does not send the patient in good timenot until the peritonitis and intoxication have caused irreparable harm? Most of you are sure to meet with such a case sooner or later, and the patient's life will then depend, first and foremost, on your diagnosis and activity.

Then, can ulcus perforans be diagnosed with certainty? In other words, does the stomach ulcer perforation present a typical aspect, characteristic of this disease? To this we must unfortunately reply: No. The picture is, indeed, somewhat characteristic, but, on the whole, is that peculiar to perforative peritonitis: sudden, violent pains in the abdomen, often accompanied by a state of collapse, fainting, feeble pulse, and cold extremities; the abdomen is tense, often as hard as a board, the result of extensive défense musculaire, and most frequently sensitive everywhere to pressure; if the tenderness is confined to any particular spot, it is more frequently in the left than the right side. As you perceive, this is the aspect of perforative peritonitis as we may see it with perforative appendicitis, with the breaking of a pyosalpinx, of a gall-bladder, as well as with perforation of a stomach ulcer. In many cases, by a careful examination of the anamnesia, one will now be relatively certain that it really concerns a stomach ulcer,that is, where the patient's history shows typical ulcer symptoms: hæmatemesis, melæna, and cardialgia; but in many cases certain symptoms are wanting in this direction. Therefore it has been maintained that peritonitis in cases of perforating stomach ulcer ought to be distinct from other perforating peritonitis, owing to the absence of vomitings. It was thought that the perforation itself would make vomiting impossible. This, however, is incorrect, as the Edinburgh surgeon, Dr. Caird, has proved to us by his observations, inasmuch as he observed vomiting in eighteen of the twenty-five cases on which he has operated for perforating stomach or duodenal ulcers. As being most sug- 
gestive of the stomach perforation, I think one ought to emphasize the extreme tension of the abdominal muscles, which is often so pronounced, that one sees inscriptions tendinea depicted as furrows, and next the intense pain caused by the least touch, which at first is specially located in the epigastrium and the left hypochondrium. Finally, I think one is right in mentioning as characteristic the symptom indicated by Symonds: that often, with perforated stomach ulcer, after the first violent attacks have passed, a stage of relative well-being sets in, which is free from pain, and in which all of the general symptoms are less marked.

In a great number of cases you will be in doubt as to the source, even after the most careful examination, but there will be no doubt that you have a perforative peritonitis before you, and this should be sufficient to warn you that surgical aid is necessary. In such cases you will nearly always meet with opposition from the patient or his family when you demand that the patient-who has, perhaps, been ill only for an hour or so-be taken to a hospital or clinic. Here you will need experience and firmness. One sees physicians who give in too frequently, yield to the unreasonable protest of the patient or the family against an operation. You should then explain quietly and earnestly that the situation is hopeless without an operation, and how precious every hour is, every minute, and how great a responsibility the relatives incur by opposing the operation. If, after this, the patient and his relatives continue to resist your advice, you should state that you dare not or will not undertake the responsibility of the further treatment of the patient. In the face of such a determined stand, where the physician is not moved from his position in the interest of the patient, I have generally found that the resistance gives way.

When you have completed your examination, the first thing to do is to relieve the pain and to stimulate the heart 
in order to counteract the collapse. You fulfil both objects by giving the patient an injection of morphia and oil of camphor. Caffein also has a very stimulating effect, and is well adapted to be used alternately with oil of camphor. If possible, you telephone at once to the hospital and announce the coming of the patient, so that everything can be prepared for the operation, while you arrange and carry out the careful transportation of the patient to the hospital. Remember, above all, that these patients must have nothing to drink! They are often very thirsty and demand something to drink, and if one does not expressly impress upon the relatives the danger of its bringing on renewed outflow of the contents of the stomach through the perforation into the abdcmen, they naturally supply the patient with something whenever he asks for it.

From the moment the patient arrives at the hospital your responsibility ends and that of the surgeon begins. First of all, he must judge of the patient's condition. If the patient is in a state of collapse, owing to his transportation to the hospital, it may be necessary to stimulate him with injections of camphor, caffein, ether, preparations of digitalis, or whatever one thinks suitable under the circumstances. But if the patient does not pick up rapidly with these expedients, then it signifies that the state of collapse is not due to moving the patient, emotion, etc., but to the advancing peritoneal intoxication and infection. With this type of collapse there is only one thing to be done,-i.e., an immediate and rapid operation. In order to counteract the collapse during the operation, one ought simultaneously to institute a salt-water infusion, preferably intravenously if it can be conveniently given while the operation is progressing. The operator has now a number of problems before him: to close or render harmless the perforation; to neutralize the consequences of the perforation by cleansing the peritoneal cavity of the inflowing contents of the stomach 
and the purulent exudation formed therein; lastly, to protect the patient against diffuse peritonitis by drainage. The operation must be done immediately and without unnecessary delay in carrying out the details of the work. In my opinion, the best method is to expose the stomach by an incision from processus ensiformis to the umbilicus, search out the ulcer, and, if the condition of the patient permits it, to excise it, and then join the sound resected edges of the . stomach, as I did with this patient. But in most cases the patient will be too exhausted for such a protracted encroachment, and one should then be content to sew the edges of the wound with silk in two layers. If the suture seems unsafe, it is an additional safeguard to sew a piece of the omentum over the suture line. If the small omentum hangs freely, it can be drawn down over the wound, but if it cannot be employed, one simply extirpates a suitable piece of the large omentum and transplants it freely, sewing it to the serosa of the stomach over the area of the perforation. In individual cases it may prove quite impossible to suture the perforation, either on account of the denseness of the adhesions or on account of the consistency of the tissue. In such cases Lennander advises us to introduce a drain through the perforation, and, further, through the pylorus into the duodenum. Then tampon around the drain and nourish the patient for the time by injecting liquid food into the duodenum through the drain. If I were faced with such a case I should prefer to pass a thick Pezzer catheter into the stomach and pull the button tight up against that part of the wall of the stomach surrounding the perforation. This provides far greater safety against the oozing out of the contents of the stomach. If there is a pyloric stenosis or hour-glass stomach, it will be necessary to add a gastroentero-anastomosis.

For the rinsing and draining of the peritoneal cavity I consider it best to have two incisions-one in eachifossa 
iliaca-as was the case with our patient. In this way we obtain access to the deep cavities on each side of the mesentery, but if, on account of the patient's condition, the object is to conclude the operation as quickly as possible, one can rest satisfied with one incision in the median line from the symphysis upward. With regard to the cleansing of the peritoneum, opinions are very diverse, because some surgeons deem it best to mop out the free fluid by the dry methodsponges or sterile gauze-while others lay particular stress on a continuous rinsing out, " lavage " of the entire peritoneal cavity with sterile warm salt water until the rinsewater returns quite clear. While with most other forms of peritonitis I am a firm supporter of the dry method-because by rinsing $I$ am afraid of spreading the infection to hitherto sound parts of the peritoneal cavity-but in cases of perforative peritonitis originating in stomach ulcer I am thoroughly convinced that rinsing is not only justified, but of the greatest importance. Here the conditions are quite different than in other forms of peritonitis, because we have to deal both with the contents of the stomach and the results of suppuration. In fact, the stomach contents containing mucus, hydrochloric acid, pepsin, etc., are often of more importance than infection itself, and this secretion may be sterile itself. So in these cases we do not run any risk of spreading the infection by washing out, but we do attain the more important object of removing the irritating toxic juice from the gastroperitoneal cavity. As to drainage, some surgeons prefer to drain through the vault of the vagina in women. From a standpoint of gravity, a tube in the vagina is better, but from my own experience $I$ find that nothing is preferable to the bag drainage filled with strips of silver nitrate gauze. The patient whom I have presented to you here was treated in this way, and you see how well she has done in spite of the fact that the operation was delayed thirteen hours after the perforation. The mortality 
at such a time is usually 50 per cent., and every hour that is added to the interval between the perforation and the operation adds to the mortality.

A few days ago a patient was admitted sixteen hours after the perforation, but in spite of an immediate operation, just as was done in this case, the patient died a few hours after the operation. This man had suffered for many years with typical attacks pointing to a perforating ulcer, and I believe thoroughly that, if the case had been properly diagnosed, the patient could have been saved by operation if he had only arrived two or three hours earlier. 


\section{LECTURE XXI}

\section{CANCER OF THE STOMACH}

THE patient who occupies our attention to-day is a married woman, scarcely forty years of age, who was admitted to this ward a few days ago for the treatment of a stomach complaint which had existed for five weeks. The history shows that twenty years ago she was treated for some gastric disturbance which manifested itself by attacks of cardialgia without vomiting. Otherwise she has been well. She recovered after being placed on a milk diet. She has passed through four normal confinements. She has always been slender, but has noticed during the last year or two that she has become considerably thinner than formerly, and has felt tired and less persevering with her work, but she was not really ill until about five weeks ago, when she began to feel tension and oppression in the region of the cardia after eating, especially after heavy food: rye bread, pork, potatoes, etc. There has been no vomiting, and her physician, like ourselves, has looked in vain for blood in the frces. When you look at her now, the first thing which strikes you is her paleness and thinness. When you regard the abdomen, you see that this has the shape which I have described as being typical with enteroptosis patients, because the epigastrium shows a hollow where one sees and feels a strong pulsation of the aorta, while the part below the umbilicus is dome-shaped. You see also the characteristic phenomenon of the intestinal coils being depicted through the abdominal wall in peristaltic movements, especially in the central part where musculi recti have spread apart to a great extent.

If we now investigate by scraping auscultation, it appears, also, that the stomach lies considerably sunken, so 
that the greater curvature seems to reach to a level between the symphysis and the umbilicus. Can the entire condition be due simply to enteroptosis? You know that gas-

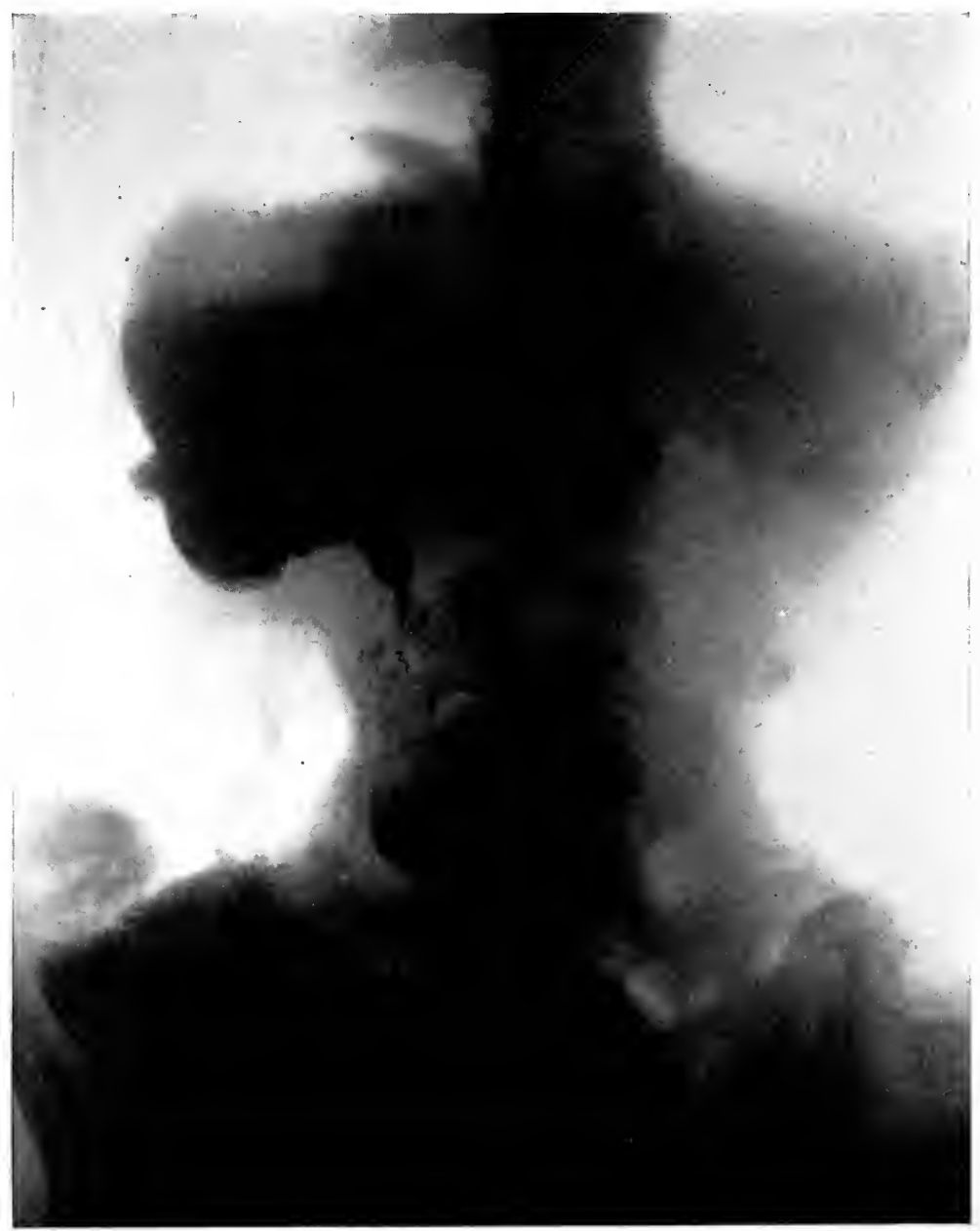

FIG. 116.-Radiograph of a case of extensive cancer of the stomach showing the irregularity of the Röentgen picture.

troptosis often manifests itself in cardialgia and dyspeptic attacks; that these patients often become extremely thin, and so cachectic, indeed, that they quite remind one of 
cancer patients. But on further inspection this explanation seems to me doubtful, because the stomach attacks in this case commenced only five weeks ago. If gastroptosis were the cause of the cachexia, it would be accompanied by more or less gastric distress lasting over a period of years -pain and vomiting which have made nourishment impossible. But in this case we find nothing of the sort, and the dyspeptic symptoms, beginning only five weeks ago, which have brought the patient here are of such a mild nature that they can in no way explain to us the emaciation and very anæmic condition of the patient. This is somewhat concealed by the coating of tan on the skin, which often gives a deceptive impression with patients from the country. In this case the blood examination shows that the quantity of hæmoglobin has fallen to 58 per cent.! Under these circumstances you will understand that we must look for another explanation, and with this patient who is so easily examined I found something very suspicious, even at the first examination. While palpating the epigastrium I asked the patient to draw a deep breath, and I felt a firm, rough tumor the size of a hen's egg protruding under the right rib curvature toward the median line, so that it appeared in the diastasis between musculi recti. I can easily show you this tumor by letting the patient take another deep breath. You notice how a roundish body pushes itself down, revealing itself plainly through the thin abdominal wall. And you see that I can take hold of this tumor and move it freely up and down and from side to side. You notice that I can push it down to the umbilicus, and over beneath the left rib curvature. Such a freely movable tumor of this shape and in this position can scarcely be anything but a tumor of the pylorus, and this suspicion is confirmed by our examination of the function of the stomach, for it appears that there is a delay in the emptying of the stomach. After administering a Bourget 
experimental meal we find, by the withdrawal of this, remains of prunes and cowberries even nine to ten hours afterward. After twelve hours, however, we find the stomach empty. There is then a distinct, if not a considerable, retention present. If we make a chemical examination of the stomach contents after giving an Ewald test-meal, it appears that there is a total deficiency of hydrochloric acid-achylia gastrica. No distinct mucus is observed in the withdrawn experimental meal or in the wash water.

There is no doubt, then, that this tumor which we feel pertains to the pyloric area, and, to a certain degree, obstructs the passage from the stomach into the duodenum. The only question is, is this tumor benign or malignant? Is this what we term ulcer tumor or cancer? Clinically it is extremely difficult and often impossible to decide this question, and this is all the more lamentable because certainty in either case is so important a factor in deciding the correct treatment. Should one be assured of an ulcer tumor, one would be justified in being content with the relatively safe encroachment gastro-enterostomy, while with cancer a radical extirpation of the diseased part would be absolutely indicated. By ulcer tumor we understand cases where the ulcer has become the source of an inflammatory infiltration, which has developed to such an extent that it resembles closely the conditions found in malignant disease, most frequently with carcinoma, and more rarely with sarcoma. You can appreciate how deceptive this condition is when you realize that its existence was first discovered in cases which were operated on under the diagnosis inoperable carcinoma, with the employment of a gastroentero-anastomosis. It was the surprising after-course which gave rise to the diagnosis, inasmuch as the tumor, instead of growing and spreading as was expected, diminished or vanished entirely at the same time that the 
doomed patient grew better and better. The first to draw attention to this, in a long series of cases, was the eminent Swedish.surgeon, John Berg, who deserves great credit for developing gastric surgery in Scandinavia. Even in 1898 he was able to report no less than ten cases of tumorlike ulcer, where gastro-enterostomy or pyloroplasty resulted in recovery. Later experiences have shown, however, that one must be somewhat careful when inferring a simple ulcer tumor, because the tumor vanishes after a gastro-enterostomy, for it has been found that, even in cases of malignant tumors which originate either from a simple ulcer or themselves become infected and ulcerate, a great part of the tumor found is due to simple inflammatory infiltration, which is reabsorbed when a gastro-enterostomy provides a new outlet from the stomach, thereby diminishing the inflammation. In such cases the diminution in the size of the tumor, as well as the improvement in the patient's condition, is only temporary and deceptive. How difficult the diagnosis may be, not only clinically, but even for the histologist who has the tumor for examination, is best shown by a case which I operated on, and published an account of, in 1898. It concerned a man, thirty-one years of age, who was admitted to my clinic to undergo an operation for cancer of the stomach. Achylia, retention, and a tumor were found. It spoke in favor of the cancer having developed on the bases of an ulcer. For more than a year the patient had suffered from violent pains in the cardia after each attempt to eat. At the operation a spindleshaped, sarcoma-like tumor was found in the neighborhood of the pylorus, which had extensively infiltrated the wall of the stomach, so that two-thirds of curvature minor and one-third of curvature major were involved in the change. I then removed three-quarters of the stomach by resection, and following Kocher's plan, implanted the duodenum into the posterior wall of the stomach. You see here some re- 
mains of the tumor which was removed. It still gives a fair idea of the deceptive appearance of this tissue and its resemblance to sarcoma tissue. Nevertheless, the first investigator, Professor Dahl, found in all the sections of the tumor which he examined that this was a simple inflammation tumor without signs of malignancy. But when I demonstrated the tumor as a typical ulcer tumor before the medical society, the pathological anatomists present protested against the diagnosis, asserting that, according to the macroscopical appearance, it must undoubtedly be a sarcoma. I then distributed pieces of the tumor among these colleagues for microscopic investigation, the result being that one and all confirmed Professor Dahl's findings, inasmuch as the tumor was not in reality a sarcoma, but simply an inflammatory infiltration, though one of the investigators found by chance a spot in the tumor where an adenocarcinoma was beginning.

The patient recovered and is still perfectly well, twelve years after the operation, which would certainly not have been the case if we had been content with a gastro-enterostomy.

Furthermore, I have had cases which at the time of operation I considered were unquestionably inoperable scirrhous carcinoma, but where the patients, even after the course of five and six years, seem completely cured by a simple gastro-enterostomy. I am reminded, to my great joy, by a yearly letter from London, of a seventy-four-yearold Danish-born engineer, on whom I did a gastro-enterostomy six years ago, after having "substantiated" a scirrhous cancer of the pylorus, which had attached itself to the under side of the liver.

On the other hand, in several cases, the sad course of the disease has shown me that cancer was present where I had contented myself with a gastro-enterostomy, believing that $I$ was confronted with simple ulcer tumor. 


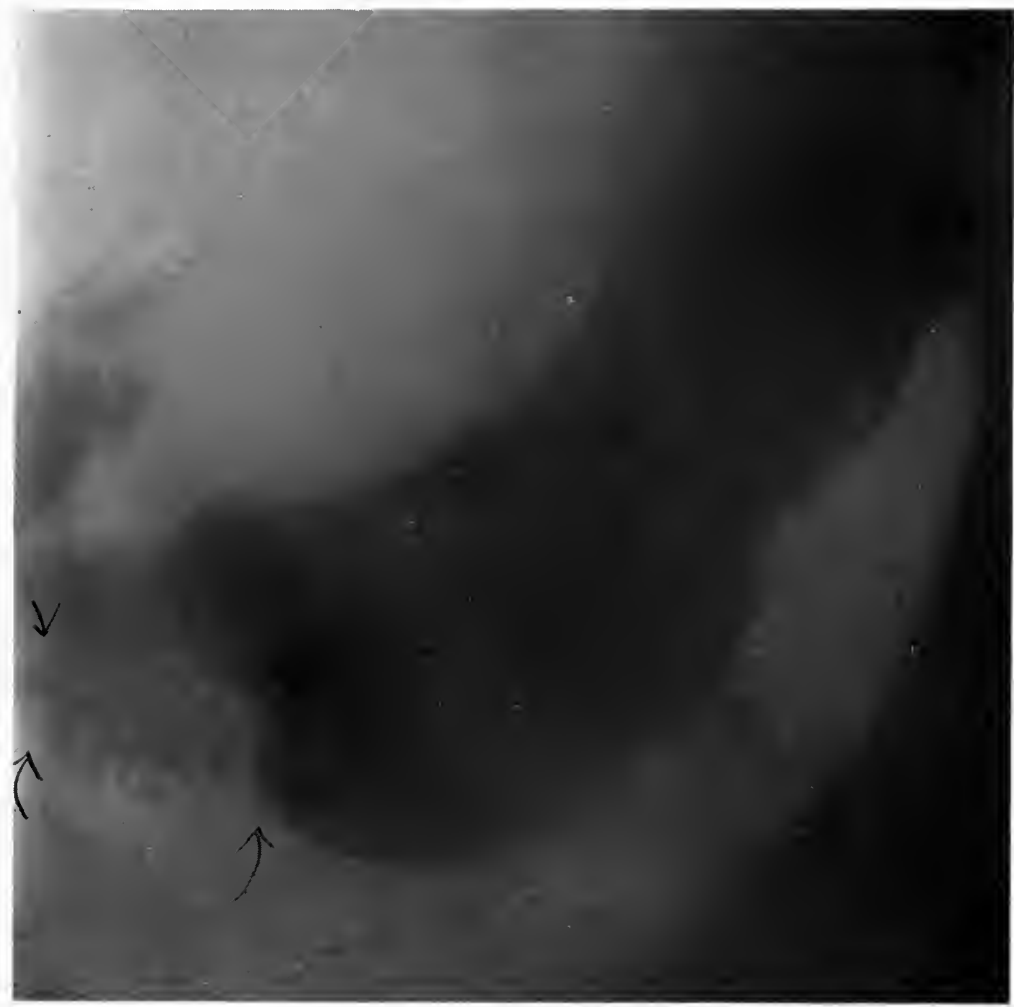

Carcinoma of the stomach showing filling defect to the left of the picture in which there was at operation an extensive carcinoma of the stomach. 

A priori, one would expect to be assisted toward a diagnosis by the history of the symptoms during the development of the tumor, because one would expect an ulcer to betray itself by the ordinary ulcer symptoms-cardialgia, hæmatemesis, and melæna-while a more insidious, symptomless development would speak in favor of cancer, which naturally is the case to a great extent. But it must not be forgotten that most of the gastric cancers develop on an ulcer base, so that a tumor which develops in connection with ulcer symptoms will just as frequently be a real cancer as a simple ulcer tumor; on the other hand, there are ulcers which give no symptoms at all.

With regard to our patient, she might belong to just this class, because she did not present any stomach symptoms until five weeks ago. I venture, nevertheless, to diagnose cancer as being almost certain in this case, because of two symptoms, which in themselves are of little account, but which, when taken in conjunction with the other signs, seem to me to speak emphatically in favor of cancer: I refer to the anæmia and the achylia. It is quite true that anæmia is very frequent with ulcer patients also, but then it is due to hemorrhages-loss of blood from the ulcer. Where, as with this patient, you find a severe anæmia without any signs of hemorrhage, the anæmia becomes a most important indication in favor of cancer.

With regard to the achylia, here again we are faced with a phenomenon which is frequently found in benign cases, but at the same time with a phenomenon the explanation and value of which have been and are still the subject of dispute and confusion. You all know that, while we formerly considered achylia gastrica to be partly a nervous phenomenon, the outcome of disturbances in the influence of the nerve system on the secretion, and partly a toxic phenomenon, Professor Faber and his pupils asserted that achylia is always due to a diffuse inflammation of the 
mucous membrane of the stomach,-i.e., gastritis. This assertion.was based on a series of original and significant experiments and microscopical examinations of the stomachs of patients who presented the symptoms of achylia. Excellent and unassailable as these examinations of Faber and Lange are in themselves, the conclusions which they drew from them seem to me to be questionable, especially the justifiableness of the sentence: "No gastritis, no achylia." I wonder whether it will not be as it was with gastrosuccorrhœa-the reverse of achylia-which we once thought an infallible symptom of the presence of a stomach ulcer, and later found to be present with ulcers.

As I have shown you in a former lecture, this is not correct: Other affections of the stomach, such as gastroptosis, can also cause a gastro-succorrhœa, and it is unreasonable to suppose that other forces should not also be able to irritate the secretory nerves-whether the irritants are mechanical or toxical. But the same might happen with the secretion's anomaly, achylia. Faber's definition is apparently so clear and simple that I should not attempt to change it if it did not leave us in the dark with regard to a great and important phase of achylia, and just that phase which interests us to-day-the relation of achylia to cancer. You all know that achylia gastrica is so very often present with cancer of the stomach that some have considered it pathognomonic when one is in doubt, and with great justice. The frequency of achylia is remarkable in these cases, for in a series of about 150 cases of cancer of the stomach I have only twice found free hydrochloric acid in the stomach. Without contesting Faber's theory, we consider that there may be two ways in which cancer of the stomach ean produce achylia. First, one can quite understand that a cancer which infiltrates all that portion of the stomach which bears the glands which produce hydrochloric acid will destroy these glands and naturally produce an 
achylia. Where the more localized tumors are concerned we might, in the next place, imagine that these might become the source of an infectious gastritis, which again might produce a real Faber's achylia. There is, however, a discrepancy here, for, as a rule, ulcerations in the stomach are not usually associated with achylia, but, on the contrary, a hypersecretion of hydrochloric acid. You know, moreover, that the majority of cancers of the stomach originate from an ulcer base, and, in my opinion, by infection of the wound with accidentally-transmitted cancer germs. How is one to explain this apparent contradiction that a simple ulcer, or, as you saw the other day, a tubercular ulcer, gives increased production of hydrochloric acid, but that, as soon as an ulcer is infected with cancer, the hydrochloric acid vanishes? In my opinion, only two explanations are possible. The first is that the achylia makes cancer infection possible,-in other words, that ulcers cannot be affected by cancer until they have become the source of a diffuse gastritis. The second is that the presence of cancer affects the secretion of the stomach, per se.

The first explanation is most attractive, not only because it falls in with Faber's theory, but also because it fits in with our conception of the hydrochloric acid as being a wonderful antiseptic, affecting the contents of the stomach and the upper part of the intestinal canal, so that they are almost free from bacteria. It was by no means unreasonable to imagine that the hydrochloric acid had a destructive effect on the cancer germs also, and protected the mucous membrane against their attack. In these cases where the cancer develops in the base of an old ulcer, which has caused stenosis and retention for a long time, the explanation might, indeed, seem sound enough, but one cannot deny that there are certain forms of cancer of the stomach which do not seem compatible with it, and preclude 
its general validity. I will mention some examples from my clinic, which some of you will perhaps remember.

Some time ago we had as the subject of our lecture on gastric ulcer a man who for sixteen years had presented symptoms of gastric ulcer, which we localized in the cardial part, because the pains always appeared immediately after the passing of the food into the stomach, and were very severe. This man had for many years been under the treatment of a specialist, and, until a few months before his admittance here, had had a goodly amount of hydrochloric acid in the gastric juice. Later we found achylia, although there was no mucous secretion or retention of food at all. You remember how I told you that the rapid transition from hyperhydrochloric acid to achylia directed my suspicions towards cancer, and the operation showed, true enough, that the ulcer had become the seat of cancer, inoperable because of its high position. The remaining part of the stomach appeared quite normal. You can understand how the symptoms in this case strengthen the belief that the achylia was the result of the cancer, which was rather advanced, while the achylia was of recent date.

Those cases in which a cancer develops on a limited portion of the stomach without there previously having been any history of ulcer or any digestive disturbance at all, and where the tumor does not cause retention and where there is no ulceration and still we find achylia, makes the theory of gastritis as the cause of this condition seem rather impracticable. An example of this was seen in a patient, fifty-eight years of age, who came for an operation on a small epigastric hernia which occasionally was very painful. The man was pale and thin, with hæmoglobin of 80 per cent., so that I suspected some more serious condition to be present. He had had some constipation alternating with diarrhœa, and on examination of the stomach we found achylia to be present, but otherwise no abnormality. Upon 
operating for the hernia I decided to examine the stomach thoroughly, and on the anterior wall of the stomach I found a flat tumor, about six centimetres long and four centimetres broad, which projected into the lumen of the stomach. At the greater curvature were found some lymphatic glands. I removed the entire diseased portion of the stomach together with the enlarged glands. These glands as well as the tumor showed a typical adenocarcinoma. At no point

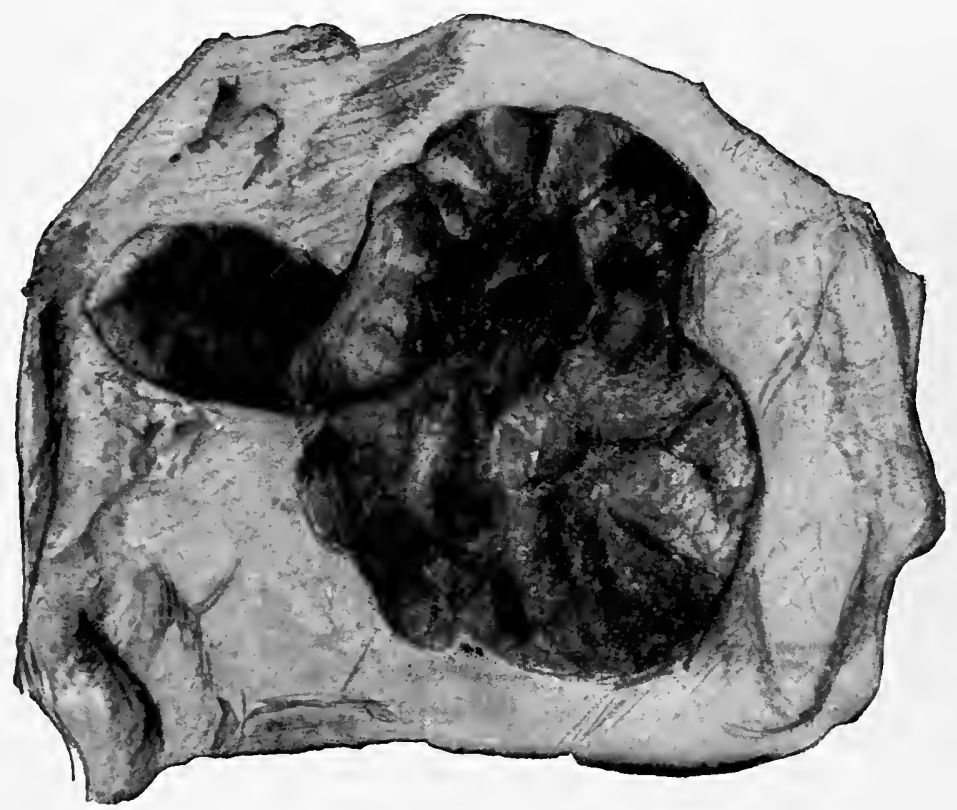

Fig. 117.-Adenocarcinoma of the anterior wall of the stomach removed by resection.

was there any ulceration of the tumor. Diaphanoscopy and gastroscopy showed that the wall of the stomach was free from gastritis. Fig. 117 shows the appearance of the tumor.

This specimen seems to speak in favor of construing the achylia as the result of cancer and not as the result of gastritis. In the patient who is now before you it does not seem that the gastritis symptoms have lasted long enough to explain the achylia, and we therefore must assume that the 
tumor which is perceptible is the cause of the achylia as well as the slight cardialgia and the dyspeptic symptoms which have been present for the last five weeks. Viewing the entire subject broadly, there is much which makes me doubt Faber's theory. Would it not be fully as natural to imagine the reverse; that is, that the achylia which arises from other cause is the cause of the gastritis in this case? I personally think so, and will tell you why. A diffuse chronic gastritis with its round-celled infiltration must naturally be due to infection, a point which Faber's school avoids in a striking manner; and the fact of the matter is that the normal hydrochloric acid contained in the gastric juice is a powerful antiseptic which seems not only to impede but naturally to prevent infection of the surface of the mucous membrane of the stomach. A number of facts bear this out: First, that during the time that food is in the stomach the gastric juice is capable of destroying the numerous germs which are swallowed with the food; second, that it is impossible to produce an ulceration or inflammation of the mucous membranes of the stomach by a traumatic lesion; third, that even a chronic embolic ulcer of the stomach only becomes the source of a gastritis in extremely rare cases. As you know, wounds and ulcerations in all other parts of the body, such as the skin, mouth, nose, and the intestinal mucous membrane, almost invariably become the starting point of diffuse inflammatory processes, while we find quite the reverse state of affairs in the stomach. Ulcer and gastritis seem to be quite uncomplicated, and naturally so, because an ulcer produces irritation which reflects the causes and increases the secretions of the gastric juices, which prevents infection and inflammation. It follows, then, that the mucous membrane of the stomach lies defenceless against the infectious materials which are constantly being taken into the stomach as soon as the production of hydrochloric acid ceases for any reason. 
Therefore, a gastritis must develop where there is achylia. Furthermore, with a total achylia there should be a corresponding advanced gastritis with destruction of all the hydrochloric glands. But this is only the case in some of the stomachs, while in others larger or smaller portions of the glandular layer itself were preserved. ${ }^{1}$ That other factors certainly are concerned in the production of achylia would seem to be suggested by its occurrence in pernicious anæmia, tuberculosis, and cancer. Achylia is also found in cases of secondary anæmia caused by botriocephalus latus. As soon as the anæmia disappears the normal conditions return. Rosenquist observed a patient who suffered from secondary anæmia for more than six months in which achylia was present and in whom anæmia was cured by expelling the worm, and within a month a normal secretion was found in the stomach with a total acidity of 58, hydrochloric acid 38.

In commenting upon this case Faber says: "It is true enough, therefore, that we can have a long-standing achylia without complete destruction of the glands, but surely no one would conclude from this that the achylia in such cases was due to the severe gastritis."

I must confess that I find it quite impossible in such cases to explain the achylia as a result of such a restricted and temporary gastritis, while I find it most natural to construe the achylia as the result of the anæmia or of the same intoxication which gives rise to the anæmia. The gastritis then becomes the natural consequence of the achylia, and disappears with it when the intoxication is removed.

If you start with this conception, you will find that all problems solve themselves, and all that which is unintelligible becomes intelligible, and, last but not least, achylia appearing as a symptom with cancer, because this also

"See FABER: "Chronic Gastritis." Introductory Lecture at the 5th Congress for Internal Medicine. Hospitalistidende, Nos. 35 and 36, 1904. 
gives a general state of intoxication which leads to severe anæmia. How could we otherwise understand that an ulcer of the stomach, which for twenty years has given hyperhydrochloric acidity, produces achylia when it becomes the seat of cancer? It is certain that Faber's theory with regard to the relation of achylia to gastritis is by no means sufficient to explain to us the relation of achylia to cancer of the stomach. I think that the explanation will be found in the direction indicated by me, but new and thorough investigations are needed to obtain proof of this.

But, whatever the real facts of the matter may be with regard to this interesting question, there is no doubt that achylia, especially when it coincides with emaciation and a considerable fall in the quantity of hæmoglobin which cannot be explained by any hemorrhage, is of the greatest significance for the diagnosis of cancer of the stomach at a somewhat early stage. The case which I have just described to you, where the achylia and the anæmia formed the whole basis for our explorative encroachment, led. to the discovery of a removable cancer. I could give you many examples bearing upon this subject where patients suffering from gastric disturbances have been treated for months and years and finally sent to the surgeon when at last the tumor had appeared in the epigastrium. As a rule, when this symptom is present, the case is already inoperable. As an example of this, a lady sixty-three years of age came to me who had suffered from alternating diarrhœa and constipation for twelve months. At the clinic it appeared that achylia had been found without retention. She was confined to bed for ten weeks and was treated for gastritis and intestinal dyspepsia. She lost steadily in weight and did not improve, but she was instructed to continue the treatment at home, and she still continued to suffer. On examining the patient I found a large, flat tumor in the epigastrium, which moved with 
respiration. At operation the whole stomach was found to be infiltrated with cancer, so that not even a gastro-enterostomy could be performed. I lay stress upon this case in order to impress you with the fact that if achylia is present you must always think of cancer and repeatedly examine the abdomen for a tumor. Remember, also, that most cancers of the stomach at first show intestinal symptoms, diarrhœa alternating with constipation probably just because the achylia is present. Always apply a functional test to the stomach, and, if you find achylia, do not rest upon a diagnosis of gastritis; think of cancer! Determine the amount of hæmoglobin in the blood, keep a sharp eye on the patient's weight, and if, in spite of a suitable diet for several weeks, these should fall continuously, then send your patient to a surgeon, even if you yourself cannot feel a tumor; because, as I have said, when you can feel a tumor it is too late!

Statistics of 131 cases of eancer of the stomach, which at one time or another have been referred to me as operationis causa, will give you a clear idea of how desperately late patients with cancer of the stomach may be brought for operative treatment. Twenty had to be refused as absolutely inoperable on account of the size of the tumor, and metastasis, and far-advanced cachexia; nineteen proved to be quite inoperable after the stomach had been laid bare by laparotomy; sixty-seven were too far advanced for an attempt at a radical removal: gastro-enterostomy was performed with fifty-six of these, twenty-two dying at the hospital. Eight cancers of the cardia were treated with gastrostomy: four died; jejunostomy was performed with three, because the entire stomach was cancerous: three died; twenty-five offered a possibility of a radical operation: resection of the stomach was performed with twentytwo, of whom twelve died. With one, total extirpation with ensuing œsophagus-jejunostomy was performed: lived six 
months. With two resection of the stomach and transverse colon was performed: both died.

When you compare these statistics, where the gastroenterostomy gives a mortality of 39 per cent. and the resection a mortality of almost 50 per cent., with our statistics of the corresponding operations in benign diseases of the stomach (see page 278), where the total mortality was 8 per cent., it will at once be obvious that the great mortality in cancer patients is not due to the operation as such, but to the nature of the disease, and, above all, depends on the lowered resistance of the patients. In my own opinion, you cannot blame any deaths of those operated upon for cancer of the stomach solely to the fact that they are brought to you for operation when the disease is in an advanced stage, because, other things being equal, every operation is more dangerous when undertaken for cancer than when undertaken for ulcer, for in the latter case also the patients are brought to us quite late enough. The reason is that cancer patients have achylia and, consequently, infectious gastritis with a highly virulent bacterial flora, on account of the condition of retention; while stomachs with normal or hyperacid secretions-as with ulcers-are sterile, and thereby fortified by the gastric juice against infection. In consequence of this circumstance, the gastro-enterostomy wound and the resection wounds are infected from the mucous membrane side, which causes intoxication and high temperature, which can often prove fatal to these patients, who are not only famished and anæmic, but who often suffer from enfeebled action of the heart. Consequently, also, a serious infection of the peritoneum often occurs from the outflow of the intestinal contents during the operation, and, therefore, in operating for cancer the surgeons must take care to avoid any oozing of the stomach and intestinal contents during the operation.

The large operative mortality is essentially due, how- 
PLATE VIII

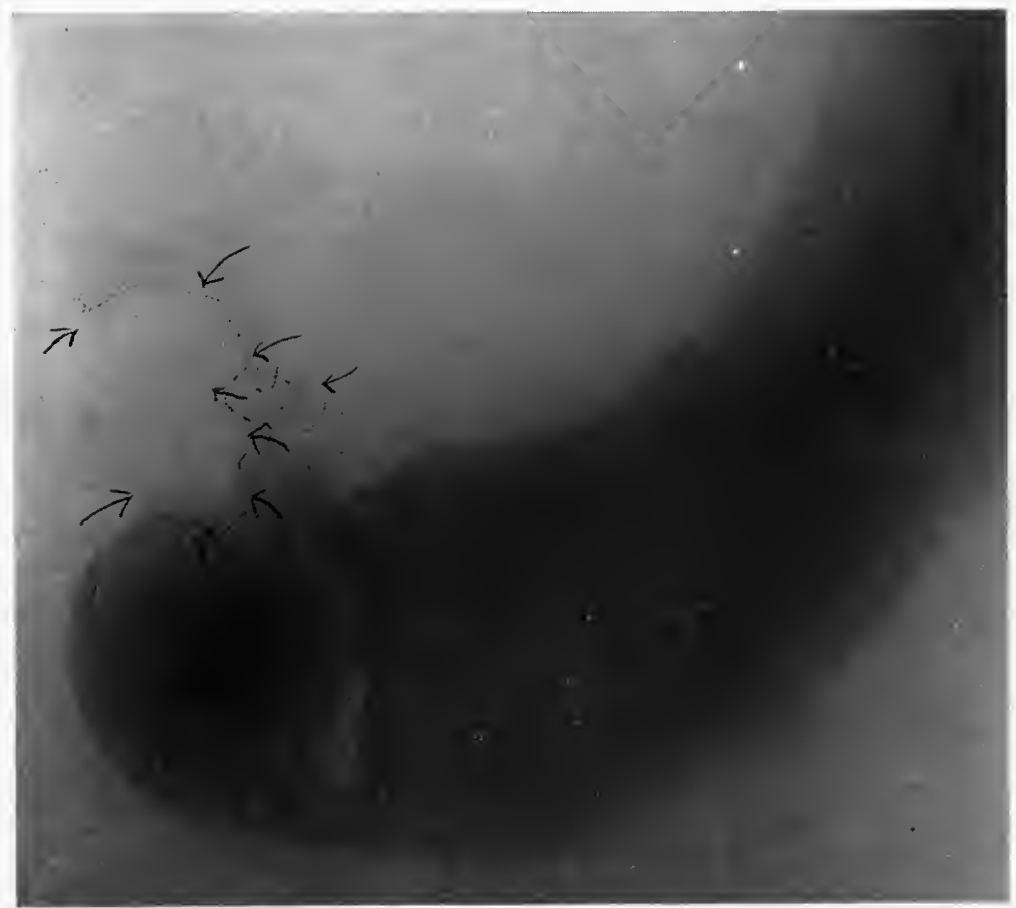

Radiograph showing filling defects of pyloric antrum and signet ring deformity of duodenum by extension, as is shown in dotted outline. This patient was operated upon by Dr. L. S. Pilcher and an extensive carcinoma of this area was discovered, necessitating a resection of the pyloric end of the stomach, first portion of the duodenum and a portion of the pancreas. 

ever, to the miserable condition of the patient, who is usually quite unfit for an operation. Why, then, do we operate on such patients? you might justly ask. Well, the explanation must be sought for in two circumstances: Firstly, that in the case of an obstructing tumor we are often in doubt as to whether it is due to an ulcer or a cancer, and as the prognosis in the case of an ulcer, even with extremely emaciated and exhausted patients, is relatively good, one ought naturally to operate when one is in doubt; because the misfortune that a doomed cancer patient dies some days sooner than would otherwise be the case counts as nothing compared with the misfortune of having omitted an operation which could have completely cured a patient with ulcer. I have already mentioned one instance where the course, after a gastro-enterostomy in the case of an old man with a supposed scirrhus, has shown that in reality it was only an ulcer with extensive infiltration, and I have a series of six cases in which the patients were so utterly debilitated, and where the enormous tumors were so extensive, adherent, and cancer-like, that at the most I expected only a temporary relief as a result of the gastro-enterostomy which was performed; but where, contrary to all expectation, the patients have flourished, are alive and in perfect health three to six years after the operation. The other circumstance which often forces us into an operation in apparently hopeless cases is the duty of relieving the fearful pain and sufferings which the state of retention with stenosis of the pylorus involves in some cases. But it must be emphasized that, pleasant as the palliative effect is which we can obtain for the moment by a gastro-entero-anastomosis in the case of cancer patients, just as sadly disappointing is the effect in the long run; because, sooner or later-often very quickly-the cancer reaches the anastomosis opening, and the sufferings due to the stenosis return. The two other palliative operations, gastrostomy and 
jejunostomy, have yielded us still less satisfactory results. Gastrostomy is indicated in an inoperable cancer situated high up near the cardia, when, in an otherwise healthy patient, it hinders the passage of the food into the stomach, and especially when it causes intense pain. Jejunostomy is essentially indicated where a stomach cancer is so extensive that a gastrostomy or a gastro-enterostomy cannot be performed at all, or where it is estimated that the area of operation will become quickly infiltrated by cancer.

You will see that it is a poor satisfaction for the surgeon that you send him a majority of the patients with stomach cancer in such good time that one of these palliative operations can still be performed, because only in rare cases do we prolong life for some weeks or months. No! the surgeon's devout wish is that he may succeed in having the patients brought to him while there is still some hope that radical extirpation of the cancerous part of the stomach may be done. This operation alone holds out the possibility of a cure, or, failing this, a palliative effect of long duration. I do not believe that we shall ever succeed in obtaining such an early diagnosis in these cases, for the simple reason that, in a certain number of cases, cancer develops without any symptoms, or with so few that it does not occur to the patient to consult a physician until the cancer has attained such dimensions that a radical removal is inconceivable. But there is no doubt that many, many of the cases which are now brought to us after having been treated medically for years, both can and should be brought to the surgeon sooner. What will help most to accomplish this is a more widespread acceptance of the great diagnostic importance of achylia. But here you must remember that, clinically, it is unusual for achylia to betray its presence through gastric symptoms, but, on the contrary, by intestinal disturbances, especially diarrhœa, which undoubtedly is due to the fact that the gastric juice has lost its antiseptic effect, 
so that pathogenic microbes pass through the stomach unhindered, and also to the fact that the food has not been properly digested in the stomach. You must remember, therefore, that an examination of the stomach is always necessary when you meet with intestinal disturbances, even if the patient does not present any symptoms referable to the stomach. Remember that most cases of cancer of the stomach begin with diarrhœa, and if, by examining the stomach, you find that achylia exists, your suspicion of cancer of the stomach must be aroused. If, further, you find retention in the stomach, the suspicion increases, and here you must particularly note that even a relatively small, perhaps microscopic retention-such as Alfred Madsen has drawn our attention to-is a frequent symptom in cases of incipient cancer. Then you should examine the patient's hæmoglobin. If this is reduced to any considerable extent, without signs of hemorrhage, then the situation is really so serious that a surgeon slould be consulted as to the advisability of an explorative encroachment. Secondly comes the importance of carefully palpating the epigastrium for signs of a tumor, using the methods which I have taught you. I say that this stands second, because where a tumor is palpable the case is often so far advanced that there is little hope for a complete removal. In most cases where a palpable tumor is present, other symptoms have already appeared, but, as in the case of the patient who is the subject of our lecture to-day, the presence of a tumor may be the first conspicuous symptom.

I think that if all physicians would follow these suggestions the number of patients admitted with stomach cancer, suitable for radical operation, would be far greater than it is at present.

In considering the indications for a radical operation in a given case we must first determine whether the growth is confined to the stomach or not. If the cancer is adherent 
to the liver or to the pancreas, or if it has affected the colon transversum, then we know that any attempt at a radical operation is contra-indicated. The same is the case if we find metastases in the liver or in the other parenchymatous organs, or secondary growths on the peritoneum, which are readily and immediately betrayed by an outpouring of ascitic fluid when the abdomen is opened. Finally, if there are metastases in the deep-lying glands which cannot be removed. With regard to the extent of the growth in the

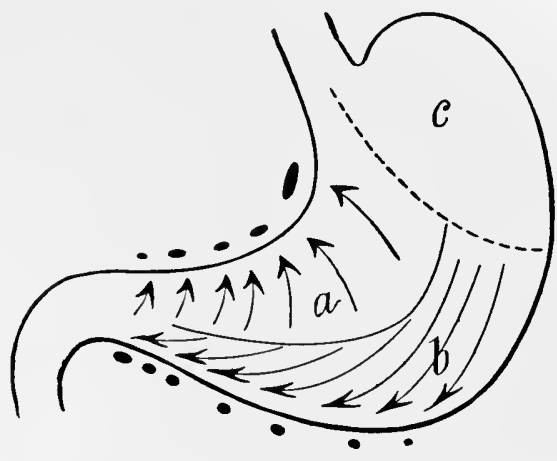

FIG. 118.-Sketch of lymphatic glands of the stomach. (Moynihan.). stomach itself, it is essential that there should be at least four centimetres of healthy tissue between the œsophagus and the edge of the growth if any radical operation is to be considered. Involvement of the pylorus does not give rise to any technical difficulties; in two of the cases in which $I$ did a radical operation a part of the duodenum was involved in the cancer. In other words, the entire stomach can be extirpated.

In resecting the stomach for cancer it is essential that sufficient healthy tissue be removed with it to insure the destruction of any outlying cancer-cells, and at the same time an effort should be made to remove all of the infected lymphatic ducts and glands which are known to drain the area which is infected. Naturally, we must know exactly the distribution of the lymphatic chains which drain the stomach. Fig. 118 is a sketch of the lymphatic vessels and glands connected with the stomach. The black dots along the greater and lesser curvatures indicate the lymphatic glands, and the arrows show the direction in which the 


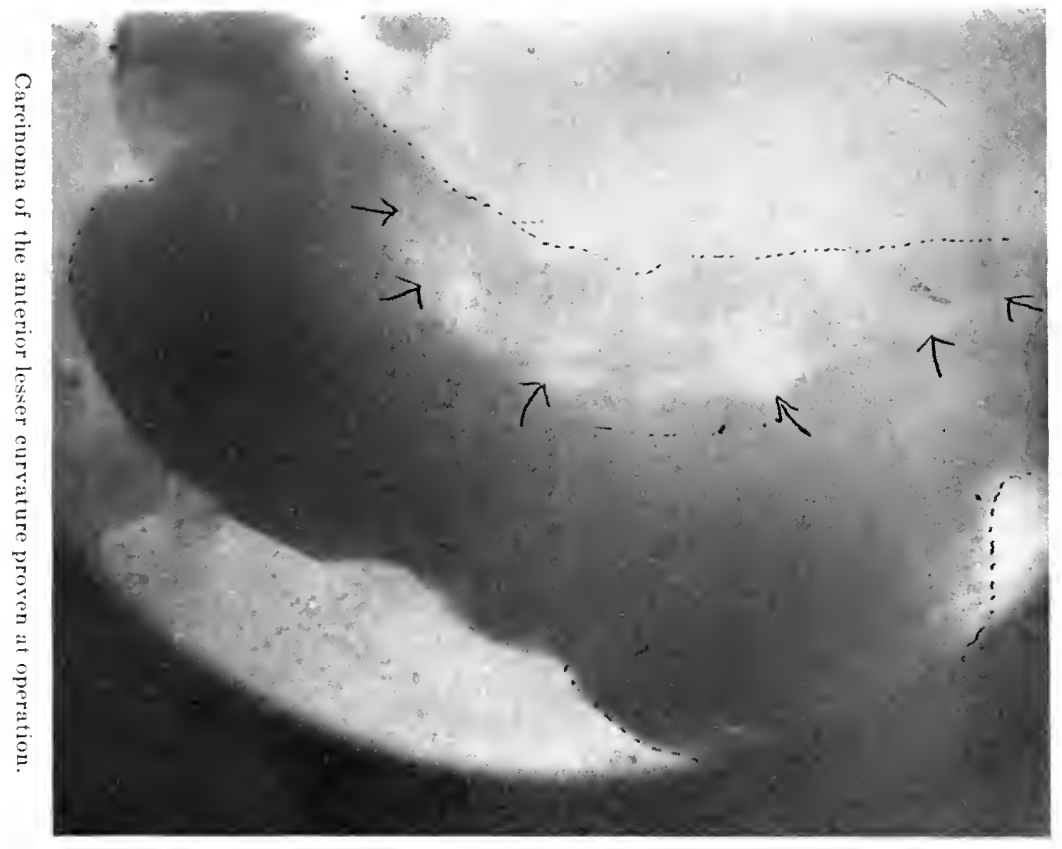

焉

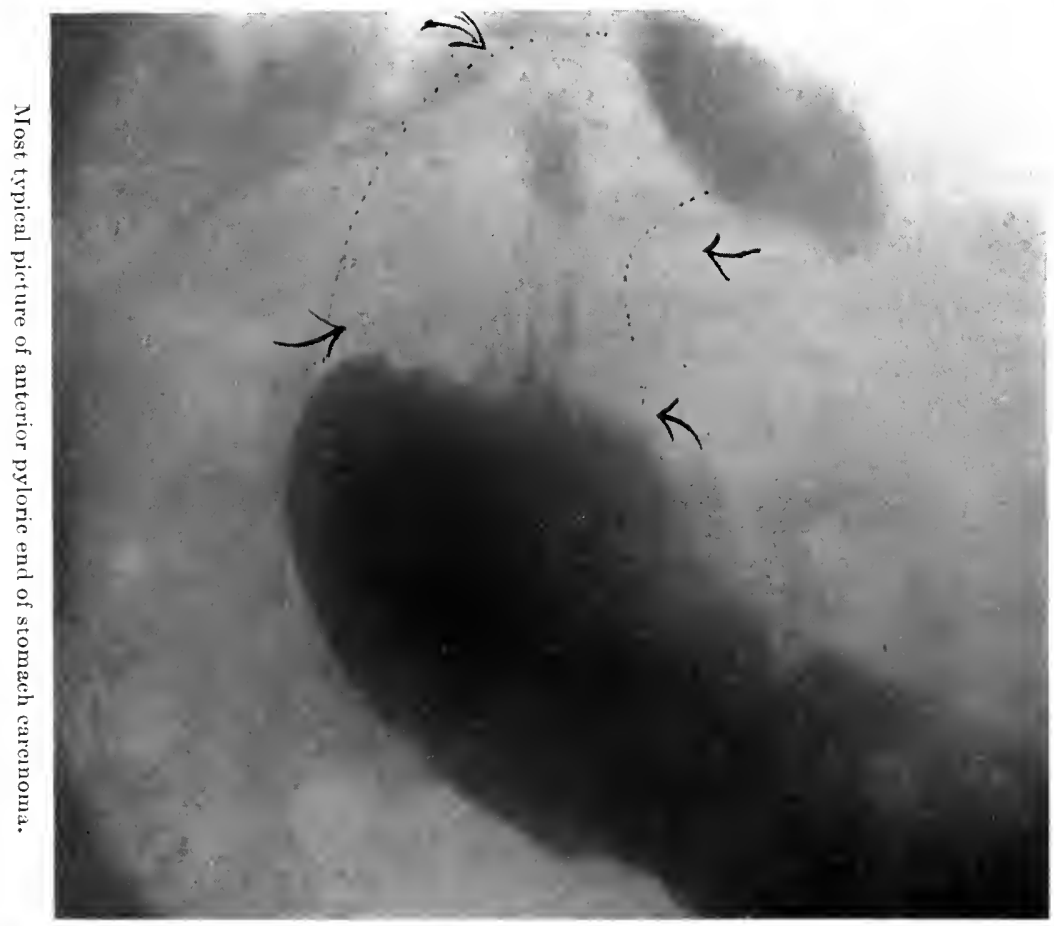



lymphatic vessels extend. The lymphatic vessels from the pars pylorica, which is the most common seat of cancer, pass upward toward the left and downward toward the right. The consequence of this is that the lymphatic chain in the lesser curvature becomes involved early, while the cancer does not spread along the greater curvature on account of the distribution of the lymphatic ducts (Fig. 118). Therefore, in operating for cancer of the pylorus it is necessary to remove the entire lesser curvature, while most of the greater curvature can be preserved. In other words, the resection line is an oblique wound starting high up in the cardial part and ending at the greater curvature considerably to the right. On the distal side of the growth the line of resection is through the duodenum at a point which is distant from the growth.

The two illustrations taken from Moynihan's book on abdominal operations, second edition, show the steps of the operation. The stomach is first drawn out through the median abdominal incision. This, of course, is much easier where the stomach is not bound down by adhesions to other organs. The first step is dividing the attachments of the stomach above and below that part of the stomach which is to be removed. After placing a double ligature, we cut ligamentum gastrohepaticum above and ligamentum gastrocolicum below. Arteria coronaria and arteries gastroduodenalis are carefully tied near the pylorus. We now apply the four long pressure forceps, which should make it possible for us to complete the resection, without allowing any of the stomach contents to escape, either from that part which has to be removed or from the remaining portion of the stomach. Three to four centimetres to the left of the tumor a pair of large, naked surgical forceps is applied. This should be large enough to grip the entire width of the stomach. Four to five centimetres from this, again, similar (but rubber covered) forceps are applied. On the 
duodenum, likewise, two small clamps are applied, the one nearest the pylorus being naked, the other, four centimetres from this, being covered with rubber. The duodenum is now cut between the clamps. Then with a continuous catgut suture (lapis catgut) which passes through all the layers I close the duodenum before removing the rubber-covered forceps, and invaginate the first suture line, using one or two silk sutures. In the same way the stomach

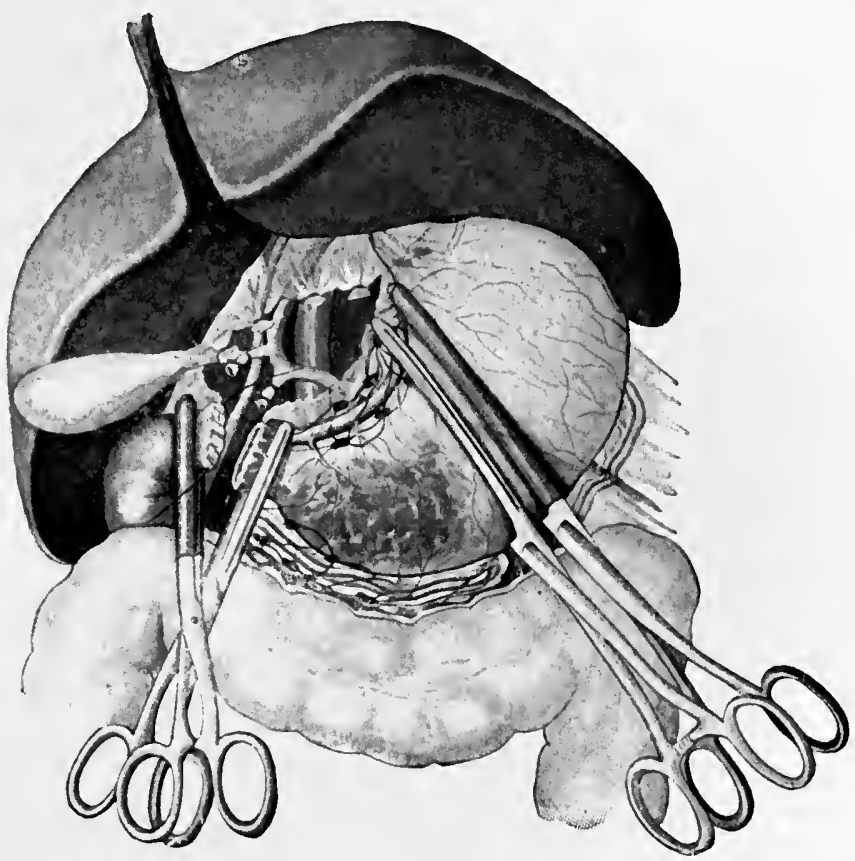

Fig. 119.-Resection of stomach. Duodenum severed between the two forceps and first suture placed in the duodenum.

is cut between the two large forceps, after which the entire diseased part of the stomach, held between the two naked forceps, is removed. Now the remaining portion of the stomach is closed with nitrate of silver catgut before the rubber-covered forceps are removed, after which a strong silk suture is used to close the peritoneum over the first suture line. The duodenum is depressed, and, finally, a 
gastro-entero-anastomosis is performed between the fundus and a coil of the jejunum (see Fig. 120). Sometimes one has to remove far more than is here described. I show you this specimen (Fig. 121), which consists of three-quarters of the stomach plus five centimetres of the duodenum. You notice how the cancer which originated near the pylorus has not only involved two-thirds of the stomach, but has also extended along the duodenum, and you see tumor masses protruding into the lumen.

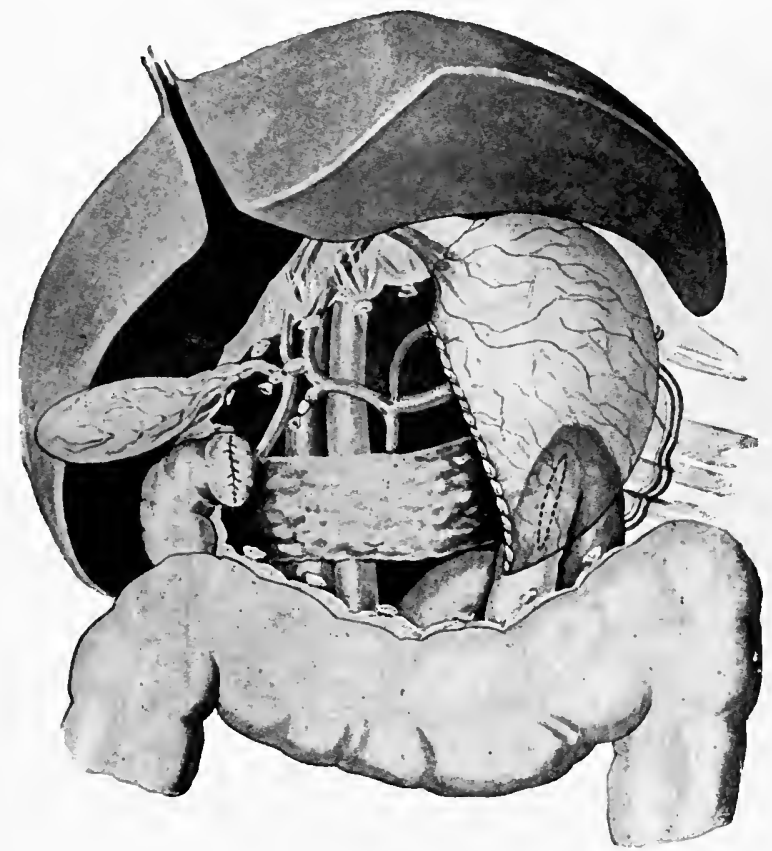

Fia. 120.-Resection of stomach completed. Duodenum depressed. The gastro-entero-anastomosis indicated.

On the other hand, less extensive resections are sometimes sufficient, as in the case which I showed you before (Fig. 105, p. 354), where I removed an adenocarcinoma confined to a portion of the anterior wall, by resecting the anterior wall only. This patient has enjoyed good health for two years since the operation. ${ }^{2}$ In another case I performed

${ }^{2}$ Patient returned later with a recurrence which was inoperable. 
a ring-shaped resection of pars cardiaca for a cancer which, as you see in the illustration (see Fig. 122), involved fourfifths of the circumference. This patient recovered and has now enjoyed good health for two and one-half years.

Finally, I can show you an entire stomach diffusely infiltrated by cancer from the pylorus to within three centimetres of the cardia. Here I performed the very rare operation: a genuine total extirpation of the stomach. ${ }^{3}$ The

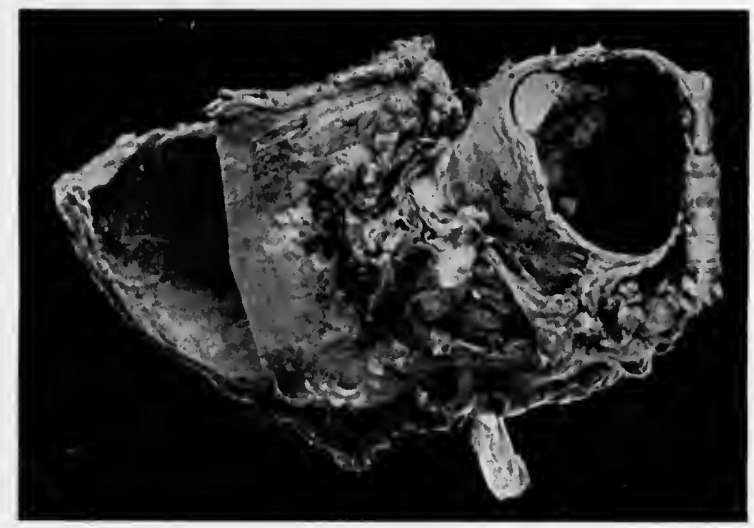

FIG. 121.-Three-quarters of stomach removed together with $5 \mathrm{~cm}$. of duodenum. The cancerous involvement of the duodenum is to the right in the illustration. Personal case.

pylorus as well as the cardia is seen in the specimen. This operation, which took place on October 18, 1900, I performed in the following manner: After I had severed the duodenum and depressed it in the way previously described, the stomach was freed as far up as the diaphragm by successively dividing the ligaments between double ligatures. By pulling strongly on the stomach, the lowest part of the œsophagus could be pulled so far down that I was able to apply a pair of Kocher's intestinal clamps above the cardia. The stomach was then removed. Through a slit in the

${ }^{3}$ Announced in the Medical Society, Fehruary 5, 1891. See Hospitalstidende, Nos. 14, 15, 16, 1901, Contribution to gastric surgery. 
mesocolon, I then drew a coil of the jejunum up to the œsophagus and performed an œsophago-jejuno-anastomosis by incising the jejunum longitudinally on the convex side, and then joined the opening in the intestine to the end of the osophagus by two continuous circular sutures, the inner of catgut, including all layers, and the outer of silk, including only the peritoneum. The after-course was satisfactory, and the result surprising. The extremely debilitated, cachectic patient thrived and developed a good appetite. It was most astonishing to see how well she could digest even solid food without a stomach. This is fully as surprising as it is that a total extirpation can be successfully accomplished. We already knew, however, from Carvalho a $\mathrm{n} \mathrm{d}$ Pachon's experiments upon animals, which were performed in 1893,

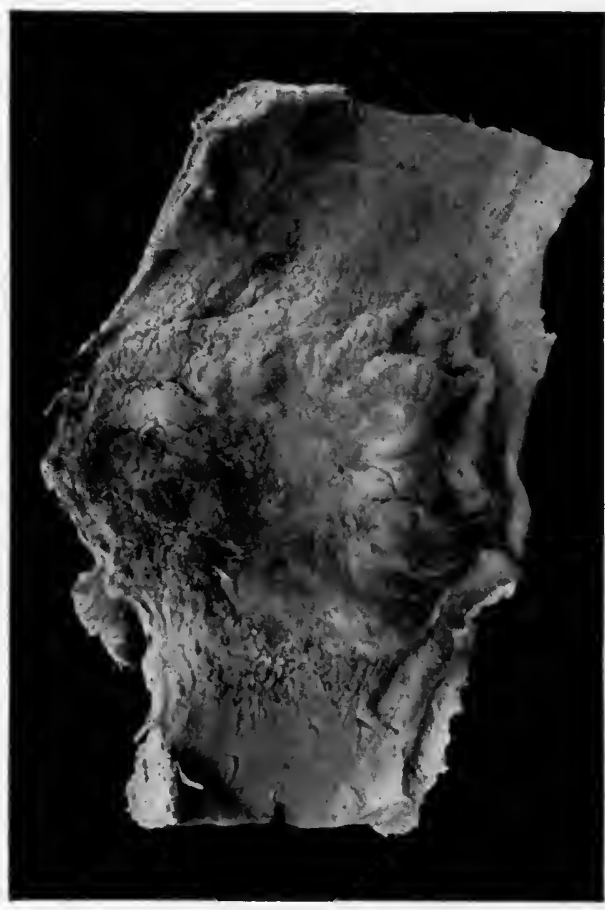

Fig, 122,-Cancer partis cardiacæ ventriculi removed by ring-shaped resection of the stomach. Split open to show extension of tumor. Personal case. that nutrition was possible, because a cat, whose stomach had been removed, recovered its normal weight after a temporary reduction, and was able to digest carbohydrate and proteid matter. The first time that the operation was performed on a human being was September 6, 1897, when Krönlein's assistant, Schlatter, removed a stomach infiltrated with cancer from a woman fifty-six years of age. Here the nourishment took place so satisfactorily 
that two months after the operation she had added eight pounds to her weight. During the first five days my patient was nourished mainly by nutritive enemata, while she was allowed to drink only sterile water; but later she could take milk, gruel, peptonized meat, and porridge by mouth, and on the tenth day began to eat meat, and then was soon put on a full light diet. Before her discharge, six weeks after the operation, she had put on several pounds in weight (she only weighed about one and one-fourth pounds more than when admitted, but it must be remembered that patients always lose several pounds in weight during the preparation for the operation and during the following days). In my case, as in all the other reported successful cases of total extirpation of the stomach, after six months of good health and general improvement rapid emaciation and exhaustion took place, and death occurred, as the result of metastases in the lymphatic glands and other organs.

So far no radical cure has followed total extirpation. It is also true that the majority of cases of resections of the stomach are followed by recurrences, but in both instances a lasting palliative effect has been achieved, which stands in sharp contrast to the unsatisfactory conditions which follow gastro-enterostomy. In the latter case there is only slight improvement in the general condition, because the large cancer masses remain in the organism, while with the radical operations we always have the satisfaction of seeing the patients thrive for a shorter or longer period, free from the original growth; and when death occurs it is less terrible, for the patients themselves usually are not conscious that a recurrence of the disease is in question. If we now ask how many cases have remained free from recurrence-the ideal result of a radical operation-we must admit that our results have not been satisfactory, though by no means inferior to those in other localities. Of the twelve patients treated by resection of the stomach, 
who were discharged as temporarily cured, six died at periods varying from six months to three years. The remaining six are still alive, but in three instances it is too soon to claim a victory, as only one, two, and two and a half years respectively have passed since the operation. The other three, however, we may report as radically recovered cancer patients, if one is ever justified in using such an expression, because now, six, nine, and twelve years respectively after operation, they are apparently in good health. The patient who is quite well after the lapse of twelve years is the man, previously mentioned, from whom I removed the greater part of the stomach on account of an enormous tumor, which consisted chiefly of an ulcer with inflammatory infiltration, but where a small part was a d e $\mathrm{n}$ o carcinomatous.

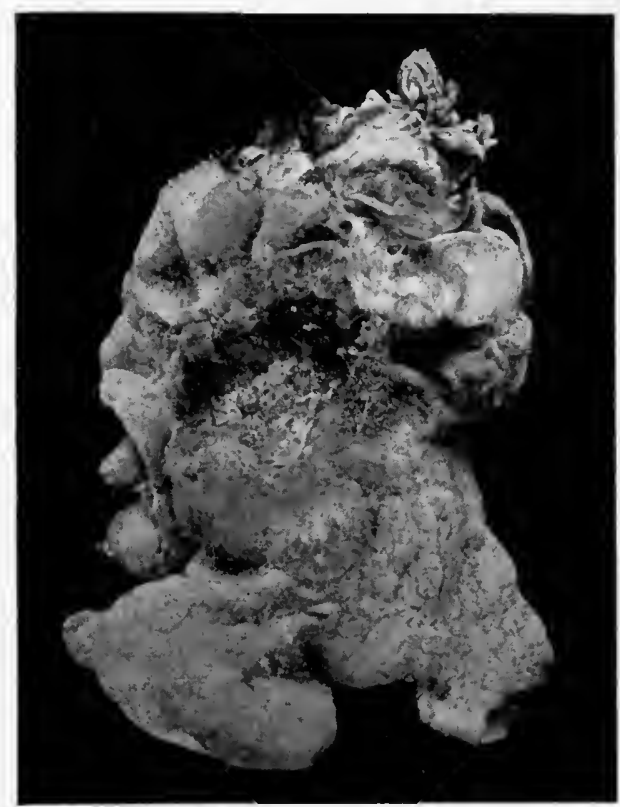

FIG. 123.-Cancer pylori removed by resection. Personal observation.

The other two were women with scirrhous cancer pylori, where there was only distinct metastasis in the glands along the greater curvature-cases which closely resemble clinically the case on which we are about to operate to-day. Everything considered, these cases show that there is a possibility of a radical cure of cancer of the stomach, and when we further consider that all these cases have been very far advanced, and have been brought to the surgeon later than they should have been. We hope that in the future, when 
more attention is paid to exact diagnosis, more patients will come to us in a position to have a radical operation done.

The operation on the patient who was the subject of our last lecture progressed very much as had been expected. The cancer of the pylorus was found, which was larger than we had expected. A tumor always feels smaller than it really is, even when the patient is thin and the mass is easily felt. Fig. 123 shows a specimen which illustrates very well the course of the lymphatic tracts in the stomach. The lymphatic glands along the lesser curvature were already infiltrated by cancer, while along the greater curvature only two glands situated near the pylorus were infiltrated. The anterior lesser curvature was removed.

The resection was accomplished after the method of Billroth, which was recently described to you. The end of the duodenum was closed and a gastro-jejuno-anastomosis was done. As is the case with most patients who have achylia, the patient had considerable fever for the first few days. After the operation this was controlled by the administration of nitrate of silver, and the patient has made a good recovery. 


\section{LECTURE XXII}

\section{PEPTIC ULCER OF THE JEJUNUM-TECHNIC OF GASTRO-ENTEROSTOMY}

During the course of these lectures we have often spoken of the gastro-enterostomy, the indications and contra-indications for its employment, of the benefit which we often reap from this operation, and of the dangers and drawbacks which attend it. One of the most serious dangers attending this operation is the possibility of the development of an ulcus pepticum. We have a patient at present in the ward in whom this complication occurred and whom I wish you to see before he is discharged. His history is as follows:

$\mathrm{He}$ is a farmer, thirty-six years of age, who was admitted to this ward two years ago with the diagnosis of appendicitis. He had then suffered for some time from attacks of violent pain in the right side of the abdomen, which pain was generally accompanied by nausea and vomiting. On further examination it seemed that the pain was situated high up in the right side, close below the rib curvature; there was also some sensitiveness to pressure, while there was no pain over MacBurney's point, either upon direct or indirect pressure. A functional test of the stomach showed that there was considerable retention, both on fasting and ten hours after a Bourget's test-meal. The gastric juice showed a high degree of acidity. I diagnosed a contracting ulcer, which I imagined to be in the upper part of the duodenum, judging by the character of the pain. At the operation this diagnosis proved to be correct. We found the stomach greatly dilated but otherwise normal, and the pylorus so dilated that one could easily insert two fingers into it. On the other hand, we found the duodenum con- 
tracted, due to the presence of an ulcer the size of a shilling, while the inflammation had spread to the peritoneal covering and had cemented the duodenum to the surrounding tissues. A radical operation was, therefore, deemed inadvisable, and I contented myself with a gastro-enteroanastomosis after Wölfler's method, using the anterior wall of the stomach, supplementing it with an entero-anastomosis ad modum Braum, in order to insure the patient against a circulus vitiosus.

The after-course was quite ideal, and the patient quickly recovered, added twenty-two pounds to his weight in three months, and was perfectly well until about a month before his return, when he suddenly had an attack of violent pain in the right side of the epigastrium and in the right hypochondrium. These attacks were repeated with increased frequency and varying strength. There was some fever from time to time, and during the attacks he could take no food, but in the interim the food did not seem to inconvenience him. He again became greatly emaciated, and was admitted here three weeks ago in a very deplorable condition, complaining of pains in the right side of the epigastrium. When examined he was found to be very sensitive to pressure over the entire region between the right rib curvature and linea media, and whenever I attempted to palpate he contracted musculus rectus so violently that I was unable to feel thoroughly in the depth. By palpating very carefully and gently I succeeded in making out a diffuse distention corresponding to the sensitive portion. It was quite evident to me that here we had before us a perforating ulcer, but the most important question was, whether it was the old ulcus duodeni, which had eaten its way farther along, in spite of the gastro-enterostomy, or if we were here confronted with an ulcus pepticum jejuni, which is greatly feared when it follows.

This lesion consists of a terrace-shaped ulcer, greatly 
resembling the circular stomach ulcer, which, sooner or later after a gastro-enterostomy, develops at or a few centimetres from the anastomosis in the jejunum. This spot otherwise is never the seat of ulcus simplex. As these ulcers only occur after gastro-enterostomy on patients with normal gastric juice or hyper-hydrochloric acidity, and, on the other hand, never among patients with achylia, we naturally supposed that these ulcers are due to some digesting effect of the gastric juice on a portion of the intestinal mucous membrane which has been injured in some way or another, and which is, therefore, less capable of resistance. Probably this ulcer forms rapidly after the operation of anastomosis as a result of a lesion of the mucous membrane caused during the operation, and may be symptomless until it reaches the peritoneum or perforates, causing peritonitis -local or diffuse.

Jorgen Jensen, who has lately (Hospitalstidende, Nos. 47 and 48,1908 ) published the account of two cases here in Denmark operated on by Kraft and Pers, has been able to collect from the literature fifty-eight cases, all of which have been perforated ulcers. Therefore we do not know how frequent the disease is, how frequently such ulcers heal spontaneously, or how often they may possibly exist without perforating. It is remarkable that this drawback to gastro-enterostomy was first noticed eighteen years after the performance of the first gastro-enterostomy, when Braun in 1899 reported the first case of diffuse perforative peritonitis resulting from ulcus pepticum jejuni. The perforation, which has been known to occur from a few weeks to seven years after the operation of anastomosis, presents various symptoms, dependent on whether the ulcer perforates freely into the abdominal cavity, or is limited by adhesions and causes only a local peritonitis.

In the first case we at once have a diffuse peritonitis and a rapid fatal progress, unless a successful surgical en- 
croachment is promptly undertaken. If, on the other hand, the perforation is temporarily confined, the ulcer perforates into a shut-off portion of the abdominal cavity, where an abscess forms, which generally spreads upward and becomes subphrenic; the most important factor then is to make the right diagnosis, and to operate before the abscess breaks its boundaries and invades the peritoneal cavity, causing a dangerous diffuse peritonitis. In this case the diagnosis lay between ulcus pepticum perforans, as mentioned above, and a perforation of the old duodenal ulcer. The location of the pain on the right side spoke in favor of the latter, as did also the fact that examination of the stomach showed no retention. The patient had, however, been free from the old symptoms of the duodenal ulcer since the operation, and this fact favored the theory of ulcus pepticum. In order to obtain a sufficient access to the duodenum, I made use of a bayonet-shaped incision, as you see here on the cicatrix. I began in the centre line close under processus ensiformis, but six centimetres down. I continued the incision obliquely across the left musculus rectus, in order to continue it longitudinally along the outer edge of this. After I had opened the peritoneal cavity, I came upon an abscess surrounded by a quantity of adhesions, which cemented the intestines partly to one another, partly to the stomach, and partly up to the liver. To find my bearings, I was obliged to loosen all these adherences cautiously, while I naturally absorbed and wiped away the contents of the abscess most carefully. It then appeared that we were faced by an ulcus pepticum, which had perforated just below the anastomosis and had formed an enclosed abscess, the upper boundary of which was the liver, on the under side of which the abscess had formed a cupshaped hollow, while the remaining walls consisted of accreted intestinal coils. I excised the remains of the ulcer, and then passed my finger into the stomach and on through 
the pylorus into the duodenum. It appeared that our gastroentero-anastomosis had so far been extremely successful in its effect, inasmuch as the duodenal ulcer had healed completely, with a smooth, fibrous cicatrix. I had nothing else to do then but to rejoin the jejunum to the stomach at the point corresponding with the perforation. I then drained the abscess cavity with a cigarette drain (nitrate of silver gauze wrapped in oilsilk), which was. led out beneath the edge of the liver, while the whole laparotomy wound was joined in the usual manner. The progress of the wound has been good. The cigarette drain acted well, and could be removed on the eighth day. The rest of the wound healed per primam intentionem.

Since the operation the patient has been entirely freed from his pains. One naturally asks one's self: Will not the ulcus pepticum recur? Of course we cannot be quite certain that it will not, but we have tried to prevent it by administering from the moment of operation bicarbonate of soda in large doses to neutralize the acid in the gastric juice, and we have instructed the patient to continue to do this at his home.

Far more serious still, of course, is the prognosis with the second and, fortunately, smaller group of cases, where the perforation takes place acutely into the free peritoneal cavity. Most of these patients die because they never come under surgical treatment at all, and even of those who are brought for operation quickly and with a correct diagnosis many do not recover.

This is, then, a very serious result of the gastro-enteroanastomosis, and calls for the greatest possible restriction in the employment of the operation in cases of gastric ulcer and other diseases of the stomach where the gastric juice is normal, but which must also lead to a revision of the technic of the operation itself, which seemed to have reached perfection just at that time when the surgeons 
realized this new danger. A lively discussion arose and great depression was caused when one had a suspicion that those devices by which one had thought to avoid the hitherto

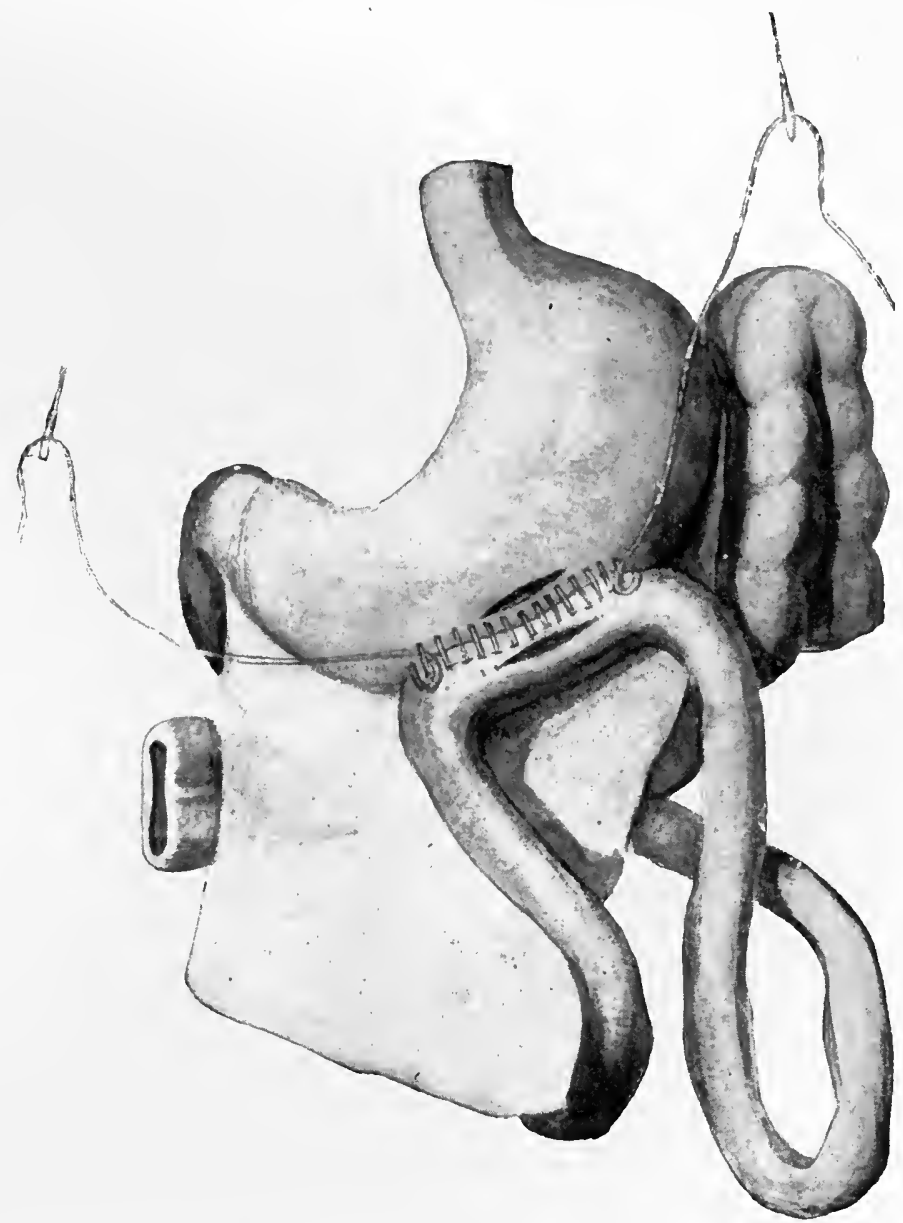

Fig. 124.-Wölflers gastro-enterostomy. Hartmann's suture method. First step.

known dangers incurred fresh risk for the patient by favoring the development of ulcus pepticum.

In order that you may understand this rightly, I will review with you the various methods of gastro-enterostomy and the general technic of the operation. 
The first gastro-enterostomy, performed by Wölfler in 1881, was the later so-called gastro-enterostomia antecolica anterior, by which a coil of the jejunum forty to fifty centimetres from its source in fossa duodeno-jejunalis is brought up in front of the omentum and colon transversum and joined to the stomach close above the greater curvature

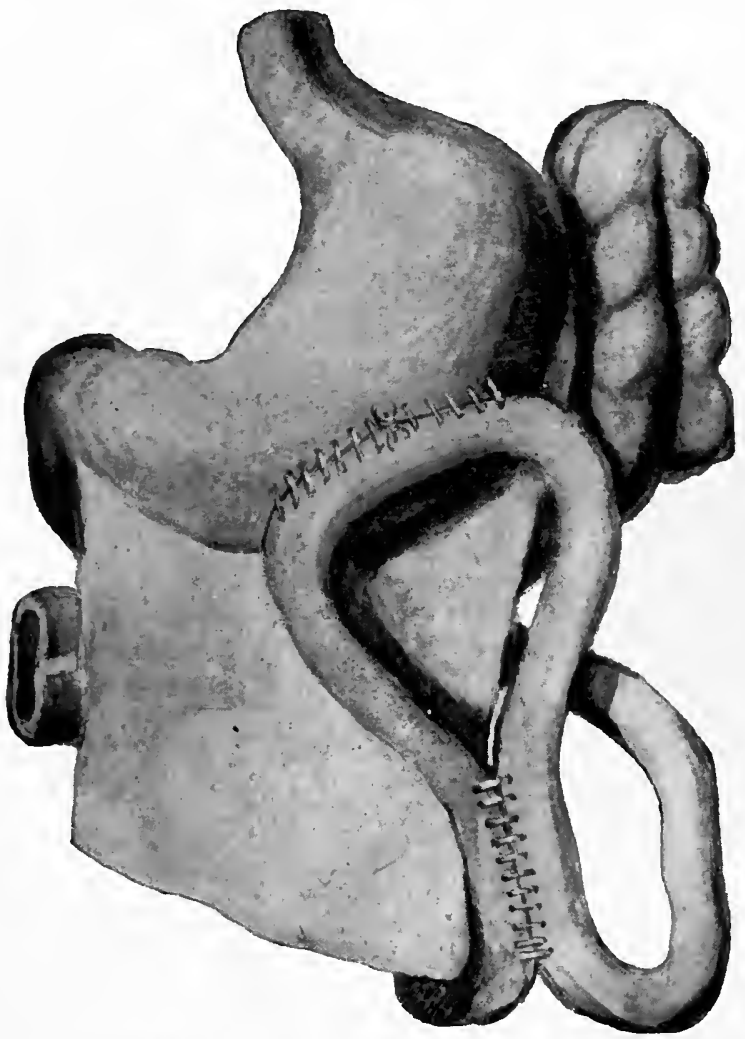

FrG. 125.-Wölflers gastro-enterostomy, second step, and Braun's entero-anastomosis.

(see Figs. 124 and 125) in such a manner that the direction of the intestine is isoperistaltic with that of the stomach; that is to say, that the supplying leg of the intestinal coil lies to the left, and the discharging one to the right. It soon became clear that otherwise the so-called circulus vitiosus occurs very easily, partly by the stomach driving the food 
into the admitting tube, and partly by the passage through the emitting tube being hindered by the twists and bends caused by the unnatural direction in which the intestine is sewn. But, even where these rules were carefully observed, one sometimes saw after the operation circulus vitiosus occurring with vomiting of bile,-in short, ileus of the stomach. One first attempted to remedy this by small technical alterations. One attached the intestinal coil broadly to the stomach on a considerably longer portion than that to which the anastomosis was confined, in order

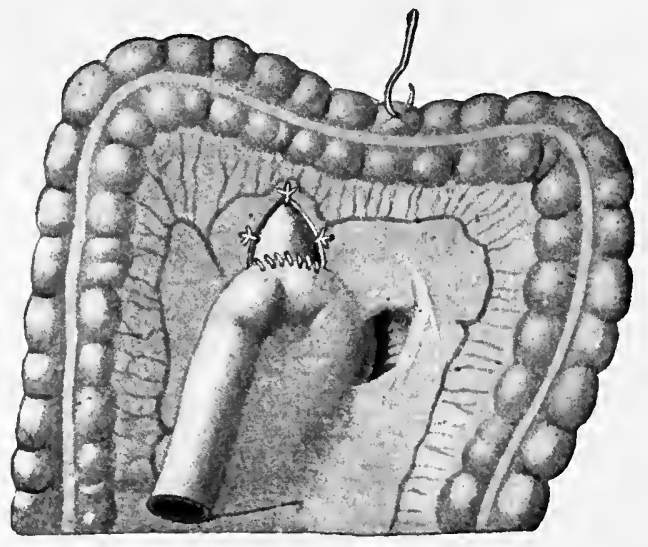

FIG. 126.-Von Hacker's gastro-enterostomia retrocolica.

thus to prevent bends. Kocher took it into his head to make the incision in the stomach and intestine bow-shaped, so that a kind of flap was formed, which was to prevent the contents of the stomach from returning to the supplying part of the jejunum. This last modification was quickly discarded, as it appeared that the flap was not stable.

The more radical modification introduced by von Hacker reeived far greater sympathy. He made (see Fig. 126) the anastomosis on the posterior side of the stomach, while he obtained entrance to this by raising the colon and the omentum, and then incised the mesocolon. When a sufficiently large portion of the stomach is exposed it is necessary to sew the edge of the incision in the mesocolon to the serosa of the stomach, so that the anastomosis shall not be squeezed in this opening later on. There should be essentially two advantages with this procedure: first, that 
the discharge of the stomach ought to take place far more quickly and safely through this anastomosis, which is situated in a more declivitous portion of the stomach than that of Wölfler's; and, secondly, that one is spared the long coil of the jejunum, because, as you see in the picture, one here makes the anastomosis a few centimetres from fossa duodeno-jejunalis without any coil-formation whatever. This reasoning really appeared very seductive and enticing, and there was a great turn of the tide in favor of von Hacker's operation. I myself was among those who were at once attracted by the method, and tried it in a series of eases; but I quickly discarded it, greatly disappointed in its results. The result was that the contents of the stomach did not empty themselves more quickly into the intestine, but the intestinal contents had the most inconvenient inclination to enter the stomach. A number of these patients had vomiting of bile, especially in the morning, because the stomach became almost filled with bile during the night, and those who did not suffer from vomiting had nausea and indisposition, which proved always to be due to an influx of bile into the stomach. Moreover, I, who have never seen circulus vitiosus with Wölfler's method, experienced two such cases with von Hacker's, and thereby saw the great drawback with this operation: that the anastomosis is extremely difficult of access as compared with Wölfler's when such a dire complication necessitates a re-laparatomy. After having attempted von Hacker's operation some half-dozen times, I returned, like so many other surgeons, to Wölfler's.

As von Hacker's modification had thus disappointed the expectations with regard to the only great danger of the gastro-enterostomy then known-circulus vitiosus-one endeavored to avoid the danger in some other way; namely, by leading the contents of the supplying intestine into the discharging one at some distance from the gastro-anasto- 
mosis. The Swiss surgeon, Roux, and the German surgeon, Braun, have solved this problem in the following two rather different fashions.

Roux, whose method is especially intended for gastroenterostomia-posterior, even though it can be used with anterior as well, is called the $\mathrm{Y}$-shaped entero-anastomosis, and will be quickly comprehended by a glance at these illustrations (Figs 127 and 128). About ten centimetres below

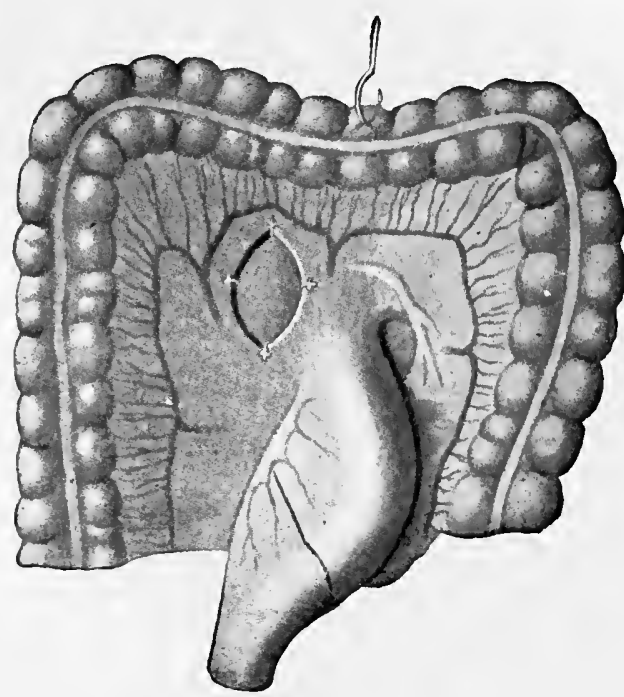

Fig. 127.-Roux's Y-entero-anastomosis. First step. fossa duodeno-jejunalis he cuts through the jejunum and elongates the incision down in the mesentery, but not far enough to sever the nourishing arterial arch. He then implants the peripheral end of the jejunuminto the stomach, and afterwards plants the central end of the jejunum into the peripheral end somewhat below the gastro-anastomosis- "end to side," as the Englishman says. The slit in the mesentery also is carefully stitched up.

The other method, Braun's entero-anastomosis (see Fig. 125), is far less complicated, because here "side to side" one simply makes an anastomosis between the two legs of the coil of the jejunum at a distance of about ten centimetres from the gastro-entero-anastomosis. Besides being far less complicated and more quickly performed than Roux's, this operation seems to me to offer double security for the passage, because the contents from the supplying 
leg have two routes to the discharging leg. It really seems, also, to be quite an ideal method for avoiding circulus vitiosus. With the two hundred gastro-enterostomies where I have employed Braun's entero-anastomosis I have never experienced circulus vitiosus.

But the joy over Roux's and Braun's methods was greatly dampened by the discovery that ulcus pepticum jejuni constitutes a somewhat frequent and very dangerous result of the gastro-enterostomy, because it must at once be clear to us that the diversion of the bile from the upper part of the intestinal coil must favor the development of a peptic ulcer, because the hydrochloric acid secretion of the stomach then has free play in this part of the intestine. It is true Kocher thinks that, even where there is no entero-anastomosis, a sort of sphineter always forms in

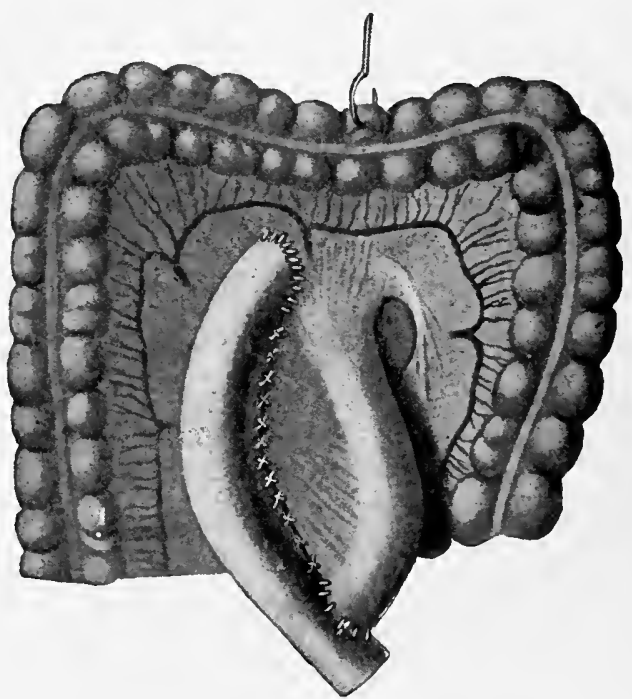

FIg. 128.-Roux's Y-shaped entero-anastomosis. Second step.

the jejunum by muscular contraction, a little below the anastomosis, so that the upper part must be exposed often and long to the effect of the gastric juice. This theory, however, is not wholly convincing when one remembers how frequently before the use of the entero-anastomosis one used to find a constant influx of bile into the stomach, which neutralized the gastric juice. Undoubtedly, the want of bile in the upper portion of the intestine must favor the development of ulcus pepticum, if the other conditions for its development are present. 
The question then is: Shall we dispense with Roux and Braun's operations which insure us against circulus vitiosus, or risk this in order to diminish the danger of ulcus pepticum? I have no doubt that Roux's operation should be consigned to oblivion, and Roux himself seems to have given it up just on account of this danger. On the other hand, I think the employment of Braun's anastomosis should be retained in its present form as a necessary security against circulus vitiosus, and as the entero-anastomosis which is least disposed to peptic ulcer. The fact is that, while Roux leads the biliary secretion entirely away from the upper ten centimetres of the intestine communicating with the stomach, with Braun's operation the alkaline intestinal contents, when occurring in fair abundance, can also escape upward and pass the gastro-anastomosis. That this happens in fact I have been able to substantiate on several occasions by probe examination of the stomach contents. There is every reason to believe that, when the patient lies down, especially of an evening and at night, when the production of bile is specially active, the parts nearest to the stomach and the gastro-enterostomy wound itself will be moistened by bile. Therefore I believe that Braun's anastomosis is as far from favoring the development of ulcus pepticum as Roux's operation is. We cannot, however, altogether reason ourselves away from the fact that the entero-anastomosis involves some risk of peptic jejunal ulcer, and it is, therefore, important, if this calamity occurs, to have easy access to the anastomosis. It is my opinion, therefore, that one ought to abandon von Hacker's gastro-enterostomia-posterior, because if, after this, an ulcus pepticum develops, the intestinal and stomach contents will, in the event of free perforation, be dispersed diffusely between the coils of the small intestine, and, under the more favorable circumstances, where there is accretion and abscess formation it will generally be diagnosed too 
late, because it lies so deep. In all circumstances an operation-whether in the presence of an acute perforation or a more chronic progress-will be far more difficult than with Wölfler's anastomosis, which lies close to the anterior abdominal wall, and is confined at the back, to a great extent, by the omentum, whereby not only are adherence formation and decapsulation favored, but diagnosis and treatment are equally facilitated. Just think how difficult the operation would have been with our patient, and how difficult, not to say impossible, would have been the problem, which we have so successfully solved, of draining the abscess effectively, if this had been situated belind the stomach, colon, and omentum. There are many who still hold fast to von Hacker's operation, in the belief that the emptying of the stomach should take place quickly-immediately after the food has entered the stomach, so to speak. In this way Pers and Fisher are of the opinion that they have observed, by means of Röntgen rays, that bismuth-porridge disappears in the intestine through the anastomosis a few minutes after it is swallowed. As Pers's results are opposed to previous investigations of the same kind, especially those of Blake and Cannon (see p. 248), who have occupied themselves with experimental investigations as to the conditions of discharge after gastro-entero-anastomosis, the future must prove whether Pers's investigations are valid and on what the incongruity rests. But, all things considered, it appears to me very doubtful whether it can be termed an advantage with von Hacker's operation that the stomach empties its contents so quickly into the intestine, because thereby the food would be deprived of physiological treatment in the stomach, which otherwise takes hours, and the sour gastric juice, having no food to act upon, will then attack with increased strength the stomach ulcer, for which one wishes to procure peace by means of the gastro-enterostomy. With our Wölfler gastro-enterostomies the stom- 
ach empties itself much more slowly, as Blad has proved, and $I$ think this is an advantage.

But, in my opinion, in addition to the placing of the gastro-entero-anastomosis, and the question of enteroanastomosis, there are other little heeded points in the technic of the operation which are of importance for the pathogeny of the peptic jejunal ulcer. It is quite clear that we cannot have such an ulcer solely because a little hydrochloric gastric juice comes in contact with the mucous membrane of the jejunum. The presence of a lesion or a reduced vitality of the mucous membrane is required for the gastric juice to produce an ulcer. In my opinion, one should here think, above all, of (1) deficient hæmastasis, and consequent suggilations and lımatomas in mucosa and submucosa, and (2) of necrosis from pressure. In my opinion both these are not unfrequently the result of the closing off the stomach and the intestine by using forceps during the performance of gastro-enterostomy as practised at present by most surgeons. These clamps grip so tightly that they partly act as hæmastats and partly block off completely the intestine and stomach contents.

Hereby two very great advantages are apparently attained, but back of these two great dangers are concealed. When one does not see any vessel bleeding during the suturing no hæmastasis is performed, and one closes the entire wound without knowing whether the suture has stopped all bleeding. The reason why I discarded the use of clamps while doing a gastro-enterostomy was on account of the danger of bleeding. Secondary hemorrhage nearly carried off a young lady on whom I had performed gastro-enteroanastomosis for gastric ulcer. We only just succeeded in saving her by reopening the abdomen and finding that a spurting artery had penetrated the lumen of the intestine between two suture stitches, and had quietly filled most of the intestinal canal with blood, causing an extremely 
dangerous anæmia. The other danger is that the strong and protracted pressure of the clamp may cause necrosis of the mucous membrane, with bruises and contusions which may become the source of a peptic ulceration.

In performing gastro-enterostomy, therefore, I never use clamps to close off the intestine and stomach, but freely incise these, after having first joined their peritoneal surfaces posteriorly. One then has the advantage of seeing where the bleeding is and can accomplish an exact hæmastasis with the aid of small, suitable péans and catgut ligature. The dreaded effusion of intestinal and stomach contents is minimized, as a rule, in well-prepared patients; but with ulcer patients, with an abundance of hydrochloric acid secretion, the contents are sterile, and their effusion, therefore, involves no danger of infection and peritonitis. Only where there is achylia and gastritis is there danger of infection, and here there might be cause for closing off the intestine and stomach with rubber-covered clamps; all the more so because there is no danger of ulcus pepticum developing. But it is not necessary for one who employs Wölfler's method, because, after having previously protected the peritoneal cavity with gauze, one can almost always draw the stomach and jejunum outside the wound. For the rest, I think that, with the after-treatment of a gastro-enterostomy, one can do much to prevent the development of ulcus pepticum when, by administering bicarbonate of soda and ordering a suitable diet, one takes care to neutralize the hydrochloric acid in the gastric juice.

As I have trenched so much on the technic of the gastroenterostomy on account of this ulcus pepticum, I shall conclude by showing you the method by which we perform anastomosis with suture here in this ward. I employ the double circular suture indicated by the French surgeon, Hartmann (see Figs. 124, 125; pp. 428, 429), which I will 
recommend to you because it is quick and safe. I commence in fossa duodeno-jejunalis by drawing forth with two fingers the beginning of the jejunum, which is easily distinguished because it is fixed. Fifty centimetres from the source of the intestine I now pull the jejunum forth in front of the omentum and colon, and place it close above the curvature, while for the present I fix the serosa of the intestine at each end to that of the stomach with the small forceps suggested by Rutherford Morison. While an assistant draws these forceps out to each side, the peritoneal surfaces of the intestine and the stomach lie smoothly against each other, and are then easily sutured from one forceps to the other with continuous silk thread. The thread is now tied temporarily, after which the two ends, with their respective needles, are wrapped carefully in gauze. The stomach and intestine are then incised one-half centimetre from the peritoneal suture, and in such a manner that the incisions on both sides stop within two centimetres of the end of the peritoneal suture. The bleeding vessels are caught and tied, and the anastomosis is now sutured the whole way round with nitrate of silver catgut, comprising all layers. The suture is continuous. As soon as the two lumina have been thus joined, the silk thread, with its needles, is unwrapped, and the serosa suture is now continued in front of the anastomosis until the circle is completed. I emphasize the employment of catgut for the innermost suture because here, where there is a possibility of infection, the suture material should be resorbent, so as not to retain the infectious material. The outer suture, however, is of silk, in order to secure the joining until this becomes firm. One performs entero-anastomosis on quite the same principle. The Murphy button, which was at one time the universal method for forming an anastomosis, is now used so seldom that possibly you have never seen one, nor know how it is employed. I shall, therefore, show you this in- 
geniously-devised instrument, which is too valuable to be entirely consigned to oblivion or to vanish from our armamentarium. You see (Fig. 129) that it consists of a metal button in two halves, a male and a female part, which can be pushed close together by a simple pressure at the ends, but which, thanks to two small barbs on the male half, can only be unscrewed by a number of revolutions, because the two barbs work into a long worm inside the female part. There is quite a considerable lumen in the centre, but, besides, the buttons are perforated for drainage.

The mode of application is as follows: After having

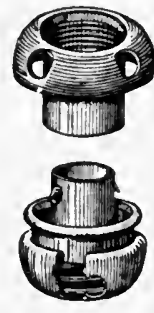

FIG. 129.

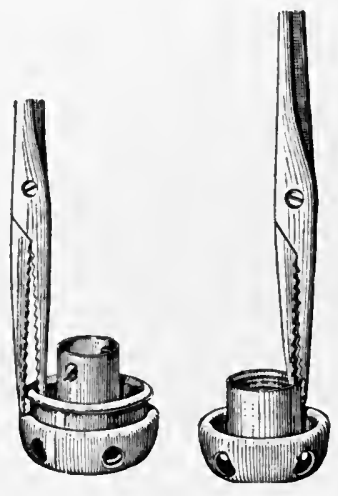

FrG. 130.

made an incision in the stomach and the intestine just large enough to allow the button to pass, you seize each half of the button with squeezing forceps (see Fig. 130) and pass them into the stomach and the intestine respectively, and, while an assistant holds them fixed by means of the forceps, you sew the wall of the stomach and that of the intestine together with a tobacco-pouch suture round the neck of the button, so that by pushing the halves together the wall of the stomach and the wall of the intestine with the serosa surfaces are pressed together between the broad surfaces of the button. In order that the pressure shall not be too strong and gangrene occur too quickly, the button is pro- 
vided with an elastic rim; when, in the course of six to eight days, the necrosis is accomplished, the idea is that the button should leave the body through the intestinal canal. What brought the Murphy button into discredit after it had proved a success was the fact that its manufacture, which was so excellent to begin with, passed from American hands to German hands, and became cheaper but very unreliable, so that one could no longer rely upon the two parts holding fast, or on the gangrene occurring sufficiently slowly. In the next place, there was the fact that, instead of passing out through the intestine, the button in a number of cases remained lying in the stomach, generally, it is true, without causing serious harm. Another most unpleasant occurrence is when the button remains fast in situ. Then it may long afterward cause ileus, either because the passage in the button is rendered futile by solid deposits or by the button, turning crosswise in the intestine, becoming fixed to a portion of the wall. In this way I have had a patient with ileus years after a gastro-enterostomy, and found the cause to be the Murphy button wearing out the intestine in this manner. I removed it by a simple incision and the patient recovered. In spite of all this, the Murphy button is an admirable and, in certain cases, invaluable instrument. When you have made a large and protracted resection of the stomach, and have yet to perform a gastro-enterostomy on the extremely exhausted patient, the use of the Murphy button may save you twenty most valuable minutes. On the whole, with cancer patients who are greatly run down, one should often make use of the Murphy button, which enables one to complete the operation in a few minutes. 


\section{LECTURE XXIII}

\section{ECHINOCOCCUS OF THE LIVER}

I sHALL speak to you to-day about a patient who is well adapted to supplement those cases which I have lately made the subject of my lectures on abdominal surgery. She is a married woman, about thirty-nine years of age, sallow and thin, with anxious mien. She has been admitted here for examination and eventual operation for a tumor in the epigastrium. When you see the patient's uncovered abdomen you cannot at first observe any tumor, in spite of the very thin abdominal wall. The epigastrium seems sunken, as compared with the convex subumbilical part of the abdomen. You also see and feel the pulsation in the epigastrium, and, as I have told you, ought at once to suspect gastroptosis, a suspicion which is confirmed on further investigation, because the scraping auscultation shows that the greater curvature lies twelve centimetres and the lesser curvature six centimetres below the umbilicus. A simple palpation shows the abdomen everywhere soft, and by such a superficial examination nothing is felt of the tumor for which the patient has been admitted. But if you will regard the epigastrium when I let the patient inspire deeply you will see a roundish tumor about the size of an orange protruding in the epigastrium from under the left rib curvature, behind which it disappears again during expiration. Perhaps you remember that, at our last lecture but one, we had a patient who resembled this one, inasmuch as she was a woman of the same age, with severe gastroptosis, and, like this one, greatly emaciated. In that patient, also, upon deep inspirations you saw a tumor protruding in the epigastrium, but that tumor appeared under the right rib curvature. It was the size of a hen's egg, solid, slightly irregular, 
and very mobile. It proved to be a cancer pylori as was supposed, and was removed by resection of the stomach. It is not unnatural, then, to think that possibly we are dealing with a tumor of the stomach in this case, but, if so, we must not think of the pylorus, but rather of the cardial part of the stomach or the fundus as the seat of the tumor, which here protrudes from the opposite side. If we now feel the tumor while it is protruding, it appears in this case to have a smooth surface, and impresses one as being tense and elastic. It is, however, impossible to judge accurately a tumor which retreats up behind the wall of the thorax when pressed upon. The point is to fix it, but this can only be attained by asking the patient to hold her breath after the tumor has been forced out by a full inspiration. You see that the tumor now remains, and while I palpate I distinctly feel fluctuation. This discovery would tend to lessen the possibility of cancer of the stomach, even if it does not exclude the idea of the tumor belonging to the stomach. For instance, we might suppose it to be an hour-glass stomach, the upper part of which is so dilated with fluid that it presents itself like a large cyst. One might also think of a sub-diaphragmatic abscess originating from a perforating ulcer of the stomach, but the smooth surface and great mobility of the tumor do not fit in with this. Such an abscess would be fixed between the stomach and peritoneum parietale by broad adherences. One might think possibly of a cystic tumor which had developed in the wall of the stomach itself. Everything considered, we must try to determine whether this tumor has anything to do with the stomach or not. If we question the patient with regard to stomach symptoms, she states that she has had pricking and oppressive pains in the region of the cardia since she was sixteen years of age; even then she thought at times that she could feel a distention. But these attacks seem to have been quite independent of her meals, and she has not suffered from 
nausea, eructations, or vomiting, nor has any melæna been observed. When we introduce a stomach-tube into the stomach it appears that there is no retention, and when the stomach is inflated the tumor does not change its position with the wall of the stomach, but can be palpated in front of it. This finally convinces us that the tumor has nothing to do with the stomach, because retention in an hourglass stomach is at once excluded, and a tumor in the anterior wall of the stomach would be drawn downward by the inflation of the stomach and become more palpable, while a tumor pertaining to the posterior wall of the stomach would disappear entirely. Now, however, we feel the tumor lying unaffected above and in front of the stomach. We must think of a cystic tumor in one of the organs bordering on the stomach. Here we must first think of the liver, the spleen, and the pancreas, all of which may be the seat of cystic tumors, and, secondly, of the left kidney, which may thrust itself up here in between the stomach and the pancreas.

In this way I once extirpated a hydronephrotic kidney which had coalesced so intimately with the pancreas that cauda pancreatis had to be extirpated together with the tumor (published by H. Koster in Hospitalstidende, 1901, as a contribution to "Fat-necrosis with Pancreatic Lesions"'). In this case, however, I think I can decidedly exclude hydronephrosis, as the tumor so evidently comes from above, and does not extend deeply into the lumbar region. I think I may also exclude a pancreatic eyst. It is true that a pancreatic cyst can appear above the stomach, especially when, as in this case, there is gastroptosis; but then it would appear either in the median line just above the lesser curvature, or, if it should be to the left, as in this case, it should be behind the stomach, whereas this lies in front. Finally, the fact that it moves with respiration does not fit in with a tumor in the pancreas, but rather with 
one in the liver or the spleen, both of which lie just under the diaphragm. In both these organs we may have cystic tumors, but here, I think, we may disregard the spleen, because there is a distinct space between the tumor dulness and the splenic dulness on percussion. I therefore think that this tumor pertains to the liver, and, to be more exact, to the left lobe of the liver. The question then remains, what cystic tumors are found in the liver? We can, as in other glandular organs, have simple retention cyst, and we may have genuine, eystic new formations. For instance, we know of a very peculiar cystic degeneration of the liver which occurs with cystic degeneration of the kidney, but here, as a rule, so far as the liver is concerned, it is a question of multiple, scattered, small cysts. In the next place, we may also have cystic degeneration of malignant tumors, cystic sarcomata, or cystic carcinomata. Finally, we may have abscesses in the liver which may develop either from a canalicular infection, which occurs as a result of blocking the gall-duct with gall-stones, or with simple infectious cholangitis, or from hæmatogenous infection originating in the region of the portal vein, most frequently due to appendicitis, or, in the tropics, to dysentery. Lastly, we have also a cystic formation, which, on account of its relative rarity in this country, we are inclined to forget, namely, the echinococcus cyst.

You all know that this is a parasitic infection which enters through the alimentary canal, inasmuch as the egg of the parasite is generally conveyed to human beings from dogs. Owing to the effect of the gastric juice, the egg is freed from its cuticle. The fotus is liberated and, with the aid of its hooks, makes its way through the intestinal wall into the circulation, and then, in most cases (69 per cent.), is conveyed to the liver by the portal vessels. But they may also penetrate the lymphatic vessels and creep into the pulmonary circulation through ductus thoracicus, so that 
we may have echinococcus in the heart and lungs, and also in the various organs supplied by the systemic circulation.

If we now consider which of these conditions is most likely to be present in our patient, I think we are justified in excluding a cystic malignant tumor in spite of the fact that the patient looks eachectic. One striking fact is that the patient maintains that she has felt some tumefaction for at least sixteen years. This fact, together with the relatively high percentage of hæmoglobin (85 per cent.), makes me certain that we are dealing with a benign tumor. There is no ground for supposing this to be either a multilocular cyst or an abscess. It is true that the patient has from time to time a slight rise in temperature and sticking pains, but her history does not show any attack of dysentery, appendicitis, or other intestinal inflammation, and, therefore, an abscess is not likely. Such abscesses, as a rule, are found in the right lobe of the liver. It is my opinion, therefore, that the diagnosis rests between the very rare, solitary retention cyst and echinococcus. When you hear that the patient comes from Iceland, where echinococcus is a common disease (according to Finsen's excellent book, one in sixty of the Icelanders is afflicted by it), and that both her parents died of echinococcus of the liver, you will understand that our suspicion is changed to a conviction and that here we have echinococcus. A symptom which is often mentioned in the text-books as characteristic of this is the so-called "hydatid-tingle," a peculiar tingling sensation which is communicated to the fingers of the examiner when one is palpating an echinococcus cyst which lies close to the abdominal wall (fine peritoneal adhesions?). This we have not been able to substantiate here, but I think that it is a symptom of very dubious value. One might possibly assure one's self of the diagnosis by simply puncturing the cyst and examining the contents, because in the echinococcus fluid, which consists of a watery solu- 
tion free of albumin, one often finds the characteristic hooks of echinococcus. We will not make such a puncture, however, because the fluid is toxic and would produce peritonitis if it leaked into the peritoneal cavity. At times also the puncture may become the starting point of a large tear in the cystic wall, and we may then have an efflux of daughter cysts and echinococcus formation in the peritoneal cavity. As all the circumstances in this particular case favor a laparotomy, we will not venture to compromise the result of a radical operation by previously performing an experimental puncture. You may ask if an echinococcus cyst can exist for so many years-twenty years, as it appears here-without giving more serious symptoms than with this patient. My answer is that experience shows that the course may vary greatly. But with regard to echinococcus of the liver, in particular, it seems very common for it to develop quite without symptoms. A small cyst is formed, from the inside of which fresh echinococcus buds grow out, and from these the so-called daughter cysts develop. Only when the tumor has reached a considerable size or when it ruptures does it present tangible symptoms. Sometimes, it is true, a pause in the growth and a spontaneous recovery may be effected by a calcification on the wall of the cyst.

I shall now show you a completely ossified echinococcus cyst the size of a hen's egg, which I accidentally found and removed some years ago from a patient who was suffering from gall-stones. After having removed a cholesterin stone from the gall-bladder, I proceeded to palpate the gall-ducts, and I found, to my surprise, a very hard, round body, the size of a hen's egg, situated at the hilus. In the belief that it was a large gall-stone which had distended the bile ducts and penetrated the liver tissue, I scooped it out and found, to my surprise, that it was a cyst in the liver itself, and had nothing to do with the gall-ducts. Further examination showed that it was a calcined echinococcus cyst. Inside 
the rind, which was about five millimetres in thickness, we found a slimy, purulent fluid containing the remains of daughter cysts and scolices. Sometimes a partial calcination of the wall of the echinococcus cyst may prevent healing after an operation which consists of incision and drainage, because the calcified part prevents the wall from collapsing and obliterating the cavity, and forms a fistula. which easily becomes infected, and the constant oozing and loss of fluid irritate and exhaust the patient and frequently cause recurring attacks of fever.

I was lately consulted by a young farmer from Iceland who suffered from similar attacks. He had been operated on in Iceland nine years ago for a large echinococcus cyst in the right lobe of the liver, but the fistula had never closed, and the constantly-recurring attacks of fever, which occurred when the free drainage was interfered with by contraction of the fistula, had exhausted him greatly and made his condition quite precarious. Exploiting the fistula with a probe, I reached a cavity sixteen centimetres from the surface which was surrounded by resonant, bony-hard tissue. By an incision along the rib curvature I freely exposed the liver, and, guided by a probe inserted in the fistula, I made my way up to the calcified echinococeus cyst and extirpated it. I inserted a cigarette drain, and the cavity, the walls of which could now fall together, gradually closed.

The question now is: How shall we operate on this patient? Because we can have no doubt that our duty is to operate on her, and no one knows when this cyst, which is probably very large, may burst into the peritoneal cavity and cause the patient's death. There has been a great diversity of opinion as to how one should operate in such a case. The operation most generally employed to-day is the two-stage operation indicated by the late famous surgeon, Volkmann. The first step consists in opening the peritoneal cavity in that region where the tumor protrudes, 
and, while keeping the centre of the wound open with tampons, one sews the organ in which the echinococcus cyst is found to peritoneum parietale. In this way, when the abdominal cavity is safely shut off by adhesions from the

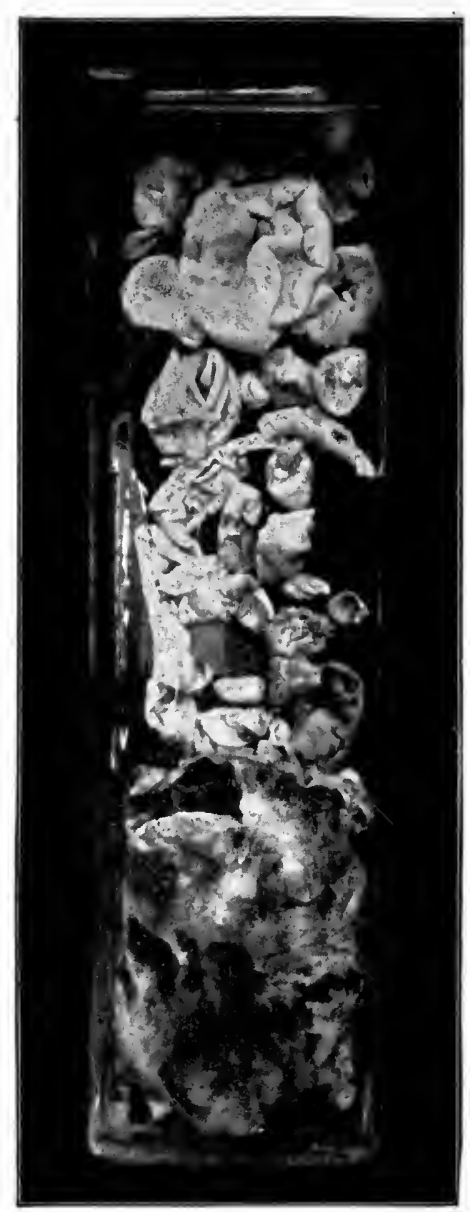

Fia. 131.-The removed echinococglass, and the daughter blisters above. One-fifth life size. cus cyst is seen at the bottom of the

field of operation, the cyst is incised and the contents evacuated. This method of operating, appearing as it did in the early days of antisepsis, was a great step in advance, because an incision of the cyst in one séance involved at that time a fatal peritonitis. This is safely avoided by Volkmann's method, and most patients recover, but only after a long confinement to bed necessitated by the frequent occurrence of suppuration within the cavity. The fact is that it is impossible, as a rule, to completely empty an enormous echinococcus cyst which contains daughter cysts and fragments of membrane, through the small, deep-lying, secondary incision. Nowadays, when we liave learned to protect the peritoneum during the operation, many surgeons prefer to operate in one séance, because, after one has carefully emptied the cyst of its contents, one either sews this into the wound and drains it, or tries to extirpate the entire tumor, in order then to obliterate the remaining 
cavity with suture. We owe this radical operation to the famous English surgeon, Knowsley Thornton, who pointed out that a pericyst wall of fibrous tissue is almost always found around the large echinococcus cyst, and that this can, as a rule, be easily scooped out of the pericyst, without injuring the liver tissue. This operation, which was first performed in 1883, has been widely propagated, and with many fine results. I trust that we shall succeed in performing Thornton's operation in this patient who is about to be operated on, because you will understand what an advantage it is, with such a weak and debilitated patient as ours, if we can remove all diseased parts at once, and obtain a healing per primam intentionem, instead of exposing her to the protracted course incurred by leaving a fistula and a large cavity which easily becomes the seat of suppuration.

At the operation on the patient who was the subject of our last lecture it appeared that our diagnosis was correct enough, because the left lobe of the liver was the seat of an enormous echinococcus cyst, which you see here preserved in this glass (Fig. 131). At the bottom you see the large, partly-calcined cyst, while above, in a more or less crumpled condition, you see the quantities of daughter cysts which the cyst contained. As you see, I succeeded in extirpating the entire tumor, but not according to the method indicated by Thornton. When we opened the peritoneum it appeared that the tumor did not present itself on the surface of the liver at all, but protruded, and was only accessible from the under surface. For this reason, Volkmann's method was at once excluded, because there was absolutely no possibility of sewing the liver or the tumor to peritoneum parietale. After having carefully protected the peritoneal cavity with pieces of gauze, I punctured the cyst with a thick trocar. Only a very little fluid was discharged, because impertinent daughter cysts clogged the opening of the 
cannula. There was nothing to be done but make a broad incision. This was done, and large quantities of daughter cysts poured forth. It was now evident that only at the lowest part where we had felt the fluctuation was the cyst wall somewhat normal; all the remainder was completely calcified and almost as hard as bone. The pouch was enormous, reaching high up under the diaphragm, and it was evident that the walls would never collapse and heal through simple drainage. I then endeavored to curette it out after Thornton's method, but this was only possible at the lower pole, where the pouch was still soft, while all the calcified part was indissolubly coalesced with the liver tissue. I then decided to cut the Gordian knot by resecting the liver. By a cuneiform section of the liver I removed the entire portion containing the cyst, and then joined the edges of the resection wound with a continuous catgut suture, which included the peritoneal covering with the liver tissue. I inserted a cigarette drain-nitrate of silver gauze wrapped in oilsilk-under the liver, and sutured the entire laparotomy wound round it. The course since the operation has so far been without reaction.

The patient was discharged after six weeks, cured, and has been quite well during the twelve months which have elapsed since. 


\section{LECTURE XXIV}

\section{ABSCESS OF THE LIVER}

The young man whom you see lying here is to be operated on to-day for a disease which you rarely have an opportunity to see treated in this country. As the disease in itself is very interesting and quite worthy of your attention, while the whole aspect of the disease is of great diagnostic importance, I have thought fit to make him the subject of our lecture to-day. When, at the end of the lecture, we have discussed his disease, I will operate on him, in order that you may have the opportunity of convincing yourselves that our diagnosis has been correct.

The patient is a seaman, twenty-four years of age and unmarried, who, on the whole, enjoyed good health as a child, while there appears to have been no morbid dispositions in his family. He performed his military service without any sign of disease, and has been completely free from genital affections. He states that he had never been really ill until he had severe enteritis when travelling through Australia; during which time, as he tells us, he suffered from diarrhœa with bleeding. He was considerably indisposed and was confined to his bunk for several weeks, but afterward recovered. A year later, in 1902, while travelling along the coast of China, he again had acute diarrhœa with bleeding, which, in the course of a month, developed into a chronic intestinal catarrh. For the past seven years the trouble has shown itself by three or four loose movements a day. In spite of this, he has felt fairly well and has been able to attend to his work. In August, 1909, when his ship was lying at St. Petersburg, he suddenly became seriously ill, with acute pains in the right side of the abdomen, corresponding with the position of the gall- 
bladder. These pains radiated up into the right side of the chest as high as the region of the right shoulder. They came intermittently with subjective sensations of fever, and, according to the description, appear to have exactly resembled gall-stone pains. The attacks passed off quickly, but between times he suffered from pains in the right side, especially when inspiring deeply. It is very difficult to ascertain if there has been any jaundice. His comrades declared that his face was yellow and green; but he himself is decidedly of the opinion that the skin on his body was not yellow, and he maintains that the diarrhœa from which he had suffered for seven years ceased entirely during this attack in St. Petersburg. A week after the pains had ceased he suffered from vomiting. After three weeks he recovered somewhat, but felt slack and tired. Early in December, 1909, he had a second attack of pain similar to that which he had in St. Petersburg, though this time it was much worse when he respired deeply. There was high fever, and the diarrhœa increased and became more frequent. He was then admitted to the medical ward in this hospital, where he lay for two months, until, eight days ago, he was transferred to this ward. In the medical ward his diarrhœa was controlled by diet and medicine, and his bowel movements are now quite normal, but, on the other hand, the pains in the right side of the chest and the abdomen have increased. Percussion at first showed a normal note as far down as the tenth rib; but on January 17, upon examination, a marked dulness was found on the right side posteriorly. No friction sound was heard, but the vocal resonance was increased. On January 19, two days later, an experimental puncture was made in the eighth intercostal space, and only a small amount of clear yellow fluid containing a number of lymphocytes was obtained; neither by microscopy nor by cultivation were any microbes found. A radiograph of the right half of the chest showed a shadow obscuring a 
portion of the right lung, beginning at the level of the sixth rib, and growing denser and denser until it passed over into the liver shadow. The diagnosis then pointed most strongly toward a tuberculous affection of the lung, with pleuritis, and it was thought at first that the diarrhœa of many years' standing was due to an intestinal tuberculosis. Later a von Pirquet's test was made. This gave a negative result, and, while a positive one does not mean so very much, because so many people go about with an encysted tuberculosis in some part or another, so that the reaction does not necessarily mean that the illness which is evident is tubercular, a negative Pirquet's test, however, means much, because it tells us, as a rule, that the patient is not at all tuberculous. An aspirating needle was again introduced in the eighth intercostal space, and this time the syringe was filled with pus. Immediately after this puncture the patient, who had previously been free from fever, had high fever-temperature over $40^{\circ}$-and was at once transferred to the surgical ward under the diagnosis empyema in the right pleura cavity (abscessus hepatis?), in order that we might operate on his empyema and eventually on a liver abscess, if we found one. When the patient came here I found him too weak for an operative encroachment; his temperature, as mentioned, was very high; his pulse very bad; and the sallow, debilitated patient looked most deplorable. Moreover, the aspect of the disease seemed obscure to me, and from the preliminary examination I did not feel convinced that it was a question of empyema; on the contrary, many things seemed to favor the idea that the patient had an enormous abscess on the liver. Therefore, I began by trying to improve the patient's general condition. In order to counteract the intoxication, I administered daily subcutaneous infusions of salt water; reduced the temperature with phenacetin; strengthened the heart with digitalis and caffein; and put him on a light, strength- 
ening diet. Under this treatment he has recovered well from the state of collapse. His temperature is now almost normal and the leart's action so vigorous that to-day I dare perform the operation.

But in order to operate on this weak patient with any chance of success we must know what we have before us: whether it is empyema or an abscess of the liver, or perhaps both. While he has been under my observation and consideration I think I have arrived at the correct diagnosis, and have laid down a plan of operation accordingly.

I will now shortly relate to you my investigations and considerations. When you see the patient sitting here with his trunk uncovered he will remind you mostly of a patient in the last stage of phthisis: his pale, wan countenance, his dyspnœa, and the domical nails on his swollen, drumstickshaped finger-tips. When you regard his chest more exactly, either from in front or behind, you will at once notice a striking difference in the two sides: while the left half is shaped normally, though thin and with sunken intercostal spaces, the right half from spina scapulæ downward distends increasingly in the shape of a barrel, with a slight sphericity of the broad intercostal spaces. Corresponding with this you now hear that my percussion gives quite a dulled resonance from the right spina scapulæ down to the basis. This might fit in very well with a large tuberculous empyema. Now you have heard, however, that Pirquet's reaction was negative, and you have also heard, I think, that this examination may fail in a very advanced and hopeless stage of phthisis, for which reason you dare not rely on it. On the other hand, you will doubt the presence of an advanced phthisis in a patient who has never suffered from coughing or spitting of phlegm, and still more doubtful at not finding signs of cavities in the lungs, and not even rattling or impaired respiration over the apices of the lung.

If we examine the condition of the prolapsed liver 
downward, this is found to extend five to six centimetres below the rib curvature in front and three centimetres below the curvature in the axillary line, and we accordingly feel the edge of the liver. This might simply mean that the liver is thrust down by an empyema, which presses on the diaphragm, if a further examination of the chest did not point in another direction. If I measure the increase in the thorax at various heights, it appears to measure most between the spinal process of the ninth dorsal vertebra at the back and the point of processus ensiformus in front. Here the increase is seven centimetres, compared with the healthy side, and grows less above and below. This tells us that the largest increase, even if we think of the liver as depressed, is in the liver, and not in that of the $\mathrm{lung}$ and

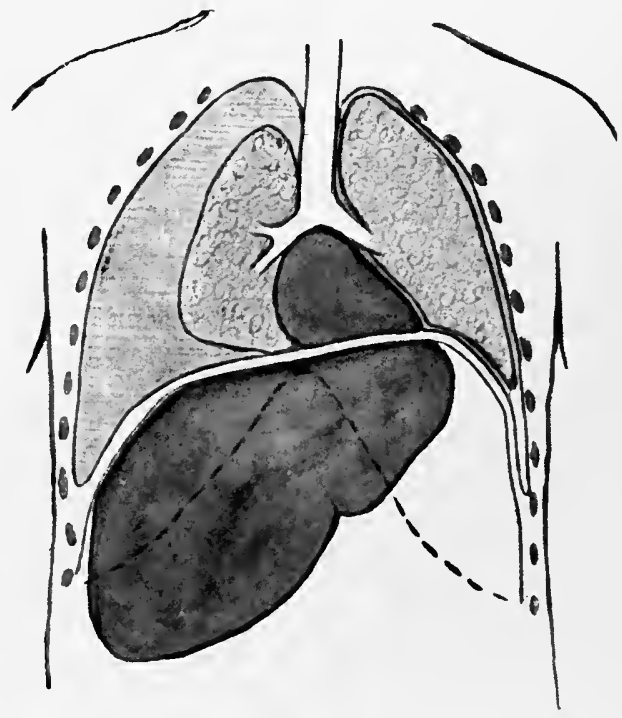

Frg. 132.-Dilatation of chest cavity by empyema as contrasted with dilatation by liver abscess.

pleura (see Figs. 132 and 133), and, therefore, it is more reasonable to think that we are concerned with an enlarged than with a prolapsed liver. That the puncture of the pleura, which was performed when we first found dulness from spina scapulæ to the base of the lungs, gave only very little clear serous fluid confirms this. At that time there was no empyema-indeed, no quantity of fluid which could be thought to dislocate the diaphragm and the liver.

But the last puncture, which disclosed pus, does not tell us with certainty that there is an empyema now. You will 
understand this by looking at the sketch which I have drawn on the board to illustrate my view of the case. I have in mind an enormous abscess in the right lobe of the liver, extending high up toward the upper convex surface. The enormously enlarged liver raises the diaphragm and right lung, and presses the diaphragm and the two leaves of the pleura together against the chest-wall in an outward direction. You will understand that a puncture needle which

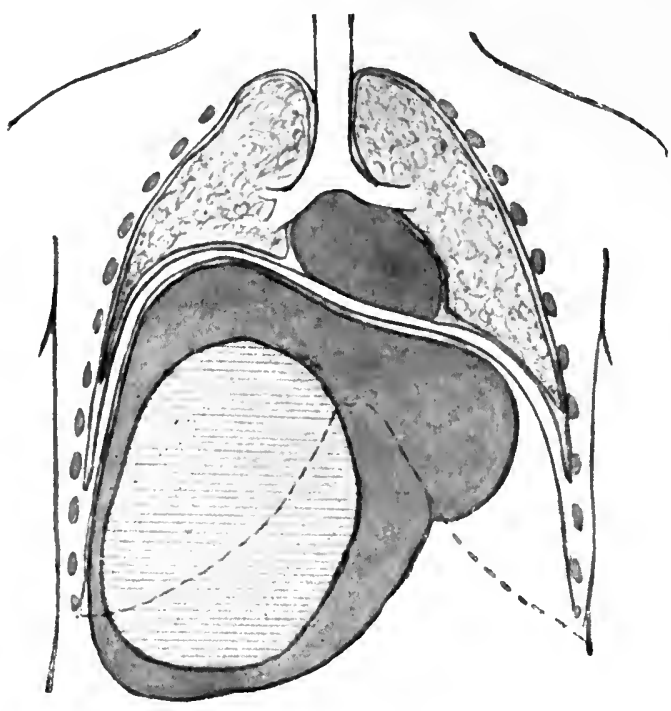

FIG. 133.-Diaphragm raised, and cavity of right lung and pleura compressed by liver abscess and enlarged liver. Boundary of pleural cavity seen in posterior axillary line. is $\mathrm{thrust}$ into the eighth intercostal space may very easily enter the liver abscess without one's suspecting that one has passed through the pleura. If, in this case, I feel $q \mathrm{u}$ it e convinced that this has occurred, it is essentially due to the microscopic examination of the discharged "pus," which was proved to consist exclusively of detritus and degenerate cells, without any genuine pus-cells whatever-in short, pus from an old abscess, and pus which one would never find in an empyema of the pleura of such recent date as this is supposed to be. I do not think, therefore, that this pus dates from an empyema, but there might be good reason to fear that an empyema might have developed as a result of the puncture, because the cannula, when removed, might have carried infectious matter from the liver abscess into the pleural cavity. That an infection has taken place is evi- 
dent from the high fever which followed on the heels of the puncture, but I do not think it has led to the formation of any empyema, because the reaction would then have been more acute, with pain and dyspnœea, and the fever would not have ceased.

How does the diagnosis, built on the objective examination, agree with the patient's anamnesia with the earlier course of the disease? In reality, very well indeed, because the disease, which the patient himself dates back to his stay in St. Petersburg in August of last year, distinctly began with violent pains in the vicinity of the gall-bladder and the liver, occurring intermittently and greatly resembling gall-stone colic.

Then can gall-stones cause abscess in the liver? Yes, in our latitudes it is perhaps the most frequent cause thereof, and most often in this way, that a choledochus stone stops the outflow from the previously-infected gall-ducts, the microbes spread up into the dilated gall-passages, and, if their virulence and pyogenic qualities are strong enough, the result may be larger or smaller abscesses. In this case, however, this does not seem to apply, because there does not seem to have been any icterus-the first necessary consequence of such choledochus blockage. If we should maintain the idea of gall-stone as one of the causes, we should be reduced to supposing that either a metastatic propagation of the infection from the gall-bladder or ductus eysticus to the liver had taken place, or a direct attack on the liver tissue, as, for instance, by a stone in ductus cysticus gnawing through this into the liver substance. But this is not very prohable, because here the abscess is so tremendous, and is not prominent in the region of the gall-bladder, but, to judge from the mensuration and puncture, it has its seat upward and outward in the liver tissue, and approaches the surface out toward the wall of the thorax. But there is one symptom present in this patient, to which I have al- 
ready drawn your attention, and which induces me to think that the pains with which the disease apparently began at St. Petersburg were not gall-stone pains at all. This symptom is the domical nails and swollen finger-tips, a symptom which means that he has suffered for a long timeprobably many years-from a suppurative complaint. A suppuration dating from August of last year could not have caused this. I think, therefore, that the attacks at St. Petersburg were merely the bursting of a tense abscess in the liver, with temporary, acute attacks of inflammation in the surrounding liver tissue. If we consider what such an old suppuration can be due to, we naturally think of the first and only illness which he has gone through in the past; namely, the bleeding diarrhœa which overtook him for the first time in Australia in 1901, and for the second time on the coast of China in 1902, and left behind it a chronic enteritis with daily diarrhœas. You ought further to know that the most frequent cause of abscesses in the liver is tropical, intestinal infection, especially the so-called dysentery, a disease the etiology of which is not yet fully clear to us; possibly because many various infections are classed together as tropical, epidemic dysentery. It was thought that this dysentery was due to amœba (Kartuli), but later it was possible to distinguish between amœbic dysentery and a "real epidemic" dysentery, due to a particular bacillus having a certain resemblance to the typhus bacillus. Krause found this bacillus during an epidemic of dysentery in Germany, and the Japanese Shiga is said to have found a similar one in tropical dysentery. I think, however, that one should be very careful in accepting this specific bacillus as the cause of this disorder, even if this produces inflammation of the intestines when injected into animals, because we must remember that, with all inflammations of the intestine, the ordinary and otherwise harmless intestinal microbes often assume a virulence which 
they do not possess normally. One thing is particularly suspicious, namely, that, while this bacillus is extremely easy to cultivate from the intestinal contents, no one has ever been able to demonstrate it, or any other bacteria, in the abscesses of the liver resulting directly from dysentery. Although this class of liver abscess is essentially a tropical disease, it still holds considerable interest for us here in Denmark; not so much because we possess a small colony in the West Indies as because we are a seafaring nation, whose inhabitants navigate the tropical ports and may there contract a dysentery which may give rise to a subtle, insidious development of an abscess in the liver. Often it is only after many years that such an abscess gives symptoms, the cause of which it may then be very difficult to recognize, if one is not familiar with the existence and nature of the disease; and this may prove fatal, on account of the dangerous character of the disease, if not treated correctly. You are aware that a number of Danish physicians go to the Dutch colonies, enticed by the relatively large salaries, which, however, are scarcely commensurate with the various risks to life which are there involved. Among these are liver abscesses, and several clever young physicians have fallen victims to this disease, even if it is not so bad as an old colleague of mine used to say: that the doctors always returned from the Dutch Indies "with a small pension and a large liver abscess." I have no doubt whatever, therefore, that here we have just such a tropical, dysenteric abscess of the liver. The only question is, then, whether it is still confined to the liver, or has burst and formed a sub-diaphragmatic, decapsulated abscess, or whether it has possibly proceeded farther through the diaphragm to attack the lung and the pouch of the pleura. This happens by no means rarely with neglected liver abscesses. I have seen instances of both-one where the abscess had broken into the lung and where the patient 
coughed up large quantities of peculiar liver-abscess pus, reminding one of reddish fruit soup, which was distinguishable from the pus in the ordinary lung abscesses by being odorless; and another where the pus had penetrated the pleura, and here, accompanied by acute fever and pain, had caused the formation of a large empyema. The bursting of a liver abscess into the lung or the pleura is a very serious matter, and, as a rule, gives very serious symptoms. Therefore I do not think that this has happened in our case, where no symptoms at all can be substantiated beyond the suppression, which may just as well be due to the liver itself.

We now come to the question: How are we to treat this patient, and, at the same time, the dysenteric liver abscesses which generally have their seat high up and towards the posterior surface of the liver? The former mode of treatment consisted of a simple puncture with aspiration and siphon drainage, and this method still has advocates among eminent tropical physicians, such as James Canthie, mostly because the method is so simple, and does not demand a schooled surgeon. This method is somewhat reckless, however, and by no means free of danger, because, whether the puncture is made through the abdomen or intercostally through the wall of the thorax, the puncture cannula and the pus alongside of it pass a cavity, the infection of which is extremely dangerous, and many patients have died from the resulting peritonitis or empyema. But, apart from this, the drainage was often insufficient, the fistula became chronic and mixed infection set in. Strohmeyer-Little, an English physician in Shanghai, was the first, with an eye to these dangers, to propose a more radical mode of procedure, by first making an incision down to the liver through the overlying tissues, and then puncturing the liver abscess, while widening with a blunt knife the puncture opening in order to allow 
of the introduction of a thick drain into the abscess cavity. This method naturally results in a far more effective emptying and drainage, but one must admit to the advocates of the puncture that the danger of infecting the pleura and the peritoneum is in no wise diminished. The radical incision method becomes an assured step forward only when, previous to opening the abscess, one closes the pleural cavity and the peritoneal cavity respectively so that it cannot be soiled by the pus when the abscess is opened. Godlee was the first to do this, when, after resecting one or two ribs, he opened the pleural cavity and sewed the leaves of this together before he punctured and drained the abscess. This method is, however, far from safe, because you will understand that a suture can easily break or cut out, and there results not only an empyema but also a pyopneumothorax, which might easily cause death in such an enfeebled patient. I must do my

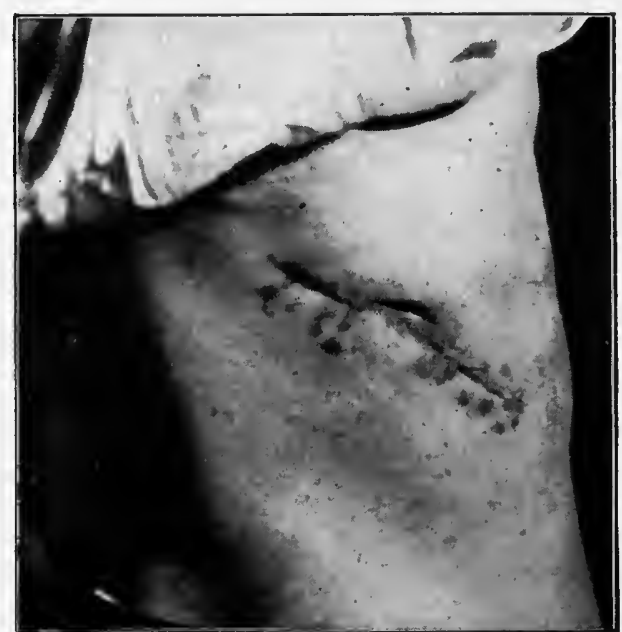

Fig. 134.-Shows the cicatrix after author's subpleural incision and resection of the tenth rib. Drainage spot in centre.

utmost, therefore, to avoid the pleural cavity, as you will now witness with the anæsthetized patient. You know that the pleura reaches only to the ninth rib in the posterior axillary line. When I have placed the patient in a slightly left-sided position, I make an incision across the tenth rib (see Fig. 134), in such a way that the centre of the incision meets the point of intersection between the posterior axillary line and the ribs. After having extir- 
pated seven centimetres of the tenth rib subperiosteally, I work in under the fold of the pleura, incise the diaphragm, and now have the liver lying before me. I sew the serosa of the liver to the peritoneal covering of the diaphragm with silver catgut, so that a portion of the liver surface, the size of a half-crown, lies accessible at the bottom of the wound. I now puncture the liver in an upward and backward direction with a thick and slightly-curved trocar, and you see that a quantity of inspissated, greenish-yellow pus immediately streams forth. We collect the pus in a measuring glass. You see that we have now emptied out 1500 cubic centimetres, and that the fluid now begins to grow thin and bile-colored. I remove the trocar and with the aid of this long instrument, which has the same curve, I insert a Pezzer eatheter through the six-centimetres-long canal in the abscess cavity. When the catheter is inserted, the button unfolds, and by drawing this tight down toward the bottom of the abscess I hope here, as in other cases, to be able to prevent any oozing out of pus alongside the drain. I now sew the wound together with aluminum bronze around the drain, and cover the line of suture with collodion and cotton wool. The dressing consists of a small piece of silver nitrate gauze around the drain, and a piece of non-absorbent cotton wool through which the drain is led out. The drain is connected with another tube which leads down to a glass container, which is placed at the side of the bed, and into which it empties itself under phenosalyl water.

During the first forty-eight hours, 3000 cubic centimetres of pus were emptied out, but then the quantity diminished fairly rapidly, and has now fluctuated for a long time between 400 and 500 cubic centimetres in the twenty-four hours. From the 16th of March it diminished slowly, until the secretion ceased early in April. On the 5th of April Pezzer's catheter was removed; on the 13 th the patient was dis- 
charged, and on the 26th was exhibited at the concluding lecture, completely cured, difficult to recognize on account of his ruddy and well-nourished appearance. The wound was completely healed (see Fig. 134). With regard to the conditions in the right chest, it should be remarked, in conclusion, that the lung gradually moved down as the liver tumor disappeared. There was no sound of friction or compression. In short, the lung was quite normal, and the course of the disease proved us to be quite correct in our supposition that the pleura and the lung were really unaffected by the disease. 


\section{LECTURE XXV *}

\section{CHRONIC JAUNDICE. XANTHOMA XANTHELASMA MULTIPLEX}

To-DaY I wish to conclude this term's lectures by showing you a very interesting but, from a diagnostic standpoint, a very difficult case of icterus. To diagnose chronic icterus is not difficult: you can do that by merely glancing at the patient, whose deeply yellow scleræ and extraordinarily dark, almost black, greenish-yellow skin, with its numerous lesions caused by scratching, show you that this icterus has lasted for many years. But what makes this case so difficult is that we have to ascertain which form of icterus it pertains to, what cause is behind it, and whether it is possible to cure the patient by an operation.

The patient, who is a married tram conductor, thirtyeight years of age, has been transferred to us from the medical ward with the diagnosis stenosis ductus choledochi ex ulcera duodeni. You will understand why our medical colleagues have given this diagnosis when I give you a short résumé of his history.

The patient, whose family is not morbidly disposed in any particular way, states that he enjoyed good health until his present illness began, two years ago, when, without previous pain or dyspepsia, and entirely without any symptoms referable to the gastro-intestinal tract, and without any fever, an icterus developed which quickly attained considerable proportions.

This jaundice has increased steadily during the last two years, and all this time the excretion has been clay-colored, except on two occasions, some four or five months ago, when for one day it was tar-colored. The urine has been

* Last lecture delivered at the old hospital, April 26, 1910. 464 
bile-colored, though of somewhat varying intensity. The patient, who was treated by a homœopathist with diet and some medicine during the first eighteen months, had, apart from an increasing itching of his skin, been quite well and able to attend to his work until January of this year. Only during the last three months does he think that he has lost weight. Quite a fortnight ago he was admitted to the medical ward. On examination there the urine was found free of albumin, blood, or saccharine matter; the chemical and motory function of the stomach proved normal; Wassermann's reaction gave a negative result, and the heart and lungs were found normal. A chemical examination of the fæces, however, revealed blood on two occasions.

It is easy, then, to understand the reasons for assuming this to be a case of stenosis of the ductus choledochus. We have before us a completely painless development of severe jaundice, the most frequent cause of which is cancer capitis pancreatis, which, by its pressure, closes ductus choledochus; but this diagnosis has been abandoned because of the patient's good general condition and strength, which do not harmonize with the thought of a cancer of such long duration. A syphilitic stricture, which at times has been known to cause a chronic icterus, seems excluded by the negative result of Wassermann's reaction. Sometimes a simple catarrhal, infectious icterus develops into a chronic one, but, if this had been the case, one would have expected attacks of fever, with a rapidly-rising temperature alternating with a subnormal one.

But in this case there seems to have been no attacks of fever at all. One's thoughts then turn to the rarer cause, a chronic ulcus duodeni situated on or close to the papilla, so that its cicatricial processes may have produced a contraction or obliteration of the choledochus orifice. This diagnosis is strengthened by finding blood in the fæces, in conjunction with the patient's statement that he has re- 
peatedly noticed a tar-colored excretion. I do not deny that this diagnosis may be correct, but I will not hide from you that I consider it very doubtful. For complete reliance on the diagnosis ulcus duodeni the symptom pain is lacking. Some of you will remember, perhaps, that last year I had a patient, a woman, forty-eight years of age, with chronic icterus, in whom I diagnosed contracting ulcus duodeni, basing my opinion on two symptoms: melæna and pains in the right hypochondrium, and at the operation this diagnosis proved to be correct. Now, duodenal ulcers may, of course, be quite painless at times, but this is rare, and I think especially that an ulcer near the papilla, where bile and pancreatic juice continually press forward to make their way into the intestines, can scarcely progress entirely without pain. Moreover, the symptom melæna must be regarded with the greatest caution when it is observed only during an advanced stage of chronic icterus-in this case not until twenty-one months after the commencement of the jaundice; because one of the most dreaded results of chronic jaundice is the tendency to bleeding, which breaks out sometimes from the nose and sometimes from the intestines, a fact of which we surgeons are unpleasantly aware from having to operate on such patients. I therefore think that one should be careful in diagnosing an ulcer basing his opinion on the presence of blood in the fres. In any case we must take other possibilities into consideration, and such there are. You must first bear in mind that you must not reject the thought of gall-stones because there have been no pains. For instance, when I was an assistant surgeon in this ward I saw a very instructive case. It was that of a young girl who was transferred here from the medical ward with a chronic icterus which had developed quite painlessly. This happened during the infancy of gall-duct surgery, and, as she had no pains, we thought we might exclude gall-stone. But, after her death from icterus, it appeared at the dis- 
section that death was due to a small stone which was jammed in the lowest part of ductus choledochus, but which I was able to push into the intestine without any difficulty. This case taught me that a painless icterus can be due to the wedging of a gall-stone in the choledochus, and since then I have been successful in three cases in curing by a choledoco-lithotomy patients who for years had suffered from totally painless icterus, and had, therefore, been regarded as cancer patients. In this case, therefore, we ought to bear in mind the possibility of a calculus.

But, in addition to this, we can have benign or ductus choledochus tumors in the pancreas which obstruct the outlet of the bile. I have encountered two such cases in this ward. In the one case I found a fluctuating tumor the size of an orange in the pancreas, which disappeared spontaneously

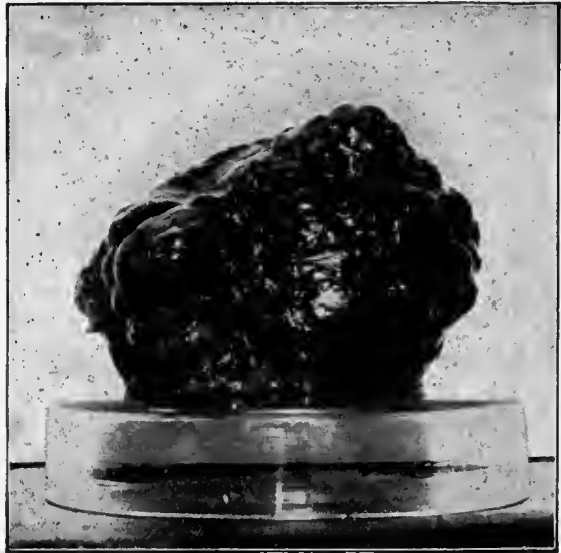

FIG. 135.-Multilocular cystoma in caput pancreatis as a cause of chronic icterus. Removed by author's operation.

after I had performed cholecystostomy, enabling the bile to pass into the intestine again. With the other patient, the cause of the icterus was found to be a multilocular cystoma in the pancreas (see Fig. 135), which I succeeded in enucleating. If, with these questions in our minds' eye, we now examine our patient, we must first observe the condition of the gall-bladder-whether it feels tense and dilated with gall or not. You must know that the first speaks for a tumor or stricture but against gall-stone, because with gall-stone icterus one generally finds the gall-bladder shrunken, inasmuch as it is just this process of shrinking 
which forces the stone over into ductus choledochus. Next we will naturally ascertain whetler any tumor can be felt. As you see, the abdomen is, as it were, a little tense toward the top. In the epigastrium and under the right rib curvature I feel the edge of the liver protrude the breadth of a little finger, and corresponding with the seat of the gallbladder at the tip of the ninth rib. I think I feel a pearshaped, tense distention below the edge of the liver. I think this is the dilated gall-bladder, but I cannot feel any tumorlike distention in the depth to

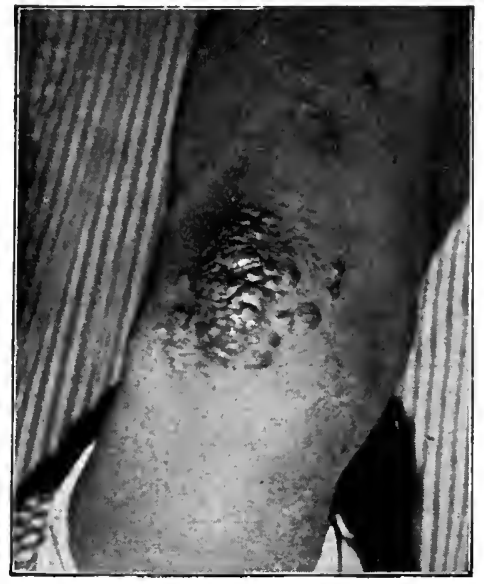

Fia. 136.-Group of xanthoma tumors on patient's elbow. the right of the vertebral column. There is no sign of ascites, and the abdomen is, on the whole, soft and natural. But because there is no palpable tumor, the choledochus may quite well be blocked by a tumor which cannot be felt from outside. But this patient presents a peculiar affliction, which has been noticed and described in the medical ward, though only as an affliction irrelevant to the main disease. Yet it is an affliction well worthy of our attention, because it stands possibly in intimate relation to the chronic icterus. This affliction consists, as you see, of small tumors in the skin, dispersed over this, but located particularly in the skin of the face, especially in the eyelids and on the elbows of the patient (see Fig. 136). The tumors are whitish-yellow, partly flat and bud-shaped, and I think that most of you will have seen them on the eyelids, where they occur in old people and are termed Xanthoma. With regard to these tumors, we only know that under the microscope they appear to be formed 
by fatty degeneration of the fibrous tissue cells, but we know nothing of what they really signify. When, as is usual, they appear singly or in small numbers on the eyelids, they seem to be very benign, but during the last decade one has noticed a series of cases where the xanthomas have appeared multiple and dispersed over the organism, and not on the skin only, but also on the internal organs-for instance, on the valves of the heart and on the liver. And what especially interests us to-day is that this so-called Xanthelasma multiplex is in a large number of cases combined with a chronic icterus. Thus, the dermatologist, Louis Török (Budapest), has been able to collect thirty cases with chronic icterus. (De la nature des xantomes, Annales de dermatologie et de syphiligraphie, t. iv, 1893.)

I have studied the published account of these cases in order, if possible, to ascertain the relationship of icterus to xanthoma, a matter which is of such importance to us. You will understand that there are two diametrically opposed possibilities. One might first think of the xanthoma as a product of the jaundice, resulting from the poisoning and irritation produced in the skin and internal organs by the bile elements, but one might also imagine that the xanthomas were the cause of the icterus, and, so far as I can perceive, this conception is dominant among the dermatologists, to whom we are essentially indebted for our knowledge of this peculiar disease. Some have imagined that icterus might be due to multiple xanthoma formation in the liver. Whether the one explanation or the other is correct it is impossible to decide by studying the history of the disease as collected in literature, because in only a very few cases do we find a post-mortem description. It seems, however, from the histories of the patients as if the xanthomas have usually been present when the jaundice commenced. If the theory were correct, that the xanthomas were due to the jaundice, then one would expect 
them to develop at an advanced stage of this, and, at any rate, that they would grow and increase in number. I have questioned our patient repeatedly. with regard to this, and he, who is an intelligent and sensible man, insists that he noticed the tumors simultaneously with the commencement of the jaundice. Therefore I am mostly inclined to think that, if the xanthomas have anything at all to do with the jaundice, they must have caused it. But the question then is whether, as the dermatologists assert, this is really due to diffuse xanthomas in the liver tissue. You will understand that if this is so it will be impossible to relieve the patient by an operation. I think, however, that one is justified in hoping that it concerns a blockage of ductus choledochus with xantloma tumors, because, if it only concerned tumors in the liver, why should the fæces be quite clay-colored, and the jaundice so intense and total? Part of the bile should then have a free outlet into the intestine. My hope is increased by the fact that Moxon, in one of the few dissected cases, found the choledochus blocked by xantliomas. It is my opinion, therefore, that one is not only justified but is in duty bound to make an explorative operation on this man whose condition is so good, in order to see if there is any indication for an operation on the gallducts. What I shall at once fix my attention on is whether the gall-bladder and large gall-ducts are distended or not. This is, in reality, the deciding factor. If there is no distention, I at once close the wound, because in such a case the jaundice is due to morbid processes in the parenchyma of the liver, or in the small gall-ducts, which we cannot reach. If, however, the gall-bladder, ductus cysticus, hepaticus, and choledochus are dilated one naturally will first ascertain whether, contrary to expectation, a wedged gall-stone or a tumor of some sort blocks the biliary outlet. In the first case I shall remove the stone by choledochotomy, and in the latter I shall form a new passage for the bile over 
into the intestine by performing an anastomosis between the gall-bladder and the duodenum or a coil of the jejunum.

P. S.-At the operation it appeared that the edge of the liver was plump, and protruded two centimetres below the rib curvature. The gall-bladder protruded several centimetres below the edge of the liver. This was dilated with bile, just as ductus cysticus, ductus hepaticus, and ductus choledochus were distended to the thickness of a finger. The serosa was everywhere glossy; there was no sign of inflammation, while no swollen lymphatic glands were felt along the gall-ducts. Just where ductus choledochus enters the pancreas I felt a soft lobate tumor in the lumen. The pancreas did not appear to be the seat of any tumor. The duodenum was everywhere normal to the touch. The liver presented the aspect of a gall-stasis liver in the cirrhotic stage. As I had no doubt that the obstruction was situated in ductus choledochus, I performed an anastomosis between the gall-bladder and a coil of the jejunum. To prevent the intestinal contents from entering the gall-bladder, an enteroanastomosis was performed between the legs of the jejunum about ten centimetres from the gall-bladder. The course was reactionless after the operation. The fæces grew more and more bile-colored, and the urine has now become almost normal in color, though, naturally, the icteric color of the skin and scleræ has by no means disappeared. The patient was discharged seven weeks after the operation, and is still enjoying good health three months afterwards. The xanthomata on the skin are practically unchanged. 



\section{N D E X}

Abdominal support, Curtis, 223

Abscess following perforation of stomach ulcer, 385

of the liver, 451

subphrenic, 384

Achylia, cause of, 198

gastrica in cancer of the stomach, 401

occurrence in cancer of the stomach, 398

significance of, 402

Aluminum bronze, for prevention of hernia, 63

thread for sutures, 62

Anæsthesia, cocaine, 101

ethylchloride local, 98

general, chloroform, 74

ether, 74

history of, 72

introduction of laughing gas, 73

infiltration, 107

local, 96

indication for, 113

refrigeration, 97

regional, Oberst's method, 104

spinal-cord, 113

Wanscher's apparatus for, 84

Antiseptic methods, 35

history of, 21,24

Appendicitis, explanation of gastric symptoms in chronic cases of, 290

Aseptic method of wound treatment, 26

Belt, Vermehren's abdominal, 222

Belts, abdominal, 221, 223

Billroth method of pylorectomy, 317

Blood, occult, significance of, 295

in the stool, as symptom of gastric ulcer, 287

examination indicated in, 273

in vomitus in cases of ptosis, 294

Bougies, ivory-knobbed, for œsophagus, 127

rubber, 127

the use of, in stricture of œsophagus, 165

Bourget's experimental meals, 194

Burns, antiseptic treatment of, 50

Cancer of stomach, 395 (see stomach) development of diagnosis, 397

Carbolic acid, its early use, 25 toxic effects from use, 25
Cardialgia, due to chronic ulcer, 292

in gastroptosis, 228

Catgut, handling of, 65

its uses, 63

preparation of, 64

preservation of, 65

Chloroform, collapse under, 78

primary collapse under, 78

primary death from, 78

secondary collapse under, 78

versus ether, 80

Circulus vitiosus, prevention of, 337 , 430

with anterior gastro-enterostomy, 431

Cocaine, injection into the spinal canal, 114

poisonings, acute, 101

prevention, 102

Coffey's operation for gastroptosis, 259

Coin catcher, uses and dangers of, 135

Collodion dressing, application, 43 to prevent infection, 49

Constipation, as symptom of gastroptosis, 215

in gastroptosis, 243

Cotton gloves, use of, 39

Curtis's abdominal support, 223

Deaths following gastropexy, 263

operation for gastric ulcer, 277

Diagnosis, exploratory incision for, 13 sample incision for, 15

Diaphano-gastroscopy in hæmatemesis, 368

Diaphanoscopy, 201

in diseases of the stomach, 199

Disinfection, of the skin, 36

cultural experiments, 36

of surgeon's hands, 37,70

rules for, 70

Drainage methods, Mikulicz bag, 46 tube, 53

Drains, cigarette, 53

glass, 54

rubber, 53

wicking, 53

Duodenal ulcer, character of pain in, 307

difficulties of resection of, 285

operative statistics, 278

relation of, to chronic jaundice, 466 
Duodeno-gastroscopy, 201

Duret, method of gastropexy, 254

Dysentery a symptom of liver abscess, 459

Echinococcus of the liver, 441 choice of operation for, 447 establishing diagnosis of, 442 method of infection of, 442

Thornton operation for, 449 Volkmann operation for, 448

Epigastric pulsation, in gastroptosis, 213

Ether, action of, on air passages, 86 administration in cases of lung disease, 87

advantages over chloroform, 90 mask, Sudeck's, 82

narcosis, death from, 80 instructions for inducing with

Eucaine, 112

Wanscher's apparatus, 94

Ewald's test-meal, 193

Examination of patient, rules for, 4

Feeding after pylorectomy, 315

Fish-bones, swallowing of, 125

Food, partaking of, after pylorectomy, 315

retention of, in gastric ulcer, 288

Fowler's position, 379

Freezing mixture, application of, 100 disadvantage of, 100

Gastric ulcer, case of symptom, 299 characteristies and types of, 301

characteristic types of, presentation of patient, 311 confusion with gastroptosis, 290

diagnosis of, 287,389

differential diagnosis of, 297

differentiation from appendicitis, 289

developing diagnosis of cases of, 276,384

excision of, 283

failure of gastro-enterostomy for, 280

Hacker's operation for, 276

immediate operation for, 392

its differentiation from cancer of the pylorus, 305

occurrence of tubercular, 329

of long standing, 303

operative statistics, 278

pain in, 305

perforating, 375,387

perforating, statistics of, 383 , 387
Gastric ulcer, prognosis and operative treatment, 309

pylorectomy for, 313

sources of error in diagnosis, 298

statistics of, 277

surgical indications for, 388

theories of production, 353

tubercular, 324

tubercular, developing in diagnosis of, 327

tuberculous, theory of production of, 335

with carcinoma developing in cases of, 311

Wölfler's operation for, 275

Gastro-anastomosis, 355

Gastrocolic ligament, method of shortening, 252

Gastro-duodenostomy for ulcer, 319

Gastro-entero-anastomosis, 355

Gastro-enterostomy, after-treatment, 314

anterior, 429

course of food following, 281

exaggerated relief in, 276

failure of, for gastric ulcer, 280

Hacker's posterior, 276

infection following, 314

posterior, 430

Roux's method, 432

technic of, 423,428

Gastro-jejuno-cesophagostomy, 181

Gastropexy, deaths following, 263

Duret's method, 254

fixation of fundus only, 256

glass plate for use in, 256

preliminaries to, 251

Rovsing's method, 255

statistics of, 262

Gastroplasty, 354

Gastroptosis, advanced, resemblance to cancer, 236

argument for, in case of, 241

atypical cases of, 227

bandage treatment of, 221

cachexia, 237

constipation in, 243

explanation of symptoms in, 241

Glénard's theory of, 206

intestinal symptoms in, 243

maternal, 210

Relation of, to menstruation, 245

results of operations for, 265

shortening ligaments for, 258

Stiller's theory, 207

treatment of, 239

virginal, 210, 215 
Gastroscope, application of, 247

the use of in case of stomach ulcer, 272

Gastroscopy, disadvantages of, 200

Rovsing's, 202

value of, 205

Gastrostomy, for stricture of œsophagus, 150

Kocher's method, 177

Rovsing's method, 177

Witzel's method, 176

Gastrosuccorrhœa, 230

Gauze, antiseptic dressings, 60

Glénard's theory of enteroptosis, 206

Gloves, cotton, use of, 39 rubber, 38

Hacker's posterior gastro-enterostomy for ulcer, 276

Hæmatemesis, examination of stomach in presence of, 273

as symptom of gastric ulcer, 287

causes other than ulcer, 361

control of hemorrhage by suture, 369

due to cirrhosis, 361

employment of diaphano-gastroscopy in, 368

examination of stomach in presence of, 273

extensive, from ulcer, 358

indications for treatment, 371

lack of, in gastric ulcer, 288

perilous, indications for, 363

ptosis cases, explanation of, 294

question of surgical intervention in, 365

responsibility of physicians in, 373

treatment in case of, 364

Hæmoglobin, operation in presence of low percentage of, 275

Hæmophilia, hæmatemesis in, 361

"Hair-balls," in stomach, 139

Heinicke-Mikulicz operation, 319

Hemorrhage from ulcer, examination of stomach in presence of, 274

into stomach, control, by suture, 369

perilous, from ulcer, 358

Hepatopexy as preliminary to gastropexy, 247

liver, resection of, for hepatoptosis, 249

technic of, 248

technic of operation, 249

technic of, 267

Hepatoptosis, determining the presence of, 234
Hour-glass stomach, 339

cause of, 342

developing the diagnosis in cases of, 340

development of, 342

in women, 345

occurrence of, 351

pathogenesis of, 346

Röntgen ray in diagnosis of, 350

treatment of, 353

Hydatid tingle as symptom of echinococcus, 445

Ileo-sigmoideostomy, indication for, 267

Infection, atmospheric, 52

catgut, 62

of suture line in gastro-enterostomy, 314

Instruments, sterilization of, 67

Intestines, foreign substances in, 140

Iodoform, as an antiseptic, 27 poisoning, 30

Jaundice, chronic, 464

benign tumor, cause of, 467

development of diagnosis in cases of, 465

Jejunostomy, in cases of stricture of œsophagus, 179

Kammerer's operation, 355

Kelene, 98

Kocher's gastrostomy, 177

Lister and antisepsis, 22

Liver, abscess of, 451

consideration of treatment, 460

development of diagnosis, 452

dysentery as symptom, 459

echinococcus of the, 441

etiology of, 457

examination of patient, 455

prolapse of, operation for, 247-249

rupture into lung, 460

subpleural incision in, 461

use of needle puncture, 456

Local anæsthesia, technic of, 106

Lumbar anæsthesia, 113

Lung, collapse of the, methods for preventing, 172

infection from liver abscess, 460

Melæna as symptom of gastric ulcer, 287

examination of stomach in presence of, 273 
Menstruation, relation to gastropexy and nephropexy, 245

relation of gastroptosis to, 245

Mikulicz's rib separator, 173

Murphy button, in anastomosis of œesophagus and stomach, 174 use of for gastro-enterostomy, 439

Narcosis, morphine-ether, 83

obstetric, 92

"pneumonia," 85

primary ether, 91

statistics of, 76

Nitrate of silver, preparation of, 67

Oberst's method of regional anæsthesia, 104

Occult blood, significance of, 295

Esophagoscope, with Casper's panelectroscope, 132

Esophagoscopy, 133

Esophagus, anastomosis with stomach, 174

caustic stricture of, 143

congenital strictures of the, 162

dilatation of the, 159

dilatation of, palliative treatment, 169

dilatation of the, use of bougies in, 164

diverticulum of, 161

elastic stricture, 151

foreign substance in, 122 management of, 126

methods of diagnosis, 133 operative methods, 136,137 suffocation from, 124

value of Röntgen ray, 129

operative treatment of stricture of, 170

resection of, 173

stricture of the, 141

gradual retrograde dilatation, tendency to retraction, 148 retrograde dilatation for, 147 methods of treatment, 145

treatment of cancer of the, 175

Pain in duodenal ulcer, characteristics of, 307

in gastric ulcer, 305

in gastroptosis, 230

left sided, abdominal, in gastroptosis, 243

Pasteur and antisepsis, 22

Peptic ulcer, 423

case of, 277

development of, 332

occurrence of, 425

production of, 436

prognosis of, 427
Peritoneal cavity, toilet of, following perforating ulcer, 393

Peritonitis from perforating gastric ulcer, 386

prevention of general, 379

Pezzer catheter, in gastrostomy, 150 use of in gastrostomy, 178

\section{Phenosalyl, 58}

Pneumonia, postoperative, 84

Poisoning, cocaine, 101

Prolapse of stomach, operation for (sec gastropexy and gastroptosis)

Pylorectomy, Billroth method of, 317 feeding after, 315

for gastric ulcer, 313

indications for, 320

Pyloroplasty, methods of, 319

Pylorus, cancer of, differential diagnosis of, from gastric ulcer, 305

resection of, deaths following, 248

Resection of stomach, 355

for cancer, 414

for cancer, technic, 415

statistics of, 421

Retention of food in cases of gastric ulcer, 288

Röntgen ray, a diagnostic aid in locating foreign bodies in alimentary tract, 129

in diagnosis of diseases of the stomach, 190

in diagnosis of hour-glass stomach, 350

use in diagnosis of dilatation, 166

Roux's angiotribe, use of, in resection of liver, 249

gastro-jejuno-œsophagostomy, 180

Rovsing's gastrostomy, 177

method of gastropexy, 255

operation, 356

Rubber gloves, use of, 38

Salt solution, infusion of, in peritonitis, 379

Scraping ausculation of the abdomen, 189

Silk, use of as suture, 67

Silver nitrate, qualifications as an antiseptic, 56

use of, to prevent infection following gastro-enteros-

Skin, cleaning, 70 tomy, 314

protection of patients, during operation, 42

Spinal anæsthesia, fatalities from, 117, 118

justification for, 120

technic of, 114 
Stiller's theory of gastroptosis, 207

Stomach, achylia in cancer of, 401-402 cancer of, 395-397

cancer of, anæmia and achylia in, 400

cancer of, developing on ulcer base, 311

cancer of, operative considerations, 411

chronic ulcer of, 269

diagnosis of diseases of the, 183

foreign substances in, gastrotomy for, 138,139

hour-glass, 339

inflammatory tumors simulating cancer, 399

methods of examination in diseases of, 188

perforating ulcers of, 375

resection of for cancer of, 414

statistics, 409

total extirpation of, 418

tube, use of, in gastic ulcer, 273

ulcer, choice of operation for, 276

danger of using stomach tube in case of, 273

deaths following operations for, 277

diagnosis of, 271

gastroscope in, 272

of lesser curvature, 384

of posterior wall, 384

(see gastric ulcer)

statistics of, 277

Stovain, for spinal anæsthesia, 118
Subphrenic abscess, development from perforating ulcer, 384

Suture line, protection of after operation, 43

Test-meals, 193

method of withdrawing, 195

Thornton operation for echinococcus of the liver, 449

Tubercular ulcer of the stomach, 324,327 recognition of, 331

Ulcer, chronic, of stomach, 269

dangerous hemorrhage from, 358

duodenal (see duodenal ulcer)

gastro-intestinal, location of, 363

of stomach, diagnosis of, 287

(see gastric ulcer)

peptic, case of, 277

Vermehren's abdominal belt, 222

Virginal ptosis, method of enlarging abdominal wall in, 266

Volkmann method of operation for echinococcus of the liver, 448

Wanscher's apparatus for anæsthesia, 84

inducing narcosis with, 94

Witzel's gastrostomy, 176

Wölfler's operation for ulcer of stomach, 275

Wound dressings, now used, 34

Bloch's bandage, 31

treatment, rules for, 70

Xanthelasma, multiplex, 469

Xanthoma, 464 relationship of icterus to, 469 







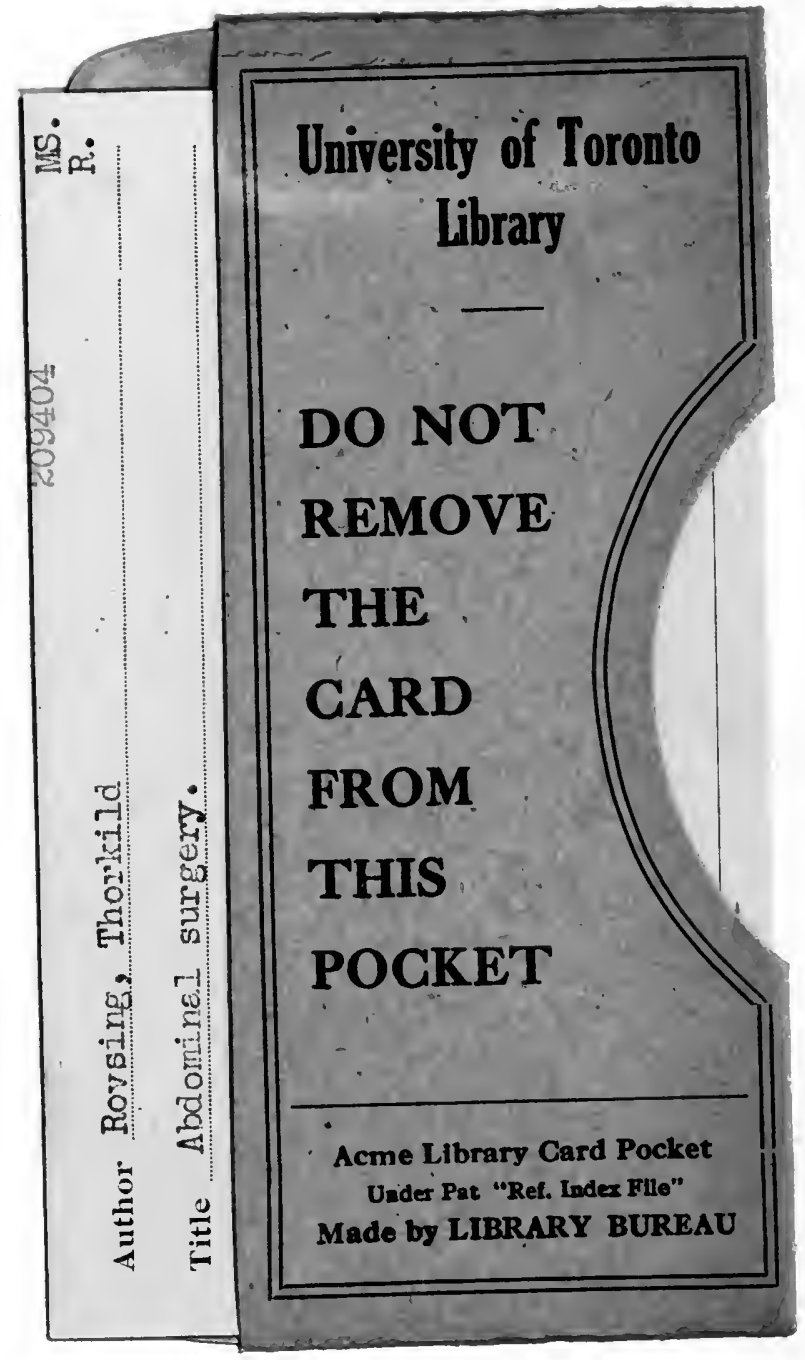


